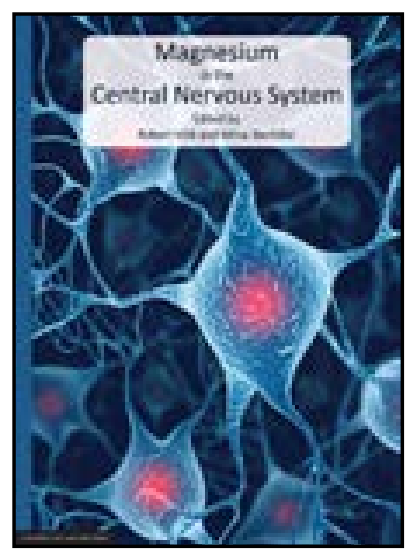

Welcome to the electronic edition of Magnesium in the Central Nervous System.

The book opens with the bookmark panel and you will see the contents page. Click on this anytime to return to the contents. You can also add your own bookmarks.

Each chapter heading in the contents table is clickable and will take you direct to the chapter. Return using the contents link in the bookmarks.

The whole document is fully searchable.

Enjoy.

Click here to help us know more about our readers with this one-minute questionnaire 


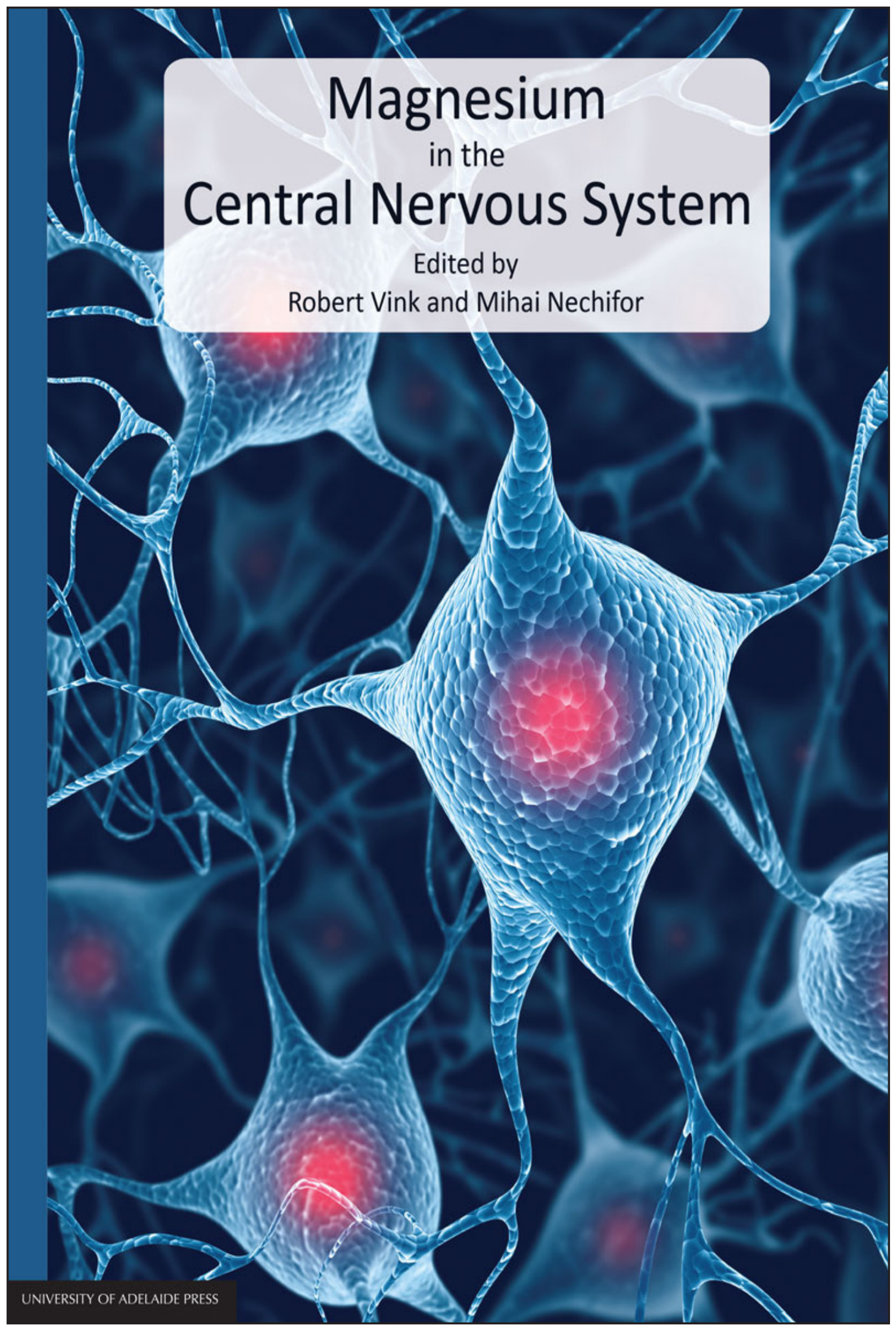




\section{Magnesium in the Central Nervous System}

\section{Edited by Robert Vink and Mihai Nechifor}

Our understanding of the physiology and biochemistry of the brain has improved dramatically in the last two decades. In particular, the critical role of cations, including magnesium, has become evident, even if incompletely understood at a mechanistic level.

The exact role and regulation of magnesium, in particular, remains elusive, largely because intracellular levels are so difficult to routinely quantify. Nonetheless, the importance of magnesium to normal central nervous system activity is self-evident given the complicated homeostatic mechanisms that maintain the concentration of this cation within strict limits essential for normal physiology and metabolism.

There is also considerable accumulating evidence to suggest alterations to some brain functions in both normal and pathological conditions may be linked to alterations in local magnesium concentration.

This book, containing chapters written by some of the foremost experts in the field of magnesium research, brings together the latest in experimental and clinical magnesium research as it relates to the central nervous system. It offers a complete and updated view of magnesium's involvement in central nervous system function and in so doing, brings together two main pillars of contemporary neuroscience research, namely providing an explanation for the molecular mechanisms involved in brain function, and emphasizing the connections between the molecular changes and behavior.

It is the untiring efforts of those magnesium researchers who have dedicated their lives to unraveling the mysteries of magnesium's role in biological systems that has inspired the collation of this volume of work. 


\section{Magnesium in the \\ Central Nervous System}





\title{
Magnesium in the \\ Central Nervous System
}

\author{
EDITED BY
}

\section{ROBERT VINK}

Discipline of Anatomy and Pathology \& Adelaide Centre for Neuroscience Research, School of Medical Sciences, The University of Adelaide, Adelaide, South Australia, Australia

\section{MIHAI NECHIFOR}

Department of Pharmacology, "Gr. T. Popa" University of Medicine and Pharmacy, Iasi, Romania 


\section{Published in Adelaide by}

University of Adelaide Press

Barr Smith Library

The University of Adelaide

South Australia

5005

press@adelaide.edu.au

www.adelaide.edu.au/press

The University of Adelaide Press publishes externally refereed scholarly books by staff of the University of Adelaide. It aims to maximise the accessibility to its best research by publishing works through the internet as free downloads and as high quality printed volumes on demand.

Electronic Index: this book is available from the website as a down-loadable PDF with fully searchable text. Please use the electronic version to serve as the index.

(C) 2011 The Authors

This book is copyright. Apart from any fair dealing for the purposes of private study, research, criticism or review as permitted under the Copyright Act, no part may be reproduced, stored in a retrieval system, or transmitted, in any form or by any means, electronic, mechanical, photocopying, recording or otherwise without the prior written permission. Address all inquiries to the Director at the above address.

For the full Cataloguing-in-Publication data please contact National Library of Australia

Magnesium in the central nervous system / edited by Robert Vink and Mihai Nechifor.

I Vink, Robert.

II Nechifor, Mihai.

1 Magnesium - Physiological effect.

2 Magnesium in the body.

3 Central nervous system.

ISBN (electronic) 978-0-9870730-5-1

ISBN (paperback) 978-0-9870730-6-8

Book design: Robert Vink

Cover design: Emma Spoehr. Photograph with permission, istockphoto.com

Paperback printed by Griffin Press, South Australia 


\section{List of Contributors}

\section{Bulent Ahishali}

Istanbul University, Istanbul Faculty of Medicine, Department of Physiology \& Department of Histology and Embryology, Capa 34093 Istanbul, Turkey

Jean-Pierre Bali

Université Montpellier I, 34000 Montpellier, France

Jean-Marie Billard

Université Paris Descartes, Faculté de Médecine René Descartes, Centre de Psychiatrie et

Neurosciences, UMR 894, Paris, 75014, France

\section{Kym Campbell}

Centre for Neuromuscular and Neurological Disorders, University of Western Australia \& Australian Neuromuscular Research Institute, Department of Neurosurgery, Sir Charles Gairdner Hospital,

Nedlands, Western Australia, Australia

Yves Cazals

UMR6231, Université Paul Cézanne, Marseille, France

\section{Zheng Chen}

Department of Psychiatry \& Institute for Geriatric Clinic and Rehabilitation, Beijing Geriatric Hospital,

\section{Dehua Chui} Beijing 100095, China

Neuroscience Research Institute \& Department of Neurobiology, Key Laboratory for Neuroscience, Ministry of Education \& Ministry of Public Health, Health Science Center, Peking University, Beijing 100191, China

\section{Naomi L. Cook}

Discipline of Anatomy and Pathology, School of Medical Sciences \& Adelaide Centre for Neuroscience Research, The University of Adelaide, Adelaide, South Australia, Australia

\section{Frances Corrigan}

Discipline of Anatomy and Pathology, School of Medical Sciences \& Adelaide Centre for Neuroscience Research, The University of Adelaide, Adelaide, South Australia, Australia

Magdalena D. Cuciureanu

Department of Pharmacology, "Gr. T. Popa" University of Medicine and Pharmacy 16, Iasi, Romania

\section{Sang-Hwan Do}

Department of Anesthesiology and Pain medicine, Seoul National University Bundang Hospital, Seongnam, South Korea

George A. Eby III

George Eby Research Institute, 14909-C Fitzhugh Road, Austin, Texas, USA

\section{Karen L. Eby}

George Eby Research Institute, 14909-C Fitzhugh Road, Austin, Texas, USA

\section{Mounir N. Ghabriel}

Discipline of Anatomy and Pathology, School of Medical Sciences \& Adelaide Centre for Neuroscience Research, The University of Adelaide, Adelaide, South Australia, Australia

Tomoyo Hashimoto

Department of Internal Medicine (Neurology and Rheumatology), Shinshu University School of Medicine, Nagano, Japan

\section{Michael R. Hoane}

Restorative Neuroscience Laboratory, Brain and Cognitive Science Program, Department of Psychology Southern Illinois University, Carbondale, Illinois, USA

\section{Xavier Holy}

Institut de Recherche Biomédicale des Armées, Antenne de Brétigny, Brétigny-sur-Orge, France

\section{Stefano lotti}

Dipartimento di Medicina Interna, dell'Invecchiamento e Malattie Nefrologiche, Università di Bologna, Italy and Istituto Nazionale di Biostrutture e Biosistemi, Roma, Italy 


\section{Mehmet Kaya}

Istanbul University, Istanbul Faculty of Medicine, Department of Physiology \& Department of Histology and Embryology, Capa 34093 Istanbul, Turkey

Neville W. Knuckey

Centre for Neuromuscular and Neurological Disorders, University of Western Australia, \& Australian Neuromuscular Research Institute, Department of Neurosurgery, Sir Charles Gairdner Hospital, Nedlands, Western Australia, Australia

\section{Marzia Leidi}

Università di Milano, Dipartimento di Scienze Cliniche Luigi Sacco, Via G.B. Grassi 74, 20157 Milano, Italy

Yi Liu

Neuroscience Research Institute \& Department of Neurobiology, Key Laboratory for Neuroscience, Ministry of Education \& Ministry of Public Health, Health Science Center, Peking University, Beijing 100191, China

Jeanette A.M. Maier

Università di Milano, Dipartimento di Scienze Cliniche Luigi Sacco, Via G.B. Grassi 74, 20157 Milano, Italy

Emil Malucelli

Dipartimento di Medicina Interna, dell'Invecchiamento e Malattie Nefrologiche' Università di Bologna, Italy

Lucia Mastrototaro

Istituto di Patologia Generale e Centro di Ricerche Oncologiche Giovanni XXIII, Università Cattolica del Sacro Cuore, Rome, Italy

Alexander Mauskop

The New York Headache Center, 30 East 76th Street, New York, NY, USA

Bruno P. Meloni

Centre for Neuromuscular and Neurological Disorders, University of Western Australia, \& Australian Neuromuscular Research Institute, Department of Neurosurgery, Sir Charles Gairdner Hospital, Nedlands, Western Australia, Australia

Marianne Mousain-Bosc

Ecole de l'ADN, Museum d'Histoire Naturelle de Nîmes, 19, Grand'rue, 30000 Nimes, France

Harald Murck

Clinic of Psychiatry and Psychotherapy, University of Marburg, Marburg, Germany

Hyo-Seok $\mathrm{Na}$

Department of Anesthesiology and Pain medicine, Seoul National University Bundang Hospital, Seongnam, South Korea

Mihai Nechifor

Department of Pharmacology, "Gr. T. Popa" University of Medicine and Pharmacy, lasi, Romania

\section{Kiyomitsu Oyanagi}

Department of Brain Disease Research, Shinshu University School of Medicine, Nagano Japan

Victoria Papadopol

Regional Centre of Public Health Iaşi, Romania

\section{Florent Raffin}

Institut de Recherche Biomédicale des Armées, Antenne de la Tronche, La Tronche, France

Andrea M.P. Romani

Department of Physiology and Biophysics, School of Medicine, Case Western Reserve University,

Cleveland, Ohio USA

\section{Harry Rubin}

Department of Molecular and Cell Biology, Life Sciences Addition, University of California, Berkeley, CA, USA

\section{Jung-Hee Ryu}

Department of Anesthesiology and Pain medicine, Seoul National University Bundang Hospital, Seongnam, South Korea 
Jeffrey L. Saver

UCLA Stroke Center, Geffen School of Medicine of the University of California, Los Angeles, CA, USA

Isabelle Sendowski

Institut de Recherche Biomédicale des Armées, Antenne de la Tronche, La Tronche, France

Christian Siatka

Ecole de l'ADN, Museum d'Histoire Naturelle de Nîmes, 19, grand'rue, 30000 Nimes, France

Yuetao Song

Department of Psychiatry \& Institute for Geriatric Clinic and Rehabilitation, Beijing Geriatric Hospital, Beijing 100095, China

\section{Sidney Starkman}

UCLA Stroke Center, Geffen School of Medicine of the University of California, Los Angeles, CA, USA

Valentina Trapani

Istituto di Patologia Generale e Centro di Ricerche Oncologiche Giovanni XXIII, Università Cattolica del Sacro Cuore, Rome, Italy

Walter M. van den Bergh

Department of Intensive Care, Academic Medical Center, 1100 DD Amsterdam, The Netherlands

\section{Corinna van den Heuvel}

Discipline of Anatomy and Pathology, School of Medical Sciences \& Adelaide Centre for Neuroscience Research, The University of Adelaide, Adelaide, South Australia, Australia

\section{Robert Vink}

Discipline of Anatomy and Pathology, School of Medical Sciences \& Adelaide Centre for Neuroscience Research, The University of Adelaide, Adelaide, South Australia, Australia

Weishan Wang

Department of Psychiatry \& Institute for Geriatric Clinic and Rehabilitation, Beijing Geriatric Hospital, Beijing 100095, China

\section{Federica I. Wolf}

Istituto di Patologia Generale e Centro di Ricerche Oncologiche Giovanni XXIII, Università Cattolica del Sacro Cuore, Rome, Italy

Lisa A. Yablon

The New York Headache Center, 30 East 76th Street, New York, NY, USA

\section{Huan Yang}

Neuroscience Research Institute \& Department of Neurobiology, Key Laboratory for Neuroscience, Ministry of Education \& Ministry of Public Health, Health Science Center, Peking University, Beijing 100191, China

Jia Yu

Department of Psychiatry \& Institute for Geriatric Clinic and Rehabilitation, Beijing Geriatric Hospital, Beijing 100095, China

\section{Honglin Zhang}

Department of Psychiatry \& Institute for Geriatric Clinic and Rehabilitation, Beijing Geriatric Hospital, Beijing 100095, China 


\section{Preface}

The brain is the most complex organ in our body. Indeed, it is perhaps the most complex structure we have ever encountered in nature. Both structurally and functionally, there are many peculiarities that differentiate the brain from all other organs. The brain is our connection to the world around us and by governing nervous system and higher function, any disturbance induces severe neurological and psychiatric disorders that can have a devastating effect on quality of life.

Our understanding of the physiology and biochemistry of the brain has improved dramatically in the last two decades. In particular, the critical role of cations, including magnesium, has become evident, even if incompletely understood at a mechanistic level. The exact role and regulation of magnesium, in particular, remains elusive, largely because intracellular levels are so difficult to quantify routinely. Nonetheless, the importance of magnesium to normal central nervous system activity is self-evident given the complicated homeostatic mechanisms that maintain the concentration of this cation within strict limits essential for normal physiology and metabolism. There is also considerable accumulating evidence to suggest that alterations to some brain functions in both normal and pathological conditions may be linked to alterations in local magnesium concentration.

This book, containing chapters written by some of the foremost experts in the field of magnesium research, brings together the latest in experimental and clinical magnesium research as it relates to the central nervous system. It offers a complete and updated view of magnesium's involvement in central nervous system function and in so doing, brings together two main pillars of contemporary neuroscience research, namely providing an explanation for the molecular mechanisms involved in brain function, and emphasizing the connections between the molecular changes and behaviour.

It is the untiring efforts of those magnesium researchers who have dedicated their lives to unravelling the mysteries of magnesium's role in biological systems that has inspired the collation of this volume of work.

Robert Vink

Mihai Nechifor 
Section 1: Magnesium in Normal Brain

Chapter 1 Free magnesium concentration in human brain

Stefano lotti and Emil Malucelli

Chapter 2 Intracellular magnesium homeostasis

Andrea M.P. Romani

Chapter 3 Magnesium transport across the blood-brain barriers

Mounir N. Ghabriel and Robert Vink

Chapter 4 Intracellular free $\mathrm{Mg}^{2+}$ and $\mathrm{MgATP}^{2-}$ in coordinate control of protein synthesis and cell proliferation Harry Rubin

Chapter 5 Magnesium and the Yin-Yang interplay in apoptosis

Chapter 6 Brain magnesium homeostasis as a target for reducing cognitive ageing

Jean-Marie Billard

Section 2: Magnesium in Neurological Diseases

Chapter 7 The role of magnesium therapy in learning and memory Michael R. Hoane

Chapter 8 The role of magnesium in headache and migraine Lisa A. Yablon and Alexander Mauskop

Chapter 9 Magnesium in edema and blood-brain barrier disruption Mehmet Kaya and Bulent Ahishali

Chapter 10 Magnesium and hearing loss Isabelle Sendowski, Xavier Holy, Florent Raffin and Yves Cazals

Chapter 11 The role of magnesium in pain Hyo-Seok $\mathrm{Na}$, Jung-Hee Ryu and Sang-Hwan Do

Chapter 12 The role of magnesium in traumatic CNS injury Naomi L. Cook, Frances Corrigan and Corinna van den Heuvel

Chapter 13 The use of magnesium in experimental cerebral ischaemia Bruno P. Meloni, Kym Campbell and Neville W. Knuckey

Chapter 14 Magnesium in subarachnoid hemorrhage Walter M. van den Bergh

Chapter 15 Magnesium in clinical stroke Jeffrey L. Saver and Sidney Starkman

Chapter 16 Magnesium in cancer: more questions than answers

Chapter 17 Magnesium in Parkinson's disease: an update in clinical and basic aspects Kiyomitsu Oyanagi and Tomoyo Hashimoto 


\section{Section 3: Involvement of Magnesium in Psychiatric Diseases}

Chapter 18 Magnesium and Alzheimer's disease

Dehua Chui, Zheng Chen, Jia Yu, Honglin Zhang, Weishan Wang Yuetao Song, Huan Yang and Yi Liu

Chapter 19 Magnesium and stress

Magdalena D. Cuciureanu and Robert Vink

Chapter 20 Magnesium in neuroses

Victoria Papadopol and Mihai Nechifor

Chapter 21 Magnesium, hyperactivity and autism in children Marianne Mousain-Bosc, Christian Siatka and Jean-Pierre Bali

Chapter 22 Magnesium in psychoses (schizophrenia, bipolar disorder)

Chapter 23 Magnesium and major depression

George A. Eby III, Karen L. Eby and Harald Murck

Chapter 24 Magnesium in drug abuse and addiction

Mihai Nechifor

Electronic Index: this book is available from the website adelaide.edu.au/press as a free downloadable PDF with fully searchable text. Please use the electronic version to serve as the index. 


\section{Section 1}

Magnesium in Normal Brain 



\title{
Free magnesium concentration in the human brain
}

\author{
Stefano lotti ${ }^{1,2, \square}$ and Emil Malucelli ${ }^{1}$ \\ ${ }^{1}$ Dipartimento di Medicina Interna, dell'Invecchiamento e Malattie Nefrologiche, Università di Bologna, Italy. \\ ${ }^{2}$ Istituto Nazionale di Biostrutture e Biosistemi, Roma, Italy. \\ $\triangle$ stefano.iotti@unibo.it
}

\section{Abstract}

The cytosolic free magnesium concentration can be assessed in vivo in the human brain by phosphorus magnetic resonance spectroscopy. This technique has been employed in the human brain, providing new hints on the $\mathrm{Mg}^{2+}$ homeostasis and on its involvement on the cellular bioenergetics. The free cytosolic $\left[\mathrm{Mg}^{2+}\right]$ measured in the human brain is about half of that assessed in the human skeletal muscle. This result is likely related to the lower ATP concentration of brain tissue. The possibility to assess the cytosolic $\left[\mathrm{Mg}^{2+}\right]$ in the human brain opened the chance to study the involvement of $\mathrm{Mg}^{2+}$ in different neurological pathologies, and particularly in those where the defective mitochondrial energy production represents the primary causative factor in pathogenesis. The results obtained, studying patients affected by different types of mitochondrial cytopathies, helped to clarify the functional relationship between the energy metabolism and free $\left[\mathrm{Mg}^{2+}\right]$, providing evidence that the cytosolic $\left[\mathrm{Mg}^{2+}\right]$ is a function of the energy charge of brain cells and a defective mitochondrial respiration causes a derangement of cytosolic $\left[\mathrm{Mg}^{2+}\right]$ homeostasis. A reduced cytosolic $\left[\mathrm{Mg}^{2+}\right]$ has been also found in the occipital lobes of patients with different types of migraine and cluster headache, showing among migraine patients a trend in keeping with the severity of clinical phenotype. In addition, the assessment by phosphorus magnetic resonance spectroscopy of brain $\left[\mathrm{Mg}^{2+}\right]$ can help in the differential diagnosis of Multiple System Atrophy and Parkinson's disease, offering a new diagnostic tool that may help to differentiate neurodegenerative diseases sharing common clinical features.

\section{Introduction}

Inorganic phosphate $(\mathrm{Pi})$, phosphocreatine $(\mathrm{PCr})$, and ATP are the principal $\mathrm{Mg}^{+2}$ ligands among the phosphorylated molecules present in the cell cytosol detectable by phosphorus magnetic resonance spectroscopy ( $\left.{ }^{31} \mathrm{P}-\mathrm{MRS}\right)$ (Figure 1). Nevertheless, in the cytosol matrix, magnesium is primarily bound to ATP. As a consequence, in the living organism, ATP is present mainly in the form MgATP $^{2-}$ (lotti et al., 1996) (Figure 2) that is the active species in enzyme binding (Kuby and Noltman, 1959), as well as the energy producing form in active transport (Skou, 1982; Repke, 1982) and muscular contraction (Ramirez and Marecek 1980; Wells et al., 1980).

The amount of magnesium bound to ATP shifts the resonance frequencies measured by ${ }^{31} \mathrm{P}-\mathrm{MRS}$ of signals coming from the three phosphoric groups $\alpha, \beta, \gamma$ of the molecule (chemical shift). Due to the chemical equilibrium between the $\mathrm{Mg}$ bound to ATP and free $\mathrm{Mg}^{2+}$, the chemical shift of ATP signals is function of free $\mathrm{Mg}^{2+}$ concentration
(Figure 3). The in vivo assessment of cytosolic $\mathrm{Mg}^{2+}$ concentration $\left(\left[\mathrm{Mg}^{2+}\right]\right)$ in the human brain needs a specific calibration curve that should satisfy precise criteria. In particular, the calibration curve should take into account: i) other ions present in the cell cytosol competing with $\mathrm{Mg}^{2+}$ in binding ATP such as $\mathrm{H}^{+}, \mathrm{Na}^{+}, \mathrm{K}^{+}$, and ii) other ligands such as $\mathrm{ADP}, \mathrm{PCr}$ and $\mathrm{Pi}$ competing with ATP in binding $\mathrm{Mg}^{2+}$ (lotti et al., 1996).

Operatively, $\left[\mathrm{Mg}^{2+}\right]$ is assessed by ${ }^{31} \mathrm{P}-\mathrm{MRS}$ from the chemical shift of $\beta$-ATP being measured from the signal of either $\alpha$-ATP (Gupta et al., 1984; Williams and Smith, 1995) or PCr (Taylor et al., 1991; Halvorson et al., 1992). The reliability of the in vivo measurements depends on the availability of an appropriate in vitro calibration method to determine the limits of chemical shifts of unbound ATP and Mg-ATP complexes. As a result, the calibration method has to take into account the two issues mentioned above together with other variables such as the ionic strength, temperature and $\mathrm{pH}$ of the medium. Besides, to have a precise measurement of $\beta$-ATP 


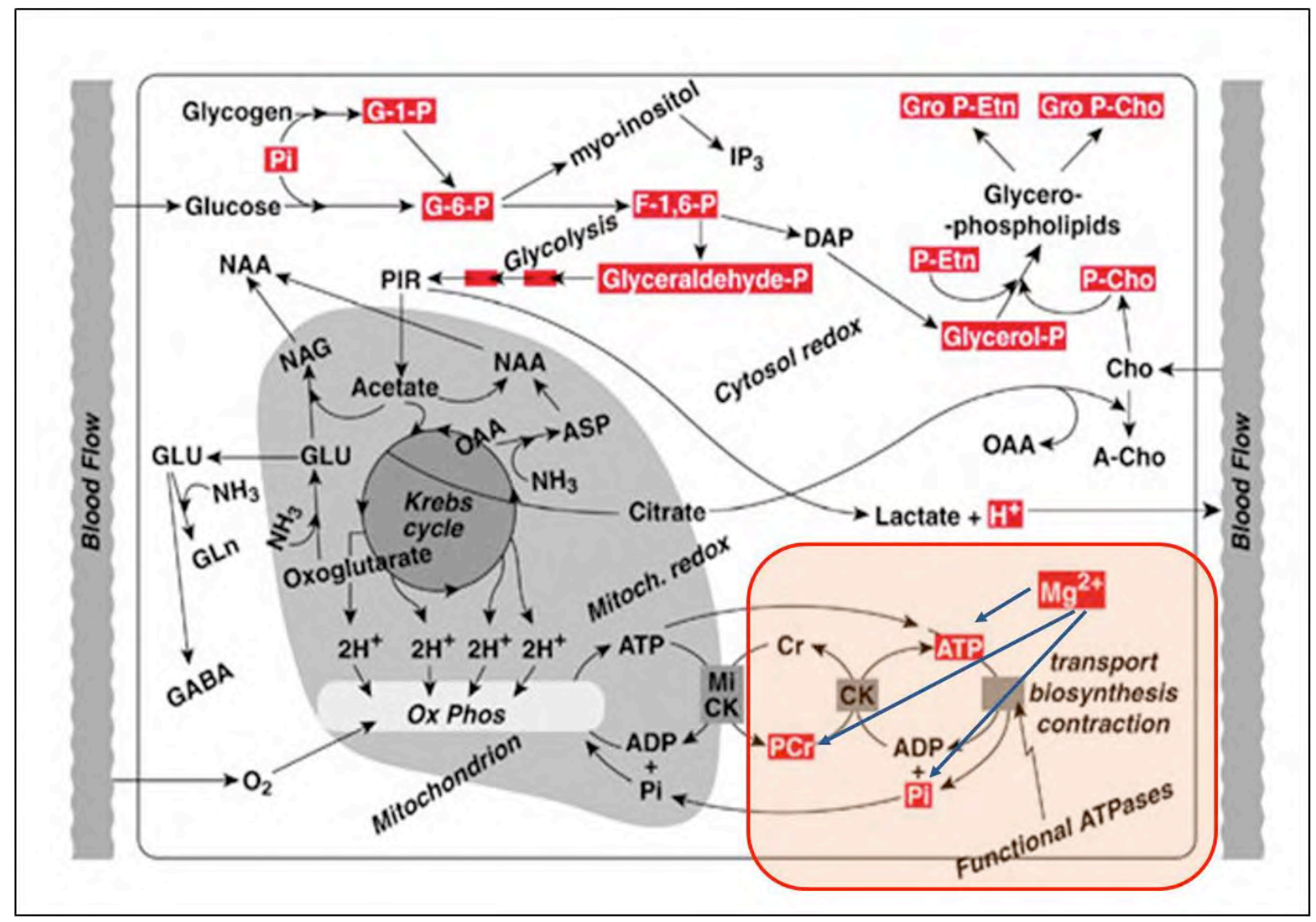

Figure 1. Scheme of the main phosphorylated molecules present in the brain cell cytosol detectable by phosphorus magnetic resonance spectroscopy. Inorganic phosphate (Pi), phosphocreatine (PCr), and ATP are the principal magnesium ligands.

chemical shift it must be taken into account that the shifts of both $\alpha$-ATP and PCr change as a function of $\left[\mathrm{Mg}^{2+}\right]$ (lotti et al., 1996). The earlier works of Gupta (Gupta et al., 1978; Gupta et al., 1983; Gupta et al., 1984) were based on the assumption of the presence of only one ATP-Mg complex. In the 1990s several studies were published proposing more accurate calibration methods taking into account equilibria involving few different chemical species (Mosher et al., 1992; Halvorson et al., 1992; Golding and Golding, 1995). Nevertheless, the influence of the ionic strength (I) of the medium on the equilibrium constants of the species present in the calibration solutions must be taken into account as discussed in one report published in those years (Mottet et al., 1994).

In the next section the methodological approach used to build the calibration curve for the $\left[\mathrm{Mg}^{2+}\right]$ determination in human brain by ${ }^{31} \mathrm{P}-\mathrm{MRS}$ is analytically presented.

\section{Building a calibration curve for the $\left[\mathrm{Mg}^{2+}\right]$ determination in human brain by ${ }^{31} \mathrm{P}$ MRS}

The accuracy and reliability of in vivo assessment of $\left[\mathrm{Mg}^{2+}\right]$ mainly depend on the availability of calibration curves based on precise in vitro NMR measurements performed on appropriate solutions that mimic, as far as possible, the in vivo conditions of the tissue to be studied. As a consequence, these solutions will contain molecules involved in multiple equilibria in which several ligands (Lewis base) interact with metal ions (Lewis acid), and among them $\mathrm{Mg}^{2+}$ and $\mathrm{H}^{+}$. Typically, to build a calibration curve, a titration of the species of interest must be performed, linking its concentration to the experimental NMR measurement. $\left[\mathrm{Mg}^{2+}\right]$ can be assessed by ${ }^{31} \mathrm{P}$ MRS from the chemical shift of $\beta$-ATP signal, which in turn depends on the fraction of total ATP bound to $\mathrm{Mg}^{2+}$. Quite problematic is the use of a $\mathrm{Mg}$ electrode to measure $\left[\mathrm{Mg}^{2+}\right]$ in a complex 


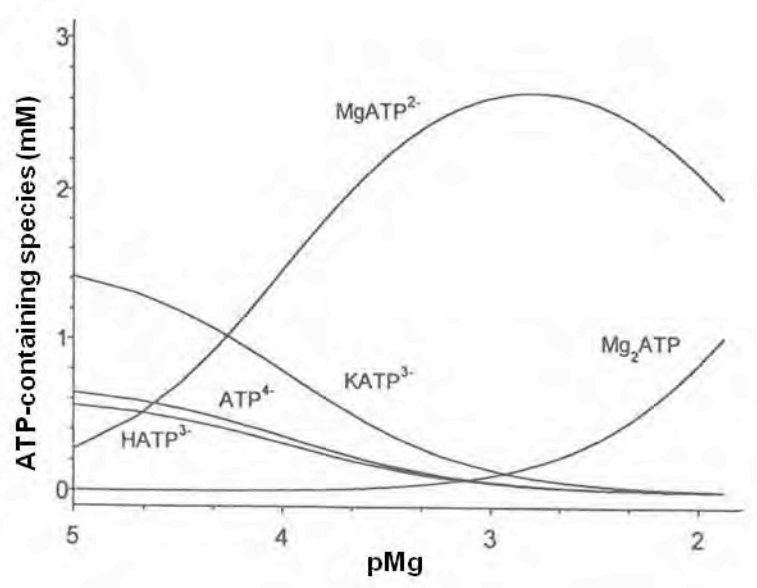

Figure 2. Concentration of the major ATPcontaining species present in solutions mimicking the cell brain cytosol plotted versus $\mathrm{pMg}$. Solutions composition were: ATP $=3 \mathrm{mM}$, phosphocreatine $=4.5 \mathrm{mM}, \mathrm{K}_{2} \mathrm{HPO}_{4}=1.0 \mathrm{mM}$, and variable amounts of $\mathrm{MgCl}_{2}$ at $\mathrm{pH}=7.00,37^{\circ} \mathrm{C}$ and ionic strength $0.25 \mathrm{M}$. Adapted from lotti et al., 1996.

mixture, having to deal with interferences and lack of accuracy.

A convenient approach to measure the $\left[\mathrm{Mg}^{2+}\right]$ in a multi-equilibrium system is using appropriate algorithms and software, which are currently available, allowing a quantitative chemical speciation of any component present in solution, given a set of stability and acidic dissociation constants. In this approach, a pivotal step is the definition of the chemical model better describing the multi-equilibrium system present in solution. After the characterization of ligands and Lewis acids as the basic species, the next step is the delineation of all the possible equilibria between them.

In the calibration curve specifically developed for the in vivo assessment of $\left[\mathrm{Mg}^{2+}\right]$ in human brain (lotti et al., 1996) the chemical model was defined by choosing four ligands $-\mathrm{ATP}^{4-}, \mathrm{PO}_{4}{ }^{3-}, \mathrm{Cl}^{-}$ and $\mathrm{PCr}^{2-}$ and four Lewis acids $-\mathrm{Na}^{+}, \mathrm{K}^{+}, \mathrm{Mg}^{2+}$ and $\mathrm{H}^{+}$as basic species, giving raise to the multiequilibrium system shown in Figure 4.

An obligatory prerequisite for the analysis of spectra recorded in vitro is a reliable and homogeneous set of equilibrium constants to be used in the quantitative speciation of the experimental solutions. Therefore, if the equilibrium
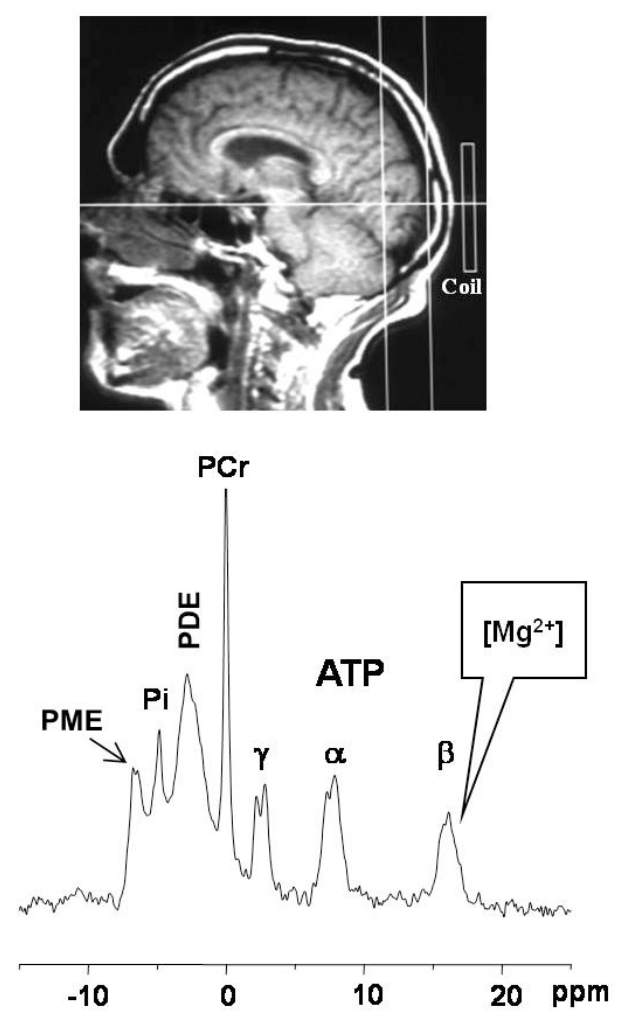

Figure 3. Top: Sagittal slice image of human brain acquired by magnetic resonance imaging. The brain volume within vertical axes defines the region of occipital lobes. Bottom: ${ }^{31} \mathrm{P}$-MRS spectra acquired from occipital lobes placing the surface coil directly on the skull. Abbreviations: Pi, inorganic phosphate; $\mathrm{PCr}$, phosphocreatine; $\alpha, \beta, \gamma$, ATP phosphoric groups; PME, phosphomonoesters; PDE, phosphodiesters. Brain cytosolic $\left[\mathrm{Mg}^{2+}\right]$ is assessed from the chemical shift of $\beta$ ATP.

constants reported in the literature do not conform to the conditions of temperature and ionic strength used in the experiments, suitable corrections, using Van't Hoff and/or Davies equations, have to be applied to account for the different conditions of NMR experiments (lotti et al., 1996).

Furthermore, the literature is rich with data pertaining to interactions between metal ions and ligands in solution, but the reported values are often characterized by extremely variable levels of precision and accuracy. Williams and colleagues (Duffield et al., 1991) discussed the problems related to the determination of the magnitude of quantities of biological/environ- 


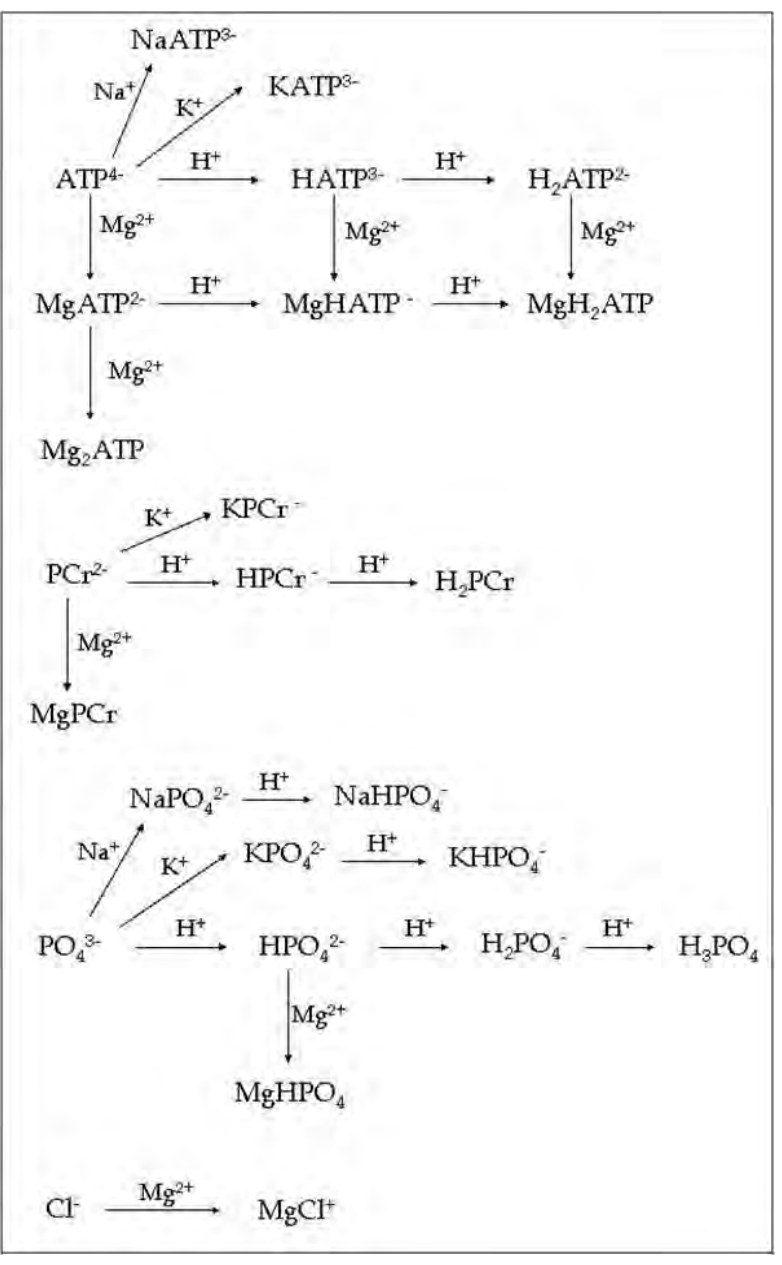

Figure 4. Scheme of the chemical model used to build the calibration curve for the assessment of brain cytosolic $\left[\mathrm{Mg}^{2+}\right]$ by ${ }^{31} \mathrm{P}-\mathrm{MRS}$. Four ligands $\left(\mathrm{ATP}^{4-}, \mathrm{PO}_{4}{ }^{3-}, \mathrm{Cl}^{-}\right.$, and $\left.\mathrm{PCr}^{2-}\right)$ and four Lewis acids $\left(\mathrm{Na}^{+}, \mathrm{K}^{+}, \mathrm{Mg}^{2+}\right.$, and $\left.\mathrm{H}^{+}\right)$were chosen as basic species.

mental interest and to its associated error. When these quantities are used for the chemical speciation of natural systems, the results bear an uncertainty directly related to the errors which affect the original quantities. Hence, a careful examination of the published values of equilibrium constants for the reactions involved in the chemical model chosen, must be performed.

Another consideration to bear in mind is when using protonation constants that have been determined with the so-called "incomplete convention" (Halvorson et al., 1992), in which hydrogen ion activity is used instead of hydrogen ion concentration in the expression used to define the equilibrium constants. This can generate confusion when such constants are used together with others that are expressed solely in terms of concentration. Therefore, a coherent and internally consistent set of equilibrium constants has to be collected, which are valid at $37^{\circ} \mathrm{C}$ and at $\mathrm{I}=0.25 \mathrm{M}$, the conditions of solutions that mimic the in vivo environment.

To handle the relevant number of different equilibria taken into consideration, an algorithm was developed that allowed a quantitative chemical speciation of the $\mathrm{Mg}^{2+}$-binding molecules with the goal of building a semiempirical equation that correlates the chemical shift of the $\beta$-ATP signal measured by in vitro NMR measurements in the calibration solutions to $\left[\mathrm{Mg}^{2+}\right]$ calculated, taking into account the amount of $\mathrm{Mg}^{2+}$ bound to all constituents in solution. As a result, the concentrations of all species present under a given set of conditions (i.e. total concentrations of all reagents) were determined by the program HYSS (Alderighi et al., 1999), a specific software program, utilized for chemical speciation. Using this software it was possible to derive the concentration of free magnesium in any condition. HYSS requires two sets of data: a chemical model and the experimental conditions. The model was the one reported in Figure 4, by specifying values for the equilibrium constants $\beta_{a b c \ldots . .}$ and the corresponding stoichiometric coefficients $a, b, c, \ldots .$. considering that the general reaction between reagents $A, B, C \ldots$ to give a chemical species of formula $\mathrm{A} a \mathrm{~B} b \mathrm{C} C \mathrm{c}$...

$$
a A+b B+c C+\ldots \leftrightarrows A a B b C c
$$

is associated with an equilibrium constant $\beta_{a b c . . .}$ :

$$
\beta_{a b c}=\frac{\left[\mathrm{A} a \mathrm{~B} b \mathrm{C}_{c} \ldots . .\right]}{[\mathrm{A}]^{a}[\mathrm{~B}]^{b}[\mathrm{C}]^{c}}
$$

Electric charges have been omitted for the sake of simplicity. By using this approach and different calibration solutions prepared to mimic the in vivo metabolic conditions of brain, it has been possible to obtain, following an heuristic procedure, the following equation:

$$
p M g=x_{1}-\log _{10}\left[\left(d-x_{2}\right)^{x_{3}} /\left(x_{4}-d\right)^{x_{5}}\right]
$$

where $x_{1}, x_{2}, x_{3}, x_{4}, x_{5}$ are the calculated parameters, $\delta$ is the measured chemical shift, and $\mathrm{pMg}=-\log _{10}\left[\mathrm{Mg}^{2+}\right]$. This equation can be seen as 
a more general expression of the typical titration curve in which $x_{1}$ is the $p K$ of the equilibrium involved and with $x_{3}, x_{5}=1$. More precisely, two different equations were obtained, where $\mathrm{pMg}$ is expressed as a function of the chemical shifts of $\beta$-ATP from $\operatorname{PCr}\left(\delta_{\beta}\right)$ or from $\alpha$-ATP $\left(\delta_{\alpha \beta}\right)$ (lotti et al., 1996). However, the calibration curve obtained using $\delta \beta$ as experimental variable showed to be more accurate than that obtained using $\delta \alpha \beta$ (lotti et al., 1996). This is because the measurements of $\delta \alpha \beta$ in spectra obtained in vivo are usually less accurate than those of $\delta_{\beta}$, due to the unresolved resonances of $\alpha-A D P, N A D, N A D H$ underlying upfield the $\alpha$-ATP peak. Furthermore, $\mathrm{PCr}$ resonates alone and is symmetric, allowing a more accurate identification of the centroid of the peak. Therefore, $\delta \beta$ should be preferable to $\delta \alpha \beta$ as experimental variable for the in vivo measurements of brain cytosolic free $\left[\mathrm{Mg}^{2+}\right]$. According to this, the equation to be used is:

$$
\mathrm{pMg}=4.24-\log _{10}\left[\left(\delta_{\beta}-18.58\right)^{0.42} /\left(15.74-\delta_{\beta}\right)^{0.84}\right]
$$

The specific software package MAGIC-BC based on the equation reported above has been made available at http://www.cermiv.unibo.it. It is worth underlying that, in principle, by this approach it is possible to assess the concentration of any species independently of the degree of complexity of the system under investigation, with the restriction of only two requisites: i) a precise definition of the chemical model and ii) the knowledge of the equilibrium constants of the chemical reactions involved.

\section{Cytosolic $\left[\mathrm{Mg}^{2+}\right]$ in human brain}

The cytosolic $\left[\mathrm{Mg}^{2+}\right]$ assessed by ${ }^{31} \mathrm{P}-\mathrm{MRS}$ in the occipital lobes of human brain of 36 control subjects (22 men and 14 women) of mean age $36.4 \pm 17.1$ (SD) (16 - 67 years) was $0.182 \mathrm{mM}$ (lotti et al., 1996). Brain studies were performed on occipital lobes by placing the surface coil directly on the skull in the occipital region and precisely positioned by imaging the brain as shown in Figure 3. The free cytosolic $\left[\mathrm{Mg}^{2+}\right]$ measured in the human brain is about half of that assessed in the human calf muscle (lotti et al., 2000; lotti and Malucelli, 2008). This result is likely related to the lower ATP concentration of brain tissue ( $3 \mathrm{mM})$ compared to that of skeletal muscle ( $8 \mathrm{mM})$, ATP being the major binding site present in the cellular milieu. Nevertheless, the value found of $0.182 \mathrm{mM}$ is lower than that obtained by others (Taylor et al., 1991; Halvorson et al., 1992). This is not a surprising result as the model considered in building this calibration curve contains more species that bind $\mathrm{Mg}^{2+}$ than in all other approaches published. As a consequence, a lower value of cytosolic free $\left[\mathrm{Mg}^{2+}\right]$ has to be expected. If the model described previously, upon which the cytosolic $\left[\mathrm{Mg}^{2+}\right]$ assessment is based, had taken into account more species binding $\mathrm{Mg}^{2+}$, the resulting value of brain $\left[\mathrm{Mg}^{2+}\right]$ would be even lower. Therefore, we believe that the present brain $\left[\mathrm{Mg}^{2+}\right]$ value is a good estimate of the cytosolic free ion concentration, which however could still be slightly overestimated.

In the cohort of subjects studied, there was no significant difference in cytosolic $\left[\mathrm{Mg}^{2+}\right]$ as a function of age and sex. This shows that the sum of anions binding $\mathrm{Mg}^{2+}$ in vivo does not change in the brain cortex during the life span studied and/or that regulatory mechanisms exist to maintain the cytosolic free $\left[\mathrm{Mg}^{2+}\right]$ constant. The possibility to assess the free cytosolic $\left[\mathrm{Mg}^{2+}\right]$ in the human brain opened the chance to study the involvement of $\mathrm{Mg}$ in different neurological pathologies. Particularly interesting are mitochondrial cytopathies and migraines in which the defective mitochondrial energy production is respectively the primary causative or putative pathogenetic factor.

\section{Brain $\left[\mathrm{Mg}^{2+}\right]$ in mitochondrial cytopathies}

${ }^{31} \mathrm{P}-\mathrm{MRS}$ was used to assess the free $\left[\mathrm{Mg}^{2+}\right]$ in the occipital lobes of patients affected by different types of mitochondrial cytopathies due to known enzyme and/or mitochondrial DNA defects to clarify the functional relationship between the energy metabolism and the concentration of cytosolic free magnesium (Barbiroli et al., 1999a). In particular, 19 subjects ( 9 women and 10 men) of mean age $43 \pm 19$ (SD) (13-75 years) with mitochondrial cytopathies were studied (see Table 1). Treatment with oral CoQ10 (150 $\mathrm{mg} /$ day) was given for six months to 9 patients. All patients displayed significantly low cytosolic $\left[\mathrm{Mg}^{2+}\right]$, well below the $95 \%$ confidence interval of control values (Figure 5). Since the mtDNA mutations and enzyme defects found in these patients (Table 1 ) are known to be primarily responsible for brain defective mitochondrial respiration, this outcome strongly suggests that 
Table 1. Patients with mitochondrial cytopathies due to known enzyme/mtDNA defect/s. cOX = cytochrome oxidase. Patients 1-4, 10, 12-14 and 16 were treated with CoQ10 (150 mg/day for six months). Adapted from Barbiroli et al., 1999a.

\begin{tabular}{|c|c|c|c|}
\hline $\begin{array}{l}\text { Patients } \\
\text { numbers }\end{array}$ & Sex/age (years) & Diagnosis & $\begin{array}{c}\text { Enzyme defect or mtDNA bp } \\
\text { mutation/deletion }\end{array}$ \\
\hline $1^{*}$ & $F / 61$ & CPEO & COX \\
\hline $2^{*}$ & $M / 75$ & CPEO & $\operatorname{cox}$ \\
\hline $3^{*}$ & $F / 69$ & CPEO & mtDNA deletion \\
\hline $4^{*}$ & $M / 66$ & CPEO & $\operatorname{cox}$ \\
\hline 5 & $F / 21$ & LHON & 3460 \\
\hline 6 & $M / 26$ & LHON & 11778 \\
\hline 7 & $M / 33$ & LHON & 11778 \\
\hline 8 & $F / 61$ & LHON & 11778 \\
\hline 9 & $\mathrm{~F} / 54$ & LHON & 11778 \\
\hline $10^{*}$ & $M / 33$ & LHON & 3460 \\
\hline 11 & $M / 51$ & LHON & $11778+4216+13708$ \\
\hline $12^{*}$ & $M / 56$ & LHON & 11778 \\
\hline $13^{*}$ & $M / 36$ & LHON & 11778 \\
\hline $14^{*}$ & $M / 25$ & LHON & 11778 \\
\hline 15 & $\mathrm{~F} / 41$ & NARP & 8993 \\
\hline $16^{*}$ & $F / 13$ & NARP & 8993 \\
\hline 17 & $\mathrm{~F} / 16$ & MERFF & 8344 \\
\hline 18 & $M / 49$ & MELAS & 3243 \\
\hline 19 & $F / 38$ & MEM & mtDNA deletion \\
\hline
\end{tabular}

Abbreviations: * treated with CoQ10; CPEO, chronic progressive external ophthalmoplegia; LHON, Leber's hereditary optic neuropathy; NARP, neuropathy ataxia and retinitis pigmentosa; MEM, mitochondrial encephalomyopathy; MERF, myoclonic epilepsy with ragged red fibres; MELAS, mitochondrial myopathy encephalopathy, lactic acidosis, and stroke.

the low cytosolic $\left[\mathrm{Mg}^{2+}\right]$ found in these patients was secondary to failure of the respiratory chain.

Nine of the 19 patients investigated were treated with $\mathrm{CoQ}$, which improved the efficiency of the respiratory chain (Barbiroli et al., 1999a). Administration of $\mathrm{CoQ}$ also increased cytosolic $\left[\mathrm{Mg}^{2+}\right]$ in all treated patients (Figure 5). The increased efficiency of oxidative phosphorylation following treatment with CoQ accompanied by an increased cytosolic free $\left[\mathrm{Mg}^{2+}\right]$ gives further support to the hypothesis that changes in brain cytosolic $\left[\mathrm{Mg}^{2+}\right]$ were secondary to the improved efficiency of mitochondrial energy production.
According to this view, the cytosolic $\left[\mathrm{Mg}^{2+}\right]$ is a function of the energy charge of brain cells and a defective mitochondrial respiration causes a derangement of cytosolic $\left[\mathrm{Mg}^{2+}\right]$ homeostasis. This interpretation is consistent with the observations of several reports that indicate mitochondria as the primary pool for the increase and decrease in cellular magnesium content, suggesting that mitochondria might act as magnesium stores (Kubota et al., 2005; Farruggia et al., 2006) and play a key role in regulating magnesium homeostasis (Fatholahi et al., 2000; Murphy, 2000). 


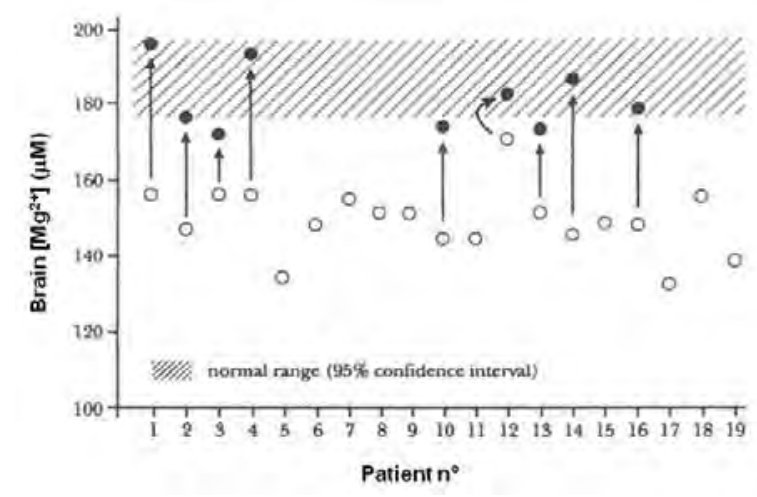

Figure 5. Brain cytosolic $\left[\mathrm{Mg}^{2+}\right]$ assessed in the occipital lobes of patients with mitochondrial cytopathies (open symbols). The values from nine of these patients after treatment with CoQ are shown by closed symbols. Dashed areas represent the $95 \%$ confidence intervals of 36 healthy control subjects. Adapted from Barbiroli et al., 1999a.

\section{Brain $\left[\mathrm{Mg}^{2+}\right]$ in different types of migraine and cluster headache}

Low cytosolic free magnesium has been found in the brains of patients with migraine with and without aura during attacks (Ramadan et al., 1989) and interictally in young patients with migraine with aura (Lodi et al., 1997). It has been suggested that low free magnesium may contribute to the bioenergetic deficit in headache patients as magnesium is essential for mitochondrial membrane stability and coupling of oxidative phosphorylation (Welch and Ramadan, 1995). Migraine headache is a common feature in patients with mitochondrial encephalomyopathies where deficient brain mitochondrial oxidation is due to mutations of mitochondrial DNA. In fact, several studies contributed to disclose an altered energy metabolism in the brain of patients with different types of migraine and cluster headache (Barbiroli et al., 1992; Montagna et al., 1994; Montagna et al., 1997), although the molecular mechanisms leading to oxidative deficit in migraine and cluster headache are unknown. On the other hand, as shown in the previous section, the reduction in cytosolic $\left[\mathrm{Mg}^{2+}\right]$ seems to be secondary to the bioenergetics deficit in the brains of patients with different mitochondrial encephalomyopathies. These outcomes provided the rationale to perform an extensive study to assess the brain $\mathrm{Mg}^{2+}$ by ${ }^{31} \mathrm{P}-\mathrm{MRS}$ in different form of migraines and in cluster headache (Lodi et al., 2001). The study was performed in 78 patients with different forms of migraine in attack-free periods ( 7 with migraine stroke, 13 with migraine with prolonged aura, 37 with migraine with typical aura or basilar migraine, 21 with migraine without aura), and 13 patients with cluster headache. In the occipital lobes of all subgroups of migraine and in cluster headache patients cytosolic $\left[\mathrm{Mg}^{2+}\right]$ was significantly reduced. Among migraine patients the level of cytosolic free $\left[\mathrm{Mg}^{2+}\right]$ correlated with the severity of clinical phenotype, showing the lowest values in patients with migraine stroke and the highest in patients with migraine without aura, as shown in Figure 6 (Lodi et al., 2001). Again, the results of this study sustain the hypothesis that the reduction of cytosolic $\left[\mathrm{Mg}^{2+}\right]$ in tissues with defective mitochondrial functionality is secondary to the bioenergetics deficit, hence the reduction of brain cytosolic free $\left[\mathrm{Mg}^{2+}\right]$ is unlikely to be a direct consequence of hypomagnesemia.

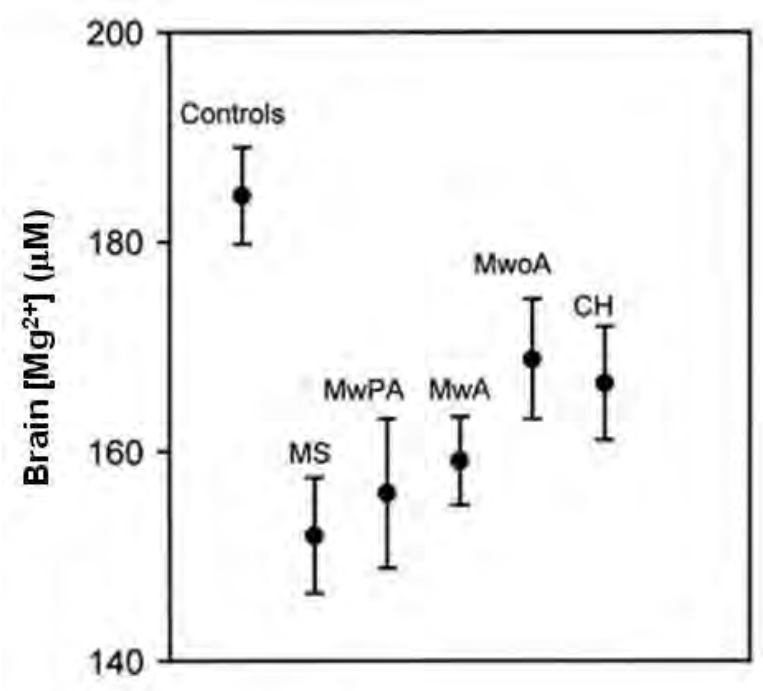

Figure 6. Concentration of cytosolic $\left[\mathrm{Mg}^{2+}\right]$ in the occipital lobes of patients with migraine and cluster headache compared with healthy control subjects. Abbreviations: MS, migraine stroke; MwPA, migraine with prolonged aura; MwA, migraine with typical aura or basilar migraine; MwoA, migraine without aura; $\mathrm{CH}$, cluster headache. All values are reported as mean $\pm \mathrm{SE}$. Adapted from Lodi et al., 2001. 


\section{Brain $\left[\mathrm{Mg}^{2+}\right]$ in the differential diagnosis of multiple system atrophy and idiopathic Parkinson's disease}

An in vivo study by ${ }^{31} \mathrm{P}-\mathrm{MRS}$ showed that the combined measurement of $[\mathrm{PCr}]$ and free $\left[\mathrm{Mg}^{2+}\right]$ could help to differentiate patients with Multiple System Atrophy (MSA) from those with Parkinson's disease (PD) (Barbiroli et al., 1999b). MSA is a group of multisystem degenerative diseases that have several clinical features of PD. Furthermore, not all the signs in MSA become evident at the same time, rendering difficult the differentiation from PD. Therefore, differentiating MSA from PD can be complicated and the diagnosis of MSA represents a clinical challenge. The study was carried out on the occipital lobes of 15 patients with MSA and 13 patients with idiopathic PD.

In the occipital lobes of MSA patients we found a ${ }^{31} \mathrm{P}$-MRS spectroscopy pattern suggestive of defective brain mitochondrial respiration with low $[\mathrm{PCr}]$ and high [Pi]. However, the brain cytosolic $\left[\mathrm{Mg}^{2+}\right]$ of MSA patients did not differ significantly from control values, displaying a peculiar pattern compared to mitochondrial cytopathies. On the other hand, PD patients did not exhibit in their occipital lobes the metabolic pattern typical of mitochondrial malfunction, showing an increased [Pi], decreased cytosolic $\left[\mathrm{Mg}^{2+}\right]$ and unchanged $[\mathrm{PCr}]$ and $\mathrm{pH}$. Although there is a highly significant difference in both [PCr] and $\left[\mathrm{Mg}^{2+}\right]$ between MSA and PD patients when compared as groups, there is a small overlap of $[\mathrm{PCr}]$ and $\left[\mathrm{Mg}^{2+}\right]$ values between the two groups when considering single individuals (Barbiroli et al., 1999b). However, taking into consideration both variables at the same time, the individual patients with MSA can be separated from the individual patients with PD

\section{References}

Alderighi L, Gans P, lenco A, Peters D, Sabatini A, Vacca A (1999) Hyperquad simulation and speciation (HySS): a utility program for the investigation of equilibria involving soluble and partially soluble species. Coord Chem Rev 184:311-8.

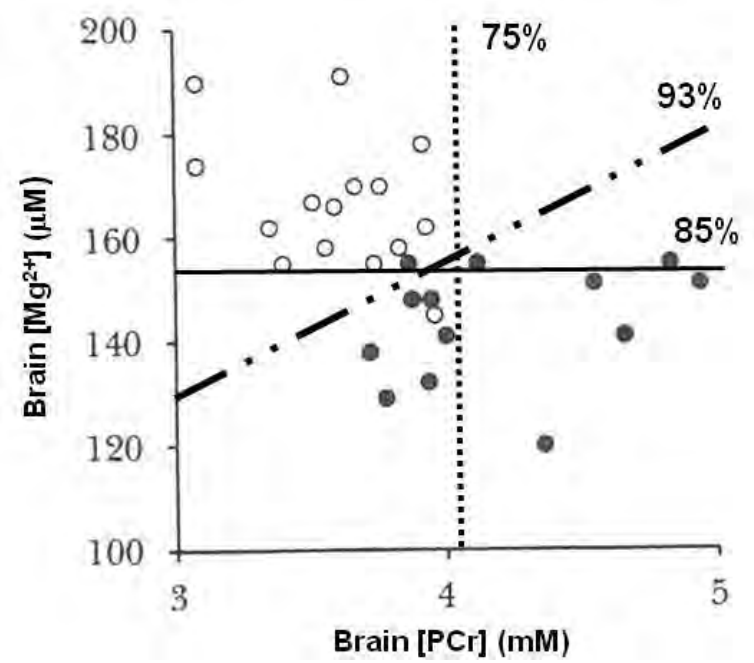

Figure 7. Plot of the distribution patterns of 13 patients with Idiopathic Parkinson's Disease (closed symbols) and 15 patients with Multiple System Atrophy (open symbols) as a function of brain $[\mathrm{PCr}]$ and free $\left[\mathrm{Mg}^{2+}\right]$ assessed by ${ }^{31} \mathrm{P}-\mathrm{MRS}$. $[\mathrm{PCr}]$ and $\left[\mathrm{Mg}^{2+}\right]$ alone were able to classify respectively $75 \%$ and $85 \%$ of the patients. Discriminant analysis using contemporarily these two independent parameters correctly classified 93\% of cases. Adapted from Barbiroli et al., 1999b.

with a probability of $93 \%$, the degree of uncertainty being due to two cases (one case in each group, Figure 7). The results of the study revealed an abnormal bioenergetics in MSA and a decreased cytosolic $\left[\mathrm{Mg}^{2+}\right]$ content in PD, offering a new diagnostic tool that may help to differentiate MSA from PD.

\section{Acknowledgments}

This work was supported by an RFO grants from the University of Bologna and PRIN 2007ZT39FN from MIUR to Stefano lotti.
Barbiroli B, Montagna P, Funicello R, lotti S, Monari L, Pierangeli G, Zaniol P, Lugaresi E (1992) Abnormal brain and muscle energy metabolism shown by ${ }^{31} \mathrm{P}$ magnetic resonance spectroscopy in patients affected by migraine with aura. Neurology 42:1209-14. 
Barbiroli B, lotti S, Cortelli P, Martinelli P, Lodi R, Carelli V, Montagna P (1999a) Low brain intracellular free magnesium in mitochondrial cytopathies. J Cereb Blood Flow Metab 19:528-32.

Barbiroli B, Martinelli P, Patuelli A, Lodi R, lotti S, Cortelli P, Montagna P (1999b) Phosphorus magnetic resonance spectroscopy in multiple system atrophy and Parkinson's disease. Mov Disord 14:430-5.

Duffield JR, Marsicano F, Williams D R (1991) Chemical speciation modelling and thermodynamic database compilation-I. Data uncertainties. Polyhedron 10:1105-11.

Farruggia G, lotti S, Prodi L, Montalti M, Zaccheroni N, Savage PB, Trapani V, Sale P, Wolf FI (2006) 8Hydroxyquinoline derivatives as fluorescent sensors for magnesium in living cells. J Am Chem Soc 128:34450.

Fatholahi M, LaNoue K, Romani A, Scarpa A (2000) Relationship between total and free cellular $\mathrm{Mg}\left({ }^{2+}\right)$ during metabolic stimulation of rat cardiac myocytes and perfused hearts. Arch Biochem Biophys 374:395401.

Golding EM, Golding RM (1995) Interpretation of ${ }^{31} \mathrm{P}$ MRS spectra in determining intracellular free magnesium and potassium ion concentration. Magn Reson Med. 33:467-74.

Gupta RK, Benovic JL, Rose Z B (1978) The determination of the free magnesium level in the human red blood cell by ${ }^{31}$ P NMR. J Biol Chem 252:6172-6.

Gupta RK, Gupta P, Yushok WD, Rose ZB (1983) Measurements of the dissociation constant of MgATP at physiological nucleotide levels by a combination of ${ }^{31}$ P NMR and optical absorbance spectroscopy. Biochem Biophys Res Commun 117:210-6.

Gupta RK, Gupta P, Moore RD (1984) NMR studies of intracellular metal ions in intact cells and tissues. Ann Rev Biophys Bioeng 13:221-46.

Halvorson HR, Vande Linde AMQ, Helpern JA, Welch KMA (1992) Assessment of magnesium concentration by ${ }^{31} \mathrm{P}$ NMR in vivo. NMR Biomed 5:53-8.

lotti S, Frassineti C, Alderighi L, Sabatini A, Vacca A, Barbiroli B (1996) In vivo assessment of free magnesium concentration in human brain by ${ }^{31} \mathrm{P}-\mathrm{MRS}$. A new calibration curve based on a mathematical algorithm. NMR Biomed 9:24-32. lotti S, Frassineti C, Alderighi L, Sabatini A, Vacca A, Barbiroli B (2000) In vivo ${ }^{31} \mathrm{P}-\mathrm{MRS}$ assessment of cytosolic $\left[\mathrm{Mg}\left({ }^{2+}\right)\right]$ in the human skeletal muscle in different metabolic conditions. Magn Reson Imag 18:607-14.

lotti S, Malucelli E (2008) In vivo assessment of $\mathrm{Mg}^{2+}$ in human brain and skeletal muscle by ${ }^{31} \mathrm{P}-\mathrm{MRS}$. Magnesium Research 21:157-62.

Kubota T, Shindo Y, Tokuno K, Komatsu H, Ogawa $\mathrm{H}$, Kudo S, Kitamura Y, Suzuki K, Oka K (2005) Mitochondria are intracellular magnesium stores: investigation by simultaneous fluorescent imagings in PC12 cells. Biochim Biophys Acta 1744(1):19-28.

Kuby SA, Noltman EA (1959) ATP-Creatine Transphosphorylase, in: P.D. In: The Enzymes, (Boyer Ed), 2nd ed, New York, PA: Academic Press, 515-603.

Lodi R, Montagna P, Soriani S, lotti S, Arnaldi C, Cortelli $P$ et al. (1997) Deficit of brain and skeletal muscle bioenergetics and low brain magnesium in juvenile migraine: an in vivo ${ }^{31} \mathrm{P}$ magnetic resonance spectroscopy interictal study. Pediatr Res 42:866-71.

Lodi R, lotti S, Cortelli P, Pierangeli G, Cevoli S, Clementi V, Soriani S, Montagna P, Barbiroli B (2001) Deficient energy metabolism is associated with low free magnesium in the brains of patients with migraine and cluster headache. Brain Res Bull 54:437-41.

Montagna P, Cortelli P, Monari L, Pierangeli G, Parchi $P$, Lodi $R$, lotti $S$, Frassineti $C$, Zaniol $P$, Lugaresi E $(1994){ }^{31} \mathrm{P}$-magnetic resonance spectroscopy in migraine without aura. Neurology 44:666-9.

Montagna P, Lodi R, Cortelli P, Pierangeli G, lotti S, Cevoli S, Zaniol P, Barbiroli B (1997) Phosphorus magnetic resonance spectroscopy in cluster headache. Neurology 48:113-8.

Mosher TJ, Williams GD, Doumen C, LaNoue KF, Smith MB (1992) Errors in the calibration of MgATP chemical shift limit: effects on the determination of free magnesium by ${ }^{31} \mathrm{P}$ NMR spectroscopy. Magn Reson Med 24:163-9.

Mottet I, Demeure R, Gallez B, Grandin C, Van Beers $B E$, Pringot J (1994) Experimental ${ }^{31}$ P NMR study of the influence of ionic strength on the apparent dissociation constant of MgATP. Magma 2:101-7.

Murphy E, (2000) Mysteries of Magnesium Homeostasis. Circ Res 86:245-8. 
Ramadan NM, Halvorson $\mathrm{H}$, Vande-Linde A, Levine SR, Helpern JA, Welch KMA (1989) Low brain magnesium in migraine. Headache 29:590-3.

Ramirez F, Marecek JF (1980) Coordination of magnesium with adenosine 5'-diphosphate and triphosphate. Biochim. Biophys Acta 589:21-9.

Repke KRH (1982) On the mechanism of energy release, transfer, and utilization in sodium-potassium ATPase transport work: old ideas and new findings. Ann N Y Acad Sci 402:272-86.

Skou C (1982) The sodium-potassium ATPase: coupling of the reaction with ATP to the reaction with sodium ion and potassium ion. Ann N Y Acad Sci 402:169-84.
Taylor JS, Vigneron DB, Murphy-Boesch J, Nelson SJ, Kessler HB, Coia L, Curran W., Brown TR (1991) Free magnesium levels in normal human brain and brain tumors: ${ }^{31} \mathrm{P}$ chemical-shift imaging measurements at 1.5 T. Proc Natl Acad Sci USA 88:6810-4.

Welch KMA, Ramadan NM (1995) Mitochondria, magnesium and migraine. J Neurol Sci 134:9-14.

Wells JA, Knoeber C, Sheldon MC, Werber MM, Yount RG (1980) Cross-linking of myosin subfragment 1. Nucleotide-enhanced modification by a variety of bifunctional reagents. J Biol Chem 255:11135-40.

Williams GD, Smith MB (1995) Application of the accurate assessment of intracellular magnesium and $\mathrm{pH}$ from the ${ }^{31} \mathrm{P}$ shift of ATP to cerebral hypoxiaischemia in neonatal rat. Magn Reson Med 33:853-7. 


\title{
Intracellular magnesium homeostasis
}

\author{
Andrea M.P. Romani ${ }^{\square}$ \\ Department of Physiology and Biophysics, School of Medicine, Case Western Reserve University, Cleveland, Ohio, USA. \\ $\triangle$ amr5@po.cwru.edu
}

\begin{abstract}
Magnesium $\left(\mathrm{Mg}^{2+}\right)$ is the fourth most abundant cation in the whole body and the second most abundant cation within the cell. Numerous cellular functions and enzymes, including ion channels, metabolic cycles, and signalling pathways are regulated by $\mathrm{Mg}^{2+}$. Our understanding of how cells regulate $\mathrm{Mg}^{2+}$ homeostasis and transport has registered significant progress in recent time. Yet, several aspects of $\mathrm{Mg}^{2+}$ homeostasis within cellular organelles, and the nature of the $\mathrm{Mg}^{2+}$ extrusion mechanisms at the cell membrane, are still undefined. The present work attempts to provide a comprehensive and updated review of the mechanisms regulating cellular $\mathrm{Mg}^{2+}$ homeostasis in eukaryotic cells under physiological conditions and the modifications these mechanisms undergo in various human and animal pathologies.
\end{abstract}

\section{Introduction}

Mammalian cells contain high concentrations of total and free magnesium ion $\left(\mathrm{Mg}^{2+}\right)$. These concentrations are essential to regulate numerous cellular functions and enzymes, including ion channels, metabolic cycles, and signalling pathways. While the increasing number of observations supports a key regulatory role for $\mathrm{Mg}^{2+}$ within the cell, our understanding of how $\mathrm{Mg}^{2+}$ homeostasis is regulated at the cellular and subcellular level remains sketchy and incomplete. There are both conceptual and methodological reasons for this limitation. The relative slow turnover of $\mathrm{Mg}^{2+}$ across the plasma membrane or other biological membranes in the absence of metabolic and hormonal stimuli, the absolute abundance of total and free $\mathrm{Mg}^{2+}$ within the cell, and the limited occurrence of significant changes in free $\left[\mathrm{Mg}^{2+}\right]$ have all contributed for a long time to the assumption that cellular $\mathrm{Mg}^{2+}$ concentration does not change significantly, and is consistently at a level adequate for its role as a co-factor for various cellular enzymes and proteins. Consequently, this conceptual assumption has limited the interest to develop techniques and methodologies able to rapidly and accurately quantify changes in cellular $\mathrm{Mg}^{2+}$ content. In the last two decades, however, an increasing number of experimental and clinical observations have challenged this assumption. More than 1000 entries in the literature highlight the regulatory role of $\mathrm{Mg}^{2+}$ on various cellular functions and cycles, and indicate the occurrence of major fluxes of $\mathrm{Mg}^{2+}$ across the plasma membrane of various mammalian cells under a variety of metabolic or hormonal stimuli. In turn, these fluxes have resulted in appreciable changes in cytosolic free $\left[\mathrm{Mg}^{2+}\right]$ and total $\mathrm{Mg}^{2+}$ content within the cell and cellular organelles. Furthermore, genetic and electrophysiological approaches in bacteria, yeast and mammalian cells have identified several $\mathrm{Mg}^{2+}$ entry mechanisms that operate either at the cell membrane level or in the membrane of cellular organelles such as mitochondria and Golgi. At the same time, the increased interest in biological functions regulated by $\mathrm{Mg}^{2+}$ has stimulated the development of methodological approaches aimed at better detecting and quantifying variations in cellular $\mathrm{Mg}^{2+}$ level, and renewed interest in relating alterations in $\mathrm{Mg}^{2+}$ homeostasis with the onset of specific pathologies and complications in human patients. All these different aspects will be elucidated in the present review to provide a framework as comprehensive as possible to correlate changes in cellular $\mathrm{Mg}^{2+}$ homeostasis and content with variations in the function of specific enzymes, which ultimately affect the modus operandi of different cellular organelles and cell types.

\section{Cellular $\mathrm{Mg}^{2+}$ distribution}

Direct and indirect measurement of total cellular $\mathrm{Mg}^{2+}$ content by various techniques consistently 
indicates that total $\mathrm{Mg}^{2+}$ concentration ranges between 17 to $20 \mathrm{mM}$ in the majority of mammalian cell types examined (Romani and Scarpa, 1992; Wolf et al., 2003). Determinations of total and free $\mathrm{Mg}^{2+}$ concentrations by electron probe $\mathrm{X}$-rays microanalysis (EPXMA), ${ }^{31} \mathrm{P}-\mathrm{NMR}$, selective $\mathrm{Mg}^{2+}$-electrode, ${ }^{13} \mathrm{C}-\mathrm{NMR}$ citrate/isocitrate ratio or fluorescent indicators (Table $I$ in Romani and Scarpa, 1992, and in Wolf and Cittadini, 2003) localize major amounts of $\mathrm{Mg}^{2+}$ within mitochondria, nucleus, and endo-(sarco)plasmic reticulum, with total concentrations ranging between 15 to $18 \mathrm{mM}$ in each of these organelles. Binding of $\mathrm{Mg}^{2+}$ by phospholipids, proteins, nucleic acids, chromatin and nucleotides has been invoked to explain the persistence of such a high $\mathrm{Mg}^{2+}$ concentration within these organelles. Although the specific modality and nature of these bindings have not been fully investigated, experimental evidence indicates that only a small fraction of such a large $\mathrm{Mg}^{2+}$ content is actually free in the lumen of these structures. Concentrations of 0.8 and 1.2 $\mathrm{mM}$ free $\left[\mathrm{Mg}^{2+}\right]$ have been measured in the matrix of cardiac and liver mitochondria (Jung et al., 1990; Rutter et al., 1990). No determinations of free $\left[\mathrm{Mg}^{2+}\right]$ are available for the nucleus and the endo-(sarco)-plasmic reticulum. The porous structure of the nuclear envelope makes it reasonable to envision that the intranuclear free $\left[\mathrm{Mg}^{2+}\right]$ is similar to the concentration measured in the cytoplasm. The free $\left[\mathrm{Mg}^{2+}\right]$ within the endo(sarco)-plasmic reticulum lumen cannot be reliably determined due to the elevated millimolar concentration of $\mathrm{Ca}^{2+}$ inside the organelle (Somlyo et al., 1985), and the high affinity of the fluorescent dyes Mag-Fura or Mag-Indo for $\mathrm{Ca}^{2+}$ (50mM) as compared to $\mathrm{Mg}^{2+}(1.5 \mathrm{mM})$ (Hofer and Machen, 1993). A third considerable pool of $\mathrm{Mg}^{2+}(\sim 4-5 \mathrm{mM})$ is present in the cytoplasm in the form of a complex with ATP, and other phosphonucleotides and phosphometabolites (Scarpa and Brinley, 1981). Because of its abundance ( 5mM) and $\mathrm{Mg}^{2+}$ binding affinity $\left(\mathrm{K}_{\mathrm{d}} \sim 78 \mu \mathrm{M}\right)$, ATP constitutes the largest metabolic pool able to bind $\mathrm{Mg}^{2+}$ within the cytoplasm and the mitochondria matrix as well (Luthi et al., 1999). The binding/buffering capacity of ATP, phosphonucleotides and phosphometabolites, and possibly proteins, maintains cytosolic free $\left[\mathrm{Mg}^{2+}\right]$ between $0.5-1 \mathrm{mM}$, or less than $5 \%$ of total cellular $\mathrm{Mg}^{2+}$ content in almost all the cells and tissues examined (Table I in Romani and Scarpa,
1992). Similar values have been obtained using fluorescent dyes, ${ }^{31} \mathrm{P}-\mathrm{NMR}$ and citrate/isocitrate ratio (Romani and Scarpa, 1992). Overall, these results support the presence of a very limited chemical $\mathrm{Mg}^{2+}$ gradient across the cell membrane, and across the membrane of cellular organelles. In erythrocytes, which lack cellular $\mathrm{Mg}^{2+}$ compartmentation, Flatman and Lew (1981) have observed three kinetically distinct binding pools for $\mathrm{Mg}^{2+}$ : a low capacity, high affinity pool represented by cell proteins, and two pools that correspond reasonably well to ATP and 2,3diphosphoglycerate (2,3-DPG) respectively (Gunther et al., 1995). This model has been further refined by Raftos et al., (1999) to take into account $\mathrm{Mg}^{2+}$ binding by hemoglobin under oxygenated and not oxygenated conditions.

Limited information is available about the ability of proteins to bind $\mathrm{Mg}^{2+}$ within the cell and cellular organelles. Aside from calmodulin (Oki et al., 1997), troponin C (Wang et al., 1998), parvalbumin (Allouche et al., 1999), and S100 protein (Ogoma et al., 1992), there is no indication as to whether other cytosolic or intraorganelle proteins can bind substantial amounts of $\mathrm{Mg}^{2+}$ and contribute to the elevated concentrations of total $\mathrm{Mg}^{2+}$ measured within the mitochondria or discrete regions of the endo(sarco)-plasmic reticulum. An early report by Bogucka and Wojitczak (1971) has suggested the presence of two proteins able to bind $\mathrm{Mg}^{2+}$ with high affinity/low capacity and high capacity/low affinity, respectively, in the intermembraneous space of the mitochondrion. However, no subsequent study has confirmed their presence or identified the proteins. The presence of $\mathrm{Mg}^{2+}$ binding sites has been indicated for several other cellular proteins, but no clear information is available as to whether these proteins do bind $\mathrm{Mg}^{2+}$ under basal conditions and whether binding changes to a significant extent following hormonal or metabolic stimuli, or under pathological conditions. Moreover, the potential physiological relevance of $\mathrm{Mg}^{2+}$ binding by any of the mentioned proteins has been called into question by the observation that parvalbumin null mice do not exhibit hypomagnesaemia or significant changes in tissue $\mathrm{Mg}^{2+}$ handling and homeostasis (Belge et al., 2007).

Taking into account the cellular distribution and assuming a $\mathrm{Mg}^{2+}$ concentration in plasma and 
extracellular fluid of 1.2-1.4 mM, one-third of which is binding extracellular proteins (e.g. albumin) or other biochemical moieties (Geigy, 1984), it appears that most mammalian cells are at or near zero trans condition as far it concerns the chemical free $\left[\mathrm{Mg}^{2+}\right]$ concentration across the cell membrane or the biomembrane of cellular organelles (e.g. mitochondria). Because the electrochemical equilibrium potential for cellular free $\left[\mathrm{Mg}^{2+}\right]$ is approximately $50 \mathrm{mM}$ in most mammalian cells under resting conditions (Flatman, 1984), it is evident that mechanisms must operate in the cell membrane to maintain cytosolic free $\mathrm{Mg}^{2+}$ and total cellular $\mathrm{Mg}^{2+}$ content within the measured levels.

\section{$\mathrm{Mg}^{2+}$ transport mechanisms}

Eukaryotes retain their cellular $\mathrm{Mg}^{2+}$ content virtually unaltered under resting conditions even when exposed to a significant gradient across the cell membrane (e.g. culturing in virtually zero extracellular $\mathrm{Mg}^{2+}$ content) (Wolf et al., 2003; Romani, 2007). Atomic absorbance spectrophotometry determinations and radioisotopic equilibrium indicate $\mathrm{Mg}^{2+}$ turnover rates ranging from 1 hour (adipocytes) to several days (lymphocytes) as a result of structural and functional specificity of different tissues and cells (Romani, 2007). Within the same cell types discrepancies can be observed based upon the experimental conditions or the modality of isolation, e.g. cells in situ versus freshly isolated cells versus cells in culture. For example, cardiac ventricular myocytes attain ${ }^{28} \mathrm{Mg}$ equilibrium within 3 hours in the whole animal but require $72-80$ hours, as dispersed cells incubated at $37^{\circ} \mathrm{C}$, or even a longer period of times when exposed to $20^{\circ} \mathrm{C}$ (Polimeni and Page, 1974; Rogers et al., 1960; Rogers, 1961). Lymphocytes also present differences in amplitude (or operation) of $\mathrm{Mg}^{2+}$ transport depending on whether they are freshly isolated (Wolf et al., 1997) or cultured (Maguire and Erdos, 1978) cells.

For a long time, the slow $\mathrm{Mg}^{2+}$ turnover observed in various tissues or cells has contributed to the erroneous idea that $\mathrm{Mg}^{2+}$ content in mammalian cells does not change, or changes at such a slow pace that it lacks physiological significance. In the last twenty years, this notion has been completely reverted by a large body of experimental evidence, which indicates that large fluxes of $\mathrm{Mg}^{2+}$ can cross the plasma membrane of eukaryotic cells within minutes from the application of metabolic or hormonal stimuli, with relatively small changes in free $\mathrm{Mg}^{2+}$ level (Romani, 2007; Grubbs and Maguire, 1987; Romani and Scarpa, 2000; Romani and Maguire, 2002). Lymphocytes (Gunther and Vormann, 1990; Wolf et al., 1997), erythrocytes (Matsuura, 1993), cardiac myocytes (Vormann and Gunther, 1987; Romani and Scarpa, 1990a) and liver cells (Jakob et al., 1989; Romani and Scarpa, 1990b; Gunther et al., 1991) are just a few examples of the mammalian cells that have been reported to extrude $10 \%$ to $20 \%$ of their total cellular $\mathrm{Mg}^{2+}$ content in less than $10 \mathrm{~min}$ from the application of adrenergic or metabolic stimuli. The amplitude and rapidity of these fluxes presuppose the presence and operation of powerful transport mechanisms able to move large amounts of $\mathrm{Mg}^{2+}$ in and out of mammalian cells following various stimuli (for a list of experimental models and conditions see Romani and Scarpa, 2000). In addition, the operation of $\mathrm{Mg}^{2+}$ entry mechanisms appears to be tightly coupled with the ability of the cell to rapidly and efficiently buffering the magnesium ions accumulated, as suggested by the limited changes in cytosolic free $\left[\mathrm{Mg}^{2+}\right]$ in the large majority of conditions tested (Fatholahi et al., 2000; Kubota et al., 2005).

The $\mathrm{Mg}^{2+}$ transport mechanisms operating at the level of the cell membrane or in the membrane of cellular organelles are represented by channels and exchangers. While channels are predominantly involved in $\mathrm{Mg}^{2+}$ accumulation, exchangers mediate essentially $\mathrm{Mg}^{2+}$ extrusion. The majority of recently identified $\mathrm{Mg}^{2+}$ entry mechanisms operate in the cell membrane but two of them have been located in the mitochondrial membrane and the Golgi system, respectively. For the most part, these entry mechanisms present a modest selectivity for $\mathrm{Mg}^{2+}$, and do not appear to be involved in $\mathrm{Mg}^{2+}$ extrusion. No information is currently available about the mechanisms that contribute to maintain an elevated $\mathrm{Mg}^{2+}$ concentration within the lumen of the endoplasmic (sarcoplasmic) reticulum.

What it follows is a general description of our current knowledge about the channels and exchangers involved in $\mathrm{Mg}^{2+}$ transport across biological membranes (summarized in Table 1). 


\section{Channels}

Magnesium ions enter the cell via channels or channel-like mechanisms. Channels able to transport $\mathrm{Mg}^{2+}$ into the cell have been originally described in prokaryotes (Kehres et al., 1998; Moncrief and Maguire, 1999), including protozoans (Preston, 1990), but recently several $\mathrm{Mg}^{2+}$ entry mechanisms with channels or channels-like features have been identified in eukaryotic cells. Some of these mechanisms exhibit a relatively high specificity for $\mathrm{Mg}^{2+}$ but they can permeate other divalent cations as well. Whereas the majority of these channels are located in the cell membrane or perhaps translocate between early endosomal vesicles and the cell membrane, other are specifically located in the mitochondrial membrane or in the Golgi cysternae. Because the identification and characterization of these eukaryotic $\mathrm{Mg}^{2+}$ transporting channels is far from being complete, information relative to their regulation is still largely fragmentary. The abundance of mechanisms favouring $\mathrm{Mg}^{2+}$ entry into the cell also raises the question as to what extent the different mechanisms cooperate to modulate $\mathrm{Mg}^{2+}$ entry or exert an absolute (or relative predominance) in specific cells under well defined conditions.

\section{TRPM Channels}

TRPM7 (Nadler et al., 2001) and TRPM6 (Schlingmann et al., 2002) were the first channels identified as being able to transport $\mathrm{Mg}^{2+}$ into mammalian cells. While Fleig and her group (Nadler et al., 2001) reported a preferential $\mathrm{Mg}^{2+}$ permeation through the LTRPC7 channel (i.e. TRPM7 based on the current nomenclature), genetic analysis (Schlingmann et al., 2002) indicated, more or less at the same time, that TRPM6, another member of the melastatin subfamily of TRP channels, exhibits a selective $\mathrm{Mg}^{2+}$ permeation. At variance of the ubiquitous nature of TRPM7, TRMP6 is specifically localized in the colon and the distal convolute tubule of the nephron, a distribution that strongly emphases the role of TRPM6 in controlling whole body $\mathrm{Mg}^{2+}$ homeostasis via intestinal absorption and renal resorption. In contrast, it would appear that TRPM7 is more in control of $\mathrm{Mg}^{2+}$ homeostasis in individual cells.

The original observations have led to a flurry of studies aimed at better understanding the role, regulation and interaction of these channels with other cellular components involved to a varying degree in $\mathrm{Mg}^{2+}$ homeostasis, and presently more than 190 publications relative to TRPM7 and 110 publications relative to TRPM 6 can be found in the literature. Although sharing several similarities in terms of structure and operation, these two channels differ in various aspects ranging from location to hormonal modulation.

\section{- TRPM7}

The key role of TRPM7 in transporting $\mathrm{Mg}^{2+}$ into cells and modulating cell growth was first evidenced by Nadler et al., (2001). At the time, the channel was identified as LTRPC7 or long TRP channel 7 owing to the presence of a particularly long extension outside the channel segment (Yamaguchi et al., 2001). Due to the presence of an alpha-kinase domain at the C-terminus (Ryazanova et al., 2001) and its functional homology to eEF2-kinase (Ryazanov, 2002), this protein was already known as CHAK1 (channel kinase 1) (Ryazanova et al., 2001). Shortly after the observation of Nadler et al., (2001), Runnels et al., (2001) evidenced the peculiar structure of TRPM7, combining a channel structure with an alpha-kinase domain at the C-terminus. Although originally investigated for a possible role in $\mathrm{Ca}^{2+}$ signalling in lymphocyte, it became rapidly apparent that the channel would preferentially carry $\mathrm{Mg}^{2+}$ and $\mathrm{Ca}^{2+}$ (Nadler et al., 2001) but also trace amounts of divalent cations such as $\mathrm{Ni}^{2+}$ and $\mathrm{Zn}^{2+}$ (Bessac and Fleig, 2007; Monteilh-Zoller et al., 2003).

Located on the human chromosome 15 at the locus $15 q 21$, the protein is formed by 1865 amino acids arranged to possibly form 10 transmembrane domains, with both the $\mathrm{C}$ - and $\mathrm{N}$ termini internalized. The protein is ubiquitously expressed albeit to a varying extent in all mammalian cells tested so far. The functional structure is supposed to be a tetramer but disagreement exists as to whether it is formed by 4 identical monomers or by a combination of TRPM7 and TRPM6 (see following section) arranged with a varying stoichiometry in different portion of the cell membrane or in different cell types. Voets and colleagues reported the functional expression of TRPM6 channels in HEK293 cells with electrophysiological properties similar to those of TRPM7 (Voets et al., 2004). In contrast, Chubanov et al., (2004) reported the absence of functional currents through TRPM6 


\section{Table 1. $\mathrm{Mg}^{2+}$ transporters in eukaryotes}

\begin{tabular}{|c|c|c|c|c|c|}
\hline & Family & Members & Apparent $\mathrm{K}_{\mathrm{m}}$ & Type of Transporter & Reference \\
\hline \multicolumn{6}{|l|}{ Entry Mechanisms } \\
\hline \multirow[t]{15}{*}{ Cell Membrane } & TRPM & TRPM6 & $\sim 0.7 \mathrm{mM}$ & Channel & Schlingmann et al., 2002 \\
\hline & & TRPM7 & $\sim 0.7 \mathrm{mM}$ & Channel & Nadler et al., 2001 \\
\hline & Claudins & Claudin 16 (PCLN-1) & $\sim 0.7 \mathrm{mM}$ & Channel & Simon et al., 1999 \\
\hline & & Claudin-19 & $\sim 0.7 \mathrm{mM}$ & Channel & Hou et al., 2009 \\
\hline & MagT1 & MagT1 & $0.2 \mathrm{mM}$ & Channel & Goytain and Quamme, 2005a \\
\hline & & & & & Zou and Clapham, 2009 \\
\hline & SLC41 & SLC41A1 & $0.7-3 \mathrm{mM}$ & Carrier & Goytain and Quamme, 2005b \\
\hline & & SLC41A2 & $0.7-3 \mathrm{mM}$ & Carrier & Goytain and Quamme, 2005c \\
\hline & & & & & Sahni et al., 2007 \\
\hline & ACDP & ACDP1 & $\sim 0.7 \mathrm{mM}$ & Carrier & Goytain and Quamme, 2005d \\
\hline & & ACDP2 & $\sim 0.5 \mathrm{mM}$ & Carrier & Goytain and Quamme, 2005d \\
\hline & NIPA & NIPA1 (SPG6) & $0.7 \mathrm{mM}$ & Carrier & Goytain et al., 2007 \\
\hline & & NIPA2 & $0.7 \mathrm{mM}$ & Carrier & Goytain et al., 2008a \\
\hline & Huntingtin & Huntingtin1 (HIP14) & $0.87 \mathrm{mM}$ & Carrier & Goytain et al., 2008b \\
\hline & & HIP14L & $0.74 \mathrm{mM}$ & Carrier & Goytain et al., 2008b \\
\hline Mitochondria & Mrs2 & Mrs2/AtMrs2, Lpe10 & $\sim 1.5 \mathrm{mM}$ & Channel & Koliske et al., 2003 \\
\hline \multirow[t]{2}{*}{ Golgi } & MMgt & MMgT1 & $1.5 \mathrm{mM}$ & Channel & Goytain and Quamme, 2008 \\
\hline & & MMgT2 & $0.6 \mathrm{mM}$ & Channel & Goytain and Quamme, 2008 \\
\hline
\end{tabular}




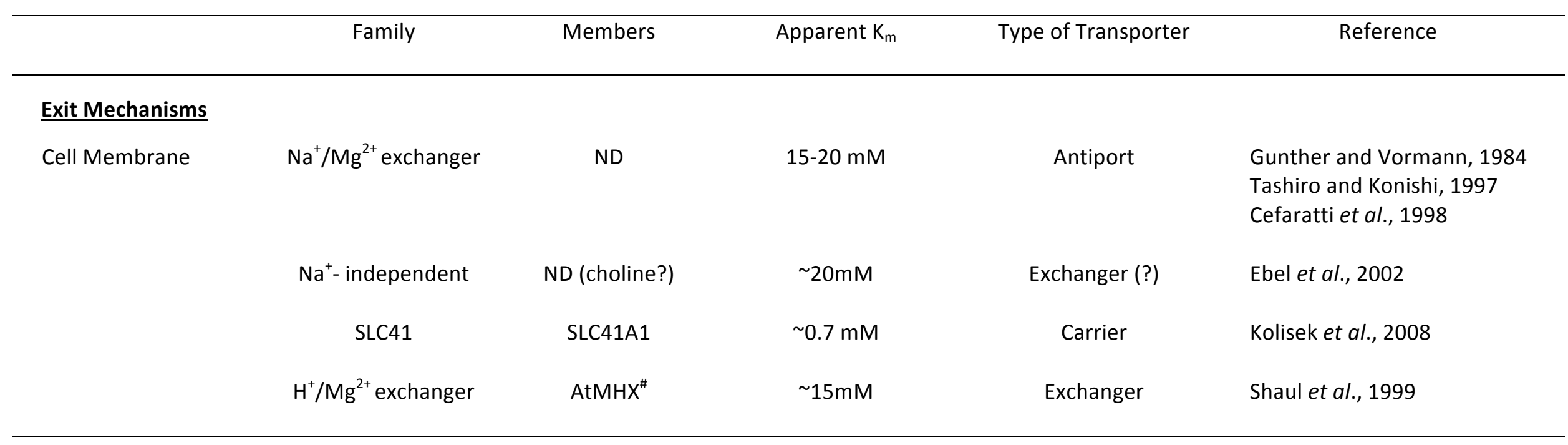

\# Identified only in plants and yeast and not in mammalian/human cells. 
when the channel is expressed by itself in either HEK-293 cells or $X$. Laevis oocytes, and suggested that TRPM7 co-expression was required for TRPM6 to incorporate into channel complexes at the plasma membrane level. Schmitz et al., (2005) subsequently confirmed the association of TRPM6 and TRPM7 channel proteins to obtain a functional structure. Yet, a detailed functional characterization of the TRPM6/7 chimeric channel remained undefined (Chubanov et al., 2005) until Yue and collaborators addressed the issue in two elegant electro-physiology studies (Li et al., 2006; Li et al., 2007). In these studies, the authors demonstrate that TRPM6 6 and TRPM7 can indeed form a chimeric heterotetramer, and that TRPM6, TRPM7, and TRPM6/7 constitute three distinct ion channels with different divalent cation permeability, $\mathrm{pH}$ sensitivity, and unique single channel conductance. In addition, these authors reported that 2-APB can differentially regulate the channel activities of TRPM6, TRPM7, and TRPM6/7, markedly increasing $\mathrm{Mg}^{2+}$ and $\mathrm{Ca}^{2+}$ entry through TRPM6 (Li et al., 2006). Based on these results, it would then appear that TRPM6 can form either functional homotetrameric channels or hetero-tetrameric TRPM6/7 channels (Gwanyanya et al., 2004). A corollary of this observation would be that TRPM6, TRPM7, and TRPM6/7 channels may play different roles under various physiological or pathological conditions in different tissues. A detailed mapping of the distribution of homomeric TRPM7 versus heteromeric TRPM6/7 channels in various tissues, however, is still lacking, leaving their relative role largely undefined.

More recently, some light has been shed on the modality of TRPM7 regulation. At the direct channel level, TRPM7 inward current is markedly enhanced by protons, which compete with $\mathrm{Ca}^{2+}$ and $\mathrm{Mg}^{2+}$ for binding sites, most likely at the level of the channel pore, thereby releasing the blockade of divalent cations on inward monovalent currents (Bessac and Fleig, 2007; MonteilhZoller et al., 2003). Not only extracellular protons significantly increased monovalent cation permeability, but higher proton concentrations are required to induce $50 \%$ of maximal increase in TRPM7 currents under conditions in which extracellular $\mathrm{Ca}^{2+}$ and $\mathrm{Mg}^{2+}$ concentrations are increased. Following the increase in extracellular $\mathrm{H}^{+}$concentration, in fact, the apparent affinity for $\mathrm{Ca}^{2+}$ and $\mathrm{Mg}^{2+}$ is significantly diminished. This set of observation suggests that at physiological $\mathrm{pH}$, $\mathrm{Ca}^{2+}$ and $\mathrm{Mg}^{2+}$ bind to TRPM7 and inhibit the monovalent cation currents. At higher $\mathrm{H}^{+}$ concentrations, instead, the affinity of TRPM7 for $\mathrm{Ca}^{2+}$ and $\mathrm{Mg}^{2+}$ is decreased, thus allowing monovalent cations to permeate the channel (Jiang et al., 2005). Another level of regulation appears to be provided by PIP2, as initially reported by Clapham and his collaborators (Runnels et al., 2002). This observation was not confirmed by Fleig's group, which instead reported a regulatory role by CAMP (Takezawa et al., 2004). More recently, however, Langeslag et al., (2007) have observed that the depletion in PIP2 level resulting from PLC-activation counteracts TRPM7 activation. It would therefore appear that either PLC-activation accelerates TRPM7 'rundown' via PIP2 depletion or PIP2 depletion plays a feedback regulatory role on the channel activation by PLC (Langeslag et al., 2007). Additional evidence for a regulatory role by PIP2 on TRPM7 has been provided by Mubagwa's group (Gwanyanya et al., 2006; Macianskiene et al., 2008). This group, in fact, has reported that inhibition of phospholipase $\mathrm{C}$ or addition of exogenous PIP2 decreases the run-down of the channel whereas the extracellular addition of phenylephrine accelerates it (Macianskiene et al., 2008). In addition, this group has observed that both ATP (Gwanyanya et al., 2006) and nonhydrolysable GTP analogs modulate the channel activity, most likely by forming MgATP and by accelerating the channel run-down via phospholipase-C activation, respectively (Macianskiene et al., 2008). The regulatory role of PIP2 on TRPM7 is further emphasized by the experimental evidence that agonists like bradykinin or angiotensin-II, which activate phospholipase-C coupled receptors via $\mathrm{Gq}$ signalling (Touyz et al., 2006; Langeslag et al., 2007) can modulate the channel activity via PIP2 metabolism. Interestingly, TRPM7 activation only takes place in the presence of a physiological cellular $\left[\mathrm{Mg}^{2+}\right]_{i}$, whereas reducing this concentration below its physiological level with EDTAAM results in a PLC-mediated inactivation of TRPM7 activity, most likely via PIP2 depletion (Langeslag et al., 2007).

The interaction between TRPM7 and phosphatidyl-inositol metabolites is further supported by the observation that TRPM7 is required for a sustained phosphoinositide-3- 
kinase signalling in lymphocytes (Sahni and Scharenberg, 2008). In the presence of a physiological concentration of extracellular $\mathrm{Mg}^{2+}$, TRPM7-deficient cells rapidly down-regulate their rate of growth as a result of a signalling deactivation downstream PI3-Kinase (Sahni and Scharenberg, 2008), the phenotype being rescued by supplementing the culture medium with $\mathrm{Mg}^{2+}$ (Sahni and Scharenberg, 2008).

A structural peculiarity of TRPM7 is the presence of an alpha-kinase at the C-terminus, which specifically phosphorylates serine and threonines located in an alpha-helix (Middelbeek et al., 2010). Initial experimental evidence (Runnels et al., 2002) invoked an essential role of this kinase domain in modulating channel activation and gating. Subsequent studies, however, failed to fully support this initial claim, as they indicated that TRPM7 channels lacking the kinase domain could still be activated by internal $\mathrm{Mg}^{2+}$ depletion (Schmitz et al., 2003). One consequence of lacking the kinase domain, however, is the inability of properly phosphorylating and consequently activating downstream cellular components. Yet, little is know about the molecular mechanisms activating the kinase domain. A recent study by Clark et al., (2006) strongly suggests that autophosphorylation plays a significant role in target recognition by the TRMP7 kinase domain. Phosphomapping by mass spectrometry has confirmed the massing autophosphorylation of TRPM7 kinase domain, which - in turn - increases the rate of substrate phosphorylation. The phosphomapping has also identified the majority (37 out of 46) of the autophosphor-ylation sites in a Ser/Thr rich region immediately preceding the kinase catalytic domain (Clark et al., 2008). Deletion of this region does not affect the intrinsic catalytic activity of the kinase but prevents substrate phosphorylation, confirming the role of this region in substrate recognition (Clark et al., 2008). Although this Ser/Thr region is poorly conserved at the amino acid sequence in TRPM6, the kinase domain of this channel appears to require a similar massive autophos-phorylation of its $\mathrm{Sr} / \mathrm{Thr}$ residues for proper substrate recognition and efficient target phosphorylation (Clark et al., 2008).

So far, only annexin I (Dorovkov and Ryazanov, 2004), myosin IIA heavy chain (Clark et al., 2008a;
Clark et al., 2008b), and calpain (Su et al., 2006) have been clearly identified as substrates phosphorylated by TRPM7 kinase domain. Although the number of targets is rather restricted, it appears that TRPM7 is playing a double role within cells by regulating $\mathrm{Mg}^{2+}$ homeostasis on the one hand, and cellular functions centered on cell adhesion, contractility and (anti)-inflammatory processes on the other hand. This double role of TRPM7 within cells, in particular smooth muscle cells, is emphasized by a recent observation by Touyz and colleagues (Paravicini et al., 2009). In this study, the authors report that aortic segments of mice exhibiting low intracellular $\mathrm{Mg}^{2+}$ levels present increased medial cross-section and increased TRPM7 expression but decreased levels of annexin-I expression. As annexin-I has a major antiinflammatory role (Parente and Solito, 2004), the results of this study suggest a potential regulatory role of TRPM7 in regulating vascular structure and integrity, as well as inflammation.

As our understanding of TRPM7 expression and regulation has improved, evidence of a major functional role of the channel in neuronal function and survival under hypoxia or ischemicreperfusion conditions has increased. Owing to its ability to transport either $\mathrm{Ca}^{2+}$ or $\mathrm{Mg}^{2+}$, TRPM7 exhibits an ambivalent role based upon the permeating cation. Following activation by reactive oxygen/nitrogen species and prolonged oxygen and glucose deprivation, TRPM7 favours $\mathrm{Ca}^{2+}$ fluxes that result in a toxic event for neurons (Aarts et al., 2003). In contrast, $\mathrm{Mg}^{2+}$ permeation of the channel enhances anti-apoptotic and cellsurvival mechanisms, preventing the anoxic death of neurons (Clark et al., 2006). The essential role of TRPM7 in detecting extracellular divalent cations is supported by a recent study by Wei et al., (2007), which indicates that activation of the channel by low extracellular divalent cations is lethal to the cell. At the same time, Jiang et al., (2008) have reported that occlusion of the middle cerebral artery for 1 hour enhances TRPM7 expression in ipsilateral hippocampus, with deleterious consequences for the neurons. The increased expression of TRPM7 and its consequences are largely counteracted by pretreatment with nerve growth factor via activation of TrkA pathway (Jiang et al., 2008). More recently, application of 5-lipoxygenase inhibitors can block TRPM7 current without affecting 
protein expression and cell membrane concentration, de facto preventing cell death (Chen et al., 2010).

The involvement of TRPM7 is not restricted to the sympathetic nervous system but extends to the parasympathetic system as well, in which the channel facilitates the fusion of cholinergic vesicles with the plasma membrane without affecting large dense core vesicle secretion (Brauchi et al., 2008).

Despite the data accumulated since its identification as a preferential $\mathrm{Mg}^{2+}$ channel, a recent report by Clapham's group has cast some concern about the effective role of TRPM7 in regulating $\mathrm{Mg}^{2+}$ homeostasis (Jin et al., 2008). In their report, the authors indicate that TRPM7 null mouse present an altered embryonic development, and that tissue specific deletion of the channel in T cell lineage disrupts thymopoiesis, leading to the progressive depletion of thymic medullary cells. Deletion of TRPM7, however, did not affect acute accumulation of $\mathrm{Mg}^{2+}$ nor impacted total cellular $\mathrm{Mg}^{2+}$ content in $\mathrm{T}$ cells. The synthesis of several growth factors, however, was significantly dysregulated, resulting in an altered differentiation of thymic epithelial cells (Jin et al., 2008). TRPM7, therefore, appears to be the first TRP channel with a non-redundant and actually essential role in embryogenesis and thymopoiesis. Whether these defects are the result of an altered $\mathrm{Ca}^{2+}$ rather than $\mathrm{Mg}^{2+}$ homeostasis is presently undefined. It is also unclear how the removal of this protein results in an altered cellular differentiation process.

\section{- TRPM6}

The unique localization of TRPM6 channels in the colon and the renal distal convolute tubule, two epithelia otherwise highly impermeable to salt reabsorption, highlights the specific role of this channel in controlling intestinal $\mathrm{Mg}^{2+}$ absorption and renal $\mathrm{Mg}^{2+}$ resorption, and consequently contributing to whole-body $\mathrm{Mg}^{2+}$ homeostasis.

The TRPM6 gene was originally identified as the site of various mutations responsible for Hypomagnesaemia with Secondary Hypocalcaemia (HSH), a rare autosomal recessive disease characterized by $\mathrm{Mg}^{2+}$ and $\mathrm{Ca}^{2+}$ wasting, whose symptoms could be ameliorated by massive intra- venous $\mathrm{Mg}^{2+}$ administration followed by oral $\mathrm{Mg}^{2+}$ supplementation (Schlingmann et al., 2002). Surprisingly, while hypocalcaemia is completely alleviated by this treatment, the patients continue to present serum $\mathrm{Mg}^{2+}$ level around 0.5$0.6 \mathrm{mmol} / \mathrm{L}$, i.e. about half the physiological level (Schlingmann et al., 2002). Because the primary defect in these patients is at the level of the TRPM6 expressed in the intestine (Schlingmann et al., 2002), the excess $\mathrm{Mg}^{2+}$ supplementation is rapidly filtered at the glomerular level and increases passive renal absorption via paracellin1 (see next section). Trans-cellular absorption via renal TRPM6, however, remains depressed and unable to restore physiological serum $\mathrm{Mg}^{2+}$ level (Schlingmann et al., 2002).

At the functional level, experimental evidence suggests that the channel forms a tetramer within the plasma membrane. As indicated in the previous section, questions remain as to whether the channel forms a homo-tetramer, or a heterotetramer with TRPM7, with a varying stoichiometry. Irrespective of the possibilities, several TRPM6 mutations have been identified (Walder et al., 2002). The majority of these mutations result in the expression of a truncated and nonfunctional channel (Walder et al., 2002). The missense mutation $S^{141} \mathrm{~L}$, on the other hand, occurs at the $\mathrm{N}$-terminus of the channel and prevents its proper assembly as a homotetramer, or a hetero-tetramer with TRPM7 (Walder et al., 2002). Another missense identified in humans is the $\mathrm{P}^{1017} \mathrm{R}$ mutation (Walder et al., 2002), which appears to occur in a region putatively identified as the pore region of the channel. Yet, this mutation affects negatively and more significantly TRPM7 function when this protein is co-expressed with TRPM6 (Walder et al., 2002). More recently, TRPM6 null mice have been developed by Sheffield and his collaborators (Walder et al., 2009). The heterozygous Trpm6 $6^{+/-}$ have for the most part normal electrolyte levels aside for a modest low plasma $\mathrm{Mg}^{2+}$ level ( 0.67 vs. 0.75, (Walder et al., 2009). The majority of the homozygous $\mathrm{Trpm}^{-/}$animals die by embryonic day 12.5. Most of the few animals that survive to term present significant neural tube defects, consisting primarily of both exencephaly and spina bifida occulta. Administration of high $\mathrm{Mg}$ diet to dams improves offspring survival to weaning (Walder et al., 2009). 
A peculiarity TRPM6 shares with TRPM7 is the presence of an alpha-kinase domain at the Cterminus. Originally, TRPM6 was known as CHAK2 (channel kinase 2) (Ryazanov, 2002) due to the presence of this kinase domain, which presents a functional homology to eEF2-kinase (Ryazanov, 2002). At variance of other kinases, this domain phosphorylates serine and threonine residues located within an alpha-helix instead of a betasheet (Ryazanova et al., 2001; Ryazanov, 2002; Middelbeek et al., 2010). Owing to their dual function as a channel and a kinase, TRPM6 and TRPM7 are currently referred to as chanzymes. As in the case of TRPM7, removal of the kinase domain does not abolish entirely the channel activity but modulates the extent to which the channel is regulated by intra-cellular free $\mathrm{Mg}^{2+}$ or MgATP complex (Chubanov et al., 2004; Schmitz et al., 2005; Chubanov et al., 2005; Li et al., 2005; Thebault et al., 2008). Hence, the targets phosphorylated by the kinase must be located downstream from the protein. At variance of what reported for the kinase domain of TRPM7 (see previous section) no phosphorylation substrate for the TRPM6 kinase has been clearly identified up-to-date, with the exception of TRPM7 itself. Data from the Ryazanov's group clearly indicate that the TRPM6 kinase domain can phosphorylate TRPM7 channel within a heterotetramer structure while the opposite does not occur (Schmitz et al., 2005). Due to the limited information available, it is largely undefined as to whether the kinase domain of TRPM7 and TRMP6 phosphorylate similar or different substrates in the tissues in which these chanzymes are specifically expressed.

How exactly the expression and/or activity of TRPM6 channel are modulated in vivo is slowly being elucidated. Estrogens (17ß-estradiol) markedly upregulate TRPM 6 mRNA in both colon and kidney while having no effect on TRPM7 mRNA (Groenestege et al., 2006; Cao et al., 2009). In the absence of estrogen, the repressor of estrogen receptor activity (REA) binds to the $6^{\text {th }}, 7^{\text {th }}$ and $8^{\text {th }}$ beta-sheets of TRPM 6 kinase domain in a phosphorylation-dependent manner and inhibits TRPM6 activity (Cao et al., 2009). Short-term estrogen administration dissociates the binding between REA and TRPM6, resulting in an increased channel activity (Cao et al., 2009). Dietary $\mathrm{Mg}^{2+}$ restriction also upregulates TRPM6 mRNA in both colon and kidney but does not affect TRPM7 mRNA (Groenestege et al., 2006; Rondon et al., 2008). In contrast, an $\mathrm{Mg}^{2+}$ enriched diet upregulates TRPM 6 mRNA only in the colon, in keeping with an increased intestinal absorption (Groenestege et al., 2006). Mice selected for the their low erythrocyte and plasma $\mathrm{Mg}^{2+}$ status exhibit hypomagnesaemia and hypomagnesuria, and increased TRPM6 expression in kidney and intestine when fed a severely $\mathrm{Mg}^{2+}$-deficient diet (Rondon et al., 2008). Feeding the mice an $\mathrm{Mg}^{2+}$ adequate diet resulted, instead, in hypomagnesaemia and hypermagnesuria, and lower intestinal and renal TRPM6 expression (Rondon et al., 2008). These changes in TRPM6 expression and $\mathrm{Mg}^{2+}$ level in blood and urine were not observed in mice exhibiting normal or high erythrocyte and plasma $\mathrm{Mg}^{2+}$ level (Rondon et al., 2008). It is becoming progressively apparent, therefore, that genetic factors control TRPM6 expression and activity, and that dietary $\mathrm{Mg}^{2+}$ restriction increases $\mathrm{Mg}^{2+}$ resorption, the process correlating well with an increased TRPM6 expression in both intestine and kidney (Groenestege et al., 2006; Rondon et al., 2008).

As already observed for TRPM7, intracellular ATP specifically decreased TRPM6 current (Chubanov et al., 2004; Schmitz et al., 2005; Chubanov et al., 2005; Li et al., 2005; Thebault et al., 2008). The inhibitory site resides in the conserved ATPbinding motif $G X G(A) X X G$ within the alpha-kinase domain (Thebault et al., 2008). Either the full deletion of the kinase domain or point mutations within the ATP-binding motif $\left(G^{1955} D\right)$ completely prevents the inhibitory effect of intracellular ATP. The effect of ATP, however, is independent of alpha-kinase autophosphorylation activity (Thebault et al., 2008).

The activity of TRPM6 channels can also be modulated by cellular signalling molecules. Bindels and collaborators (Cao et al., 2008) have reported that over-expression of RACK1 (receptor for activated protein kinase $\underline{C}$ ) results in a direct binding of this protein to the alpha-kinase domain of TRPM6, and possibly TRPM7 due to the high homology (>84\%) between the two kinase domains. The TRPM6 site binding RACK1 is restricted to the region between amino acids 1857 and $1885\left(6^{\text {th }}, 7^{\text {th }}\right.$ and $8^{\text {th }}$ b sheets $)$. Interestingly, these are the same sheets involved in REA regulation (Cao et al., 2009). The interplay between REA and RACK1 in modulating the 
channel activity, however, still remains undefined. Accessibility analysis of the RACK1 binding site suggests that 18 of the 28 amino acids of this site are localized at the surface of the TRPM6 alpha-kinase domain (Cao et al., 2008). As a result of this interaction, the channel activity of TRPM6 6 and TRPM7 are inhibited. As it could be anticipated, co-expression of RACK1with a alphakinase deleted TRPM6 mutant fails to suppress channel activity. The inhibitory effect of RACK1 fully depends on the autophosphorylation of threonine $1851\left(\mathrm{~T}^{1851}\right)$ within the kinase domain. This residue is localized at the end of the $4^{\text {th }}$ alpha-helix adjacent to the RACK1 binding site. Mutation of $\mathrm{T}^{1851}$ to alanine $\left(\mathrm{T}^{1851} \mathrm{~A}\right)$ or to aspartate $\left(T^{1851} D\right)$ significantly decreases TRPM6 autophosphorylation while leaving unaltered RACK1 binding. The inhibitory effect of RACK1 on the channel activity, however, is completely abolished only in the case of $\mathrm{T}^{1851} \mathrm{~A}$ mutation while it persists in the case of $T^{1851} D$ mutation (Cao et al., 2008). Interestingly, $T^{1851} \mathrm{D}$ autophosphorylation strongly depends on $\mathrm{Mg}^{2+}$ concentration, steadily increasing for concentrations between 0.1 to $1 \mathrm{mM}$. In contrast, $\mathrm{T}^{1851} \mathrm{~A}$ mutant is less sensitive to intracellular $\mathrm{Mg}^{2+}$ concentrations as compared to the wild type ( $\mathrm{IC}_{50} \sim 0.7$ vs. $0.5 \mathrm{mM}$, respectively). Under conditions in which protein kinase $\mathrm{C}$ is activated (e.g. pre-incubation with PMA), the inhibitory effect of RACK1 on TRPM6 channel activity is completely prevented (Cao et al., 2008). The inhibition, however, can be restored by pretreatment with the PKC inhibitor chelerythrine (Cao et al., 2008), suggesting a competing effect of PKC for RACK1.

A recent study of Groenestege et al., (2007) has evidenced the role of EGF as an autocrine/ paracrine magnesiotropic hormone. By engaging its receptor in the basolateral domain of the distal convolute tubule, EGF is able to activate TRPM6 at the apical domain of the cell and induce cellular $\mathrm{Mg}^{2+}$ accumulation. Point mutation in the pro-EGF $\left(\mathrm{P}^{1070} \mathrm{~L}\right)$ retains EGF secretion to the apical membrane and disrupts this cascade of events, ultimately resulting in the $\mathrm{Mg}^{2+}$ wasting typical of isolated recessive renal hypo-magnesemia (IRH). An alteration of the axis EGF/TRPM6/ $\mathrm{Mg}^{2+}$ reabsorption/renal $\mathrm{Mg}^{2+}$ wasting is also observed in cancer patients undergoing treatment with antibodies anti-EGFR (Cunningham et al., 2004; Dimke et al., 2010), as the antibody antagonizes the stimulation of TRPM6 activity via EGF. The modality by which EGF modulates TRPM6 activity and/or expression appears to involve ERK1/2 signalling. A report by Ikari et al., (2008) indicates that the stimulation of NRK-52E cells by EGF results in an increased phosphorylation of ERK1/2 and an increased expression of TRPM6 in a time-dependent manner (Ikari et al., 2008) via modulation by adaptin protein-1 (AP-1) (Ikari et al., 2010). The process is prevented by the use of antagonists for integrin $a_{v} b 3$ or for MEK1/MEK2 activity, or by the use of siRNA for TRPM6 (Ikari et al., 2008). How exactly EGF, integrin, and ERK1/2 interact to enhance TRPM6 expression needs further elucidation. It is in fact unclear whether the activation of this signalling axis is connected to the release of RACK1-mediated inhibition of TRPM6 activity mentioned previously (Cao et al., 2008). The modality by which apically accumulated $\mathrm{Mg}^{2+}$ is transported across the cell to be delivered to basolateral domain of the cell to be extruded into the blood stream also needs further elucidation. One hypothesis is that parvalbumin and calbindin- $D_{28 k}$, two proteins abundantly present within cells of the distal convolute tubule of the nephron, can operate the transcellular transport of $\mathrm{Mg}^{2+}$ accumulated at the apical domain, or at least accelerate the rate of delivery at the basolateral domain. However, as mentioned previously, parvalbumin null mice do not show detectable defects in $\mathrm{Mg}^{2+}$ excretion or homeostasis (Belge et al., 2007), de facto questioning whether parvalbumin does play a role in the process or other proteins can compensate for its absence in the null model.

\section{Claudins}

Paracellin 1 (claudin 16) was the first $\mathrm{Mg}^{2+}$ transporting protein to be identified in mammals (Simon et al., 1999). The identification was rendered possible by the genetic analysis of patients affected by Familial Hypomagnesaemia with Hypercalciuria and Nephrocalcinosis (FHHNC), a disease characterized by massive renal $\mathrm{Mg}^{2+}$ and $\mathrm{Ca}^{2+}$ wasting that leads rapidly and irreversibly to renal failure (Simon et al., 1999). At variance of what is described for patients with TRPM6 mutations, the symptoms and the progressive renal deterioration in FHHNC patients are not ameliorated by $\mathrm{Mg}^{2+}$ supplementation (Simon et al., 1999). The gene responsible for this disease was identified by 
Lifton and collaborators in 1999 and named Paracellin-1 (PCLN-1) (Simon et al., 1999). More than 20 mutations affecting paracellin-1 trafficking or permeability have been identified up-to-date (Kausalya et al., 2006). PCLN-1 encodes for paracellin-1 (PCLN-1), also termed claudin-16. This protein is a member of the claudin family ((Lal-Nag and Morin, 2009), which comprehends a group of tight junction proteins that present 4 transmembrane spans coordinated by 2 extracellular loops, and both $\mathrm{C}$ - and $\mathrm{N}$ termini on the cytoplasm side.

Claudin-16 mediates paracellular $\mathrm{Ca}^{2+}$ and $\mathrm{Mg}^{2+}$ fluxes throughout the nephron. Yet, discrepancy exists about the modality by which these fluxes are generated. Data obtained in LLC-PK1 (a porcine renal cell line) suggest that claudin-16 mediates paracellular $\mathrm{Na}^{+}$-permeation which, in turn, generates a positive potential within the lumen that acts as the driving force for $\mathrm{Mg}^{2+}$ and $\mathrm{Ca}^{2+}$ resorption (Hou et al., 2005). In contrast, data in MDCK cells indicate a decrease in $\mathrm{Na}^{+}-$ permeability and an increase in $\mathrm{Mg}^{2+}$ permeability (Ikari et al., 2006). It is unclear whether these discrepancies reflect a different modus operandi in cell lines of differing origin, or depend on the experimental conditions utilized in the two studies. It is evident, however, that $P C L N-1$ expression is modulated based upon the magnesium concentration present in the extracellular medium (Efrati et al., 2005).

At the functional level, claudin-16 has to be delivered correctly to the tight junction where it interacts with the scaffolding protein ZO-1 (Muller et al., 2003). The association and dissociation of claudin-16 and ZO-1 appear to be regulated via PKA-mediated phosphorylation of $\mathrm{Ser}^{217}$ in claudin-16 (Muller et al., 2003). The dephosphorylation of this residue, as it occurs upon activation of the Calcium Sensing Receptor (CaSR) (Khan and Conigrave, 2010) results in the dissociation of claudin-16 from ZO-1 and its accumulation in the lysosomal compartment (Ikari et al., 2006). Mutations of Ser 217 can therefore accelerate claudin-16 turnover and modulate its function. Mutation of Threo233 (T233R) also impairs the interaction between claudin-16 and ZO-1, and favours the accumulation of claudin-166 into lysosomes (Muller et al., 2003; Ikari et al., 2006). More recently, evidence has emerged indicating the involvement of another claudin isoform, claudin19, in mediating $\mathrm{Mg}^{2+}$ and $\mathrm{Ca}^{2+}$ resorption (Hou et al., 2009) by forming a head-to-head cationselective complex with claudin-16 at the level of the tight junction. While the channel function of claudin-16 may not depend on its association with claudin-19, claudin-19 plays an indispensable role in recruiting claudin-16 to form a co-polymer at the level of the tight junction and in switching the channel from anion to cation selective (Hou et al., 2009). The heteromeric association between claudin-16 and claudin-19 is dramatically affected by point mutations in claudin-16 $\left(\mathrm{L}^{145} \mathrm{P}, \mathrm{L}^{151} \mathrm{~F}, \mathrm{G}^{191} \mathrm{R}, \mathrm{A}^{209} \mathrm{~T}\right.$, and $\left.\mathrm{F}^{232} \mathrm{C}\right)$ and claudin-19 $\left(\mathrm{L}^{90} \mathrm{P}\right.$ and $\left.\mathrm{G}^{123} \mathrm{R}\right)$, which abolish the physiological synergism between the two proteins and result in the development of FHHNC.

\section{MagT1}

This protein was identified by Goytain and Quamme (2005a) in human epithelial cells that upregulate the encoding gene following exposure to low- $\mathrm{Mg}^{2+}$ concentrations in the culture medium. The protein encoded by this gene has an estimated molecular weight of $38 \mathrm{KDa}$ and 5 transmembrane domains. The mature MagT1 protein, however, would contain 4 transmembrane spans owing to the cleavage of the first transmembrane segment located near the C-terminus. At variance of SLC41 (Section 3.3.1) and Mrs2 (discussed in the next Section), MagT1 does not exhibit any significant degree of homology to prokaryotic $\mathrm{Mg}^{2+}$ transporters, but has some similarities with the oligosaccharide transferase complex OST3/OST6 that regulates protein glycosylation in the endoplasmic reticulum in yeast (Shibatani et al., 2005). The murine orthologue of MagT1 is highly expressed in liver, heart, kidney and colon, with detectable levels in lung, brain and spleen (Goytain and Quamme, 2005a). For the most part, MagT1 levels in these tissues are consistent with the mRNA levels, the only exception being the liver in which a low protein level is detected (Goytain and Quamme, 2005a). At variance of the other transporters described in this section, MagT1 appears to possess high specificity for $\mathrm{Mg}^{2+}\left(\mathrm{K}_{\mathrm{m}}=\right.$ $0.23 \mathrm{mM})$. The $\mathrm{Mg}^{2+}$-elicited currents are not inhibited by $\mathrm{Ca}^{2+}$ but can be inhibited by $\mathrm{Ni}^{2+}, \mathrm{Zn}^{2+}$ and $\mathrm{Mn}^{2+}$, although the required concentrations $(>0.2 \mathrm{mM})$ far exceed the physiological concentrations of these cations in extracellular 
fluids. Interestingly, nitrendipine at $\sim 10 \mathrm{mM}$ can inhibit the $\mathrm{Mg}^{2+}$ current whereas the more common nifedipine does not, even at much higher concentrations (Goytain and Quamme, 2005a). Virtually no information is available about N33, a second member of the MagT family. Although able to transport $\mathrm{Mg}^{2+}$, this protein does not show the same high specificity presented by MagT1 for the cation, and can also mediate the transport of $\mathrm{Fe}^{2+}, \mathrm{Mn}^{2+}$ and $\mathrm{Cu}^{2+}$ (Goytain and Quamme, 2005a).

Based upon these observations, MagT1 appears to possess channel-like characteristics and a high selectivity for $\mathrm{Mg}^{2+}$. The latter evidence strongly suggests that this transporter can play an essential role in regulating $\mathrm{Mg}^{2+}$ homeostasis in mammalian cells. Support to this hypothesis is provided by the report of Zhou and Clapham (2009) that knock-out of MagT1, and its human homolog TUSC3, in HEK-293 cells results in a major reduction of cellular $\mathrm{Mg}^{2+}$ content. These authors also provide evidence that either protein can complement the yeast $\mathrm{Mg}^{2+}$ transporter ALR1 (Zhou and Clapham, 2009). Interestingly, the mRNA levels of MagT1 but not those of TUSC3 increase markedly following exposure of expressing cells to low extracellular $\mathrm{Mg}^{2+}$ concentrations for 1 day and 2 days. Incubation of the cells in high extracellular $\mathrm{Mg}^{2+}$ concentration has no effect on the expression of either protein (Zhou and Clapham, 2009).

\section{Mrs2}

This protein was identified during a screening aimed at isolating nuclear genes suppressing RNA splicing defects in yeast mitochondrial introns (Wiesenberger et al., 1992). The three main characteristics observed in yeasts deficient in Mrs2 are: 1) a splicing phenotype, 2) a significant reduction in cytochromes, and 3 ) a deficit in mitochondria respiration to the point that the yeasts become unable to grow on nonfermentable substrates.

Structurally, Mrs2 shows short regions of homology to the bacterial transporter CorA (Bui et al., 1999), and shares a similar membrane topology with 2 transmembrane domains. Mutants lacking Mrs2 can be rescued by CorA fused to the mitochondrial $\mathrm{N}$-terminus leader sequence of Mrs2, which guarantees proper insertion in the mitochondrial membrane. These mutants also present a decrease in mitochondrial $\mathrm{Mg}^{2+}$ content, which strongly supports a key role of $\mathrm{Mrs} 2$ in regulating mitochondrial $\mathrm{Mg}^{2+}$ homeostasis. Studies carried out with the fluorescent indicator Mag-Fura indicate a marked decrease in mitochondrial matrix $\mathrm{Mg}^{2+}$ level in yeast lacking Mrs2 protein whereas the overexpression of the protein results in a rapid and marked increase in matrix free $\mathrm{Mg}^{2+}$ (Kolisek et al., 2003). Mrs2 apparently functions as a channel, and its function is modulated by mitochondrial Dy as well as by inhibitors of FO-F1ATPase or ANT, which substantially decrease $\mathrm{Mg}^{2+}$ influx. Highly conserved motifs in the middle region of the protein, corresponding to the coiled-coil portion of the channel, appear to be essential to form functional channels, or to gate the channel. More recently, Schweyen and his collaborators (Piskacek et al., 2009) have confirmed in HEK293 cells some of the mitochondrial modifications observed in yeasts. These authors, in fact, have reported that HEK 293 cells deprived of Mrs2 (Piskacek et al., 2009) lack complex I expression in mitochondria and present reduced level of mitochondrial $\mathrm{Mg}^{2+}$. Furthermore, the cells show a change in configuration as well as an increased incidence in apoptosis, which within 2 weeks results in a complete loss of cell viability (Piskacek et al., 2009). It still remains to be elucidated whether the decrease in mitochondrial $\mathrm{Mg}^{2+}$ simply depends on the absence of Mrs2, or is related to some extent to the absence of complex I, which affects mitochondrial Dy and consequently $\mathrm{Mg}^{2+}$ retention within the organelle (Akerman, 1981).

Mammalian cells express a single orthologue of Mrs2, which can rescue $\mathrm{Mg}^{2+}$ deficient yeast strain (Zsurka et al., 2001). Hence, it appears that the mammalian/human Mrs2 homologue functions in a manner similar to the yeast homologue in mediating $\mathrm{Mg}^{2+}$ entry in mitochondria. Under conditions in which Mrs2p is absent, the operation of an alternative but much slower mitochondrial $\mathrm{Mg}^{2+}$ entry mechanism is observed. Although this pathway restores $\mathrm{Mg}^{2+}$ homeostasis only partially, it does rescue the phenotype of Mrs2 deficient yeast, ensuring their survival. No information is presently available about the identity, abundance, and regulation of this alternative transporter in mitochondria. Taken together, the data on Mrs2 suggest that $\mathrm{Mg}^{2+}$ is dynamically regulated within the 
mitochondrion, in which it plays a significant role in modulating mitochondrial dehydrogenases and oxygen consumption (Panov and Scarpa, 1996a; Panov and Scarpa, 1996b).

\section{MMgTs}

This gene family comprehends two proteins termed MMgT1 and MMgT2 (for membrane $\mathrm{Mg}^{2+}$ transporter 1 and 2) by Goytain and Quamme, who identified them by microarray analysis screening (Goytain and Quamme, 2008). The chromosomal location of these proteins in the mouse is XA5 for MMgT1 and 11B2 for MMgT2. In the rat, the respective locations are Xq36 for MMgT1 and 10q23 for MMgT2. Human MMgT1 is instead located on Xq26.3 (Goytain and Quamme, 2008). Immunohistochemistry assessment indicates that MMgT1 and MMgT2 are essentially located in the Golgi complex and post-Golgi vesicles, where they may contribute to the regulation of $\mathrm{Mg}^{2+}$ dependent enzymes involved in protein assembly and glycosylation (Goytain and Quamme, 2008). This localization, however, does not exclude that these proteins may play a role in modulating $\mathrm{Mg}^{2+}$ homeostasis at sites downstream the Golgi network. Widely distributed in tissues, these proteins are formed by 131 (MMgT1) and 123 (MMgT2) amino acids assembled into two predicted transmembrane domains. This suggests that these proteins can form homo-oligomeric and possibly heterooligomeric channels to favour $\mathrm{Mg}^{2+}$ permeation. MMgT-mediated $\mathrm{Mg}^{2+}$ uptake is saturable with a $\mathrm{K}_{\mathrm{m}} \sim 1.5 \mathrm{mM}$ for MMgT1 and $\sim 0.6 \mathrm{mM}$ for MMgT2, and these values do not vary significantly with voltage. However, MMgT1 and MMgT2 are not specific for $\mathrm{Mg}^{2+}$ but they can transport other cations as well. Some slight differences in cation permeation exist between the two isoforms. Whereas MMgT1 mediates $\mathrm{Sr}^{2+}, \mathrm{Fe}^{2+}, \mathrm{Co}^{2+}$ and $\mathrm{Cu}^{2+}$ transport in addition to $\mathrm{Mg}^{2+}, \mathrm{MMgT2}$ favours $\mathrm{Sr}^{2+}, \mathrm{Co}^{2+}, \mathrm{Cu}^{2+}, \mathrm{Ba}^{2+}$ and $\mathrm{Mn}^{2+}$ transport (Goytain and Quamme, 2008). Electrophysiological experiments indicate that $\mathrm{Mg}^{2+}$-generated current in $\mathrm{MMgT1}$ are inhibited by $0.2 \mathrm{mM} \mathrm{Mn}^{2+}$ but not by $\mathrm{Gd}^{3+}$ or $\mathrm{Ni}^{2+}$. Consistent with what reported for other $\mathrm{Mg}^{2+}$ transport mechanisms, MMgT1 mRNA increases $\sim 2.5$ fold in the kidney cortex of mice on low$\mathrm{Mg}^{2+}$ diet and $\sim 3.5$ fold in MDCT epithelial cells culture in low $\mathrm{Mg}^{2+}$ medium. Under similar experimental conditions MMgT2 mRNA increases $\sim 1.5$ fold in kidney cortex and $\sim 3$ fold in MDCT cells (Goytain and Quamme, 2008). We refer the interested audience to reviews by Schmitz et al., (2007), Bindels' group (Alexander et al., 2008), and Quamme (2010) for a more in-depth elucidation of the specifics of TRPM6/7 channels and other $\mathrm{Mg}^{2+}$ entry mechanisms summarily described in this section.

\section{Exchangers}

While $\mathrm{Mg}^{2+}$ entry appears to be mediated by channels or channels-like mechanisms, $\mathrm{Mg}^{2+}$ extrusion is mediated by two mechanisms operating as exchangers. Based upon the electrochemical requirements favouring $\mathrm{Mg}^{2+}$ extrusion, these mechanisms are referred to as $\mathrm{Na}^{+}$-dependent and $\mathrm{Na}^{+}$-independent $\mathrm{Mg}^{2+}$ exchanger, respectively (Table 1). Because neither of these two mechanisms has been cloned, information about their operation, abundance and tissue specificity remains mostly circumstantial or indirect based upon experimental conditions or pharmacological inhibition.

\section{Na-dependent $\left(\mathrm{Na}^{+} / \mathrm{Mg}^{2+}\right.$ Exchanger)}

The first evidence of the operation of a $\mathrm{Mg}^{2+}$ transport mechanism in mammalian cells was provided by Gunther et al., (1984). In this and a subsequent publication (Gunther and Vormann, 1985), these authors detailed the presence and operation of an amiloride-inhibited, $\mathrm{Na}^{+}$dependent, $\mathrm{Mg}^{2+}$ extrusion mechanism in chicken red blood cells. This initial observation has been subsequently confirmed by other groups in mammalian red blood cells (Feray and Garay, 1986; Flatman and Smith, 1990; Xu and Willis, 1994) including human erythrocytes (Ludi and Schatzmannm 1987; Vormann et al., 1984; Raftos et al., 1999), and in a variety of mammalian cell types (see Romani and Scarpa, 2000 for a list). In addition, observation from Vormann and Gunther (Vormann and Gunther, 1987; Gunther and Vormann, 1992a), Wolf and collaborators (Wolf et al., 1996; Wolf et al., 1997), and our laboratory (Romani and Scarpa, 1990a; Romani and Scarpa 1990b; Romani et al., 1993a; Romani et al., 1993b; Fagan and Romani, 2000; Fagan and Romani, 2001; Cefaratti and Ruse, 2007; Cefaratti and Romani, 2007) has provided compelling evidence that this $\mathrm{Na}^{+}$-dependent, amilorideinhibited $\mathrm{Mg}^{2+}$ extrusion mechanisms is specifically coupled to cAMP-production within the cells. From the experimental stand-point it is irrelevant whether cellular CAMP increases via 
stimulation of $\beta$-adrenergic, glucagon, or PGE2 receptors, or via administration of forskolin or cell-permeant cyclic-AMP analogs. All these conditions, in fact, result in the activation of the $\mathrm{Na}^{+}$-dependent $\mathrm{Mg}^{2+}$ extrusion mechanism via phosphorylation. Conversely, pre-treatment of cells with inhibitors of adenylyl cyclase (e.g. RpcAMP) completely blocks $\mathrm{Mg}^{2+}$ mobilization irrespective of the modality utilized to enhance cAMP level (Wolf et al., 1997).

Because the $\mathrm{Mg}^{2+}$ extrusion mediated by this exchanger strictly depends on the presence of a physiological concentration of $\mathrm{Na}^{+}$in the extracellular milieu (Romani et al., 1993a; Fagan and Romani, 2000), it is generally accepted that the $\mathrm{Na}^{+}$-dependent $\mathrm{Mg}^{2+}$ extrusion mechanisms is a $\mathrm{Na}^{+} / \mathrm{Mg}^{2+}$ exchanger. As this $\mathrm{Mg}^{2+}$ extrusion mechanism has not been functionally cloned, we lack detailed information about its membrane abundance, structure, proximity to other cellular transporters with whom it may possibly interact, and stoichiometry. Early reports by Gunther and Vormann (1985) suggest the operation of electroneutral bases $\left(2 \mathrm{Na}^{+}{ }_{\text {in }}: 1 \mathrm{Mg}^{2+}{ }_{\text {out }}\right)$ at least in chicken or turkey erythrocytes. This notion has not been confirmed in mammalian erythrocytes including human red blood cells, in which the exchanger appears to operate electrogenically on a $1 \mathrm{Na}^{+}{ }_{\text {in }}: 1 \mathrm{Mg}^{2+}{ }_{\text {out }}$ ratio (Ludi and Schatzmann, 1987; Flatman, 1990; Xu and Willis, 1994). The discrepancy between these reports is not apparent although it may depend on the experimental model (i.e. cell isolation vs cultured cells), composition of incubation medium, or modality of cellular $\mathrm{Mg}^{2+}$ loading. Irrespective of the stoichiometry of exchange and the experimental model, however, all the obtained results consistently indicate a $\mathrm{K}_{\mathrm{m}}$ for $\mathrm{Na}^{+}$between 15 to 20 mM (Gunther, 1996; Tashiro and Konishi, 1997; Cefaratti et al., 1998). Pharmacological inhibition has done little to enhance our understanding of the modality of operation of the putative $\mathrm{Na}^{+} / \mathrm{Mg}^{2+}$ exchanger. Amiloride, imipramine and quinidine represent the three most widely utilized inhibitors of $\mathrm{Na}^{+}$-dependent $\mathrm{Mg}^{2+}$ extrusion (Gunther and Vormann, 1984; Feray and Garay, 1988; Gunther and Vormann, 1992a). However, because of their limited specificity, it is unclear as to whether they inhibit the $\mathrm{Na}^{+} / \mathrm{Mg}^{2+}$-exchanger directly, or indirectly by operating on other transport mechanisms including $\mathrm{Na}^{+}$and $\mathrm{K}^{+}$channel, ultimately altering the cell membrane potential and the driving force for $\mathrm{Mg}^{2+}$ transport across the plasma membrane.

Despite intense research, the $\mathrm{Na}^{+} / \mathrm{Mg}^{2+}$ exchanger is not cloned as yet. Work by Schweigel, Martens and colleagues (Schweigel et al., 2000) in mammalian rumen support the operation of a $\mathrm{Na}^{+} / \mathrm{Mg}^{2+}$ exchanger with kinetic parameters and characteristics similar to those described by other groups in a various mammalian cell types. Furthermore, by using a hybridoma screening procedure, this group has generated monoclonal antibodies against the $\mathrm{Na}^{+} / \mathrm{Mg}^{2+}$ exchanger present in porcine red blood cells. Incubation of sheep rumen epithelial cells in the presence of these antibodies has resulted in a significant inhibition of $\mathrm{Mg}^{2+}$ extrusion via this exchanger (Schweigel et al., 2000). Western blot analysis utilizing these antibodies has evidenced a protein band of $\sim 70 \mathrm{KDa} \mathrm{mr}$, which could tentatively correspond to the $\mathrm{Na}^{+} / \mathrm{Mg}^{2+}$ exchanger (Schweigel et al., 2000). This is the first time that information about the molecular size of the elusive $\mathrm{Na}^{+} / \mathrm{Mg}^{2+}$ exchanger is obtained, and the utilization of these antibodies could represent an ideal tool to identify and recognize this transporter in mammalian tissue.

\section{$\mathrm{Na}^{+}$-independent}

Under conditions in which no extracellular $\mathrm{Na}^{+}$is available to exchange for intracellular $\mathrm{Mg}^{2+}$, an alternative $\mathrm{Na}^{+}$-independent $\mathrm{Mg}^{2+}$ extrusion mechanism becomes evident. The specificity of this transporter, however, is far from being characterized. Different cations, including $\mathrm{Ca}^{2+}$ or $\mathrm{Mn}^{2+}$, as well as anions (e.g. $\mathrm{HCO}_{3}{ }^{-}, \mathrm{Cl}^{-}$, or choline) (Gunther, 1993; Ebel et al., 2002) have been reported to be utilized by this mechanism to extrude $\mathrm{Mg}^{2+}$ from the cell. Hence, it remains unclear whether we are in the presence of distinct transport mechanisms, or in the presence of a transporter that can operate as an antiporter for cations or a synporter for cations and anions based upon the experimental conditions. Also, it is unclear whether the $\mathrm{Na}^{+}$-independent pathway is activated by hormonal stimulation. Results obtained in liver cells (Keenan et al., 1996; Fagan and Romani, 2000; Fagan and Romani, 2001) indicate that the stimulation by mix adrenergic agonists (e.g. epinephrine) elicit a $\mathrm{Mg}^{2+}$ extrusion that is equivalent to the sum of the amounts of $\mathrm{Mg}^{2+}$ mobilized by the separate stimulation of $\mathrm{a}_{1}$ and $b$-adrenergic receptors. More specifically, the 
selective stimulation of $\mathrm{a}_{1}$-adrenergic receptors by phenylephrine requires the presence of physiological concentrations of both $\mathrm{Na}^{+}$and $\mathrm{Ca}^{2+}$ in the extracellular medium to elicit $\mathrm{Mg}^{2+}$ extrusion from liver cells (Fagan and Romani, 2000; Fagan and Romani, 2001). As phenylephrine stimulation appears to operate via $\mathrm{Ca}^{2+}-\mathrm{CaM}$ to induce $\mathrm{Mg}^{2+}$ extrusion, it is undefined whether this signalling pathway represents an alternative modality of activation of the $\mathrm{Na}^{+} / \mathrm{Mg}^{2+}$ exchanger, or it activates instead a different $\mathrm{Mg}^{2+}$ extrusion mechanism that can be reconciled at least in part with the aforementioned $\mathrm{Na}^{+}$-independent mechanism. Adding to the uncertainty, Ebel and collaborators (Ebel et al., 2002) have suggested that in red blood cells and hepatocytes the $\mathrm{Na}^{+}$-independent $\mathrm{Mg}^{2+}$ extrusion occurs via the choline transporter, which can be inhibited rather specifically by cinchona alkaloids.

One controversial issue is whether the $\mathrm{Na}^{+}-$ dependent and $\mathrm{Na}^{+}$-independent mechanisms operate as ATPases or require ATP for their operation. Reports by Gunther and collaborators (Gunther et al., 1990; Ebel et al., 2004) indicate a certain dependence of $\mathrm{Na}^{+}$-dependent $\mathrm{Mg}^{2+}$ extrusion on the presence of a physiological concentration of cellular ATP, the absence or decrease in cellular ATP content resulting in a reduced $\mathrm{Mg}^{2+}$ efflux from the cell (Gunther et al., 1990; Ebel et al., 2004). In the particular case of red blood cells, ATP and 2,3 bisphosphoglycerate both contribute to $\mathrm{Mg}^{2+}$ homeostasis and transport (Gunther et al., 1995). A regulatory effect of ATP on $\mathrm{Mg}^{2+}$ extrusion is not observed in purified liver plasma membrane vesicles (Cefaratti et al., 1998). It is true that no $\mathrm{Mg}^{2+}$ extrusion is observed in alkaline phosphatasetreated basolateral liver plasma membrane vesicles in the absence of ATP (Cefaratti and Romani, 2007), but this observation can reasonably be explained by the requirement of ATP to phosphorylate and activate the $\mathrm{Na}^{+} / \mathrm{Mg}^{2+}$ exchanger in the presence of PKA catalytic subunit (Cefaratti and Romani, 2007).

\section{$\mathrm{Mg}^{2+} / \mathrm{H}^{+}$}

This exchange mechanism, originally identified in A. thaliana and termed AtMHX, appears to be present in all plants (Shaul et al., 1999). This transporter presents 11 putative transmembrane domains, is exclusively localized in the vacuolar membrane of the plant, and electrogenically exchanges protons with $\mathrm{Mg}^{2+}$ or $\mathrm{Zn}^{2+}$. Interestingly, the ectopic overexpression of the transporter in tobacco plants sensitizes the plant to grow in the presence of elevated concentrations of $\mathrm{Mg}^{2+}$ (or $\mathrm{Zn}^{2+}$ ) (Shaul et al., 1999). Presently, no corresponding gene and transporter have been identified in mammalian cells, although evidence for a direct or indirect exchange of $\mathrm{Mg}^{2+}$ for $\mathrm{H}^{+}$under certain conditions has been provided by Gunther (Gunther and Vormann, 1990a). An enhanced extrusion of cellular $\mathrm{Mg}^{2+}$ has been observed in cells incubated in an acidic extracellular environment, in which an inwardly oriented $\mathrm{H}^{+}$gradient is imposed, provided that extracellular $\mathrm{Na}^{+}$is present (Gunther and Vormann, 1990a; Dalal and Romani, 2010). Amiloride derivates that inhibit with high affinity the $\mathrm{Na}^{+} / \mathrm{H}^{+}$exchanger are ineffective at blocking $\mathrm{Mg}^{2+}$ extrusion under these experimental conditions (Gunzel and Schlue, 1996), thus excluding that $\mathrm{Mg}^{2+}$ extrusion depends on the operation in reverse of the $\mathrm{Na}^{+} / \mathrm{H}^{+}$exchanger in parallel with the forward operation of the $\mathrm{Na}^{+} / \mathrm{Mg}^{2+}$ exchanger.

\section{Carriers}

This section groups several novel $\mathrm{Mg}^{2+}$ transport mechanisms of murine or human origin identified as a result of diet restriction (i.e. $\mathrm{Mg}^{2+}$-deficient diet) or medium restriction (i.e. low extracellular $\mathrm{Mg}^{2+}$ content). Due to the limited information available and controversies in their modus operandi, these transport mechanisms are nondescriptively classified as carriers.

\section{SLC41}

This family of $\mathrm{Mg}^{2+}$ transport mechanisms includes three members (A1, $A 2$, and $A 3)$ that are distantly related to the prokaryotic MgtE channel identified by Maguire (Smith et al., 1995). We will discuss predominantly SLC41A1 and A2 isoforms since no study has addressed function and structure of the SLC41A3 isoform.

SLC41A1 was the first member of this family to be identified (Wabakken et al., 2003). Based on the hydrophobic profile, this protein of $\sim 56 \mathrm{kDa} \mathrm{Mr}$ was predicted to possess 10 transmembrane domains, two of which presented a discrete level of homology with MgtE (Wabakken et al., 2003). Northern blot analysis indicates a wide distribution of the SLC41A1 gene, but its 
abundance varies markedly among tissues, the highest expression being in heart and testis and the lowest being in hematopoietic tissues and cells (Wabakken et al., 2003). While modest under basal conditions, the expression of this gene is markedly up-regulated in the renal cortex of mice fed low $\mathrm{Mg}^{2+}$ diet for several days (Goytain and Quamme, 2005b). Functional expression of mouse SLC41A1 in $X$. oocyte indicates that this protein can transport $\mathrm{Mg}^{2+}$ but also $\mathrm{Fe}^{2+}, \mathrm{Cu}^{2+}, \mathrm{Zn}^{2+}$ and $\mathrm{Cd}^{2+}$ while $\mathrm{Ca}^{2+}$ is not transported nor inhibits $\mathrm{Mg}^{2+}$ transport (Goytain and Qaumme, 2005b). The original characterization of $\mathrm{Mg}^{2+}$ generated current, which would be tentatively consistent with SLC41A1 operating as a channel (Goytain and Qaumme, 2005b) or an electrogenic exchanger similar to the $\mathrm{Na} / \mathrm{Ca}$ exchanger (Quednau et al., 2004), contrasts with a recent report by Kolisek et al., (2008). In this study the authors strongly advocate for SLC41A1 operating as a carrier and predominantly favouring $\mathrm{Mg}^{2+}$ efflux rather than influx. Following overexpression of SLC41A1 in HEK293 cells 1) no detectable $\mathrm{Mg}^{2+}$ currents is observed; 2) incubation of cells in $\mathrm{Mg}^{2+}$-free media results in a significant reduction of total $\mathrm{Mg}^{2+}$ content and $\left[\mathrm{Mg}^{2+}\right]_{i} ; 3$ ) the amplitude of $\mathrm{Mg}^{2+}$ loss depends on the number of SLC41A1 molecules expressed in the membrane and the induction time, and 4) the changes in $\left[\mathrm{Mg}^{2+}\right] \mathrm{i}$ are temperature sensitive but insensitive to the $\mathrm{Mg}^{2+}$ channel blocker CoHexamine (Kolisek et al., 2008). Furthermore, Kolisek and collaborators suggest that SLC41A1 forms high molecular weight complexes within the cell membrane with masses ranging from $1236 \mathrm{KDa}$ to $\sim 360 \mathrm{kDa}$, in stark contrast to the $56 \mathrm{KDa} \mathrm{Mr}$ of the monomer (Kolisek et al., 2008). Whether this observation indicates that the SLC41A1 monomer forms large multimeric complexes and/or interacts with auxiliary proteins is presently undefined. The reason for the absence of $\mathrm{Mg}^{2+}$-generated currents in this study as compared to the original observation by Goytain and Quamme (2005b) is not apparent. One possibility is that the murine (Goytain and Qaumme, 2005b) and human orthologs (Kolisek et al., 2008) operate differently. Based upon their high degree (>90\%) of homology, the mouse and human SLC41A1 are expected to operate in a similar manner. Yet, the possibility that point mutations can dramatically alter SLC41A1 ion specificity and modality of function cannot be completely dismissed. In this respect, it has to be noted that Goytain and Quamme (2005b) did not report a dependency of SLC41A1 operation on $\mathrm{Na}^{+}$or other cations or anions following expression in $X$. oocytes, whereas Kolisek and collaborators (Kolisek et al., 2008) observed a marked $\mathrm{Cl}^{-}$conductance following expression in HEK293 cells, which was abolished by DIDS. Whether this reflects the operation of additional transport mechanisms or the presence of structural differences in the cell membrane of HEK293 cells as compared to $X$. oocyte are possibilities that need further investigation.

Two additional SLC41 isoforms were identified in both humans and mice. SLC41A2 also transport $\mathrm{Mg}^{2+}$ as well as other divalent cations albeit with a different selectivity and inhibition profile than SLC41A1 (Goytain and Qaumme, 2005c). Aside from $\mathrm{Mg}^{2+}, \mathrm{SLC4} 1 \mathrm{~A} 2$ can carry $\mathrm{Ba}^{2+}, \mathrm{Ni}^{2+}, \mathrm{Co}^{2+}, \mathrm{Fe}^{2+}$ and $\mathrm{Mn}^{2+}$ but not $\mathrm{Ca}^{2+}, \mathrm{Cu}^{2+}$ or $\mathrm{Zn}^{2+}$. At variance of SLC41A1, $\mathrm{Mg}^{2+}$ transport via SLC41A2 is inhibited by $\mathrm{Ca}^{2+}$ (Goytain and Qaumme, 2005c). Both SLC41A1 and $\mathrm{A} 2$ generate $\mathrm{Mg}^{2+}$ currents in $X$. oocyte, and the ionic uptake is voltage dependent with an apparent affinity of $0.75 \mathrm{mM}$ and 0.31 $\mathrm{mM}$, respectively (Goytain and Qaumme, 2005b; Goytain and Qaumme, 2005c). Also SLC41A2 is widely expressed in mammalian tissues, but the expression is not affected by low $\mathrm{Mg}^{2+}$ diet (Goytain and Qaumme, 2005c). At the structural level, SLC41A2 shares $>70 \%$ homology with SLC41A1, and is also thought to have 10 transmembrane domains, although this hypothesis is not supported by a recent study (Sahni et al., 2007) that instead suggests a structural arrangement of $2 \times$ five trans-membrane spans linked together by a supplementary span motif. Hydrophobicity analysis indicates that the C- and $\mathrm{N}$ - termini are located on different sites of the cell membrane (Sahni et al., 2007), a configuration that will be consistent with a total of 11 trans-membrane segments.

\section{ACDP2}

The human ACDP gene family was identified by Wang and collaborators (Wang et al., 2003a) as a possible candidate of the urofacial syndrome. Mapped to 10q23-10q24 chromosome, this gene family comprises 4 isoforms differentially located in human tissues. ACDP1 is essentially restricted to brain. ACDP2 is more widely expressed, but still retains the highest expression in brain while 
being absent in skeletal muscle. Both ACDP3 and ACDP4 are ubiquitous, but have the highest expression in the heart (Wang et al., 2003b). The murine distribution of ACDP isoforms is very similar to that observed in humans (Wang et al., 2004). Termed ancient conserved domain protein because all isoforms have one domain in common that appears to be phylogenetically conserved from bacteria to man (Wang et al., 2003a), these proteins share $>50 \%$ homology to the CorC transporter, which together with CorB and CorD plays a role in $\mathrm{Mg}^{2+}$ efflux in prokaryotes. Over-expression of ACDP2 in $X$. oocytes indicates that this protein can transport a broad range of divalent cations including $\mathrm{Mg}^{2+}, \mathrm{Co}^{2+}$, $\mathrm{Mn}^{2+}, \mathrm{Sr}^{2+}, \mathrm{Ba}^{2+}, \mathrm{Cu}^{2+}$, and $\mathrm{Fe}^{2+}$ while $\mathrm{Zn}^{2+}$ can inhibit its activity (Goytain and Quamme, 2005d). $\mathrm{Mg}^{2+}$ transport via ACDP2 is voltage dependent and occurs with a $\mathrm{K}_{\mathrm{m}}$ of $\sim 0.5 \mathrm{mM}$. The transport, however, operates independently of $\mathrm{Na}^{+}$or $\mathrm{Cl}^{-}$ ions (Goytain and Quamme, 2005d). As in the case of SLC41A1, the ACDP2 gene becomes overexpressed following a $\mathrm{Mg}^{2+}$ deficient diet (Goytain and Qaumme, 2005).

\section{NIPA}

Located in the SPG6 locus of chromosome 15q11q13, the NIPA1 gene is so called for 'nonimprinted in Prader-Willi/Angelman syndrome, a disease characterized by a complex developmental and multisystem disorder (Butler, 1990). Located among about 30 genes linked to this disease (Butler, 1990), NIPA1 has also been implicated in autosomal dominant hereditary spastic paraplegia (HSP). The human and mouse genome contain four members of the NIPA family, termed NIPA1 trough NIPA4, with an overall similarity of $\sim 40 \%$. Homology between human and mice proteins is high at around $98 \%$. Studies conducted by Goytain and Quamme indicate that both NIPA1 and NIPA2 (Goytain et al., 2007; Goytain et al., 2008a) can operate as $\mathrm{Mg}^{2+}$ transporter. Characterized by a sequence of 323 (NIPA1) and 359 amino acids (NIPA2) arranged to form 9 and 8 transmembrane spans, respectively, these two proteins can be distinguished based on their $\mathrm{K}_{\mathrm{m}}$ and specificity for $\mathrm{Mg}^{2+}$. While both proteins transport $\mathrm{Mg}^{2+}$ in a saturable fashion, NIPA1 has a $\mathrm{K}_{\mathrm{m}}$ for $\mathrm{Mg}^{2+}$ of $\sim 0.66 \mathrm{mM}$ (Goytain et al., 2007) as compared to $0.31 \mathrm{mM}$ for NIPA2 (Goytain et al., 2008a). In addition, NIPA2 is highly specific for $\mathrm{Mg}^{2+}$ while NIPA1 can also transport $\mathrm{Sr}^{2+}, \mathrm{Fe}^{2+}$ or $\mathrm{Co}^{2+}$, albeit to a lesser extent (Goytain et al., 2007). NIPA3, instead, transports $\mathrm{Sr}^{2+}, \mathrm{Ba}^{2+}, \mathrm{Fe}^{2+}$ and $\mathrm{Cu}^{2+}$ whereas NIPA4 transport $\mathrm{Sr}^{2+}$ and $\mathrm{Ba}^{2+}$. Interestingly, point mutations in NIPA1 (i.e. $G^{100} R$ or $T^{45} R$ ) represent the basis for the insurgence of autosomal dominant HSP (Rainier et al., 2003). Both the glycine and threonine residues are conserved among ortholog NIPA1 channels in different species. There are no similar consensus sites in the paralogs NIPA2, NIPA3 and NIPA4, implying that the folding of these proteins might be different. Although NIPA2 appears to be normal in HSP patients, it cannot functionally replace NIPA1 to ameliorate HSP symptoms, nor can NIPA3 or NIPA4 substitute for the defective NIPA1. This is somewhat surprising for NIPA2 as the gene encoding for this protein is part of the 30 gene clusters associated with the Prade-Willi syndrome together with NIPA1. Presently, there is no information available as to whether the Prade-Willi syndrome presents alterations in $\mathrm{Mg}^{2+}$ homeostasis.

\section{Huntingtin}

The use of oligonucleotide microarray analysis to screen for $\mathrm{Mg}^{2+}$-regulated transcripts in epithelial cells indicates Huntingtin-interacting protein 14 (HIP14) and its related protein HIP14-like (HIP14L) as significantly ( 3fold) upregulated by low-extracellular $\mathrm{Mg}^{2+}$ (Goytain et al., 2008b). Formed by approximately 532 amino acids organized in 6 transmembrane spans, HIP14 presents a strong sequence similarity to the ankyrin repeat protein Akr1p (Li and Li, 2004), and a $69 \%$ homology to HIP14L. In addition, HIP14 possesses a cytoplasmic DHHC cysteinerich domain. Defined by the Asp-His-His-Cys sequence motif, this domain confers palmitoylacyltransferase activity to the protein and gives it the ability to palmitoylate membrane components and modulate their structure. $\mathrm{Mg}^{2+}$ accumulation via HIP14 and HIP14L appears to be electrogenic, voltage-dependent, and saturable with $\mathrm{K}_{\mathrm{m}}$ of $\sim 0.87$ and $\sim 0.74 \mathrm{mM}$, respectively (Goytain et al., 2008 b). Inhibition of palmitoylation activity by 2 Br-palmitate or deletion of the DHHC domain decreases HIP14 mediated $\mathrm{Mg}^{2+}$ accumulation by $\sim 50 \%$, suggesting that palmitoylation is not required for basal $\mathrm{Mg}^{2+}$ transport. The widespread tissue distribution and intracellular localization of HIP14 (nuclear and perinuclear regions, Golgi complex, mitochondria, microtubules, endosomes, clathrin-coated and non- 
coated vesicles, and plasma membrane)(Yanai et al., 2006) has implicated this protein in numerous cellular processes including transcriptional regulation, mitochondrial bioenergetics, structural scaffolding, vesicle trafficking, endocytosis, and dendrite formation (Yanai et al., 2006). Its primary location, however, appears to be in the Golgi and post-Golgi vesicles (Yanai et al., 2006; Goytain et al., 2008b). Hence, it can be hypothesized that the ability of this protein to favour $\mathrm{Mg}^{2+}$ accumulation is linked to some extent to the role HIP14 plays in the physiological functioning of the compart-ments in which the protein is located. At the pathological level, the neuropathology of Huntington disease and the occurrence of progressive neurodegenerative disorders, cognitive deficits and choreic movements typical of this disease are linked to the abnormal expansion of glutamine residues from $<34$ to $>37$ at the $18^{\text {th }}$ amino acid position ( $\mathrm{Li}$ and $\mathrm{Li}, 2004)$. Presently, the mechanism responsible for the insurgence of these defects is unknown ( $\mathrm{Li}$ and $\mathrm{Li}, 2004$ ). Similarly unknown is whether the poly-glutamine expansion alters $\mathrm{Mg}^{2+}$ transport, and whether perturbation of $\mathrm{Mg}^{2+}$ homeostasis plays any role in the uprising of the neuronal defects typical of Huntington disease.

\section{$\mathrm{Mg}^{2+}$ transport in purified plasma membrane vesicles}

Because of the lack of functional cloning, several laboratories including ours have resorted to the use of plasma membrane vesicles to better characterize how different $\mathrm{Mg}^{2+}$ extrusion mechanisms operate in particular cell types. The plasma membrane model presents several advantages including: 1 ) the possibility to provide a well defined ionic extra- and intra-vesicular milieu composition to determine the modality of operation of the various $\mathrm{Mg}^{2+}$ transporters; and 2) the ability to investigate the operation of the different $\mathrm{Mg}^{2+}$ extrusion mechanisms in the absence of $\mathrm{Mg}^{2+}$ buffering by ATP, proteins or other cytosolic components, and partitioning within intracellular organelles. By purifying total liver plasma membrane or cardiac sarcolemmal vesicles as well as specific hepatic subpopulations enriched in basolateral or apical domains, our laboratory has been able to provide a better understanding of the selective location and specificity of the $\mathrm{Na}^{+}$- dependent and $\mathrm{Na}^{+}-$ independent $\mathrm{Mg}^{2+}$ extrusion mechanisms in liver cells and cardiac myocytes.

The $\mathrm{Na}^{+}$-dependent extrusion mechanism located in the basolateral domain of the hepatocyte is selectively activated by $\mathrm{Na}^{+}$(Cefaratti et al., 1998; Cefaratti et al., 2000), and specifically inhibited by imipramine (Cefaratti et al., 2000), but not amiloride and amiloride derivates (Cefaratti et al., 2000). Furthermore, the operation of the exchanger is completely inhibited by pretreatment of basolateral vesicles with alkaline phosphatase, and restored by loading the vesicles with ATP and PKA catalytic subunit (Cefaratti and Ruse, 2007; Cefaratti and Romani, 2007), leaning further support to the notion that this exchange mechanism becomes operative upon phosphorylation by cAMP. The $\mathrm{Na}^{+} / \mathrm{Mg}^{2+}$ exchanger continues to operate in the presence of zero trans $\mathrm{Mg}^{2+}$ across the plasma membrane (i.e., 20 $\mathrm{mM} \mathrm{Mg}^{2+}$ inside and outside the vesicles), an indication that $\mathrm{Mg}^{2+}$ extrusion does not depend on the trans-membrane gradient for $\mathrm{Mg}^{2+}$ but rather on that of $\mathrm{Na}^{+}$, with a $\mathrm{K}_{\mathrm{m}}$ lower than $20 \mathrm{mM}$ (Cefaratti et al., 1998), in good agreement with kinetic data obtained in isolated hepatocytes (Romani et al., 1993b) and other cell types (Tashiro and Konishi, 1997). Experiments based on $\mathrm{TPP}^{+}$distribution have confirmed the electrogenicity of this exchange mechanism in plasma membrane vesicles, and suggested a $1 \mathrm{Na}^{+}$in for $1 \mathrm{Mg}^{2+}$ out exchange ratio under the majority of experimental conditions tested (Cefaratti et al., 1998; Cefaratti et al., 2000; Cefaratti and Ruse, 2007). Interestingly, removal of intravesicular $\mathrm{Cl}$ switches the stoichiometric ratio of the exchanger from electrogenic to electroneutral (i.e. $2 \mathrm{Na}^{+}$in for $1 \mathrm{Mg}^{2+}$ out) (Cefaratti and Romani, 2011). Moreover, in the presence of intravesicular $\mathrm{Cl}^{-}$an extrusion of $\sim 35 \mathrm{nmol} \mathrm{Cl} / \mathrm{mg}$ protein is observed within $1 \mathrm{~min}$ from the addition of external $\mathrm{Na}^{+}$, in concomitance with the extrusion of $\mathrm{Mg}^{2+}$ and the accumulation of external $\mathrm{Na}^{+}$into the vesicles (Cefaratti and Romani, 2011). Chloride ion extrusion is not inhibited by anion transport inhibitors like DNDS, DIDS, or niflumic acid, nor is it blocked by NKCC1 inhibitors like bumetanide or furosemide (Cefaratti and Romani, 2011), thus excluding that it occurs via one of these mechanisms. The only agent able to block the $\mathrm{Cl}^{-}$extrusion is imipramine (Cefaratti and Romani, 2011), which specifically 
blocks the $\mathrm{Na}^{+} / \mathrm{Mg}^{2+}$ exchanger operating in the basolateral domain of the hepatocyte (Cefaratti et al., 2000). Hence, it would appear that $\mathrm{Cl}^{-}$can be extruded either via the $\mathrm{Na}^{+} / \mathrm{Mg}^{2+}$ exchanger or via $\mathrm{Cl}^{-}$channels for partial charge compensation (Cefaratti and Romani, 2011). The possibility of a $\mathrm{Cl}^{-}$extrusion via the $\mathrm{Na}^{+} / \mathrm{Mg}^{2+}$ exchanger has been suggested by (Rasgado-Flores et al., 1994) in dialyzed squid axons, and it would also be in good agreement with the observation by Gunther and collab-orators that intracellular $\mathrm{Cl}$ - has a stimulatory role on the activity of the $\mathrm{Na}^{+} / \mathrm{Mg}^{2+}$ antiport in red blood cells (Ebel and Gunther, 2003).

The basolateral domain of the hepatocyte is not the only site in which the operation of a $\mathrm{Mg}^{2+}$ extrusion mechanism has been observed. Experiments carried out in liver plasma membrane vesicles enriched in apical domain indicate the presence of two apparently distinct, unidirectional $\mathrm{Mg}^{2+}$ transport mechanisms, which extrude intravesicular $\mathrm{Mg}^{2+}$ for extravesicular $\mathrm{Na}^{+}$ and $\mathrm{Ca}^{2+}$, respectively (Cefaratti et al., 2000).

This apical $\mathrm{Na}^{+}$-dependent $\mathrm{Mg}^{2+}$ transporter presents a $\mathrm{K}_{\mathrm{m}}$ for $\mathrm{Na}^{+}$comparable to the basolateral transporter, and selectively uses $\mathrm{Na}^{+}$over other monovalent cations in a manner similar to the basolateral exchanger. Like the basolateral antiport, this exchanger transports electrogenically $1 \mathrm{Na}^{+}{ }_{\text {in }}: 1 \mathrm{Mg}^{2+}{ }_{\text {out }}$ (Cefaratti et al., 2000). From the pharmacological standpoint the apical and basolateral exchanger can be distinguished based on the specific inhibition of the apical exchanger by amiloride (Cefaratti et al., 2000), although it retains a significant level of sensitivity to imipramine inhibition, whereas only imipramine can block the basolateral antiport (Cefaratti et al., 2000). The apical exchanger can also be distinguished from the basolateral antiport based on its inability to operate in reverse mode (Cefaratti et al., 2000).

The $\mathrm{Ca}^{2+}$-dependent $\mathrm{Mg}^{2+}$ extrusion mechanism is specifically located in the apical domain of the hepatocytes, is activated by micromolar $\mathrm{Ca}^{2+}$ concentration $\left(\mathrm{K}_{\mathrm{m}} \leq 50 \mu \mathrm{M}\right)$, and is insensitive to alkaline phosphatase pre-treatment (Cefaratti and Ruse, 2007; Cefaratti and Romani, 2007). The $\mathrm{Mg}^{2+}$ extrusion elicited by this antiport occurs on electro-neutral basis (i.e. $1 \mathrm{Ca}^{2+}{ }_{\text {in }}: 1 \mathrm{Mg}^{2+}{ }_{\text {out }}$ ) (Cefaratti and Ruse, 2007). The exchanger, however, is not $\mathrm{Ca}^{2+}$ specific, as $\mathrm{Mg}^{2+}$ extrusion is observed following the extravesicular addition of micromolar concentrations of other divalent cations $\left(\mathrm{Ca}^{2+}>>\mathrm{Co}^{2+}=\mathrm{Mn}^{2+}>\mathrm{Sr}^{2+}>>\mathrm{Ba}^{2+}>\mathrm{Cu}^{2+}>>\mathrm{Cd}^{2+}\right)$ (Cefaratti et al., 2000). Similarly to the apical $\mathrm{Na}^{+} / \mathrm{Mg}^{2+}$ antiport, the $\mathrm{Ca}^{2+}$-dependent mechanism is inhibited by amiloride or imipramine (Cefaratti et al., 2000). This observation raises the question as to whether we are in the presence of two distinct apical mechanisms, modulated by $\mathrm{Na}^{+}$and cations, respectively. Several lines of evidence, however, do not fully support this possibility. First, the coaddition of $\mathrm{Na}^{+}$and $\mathrm{Ca}^{2+}$ in purified apical plasma membrane vesicles subpopulations does not appear to significantly enlarge $\mathrm{Mg}^{2+}$ extrusion (Romani, personal observation). Second, amiloride inhibits both exchangers to a comparable extent at a similar concentration (Cefaratti et al., 2000). Third, alkaline phosphatase treatment does not affect the $\mathrm{Mg}^{2+}$ extrusion elicited by either exchanger in apical liver plasma membrane vesicles (Cefaratti and Ruse, 2007; Cefaratti and Romani, 2007). Fourth, neither of these exchangers can operate in reverse at variance of the basolateral $\mathrm{Na}^{+} / \mathrm{Mg}^{2+}$ antiport. Taken together, these observations suggest the operation of a non-selective exchange mechanism able to utilize monovalent or divalent cations to promote $\mathrm{Mg}^{2+}$ extrusion. At the present time, the physiological implication for the operation of such an exchanger in the apical domain of the hepatocyte is not fully clear. Circumstantial evidence, however, might support a possible role of $\mathrm{Mg}^{2+}$ in limiting $\mathrm{Ca}^{2+}$ sedimentation in the bile with consequent formation of bile stones (Moore, 1990).

The operation of functionally similar $\mathrm{Na}^{+}-$and $\mathrm{Ca}^{2+}$-dependent $\mathrm{Mg}^{2+}$ extrusion mechanisms has also been observed in cardiac sarcolemma vesicles (Cefaratti and Romani, 2007). As in the case of liver plasma membrane vesicles, cardiac sarcolemma vesicles do not require intravesicular ATP for the operation of $\mathrm{Mg}^{2+}$ transporters (Cefaratti and Romani, 2007), and pretreatment of the vesicles with alkaline phosphatase specifically inhibits the $\mathrm{Na}^{+}$-dependent $\mathrm{Mg}^{2+}$ extrusion mechanism (Cefaratti and Romani, 2007). For technical reasons, it is presently unknown whether the $\mathrm{Ca}^{2+} / \mathrm{Mg}^{2+}$ exchanger in sarcolemmal vesicles can also utilize $\mathrm{Na}^{+}$to promote $\mathrm{Mg}^{2+}$ extrusion. 
The operation of specific $\mathrm{Mg}^{2+}$ accumulation mechanisms has also been observed in plasma membrane vesicles from brush border cells of rabbit ileum (Juttner and Ebel, 1998) and from the duodenum and jejunum of rat (Baillien and Cogneau, 1995). By using membrane vesicles from rabbit ileum and cell permeant and nonpermeant Mag-Fura, Juttner and Ebel have observed the operation of a saturable $\mathrm{Mg}^{2+}$ uptake mechanism when the intracellular $\mathrm{Na}^{+}$ concentration is higher than the extracellular one (Baillien and Cogneau, 1995). This process becomes inoperative when the $\mathrm{Na}^{+}$gradient is reversed (i.e., $\left[\mathrm{Na}^{+}\right]_{i}<\left[\mathrm{Na}^{+}\right]_{0}$ ), the vesicles are in zero trans condition for $\mathrm{Na}^{+}$, or external $\mathrm{Na}^{+}$is removed. At variance with the transporter observed in liver plasma membrane, the pathway in ileum vesicles is not reversible and appears to be electroneutral. Yet, it possesses a $\mathrm{K}_{\mathrm{m}}$ for $\mathrm{Na}^{+}$of $16 \mathrm{mM}$, a value similar to the $K_{m}$ calculated in liver plasma membranes (Cefaratti et al., 1998), in smooth muscle cells from guinea pig tenia caecum (Tashiro and Konishi, 1997), and in chicken erythrocytes (Schatzmann, 1993). Another similarity with the transporter operating in basolateral liver plasma membranes is the lack of inhibition by amiloride analogs. In good agreement with reports from Gunther and collaborators (Ebel and Gunther, 2003), this transporter is modulated by intravesicular anions, especially $\mathrm{Cl}^{-}$and $\mathrm{SCN}^{-}$, and markedly stimulated by antagonists of anion transport (e.g., $\mathrm{H}_{2}$-DIDS) (Juttner and Ebel, 1998).

The main difference between plasma membrane vesicles from duodenum and jejunum (Baillien and Cogneau, 1995) is that a single $\mathrm{Mg}^{2+}$ uptake mechanism operates in the duodenum with a $\mathrm{K}_{\mathrm{m}}$ of $0.8 \mathrm{mM}$, whereas two transporters operate in the jejunum with $\mathrm{K}_{\mathrm{m}}$ values of 0.15 and $2.4 \mathrm{mM}$, respectively. In both these experimental models, $\mathrm{Mg}^{2+}$ but not $\mathrm{Ca}^{2+}$ accumulation is reduced in the presence of alkaline phosphatase inhibitors (Baillien et al., 2005), suggesting that $\mathrm{Ca}^{2+}$ and $\mathrm{Mg}^{2+}$ are transported via distinct pathways. This hypothesis is further supported by the observation that $\mathrm{Mg}^{2+}$ accumulation is inhibited by amiloride but not by $\mathrm{Ca}^{2+}$ channel antagonists. Consistent with the report by Juttner and Ebel (Juttner and Ebel, 1998), $\mathrm{Mg}^{2+}$ accumulation is stimulated by an intravesicular electronegative potential or an alkaline $\mathrm{pH}_{\circ}$ (Baillien and Cogneau, 1995). The effect of external pH, however, is lost when $\left[\mathrm{Mg}^{2+}\right]_{0}>1 \mathrm{mM}$ (Baillien and Cogneau, 1995). Under the latter condition, $\mathrm{Mg}^{2+}$ accumulation is enhanced by the presence of $\mathrm{Na}^{+}$ or $\mathrm{K}^{+}$in the extravesicular space but is inhibited by the presence of divalent cations $\left(\mathrm{Co}^{2+}>\mathrm{Mn}^{2+}>\mathrm{Ca}^{2+}>\mathrm{Ni}^{2+}>\mathrm{Ba}^{2+}>\mathrm{Sr}^{2+}\right) \quad$ (Baillien and Cogneau, 1995).

\section{Regulation of $\mathrm{Mg}^{2+}$ transport and homeostasis}

While mammalian cells retains their basal $\mathrm{Mg}^{2+}$ content virtually unchanged under resting conditions, compelling evidence supports the ability of different hormones to induce the movement of large amount of $\mathrm{Mg}^{2+}$ in either direction across the eukaryotes cell membrane. As a result of these movements, changes in serum, total and to a lesser extent free $\mathrm{Mg}^{2+}$ content have been observed. Further, these changes have resulted in detectable variations in $\mathrm{Mg}^{2+}$ level within organelles, especially mitochondria, with major repercussions on cellular bioenergetics. A full understanding of the physiological relevance of these changes in cellular $\mathrm{Mg}^{2+}$ content is far from complete. Yet, a picture is slowly emerging, which relates changes in total $\mathrm{Mg}^{2+}$ content to the utilization of metabolites (e.g. glucose) or to meaningful changes in $\mathrm{Mg}^{2+}$ content within discrete portions of the cell or cellular organelles, whereby variations in total $\mathrm{Mg}^{2+}$ content translate into changes in concentrations able to modulate the activity of specific enzymes located within these compartments.

\section{$\mathrm{Mg}^{2+}$ extrusion}

Several classes of hormones induce $\mathrm{Mg}^{2+}$ extrusion from various cell types or perfused tissues. For the most part, these hormones are catecholamine or hormones that increase cellular CAMP level by activating different GPCR receptors at the cell membrane level. The extrusion elicited by these agents affects to a varying extent the $\mathrm{Mg}^{2+}$ pools present within cytoplasm as well as within cellular compartments. The extrusion across the cell membrane primarily occurs via the $\mathrm{Na}^{+} / \mathrm{Mg}^{2+}$ exchanger previously described although a (partial) contribution of the $\mathrm{Na}^{+}$independent pathway cannot be excluded. Magnesium extrusion can also be observed following metabolic treatments that decrease cellular ATP content, the main $\mathrm{Mg}^{2+}$ buffering component. Interestingly, several of the 
hormones that induce $\mathrm{Mg}^{2+}$ extrusion also elicit a glucose output from hepatocytes. Hence, it would appear that at least in this organ $\mathrm{Mg}^{2+}$ extrusion is functionally associated with glucose transport and utilization.

\section{Cyclic-AMP Dependent Extrusion}

Elliot and Rizack were the first to report an accumulation of $\mathrm{Mg}^{2+}$ in adipocytes stimulated by adreno-corticotrophic hormone in 1974, although they did not elucidate the modality of transport (Elliot and Rizack, 1974). The first extensive characterization of an hormonal effect on $\mathrm{Mg}^{2+}$ transport was provided by Maguire and colleagues in S49 lymphoma cells and primary lymphocytes stimulated by beta-adrenergic agonist or PGE1 (Bird and Maguire, 1978; Erdos and Maguire, 1980; Erdos and Maguire, 1983; Grubbs et al., 1984). Maguire and Erdos (1978) also provided the first observation that stimulation of protein kinase $\mathrm{C}$ enhanced $\mathrm{Mg}^{2+}$ influx in $\mathrm{S} 49$ cells whereas beta-adrenergic stimulation inhibited the process. Observation carried out in $\$ 49$ cells lacking protein kinase $A$ or adenylyl cyclase, however, indicated that CAMP was not mediating the inhibitory effect of beta-adrenergic agonists (Maguire and Erdos, 1978; Maguire and Erdos, 1980). At variance of what observed in primary lymphocytes (Wolf et al., 1997), $\mathrm{Mg}^{2+}$ transport in S49 cells appears to be independent of extracellular $\mathrm{Na}^{+}$concentration or membrane potential (Grubbs and Maguire, unpublished observation). Further, $\mathrm{Mg}^{2+}$ turnover in $\mathrm{S} 49$ required more than 40 hours as compared to the much faster $\mathrm{Ca}^{2+}$ turn-over, which was accomplished in less than 3 hours (Grubbs et al., 1985).

These initial observations were followed by a long series of report supporting the notion that $b$ adrenergic agonists and other hormones control $\mathrm{Mg}^{2+}$ homeostasis in mammalian cells. In the majority of eukaryotic cells, hormones or agents that increase cellular cAMP level elicit a significant extrusion of $\mathrm{Mg}^{2+}$ into the extracellular space or the circulation (Vormann and Gunther, 1987; Romani and Scarpa, 1990a; Romani and Scarpa, 1990b). This effect has been observed in cardiac ventricular myocytes (Vormann and Gunther, 1987; Romani and Scarpa, 1990a; Romani et al., 1993a; Howarth et al., 1994), liver cells (Romani and Scarpa, 1990b; Gunther et al., 1991; Romani et al., 1993b; Fagan and Romani, 2000; Fagan and Romani, 2001), red blood cells
(Matsuura et al., 1993), thymocytes (Gunther and Vormann, 1990b), and Erhlich ascites cells (Wolf et al., 1994) among other cells (see Romani and Scarpa, 2000 for a more comprehensive list), as well as in whole anesthetized animals (Gunther and Vormann, 1992; Keenan et al., 1995). In all cellular models, $\mathrm{Mg}^{2+}$ extrusion is a fast process that reaches the maximum within $8 \mathrm{~min}$ from the application of the stimulus irrespective of the hormone (catecholamine, isoproterenol, glucagon, PGE1, or arachidonic acid) (Vormann and Gunther, 1987; Gunther and Vormann, 1990a; Romani and Scarpa, 1990a; Romani and Scarpa 1990b; Matsuura et al., 1993; Howarth et al., 1994; Wolf et al., 1994) or agent (i.e. forskolin or cell permeant cyclic AMP analogs) (Vormann and Gunther, 1987; Gunther and Vormann, 1990a; Romani and Scarpa, 1990a; Romani and Scarpa, 1990b; Matsuura et al., 1993; Romani et al., 1993b; Fagan and Romani 2000; Fagan and Romani, 2001) utilized to increase cellular cAMP level. The key role of cAMP in modulating $\mathrm{Mg}^{2+}$ extrusion is further emphasized by the observation that pretreatment with hormones or agents that either decrease CAMP production, such as carbachol (Romani and Scarpa, 1990a; Romani and Scarpa, 1990b; Romani et al., 1993b) and insulin (Romani et al., 2000), or prevent PKA activation (e.g. Rp-cAMP (Wolf et al., 1997)) inhibits cellular $\mathrm{Mg}^{2+}$ mobilization. In an open perfusion system, the amount of $\mathrm{Mg}^{2+}$ extruded from the organ returns towards baseline level within $8 \mathrm{~min}$ from the application of the agonist irrespective of its dose or persistence in the perfusate (Romani and Scarpa, 1990a; Romani and Scarpa, 1990b), suggesting a rapid mobilization of $\mathrm{Mg}^{2+}$ from a well defined cellular pool that is rapidly depleted of its content. This notion is further supported by the evidence that submaximal doses of agonist sequentially infused within a few minutes from each other elicit $\mathrm{Mg}^{2+}$ extrusions of progressively decreasing amplitudes (Gunther and Vormann, 1990a). Under all these conditions, limited changes in cytosolic free $\left[\mathrm{Mg}^{2+}\right]_{i}$ are observed (Fatholahi et al., 2000; Amano et al., 2000), suggesting that $\mathrm{Mg}^{2+}$ is rapidly released from its binding and buffering sites, or form cellular organelle(s) and extruded across the cell membrane. Independent of the hormone utilized the cAMP-mediated $\mathrm{Mg}^{2+}$ extrusion occurs via the putative $\mathrm{Na}^{+} / \mathrm{Mg}^{2+}$ exchanger described previously. In fact, either the removal of extracellular $\mathrm{Na}^{+}$(Gunther, 1996) or 
the presence of agents like amiloride (Vormann and Gunther, 1987; Gunther, 1996), which inhibits $\mathrm{Na}^{+}$transport albeit in a non-specific manner, abolishes to a large extent the $\mathrm{Mg}^{2+}$ extrusion. Under either of these conditions, the amplitude of $\mathrm{Mg}^{2+}$ extrusion across the cell membrane is hampered and a more sustained rise in cytosolic free $\left[\mathrm{Mg}^{2+}\right]_{i}$ is observed (Fatholahi et al., 2000; Amano et al., 2000), supporting the notion that blocking $\mathrm{Na}^{+}$transport prevents $\mathrm{Mg}^{2+}$ from being extruded across the cell membrane but not its released from binding/buffering sites and/or cellular pool(s) into the cytoplasm.

\section{Cyclic-AMP Independent Extrusion}

In 1989, Jakob and collaborators reported the first observation that phenylephrine can also elicit $\mathrm{Mg}^{2+}$ extrusion from liver cells via alpha ${ }^{-}$ adrenergic stimulation (Jakob et al., 1989). Subsequently, our laboratory (Keenan et al., 1996; Fagan and Romani, 2000) confirmed this observation and provided the first evidence that the stimulation of $\alpha_{1}$ - and $\beta$-adrenergic receptor are not alternative but rather additive and complementary processes in eliciting $\mathrm{Mg}^{2+}$ extrusion from liver cells, especially when the two classes of receptors are stimulated by mix adrenergic agonists such as epinephrine or norepinephrine (Keenan et al., 1996; Fagan and Romani, 2000). Pre-treatment with insulin only abolishes $\beta$-adrenergic receptor mediated $\mathrm{Mg}^{2+}$ extrusion but leaves unaffected the $\mathrm{Mg}^{2+}$ mobilization mediated via $\alpha_{1}$-adrenergic receptors (Keenan et al., 1996). The inhibitory effect of insulin persists even in cells treated with cellpermeant CAMP analogs (Keenan et al., 1996). A similar inhibitory effect of insulin on b-adrenergic receptor mediated, CAMP-modulated $\mathrm{Mg}^{2+}$ extrusion has been observed in cardiac myocytes (Romani et al., 2000). This inhibition has been largely interpreted as the consequence of an inhibitory effect of insulin on the b-adrenergic receptor (Karoor et al., 1995) or a positive effect of the hormone on the cytosolic phosphodiesterase degrading cAMP (Smoake et al., 1995). A more recent report by Romero and collaborators (Ferreira et al., 2004), however, suggests that insulin can also modulate directly the $\mathrm{Na}^{+} / \mathrm{Mg}^{2+}$ exchanger, at least in red blood cells.

Fagan and Romani (2000; 2001) further investigated the modality of $\mathrm{Mg}^{2+}$ extrusion following $\alpha_{1}$-adrenergic receptor stimulation in liver cells. Their observation indicates that phenylephrine-induced $\mathrm{Mg}^{2+}$ extrusion strictly depends on the activation of capacitative $\mathrm{Ca}^{2+}$ entry (Fagan and Romani, 2001). Inhibition of $\mathrm{IP}_{3^{-}}$ induced $\mathrm{Ca}^{2+}$ release from the endoplasmic reticulum, chelation of cytosolic $\mathrm{Ca}^{2+}$, or inhibition of $\mathrm{Ca}^{2+}$ entry at the plasma membrane level all result in the complete inhibition of $\mathrm{Mg}^{2+}$ extrusion from the hepatocyte (Fagan and Romani, 2001). The scant information available about possible binding of $\mathrm{Mg}^{2+}$ by cellular proteins prevented the authors from ascertaining whether the extruded $\mathrm{Mg}^{2+}$ was mobilized from the ER or displaced from cytosolic binding sites following the massive entry of $\mathrm{Ca}^{2+}$ across the hepatocyte cell membrane (Fagan and Romani, 2001, and refs therein). Extracellular $\mathrm{Na}^{+}$and $\mathrm{Ca}^{2+}$ are both required for the phenylephrine-induced $\mathrm{Mg}^{2+}$ extrusion to occur (Fagan and Romani, 2001). The absence of extracellular $\mathrm{Ca}^{2+}$, in fact, decreases the amplitude of $\mathrm{Mg}^{2+}$ extrusion by $\sim 15 \%$ to $20 \%$ whereas extracellular $\mathrm{Na}^{+}$is responsible for the remaining $80 \%$ to $85 \%$ of the extrusion. It is presently unclear whether $\mathrm{Mg}^{2+}$ extrusion occurs via the $\mathrm{Ca}^{2+}$-activated $\mathrm{Na}^{+}$dependent mechanism observed in the apical domain of the hepatocyte, or whether $\mathrm{Na}^{+}$is required to maintain membrane potential and facilitate $\mathrm{Ca}^{2+}$ entry across the hepatocyte cell membrane. It has to be noted, however, that in the absence of receptor activation, thapsigargin administration can mimic phenylephrine stimulation and elicit $\mathrm{Mg}^{2+}$ extrusion from the hepatocyte, even in the absence of extracellular $\mathrm{Ca}^{2+}$ (Fagan and Romani, 2001), although to a lesser extent. Hence, it would appear that an optimal level of cytosolic $\mathrm{Ca}^{2+}$ has to be attained in order for $\mathrm{Mg}^{2+}$ extrusion to occur via displacement from cellular binding sites or via a $\mathrm{Ca}^{2+}$-calmodulin-activated mechanism (Fagan and Romani, 2001).

\section{$\mathrm{Mg}^{2+}$ homeostasis and glucose}

The presence of redundant $\mathrm{Mg}^{2+}$ extrusion mechanisms or modalities of activation of a common $\mathrm{Mg}^{2+}$ extrusion pathway raises the question of the physiological significance of $\mathrm{Mg}^{2+}$ mobilization in mammalian cells. In the case of cardiac myocytes, an increase in extracellular $\mathrm{Mg}^{2+}$ level has been associated with a modulatory effect on the open probability of the L-type $\mathrm{Ca}^{2+}$ channels (Wang and Berlin, 2006) and a 
temporary decrease in SA node action potential (Howarth et al., 1994). In the case of liver cells, instead, $\mathrm{Mg}^{2+}$ transport has been associated with a regulatory role on glucose transport and utilization. Under conditions in which hormones like catecholamine (Keenan et al., 1996; Fagan and Romani, 2000), glucagon (Fagan and Romani, 2000), or phenylephrine (Keenan et al., 1996; Fagan and Romani, 2000) elicit $\mathrm{Mg}^{2+}$ extrusion from liver cells, a concomitant release of hepatic glucose, mostly via glycogenolysis, has been observed (Fagan and Romani, 2000). Interestingly, inhibition of $\mathrm{Mg}^{2+}$ extrusion by amiloride or imipramine also results in a marked inhibition of hepatic glucose output (Fagan and Romani, 2000). The converse is also true. Inhibition of glucose transport activity by phlorethin results in a qualitatively similar inhibition of $\mathrm{Mg}^{2+}$ extrusion from liver cells (Fagan and Romani, 2000). The presence of a close functional 'link' between glucose and $\mathrm{Mg}^{2+}$ homeostasis is further emphasized by the observation that overnight starvation results in the complete depletion of hepatic glycogen and glucose as well as in a marked decrease (minus $15 \%)$ of total $\mathrm{Mg}^{2+}$ content as a consequence of the activation of the pro-glycemic hormones catecholamine and glucagon (Torres et al., 2005). Noteworthy, this decrease in hepatic $\mathrm{Mg}^{2+}$ content is equivalent to that elicited via in vitro stimulation of perfused livers by the same hormones (Torres et al., 2005), or that observed in the liver of type-I diabetic animals (Fagan et al., 2004), which are markedly decreased in cellular glycogen. The functional link between glucose and $\mathrm{Mg}^{2+}$ homeostasis is also observed under conditions in which glucose accumulation is stimulated by insulin or similar hormones in cardiac ventricular myocytes (Romani et al., 2000) or pancreatic beta cells (Henquin et al., 1983). In both experimental models, the amount of $\mathrm{Mg}^{2+}$ accumulated within the cells is directly proportional to the amplitude of glucose accumulation. Conversely, decreasing extracellular $\mathrm{Mg}^{2+}$ concentration directly reduces the amount of glucose accumulated within the cells (Fagan and Romani, 2000; Romani et al., 2000).

Although indirect, a clear proof of the glucose $/ \mathrm{Mg}^{2+}$ relationship is provided by diabetic conditions. Work by Altura's group (Resnick et al., 1993) and more recently by Resnick (Resnick, 1993) and Barbagallo (Barbagallo and Dominguez,
2007) indicate that cellular $\mathrm{Mg}^{2+}$ content is markedly decreased under type-I and type-II diabetes. Originally observed in red blood cells (Resnick et al., 1993; Resnick, 1993; Barbagallo and Dominguez, 2007), the decrease has also been reported in various other tissues including muscles, liver (Fagan et al., 2004), and cardiac myocytes (Reed et al., 2008). Interestingly, $\mathrm{Mg}^{2+}$ extrusion via $\beta$-adrenergic signalling remains operative and is actually up-regulated in liver cells from diabetic animals (Fagan et al., 2004) while it is markedly inhibited in cardiac myocytes from the same animals (Reed et al., 2008). Whether this reflects a differential operation and modulation of $\beta_{2}$-adrenergic receptors in liver cells vs $\beta_{1}$-adrenergic receptors in cardiac cells is not completely clear. Both cell models show a marked inhibition of the $\mathrm{Mg}^{2+}$ entry mechanism(s), which persists also in liver plasma membrane vesicles (Fagan et al., 2004; Cefaratti et al., 2004). Addition of glucose or glycogen to plasma membrane vesicles from diabetic animals renormalizes the amplitude of $\mathrm{Mg}^{2+}$ extrusion, but is ineffective at restoring $\mathrm{Mg}^{2+}$ accumulation in vesicles (Cefaratti et al., 2004). The defect in total cellular $\mathrm{Mg}^{2+}$ content appears to be strongly associated with the decrease in protein synthesis and ATP production detected in the cells (Reed et al., 2008). Supplementation of exogenous insulin restores both these parameters as well as $\mathrm{Mg}^{2+}$ homeostasis and extrusion provided that insulin is administered for at least two weeks (reed et al., 2008). As indicated previously, it appears that the role of insulin in modulating $\mathrm{Mg}^{2+}$ homeostasis is not restricted to controlling glucose homeostasis and accumulation or the release of pro-glycemic hormones like glucagon, but extends to a direct modulation of the $\mathrm{Na}^{+} / \mathrm{Mg}^{2+}$ exchanger (Ferreira et al., 2004). The latter effect would not only directly increase cellular $\mathrm{Mg}^{2+}$ content but it could also reverberate on the insulin receptor itself. Data obtained in animals maintained on low $\mathrm{Mg}^{2+}$ diet indicate that a decrease in cellular $\mathrm{Mg}^{2+}$ content affects the ability of the insulin receptor to properly phosphorylate the downstream insulin receptor substrate (IRS) and propagate the signalling within muscle cells (Suarez et al., 1995). This result might be of relevance in explaining the decrease in glucose accumulation observed in skeletal muscles under diabetic conditions (Suarez et al., 1995). 
$\mathrm{Mg}^{2+}$ homeostasis and ATP

Hormonal stimuli represent the most dynamic modality by which a cell can rapidly extrude $10 \%$ to $15 \%$ of its total cellular $\mathrm{Mg}^{2+}$ content within a few minutes from the application of the agonist. $\mathrm{Mg}^{2+}$, however, can also be extruded following the treatment with various agents that impact cellular ATP content and production. Cyanide (Harman et al., 1990; Dalal and Romani, 2010), mitochondrial uncouplers (Akerman, 1981; Kubota et al., 2005), fructose (Gaussin et al., 1997), ethanol (Tessman and Romani, 1998), or hypoxia (Gasbarrini et al., 1992) are just some of the agents whose addition impact cellular $\mathrm{Mg}^{2+}$ homeostasis. All these agents, in fact, have in common that they decrease cellular ATP content by either preventing the mitochondrial electron chain from generating ATP at various levels (cyanide or uncouplers), or by acting as an ATP trap (fructose), or by altering the redox state of pyridine nucleotide within the mitochondrion or the cell (ethanol). As ATP represents the major buffering component for $\mathrm{Mg}^{2+}$ within the cell (Scarpa and Brinley, 1981; Luthi et al., 1999), a decrease in this phosphonucleotide moiety results in an increase in cytosolic free $\left[\mathrm{Mg}^{2+}\right] \mathrm{i}$, and ultimately in a detectable extrusion from the cell (Harman et al., 1990; Gasbarrini et al., 1992; Gaussin et al., 1997; Tessman and Romani, 1998; Dalal and Romani, 2010). The $\mathrm{Mg}^{2+}$ extrusion can be observed to a larger extent in erythrocytes, which possess limited cellular buffering capacity and no compartmentation (Hwa et al., 1993), but it can also be observed in cells that present additional buffering by proteins or cellular organelles in addition to phosphonucleotides (Harman et al., 1990; Gasbarrini et al., 1992; Gaussin et al., 1997; Tessman and Romani, 1998; Dalal and Romani, 2010). In the case of fructose, the changes in cytosolic $\mathrm{Mg}^{2+}$ have been associated with an activation of the glycogen phosphorylase, which ultimately results in glycogenolysis activation and glucose utilization to restore ATP levels (Gaussin et al., 1997). In the majority of these experimental conditions, the increase in cytosolic free $\left[\mathrm{Mg}^{2+}\right] \mathrm{i}$ is usually modest, and considerably lower than the increase expected to occur based upon the corresponding decrease in ATP level, which strongly supports the notion that $\mathrm{Mg}^{2+}$ is for the most part extruded from the cell. Furthermore, as ATP level decreases as a result of mitochondria poisoning or changes in pyridine nucleotide ratio, it would appear that not phosphorylation but the rise in cytosolic $\mathrm{Mg}^{2+}$ albeit modest is sufficient to activate the $\mathrm{Mg}^{2+}$ extrusion mechanism and limit the rise in cytosolic free $\mathrm{Mg}^{2+}$ concentration to approximately $100-200 \mathrm{mM}$ at the most (Harman et al., 1999).

Cellular ATP plays a key role in regulating $\mathrm{Mg}^{2+}$ extrusion. Evidence for this role has been provided in the giant squid axon (Di Polo and Beauge', 1988) as well as in mammalian hepatocytes (Gunther and Hollriegl, 1993) or erythrocytes (Gunther et al., 1995). In squid axon, the $\mathrm{Na}^{+}$-dependent $\mathrm{Mg}^{2+}$ extrusion requires a physiological level of ATP. As the phosphonucleotide level decreases, so does the amplitude of extrusion (Gunther and Hollriegl, 1993). In erythrocytes and hepatocytes, instead, ATP appears to regulate the $\mathrm{Na}^{+}$-independent $\mathrm{Mg}^{2+}$ extrusion process (Gunther and Hollriegl, 1993; Gunther et al., 1995). The exact role of ATP in regulating the process, however, is unclear as it does not appear that the extrusion process is mediated by an ATPase mechanism. This notion is supported by the observation that a decrease in cellular ATP level (as it occurs for example under diabetic or alcoholic conditions) paradoxically results in an increased extrusion of $\mathrm{Mg}^{2+}$ via the $\mathrm{Na}^{+}$-dependent mechanism in a manner directly proportional to the decrease in ATP level (Tessman and Romani, 1998; Fagan et al., 2004). Hence, it appears that the role of ATP is predominantly that of a ligand for $\mathrm{Mg}^{2+}$ both in the cytoplasm and the mitochondrial matrix (Scarpa and Brinley, 1981; Luthi et al., 1999), and that a decrease in ATP results in an increase in free $\mathrm{Mg}^{2+}$ and its consequent extrusion from the cell.

\section{$\mathrm{Mg}^{2+}$ accumulation}

The identification of several $\mathrm{Mg}^{2+}$ entry mechanisms strongly support the hypothesis that cellular $\mathrm{Mg}^{2+}$ is dynamically maintained through the operation of entry and exit mechanisms that are differentially regulated by hormones and metabolic conditions. A striking difference is there, however, between the $\mathrm{Mg}^{2+}$ exit and the $\mathrm{Mg}^{2+}$ entry mechanisms. In the case of $\mathrm{Mg}^{2+}$ extrusion mechanisms we have a good understanding of the signalling activating these mechanisms but we lack any structural information about the mechanisms themselves. In the case of $\mathrm{Mg}^{2+}$ entry mechanisms, instead, 
we do have structural information about several of these mechanisms but for the most part we lack detailed information about their individual activation by hormones or second messengers, and their possible cooperation under specific conditions.

\section{Role of Protein Kinase $C$}

Experimental evidence indicates that mammalian cells can accumulate large amounts of $\mathrm{Mg}^{2+}$ as a result of hormonal stimulation. Hormones like carbachol, vasopressin, angiotensin-II, or insulin have been indicated as able to either inhibit cAMP-mediated $\mathrm{Mg}^{2+}$ extrusion or reverse the extrusion into $\mathrm{Mg}^{2+}$ accumulation in various cell types (Romani and Scarpa, 1990a; Romani et al., 2000). The list of cells that respond to hormonal stimulation by accumulating $\mathrm{Mg}^{2+}$ is rather long [see Romani and Scarpa, 2000 for a list], and involve all kind of cells: cardiac myocytes (Romani and Scarpa, 1990a; Romani et al., 2000), smooth muscle cells (Touyz and Schiffrin, 1996), hepatocytes (Romani and Scarpa, 1990b; Romani et al., 1992), platelets (Hwang et al., 1993), lymphocytes (Grubbs and Maguire, 1986), fibroblasts (Ishijima and Tatibana, 1994), and pancreatic beta cells (Henquin et al., 1983), just to name a few. In addition to inhibiting CAMP production, several of the hormones indicated above can activate protein kinase $\mathrm{C}$ (PKC) as part of their cellular signalling. Evidence supporting a role of PKC in mediating $\mathrm{Mg}^{2+}$ accumulation has been provided by several laboratories. Maguire and collaborators have indicated that administration of phorbol-myristate acetate (PMA), which directly activates PKC, elicits a marked accumulation of $\mathrm{Mg}^{2+}$ in S49 lymphoma cells (Erdos and Maguire, 1983). A similar effect of PMA has been reported by Somogyi's group in thymocytes (Csermely et al., 1987), and by our laboratory in cardiac myocytes (Romani et al., 1992) and hepatocytes (Romani et al., 1992). Furthermore, our group has reported that downregulation of PKC by exposure to a large dose of PMA for 3 hours completely abolishes the ability of cardiac and liver cells to accumulate $\mathrm{Mg}^{2+}$ while leaving unaffected the responsiveness of these cells to adrenergic agonists (Romani et al., 1992). An inhibitory effect has also been observed following administration of the PKC inhibitors calphostin (Touyz and Schiffrin, 1996) or staurosporine (Gunther and Vormann, 1995). Alteration in PKC distribution and activity and defective accumulation of $\mathrm{Mg}^{2+}$ has been observed in arterial smooth muscle cells (Yang et al., 2001), hepatocytes of animals exposed to alcohol (Torres et al., 2010), and in liver cells of diabetic animals (Tang et al., 1993).

Protein kinase $\mathrm{C}$ activation is only part of the integral response of hormones like angiotensin-II or vasopressin. The interaction of these hormones with their receptor, in fact, activates phospholipase $\mathrm{C}$ which, in turn hydrolyses PIP2 to generate diacylglycerol (DAG) and IP3. In turn, these two molecules activate protein kinase $C$ and IP3 receptor in the ER, respectively. Activation of the latter receptor results in a marked but transient increase in cytosolic $\mathrm{Ca}^{2+}$, and in a more sustained entry of $\mathrm{Ca}^{2+}$ through the capacitative $\mathrm{Ca}^{2+}$ entry mechanism. Hence, $\mathrm{Ca}^{2+}$ signalling is an integral component of the cellular response elicited by these hormones. Yet, the contribution of this second messenger in mediating $\mathrm{Mg}^{2+}$ accumulation is poorly defined. Liver cells loaded with Bapta-AM, which effectively chelates cytosolic $\mathrm{Ca}^{2+}$, are unable to extrude and accumulate $\mathrm{Mg}^{2+}$ following stimulation by phenylephrine and PMA, respectively (Romani et al., 1993b). The artificial increase in cytosolic $\mathrm{Ca}^{2+}$ elicited by thapsigargin administration also prevents $\mathrm{Mg}^{2+}$ accumulation (Romani et al., 1993b) and actually induces a $\mathrm{Mg}^{2+}$ extrusion from the liver cell if applied for more than 3-5 min (Romani et al., 1993b; Fagan and Romani, 2001). Because of the different time-scale and amplitude of the changes in cellular $\mathrm{Ca}^{2+}$ and $\mathrm{Mg}^{2+}$ content (Romani et al., 1993b), it is difficult to properly correlate these experimental variations. Cytosolic free $\mathrm{Ca}^{2+}$ transiently increases several orders of magnitude while cytosolic free $\mathrm{Mg}^{2+}$, which is already in the millimolar or submillimolar range, increases by 10-15\% (Fatholahi et al., 2000) at the most, although in absolute terms this amount is far larger than the overall change in cytosolic $\mathrm{Ca}^{2+}$ mass.

An unresolved point of inconsistency in the role of $\mathrm{Ca}^{2+}$ and PKC signalling in regulating $\mathrm{Mg}^{2+}$ accumulation is provided by the reports that the administration of phenylephrine, which activates PKC signalling in addition to the inositol 1,4,5 trisphosphate $/ \mathrm{Ca}^{2+}$ pathway, does not elicit $\mathrm{Mg}^{2+}$ accumulation but induces a $\mathrm{Mg}^{2+}$ extrusion from liver cells (Fagan and Romani, 2001). This point 
raises the question as to what modulates the differential response of the cell to the administration of phenylephrine or vasopressin. One possibility could be that different PKC isoforms are activated under one condition and not the other. For example, hepatocytes possess 3 classical and at least 2 novel PKC isoforms (Tang et al., 1993). Thus, it is reasonable to envisage that one isoform (or class of isoforms) is involved in mediating $\mathrm{Mg}^{2+}$ accumulation whereas another isoform (or class of isoforms) is involved in modulating $\mathrm{Mg}^{2+}$ extrusion. Consistent with this hypothesis, recent data from our laboratory suggests that PKCe is essential for $\mathrm{Mg}^{2+}$ accumulation to occur (Torres et al., 2010). Under conditions in which the expression of this isoform is inhibited by antisense or its translocation to the cell membrane is prevented - for example by ethanol administration, no $\mathrm{Mg}^{2+}$ accumulation can be detected in liver cells (Torres et al., 2010). Interestingly, this PKC isoform has the highest affinity for $\mathrm{Mg}^{2+}$ among all PKC isoenzymes, with a $\mathrm{K}_{\mathrm{m}}$ of $\sim 1 \mathrm{mM}$ (Konno et al., 1989), close to the physiological free $\left[\mathrm{Mg}^{2+}\right]_{i}$ measured in the cytoplasm of the hepatocyte (Corkey et al., 1986; Fatholahi et al., 2000) and other mammalian cells as well (Touyz and Schiffrin, 1996). Although the mechanism ultimately responsible for $\mathrm{Mg}^{2+}$ accumulation into the hepatocyte has not been identified, it is worth considering the recent observation by Bindels and collaborators that in the absence of PKC activation or following RACK1 over-expression, RACK1 can bind to TRPM6, and possibly TRPM7, at the level of the kinase domain and inhibit the channel activity (Cao et al., 2008).

\section{Role of MAPKs}

These results mentioned above, however, do not exclude the possibility that additional signalling pathways (e.g. MAPKS) are involved in determining the differing response of the hepatocyte under apparently similar stimulatory conditions. In agreement with previous reports from Altura's group (Yang et al., 2000) and Touyz's laboratory (Touyz and Yao, 2003), our laboratory has evidenced that pharmacological inhibition of ERK1/2 and p38 MAPKs in liver cells abolishes PKC mediated $\mathrm{Mg}^{2+}$ accumulation (Torres et al., 2006). In this respect, it has to be noted that the inhibition of MAPKs hampers $\mathrm{Mg}^{2+}$ accumulation in vascular smooth muscle cells, and significantly affect cyclin activity (Touyz and Yao, 2003) and consequently the ability of the cells to progress in the cell cycle (Touyz and Yao, 2003). This effect may occur via changes in nuclear functions directly regulated by $\mathrm{Mg}^{2+}$, as proposed by Rubin (2005) and/or via changes in nuclear functions of ERK2, which depends on $\mathrm{Mg}^{2+}$ level to properly dimerize, translocate and activate specific nuclear targets (Waas and Dalby, 2003). The role of ERK1/2 in $\mathrm{Mg}^{2+}$ regulation is further emphasized by the recent report that increased phosphorylation of ERK1/2 and increased expression of TRPM6 has been observed upon EGF administration to renal epithelial cells (Ikari et al., 2008; Ikari et al., 2010). The role of these MAPKs, however, is far from being elucidated as this kinase appears to be involved to some extent in also mediating $\mathrm{Mg}^{2+}$ extrusion (Kim et al., 2005; Torres et al., 2006).

\section{Role of EGF}

As mentioned, direct and indirect evidence suggests a key role of EGF in modulating $\mathrm{Mg}^{2+}$ accumulation, at least in kidney cells. The administration of EGF controls proper operation of TRPM 6 in the apical domain of renal epithelial cells to promote $\mathrm{Mg}^{2+}$ accumulation (Ikari et al., 2008; Thebault et al., 2009; Ikari et al., 2010). Point mutations in EGF sequence result in limited function of TRPM6, and limited or no $\mathrm{Mg}^{2+}$ accumulation within the cells (van der Wjist et al., 2009). The modulation of TRPM6 appears to occur via MAPKs signalling, most likely ERK1/2 coupled to activator protein-1 (AP-1) (Ikari et al., 2010). Indirect evidence of EGF regulation is provided by the evidence that antibodies against EGF used in several forms of colon cancer (Cunningham et al., 2004; Dimke et al., 2010) induce $\mathrm{Mg}^{2+}$ wasting and hypomagnesaemia.

\section{Serum $\mathrm{Mg}^{2+}$ level and $\mathrm{Mg}^{2+}$ sensing mechanism}

The experimental evidence that mammalian cells accumulate or extrude $\mathrm{Mg}^{2+}$ under a variety of experimental conditions suggests the presence of a sensor for the cytosolic $\mathrm{Mg}^{2+}$ concentration, whereby the cell would operate accordingly either extruding the excess cation or accumulating it to restore the 'set-point'. Compelling evidence for the presence of such a sensor mechanism is provided by the observation that prolonged exposure to $0 \mathrm{mM}\left[\mathrm{Mg}^{2+}\right]_{\text {。 }}$ decreases cytosolic free $\mathrm{Mg}^{2+}$ concentration by approximately $50 \%$ in cardiac ventricular 
myocytes (Quamme and Rabkin, 1990), MDKC (Quamme and Dai, 1990), or MDCT cells (Dai et al., 1997). The new cytosolic $\mathrm{Mg}^{2+}$ level is maintained as long as the cells are incubated in the presence of $0 \mathrm{mM}\left[\mathrm{Mg}^{2+}\right]_{0}$, but returns to the normal level as soon as $\left[\mathrm{Mg}^{2+}\right]_{0}$ is increased (Quamme and Rabkin, 1990; Quamme and Dai, 1990; Dai et al., 1997), in a frame of time that is directly proportional to the extracellular $\mathrm{Mg}^{2+}$ concentration utilized (Quamme and Rabkin, 1990; Quamme and Dai, 1990; Dai et al., 1997). The process is prevented by the presence of the L-type $\mathrm{Ca}^{2+}$-channel inhibitors verapamil or nifedipine, or $\mathrm{La}^{3+}$ in the extracellular milieu (Quamme and Rabkin, 1990). The absence of significant changes in cytosolic free $\mathrm{Ca}^{2+}$ concentration under these experimental conditions suggests a direct effect of these inhibitory agents on the $\mathrm{Mg}^{2+}$ entry mechanism (Quamme and Rabkin, 1990). This observation led the authors to propose the operation of a specific $\mathrm{Mg}^{2+}$ channel in these cells, anticipating the identification of TRPM6 (Schlingmann et al., 2002) and TRPM7 (Nadler et al., 2001) as specific $\mathrm{Mg}^{2+}$ channels.

$\mathrm{Mg}^{2+}$ accumulation is favoured by ion redistribution. In fact, renal epithelial cells accumulate $\mathrm{Mg}^{2+}$ as a result of phosphate (Dai et al., 1997) or potassium (Dai et al., 1991) redistribution across the cell membrane. The dependence of $\mathrm{Mg}^{2+}$ accumulation on $\mathrm{K}^{+}$ redistribution across the plasma membrane suggests that $\mathrm{Mg}^{2+}$ transport is the result of changes in membrane potential, possibly for charge compensation (Schweigel et al., 1999; Tashiro et al., 2002; Schweigel and Martens, 2003). In this respect, it is interesting to note that pathological conditions characterized by a marked decrease in tissue $\mathrm{Mg}^{2+}$ content (e.g., diabetes) (Fagan et al., 2004) are also characterized by an inability to properly transport potassium (Mondon et al., 1974; Taylor and Agius, 1988). Whether the effect on $\mathrm{K}^{+}$occurs through changes in membrane potential, or indirectly via a reduced operation of the $\mathrm{Na}^{+} / \mathrm{K}^{+}$-ATPase coupled to the operation in reverse of the $\mathrm{Na}^{+} / \mathrm{Mg}^{2+}$ exchanger (Gunther and Vormann, 1995), is a matter for future investigation.

Humans and many mammals present a circulating $\mathrm{Mg}^{2+}$ level of $\sim 1.2-1.4 \mathrm{mEq} / \mathrm{L}$ (Geigy, 1984; Mudge, 1989). Clinical and experimental evidence indicates that serum $\mathrm{Mg}^{2+}$ level decreases in humans and animals in several chronic diseases (Fagan et al., 2004). Yet, there is a remarkable lack of information as to whether serum $\mathrm{Mg}^{2+}$ undergoes circadian fluctuations following hormonal or non-hormonal stimuli (e.g., fasting or exercise). The infusion of catecholamine in conscious humans (Joborn et al., 1985; Bailly et al., 1990) or ovine (Rayssiguier, 1977), or the administration of isoproterenol (Guideri, 1992) or epinephrine in the presence of $\alpha_{1}$-adrenoceptor blockade (Rayssiguier, 1977) have provided contrasting results. More recent results, however, would indicate that isoproterenol infusion elicits in a marked dose- and timedependent increase in circulating $\mathrm{Mg}^{2+}$ content (Gunther and Vormann, 1992; Keenan et al., 1995). The increase is serum $\mathrm{Mg}^{2+}$ is maximal within $20 \mathrm{~min}$ from the agent administration (Keenan et al., 1995), and remains unchanged up to 2 hours even following the removal of the agonist (Keenan et al., 1995). This time frame indicates that the increase in serum $\mathrm{Mg}^{2+}$ is not dependent on the increase in heart rate and the decrease in mean arterial pressure elicited by the $\beta$-adrenergic agonist (Keenan et al., 1995). In fact, the infusion of sodium nitroprusside, which mimics the decrease in mean arterial pressure induced by isoproterenol, has no effect on serum $\mathrm{Mg}^{2+}$ levels (Keenan et al., 1995). The persistence of an elevated serum $\mathrm{Mg}^{2+}$ level for up to 2 hours also implies that operation of secondary and not fully elucidated mechanisms activated by the stimulation of $\beta$-adrenergic receptor. Consistent with the larger distribution of $\beta_{2}$ versus $\beta_{1}$ adrenergic receptors in the body (Molinoff, 1984; Barnes, 1995), the increase in serum $\mathrm{Mg}^{2+}$ occurs via the specific activation of $\beta_{2}$-adrenergic receptor, as it is mimicked by the administration of the selective $\beta_{2}$-adrenergic agonist salbutamol, and it is inhibited by the specific $\beta_{2}$-blocker ICl118551 (Keenan et al., 1995). Due to the amplitude of the increase in $\mathrm{Mg}^{2+}$ level into the bloodstream, it is reasonable to envisage that the adrenergic agonists are mobilizing $\mathrm{Mg}^{2+}$ from more than one tissue (Keenan et al., 1995). The involvement of bone has been proposed by Gunther and co-workers (Gunther and Vormann, 1992) based on the observation that the infusion of carbonic anhydrase inhibitor prevents isoproterenol-mediated $\mathrm{Mg}^{2+}$ mobilization into the blood of anesthetized rats. Based upon the pre-infusion level of serum $\mathrm{Mg}^{2+}$, the glomerular 
filtration rate $(1.62 \mathrm{~mL} / \mathrm{min})$, and the fractional excretion (17\%) (Shafik and Quamme, 1989), changes in renal excretion do not appear to contribute significantly to the increase in serum $\mathrm{Mg}^{2+}$ level, at least during the first two hours following adrenergic agonist infusion. It is interesting to note, however, that hormones that increase plasma $\mathrm{Mg}^{2+}$ by mobilizing the cation from different organs or tissues usually increase $\mathrm{Mg}^{2+}$ resorption in the Henle's loop, de facto preventing any net loss of $\mathrm{Mg}^{2+}$.

The inconsistency of changes in serum $\mathrm{Mg}^{2+}$ level observed in the literature, however, does not have a clear explanation. Several factors may contribute to this incongruity, including the proportion of b-adrenergic receptor subtypes in different experimental models, the ability of adrenergic agonists to stimulate with various potency different adrenergic receptors subtypes, and the modality, rate and duration of drug infusion. This inconsistency has also hampered our ability to assign a physiological significance to the eventual increase in serum $\mathrm{Mg}^{2+}$ level following catecholamine infusion. The observation that serum $\mathrm{Mg}^{2+}$ level increases under certain conditions may imply that specific organs or tissues have the ability to sense these changes and act accordingly. Presently, no specific $\mathrm{Mg}^{2+}$ sensing mechanism has been identified. However, the $\mathrm{Ca}^{2+}$ sensing receptor (Brown et al., 1993) can senses changes in circulating $\mathrm{Mg}^{2+}$ level in a range of concentrations far higher than those of $\mathrm{Ca}^{2+}$ (Nemeth and Scarpa, 1987) but consistent with the increase in serum $\mathrm{Mg}^{2+}$ level reported in the literature (Gunther and Vormann, 1992; Keenan et al., 1995). The observation that in distal convoluted tubule cells (MDCT) of the mouse the $\mathrm{Ca}^{2+}$-sensing receptor can be activated with comparable sensitivity by both extracellular $\mathrm{Ca}^{2+}$ and $\mathrm{Mg}^{2+}$ (Bapty et al., 1998a) suggests interesting hypotheses in terms of whole body physiology. The activation of this sensor mechanism would inhibit glucagon- or vasopressin-mediated entry of $\mathrm{Mg}^{2+}$ into the cell (Bapty et al., 1998b) and favour the elimination of the cation with the urine. This would then explain the clinical and experimental evidence that hypermagnesemia and hypercalcemia inhibit hormone-stimulated CAMP-mediated resorption of $\mathrm{Mg}^{2+}$ and $\mathrm{Ca}^{2+}$ along the different segments of the nephron (Quamme and Dirks, 1980). Also, it would represent a distal regulatory mechanism to restore magnesemia to a physiological level following the increase observed in anesthetized animals infused with adrenergic agonists (Gunther and Vormann, 1992; Keenan et al., 1995). Whether this sensing mechanism and/or its associated modulatory components are altered under diabetic conditions in which an increased magnesuria is observed it remains to be elucidated.

At variance of an increase in calcemia, which is associated with muscle weakness and arrhythmia, an increase in serum $\mathrm{Mg}^{2+}$ level appears to be well tolerated in vivo. Rats infused with boluses of $\mathrm{Mg}^{2+}$ that increase serum $\mathrm{Mg}^{2+}$ level by $50 \%$ do not exhibit significant systemic hemodynamic changes but show a marked increase in coronary artery flow (Dipette et al., 1987). Baboons infused with pharmacological doses of $\mathrm{Mg}^{2+}$ sufficient to prevent epinephrineinduced cardiac arrhythmias show a significant attenuation of the epinephrine-induced increase in mean arterial pressure and systemic vascular resistance (Stanbury, 1948). It would appear, therefore, that an elevated level of $\left[\mathrm{Mg}^{2+}\right]_{0}$ can regulate catecholamine release from peripheral and adrenal sources (James et al., 1988) and modulate cardiac contractility (Howarth et al., 1994). Taken together, these observations suggest that an increase in serum $\mathrm{Mg}^{2+}$ level following adrenergic stimulation can: 1 ) act as a feed-back mechanism to modulate catecholamine release and activity; and 2) contribute to improved blood flow and $\mathrm{O}_{2}$ delivery to the heart and possibly other tissues at a time when an increase in energy production is expected.

\section{Physiological role of intracellular $\mathrm{Mg}^{2+}$}

The conclusions presented in the previous sections strongly suggest a key role for $\mathrm{Mg}^{2+}$ as an indispensable component for enzymes, phosphometabolites, and channel activity (Grubbs and Maguire, 1987; Romani and Scarpa, 1992). Several glycolytic enzymes, including hexokinase, phosphofructokinase, phosphoglycerate mutase, phosphoglycerate kinase, enolase and pyruvate kinase, show activation at low and inhibition at high $\mathrm{Mg}^{2+}$ concentrations (Otto et al., 1974; Garfinkel and Garfinkel, 1988). The adenylate cyclase is another example of an enzyme directly regulated by $\mathrm{Mg}^{2+}$ (reviewed in Maguire, 1984). All these processes occur at $\mathrm{Mg}^{2+}$ concentrations 
between 0.5 to $1 \mathrm{mM}$, which are well within the fluctuations in free $\left[\mathrm{Mg}^{2+}\right]_{\mathrm{i}}$ measured in the cytoplasm of various cells including hepatocyte (Corkey et al., 1986). With the exception of the glycolytic enzymes, studies attempting to evidence a regulatory role of $\mathrm{Mg}^{2+}$ for cytosolic enzymes have been disappointing, mostly because of the underlying assumption that $\mathrm{Mg}^{2+}$ would operate as $\mathrm{Ca}^{2+}$ in modulating enzyme activity. At variance from $\mathrm{Ca}^{2+}, \mathrm{Mg}^{2+}$ concentration in the cytoplasm and extracellular fluids is in the millimolar or submillimolar range. Consequently, an increase or decrease in cytosolic $\mathrm{Mg}^{2+}$ level equivalent to the changes observed for $\mathrm{Ca}^{2+}$ will go largely undetected by the common fluorescent or ${ }^{31} \mathrm{P}-\mathrm{NMR}$ techniques. Heretofore, a role of $\mathrm{Mg}^{2+}$ as transient regulator of cytosolic enzymes appears to be unlikely. It has to be noted that even under conditions in which hormonal and non-hormonal stimuli elicit major fluxes of $\mathrm{Mg}^{2+}$ across the cell plasma membrane in either direction, massive translocations of $\mathrm{Mg}^{2+}$ that increase or decrease the total cellular $\mathrm{Mg}^{2+}$ content by 1 to $2 \mathrm{mM}$ (or $5-10 \%$ of total cell content) result in minor or no changes in cytosolic free $\left[\mathrm{Mg}^{2+}\right]_{\mathrm{i}}$ (Harman et al., 1990; Romani et al., 2000). This disconnect can be explained by assuming that the source or destination of transported $\mathrm{Mg}^{2+}$ is a cellular compartment or organelle, or a major binding site, or that $\mathrm{Mg}^{2+}$ is highly buffered. Hence, regulation of cellular functions by $\mathrm{Mg}^{2+}$ should not be necessarily expected to occur in the cytosol but within organelles, and in the plasma, where $\mathrm{Mg}^{2+}$ concentration can rapidly increase or decrease more than 20\% (Gunther and Vormann, 1992; Keenan et al., 1995).

In the following pages we will highlight what is known about regulatory effects of extracellular or intracellular $\mathrm{Mg}^{2+}$ on the operation of cation channels in the plasma membrane, as well as on mitochondria respiration and volume following changes in $\mathrm{Mg}^{2+}$ concentration within the organelle.

\section{$\mathrm{Ca}^{2+}$ - and $\mathrm{K}^{+}$-channels}

The first report of a regulatory effect of intracellular free $\mathrm{Mg}^{2+}$ on calcium channels was by White and Hartzell (1988). These authors reported that increasing intracellular free $\left[\mathrm{Mg}^{2+}\right]_{\mathrm{i}}$ from 0.3 to $3.0 \mathrm{mM}$ by internal perfusion had a dual effect in cardiac ventricular myocytes. It had a small effect on basal L-type $\mathrm{Ca}^{2+}$-channels current $\left(I_{C_{a}}\right)$ but decreased by more than $50 \%$ the amplitude of $I_{\mathrm{Ca}}$ elevated by CAMP-dependent phosphorylation. The effect of $\mathrm{Mg}^{2+}$ was not due to changes in cAMP concentration or in the velocity of phosphorylation but appeared to be a direct effect on the phosphorylated channel or on the channel dephosphorylation rate (White and Hartzell, 1988). Similar results were also reported by Agus and Morad (1991) in guinea pig cardiac myocytes. The block induced by $\mathrm{Mg}^{2+}$ on the $\mathrm{Ca}^{2+}$ current depended on a direct effect on the inactivation state of the channel as the block persisted in the presence and in the absence of CAMP and was not reversed by elevation of extracellular $\mathrm{Ca}^{2+}$ concentration or addition of catecholamine (Agus and Morad, 1991). The effect of $\mathrm{Mg}^{2+}$ on $\mathrm{Ca}^{2+}$-channels is not restricted to cardiac cells or to an intra-cellular site of action. Bara and Guiet-Bara (2001) have shown that in vascular smooth muscle cells and endothelial cells in human placenta, $\mathrm{MgCl}_{2}$ (and to a lesser extent $\mathrm{MgSO}_{4}$ ) regulates the influx of $\mathrm{Ca}^{2+}$ through voltage-gated $\mathrm{Ca}^{2+}$ channels by acting at an extracellular site on the channel, thus modulating the tonus of the vessels. Evidence for a similar block by extracellular $\mathrm{Mg}^{2+}$ on $\mathrm{T}$-type $\mathrm{Ca}^{2+}$-channels has been provided by Serrano et al., (2000). The modulatory effect of $\mathrm{Mg}^{2+}$ appears to take place at the EF-hand motif of the $\mathrm{COOH}$-terminus of $\mathrm{Ca}_{v} 1.2$ channels as recently evidence by Catterall and his group (Brunet et al., 2006).

Extracellular $\mathrm{Mg}^{2+}$ also modulates the activity of store-operated $\mathrm{Ca}^{2+}$ channels. Studies in intact, pressurized rat mesenteric arteries with myogenic tone indicate that $10 \mathrm{mM}$ extracellular $\mathrm{Mg}^{2+}$ can mimic nifedipine in preventing or reversing the vasoconstriction elicited by phenylephrine administration, but not that induced by $\mathrm{K}^{+}$ depolarization (Zhang et al., 2002). Therefore, $\mathrm{Mg}^{2+}$ abolishes the vasoconstriction attributed to $\mathrm{Ca}^{2+}$ entry through store-operated channels, which are activated following phenylephrine administration, contributing to maintain both myogenic tone and $\alpha_{1}$-adrenoceptor-induced tonic vasoconstriction. These data may have direct implication to explain some of the modifications that occur under hypertensive conditions, in which a decrease in plasma $\mathrm{Mg}^{2+}$ has often been reported. 
Intracellular $\mathrm{Mg}^{2+}$ also affects he operation of store-operated calcium release-activated $\mathrm{Ca}^{2+}$ (CRAC) channels (Prakriya and Lewis, 2002). CRAC channels are highly $\mathrm{Ca}^{2+}$-selective under physiological ionic conditions whereas removal of extracellular divalent cations makes them freely permeable to monovalent cations, in particular $\mathrm{Na}^{+}$. Experimental evidence indicates that intracellular $\mathrm{Mg}^{2+}$ can modulate the activity and selectivity of these channels therefore affecting monovalent cation permeability. While an effect of intracellular $\mathrm{Mg}^{2+}$ on CRAC channels cannot be completely excluded, a report by Prakriya and Lewis (2002) suggests that the channels modulated by intracellular $\mathrm{Mg}^{2+}$ are not CRAC channels, but a different class of channels that open when $\mathrm{Mg}^{2+}$ is washed out of the cytosol. These channels have therefore been termed $\mathrm{Mg}^{2+}$-inhibited cation (MIC) channels, as they present distinctive functional parameters in terms of inhibition, regulation, ion permeation and selectivity (Prakriya and Lewis, 2002).

Potassium channels are also targets for $\mathrm{Mg}^{2+}$. Matsuda (1991) has reported that the presence of $\mathrm{Mg}^{2+}$ on the cytoplasmic side of the inwardly rectifying $\mathrm{K}^{+}$channel blocks the outward currents without affecting the inward currents. The $\mathrm{Mg}^{2+}$ block is achieved at a half-saturation concentration of $1.7 \mu \mathrm{M}$. When the $\mathrm{Mg}^{2+}$ concentration is elevated to $2-10 \mu \mathrm{M}$, the outward current fluctuated between two intermediate sublevels in addition to the fully open and closed configuration. However, these concentrations of $\mathrm{Mg}^{2+}$ are far from being physiological and it is difficult to envision the occurrence of a similar regulatory effect under normal conditions without invoking $\mathrm{Mg}^{2+}$ microcompartmentation. A regulatory role of intracellular $\mathrm{Mg}^{2+}$ on $\mathrm{K}_{\mathrm{v}}$ channels in vascular smooth muscle cells has been observed by Tammaro et al., (2005). These authors have observed that an increase in intracellular $\mathrm{Mg}^{2+}$ in a range of concentrations consistent with its physiological variations can slow down the kinetic of activation of the $K_{v}$ channel, cause inward rectification at positive membrane potentials, and shift the voltage-dependent inactivation (Tammaro et al., 2005). Overall, this represents a novel mechanism for the regulation of this channel in the vasculature. Intracellular $\mathrm{Mg}^{2+}$ also modulates large-conductance (BK type) $\mathrm{Ca}^{2+}$ dependent $\mathrm{K}^{+}$channels by either blocking the pore of BK channels in a voltage-dependent manner, or by activating the channels independently of changes in $\mathrm{Ca}^{2+}$ and voltage by preferentially binding to the channel open conformation at a site different from $\mathrm{Ca}^{2+}$ sites. Interestingly, $\mathrm{Mg}^{2+}$ may also bind to $\mathrm{Ca}^{2+}$ sites and competitively inhibit $\mathrm{Ca}^{2+}$-dependent activation (Shi et al., 2002).

The inhibitory effect of $\mathrm{Mg}^{2+}$ is not restricted to cell membrane channels. Experimental evidence by Bednarczyk et al., (2005) indicates that $\mathrm{Mg}^{2+}$ in the mitochondrial matrix can modulate gating and conductance of mitochondrial $\mathrm{K}_{\text {ATP }}$ channels, which play a key role under ischemia/reperfusion conditions.

\section{Mitochondrial dehydrogenases}

Mitochondria represent one of the major cellular pools for $\mathrm{Mg}^{2+}$, its concentration being 14 to 16 $\mathrm{mM}$ (Gunther, 1986). Evidence from this (Romani et al., 1991) and other laboratories (Zhang and Melvin, 1992; Zhang and Melvin, 1996; Kubota et al., 2005) suggests that $\mathrm{Mg}^{2+}$ can be mobilized from mitochondria under various conditions including hormone-mediated increase in cytosolic cAMP level through a mechanism(s) that has not been fully elucidated but it appears to involve the adenine nucleotide translocase (Romani et al., 1991). Several reviews have analyzed in detail how $\mathrm{Mg}^{2+}$ homeostasis is regulated in the organelle (Flatman, 1984; Gunther, 1986; Ogoma et al., 1992), and we refer to those reviews for further information. This section will focus on some recent evidence about a role of intra- and extra-mitochondrial $\mathrm{Mg}^{2+}$ on the activity of specific mitochondrial proteins.

It is commonly accepted that changes in matrix $\mathrm{Ca}^{2+}$ can affect the rate of mitochondrial dehydrogenases and therefore the rate of respiration (McCormack et al., 1990; Hansford, 1994). Yet, limited evidence supports a similar role for $\mathrm{Mg}^{2+}$ although the activity of several mitochondrial dehydrogenases has been observed to increase within minutes from the application of hormonal or metabolic stimuli despite the absence of a detectable increase in mitochondrial $\mathrm{Ca}^{2+}$ (Moravec and Bond, 1991; Moravec and Bond, 1992). The role of matrix $\mathrm{Mg}^{2+}$ as regulator of dehydrogenases and consequently mitochondrial respiration has been investigated by measuring the activity of several dehydrogenases in 
mitochondria under conditions in which matrix $\mathrm{Ca}^{2+}$ and/or $\mathrm{Mg}^{2+}$ concentration were varied. From those data, it appears that $\alpha$-ketoglutarate dehydrogenase and pyruvate dehydrogenase are not regulated by changes in mitochondrial $\mathrm{Mg}^{2+}$, whereas $\mathrm{Mg}^{2+}$ removal increases several fold the activity of succinate and glutamate dehydrogenases (Panov and Scarpa, 1996a; Panov and Scarpa, 1996b). This evidence would therefore indicate that changes in matrix $\mathrm{Mg}^{2+}$ content (in combination with or in alternative to changes in mitochondrial $\mathrm{Ca}^{2+}$ ) can control mitochondria respiration, at least under well defined conditions. In this respect, mitochondrial $\mathrm{Mg}^{2+}$ content appears to change quite significantly during transition from state 3 to state 4 (Brierley et al., 1987), affecting at the same time the amplitude of mitochondria respiration. In addition, data from our laboratory (Romani et al., 1991), Zhang and Melvin (1996), and Kubota et al (2005) would suggest that mitochondrial $\mathrm{Mg}^{2+}$ can be mobilized by catecholamine stimulation via CAMP. Hence, the enhanced mitochondrial respiration elicited by catecholamine could depend, at least in part, on CAMP-mediated modulation of mitochondrial $\mathrm{Mg}^{2+}$, which, in turn, will stimulate directly some dehydrogenases while rendering others more susceptible to the $\mathrm{Ca}^{2+}$ concentrations present in the mitochondrial matrix.

The regulatory effect of $\mathrm{Mg}^{2+}$ on mitochondrial function is not restricted to the dehydrogenases activity but affects also the anion channel present in the mitochondrial membrane (Beavis and Powers, 2004) as well as the opening of the permeability transition pore (Dolder et al., 2003). The mitochondrial inner membrane anion channel (IMAC) transports various anions, and is involved in regulating the organelle volume in conjunction with the $\mathrm{K}^{+} / \mathrm{H}^{+}$antiporter. Although its fine regulation is not completely elucidated as yet, experimental evidence suggests that matrix $\mathrm{Mg}^{2+}$ and protons inhibit IMAC, maintaining the channel in its closed state (Beavis and Powers, 2004). Kinetic studies by Beavis and collaborators further support a main role of $\mathrm{Mg}^{2+}$ in maintaining the channel in a condition that would allow fine modulation by small changes in $\mathrm{pH}$ and proton distribution under physiological conditions (Beavis and Powers, 2004).
The permeability transition pore (PTP) is a proteinaceous pore that opens in the inner mitochondrial membrane following a marked decrease in membrane potential and results in the rapid equilibration and redistribution of matrix and extramitochondrial solutes down their concentrations gradient. While it is still debated exactly which proteins participate in the pore formation and how the pore opens, it is well established that an increase in mitochondrial $\mathrm{Ca}^{2+}$ content facilitates the opening whereas an increase in mitochondrial $\mathrm{Mg}^{2+}$ antagonizes it. This effect can be appreciated well in yeasts, which do not possess a canonical PTP (Bradshaw and Pfeiffer, 2006). According to one model of regulation, creatine kinase can regulate the opening of the permeability transition pore by tightly associating to the mitochondrial membrane and remaining in an active state (Dolder et al., 2003). Both these processes are $\mathrm{Mg}^{2+}$-dependent, and $\mathrm{Mg}^{2+}$ removal from the extramitochondrial environment results in a decline in creatine kinas activity and in the opening of the permeability transition pore Dolder et al., 2003).

Considering the effect of $\mathrm{Mg}^{2+}$ on mitochondrial function and channels (see previous section), it appears that $\mathrm{Mg}^{2+}$ plays more than one role within this organelle regulating: 1) mitochondrial volume; 2) ion composition; 3) ATP production; and 4) metabolic interaction with the hosting cell.

\section{Reticular G6Pase}

The Endoplasmic Reticulum (ER) represents one of the major cellular $\mathrm{Mg}^{2+}$ pools, with a total concentration of 14 to $18 \mathrm{mM}$ (Romani and Scarpa, 1992). Yet, limited information is available about any major role of luminal $\mathrm{Mg}^{2+}$ on reticular functions other than protein synthesis (Rubin, 2005). It is also unknown whether $\mathrm{Mg}^{2+}$ is buffered or chelated by endoluminal proteins in the same way that $\mathrm{Ca}^{2+}$ is complexed. Furthermore, we do not have any information about the transport mechanism(s) that allow(s) ER (and SR) to attain and maintain such a large luminal $\mathrm{Mg}^{2+}$ concentration.

Work by Volpe and collaborators (Volpe et al., 1990; Volpe and Vezu', 1993), Gusev and Niggli (2008), and Laver and Honen (2008) suggests that cytosolic and perhaps luminal $\mathrm{Mg}^{2+}$ concentration can have a major effect in limiting $\mathrm{Ca}^{2+}$ uptake 
into the ER/SR and its release from the organelle via $\mathrm{IP}_{3}$ (Volpe et al., 1990) and ryanodine receptor (Laver and Honen, 2008). In the latter case, a direct effect of $\mathrm{Mg}^{2+}$ on the receptor has been observed (Gusev and Niggli, 2008; Laver and Honen, 2008). Less clear is whether a similar effect takes also place at the level of the $\mathrm{IP}_{3}$ receptor. More recently, our laboratory has reported that cytosolic $\mathrm{Mg}^{2+}$ can have a regulatory role on the activity of reticular glucose 6-phosphatase (G6Pase) in liver cells (Doleh and Romani, 2007). This effect is biphasic, with an optimal stimulatory effect at $\sim 0.5 \mathrm{mM}\left[\mathrm{Mg}^{2+}\right]_{i}$ and an inhibitory effect at higher concentrations (Doleh and Romani, 2007). The effect of $\mathrm{Mg}^{2+}$ appears to take place at the level of the G6Pi transport component of the G6Pase enzymatic complex in that it can be inhibited by EDTA (as $\mathrm{Mg}^{2+}$ chelating agent) or taurocholic acid, which permeabilizes the ER membrane and allows G6Pi to bypass the transport mechanism and be delivered directly to the catalytic site of the G6Pase within the ER lumen (Doleh and Romani, 2007). Investigation is in progress to determine whether $\mathrm{Mg}^{2+}$ exerts a similar modulatory effect on other ER enzymes.

\section{Cell $\mathrm{pH}$ and volume}

As mentioned earlier, exposure of the cell to cyanide (Dalal and Romani, 2010), fructose (Gaussin et al., 1997), hypoxia (Harman et al., 1990; Gasbarrini et al., 1992), ethanol (Tessman and Romani, 1998), or choline chloride (Romani et al., 1993b) results in a marked cellular acidification associated with a decrease in cellular ATP content and a major $\mathrm{Mg}^{2+}$ extrusion. This extrusion is the consequence of a decrease in buffering capacity (ATP loss) as well as binding affinity. Now, a report by Yamaguchi and Ishikawa (2008) highlights a new and important regulatory role of intracellular $\mathrm{Mg}^{2+}$ on the operation of the electrogenic $\mathrm{Na}^{+}-\mathrm{HCO}_{3}{ }^{-}$cotransporter NBCe1-B in a range of concentrations that are consistent with the variations in $\mathrm{Mg}^{2+}$ content measured within the cytoplasm of various cell types (Grubbs and Maguire, 1987). This regulatory effect is exerted by $\mathrm{Mg}^{2+}$ and not by MgATP, and requires a functional $\mathrm{N}$-terminus on the NBCe1-B transporter (Yamaguchi and Ishikawa, 2008). It is still unclear whether $\mathrm{Mg}^{2+}$ binds the $\mathrm{N}$-terminus of the transporter directly or exerts its effects via an intermediate, $\mathrm{Mg}^{2+}$ modulated regulatory protein (Yamaguchi and
Ishikawa, 2008). At a cytosolic $\left[\mathrm{Mg}^{2+}\right]$ of $\sim 1 \mathrm{mM}$ (a physiological $\mathrm{Mg}^{2+}$ concentration measured in the cytosol of various cells (Corkey et al., 1986; Grubbs and Maguire, 1987), the NBCe1-B current is inhibited by $\sim 50 \%$, and no detectable current can be measured for free $\mathrm{Mg}^{2+}$ concentrations of 3-5mM.

More or less at the same time, the group of Hirano and collaborators reported that increasing cellular $\mathrm{Mg}^{2+}$ content has a stimulatory role on the expression of aquaporin 3 in $\mathrm{CaCo}-3$ cells (Okahira et al., 2008). This isoform of aquaporin is highly expressed in the gastro-intestinal tract, in which it absorbs water, glycerol and urea. The effect of $\mathrm{Mg}^{2+}$ on aquaporin mRNA expression appears to involve cAMP/PKA/CREB signalling, as well as we MEK1/2 and MSK1 (Okahira et al., 2008), suggesting short- and long-term regulation on the activity and expression of this protein. It is presently undefined whether $\mathrm{Mg}^{2+}$ exerts a similar regulatory role on other aquaporin isoforms. Moreover, as aquaporin 3 is also expressed in brain, erythrocytes, kidney, and skin, a modulatory role of $\mathrm{Mg}^{2+}$ on the protein expression in these tissues may have major relevance for various physiological and/or pathological conditions.

Taken together, these two sets of information suggest that $\mathrm{Mg}^{2+}$ can have a major regulatory role on cellular $\mathrm{pH}$, cellular volume, and cellular cation concentration, especially $\mathrm{Na}^{+}$. Also, as aquaporin 3 mediates glycerol and urea accumulation, possible repercussions on fatty acid metabolism and urea cycle must be taken into proper consideration.

\section{Cell cycle}

Cell cycle (Maguire, 1988; Sgambato et al., 1999; Touyz et Yao, 2003), cell proliferation (Wolf et al., 2009b) and cell differentiation (Covacci et al., 1998; Wolf et al., 1998; Di Francesco et al., 1998) have all been associated with the maintenance of an optimal cellular $\mathrm{Mg}^{2+}$ level. Under conditions in which cellular $\mathrm{Mg}^{2+}$ accessibility is restricted or reduced, cell proliferation and cell cycle progression are markedly impaired. A decrease in extracellular $\mathrm{Mg}^{2+}$ content also affects cell differentiation (Covacci et al., 1998; Wolf et al., 1998; Di Francesco et al., 1998). The mechanisms by which a decrease in cellular $\mathrm{Mg}^{2+}$ content affects these cellular processes have been 
attributed to defective MAPKs (Touyz and Yao, 2003) and p27 (Sgambato et al., 1999) signalling, increased oxidative stress level (Wolf et al., 2009b), and decreased MgATP levels (Di Francesco et al., 1998; Rubin, 2005). Because cellular MgATP level is at a level optimal for protein synthesis (Rubin, 2005), any alteration in this metabolic parameter will have major repercussion on the proper functioning of the cell. In addition, extracellular $\mathrm{Mg}^{2+}$ levels regulate integrins signalling, de facto modulating the interaction among cells and between cells and extracellular matrix (Trache et al., 2010). Hence, consistent with the long-term regulatory function of $\mathrm{Mg}^{2+}$ proposed by Grubbs and Maguire (1987) several years ago, all these observations underlie the important role of $\mathrm{Mg}^{2+}$ to guarantee cell cycle progression and retention of proper cell morphology and function, avoiding undesired

\section{References}

Aarts $M$, lihara K, Wei WL, Xiong ZG, Arundine $M$, Cerwinski W, MacDonald JF, Tymianski M (2003) A key role for TRPM7 channels in anoxic neuronal death. Cell 115:863-77.

Agus ZS, Morad M (1991) Modulation of cardiac ion channels by magnesium. Annu Rev Physiol 53:299-307.

Akerman KE (1981) Inhibition and stimulation of respiration-linked $\mathrm{Mg}^{2+}$ efflux in rat heart mitochondria. J Bioenerg Biomembr 13:133-9.

Alexander RT, Hoenderop JG, Bindels RJ (2008) Molecular determinants of magnesium homeostasis: insight from human disease. J Am Soc Nephrol 19:1451-8.

Allouche D, Parello J, Sanejouand YH (1999) $\mathrm{Ca}^{2+} / \mathrm{Mg}^{2+}$ exchange in parvalbumin and other EF-hand proteins. A theoretical study. J Mol Biol 285:857-73.

Amano T, Matsubara T, Watanabe J, Nakayama S, Hotta N (2000) Insulin modulation of intracellular free magnesium in heart: involvement of protein kinase $\mathrm{C}$. Brit J Pharmacol 130:731-8.

Baillien M, Cogneau M (1995) Characterization of two mechanisms of ${ }^{28} \mathrm{Mg}$ uptake in rat jejunal brush border membrane vesicles. Magnesium 8:331-9. progression towards cell death or neoplastic destiny (Wolf et al., 2009a).

\section{Conclusions}

In the last few years, our understanding of cellular and whole body $\mathrm{Mg}^{2+}$ homeostasis has significantly advanced. While it still lags behind as compared to the knowledge available for other ions such as $\mathrm{Ca}^{2+}, \mathrm{H}^{+}, \mathrm{K}^{+}$or $\mathrm{Na}^{+}$, the identification of putative $\mathrm{Mg}^{2+}$ channels and transport mechanisms in the membrane of the cell or mitochondria, as well as an improved understanding of the signalling pathways and conditions regulating $\mathrm{Mg}^{2+}$ transport are providing new tools to address essential questions about the physiological role $\mathrm{Mg}^{2+}$ plays inside the cell and in the whole body.

Baillien M, Wang H, Cogneau M (1995) Uptake of ${ }^{28} \mathrm{Mg}$ by duodenal and jejunal brush border membrane vesicle in the rat. Magnesium 8:315-29.

Bailly C, Imbert-Teboul M, Roinel N, Amiel C (1990) Isoproterenol increases $\mathrm{Ca}, \mathrm{Mg}$, and $\mathrm{NaCl}$ reabsorption in mouse thick ascending limb. Am J Physiol 258:F1224-31.

Bapty BW, Dai LJ, Ritchie G, Canaff L, Hendy GN, Quamme GA (1998) $\mathrm{Mg}^{2+} / \mathrm{Ca}^{2+}$ sensing inhibits hormone-stimulated uptake in mouse distal convolute tubule cells. Am J Physiol 275:F353-60.

Bapty BW, Dai LJ, Ritchie G, Jirik F, Canaff L, Hendy GN, Quamme GA (1998) Extracellular $\mathrm{Mg}^{2+}$ - and $\mathrm{Ca}^{2+}$ sensing in mouse distal convoluted tubule cells. Kidney Intern 53:583-92.

Bara M, Guiet-Bara A (2001) Magnesium regulation of $\mathrm{Ca}^{2+}$ channels in smooth muscle and endothelial cells of human allantochorial placental vessels. Magnes Res 14:11-8.

Barbagallo M, Dominguez LJ (2007) Magnesium metabolism in type 2 diabetes mellitus, metabolic syndrome and insulin resistance. Arch Biochem Biophys 458:40-7. 
Barnes PJ (1995) Beta-adrenergic receptors and their regulation. Am J Respir Crit Care Med 152:838-60.

Beavis AD, Powers M (2004) Temperature dependence of the mitochondrial inner membrane anion channel: the relationship between temperature and inhibition by magnesium. J Biol Chem 279:4045-50.

Bednarczyk P, Dolowy K, Szewczyk A (2005) Matrix $\mathrm{Mg}^{2+}$ regulates mitochondrial ATP-dependent potassium channel from heart. FEBS Lett 579:1625-30.

Belge H, Gailly P, Schwaller B, Loffing J, Debaix H, Riveira-Munoz E, Beauwens R, Devogelaer JP, Hoenderop JG, Bindels RJ, Devuyst O (2007) Renal expression of parvalbumin is critical for $\mathrm{NaCl}$ handling and response to diuretics. Proc Natl Acad Sci USA 104:14849-54.

Bessac BF, Fleig A (2007) TRPM7 channel is sensitive to osmotic gradients in human kidney cells. J Physiol 582:1073-86.

Bird SJ, Maguire ME (1978) The agonist-specific effect of magnesium ion on binding by $\beta$-adrenergic receptors of S49 lymphoma cells: Interactions of GTP and magnesium in magnesium in adenylate cyclase activation. J Biol Chem 253:8826-34.

Bogucka K, Wojtczak L (1971) Intramitochondrial distribution of magnesium. Biochem Biophys Res Commun 44: 1330-7.

Brauchi S, Krapivinsky G, Krapivinsky L, Clapham DE (2008) TRPM7 facilitates cholinergic vesicle fusion with the plasma membrane. Proc Natl Acad Sci U S A 105:8304-8.

Brierley GP, Davis M, Jung DW (1987) Respirationdependent uptake and extrusion of $\mathrm{Mg}^{2+}$ by isolated heart mitochondria. Arch Biochem Biophys 253: 32232.

Brown EM, Gamba G, Riccardi D, Lombardi M, Butters R, Kifor O, Sun A, Hediger MA, Lytton J, Herbert SC (1993) Cloning and characterization of an extracellular $\mathrm{Ca}^{2+}$-sensing from bovine parathyroid. Nature 366:575-80.

Brunet S, Scheuer T, Klevit R, Catterall WA (2006) Modulation of $\mathrm{Ca}_{v} 1.2$ channels by $\mathrm{Mg}^{2+}$ acting at an EFhand motif in the COOh-terminal domain. J Gen Physiol 126:311-23.

Bui DM, Gregan J, Jarosch E, Ragnini A, Schweyen RJ (1999) The bacterial magnesium transporter CorA can functionally substitute for its putative homologue
Mrs2p in the Yeast inner mitochondrial membrane. $J$ Biol Chem 274:20438-43.

Butler MG (1990) Prader-Willi syndrome: current understanding of cause and diagnosis. Am J Med Genet 35:319-32.

Cao G, Thébault S, van der Wijst J, van der Kemp A, Lasonder E, Bindels RJ, Hoenderop JG (2008) RACK1 inhibits TRPM6 activity via phosphorylation of the fused alpha-kinase domain. Curr Biol 18:168-76.

Cao G, van der Wijst J, van der Kemp A, van Zeeland F, Bindels RJ, Hoenderop JG (2009) Regulation of the epithelial $\mathrm{Mg}^{2+}$ channel TRPM 6 by estrogen and the associated repressor protein of estrogen receptor activity (REA). J Biol Chem 284:14788-95.

Cefaratti C, McKinnis A, Romani A (2004) Altered $\mathrm{Mg}^{2+}$ transport across liver plasma membrane from streptozotocin-treated rats. Mol Cell Biochem 262:145-54.

Cefaratti C, Romani A (2007) Functional characterization of two distinct $\mathrm{Mg}^{2+}$ extrusion mechanisms in cardiac sarcolemmal vesicles. Mol Cell Biochem 303:63-72.

Cefaratti C, Romani A (2011) Modulation of $\mathrm{Na}^{+} / \mathrm{Mg}^{2+}$ exchanger stoichiometry ratio by $\mathrm{Cl}^{-}$ions in basolateral rat liver plasma membrane vesicles. Mol Cell Biochem 351:133-42.

Cefaratti C, Romani A, Scarpa A (1998) Characterization of two $\mathrm{Mg}^{2+}$ transporters in sealed plasma membrane vesicles from rat liver. Am J Physiol 275:C995-1008.

Cefaratti C, Romani A, Scarpa A (2000) Differential localization and operation of distinct $\mathrm{Mg}^{2+}$ transporters in Apical and Basolateral Sides of Rat Liver Plasma Membrane. J Biol Chem 275:3772-80.

Cefaratti C, Ruse C (2007) Protein kinase A dependent phosphorylation activates $\mathrm{Mg}^{2+}$ efflux in the basolateral region of the liver. $\mathrm{Mol}$ Cell Biochem 297:209-14.

Chen H-S, Xie J, Zhang Z, Su L-T, Yue L, Runnels LW (2010) Blockade of TRPM7 channel activity and cell death by inhibitors of 5-lipoxygenase. PLoS One 5:e11161. 
Chubanov V, Gudermann T, Schlingmann KP (2005) Essential role for TRPM6 in epithelial magnesium transport and body magnesium homeostasis. Pflugers Arch 451:228-34.

Chubanov V, Waldegger S, Mederos y Schnitzler M, Vitzthum H, Sassen MC, Seyberth HW, Konrad M, Gudermann T (2004) Disruption of TRPM6/TRPM7 complex formation by a mutation in the TRPM6 gene causes hypomagnesemia with secondary hypocalcemia. Proc Natl Acad Sci U S A 101:2894-9.

Clark K, Langeslag $M$, van Leeuwen $B$, Ran L, Ryazanov $A G$, Figdor CG, Moolenaar WH, Jalink K, van Leeuwen FN (2006) TRPM7, a novel regulator of actomyosin contractility and cell adhesion. EMBO J 25:290-301.

Clark K, Middelbeek J, Lasonder E, Dulyaninova NG, Morrice NA, Ryazanov AG, Bresnick AR, Figdor CG, van Leeuwen FN (2008a) TRPM7 regulates myosin IIA filament stability and protein localization by heavy chain phosphorylation. J Mol Biol 378:790-803.

Clark K, Middelbeek J, Morrice NA, Figdor CG, Lasonder E, van Leeuwen FN (2008b) Massive autophosphorylation of the Ser/Thr-rich domain controls protein kinase activity of TRPM6 and TRPM7. PLoS One 3:e1876 (1-10).

Corkey BE, Duszynski J, Rich TL, Matchinsky B, Williamson JR (1986) Regulation of free and bound magnesium in rat hepatocytes and isolated mitochondria. J Biol Chem 261:2567-74.

Covacci V, Bruzzese N, Sgambato A, Di Francesco A, Russo MA, Wolf Fl, Cittadini A (1998) Magnesium restriction induces granulocytic differentiation and expression of p27Kip1 in human leukemic HL-60 cells. J Cell Biochem 70:313-22.

Csermely P, Fodor P, Somogyi J (1987) The tumor promoter tetradecanoylphorbol-13-acetate elicits the redistribution of heavy metal in subcellular fractions of rabbit thymocytes as measured by plasma emission spectroscopy. Carcinogenesis 8:1663-6.

Cunningham $D$, Humblet $Y$, Siena $S$, Khayat $D$, Bleiberg $H$, Santoro A, Bets D, Mueser M, Harstrick A, Verslype C, Chau I, van Cutsem E (2004) Cetuximab monotherpay and cetuximab plus irinotecan in irinotecan-refractory metastatic colorectal cancer. $N$ Engl J Med 351:337-45.

Dai L-J, Friedman PA, Quamme GA (1991) Cellular Mechanism of chlorothiazide and cellular potassium depletion on $\mathrm{Mg}^{2+}$ uptake in mouse distal convoluted tubule cells. Kidney Inter 51:1008-17.
Dai L-J, Friedman PA, Quamme GA (1997) Phosphate depletion diminishes $\mathrm{Mg}^{2+}$ uptake in mouse distal convoluted tubule cells. Kidney Inter 51:1710-18.

Dalal P, Romani A (2010) Adenosine triphosphate depletion by cyanide results in a $\mathrm{Na}^{+}$-dependent $\mathrm{Mg}^{2+}$ extrusion from liver cells. Metabolism 59:1663-71.

Di Francesco A, Desnoyer RW, Covacci V, Wolf FI, Romani A, Cittadini A, Bond M (1998) Changes in magnesium content and subcellular distribution during retinoic acid-induced differentiation of HL60 cells. Arch Biochem Biophys 360:149-57.

Di Polo R, Beauge' L (1988) An ATP-dependent $\mathrm{Na}^{+} / \mathrm{Mg}^{2+}$ countertransport is the only mechanism for $\mathrm{Mg}^{2+}$ extrusion in squid axons. Biochim Biophys Acta 946:424-8.

Dimke $\mathrm{H}$, van der Wjist J, Alexander TR, Mejier IM, Mulder GM, van Goor H, Tejpar S, Hoenderop JG, Bindels RJ (2010) Effects of the EGFR inhibitor erlotinib on magnesium handling. J Am Soc Nephrol 21:1309-16.

Dipette DJ, Simpson K, Guntupalli J (1987) Systemic and regional hemodynamic effect of acute magnesium administration in the normotensive and hypertensive state. Magnesium 6:136-9.

Dolder M, Walzel B, Speer O, Schlattner U, Wallimann $T$ (2003) Inhibition of the mitochondrial permeability transition by creatine kinase substrates. $\mathrm{J} \mathrm{Biol} \mathrm{Chem}$ 278:17760-6.

Doleh L, Romani A (2007) Biphasic effect of extrareticular $\mathrm{Mg}^{2+}$ on hepatic G6P transport and hydrolysis. Arch Biochem Biophys 467:283-90.

Dorovkov MV, Ryazanov AG (2004) Phosphorylation of annexin I by TRPM7 channel-kinase. J Biol Chem 279:50643-6.

Ebel H, Gunther $\mathrm{T}$ (2003) Stimulation of $\mathrm{Na}^{+} / \mathrm{Mg}^{2+}$ antiport in rat erythrocytes by intracellular $\mathrm{Cl}^{-}$. FEBS Lett 543:103-7.

Ebel $\mathrm{H}$, Hollstein M, Gunther T (2002) Role of the choline exchanger in $\mathrm{Na}^{+}$-independent $\mathrm{Mg}^{2+}$ efflux from rat erythrocytes. Biochim Biophys Acta 1559:13544.

Ebel H, Hollstein M, Günther T (2004) Differential effect of imipramine and related compounds on $\mathrm{Mg}^{2+}$ efflux from rat erythrocytes. Biochim Biophys Acta 1667:132-40. 
Efrati E, Arsentiev-Rozenfeld J, Zelikovic I (2005) The human paracellin-1 gene (hPCLN-1): renal epithelial cell specific expression and regulation. Am J Physiol 288:F272-83.

Elliot DA, Rizack MA (1974) Epinephrine and adrenocorticotrophic hormone-stimulated magnesium accumulation in adipocytes and their plasma membranes. J Biol Chem 249:3985-90.

Erdos JJ, Maguire ME (1980) Independent desensitization of $\beta$-adrenergic receptor regulated magnesium transport and cyclic AMP accumulation. Mol Pharmacol 18:379-83.

Erdos JJ, Maguire ME (1983) Hormone-sensitive magnesium transport in murine S49 lymphoma cells: Characterization and specificity for magnesium. J Physiol 337:351-71.

Fagan TE, Cefaratti C, Romani A (2004) Streptozotocininduced diabetes impairs $\mathrm{Mg}^{2+}$ homeostasis and uptake in rat liver cells. Am J Physiol 286:E184-93.

Fagan TE, Romani A (2000) Activation of $\mathrm{Na}^{+}$- and $\mathrm{Ca}^{2+}$. dependent $\mathrm{Mg}^{2+}$ extrusion by $\mathrm{a}_{1}$ - and $\mathrm{b}_{1}$-adrenergic agonists in rat liver cells. Am J Physiol 279:G943-50.

Fagan TE, Romani A (2001) a $a_{1}$-adrenoceptor-induced $\mathrm{Mg}^{2+}$ extrusion from rat hepatocytes occurs via $\mathrm{Na}^{+}-$ dependent transport mechanism. Am J Physiol 280:G1145-56.

Fatholahi M, Lanoue K, Romani A, Scarpa A (2000) Relationship between total and free cellular $\mathrm{Mg}^{2+}$ during metabolic stimulation of rat cardiac myocytes and perfused hearts. Arch Biochem Biophys 374:395401.

Feray J-C, Garay R (1986) An $\mathrm{Na}^{+}$-stimulated $\mathrm{Mg}^{2+}$ transport system in human red blood cells. Biochim Biophys Acta 856: 76-84.

Feray J-C, Garay R (1988) Demonstration of a $\mathrm{Na}^{+}: \mathrm{Mg}^{2+}$ exchange in human red cells by its sensitivity to tricyclic antidepressant drugs. Naunyn-Schmied Arch Pharmacol 338:332-7.

Ferreira A, Rivera A, Romero JR (2004) $\mathrm{Na}^{+} / \mathrm{Mg}^{2+}$ exchange is functionally coupled to the insulin receptor. J Cell Physiol 199:434-40.

Flatman PW (1984) Magnesium transport across cell membrane. J Membr Biol 80:1-14.

Flatman PW, Lew VL (1981) The magnesium dependence of sodium-pump-mediated sodium- potassium and sodium-sodium exchange in intact human red cells. J Physiol 315:421-46.

Flatman PW, Smith LM (1990) Magnesium transport in ferret red cells. J Physiol 431:11-25.

Garfinkel D, Garfinkel L (1988) Magnesium and regulation of carbohydrate metabolism at the molecular level. Magnesium 7:249-61.

Gasbarrini A, Borle AB, Farghali H, Bender C, Francavilla A, van Thiel D (1992) Effect of anoxia on intracellular ATP, $\mathrm{Na}^{+}{ }_{\mathrm{i}}, \mathrm{Ca}^{2+}{ }_{i}, \mathrm{Mg}^{2+}{ }_{i}$, and cytotoxicity in rat hepatocytes. J Biol Chem 267:6654-63.

Gaussin V, Gailly P, Gillis J-M, Hue L(1997) Fructoseinduced increase in intracellular free $\mathrm{Mg}^{2+}$ ion concentration in rat hepatocytes: relation with the enzymes of glycogen metabolism. Biochem J 326:8237.

Geigy Scientific Tables. (1984) Ed.: Lentner C., CibaGeigy, Basel, Switzerland.

Goytain A, Hines RM, El-Husseini A, Quamme GA (2007) NIPA1(SPG6), the basis for autosomal dominant form of hereditary spastic paraplegia, encodes a functional $\mathrm{Mg}^{2+}$ transporter. J Biol Chem 282:8060-8.

Goytain A, Hines RM, Quamme GA (2008a) Functional characterization of NIPA2, a selective $\mathrm{Mg}^{2+}$ transporter. Am J Physiol 295:C944-53.

Goytain A, Hines RM, Quamme GA (2008b) Huntingtininteracting proteins, HIP14 and HIP14L, mediate dual functions, palmitoyl acyltransferase and $\mathrm{Mg}^{2+}$ transport. J Biol Chem 283:33365-74.

Goytain A, Quamme GA (2005a) Identification and characterization of a novel mammalian $\mathrm{Mg}^{2+}$ transporter with channel-like properties. BMC Genomics 6:48.

Goytain A, Quamme GA (2005b) Functional characterization of human SLC41A1, a $\mathrm{Mg}^{2+}$

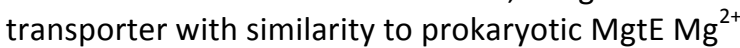
transporters. Physiol Genomics 21:337-342.

Goytain A, Quamme GA (2005c) Functional characterization of the human solute carrier, SLC41A2. Biochem Biophys Res Commun 330:701-5.

Goytain A, Quamme GA (2005d) Functional characterization of ACDP2 (ancient conserved domain protein), a divalent metal transporter. Physiol Genomics 22:382-89. 
Goytain A, Quamme GA (2008) Identification and characterization of a novel family of membrane magnesium transporters, MMgT1 and MMgT2. Am J Physiol 294:C495-502.

Groenestege WM, Hoenderop JG, van den Heuvel L, Knoers N, Bindels RJ (2006) The epithelial $\mathrm{Mg}^{2+}$ channel transient receptor potential melastatin 6 is regulated by dietary $\mathrm{Mg}^{2+}$ content and estrogens. J Am Soc Nephrol 17:1035-43.

Groenestege WM, Thébault S, van der Wijst J, van den Berg D, Janssen R, Tejpar S, van den Heuvel LP, van Cutsem E, Hoenderop JG, Knoers NV, Bindels RJ (2007) Impaired basolateral sorting of pro-EGF causes isolated recessive renal hypomagnesemia. J Clin Invest 117:2260-27.

Grubbs RD, Collins SD, Maguire ME (1985) Differential compartmentation of magnesium and calcium in murine S49 lymphoma cells. J Biol Chem 259:1218492.

Grubbs RD, Maguire ME (1986) Regulation of magnesium but not calcium by phorbol esters. J Biol Chem 261:12550-4.

Grubbs RD, Maguire ME (1987) Magnesium as a regulatory cation: Criteria and evaluation. Magnesium 6:113-27.

Grubbs RD, Wetherill CA, Kutschke K, Maguire ME (1984) Magnesium transport in murine S49 lymphoma cells: pharmacology and divalent cation selectivity. Amer J Physiol 248:C51-7.

Guideri G (1992) Catecholamine modulation of $\mathrm{Mg}^{2+}$ plasma levels in the rat. Arch Intern Pharmacodyn Therap 14:122-25.

Gunther T (1986) Functional compartmentation of intracellular magnesium. Magnesium 5:53-9.

Günther T (1993) Mechanisms and regulation of $\mathrm{Mg}^{2+}$ efflux and $\mathrm{Mg}^{2+}$ influx. Miner Electrolyte Metab 19:259-65.

Gunther T (1996) Putative mechanism of $\mathrm{Mg}^{2+} / \mathrm{Mg}^{2+}$ exchange and $\mathrm{Na}^{+} / \mathrm{Mg}^{2+}$ antiport. Magnes Bull 18:2-6.

Günther T, Höllriegl V (1993) $\mathrm{Na}^{+}$- and aniondependent $\mathrm{Mg}^{2+}$ influx in isolated hepatocytes. Biochim Biophys Acta 1149:49-54.

Gunther T, Vormann J (1985) $\mathrm{Mg}^{2+}$ efflux is accomplished by an amiloride-sensitive $\mathrm{Na}^{+} / \mathrm{Mg}^{2+}$ antiport. Biochem Biophys Res Commun 130:540-45.
Günther T, Vormann J (1990a) Induction of $\mathrm{Mn}^{2+} / \mathrm{H}^{+}$ antiport in chicken erythrocytes by intracellular $\mathrm{Mg}^{2+}$ and $\mathrm{Mn}^{2+}$. FEBS Lett 265:55-8.

Gunther T, Vormann J (1990b) $\mathrm{Na}^{+}$-dependent $\mathrm{Mg}^{2+}$ efflux from $\mathrm{Mg}^{2+}$-loaded rat thymocytes and $\mathrm{HL} 60$ cells. Magnes Trace Elem 9:279-82.

Gunther T, Vormann J (1992a) Activation of $\mathrm{Na}^{+} / \mathrm{Mg}^{2+}$ antiport in thymocytes by cAMP. FEBS Lett 297:132-4.

Gunther T, Vormann J (1992b) Mechanism of betaagonist-induced hypermagnesemia. Magnes Bull 14:122-5.

Gunther T, Vormann J (1995) Reversibility of $\mathrm{Na}^{+} / \mathrm{Mg}^{2+}$ antiport in rat erythrocytes. Biochim Biophys Acta 1234:105-10.

Gunther T, Vormann J, Forster R (1984) Regulation of intracellular magnesium by $\mathrm{Mg}^{2+}$ efflux. Biochem Biophys Res Commun 119:124-31.

Günther T, Vormann J, HöllriegI V (1990)

Characterization of $\mathrm{Na}^{+}$-dependent $\mathrm{Mg}^{2+}$ efflux from $\mathrm{Mg}^{2+}$-loaded rat erythrocytes. Biochim Biophys Acta 1023:455-61.

Gunther T, Vormann J, HollriegI V (1991) Noradrenaline-induced $\mathrm{Na}^{+}$-dependent $\mathrm{Mg}^{2+}$ efflux from rat liver. Magnes Bull 13:122-4.

Günther T, Vormann J, McGuigan JA (1995) Buffering and activity coefficient of intracellular free magnesium concentration in human erythrocytes. Biochem $\mathrm{Mol}$ Biol Int 37:871-5.

Günzel D, Schlue WR (1996) Sodium-magnesium antiport in Retzius neurones of the leech Hirudo medicinalis. J Physiol 491:595-608.

Gusev K, Niggli E (2008) Modulation of the local SR $\mathrm{Ca}^{2+}$ release by intracellular $\mathrm{Mg}^{2+}$ in cardiac myocytes. J Gen Physiol. 132:721-30.

Gwanyanya A, Amuzescu B, Zakharov SI, Macianskiene R, Sipido KR, Bolotina VM, Vereecke J, Mubagwa K (2004) Magnesium-inhibited, TRPM6/7-like channel in cardiac myocytes: permeation of divalent cations and pH-mediated regulation. J Physiol 559:761-76.

Gwanyanya A, Sipido KR, Vereecke J, Mubagwa K (2006) ATP and PIP2 dependence of the magnesiuminhibited, TRPM7-like cation channel in cardiac myocytes. Am J Physiol 291:C627-35.

Hansford RG (1994) Physiological role of mitochondrial $\mathrm{Ca}^{2+}$ transport. J Bioenerg Biomembr 26:495-508. 
Harman AW, Nieminen AL, Lemasters JJ, Herman B (1990) Cytosolic free magnesium, ATP and blebbing during chemical hypoxia in cultured rat hepatocytes. Biochem Biophys Res Commun 170:477-83.

Henquin JC, Tamagawa T, Nenquin M, Cogneau M (1983) Glucose modulates $\mathrm{Mg}^{2+}$ fluxes in pancreatic islet cells. Nature 301:73-4.

Hofer AM, Machen TE (1993) Technique for in situ measurement of calcium in intracellular inositol 1,4,5trisphosphate-sensitive stores using the fluorescent indicator mag-fura-2. Proc Natl Acad Sci USA. 90:2598602.

Hou J, Paul DL, Goodenough DA (2005). Paracellin-1 and the modulation of ion selectivity of tight junctions. J Cell Sci 118:5109-18.

Hou J, Renigunta A, Gomes AS, Hou M, Paul DL, Waldegger S, Goodenough DA (2009) Claudin-16 and claudin-19 interaction is required for their assembly into tight junctions and for renal reabsorption of magnesium. Proc Natl Acad Sci U S A. 106:15350-5.

Howarth FC, Waring J, Hustler BI, Singh J (1994) Effects of extracellular magnesium and beta adrenergic stimulation on contractile force and magnesium mobilization in the isolated rat heart. Magnes Res 7:187-97.

Hwa J, Romani A, Marfella C, Scarpa A (1993) $\mathrm{Mg}^{2+}$ transport in erythrocytes: Regulation by intracellular pH or cAMP. Biophys J 63,A307.

Hwang DL, Yen CF, Nadler JL (1993) Insulin increases intracellular magnesium transport in human platelets. J Clin Endocrinol Metab 76:549-53.

Ikari A, Matsumoto S, Harada H, Takagi K, Hayashi H, Suzuki Y, Degawa M, Miwa M (2006) Phosphorylation of paracellin-1 at Ser217 by protein kinase $A$ is essential for localization in tight junctions. J Cell Sci 119:1781-9.

Ikari A, Okude C, Sawada H, Yamazaki Y, Sugatani J, Miwa M (2008) TRPM6 expression and cell proliferation are up-regulated by phosphorylation of Erk1/2 in renal epithelial cells. Biochem Biophys Res Commun 369:1129-33.

Ikari A, Sanada A, Okude C, Sawada H, Yamazaki Y, Sugatani J, Miwa M (2010) Up-regulation of TRPM6 transcriptional activity by AP-1 in renal epithelial cells. J Cell Physiol 222:481-7.

Ishijima S, Tatibana M (1994) Rapid mobilization of intracellular $\mathrm{Mg}^{2+}$ by bombesin in Swiss 3T3 cells:
Mobilization through external $\mathrm{Ca}^{2+}$ - and tyrosine kinase-dependent mechanism. J Biochem 115:730-7.

Jakob A, Becker J, Schottli G, Fritzsch G (1989) alpha ${ }^{-}$ adrenergic stimulation causes $\mathrm{Mg}^{2+}$ release from perfused rat liver. FEBS Lett 246:127-30.

James MFM, Cork RC, Harlen GM, White JF (1988) Interaction of adrenaline and magnesium in the cardiovascular system of the baboon. Magnesium 7:37-43.

Jiang H, Tian SL, Zeng Y, Li LL, Shi J (2008) TrkA pathway(s) is involved in regulation of TRPM7 expression in hippocampal neurons subjected to ischemic-reperfusion and oxygen-glucose deprivation. Brain Res Bull 76:124-30.

Jiang J, Li M, Yue L (2005) Potentiation of TRPM7 inward current by protons. J Gen Physiol 126:137-50.

Jin J, Desai BN, Navarro B, Donovan A, Andrews NC, Clapham DE (2008) Deletion of Trpm7 disrupts embryonic development and thymopoiesis without altering $\mathrm{Mg}^{2+}$ homeostasis. Science 322:756-60.

Joborn H, Akerstrom G, Ljunghall S (1985) Effects of exogenous catecholamines and exercise on plasma magnesium concentrations. Clin Endocrinol 23:219-26.

Jung DW, Apel L, Brierley GP (1990) Matrix free $\mathrm{Mg}^{2+}$ changes with metabolic state in isolated heart mitochondria. Biochemistry 29:4121-8.

Juttner R, Ebel H (1998) Characterization of $\mathrm{Mg}^{2+}$ transport in brush border membrane vesicles of rabbit ileum studied with mag-fura-2. Biochim Biophys Acta 1370:51-63.

Karoor V, Baltensperger K, Paul H, Czech MC, Malbon CC (1995) Phosphorylation of tyrosyl residues 350/354 of the beta-adrenergic receptor is obligatory for counterregulatory effects of insulin. J Biol Chem 270:25305-8.

Kausalya PJ, Amasheh S, Gunzel D, Wurps H, Muller D, Fromm M, Hunziker W (2006) Disease-associated mutations affect intracellular traffic and paracellular $\mathrm{Mg}^{2+}$ transport function of Claudin-16. J Clin Invest 116:878-91.

Keenan D, Romani A, Scarpa A (1995) Differential regulation of circulating $\mathrm{Mg}^{2+}$ in the rat by $b_{1}$ - and $b_{2}$ adrenergic receptor stimulation. Circ Res 77:973-83.

Keenan D, Romani A, Scarpa A (1996) Regulation of $\mathrm{Mg}^{2+}$ homeostasis by insulin in perfused rat livers and isolated hepatocytes. FEBS Lett 395:241-4. 
Kehres DG, Lawyer CH, Maguire ME (1998) The CorA magnesium transporter gene family. Microb Comp Genomics 3:151-9.

Khan MA, Conigrave AD (2010) Mechanisms of multimodal sensing by extracellular $\mathrm{Ca}^{2+}$-sensing receptors: a domain-based survey of requirements for binding and signalling. Br J Pharmacol 159:1039-50

Kim SJ, Kang HS, Kang MS, Yu X, Park SY, Kim IS, Kim NS, Kim SZ, Kwak YG, Kim JS (2005) alpha ${ }_{1}$-Agonistsinduced $\mathrm{Mg}^{2+}$ efflux is related to MAP kinase activation in the heart, Biochem Biophys Res Commun 333:11328.

Kolisek M, Launay P, Beck A, Sponder G, Serafini N, Brenkus M, Froschauer EM, Martens $\mathrm{H}$, Fleig A, Schweigel M (2008) SLC41A1 is a novel mammalian $\mathrm{Mg}^{2+}$ carrier. J Biol Chem 283:16235-47.

Kolisek M, Zsurka G, Samaj J, Weghuber J, Schweyen RJ, Schweigel M (2003) Mrs2p is an essential component of the major electrophoretic $\mathrm{Mg}^{2+}$ influx system in mitochondria. EMBO J 22:1235-44.

Konno Y, Ohno S, Akita Y, Kawasaki H, Suzuki K (1989) Enzymatic properties of a novel phorbol ester receptor / protein kinase, nPKC. J Biochem 106:673-8.

Kubota T, Shindo $Y$, Tokuno K, Komatsu H, Ogawa $H$, Kudo S, Kitamura Y, Suzuki K, Oka K (2005) Mitochondria are intracellular magnesium stores: investigation by simultaneous fluorescent imaging in PC12 cells. Biochim Biophys Acta 1744:19-28.

Lal-Nag M, Morin PJ (2009) The claudins. Genome Biol 10(8):235.

Langeslag M, Clark K, Moolenaar WH, van Leeuwen FN, Jalink K (2007) Activation of TRPM7 channels by phospholipase C-coupled receptor agonists. J Biol Chem 282:232-9.

Laver DR, Honen BN (2008) Luminal $\mathrm{Mg}^{2+}$, a key factor controlling RYR2-mediated $\mathrm{Ca}^{2+}$ release: cytoplasmic and luminal regulation modeled in a tetrameric channel. J Gen Physiol 132:429-46.

Li M, Du J, Jiang J, Ratzan W, Su LT, Runnels LW, Yue L (2007) Molecular determinants of $\mathrm{Mg}^{2+}$ and $\mathrm{Ca}^{2+}$ permeability and $\mathrm{pH}$ sensitivity in TRPM6 and TRPM7. J Biol Chem 282:25817-30.

Li M, Jiang J, Yue L (2006) Functional characterization of homo- and heteromeric channel kinases TRPM6 and TRPM7. J Gen Physiol 127:525-37.
Li, S-H, Li, X-J (2004) Huntingtin-protein interactions and the pathogenesis of Huntington's disease. Trends Genet 20:146-52.

Ludi H, Schatzmann HJ (1987) Some properties of a system for sodium-dependent outward movement of magnesium from metabolizing human red blood cells. J Physiol 390:367-82.

Lüthi D, Günzel D, McGuigan JA (1999) Mg-ATP binding: its modification by spermine, the relevance to cytosolic $\mathrm{Mg}^{2+}$ buffering, changes in the intracellular ionized $\mathrm{Mg} 2+$ concentration and the estimation of $\mathrm{Mg}^{2+}$ by 31P-NMR. Exp Physiol 84:231-52.

Macianskiene R, Gwanyanya A, Vereecke J, Mubagwa $K$ (2008) Inhibition of the magnesium-sensitive TRPM7-like channel in cardiac myocytes by nonhydrolysable GTP analogs: involvement of phosphoinositide metabolism. Cell Physiol Biochem 22:109-18.

Maguire ME (1984) Hormone-sensitive magnesium transport and magnesium regulation of adenylate cyclase. Trends Pharmacol Sci 5:73-7.

Maguire ME (1988) Magnesium and cell proliferation. Ann NY Acad Sci 551: 215-7.

Maguire ME, Erdos JJ (1978) Magnesium but not calcium accumulation is inhibited by $\beta$-adrenergic stimulation in $\$ 49$ lymphoma cells. J Biol Chem 253:6633-6.

Maguire ME, Erdos JJ (1980) Inhibition of magnesium uptake by $\beta$-adrenergic agonists and prostaglandin $E_{1}$ is not mediated by cyclic AMP. J Biol Chem 255:10301035.

Matsuda H (1991) Magnesium gating of the inwardly rectifying $\mathrm{K}^{+}$channel. Annu Rev Physiol 53:289-98.

Matsuura T, Kanayama Y, Inoue T, Takeda T, Morishima I (1993) cAMP-induced changes of intracellular $\mathrm{Mg}^{2+}$ levels in human erythrocytes. Biochim Biophys Acta 1220:31-6.

McCormack JG, Halestrap A, Denton RM (1990) Role of calcium ions in regulation of mammalian intramitochondrial metabolism. Physiol Rev 70:391425.

Middelbeek J, Clark K, Venselaar HV, Huynen MA, van Leeuwen FN (2010) The alpha-kianse family: an exceptional branch on the protein kinase tree. Cell Mol Life Sci 67:875-90. 
Molinoff PB (1984) Alpha- and beta-adrenergic receptor subtypes, properties, distribution and regulation. Drugs 28:1-14.

Moncrief MB, Maguire ME (1999) Magnesium transport in prokaryotes. J Biol Inorg Chem 4:523-7.

Mondon CE, Dolkas CB, Olefsky JM, Reaven GM (1974) Insulin sensitivity of isolated perfused rat liver. Diabetes 24: 225-9.

Monteilh-Zoller MK, Hermosura MC, Nadler MJ, Scharenberg AM, Penner R, Fleig A (2003) TRPM7 provides an ion channel mechanism for cellular entry of trace metal ions. J Gen Physiol 121:49-60.

Moore EW (1990) Biliary calcium and gallstone formation. Hepatology 12:206S-14S.

Moravec CS, Bond M (1991) Calcium is released from the junctional sarcoplasmic reticulum during cardiac muscle contraction. Am J Physiol 260:H989-97.

Moravec CS, Bond M (1992) Effect of inotropic stimulation on mitochondrial calcium in cardiac muscle. J Biol Chem 267:5310-6.

Mudge GH (1989) Agents affecting volume and composition of body fluids. In: The Pharmacological Basis of Therapeutics (Goodman Gilman A, Godman LS, Rall TW, Murad F, eds.) MacMillian, NY.

Muller D, Kausalya PJ, Claverie-Martin F, Meij IC, Eggert P, Garcia-Nieto V, Hunziker W (2003) A novel claudin 16 mutation associated with childhood hypercalciuria abolishes binding to ZO-1 and results in lysosomal mistargeting. Am J Hum Genet 73:1293-301.

Nadler MJ, Hermosura MC, Inabe K, Perraud AL, Zhu Q, Stokes AJ, Kurosaki T, Kinet JP, Penner R, Scharenberg AM, Fleig A (2001) LTRPC7 is a Mg.ATPregulated divalent cation channel required for cell viability. Nature 411:590-5.

Nemeth EF, Scarpa A (1987) Rapid mobilization of cellular $\mathrm{Ca}^{2+}$ in bovine parathyroid cells by external divalent cations. J Biol Chem 262:5188-96.

Ogoma Y, Kobayashi H, Fujii T, Kondo Y, Hachimori A, Shimizu T, Hatano M (1992) Binding study of metal ions to S100 protein: ${ }^{43} \mathrm{Ca},{ }^{25} \mathrm{Mg},{ }^{67} \mathrm{Zn}$ and ${ }^{39} \mathrm{~K}$ N.M.R.. Int J Biol Macromol 14:279-86.

Okahira M, Kubota M, Iguchi K, Usui S, Hirano K (2008) Regulation of aquaporin 3 expression by magnesium ion. Eur J Pharmacol 588:26-32.
Oki S, Ikura M, Zhang M (1997) Identification of $\mathrm{Mg}^{2+}$ binding sites and role of $\mathrm{Mg}^{2+}$ on target recognition by calmodulin. Biochemistry 36; 4309-16.

Otto M, Heinrich R, Kuhn B, Jacobasch G (1974) A mathematical model for the influence of fructose 6phosphate, ATP, potassium, ammonium and magnesium on the phosphofructo-kinase from rat erythrocytes. Eur J Biochem 49:169-78.

Panov A, Scarpa A (1996a) Independent modulation of the activity of alpha-ketoglutarate dehydrogenase complex by $\mathrm{Ca}^{2+}$ and $\mathrm{Mg}^{2+}$. Biochemistry 35:427-32.

Panov A, Scarpa A (1996b) $\mathrm{Mg}^{2+}$ control of respiration in isolated rat liver mitochondria. Biochemistry 35:12849-56.

Paravicini TM, Yogi A, Mazur A, Touyz RM (2009) Dysregulation of vascular TRPM7 and annexin-1 is associated with endothelial dysfunction in inherited hypomagnesemia. Hypertension 53:423-9.

Parente L, Solito E (2004) Annexin 1: more than an anti-phospholipase protein. Inflamm Res 53:125-32.

Piskacek M, Zotova L, Zsurka G, Schweyen RJ (2009) Conditional knockdown of hMRS2 results in loss of mitochondrial $\mathrm{Mg}^{2+}$ uptake and cell death. J Cell Mol Med 13:693-700.

Polimeni PI, Page E (1974) In: Recent advances in study in cardiac cells and metabolism. Ed.: Dhalle N S, vol IV, pp. 217-232, University Park Press, Baltimore.

Prakriya M, Lewis RS (2002) Separation and characterization of currents through store-operated CRAC channels and $\mathrm{Mg}^{2+}$-inhibited cation (MIC) channels. J Gen Physiol 119:487-507.

Preston RR (1990) A magnesium current in Paramecium. Science 250:285-8.

Quamme GA (2010) Molecular identification of ancient and modern mammalian magnesium transporters. Am J Physiol 298:407-29.

Quamme GA, Dai L-S (1990) Presence of a novel influx pathway for Mg2+ in MDCK cells. Am J Physiol 259:C521-5.

Quamme GA, Dirks JH (1980) Effect of intraluminal and contraluminal magnesium on magnesium and calcium transfer in the rat nephron. Am J Physiol 238:F187-98. 
Quamme GA, Rabkin SW (1990) Cytosolic free magnesium in cardiac myocytes: Identification of a $\mathrm{Mg}^{2+}$ influx pathway. Biochem Biophys Res Commun 167:1406-12.

Quednau BD, Nicoll DA, Philipson KD (2004) The sodium/calcium exchanger family - SLC8. Pflugers Arch 447:543-8.

Raftos JE, Lew VL, Flatman PW (1999) Refinement and evaluation of a model of $\mathrm{Mg}^{2+}$ buffering in human red cells. Eur J Biochem 263:635-45.

Rainier S, Chai JH, Tokarz D, Nicholls RD, Fink JK (2003) NIPA1 gene mutations cause autosomal dominant hereditary spastic paraplegia (SPG6). Am J Hum Genet 73:967-71.

Rasgado-Flores H, Gonzalez-Serratos H, DeSantiago J (1994) Extracellular $\mathrm{Mg}^{2+}$-dependent $\mathrm{Na}^{+}, \mathrm{K}^{+}$, and $\mathrm{Cl}^{-}$ efflux in squid giant axons. Am J Physiol 266:C1112-7.

Rayssiguier Y (1977) Hypomagnesemia resulting from adrenaline infusion in ewes: its relation to lipolysis. Horm Metab Res 9:309-14.

Reed G, Cefaratti C, Berti-Mattera LN, Romani A (2008) Lack of insulin impairs $\mathrm{Mg}^{2+}$ homeostasis and transport in cardiac cells of streptozotocin-injected diabetic rats.J Cell Biochem 104:1034-53.

Resnick LM (1993) lonic basis of hypertension, insulin resistance, vascular disease, and related disorders. The mechanism of "syndrome X". Am J Hypertens 6:123S34 S.

Resnick LM, Altura BT, Gupta RK, Laragh JH, Alderman $\mathrm{MH}$, Altura BM (1993) Intracellular and extracellular magnesium depletion in type 2 (non-insulindependent) diabetes mellitus. Diabetologia 36:767-70.

Rogers TA (1961) The exchange of radioactive magnesium in erythrocytes. J Cell Comp Physiol 57:119-21.

Rogers TA, Haven FL, Mahan PE (1960) Exchange of magnesium in the rat. $J$ Natl Cancer Inst 25:887-8.

Romani A (2007) Regulation of magnesium homeostasis and transport in mammalian cells. Arch Biochem Biophys 458:90-102.

Romani A, Dowell E, Scarpa A (1991) Cyclic AMPinduced $\mathrm{Mg}^{2+}$ release from rat liver hepatocytes, permeabilized hepatocytes, and isolated mitochondria. J Biol Chem 266:24376-84.
Romani A, Marfella C, Scarpa A (1992) Regulation of $\mathrm{Mg}^{2+}$ uptake in isolated rat myocytes and hepatocytes by protein kinase C. FEBS Lett 296:135-40.

Romani A, Marfella C, Scarpa A (1993a) Regulation of magnesium uptake and release in the heart and in isolated ventricular myocytes. Circ Res 72:1139-48.

Romani A, Marfella C, Scarpa A (1993b) Hormonal stimulation of $\mathrm{Mg}^{2+}$ uptake in hepatocytes. Regulation by plasma membrane and intracellular organelles. $J$ Biol Chem 268:15489-95.

Romani A, Scarpa A (1990a) Hormonal control of $\mathrm{Mg}^{2+}$ in the heart. Nature 346:841-4.

Romani A, Scarpa A (1990b) Norepinephrine evokes a marked $\mathrm{Mg}^{2+}$ efflux from liver cells. FEBS Lett 269:3740.

Romani A, Scarpa A (1992) Regulation of cell magnesium. Arch Biochem Biophys 298:1-12.

Romani A, Scarpa A (2000) Regulation of cellular magnesium. Front Biosci 5:D720-34.

Romani AM, Maguire ME (2002) Hormonal regulation of $\mathrm{Mg}^{2+}$ transport and homeostasis in eukaryotic cells. Biometals 15:271-83.

Romani AM, Matthews VD, Scarpa A (2000) Parallel stimulation of glucose and $\mathrm{Mg}^{2+}$ accumulation by insulin in rat hearts and cardiac ventricular myocytes. Circ Res 86:326-33.

Rondón LJ, Groenestege WM, Rayssiguier Y, Mazur A (2008) Relationship between low magnesium status and TRPM6 expression in the kidney and large intestine. Am J Physiol 294:R2001-7.

Rubin $\mathrm{H}$ (2005) Central roles of $\mathrm{Mg}^{2+}$ and $\mathrm{MgATP}^{2-}$ in the regulation of protein synthesis and cell proliferation: significance for neoplastic transformation. Adv Cancer Res 93:1-58.

Runnels LW, Yue L, Clapham DE (2001) TRP-PLIK, a bifunctional protein with kinase and ion channel activities, Science 291:1043-7.

Runnels LW, Yue L, Clapham DE (2002) The TRPM7 channel is inactivated by PIP(2) hydrolysis. Nat Cell Biol 4:329-36.

Rutter GA, Osbaldeston NJ, McCormack JG, Denton RM (1990) Measurement of matrix free $\mathrm{Mg}^{2+}$ concentration in rat heart mitochondria by using entrapped fluorescent probes. Biochem J 271:627-34. 
Ryazanov AG (2002) Elongation factor-2 kinase and its newly discovered relatives. FEBS Lett 514:26-9.

Ryazanova LV, Pavur KS, Petrov AN, Dorovkov MV, Ryazanov AG (2001) [Novel type of signalling molecules: protein kinases covalently linked to ion channels]. Mol Biol (Mosk) 35:321-32.

Sahni J, Nelson B, Scharenberg AM (2007) SLC41A2 encodes a plasma-membrane $\mathrm{Mg}^{2+}$ transporter. Biochem J 401:505-13.

Sahni J, Scharenberg AM (2008) TRPM7 ion channels are required for sustained phosphoinositide 3-kinase signalling in lymphocytes. Cell Metab 8:84-93.

Scarpa A, Brinley FJ (1981) In situ measurements of free cytosolic magnesium ions. Fed Proc 40:2646-52.

Schatzmann HJ (1993) Asymmetry of the magnesium sodium exchange across the human red cell membrane. Biochim Biophys Acta 1148:15-8.

Schlingmann KP, Weber S, Peters M, Niemann NL, Vitzthum $\mathrm{H}$, Klingel K, Kratz M, Haddad E, Ristoff E, Dinour D, Syrrou M, Nielsen S, Sassen M, Waldegger S, Seyberth HW, Konrad M (2002) Hypomagnesemia with secondary hypocalcemia is caused by mutations in TRPM6, a new member of the TRPM gene family. Nat Genet 31:166-70.

Schmitz C, Deason F, Perraud A-L (2007) Molecular components of vertebrate $\mathrm{Mg}^{2+}$ homeostasis regulation. Magnes Res 20:6-18.

Schmitz C, Dorovkov MV, Zhao X, Davenport BJ, Ryazanov AG, Perraud A-L (2005) The channel kinases TRPM6 and TRPM7 are functionally nonredundant. J Biol Chem 280:37763-71.

Schmitz C, Perraud AL, Johnson CO, Inabe K, Smith MK, Penner R, Kurosaki T, Fleig A, Scharenberg AM (2003) Regulation of vertebrate cellular $\mathrm{Mg}^{2+}$ homeostasis by TRPM7. Cell 114:191-200.

Schweigel M, Lang I, Martens $\mathrm{H}$ (1999) $\mathrm{Mg}^{2+}$ transport in sheep rumen epithelium: evidence for an electrodiffusive uptake mechanism. Am J Physiol 277:G976-82.

Schweigel M, Martens H (2003) Anion-dependent $\mathrm{Mg}^{2+}$ influx and a role for a vacuolar $\mathrm{H}^{+}$-ATPase in sheep rumianl epithelial cells. Am J Physiol 285:G45-53.

Schweigel M, Vormann J, Martens H (2000) Mechanisms of $\mathrm{Mg}^{2+}$ transport in cultured ruminal epithelial cells. Am J Physiol 278:G400-8.
Serrano J, Dashti SR, Perez-Reyes E, Jones SW (2000) $\mathrm{Mg}^{2+}$ block unmasks $\mathrm{Ca}^{2+} / \mathrm{Ba}^{2+}$ selectivity of alpha1G Ttype calcium channels. Biophys J 79:3052-62.

Sgambato A, Wolf FI, Faraglia B, Cittadini A (1999) Magnesium depletion causes growth inhibition, reduced expression of cyclin D1, and increased expression of P27Kip1 in normal but not in transformed mammary epithelial cells. J Cell Physiol 180:245-54

Shafik IM, Quamme GA (1989) Early adaptation of renal $\mathrm{Mg}^{2+}$ reabsorption in response to magnesium restriction. Am J Phyiol 257:F974-7.

Shaul O, Hilgemann DW, de-Almeida-Engler J, Van Montagu M, Inz D, Galili G (1999) Cloning and characterization of a novel $\mathrm{Mg}^{2+} / \mathrm{H}^{+}$exchanger, EMBO J 18:3973-80.

Shi J, Krishnamoorthy G, Wang Y, Hu L, Chaturvedi N, Harilal D, Qin J, Cui J (2002) Mechanism of magnesium activation of calcium-activated potassium channels. Nature 418:876-80.

Shibatani T, David LL, McCormack AL, Frueh K, Skach WR (2005) Proteomic analysis of mammalian oligosaccharyltransferase reveals multiple subcomplexes that contain Sec61, TRAP, and two potential new subunits. Biochemistry 44:5982-92.

Simon DB, Lu Y, Choate KA, Velazquez H, Al-Sabban E, Praga M, Casari G, Bettinelli A, Colussi G, RodriguesSoriano J, McCredie D, Milford D, Sanjad S, Lifton RP (1999) Paracellin-1, a renal tight junction protein required for paracellular $\mathrm{Mg}^{2+}$ resorption. Science 285:103-6.

Smith RL, Thompson L, Maguire ME (1995) Cloning and characterization of MgtE, a putative new class of $\mathrm{Mg}^{2+}$ transporter from Bacillus firmus OF4. J Bacteriol 177:1233-8.

Smoake JA, Moy G-MM, Fang B, Solomon SS (1995) Calmodulin-dependent cyclic AMP phospho-diesterase in liver plasma membranes: Stimulated by insulin. Arch Biochem Biophys 323:223-32.

Somlyo AV, McClellan G, Gonzalez-Serratos H, Somlyo AP (1985) Electron probe X-ray microanalysis of posttetanic $\mathrm{Ca}^{2+}$ and $\mathrm{Mg}^{2+}$ movements across the sarcoplasmic reticulum in situ. J Biol Chem 260:6801-7.

Stanbury JB (1948) The blocking action of magnesium on sympathetic ganglia. J Pharmacol Exp Ther 93:5262. 
Su L-T, Agapito M A, Li M, Simpson W, Huttenlocher A, Habas R, Yue L, Runnels LW (2006) Trpm7 regulates cell adhesion by controlling the calcium dependent protease calpain. J Biol Chem 281:11260-70.

Suarez A, Pulido N, Casla A, Casanova B, Arrieta FJ, Rovira A (1995) Impaired tyrosine-kinase activity of muscle insulin receptors from hypomagnesaemic rats. Diabetologia 38:1262-70.

Takezawa R, Schmitz C, Demeuse P, Scharenberg AM, Penner R, Fleig A (2004) Receptor-mediated regulation of the TRPM7 channel through its endogenous protein kinase domain. Proc Natl Acad Sci USA 101:6009-14.

Tammaro P, Smith AL, Crowley BL, Smirnov SV (2005) Modulation of the voltage-dependent $\mathrm{K}^{+}$current by intracellular $\mathrm{Mg}^{2+}$ in rat aortic smooth muscle cells. Cardiovasc Res 65:387-96.

Tang EY, Parker PJ, Beattie J, Houslay MD (1993) Diabetes induces selective alterations in the expression of protein kinase $\mathrm{C}$ isoforms in hepatocytes. FEBS Lett 326:117-23.

Tashiro M, Konishi M (1997) $\mathrm{Na}^{+}$gradient-dependent $\mathrm{Mg}^{2+}$ transport in smooth muscle cells of guinea pig tenia cecum. Biophys J 73:3371-84.

Tashiro M, Tursun P, Miyazaki T, Watanabe M, Konishi $\mathrm{M}$ (2002) Effects of membrane potential on $\mathrm{Na}^{+}$dependent $\mathrm{Mg}^{2+}$ extrusion from rat ventricular myocytes. Jpn J Physiol 52:541-51.

Taylor R., Agius L (1988) The biochemistry of diabetes. Biochem J 250:625-40.

Tessman PA, Romani A (1998) Acute effect of EtOH on $\mathrm{Mg}^{2+}$ homeostasis in liver cells: Evidence for the activation of an $\mathrm{Na}^{+} / \mathrm{Mg}^{2+}$ exchanger. Am J Physiol 275:G1106-16.

Thebault S, Alexander RT, Tiel Groenestege WM, Hoenderop JG, Bindels RJ (2009) EGF increases TRPM6 activity and surface expression. J Am Soc Nephrol 20:78-85.

Thébault S, Cao G, Venselaar $\mathrm{H}, \mathrm{Xi} \mathrm{Q}$, Bindels RJ, Hoenderop JG (2008) Role of the alpha-kinase domain in transient receptor potential melastatin 6 channel and regulation by intracellular ATP. J Biol Chem 283:19999-20007.

Torres LM, Cefaratti C, Perry B, Romani A (2006) Involvement of ERK1/2 and p38 in $\mathrm{Mg}^{2+}$ accumulation in liver cells. Mol Cell Biochem 288:191-9.
Torres LM, Konopnika B, Berti-Mattera LN, Liedtke C, Romani A (2010) Defective Translocation of PKCepsilon in EtOH-Induced Inhibition of $\mathrm{Mg}$ Accumulation in Rat Hepatocytes. Alcohol Clin Exp Res 34:1659-69.

Torres LM, Youngner J, Romani A (2005) Role of glucose in modulating $\mathrm{Mg}^{2+}$ homeostasis in liver cells from starved rats. Am J Physiol 288:G195-206.

Touyz RM, He Y, Montezano ACl, Yao G, Chubanov V, Gudermann T, Callera GE (2006) Differential regulation of transient receptor potential melastatin 6 and 7 cation channels by ANG II in vascular smooth muscle cells from spontaneously hypertensive rats. Am J Physiol 290:R73-8.

Touyz RM, Schiffrin EL (1996) Angiotensin II and vasopressin modulate intracellular free magnesium in vascular smooth muscle cells through $\mathrm{Na}^{+}$-dependent protein kinase C pathways. J Biol Chem 271:24353-8.

Touyz RM, Yao G (2003) Modulation of vascular smooth muscle cell growth by magnesium-role of mitogen-activated protein kinases. J Cell Physiol 197:326-35.

Trache A, Trzeciakowski JP, Meininger GA (2010) $\mathrm{Mg}^{2+}$ modulates integrin-extracellular matrix interaction in vascular smooth muscle cells studied by atomic force microscopy. J Mol Recognit 23:316-21.

van der Wjist J, Hoenderop JG, Bindels RJ (2009) Epithelial $\mathrm{Mg}^{2+}$ channel TRPM6: insight into the molecular regulation. Magnes Res 22:127-32.

Voets T, Nilliues B, Hoefs S, van der Kemp AW, G Droogmans, Bindels RJ, Hoenderop JG (2004) TRPM6 forms the $\mathrm{Mg}^{2+}$ influx channel involved in intestinal and renal $\mathrm{Mg}^{2+}$ absorption. J Biol Chem 279:19-25.

Volpe P, Alderson-Lang BH, Nickols GA (1990)

Regulation of inositol 1,4,5-trisphosphate-induced $\mathrm{Ca}^{2+}$ release. I. Effect of $\mathrm{Mg}^{2+}$. Am J Physiol 258:C1077-85.

Volpe $P$, Vezú L (1993) Intracellular magnesium and inositol 1,4,5-trisphosphate receptor: molecular mechanisms of interaction, physiology and pharmacology. Magnes Res 6:267-74.

Vormann J, Gunther T (1987) Amiloride sensitive net $\mathrm{Mg}^{2+}$ efflux from isolated perfused rat hearts. Magnesium 6:220-4. 
Vormann J, Magdorf K, Günther T, Wahn U (1994) Increased $\mathrm{Na}^{+} / \mathrm{Mg}^{2+}$ antiport in erythrocytes of patients with cystic fibrosis. Eur J Clin Chem Clin Biochem 32:833-6.

Waas WF, Dalby KN (2003) Physiological concentrations of divalent magnesium ion activate the Serine/Threonine specific protein kinase ERK2. Biochemistry 42:2960-70.

Wabakken T, Rian E, Kveine M, Aasheim H-C (2003) The human solute carrier SLC41A1 belongs to a novel eukaryotic subfamily with homology to prokaryotic MgtE $\mathrm{Mg}^{2+}$ transporters. Biochem Biophys Res Commun 306:718-24.

Walder RY, Landau D, Meyer P, Shalev H, Tsolia M, Borochowitz Z, Boettger MB, Beck GE, Englehardt RK, Carmi R, Sheffield VC (2002) Mutation of TRPM6 causes familial hypomagnesemia with secondary hypocalcemia. Nat Genet 31:171-4.

Walder RY, Yang B, Stokes JB, Kirby PA, Cao X, Shi P, Searby CC, Husted RF, Sheffield VC (2009) Mice defective in Trpm6 show embryonic morality and neural tube defects, Human Mol Gen 18:4367-75.

Wang CY, Davoodi-Semiromi A, Shi JD, Yang P, Huang YQ, Agundez JA, Moran JM, Ochoa B, Hawkins-Lee B, She JX (2003) High resolution mapping and mutation analyses of candidate genes in the urofacial syndrome (UPS) critical region. Am J Med Genet A 119:9-14.

Wang CY, Shi JD, Yang P, Kumar PG, Li QZ, Run QG, Su YC, Scott HS, Kao KJ, She JX (2003) Molecular cloning and characterization of a novel gene family of four ancient conserved domain proteins (ACDP). Gene 306:37-44.

Wang CY, Yang P, Chi JD, Purohit S, Guo D, An H, Gu JG, Ling J, Dong Z, She JX (2004) Molecular cloning and characterization of the mouse Acdp gene family. BMC Genomics 5:7.

Wang M, Berlin JR (2006) Channel phosphorylation and modulation of L-type $\mathrm{Ca}^{2+}$ currents by cytosolic $\mathrm{Mg}^{2+}$ concentration. Am J Physiol 291:C83-92.

Wang S, George SE, Davis JP, Johnson JD (1998) Structural determinants of $\mathrm{Ca}^{2+}$ exchange and affinity in the $\mathrm{C}$ terminal of cardiac troponin C. Biochemistry 37:14539-44.

Wei WL, Sun HS, Olah ME, Sun X, Czerwinska E, Czerwinski W, Mori Y, Orser BA, Xiong ZG, Jackson MF, Tymianski M, MacDonald JF (2007) TRPM7 channels in hippocampal neurons detect levels of extracellular divalent cations. Proc Natl Acad Sci U SA 104:16323-8.
White RE, Hartzell HC (1988) Effects of intracellular free magnesium on calcium current in isolated cardiac myocytes. Science 239:778-80.

Wiesenberger G, Waldherr M, Schweyen RJ (1992) The nuclear gene MRS2 is essential for the excision of group II introns from yeast mitochondrial transcripts in vivo. J Biol Chem 267:6963-9.

Wolf Fl, Cittadini A (2003) Chemistry and biochemistry of magnesium. Mol Aspects Med 24:3-9.

Wolf FI, Cittadini AR, Maier JA (2009a) Magnesium and tumors: ally or foe? Cancer Treat Rev 35:378-82.

Wolf Fl, Covacci V, Bruzzese N, Di Francesco A, Sachets A, Cord D, Cittadini A (1998) Differentiation of HL-60 promyelocytic leukemia cells is accompanied by a modification of magnesium homeostasis. J Cell Biochem 71:441-8.

Wolf FI, Di Francesco A, Cittadini A (1994)

Characterization of magnesium efflux from Ehrlich ascites tumor cells. Arch Biochem Biophys 308:335-41.

Wolf FI, Di Francesco A, Covacci V, Cittadini A (1997) Regulation of magnesium efflux from rat spleen lymphocytes. Arch Biochem Biophys 344:397-403.

Wolf FI, Di Francesco A, Covacci V, Corda D, Cittadini A (1996) Regulation of intracellular magnesium in ascites cells: involvement of different regulatory pathways. Arch Biochem Biophys 331:194-200.

Wolf FI, Torsello A, Fasanella S Cittadini A (2003) Cell physiology of magnesium. Mol Asp Med 24:11-26.

Wolf FI, Trapani V, Simonacci M, Boninsegna A, Mazur A, Maier JA (2009b) Magnesium deficiency affects mammary epithelial cell proliferation: involvement of oxidative stress. Nutr Cancer 61:131-6.

Xu W, Willis JS (1994) Sodium transport through the amiloride-sensitive $\mathrm{Na}-\mathrm{Mg}$ pathway of hamster red cells. J Membr Biol 141:277-87.

Yamaguchi S, Ishikawa T (2008) The electgrogenic $\mathrm{Na}^{+}$$\mathrm{HCO}_{3}^{-}$cotransporter NBCe1-B is regulated by cellular $\mathrm{Mg}^{2+}$. Biochem Biophys Res Commun 376:100-4.

YamaguchiH, Matsushita M, Nairn A, Kuriyan J (2001) Crystal structure of the atypical protein kinase domain of a TRP channel with phosphotransferase activity. Mol Cell 7:1047-57. 
Yanai A, Huang K, Kang R, Singaraja RR, Arstikaitis $P$, Gan L, Orban PC, Mullard A, Cowan CM, Raymond LA, Drisdel RC, Green WN, Ravikumar B, Rubinsztein DC, El-Husseini A, Hayden MR (2006) Palmitoylation of huntingtin by HIP14 is essential for its trafficking and function. Nat. Neurosci 9:824-31.

Yang Z, Wang J, Altura BT, Altura BM (2000)

Extracellular magnesium deficiency induces contraction of arterial muscle: role of PI3-kinases and MAPK signalling pathways. Pflugers Arch 439:240-7.

Yang ZW, Wang J, Zheng T, Altura BT, Altura BM (2001) Ethanol-induced contractions in cerebral arteries: role of tyrosine and mitogen-activated protein kinases. Stroke 32:249-57.

Zhang GH, Melvin JE (1992) Secretagogue-induced mobilization of an intracellular $\mathrm{Mg}^{2+}$ pool in rat sublingual mucous acini. J Biol Chem 267:20721-7.
Zhang GH, Melvin JE (1996) $\mathrm{Na}^{+}$-dependent release of $\mathrm{Mg}^{2+}$ from an intracellular pool in rat sublingual mucous acini. J Biol Chem 271:29067-72.

Zhang J, Wier G, Blaustein MP (2002) $\mathrm{Mg}^{2+}$ blocks myogenic tone but not $\mathrm{K}^{+}$-induced constriction: Role for SOCs in small arteries. Am J Physiol 283:H2692705.

Zhou H, Clapham DE (2009) Mammalian MagT1 and TUSC3 are required for cellular magnesium uptake and vertebrate embryonic development. Proc Natl Acad Sci U S A 106:15750-5.

Zsurka G, Gregan J, Schweyen RJ (2001) The human mitochondrial Mrs2p protein functionally substitutes for its yeast homologue, a candidate magnesium transporter. Genomics 72:158-68. 


\title{
Magnesium transport across the blood-brain barriers
}

\author{
Mounir N. Ghabriel ${ }^{\bowtie}$ and Robert Vink
}

Discipline of Anatomy and Pathology \& Adelaide Centre for Neuroscience Research, School of Medical Sciences, The University of Adelaide, Adelaide, South Australia 5005, Australia.

$\triangle$ mounir.ghabriel@adelaide.edu.au

\begin{abstract}
The finding that magnesium levels are reduced in acute and chronic brain diseases has led to a recent surge in interest in the role of magnesium in the normal and injured nervous system, although the mechanisms of magnesium decline in pathological conditions and its availability in the neural tissue after administration are not fully understood. The brain has two main barrier systems: (1) the blood-brain barrier (BBB) formed by brain capillary endothelial cells which separate the blood from the extracellular fluid in the neuropil; and (2) the blood-CSF barrier (BCSFB) formed by choroidal epithelial cells which separate the blood from the CSF. Genetic studies in families with hereditary hypomagnesemia have identified mutations in two genes encoding claudin-16 (paracellin-1) and claudin-19, both localized at tight junctions between nephron epithelial cells and providing passive paracellular conductance for magnesium in the kidney. Endothelial cells of the BBB also express claudins, although whether members of the claudin family expressed at the BBB and BCSFB have similar conductance for magnesium akin to the role of claudin-16 and -19 in the nephron remains to be confirmed. Recently, the transient receptor potential melastatin (TRPM) members TRPM6 and TRPM7 have been identified as cation channels for magnesium transport. Although it is not known if choroidal epithelial cells express TRPM6 and TRPM7, these molecules are expressed by brain endothelial cells and may play a role in magnesium transport. While it is evident that magnesium enters the CNS through the BBB and is actively transported by choroidal epithelial cells into the CSF, the mechanisms of its entry into the brain will require further investigation.
\end{abstract}

\section{Introduction}

Magnesium is a critical cation and an essential nutrient for normal body functions; hence mechanisms exist in the body for its homeostasis through a highly integrated feedback system involving intestinal absorption, renal excretion, bone metabolism and the parathyroid gland (Dai et al., 2001). Magnesium is involved in a myriad of biochemical processes including acting as a cofactor in the activation of many intracellular enzymes (Aikawa, 1976; Ebel and Gunther, 1980; Gunther, 2008) and is important for protein synthesis (Terasaki and Rubin 1985) and cell membrane stabilization (Bara and Guiet-Bara 1984). Magnesium deficiency reduces protein synthesis, serum antibody activity and the immune response (McCoy and Kenney, 1975), and induces CNS epileptiform activity (Morris, 1992) and hypomagnesemic tetany (Fontenot et al., 1989). There is an inverse correlation between dietary magnesium and the level of C- reactive protein (a marker of inflammation) and the level of E-selectin (a marker of endothelial cell dysfunction) (Song et al., 2007). Recent technical developments have allowed better assessment of the levels of magnesium in the body, although the blood, which is used in routine testing, contains only $0.3 \%$ of the total body magnesium (Elin, 1987). Approximately one half of the magnesium contained in the body is stored in the bone, while the rest exists in the soft tissues, mainly in the intracellular compartment, with less that $1 \%$ being present in the extracellular compartment (Elin, 1988). Indeed, magnesium is the second most common cation in the intracellular compartment after potassium. More than one half of the plasma content of magnesium is bound to plasma proteins, and the remaining free ionized magnesium $\left(\mathrm{Mg}^{2+}\right)$, the metabolically active fraction, is held within a narrow range (0.53$0.67 \mathrm{mM}$ ) in normal healthy controls (Altura and Altura, 1991). The recom-mended daily allowance 
ranges from 320 to $420 \mathrm{mg} /$ day for women and men, respectively (Bergman et al., 2009).

There has been a recent surge in interest in the dynamics and role of magnesium in the normal and injured brain due to accumulating evidence of a reduction in the level of total and free $\mathrm{Mg}^{2+}$ in the brain in acute and chronic neurological diseases (Vink et al., 1987; 2009). In intensive care patients with traumatic brain injury (TBI) reduction of serum ionized $\mathrm{Mg}^{2+}$ correlates with the severity of TBI as determined by the Glasgow Coma Scale Score (Kahraman et al., 2003). In experimental TBI in the rat there is a sustained decline in intracellular $\mathrm{Mg}^{2+}$, detected by phosphorus magnetic resonance spectroscopy, that correlates with motor deficit (Cernak et al., 2004; Heath and Vink, 1996), while magnesium administration significantly improves motor outcome (Heath and Vink 1999; Turner et al., 2004). Magnesium administration reduces TBIinduced brain edema (Feldman et al., 1996) and restores blood-brain barrier (BBB) effectiveness to Evans blue tracer when compared to injured non-treated rats (Esen et al., 2003; Imer et al., 2009). Magnesium also reduces brain edema after cold-induced brain injury (Turkoglu et al., 2008). However, the mechanisms of magnesium decline in pathological conditions and its availability in the neural tissue after administration are still unclear (Vink et al., 2009).

Therapeutically, magnesium can be administered orally, intravenously or intramuscularly (Elin, 1988). It is most commonly used used as magnesium chloride, sulphate, gluconate, acetate (Fine et al., 1991) or lactate (Fine et al., 1991; Simoes Fernandes et al., 1985). Magnesium therapy has been utilized in numerous experimental and clinical settings including migraine (Peikert et al., 1996), asthma (Cheuk et al., 2005), depression (Eby and Eby, 2006), anxiety (Kara et al., 2002), diabetes (Resnick et al., 1993; Wester and Dyckner, 1987), hypertension (Wester and Dyckner, 1987), atrial fibrillation (Fanning et al., 1991), sleep disorders, insomnia and chronic fatigue (Takahashi et al., 1992), dementia (Glick, 1990), osteoporosis (Sojka and Weaver, 1995), fibromyalgia (Porter et al., 2010), pain (Soave et al., 2009), eclampsia (Euser and Cipolla, 2009), constipation (Guerrera et al., 2009), cerebral palsy (Rouse, 2009), lacunar stroke (Muir et al., 2004), TBI (Mclntosh et al.,
1988) and aneurysmal subarachnoid hemorrhage (van den Bergh, 2009; Yahia et al., 2005).

The current review will briefly outline the status of magnesium in the body, then will summarize current knowledge of its distribution in the CNS and discuss its transport across barrier membranes in the brain compared to its transport in other organs such as the kidney.

\section{Magnesium absorption and excretion}

Much of our knowledge about magnesium absorption and excretion was gained in the latter part of the twentieth century and particularly in the nineties. Studies of normal subjects showed that magnesium bioavailability from high magnesium containing food sources such as almonds is equal to that obtained from soluble magnesium acetate, but enteric coating of magnesium chloride impairs magnesium bioavailability (Fine et al., 1991). Magnesium absorption occurs mainly in the small intestine (Brannan et al., 1976; Graham et al., 1960; Schroeder et al., 1969). The kinetics and rate of magnesium absorption are not dependant on calcium intake (Brannan et al., 1976). Under basal conditions the small intestine absorbs $30-50 \%$ of the magnesium intake, although this percentage diminishes with senescence, chronic renal disease and increasing intake (Musso 2009). The fractional magnesium absorption appears to fall progressively so that absorption as a function of intake is curvilinear (Fine et al., 1991). The fecal magnesium appears to be primarily derived from material that is not absorbed by the body rather than magnesium secreted by the intestine (Aikawa, 1976). Approximately $80 \%$ of the absorbed magnesium passes in the glomerular filtrate (Dai et al., 2001; Quamme and de Rouffignac, 2000).

More that $95 \%$ of the magnesium filtered by the glomerulus is reclaimed mainly in the thick ascending loop of Henle (60-70\%) and to a lesser extent $(10-15 \%)$ in the proximal convoluted tubules (Brunette et al., 1974; Dai et al., 2001; Quamme 1997; Quamme and de Rouffignac, 2000). A further $10 \%$ of magnesium in the filtrate is claimed in the distal convoluted tubule (de Rouffignac and Quamme, 1994) and this segment contributes to magnesium conservation (Quamme 1997). Magnesium reabsorption within the thick 
ascending loop appears to be passive and occurs via the paracellular route (de Rouffignac and Quamme, 1994), being driven by the transepithelial voltage (Quamme, 1997; Quamme and de Rouffignac, 2000), where positive luminal charge favors movement of magnesium from the luminal to the abluminal side of nephron epithelium (Quamme, 1997). On the other hand magnesium transport in the distal convoluted tubule is active and transcellular (Quamme, 1 997). While the mechanisms of magnesium transport were unclear and speculative (Dai et al, 2001; Quamme and Dirks, 1980) more recent reports point to specific paracellular (Efrati et al, 2005; Simon et al, 1999) and transcellular (Hoenderop and Bindels, 2005) routes across barrier membranes.

\section{Barriers between the blood and the CNS}

Two main fluid compartments are associated with the brain, the extracellular fluid (ECF) that bathes neurons and glial cells, and the cerebrospinal fluid (CSF) located in the subarachnoid space and ventricles of the brain (Figure 1).

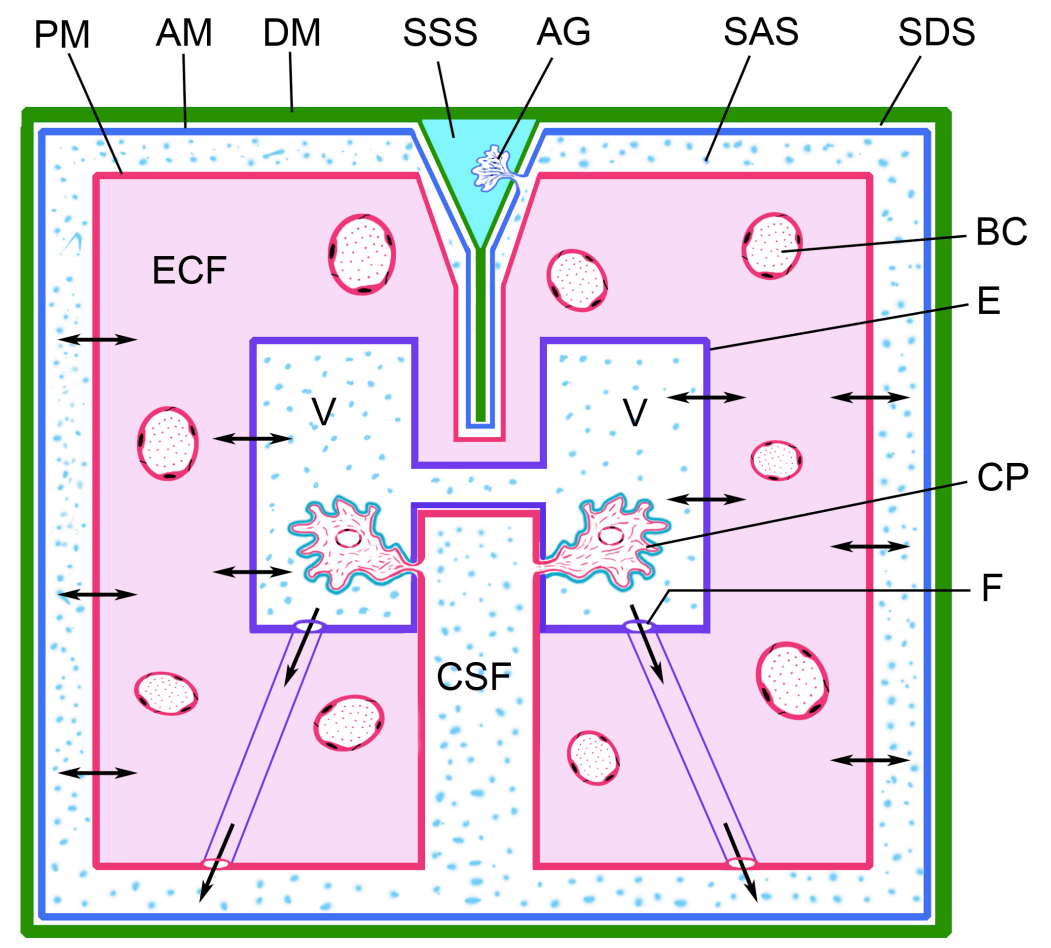

Figure 1. Diagram showing locations of the blood-brain barrier (BBB), the blood-CSF barrier (BCSFB) and the meninges. The cranial dura mater (DM) encloses all intracranial contents, and is formed of two fused layers that separate at certain places to enclose dural venous sinuses, such as the superior sagittal sinus (SSS). The subdural space (SDS) is a potential space as the dura and arachnoid mater (AM) are closely apposed. The arachnoid mater is impermeable to CSF contained in the subarachnoid space (SAS). The arachnoid mater forms the arachnoid granulations (AG) that pierce the dural wall of the superior sagittal sinus and drain the CSF into the venous blood. Brain capillaries $(B C)$ are lined with endothelial cells joined by tight junctions and form the BBB, thus they control the composition of the extracellular fluid (ECF). Ventricles (V) of the brain contain CSF produced mainly by the choroidal epithelium, which covers the choroid plexuses (CP) and form the BCSFB. The ventricles are lined with ependymal cells $(E)$, which are continuous with the choroidal epithelium at the neck of the choroid plexus. The core of the choroid plexus is formed of pia mater and contains fenestrated capillaries. CSF leaves the ventricular system via the three foramina of the fourth ventricle, represented here by two channels (F). The CSF and ECF of the brain equilibrate through the pia mater on the surface of the brain, and the ependyma lining the ventricles (double-headed arrows). 
Exchange between the blood and these fluid compartments can potentially occur at four sites, which have barrier mechanisms: (1) brain capillary endothelial cells, which separate the ECF from the blood and form the BBB; (2) the choroidal epithelial cells at the ventricular surface of the choroid plexuses, which interface between the blood and the CSF and form the blood-CSF barrier (BCSFB); (3) the arachnoid mater, which surrounds the CSF contained within the subarachnoid space; (4) the circumventricular organs of the brain, which have fenestrated capillaries. The four sites form potential entry routes for water, molecules, electrolytes, toxins, pathogens and drugs (Segal, 2000).

\section{The arachnoid mater and circumventricular organs}

The arachnoid mater forms an external barrier, the morphological substrates of which are the flat tightly packed mesothelial cells (the arachnoid barrier cell layer) with their numerous tight junctions. They separate the CSF from the subdural space and the dura (Fig.1), which has fenestrated capillaries (Nabeshima et al., 1975; Vandenabeele et al., 1996). The arachnoid is avascular (Alcolado et al., 1988) and, relative to brain capillaries, has a much less surface area, thus its contribution to transport from the subdural space to the CSF and brain is negligible. Also the circumventricular organs of the brain, although they have fenestrated capillaries (McKinley et al., 2003; Sunn et al., 2003), they are provided with an internal mechanism that contributes to a barrier between these sites and the surrounding brain. They have rapid venous return systems, which compensates for the leaky capillaries, effectively preventing the spread of marker molecules to the surrounding brain tissue (Hashimoto, 1988; Segal, 2000). Therefore, the two main routes that are likely to have greater involvement in the regulation of the ECF and CSF environments are the BBB and BCSFB (Abbott et al., 2010).

\section{The blood-brain barrier (BBB)}

The concept of a BBB has been proposed over a century ago (Bradbury 1979) and is still being developed (Wolburg et al., 2009). The BBB is formed by endothelial cells of brain capillaries, the characteristics of which are influenced by the surrounding microenvironment, including astrocytes and pericytes (Wolburg et al., 2009). Apart from capillaries in the circumventricular organs which have fenestrated endothelial cells, those in the rest of the brain are lined with specialized endothelial cells which show few endocytotic vesicles, have no fenestrations and are rich in mitochondria (Brightman and Reese, 1969; Fenstermacher et al., 1988; Reese and Karnovsky, 1967; Rubin and Staddon, 1999; Sedlakova et al., 1999). Brain endothelial cells have high electrical resistance (Butt et al., 1990) and are joined by tight junctions (TJs) (Begley and Brightman, 2003; Rubin and Staddon, 1999). These TJs have long been recognized as the sites of exclusion of protein tracers as detected by electron microscopy (Brightman and Reese 1969; Reese and Karnovsky 1967; Sedlakova et al., 1999). Freeze-fracture electron microscopy revealed that these tight junctions have an elaborate and complex arrangement of network of interconnecting strands of intramembranous particles, that particularly cleave with the P-face leaving corresponding grooves on the E-face of replicas of the split membrane (Sedlakova et al., 1999).

The BBB therefore plays a pivotal role in the tight regulation and rigorous stabilization of the chemical composition of brain ECF against fluctuations in the plasma chemistry, thus promoting normal neuronal signaling. The privileged status of the brain being protected by the BBB has a downside, in restricting access by drugs and therapeutic agents into the brain when needed (Abbott and Romero, 1996). Although the barrier nature of the BBB has been emphasized, it is obviously a selective barrier, as it should allow passage of nutrients and electrolytes. Thus endothelial cells of brain vessels are endowed with a host of receptors, enzymes and carriers in a polarized distribution to promote selective transport (Abbott et al., 2010; Betz et al., 1980; Ohtsuki and Terasaki, 2007; Roberts et al., 2008). Brain endothelial cells express the glucose transporter Glut-1 protein (Dick et al., 1984; Harik et al., 1994; Ohtsuki and Terasaki, 2007; Pardridge et al., 1990; Pardridge, 1991), which is coupled to neuronal demand (Leybaert, 2005) and its expression increases in hypoxia (Harik et al., 1994). Rat brain endothelial cells express a barrier antigen (Sternberger and Sternberger, 1987), neutralization of which leads to reversible opening of the BBB to exogenous and endogenous proteins (Ghabriel et al., 2000; 2004). Lipophilic molecules can penetrate 
endothelial cell membranes by diffusion, although some of these may be effluxed via $A B C$ transporters, such as P-glyco-protein (Begley 2004; Miller, 2010; Shen and Zhang, 2010). Macromolecules may cross the BBB via receptormediated transcytosis (Descamps et al., 1996), or adsorptive-mediated transcytosis (Villegas and Broadwell, 1993; Zlokovic et al., 1990). Watersoluble nutrients and metabolites can to a limited degree pass passively across the BBB, but greater bulks of water, hydrophilic molecules and electrolytes have to be transported via carrier systems and channels (Zhang et al., 2002). Amino acids, nucleosides, small peptides, organic anions and organic cations are transported via solute carriers (Abbott et al., 2010; Koepsell et al., 2003; Ohtsuki and Terasaki, 2007).

Unlike in other organs, such as the intestinal wall where the extracellular space is large and is occupied by loose connective tissue, that of the brain is very narrow as neuronal and glial membranes are closely apposed leaving a gap of 20-50 nm (Vanharreveld et al., 1965) with a small volume of ECF. A small change in the ECF volume may lead to large alterations in electrolyte concentrations, deleteriously affecting neuronal excitability (Amiry-Moghaddam and Ottersen, 2003). Hence water transport across the BBB is intricately linked to ions transport. Brain perivascular astrocytic foot processes, sub-pial astrocytes and the ependyma, which lines the ventricles, express the water channels AQP-4 (Amiry-Moghaddam and Ottersen, 2003; Li et al., 2009; Nielsen et al., 1997). More recent studies demonstrated that brain endothelial cells also express AQP-4 at their luminal and abluminal membranes, albeit at a much lower density than in astrocytic foot processes (Amiry-Moghaddam and Ottersen, 2003; Kobayashi et al., 2001). The link between water movements and electrolyte concentrations is further strengthened by the coexpression of AQP4 and Kir-4.1 potassium channels, and the discovery that water movement is associated with potassium fluxes in the same direction (Amiry-Moghaddam and Ottersen, 2003; Niermann et al., 2001). The BBB is relatively permeable to water, allowing a slow bulk flow (Abbott, 2004) but less so to ions (Go, 1997). Electrolytes homeostasis in the ECF is actively maintained by the BBB, as evidenced by a high $\mathrm{Na}^{+}, \mathrm{K}^{+}$-ATPase activity in brain endothelial cells (Bauer et al., 1990; Go, 1997; Seda et al.,
1984). Potassium concentration in the ECF is kept at a lower level than that of plasma (Hansen, 1985).

\section{The blood-CSF barrier (BCSFB)}

The brain has four choroid plexuses that project into the two lateral ventricles and the third and fourth ventricles. Each choroid plexus contains a core of loosely arranged stroma derived from the leptomeninges. Their ventricular surface is extremely fringed (Fig.1). The core of the plexus is richly supplied with capillaries, the endothelial cells of which have fenestrations and leaky tight junctions (Wolburg and Paulus, 2010). Large molecules such as peroxidase penetrate the choroid plexus capillaries and enter the interstitial space in the core of the plexus (Brightman, 1968). As the plexus invaginates the ventricle it acquires a covering of ependyma, the cell layer that forms the lining of the ventricle (Fig.1). This ependymal covering is known as the choroidal epithelium, which, although continuous with the rest of the ependyma at the neck of the choroid plexus, is structurally and functionally different, as it becomes modified and specialized to form the BCSFB (Brightman 1968; Redzic and Segal 2004; Tripathi 1973). The choroidal epithelium therefore separates the CSF from the milieu of the plexus core with its leaky capillaries. Choroidal epithelial cells are joined by tight junctions, which occlude the paracellular route (Wolburg and Lippoldt, 2002; Wolburg and Paulus, 2010). Choroidal epithelial cells actively produce the CSF and regulate its electrolyte, protein and water content (Bradbury et al., 1963). Choroidal epithelial cells express the water channels AQP-1 (Agre et al., 1993; Nielsen et al., 1993). In addition to their main function of CSF secretion choroidal epithelial cells transport some substances from the blood to the CSF, for example, nucleotides and ascorbic acid, and actively removes other substances from the CSF (Go, 1997).

There is no barrier between the brain and CSF located in the subarachnoid space, as the cells of the pia mater on the brain surface have gap junctions and no tight junctions (Alcolado et al., 1988). Peroxidase perfused into the subarachnoid space penetrates between pia mater cells, through the basement membrane of astrocytes at the brain surface and between astrocytes (outer glia limitans) to enter the neuropil 
(Brightman and Reese, 1969). Also free exchange occurs between the CSF located in the ventricles and the brain ECF across the ependymal lining of the ventricles, as peroxidase injected into the ventricles enter the neuropil (Brightman and Reese, 1969), thus the CSF and the ECF of the brain equilibrate.

\section{Magnesium transport across barrier membranes including the BBB and BCSFB}

\section{Magnesium transport in the kidney}

The kidney is the main contributor to magnesium homeostasis, thus it is not surprising that research efforts to understand magnesium transport were focused on epithelial cells of the nephron. During the last decade greater understanding of the transport of magnesium across cell membranes was gained through genetic studies in families with hereditary abnormal magnesium balance, positional cloning and knockout mice investigations (Hoenderop and Bindels, 2005). Such studies led to the identification of genes encoding proteins involved in the transport of magnesium. One human gene, the paracellin-1 ( $P C L N-1)$, encodes the protein paracellin-1. PCLN-1 mRNA was detected using RT-PCR in the thick ascending limb of Henle's loop and the distal convoluted tubule of the kidney in the rabbit (Simon et al., 1999) and rodents (Weber et al., 2001). The PCLN-1 protein was also detected using immunocytochemistry in the same segments in rabbit kidney using an antibody to PCLN-1 (Simon et al., 1999). Using confocal microscopy and double immunofluorescence for PCLN-1 and occuldin, a tight junction ubiquitous protein (Furuse et al., 1993), demonstrated colocalisation of the two proteins to intercellular tight junction at both segments of rabbit nephrons (Simon et al., 1999). The paracellin-1 protein is a member of the claudin family (claudin 16) (Simon et al., 1999). Families with mutation in PCLN-1 gene show hypomagnesemia and renal magnesium wasting (Simon et al., 1999). The paracellin-1 located in the tight junction between epithelial cells in the nephron acts as a passive channel for reabsorbing magnesium along the paracellular route being favored by the positive luminal charge. Because these families also show hypercalciurea and urinary calculi, it was suggested that PCLN-1 is also a conductance for calcium (Simon et al., 1999). Such discovery modified the previously prevalent view that tight junctions exist to prevent passage of molecules and are static; rather, they do provide selective barriers. Further studies on human recessive renal magnesium loss mapped a second locus on chromosome 1 p34.2 and have identified mutations in CLDN19, which encodes the TJ protein claudin-19 expressed in the nephron tubules (Konrad et al., 2006).

Recently plasma membrane cation channels that belong to the transient receptor potential (TRP) superfamily have been described and were classified into 7 subfamilies. The closely related subfamilies TRPC, TRPV, TRPM, TRPN and TRPA are classified as group 1, while group 2 includes TRPML and TRPP (Montell 2005). The TRPM subfamily includes 8 members, of which TRPM6 and TRMP7 have been recognized as highly permeable $\mathrm{Mg}^{2+}$ transporters into cells, and have been particularly studied in epithelial cells of the nephron (Aikawa 1976; Dai et al., 2001). In familial autosomal-recessive hypomagnesemia with sec-ondary hypocalcemia, affected infants suffer from seizures and tetany due to abnormal handling of $\mathrm{Mg}^{2+}$ in intestinal absorption and renal reabsorption. Genetic analysis pointed to a gene, TRPM6, which was mutated in these patients (Schlingmann et al., 2002; Walder et al., 2002). Using immunocytochemistry, it was shown that TRPM6 protein is localized to the apical membrane of renal distal convoluted tubules epithelium, the site of active reclaiming of magnesium, and also at the brush border of apical membranes of intestinal epithelium, the main site of magnesium absorption (Voets et al., 2004). Patch-clamp analysis and measurement of intracellular magnesium indicated that TRPM6 forms all or at least part of the magnesium channel in absorbing epithelia (Voets et al., 2004). It has been suggested that mammary epithelial cells in culture, with high and low magnesium content, adapt to low magnesium availability by upregulating magnesium influx via TRPM6, and to high magnesium availability by increasing magnesium efflux primarily via $\mathrm{Na}^{+} / \mathrm{Mg}^{2+}$ exchange (Wolf et al., 2010). The TRPM7 also has a central role in $\mathrm{Mg}^{2+}$ homeostasis, because TRPM7-deficient cells become $\mathrm{Mg}^{2+}$ deficient, and cannot survive (Schmitz et al., 2003).

\section{Magnesium transport across the BBB}

Magnesium entry into the brain correlates with 
maturation of the BBB. In fetal sheep and the guinea pig, there is a drop in the concentration of $\mathrm{Mg}^{2+}$ in the cerebral hemispheres, which corresponds approximately to the minimum in potassium content and the maximum in chloride and sodium contents (Bradbury et al., 1972). During rat postnatal development, brain content of magnesium shows regional variations. At day 5 , magnesium is most marked in the pons and medulla and least marked in the cerebral cortex. Magnesium levels in all regions decline after day 5 , in parallel with the decrease in water content and the increase in tissue weight, suggesting that the maturation of the BBB plays an important role in brain magnesium homeostasis (Chan et al., 1992).

Magnesium is able to cross the BBB (Sacco et al., 2007), and it is transported via the barrier with a net flux from the blood into the parenchyma. Active magnesium transport from blood to the extracellular fluid of the brain is evidenced by its higher concentration in the cortical extracellular fluid than its concentration in the plasmadialysate or cisternal CSF (Bito, 1969). Magnesium administration attenuates cell death due to cytoskeletal alteration (Saatman et al., 2001) and reduces apoptosis and the expression of p53 following TBI (Lee et al., 2004). Thus its administration rectifies the decline in intracellular $\mathrm{Mg}^{2+}$ levels and cellular functions (Saatman et al., 2001). In experimental closed head trauma, treatment with magnesium restores the polarity of astrocytes, in terms of aquaporin-4 distribution, to a preinjury state (Ghabriel et al., 2006). A similar protective effect for magnesium was demonstrated in induced hypoglycemia (Kaya et al., 2001). This indicates replenishing of magnesium in the extracellular space of the brain. However, the question remains, how does magnesium cross endothelial cells of the BBB? Is magnesium transport across the BBB similar to its transport across nephron epithelial cells? If so, does $\mathrm{Mg}^{2+}$ cross the BBB via a paracellular route or a transcellular route or both?

Concerning the paracellular route, the proposed role for PCLN-1 (claudin-16) in the nephron (Simon et al., 1999), acting as a conductance for $\mathrm{Mg}^{2+}$, and its exclusive location in the kidney (Efrati et al., 2005; Weber et al., 2001), raise the possibility that other members of the claudin family may play a similar role for paracellular conductance across other barrier membranes. Members of the claudin family are involved in the formation of TJ strands in various tissues (Morita et al., 1999). There is tissue-specific expression of claudin members in tight junctions, with a specific colocalisation of occludin with some but not all claudins (Peppi and Ghabriel, 2004). Magnesium is important for cell membrane stabilization. In the CNS, the myelin sheath is an elaboration of the cell membrane of oligodendrocytes, which express claudin-11. In claudin-11-deficient mice, the TJs are absent in the myelin sheath of oligodendrocytes and mice show demyelination (Gow et al., 1999). This indicates that claudin-11 is an important component in the stability and formation of the myelin tight junctions. Also endothelial cells of brain microvessels express several claudins, including claudin-1, $-3,-5$ and -12 (Coisne et al., 2005; Liebner et al., 2000; Matter and Balda, 2003; Morita et al., 1999; Tsukita and Furuse, 1999; Wolburg et al., 2003). Breakdown of the $\mathrm{BBB}$ in experimental autoimmune encephalomyelitis in the mouse and the leaky vessels in human glioblastoma multiforme are accompanied by selective loss of claudin- 3 in BBB TJs (Wolburg et al., 2003). Although direct link between $\mathrm{Mg}^{2+}$ permeability and claudins has not been established in the CNS similar to the link between claudin-16 and epithelial cells of the nephron, it is tempting to speculate that, in addition to their supporting role in the morphology the BBB TJs, claudins in TJs of brain endothelial cells may contribute to $\mathrm{Mg}^{2+}$ conductance at the BBB. It is also speculated here that claudin-11 in the CNS myelin, in addition to its morphological supporting role, may act as a conductance for $\mathrm{Mg}^{2+}$ across the consecutive myelin lamellae, providing a faster access route from the ECF to the periaxonal space.

Concerning the transport of $\mathrm{Mg}^{2+}$ across the BBB via a transcellular route, it may also be prudent to compare the BBB to other barrier membranes. As stated above, TRPM6 transporter protein has been localized to the apical membrane of renal distal convoluted tubules epithelium, the site of active reclaiming of magnesium (Voets et al., 2004), and TRPM7 in the kidney also has a central role in $\mathrm{Mg}^{2+}$ homeostasis (Schmitz et al., 2003). In mouse brain, using quantitative RT-PCR, TRPM3 and TRPM7 mRNA were detected at high levels, and 19 other isoforms were also present (Brown 
et al., 2008; Kunert-Keil et al., 2006). Also in human brain TRPM1, 2, 3, 6 and 7 have been detected using RT-PCR (Fonfria et al., 2006). Evidence of TRPM presence in brain endothelial cells was obtained from endothelial cell cultures. Immortalized mouse brain microvessel endothelial cells, freshly isolated cerebral microvessels and primary cultured rat brain endothelial cells express multiple TRPC and TRPV isoforms, and also TRPM2, M3, M4, and M7 mRNA (Brown et al., 2008). We would like to suggest therefore that the TRPM6 and TRPM7, the gatekeepers of magnesium (Schlingmann et al., 2007), are likely to play a main role in brain endothelial cell transport of magnesium via the transcellular route. Western blotting and immunofluorescence assays for TRPM6 6 and TRPM7 similar to those performed for TRPV4 in mouse cerebral microvascular endothelial cells (Ma et al., 2008) would support this suggestion. Also direct evidence may be obtained from measuring intracellular magnesium in endothelial cell culture similar to previous reports on mammary epithelial cells in culture (Voets et al., 2004)

\section{Magnesium transport across the blood-CSF barrier}

What has become apparent from experimental research and human studies is that magnesium concentration in the CSF is actively maintained above that of the plasma, and changes in CSF magnesium lag behind changes in its concentration in the plasma and are less pronounced (Bradbury and Sarna, 1977; Morris, 1992). Thus electrolyte composition in the CSF of adult mammals is different from that of plasma and is more stable, suggesting the existence of mechanisms for electrolyte homeostasis in the CSF, necessary for normal brain function (Bradbury et al., 1972; Somjen, 2002). The mechanisms determining the concentrations of ions in CSF appear to develop at different and largely independent rates (Bradbury et al., 1972). In the dog (Oppelt et al., 1963) and human (Nischwitz et al., 2008) the concentration of magnesium is higher in the CSF compared to plasma. Also in fetal sheep and guinea pigs magnesium concentration in the CSF is slightly higher than that of plasma (Amtorp and Sorensen, 1974; Bradbury et al., 1972). An investigation of the permeability of the human BCSFB to magnesium carried out in 29 individuals, using paired serum and CSF samples, showed a mean
CSF/serum ratio of 1.3 (Nischwitz et al., 2008). This study also compared magnesium to other metals, and suggested that low molecular weight species such as magnesium and calcium cross the BCSFB through less specific ion channels compared to high molecular weight metals such as iron, copper and zinc, which can pass the barrier only via well controlled receptor mediated pathways (Nischwitz et al., 2008)

The choroidal epithelium plays an active role in maintaining the level of magnesium in the CSF by sensing changes in the CSF and altering the rate of active magnesium secretion (Oppelt et al., 1963; Reed and Yen 1978). Physiological studies of isolated choroid plexus of sheep (Allsop 1986) and cat (Reed and Yen 1978) showed that the choroid plexus is able to transfer magnesium against a concentration gradient. An in vivo study demonstrated a directional flow of magnesium into the CNS (Allsop and Pauli, 1985). In normal cows the magnesium concentration in the ventricular CSF was found to be higher than its concentration in the lumbar CSF (Allsop and Pauli, 1985). Under hypomagnesemic conditions, magnesium concentration in the ventricle decreased more rapidly than that of the lumbar CSF, while intravenous infusion of magnesium led to increased magnesium in the ventricular CSF before changes in the lumbar CSF (Allsop and Pauli, 1985). This study indicates that the access of magnesium from the blood to the brain is mainly via the choroid plexus to CSF, which equilibrates with the CNS parenchyma. Transport of magnesium in the choroid plexus is influenced by other selective electrolytes. For example, high levels of potassium in the perfusate of the choroid plexus leads to a reduction in the transfer rate of magnesium, but higher levels of calcium does not have a similar effect on the transfer of magnesium (Allsop, 1986).

A recent clinical trial on the protective role of magnesium in traumatic brain injury (Temkin et al., 2007) failed to replicate the improved outcome detected in experimental studies on rodents (Temkin et al., 2007; Vink and Cernak, 2000). In this clinical trial, plasma magnesium was maintained at higher levels compared to the placebo group and normal plasma level, to counteract the reported decline in $\mathrm{Mg}^{2+}$ in TBI patients (Kahraman et al., 2003). Since under normal conditions choroidal epithelial cells 
actively transport magnesium into the CSF, it is likely that such activity was disrupted in TBI cases in humans leading and failure of magnesium entry to the CNS. It is relevant here to state that in the rat, $\mathrm{TBI}$ induces severe morphological changes in choroidal epithelial cells detected by scanning and transmission electron microscopy that were still evident 4 weeks after trauma (Ghabriel et al., 2010).

Although one cannot dismiss the possibility that new channels may be involved in the transport of magnesium at the choroid plexus, it is natural to look at other systems for similar magnesium transport mechanisms. As stated above the strongest evidence for magnesium transport was obtained from genetic studies in familial hypomagnesemia, positional cloning and knockout mice investigations (Konrad et al., 2006; Schlingmann et al., 2005; Schlingmann et al., 2007; Simon et al., 1999; Walder et al., 2002) which identified TRPM6 and TRPM7 as candidates for transcellular transport, and Claudin16 (Efrati et al., 2005; Simon et al., 1999) for paracellular transport in the kidney. Recently TRPM7 has also been identified in smooth muscle cells in the vasculature (Callera et al., 2009).

\section{References}

Abbott NJ (2004) Evidence for bulk flow of brain interstitial fluid: significance for physiology and pathology. Neurochem Int 45:545-52.

Abbott NJ, Patabendige AA, Dolman DE, Yusof SR, Begley DJ (2010) Structure and function of the bloodbrain barrier. Neurobiol Dis 37:13-25.

Abbott NJ, Romero IA (1996) Transporting therapeutics across the blood-brain barrier. Mol Med Today 2:106-13.

Agre $P$, Preston GM, Smith BL, Jung JS, Raina S, Moon C, Guggino WB, Nielsen S (1993) Aquaporin CHIP: the archetypal molecular water channel. Am J Physiol 265:F463-76.

Aikawa (1976) Biochemistry and physiology of magnesium. In: Trace elements in human health (D PASaO, ed). New York: Academic Press, 47-78.
Although TRPM6 and TRPM7 were detected in the brain using RT-PCR it is not clear if these channels are expressed in choroidal epithelial cells. The role of claudin-16 and claudin-19 in magnesium reabsorption from the kidney filtrate has been discussed in previous sections, and the role of claudins as selective conductance to ions through tight junction has been stated. The choroidal epithelial cells show selective expression of claudin-1, -2 and -5 at and near to their tight junctions. Also the endothelial cells within the choroid plexus show stronger expression of claudin-5 than claudin-1 and -2 (Lippoldt et al., 2000). However, claudin-16 and claudin-19 have not been reported in the choroid plexus. Whether other claudin members may contribute to magnesium conductance in the choroid plexus is speculative. Thus it appears that the channels for magnesium transport in the choroid plexus remain to be clarified.

\section{Acknowledgement}

Supported, in part, by funding from the Neurosurgical Research Foundation (Australia).
Alcolado R, Weller RO, Parrish EP, Garrod D (1988) The cranial arachnoid and pia mater in man: anatomical and ultrastructural observations. Neuropathol Appl Neurobiol 14:1-17.

Allsop TF (1986) Transfer of magnesium across the perfused choroid plexus of sheep. Aust J Biol Sci 39:161-9.

Allsop TF, Pauli JV (1985) Magnesium concentrations in the ventricular and lumbar cerebrospinal fluid of hypomagnesaemic cows. Res Vet Sci 38:61-4.

Altura BT, Altura BM (1991) Measurement of ionized magnesium in whole blood, plasma and serum with a new ion-selective electrode in healthy and diseased human subjects. Magnes Trace Elem 10:90-8.

Amiry-Moghaddam M, Ottersen OP (2003) The molecular basis of water transport in the brain. Nat Rev Neurosci 4:991-1001. 
Amtorp O, Sorensen SC (1974) The ontogenetic development of concentration differences for protein and ions between plasma and cerebrospinal fluid in rabbits and rats. J Physiol 243:387-400.

Bara M, Guiet-Bara A (1984) Potassium, magnesium and membranes. Review of present status and new findings. Magnesium 3:215-25.

Bauer HC, Tontsch U, Amberger A, Bauer H (1990) Gamma-glutamyl-transpeptidase (GGTP) and NA+K(+)ATPase activities in different subpopulations of cloned cerebral endothelial cells: responses to glial stimulation. Biochem Biophys Res Commun 168:35863.

Begley DJ, Brightman MW (2003) Structural and functional aspects of the blood-brain barrier. Prog Drug Res 61:39-78.

Begley DJ (2004) ABC transporters and the blood-brain barrier. Curr Pharm Des 10:1295-312.

Bergman C, Gray-Scott D, Chen JJ, Meacham S (2009) What is next for the Dietary Reference Intakes for bone metabolism related nutrients beyond calcium: phosphorus, magnesium, vitamin D, and fluoride? Crit Rev Food Sci Nutr 49:136-44.

Betz AL, Firth JA, Goldstein GW (1980) Polarity of the blood-brain barrier: distribution of enzymes between the luminal and antiluminal membranes of brain capillary endothelial cells. Brain Res 192:17-28

Bito LZ (1969) Blood-Brain Barrier: Evidence for Active Cation Transport between Blood and the Extraceliular Fluid of Brain. Science 165:81-3.

Bradbury MWB (1979). The concept of a blood-brain barrier. Wiley: Chichester; New York.

Bradbury MW, Crowder J, Desai S, Reynolds JM, Reynolds M, Saunders NR (1972) Electrolytes and water in the brain and cerebrospinal fluid of the foetal sheep and guinea-pig. J Physiol 227:591-610.

Bradbury MW, Sarna GS (1977) Homeostasis of the ionic composition of the cerebrospinal fluid. Exp Eye Res 25 Suppl:249-57.

Bradbury MW, Stubbs J, Hughes IE, Parker P (1963) The Distribution of Potassium, Sodium, Chloride and Urea between Lumbar Cerebrospinal Fluid and Blood Serum in Human Subjects. Clin Sci 25:97-105.
Brannan PG, Vergne-Marini P, Pak CY, Hull AR, Fordtran JS (1976) Magnesium absorption in the human small intestine. Results in normal subjects, patients with chronic renal disease, and patients with absorptive hypercalciuria. J Clin Invest 57:1412-8

Brightman MW (1968) The intracerebral movement of proteins injected into blood and cerebrospinal fluid of mice. Prog Brain Res 29:19-40

Brightman MW, Reese TS (1969) Junctions between intimately apposed cell membranes in the vertebrate brain. J Cell Biol 40:648-77.

Brown RC, Wu L, Hicks K, O'Neil R G (2008) Regulation of blood-brain barrier permeability by transient receptor potential type $C$ and type $v$ calciumpermeable channels. Microcirculation 15:359-71.

Brunette MG, Vigneault N, Carriere S (1974) Micropuncture study of magnesium transport along the nephron in the young rat. Am J Physiol 227:891-6.

Butt AM, Jones HC, Abbott NJ (1990) Electrical resistance across the blood-brain barrier in anaesthetized rats: a developmental study. J Physiol 429:47-62.

Callera GE, He Y, Yogi A, Montezano AC, Paravicini T, Yao G, Touyz RM (2009) Regulation of the novel Mg2+ transporter transient receptor potential melastatin 7 (TRPM7) cation channel by bradykinin in vascular smooth muscle cells. J Hypertens 27:155-66.

Cernak I, Vink R, Zapple DN, Cruz MI, Ahmed F, Chang T, Fricke ST, Faden Al (2004) The pathobiology of moderate diffuse traumatic brain injury as identified using a new experimental model of injury in rats. Neurobiol Dis 17:29-43.

Chan AW, Minski MJ, Lim L, Lai JC (1992) Changes in brain regional manganese and magnesium levels during postnatal development: modulations by chronic manganese administration. Metab Brain Dis 7:21-33.

Cheuk DK, Chau TC, Lee SL (2005) A meta-analysis on intravenous magnesium sulphate for treating acute asthma. Arch Dis Child 90:74-7.

Coisne C, Dehouck L, Faveeuw C, Delplace Y, Miller F, Landry C, Morissette C, Fenart L, Cecchelli R, Tremblay $P$, Dehouck B (2005) Mouse syngenic in vitro bloodbrain barrier model: a new tool to examine inflammatory events in cerebral endothelium. $L a b$ Invest 85:734-46. 
Dai LJ, Ritchie G, Kerstan D, Kang HS, Cole DE, Quamme GA (2001) Magnesium transport in the renal distal convoluted tubule. Physiol Rev 81:51-84.

de Rouffignac C, Quamme G (1994) Renal magnesium handling and its hormonal control. Physiol Rev 74:30522.

Descamps L, Dehouck MP, Torpier G, Cecchelli R (1996) Receptor-mediated transcytosis of transferrin through blood-brain barrier endothelial cells. Am J Physiol 270:H1149-58.

Dick AP, Harik SI, Klip A, Walker DM (1984) Identification and characterization of the glucose transporter of the blood-brain barrier by cytochalasin $B$ binding and immunological reactivity. Proc Natl Acad Sci U S A 81:7233-7.

Ebel H, Gunther T (1980) Magnesium metabolism: a review. J Clin Chem Clin Biochem 18:257-70.

Eby GA, Eby KL (2006) Rapid recovery from major depression using magnesium treatment. Med Hypotheses 67:362-70.

Efrati E, Arsentiev-Rozenfeld J, Zelikovic I (2005) The human paracellin-1 gene ( $\mathrm{hPCLN}-1$ ): renal epithelial cell-specific expression and regulation. Am J Physiol Renal Physiol 288:F272-83.

Elin RJ (1987) Assessment of magnesium status. Clin Chem 33:1965-70.

Elin RJ (1988) Magnesium metabolism in health and disease. Dis Mon 34:161-218.

Esen F, Erdem T, Aktan D, Kalayci R, Cakar N, Kaya M, Telci L (2003) Effects of magnesium administration on brain edema and blood-brain barrier breakdown after experimental traumatic brain injury in rats. $J$ Neurosurg Anesthesiol 15:119-25.

Euser AG, Cipolla MJ (2009) Magnesium sulfate for the treatment of eclampsia: a brief review. Stroke 40:1169-75.

Fanning WJ, Thomas CS, Jr., Roach A, Tomichek R, Alford WC, Stoney WS, Jr. (1991) Prophylaxis of atrial fibrillation with magnesium sulfate after coronary artery bypass grafting. Ann Thorac Surg 52:529-33.

Feldman Z, Gurevitch B, Artru AA, Oppenheim A, Shohami E, Reichenthal E, Shapira Y (1996) Effect of magnesium given 1 hour after head trauma on brain edema and neurological outcome. J Neurosurg 85:1317.
Fenstermacher J, Gross P, Sposito N, Acuff V, Pettersen S, Gruber K (1988) Structural and functional variations in capillary systems within the brain. Ann N Y Acad Sci 529:21-30.

Fine KD, Santa Ana CA, Porter JL, Fordtran JS (1991) Intestinal absorption of magnesium from food and supplements. J Clin Invest 88:396-402.

Fonfria E, Murdock PR, Cusdin FS, Benham CD, Kelsell RE, McNulty S (2006) Tissue distribution profiles of the human TRPM cation channel family. J Recept Signal Transduct Res 26:159-78.

Fontenot JP, Allen VG, Bunce GE, Goff JP (1989) Factors influencing magnesium absorption and metabolism in ruminants. J Anim Sci 67:3445-55.

Furuse M, Hirase T, Itoh M, Nagafuchi A, Yonemura S, Tsukita S (1993) Occludin: a novel integral membrane protein localizing at tight junctions. J Cell Biol 123:1777-88.

Ghabriel MN, Lu JJ, Tadros R, Hermanis G (2004) A narrow time-window for access to the brain by exogenous protein after immunological targeting of a blood-brain barrier antigen. J Comp Pathol 131:52-60.

Ghabriel MN, Thomas A, Vink R (2006) Magnesium restores altered aquaporin-4 immunoreactivity following traumatic brain injury to a pre-injury state. Acta Neurochir Supp/ 96:402-6.

Ghabriel MN, Zdziarski IM, Leigh C, Vink R (2010) Changes in the blood-CSF barrier in experimental traumatic brain injury. Acta Neurochir Suppl 106:23945.

Ghabriel MN, Zhu C, Hermanis G, Allt G (2000) Immunological targeting of the endothelial barrier antigen (EBA) in vivo leads to opening of the bloodbrain barrier. Brain Res 878:127-35.

Glick JL (1990) Dementias: the role of magnesium deficiency and an hypothesis concerning the pathogenesis of Alzheimer's disease. Med Hypotheses 31:211-25.

Go KG (1997) The normal and pathological physiology of brain water. Adv Tech Stand Neurosurg 23:47-142.

Gow A, Southwood CM, Li JS, Pariali M, Riordan GP, Brodie SE, Danias J, Bronstein JM, Kachar B, Lazzarini RA (1999) CNS myelin and sertoli cell tight junction strands are absent in Osp/claudin-11 null mice. Cell 99:649-59. 
Graham LA, Caesar JJ, Burgen AS (1960)

Gastrointestinal absorption and excretion of $\mathrm{Mg} 28$ in man. Metabolism 9:646-59.

Guerrera MP, Volpe SL, Mao JJ (2009) Therapeutic uses of magnesium. Am Fam Physician 80:157-62.

Gunther T (2008) Comment on the number of Mg2+ activated enzymes. Magnes Res 21:185-7.

Hansen AJ (1985) Effect of anoxia on ion distribution in the brain. Physiol Rev 65:101-48.

Harik SI, Behmand RA, LaManna JC (1994) Hypoxia increases glucose transport at blood-brain barrier in rats. J Appl Physiol 77:896-901.

Hashimoto PH (1988) Tracer in cisternal cerebrospinal fluid is soon detected in choroid plexus capillaries. Brain Res 440:149-52.

Heath DL, Vink R (1996) Traumatic brain axonal injury produces sustained decline in intracellular free magnesium concentration. Brain Res 738:150-3.

Heath DL, Vink R (1999) Improved motor outcome in response to magnesium therapy received up to 24 hours after traumatic diffuse axonal brain injury in rats. J Neurosurg 90:504-9.

Hoenderop JG, Bindels RJ (2005) Epithelial Ca2+ and $\mathrm{Mg} 2+$ channels in health and disease. J Am Soc Nephrol 16:15-26.

Imer M, Omay B, Uzunkol A, Erdem T, Sabanci PA, Karasu A, Albayrak SB, Sencer A, Hepgul K, Kaya M (2009) Effect of magnesium, MK-801 and combination of magnesium and MK-801 on blood-brain barrier permeability and brain edema after experimental traumatic diffuse brain injury. Neurol Res 31:977-81.

Kahraman S, Ozgurtas T, Kayali H, Atabey C, Kutluay T, Timurkaynak E (2003) Monitoring of serum ionized magnesium in neurosurgical intensive care unit: preliminary results. Clin Chim Acta 334:211-5.

Kara H, Sahin N, Ulusan V, Aydogdu T (2002) Magnesium infusion reduces perioperative pain. Eur J Anaesthesiol 19:52-6.

Kaya M, Kucuk M, Kalayci RB, Cimen V, Gurses C, Elmas I, Arican N (2001) Magnesium sulfate attenuates increased blood-brain barrier permeability during insulin-induced hypoglycemia in rats. Can J Physiol Pharmacol 79:793-8.

Kobayashi H, Minami S, Itoh S, Shiraishi S, Yokoo H, Yanagita T, Uezono Y, Mohri M, Wada A (2001)
Aquaporin subtypes in rat cerebral microvessels.

Neurosci Lett 297:163-6.

Koepsell H, Schmitt BM, Gorboulev V (2003) Organic cation transporters. Rev Physiol Biochem Pharmacol 150:36-90.

Konrad M, Schaller A, Seelow D, Pandey AV, Waldegger $S$, Lesslauer A, Vitzthum $H$, Suzuki $Y$, Luk JM, Becker C, Schlingmann KP, Schmid M, RodriguezSoriano J, Ariceta G, Cano F, Enriquez R, Juppner $H$, Bakkaloglu SA, Hediger MA, Gallati S, Neuhauss SC, Nurnberg P, Weber S (2006) Mutations in the tightjunction gene claudin 19 (CLDN19) are associated with renal magnesium wasting, renal failure, and severe ocular involvement. Am J Hum Genet 79:949-57.

Kunert-Keil C, Bisping F, Kruger J, Brinkmeier H (2006) Tissue-specific expression of TRP channel genes in the mouse and its variation in three different mouse strains. BMC Genomics 7:159.

Lee JS, Han YM, Yoo DS, Choi SJ, Choi BH, Kim JH, Kim YH, Huh PW, Ko YJ, Rha HK, Cho KS, Kim DS (2004) A molecular basis for the efficacy of magnesium treatment following traumatic brain injury in rats. $J$ Neurotrauma 21:549-61.

Leybaert L (2005) Neurobarrier coupling in the brain: a partner of neurovascular and neurometabolic coupling? J Cereb Blood Flow Metab 25:2-16.

Li X, Kong H, Wu W, Xiao M, Sun X, Hu G (2009) Aquaporin-4 maintains ependymal integrity in adult mice. Neuroscience 162:67-77.

Liebner S, Kniesel U, Kalbacher H, Wolburg H (2000) Correlation of tight junction morphology with the expression of tight junction proteins in blood-brain barrier endothelial cells. Eur J Cell Biol 79:707-17.

Lippoldt A, Liebner S, Andbjer B, Kalbacher H, Wolburg $H$, Haller H, Fuxe K (2000) Organization of choroid plexus epithelial and endothelial cell tight junctions and regulation of claudin-1, -2 and -5 expression by protein kinase C. Neuroreport 11:1427-31.

Ma YY, Huo HR, Li CH, Zhao BS, Li LF, Sui F, Guo SY, Jiang TL (2008) Effects of cinnamaldehyde on PGE2 release and TRPV4 expression in mouse cerebral microvascular endothelial cells induced by interleukin1beta. Biol Pharm Bull 31:426-30.

Matter K, Balda MS (2003) Holey barrier: claudins and the regulation of brain endothelial permeability. J Cell Biol 161:459-60. 
McCoy JH, Kenney MA (1975) Depressed immune response in the magnesium-deficient rat. J Nutr 105:791-7.

McIntosh TK, Faden Al, Yamakami I, Vink R (1988) Magnesium deficiency exacerbates and pretreatment improves outcome following traumatic brain injury in rats: 31P magnetic resonance spectroscopy and behavioral studies. J Neurotrauma 5:17-31.

McKinley MJ, McAllen RM, Davern P, Giles ME, Penschow J, Sunn N, Uschakov A, Oldfield BJ (2003) The sensory circumventricular organs of the mammalian brain. Adv Anat Embryol Cell Biol 172:IIIXII, 1-122.

Miller DS (2010) Regulation of P-glycoprotein and other $A B C$ drug transporters at the blood-brain barrier. Trends Pharmacol Sci 31:246-54.

Montell C (2005) The TRP superfamily of cation channels. Sci STKE 2005:re3.

Morita K, Furuse M, Fujimoto K, Tsukita S (1999) Claudin multigene family encoding fourtransmembrane domain protein components of tight junction strands. Proc Natl Acad Sci U S A 96:511-6.

Morris ME (1992) Brain and CSF magnesium concentrations during magnesium deficit in animals and humans: neurological symptoms. Magnes Res 5:303-13.

Muir KW, Lees KR, Ford I, Davis S (2004) Magnesium for acute stroke (Intravenous Magnesium Efficacy in Stroke trial): randomised controlled trial. Lancet 363:439-45.

Musso CG (2009) Magnesium metabolism in health and disease. Int Urol Nephrol 41:357-62.

Nabeshima S, Reese TS, Landis DM, Brightman MW (1975) Junctions in the meninges and marginal glia. J Comp Neurol 164:127-69.

Nielsen S, Nagelhus EA, Amiry-Moghaddam M, Bourque C, Agre P, Ottersen OP (1997) Specialized membrane domains for water transport in glial cells: high-resolution immunogold cytochemistry of aquaporin-4 in rat brain. J Neurosci 17:171-80.

Nielsen S, Smith BL, Christensen El, Agre P (1993) Distribution of the aquaporin CHIP in secretory and resorptive epithelia and capillary endothelia. Proc Natl Acad Sci U S A 90:7275-9.
Niermann H, Amiry-Moghaddam M, Holthoff K, Witte OW, Ottersen OP (2001) A novel role of vasopressin in the brain: modulation of activity-dependent water flux in the neocortex. J Neurosci 21:3045-51.

Nischwitz V, Berthele A, Michalke B (2008) Speciation analysis of selected metals and determination of their total contents in paired serum and cerebrospinal fluid samples: An approach to investigate the permeability of the human blood-cerebrospinal fluid-barrier. Anal Chim Acta 627:258-69.

Ohtsuki S, Terasaki T (2007) Contribution of carriermediated transport systems to the blood-brain barrier as a supporting and protecting interface for the brain; importance for CNS drug discovery and development. Pharm Res 24:1745-58.

Oppelt WW, Maclntyre I, Rall DP (1963) Magnesium exchange between blood and cerebrospinal fluid. Am J Physiol 205:959-62.

Pardridge WM, Boado RJ, Farrell CR (1990) Brain-type glucose transporter (GLUT-1) is selectively localized to the blood-brain barrier. Studies with quantitative western blotting and in situ hybridization. J Biol Chem 265:18035-40.

Pardridge WM (1991) Advances in cell biology of blood-brain barrier transport. Semin Cell Biol 2:419-26.

Peikert A, Wilimzig C, Kohne-Volland R (1996) Prophylaxis of migraine with oral magnesium: results from a prospective, multi-center, placebo-controlled and double-blind randomized study. Cephalalgia 16:257-63.

Peppi M, Ghabriel MN (2004) Tissue-specific expression of the tight junction proteins claudins and occludin in the rat salivary glands. J Anat 205:257-66.

Porter NS, Jason LA, Boulton A, Bothne N, Coleman B (2010) Alternative medical interventions used in the treatment and management of myalgic encephalomyelitis/chronic fatigue syndrome and fibromyalgia. J Altern Complement Med 16:235-49.

Quamme GA (1997) Renal magnesium handling: new insights in understanding old problems. Kidney Int 52:1180-95.

Quamme GA, de Rouffignac C (2000) Epithelial magnesium transport and regulation by the kidney. Front Biosci 5:D694-711.

Quamme GA, Dirks JH (1980) Magnesium transport in the nephron. Am J Physiol 239:F393-401. 
Redzic ZB, Segal MB (2004) The structure of the choroid plexus and the physiology of the choroid plexus epithelium. Adv Drug Deliv Rev 56:1695-716.

Reed DJ, Yen MH (1978) The role of the cat choroid plexus in regulating cerebrospinal fluid magnesium. $J$ Physiol 281:477-85.

Reese TS, Karnovsky MJ (1967) Fine structural localization of a blood-brain barrier to exogenous peroxidase. J Cell Biol 34:207-17.

Resnick LM, Altura BT, Gupta RK, Laragh JH, Alderman $\mathrm{MH}$, Altura BM (1993) Intracellular and extracellular magnesium depletion in type 2 (non-insulindependent) diabetes mellitus. Diabetologia 36:767-70.

Roberts LM, Black DS, Raman C, Woodford K, Zhou M, Haggerty JE, Yan AT, Cwirla SE, Grindstaff KK (2008) Subcellular localization of transporters along the rat blood-brain barrier and blood-cerebral-spinal fluid barrier by in vivo biotinylation. Neuroscience 155:42338.

Rouse DJ (2009) Magnesium sulfate for the prevention of cerebral palsy. Am J Obstet Gynecol 200:610-2.

Rubin LL, Staddon JM (1999) The cell biology of the blood-brain barrier. Annu Rev Neurosci 22:11-28.

Saatman KE, Bareyre FM, Grady MS, McIntosh TK (2001) Acute cytoskeletal alterations and cell death induced by experimental brain injury are attenuated by magnesium treatment and exacerbated by magnesium deficiency. J Neuropathol Exp Neurol 60:183-94.

Sacco RL, Chong JY, Prabhakaran S, Elkind MS (2007) Experimental treatments for acute ischaemic stroke. Lancet 369:331-41.

Schlingmann KP, Sassen MC, Weber S, Pechmann U, Kusch K, Pelken L, Lotan D, Syrrou M, Prebble JJ, Cole DE, Metzger DL, Rahman S, Tajima T, Shu SG, Waldegger S, Seyberth HW, Konrad M (2005) Novel TRPM6 mutations in 21 families with primary hypomagnesemia and secondary hypocalcemia. J Am Soc Nephrol 16:3061-9.

Schlingmann KP, Waldegger S, Konrad M, Chubanov V, Gudermann T (2007) TRPM6 and TRPM7--Gatekeepers of human magnesium metabolism. Biochim Biophys Acta 1772:813-21.

Schlingmann KP, Weber S, Peters M, Niemann Nejsum L, Vitzthum $H$, Klingel K, Kratz $M$, Haddad E, Ristoff E, Dinour D, Syrrou M, Nielsen S, Sassen M, Waldegger S, Seyberth HW, Konrad M (2002) Hypomagnesemia with secondary hypocalcemia is caused by mutations in TRPM6, a new member of the TRPM gene family. Nat Genet 31:166-70.

Schmitz C, Perraud AL, Johnson CO, Inabe K, Smith MK, Penner R, Kurosaki T, Fleig A, Scharenberg AM (2003) Regulation of vertebrate cellular Mg2+ homeostasis by TRPM7. Cell 114:191-200.

Schroeder HA, Nason AP, Tipton IH (1969) Essential metals in man. Magnesium. J Chronic Dis 21:815-41.

Seda HW, Hughes RD, Gove CD, Williams R (1984) Inhibition of rat brain $\mathrm{Na}+\mathrm{K}+-\mathrm{ATP}$ ase activity by serum from patients with fulminant hepatic failure. Hepatology 4:74-9.

Sedlakova R, Shivers RR, Del Maestro RF (1999) Ultrastructure of the blood-brain barrier in the rabbit. J Submicrosc Cytol Pathol 31:149-61.

Segal MB (2000) The choroid plexuses and the barriers between the blood and the cerebrospinal fluid. Cell Mol Neurobiol 20:183-96.

Shen S, Zhang W (2010) ABC transporters and drug efflux at the blood-brain barrier. Rev Neurosci 21:2953.

Simoes Fernandes J, Pereira T, Carvalho J, Franca A, Andrade R, Nogueira Pereira J, Rodrigues JC, Laires MJ, Halpern MJ (1985) Therapeutic effect of a magnesium salt in patients suffering from mitral valvular prolapse and latent tetany. Magnesium 4:283-90.

Simon DB, Lu Y, Choate KA, Velazquez H, Al-Sabban E, Praga M, Casari G, Bettinelli A, Colussi G, RodriguezSoriano J, McCredie D, Milford D, Sanjad S, Lifton RP (1999) Paracellin-1, a renal tight junction protein required for paracellular $\mathrm{Mg} 2+$ resorption. Science 285:103-6.

Soave PM, Conti G, Costa R, Arcangeli A (2009) Magnesium and anaesthesia. Curr Drug Targets 10:734-43.

Sojka JE, Weaver CM (1995) Magnesium supplementation and osteoporosis. Nutr Rev 53:71-4.

Somjen GG (2002) Ion regulation in the brain: implications for pathophysiology. Neuroscientist 8:254-67.

Song Y, Li TY, van Dam RM, Manson JE, Hu FB (2007) Magnesium intake and plasma concentrations of markers of systemic inflammation and endothelial dysfunction in women. Am J Clin Nutr 85:1068-74. 
Sternberger NH, Sternberger LA (1987) Blood-brain barrier protein recognized by monoclonal antibody. Proc Natl Acad Sci U S A 84:8169-73.

Sunn N, McKinley MJ, Oldfield BJ (2003) Circulating angiotensin II activates neurones in circumventricular organs of the lamina terminalis that project to the bed nucleus of the stria terminalis. J Neuroendocrinol 15:725-31.

Takahashi H, Imai K, Katanuma A, Sugaya T, Hisano K, Motoya S, Aoki S, Sugiyama T, Yachi A (1992) [A case of chronic fatigue syndrome who showed a beneficial effect by intravenous administration of magnesium sulphate]. Arerugi 41:1605-10.

Temkin NR, Anderson GD, Winn HR, Ellenbogen RG, Britz GW, Schuster J, Lucas T, Newell DW, Mansfield PN, Machamer JE, Barber J, Dikmen SS (2007) Magnesium sulfate for neuroprotection after traumatic brain injury: a randomised controlled trial. Lancet Neurol 6:29-38.

Terasaki M, Rubin H (1985) Evidence that intracellular magnesium is present in cells at a regulatory concentration for protein synthesis. Proc Natl Acad Sci U S A 82:7324-6.

Tripathi RC (1973) Ultrastructure of the arachnoid mater in relation to outflow of cerebrospinal fluid. A new concept. Lancet 2:8-11.

Tsukita S, Furuse M (1999) Occludin and claudins in tight-junction strands: leading or supporting players? Trends Cell Biol 9:268-73.

Turkoglu OF, Eroglu H, Okutan O, Tun MK, Bodur E, Sargon MF, Oner L, Beskonakli E (2008) A comparative study of treatment for brain edema: magnesium sulphate versus dexamethasone sodium phosphate. J Clin Neurosci 15:60-5.

Turner RJ, Dasilva KW, O'Connor C, van den Heuvel C, Vink R (2004) Magnesium gluconate offers no more protection than magnesium sulphate following diffuse traumatic brain injury in rats. J Am Coll Nutr 23:541S$4 \mathrm{~S}$.

van den Bergh WM (2009) Magnesium in subarachnoid haemorrhage: proven beneficial? Magnes Res 22:121-6.

Vandenabeele F, Creemers J, Lambrichts I (1996) Ultrastructure of the human spinal arachnoid mater and dura mater. J Anat 189 ( Pt 2):417-30.
Vanharreveld A, Crowell J, Malhotra SK (1965) A Study of Extracellular Space in Central Nervous Tissue by Freeze-Substitution. J Cell Biol 25:117-37.

Villegas JC, Broadwell RD (1993) Transcytosis of protein through the mammalian cerebral epithelium and endothelium. II. Adsorptive transcytosis of WGAHRP and the blood-brain and brain-blood barriers. J Neurocytol 22:67-80.

Vink R, Cernak I (2000) Regulation of intracellular free magnesium in central nervous system injury. Front Biosci 5:D656-65.

Vink R, Cook NL, van den Heuvel C (2009) Magnesium in acute and chronic brain injury: an update. Magnes Res 22:158S-62S.

Vink R, McIntosh TK, Demediuk P, Faden AI (1987) Decrease in total and free magnesium concentration following traumatic brain injury in rats. Biochem Biophys Res Commun 149:594-9.

Voets T, Nilius B, Hoefs S, van der Kemp AW, Droogmans G, Bindels RJ, Hoenderop JG (2004) TRPM6 forms the $\mathrm{Mg} 2+$ influx channel involved in intestinal and renal Mg2+ absorption. J Biol Chem 279:19-25.

Walder RY, Landau D, Meyer P, Shalev H, Tsolia M, Borochowitz Z, Boettger MB, Beck GE, Englehardt RK, Carmi R, Sheffield VC (2002) Mutation of TRPM6 causes familial hypomagnesemia with secondary hypocalcemia. Nat Genet 31:171-4.

Weber S, Schlingmann KP, Peters M, Nejsum LN, Nielsen S, Engel H, Grzeschik KH, Seyberth HW, Grone $\mathrm{HJ}$, Nusing R, Konrad M (2001) Primary gene structure and expression studies of rodent paracellin-1. J Am Soc Nephrol 12:2664-72.

Wester PO, Dyckner T (1987) Magnesium and hypertension. J Am Coll Nutr 6:321-8.

Wolburg H, Lippoldt A (2002) Tight junctions of the blood-brain barrier: development, composition and regulation. Vascul Pharmacol 38:323-37.

Wolburg H, Wolburg-Buchholz K, Kraus J, RascherEggstein G, Liebner S, Hamm S, Duffner F, Grote EH, Risau W, Engelhardt B (2003) Localization of claudin-3 in tight junctions of the blood-brain barrier is selectively lost during experimental autoimmune encephalomyelitis and human glioblastoma multiforme. Acta Neuropathol 105:586-92. 
Wolburg H, Noell S, Wolburg-Buchholz K, Mack A, Fallier-Becker $P$ (2009) Agrin, aquaporin-4, and astrocyte polarity as an important feature of the blood-brain barrier. Neuroscientist 15:180-93.

Wolburg H, Paulus W (2010) Choroid plexus: biology and pathology. Acta Neuropathol 119:75-88.

Wolf FI, Trapani V, Simonacci M, Mastrototaro L, Cittadini A, Schweigel M (2010) Modulation of TRPM6 and $\mathrm{Na}(+) / \mathrm{Mg}(2+)$ exchange in mammary epithelial cells in response to variations of magnesium availability. J Cell Physiol 222:374-81.
Yahia AM, Kirmani JF, Qureshi Al, Guterman LR, Hopkins LN (2005) The safety and feasibility of continuous intravenous magnesium sulfate for prevention of cerebral vasospasm in aneurysmal subarachnoid hemorrhage. Neurocrit Care 3:16-23.

Zhang EY, Knipp GT, Ekins S, Swaan PW (2002) Structural biology and function of solute transporters: implications for identifying and designing substrates. Drug Metab Rev 34:709-50.

Zlokovic BV, Skundric DS, Segal MB, Lipovac MN, Mackic JB, Davson H (1990) A saturable mechanism for transport of immunoglobulin $\mathrm{G}$ across the blood-brain barrier of the guinea pig. Exp Neurol 107:263-70. 


\title{
Intracellular free $\mathrm{Mg}^{2+}$ and $\mathrm{MgATP}^{2-}$ in coordinate control of protein synthesis and cell proliferation
}

\author{
Harry Rubin \\ Department of Molecular and Cell Biology, Life Sciences Addition, University of California, Berkeley, CA 94720-3200, USA. \\ $\triangle$ hrubin@berkeley.edu
}

\begin{abstract}
Specific and non-specific mitogens stimulate the proliferation of cultured fibroblasts. They also stimulate other responses that are part of a coordinate response, some members of which are essential for proliferation and others that are not. The synthesis of protein is an early response to mitogens and its continuation through the $\mathbf{G 1}$ period drives the accumulation of protein which is required for the initiation of DNA synthesis hours later. The parabola-like curve for dependence of protein synthesis on intracellular $\mathrm{Mg}^{2+}$ concentration is similar to that of cell-free ribosome preparations, and is later reflected in the initiation of DNA synthesis. Hence, DNA synthesis is dependent on the rate of protein synthesis which is regulated by the concentration of intracellular $\mathrm{Mg}^{2+}$. Presumably free $\mathrm{Mg}^{2+}$ is an indicator of the fraction of $\mathrm{ATP}^{4-}$ that is complexed with $\mathrm{Mg}^{2+}$ as $\mathrm{MgATP}^{2-}$, which is the immediate regulatory form. Uridine uptake is determined by its phosphorylation, which is also dependent on intracellular $\mathrm{Mg}^{2+}$ in the coordinate response, but unlike protein synthesis, neither influences DNA synthesis nor exhibits downturn at a high $\mathrm{Mg}^{2+}$ concentration. Intracellular free $\mathrm{Mg}^{2+}$ determines the onset of protein synthesis in activated frog oocytes and its rate in lymphocytes. $\mathrm{Mg}^{2+}$ regulation of protein synthesis is effected through the $\mathrm{PI} 3-\mathrm{K}$ pathway at $\mathrm{mTOR}$ phosphorylation of two translation-regulating proteins. Regulation of proliferation by $\mathrm{Mg}^{2+}$ is lost in transformed cells. $\mathrm{Mg}^{2+}$ and $\mathrm{MgATP}^{2-}$ apparently play a central role in the regulation of metabolism in a wide variety of cells and developmental stages across the animal kingdom.
\end{abstract}

\section{Introduction}

The study of the regulation of cell proliferation was greatly facilitated in the 1960s and 1970s by the development of monolayer cell culture. This permitted direct microscopic examination of cell populations including cellular morphology and behaviour. Techniques were also developed for suspending and accurately counting the cells. It was also possible to vary the cell population size, change the constituents of the medium, and uniformly label the cells with radioactive chemicals to study their metabolism. Some general features of cell growth stimulation have to be considered before delving into the possible mechanism(s) of its control. Most of the experiments to be described here were conducted in cell culture with fibroblasts obtained directly from chicken embryos, or with established lines of mouse fibroblasts. In most cases they were allowed to grow into confluent sheets to establish contact inhibition with low levels of DNA synthesis that represented the small proportion of cells in the S-period, and no net proliferation. Fresh medium containing serum or other growth stimulants was added and measurements of various cellular parameters were made at intervals thereafter.

After application of a stimulus to a growthinhibited culture, increase in DNA synthesis, representing the increased proportion of cells in the S-period, does not begin for 4 hours in ex vivo chicken embryo cells (Rubin and Steiner, 1975) or 10 hours in an established line of mouse cells (Rubin et al., 1978). This indicates that multiple metabolic processes are required to produce that response. These include increases in the rates of uptake of glucose analogues, and uridine prior to its incorporation into RNA (Rubin, 1975b). Although neither of these responses is required for the increased DNA synthesis, they are clearly part of a coordinate response of the cells to the growth stimulants, and have to be taken into account in understanding the intracellular mechanism of growth regulation. Most significant, there is also a fractional increase in the rate of protein synthesis that must be 
maintained throughout the period preceding DNA synthesis, and is essential for its initiation (Rubin, 2005). Small decreases of protein synthesis in cells treated with a specific inhibitor of protein synthesis result in large decreases in the fraction of cells that later initiate DNA synthesis (Brooks, 1977; and Figure 1).

Another aspect of growth stimulation concerns treatments other than serum growth factors that produce the same constellation of responses. Certain hormones such as insulin produce the coordinate response in some cell types, indicating that a specific interaction between proteins and their receptors at the cell surface can initiate the coordinate response. However, the same effects are produced by removing a strip of cells from a confluent monolayer, which allows migration and multiplication of the cells at the margin. This indicates that the coordinate response is not limited to specific receptors. A similar conclusion is suggested by the finding that subtoxic concentrations of heavy metals including zinc, cadmium, and mercury initiate the coordinate response in chick embryo fibroblasts (Rubin, 1975b). Lead stimulated DNA synthesis in some experiments, and in other experiments did not (Sanui and Rubin, 1984). Those cultures in which DNA was not stimulated showed no increase in cellular $\mathrm{Mg}^{2+}$, whereas those that were significantly stimulated exhibited a $10-13 \%$ increase in intracellular $\mathrm{Mg}^{2+}$. Stimulation of DNA synthesis by lead coincided with the formation of lead precipitate in the medium.

A striking finding made with a permanent line of cells originating from mouse embryo fibroblasts was that increasing concentrations of inorganic pyrophosphate initiated the coordinate response at precisely the concentration (about $0.2 \mathrm{mM}$ in standard medium) at which it first formed a flocculent precipitate with calcium (Rubin and Sanui, 1977). The stimulation required contact of the precipitate with the cell surface as shown by the failure of stimulation when the culture dish was inverted so that the precipitate did not contact the cells (Bowen-Pope and Rubin, 1983). The stimulation was terminated when the $\mathrm{pH}$ of the medium was slightly lowered and the precipitate dissolved, showing that the effect required the precipitate and was exerted at the surface of the cell.

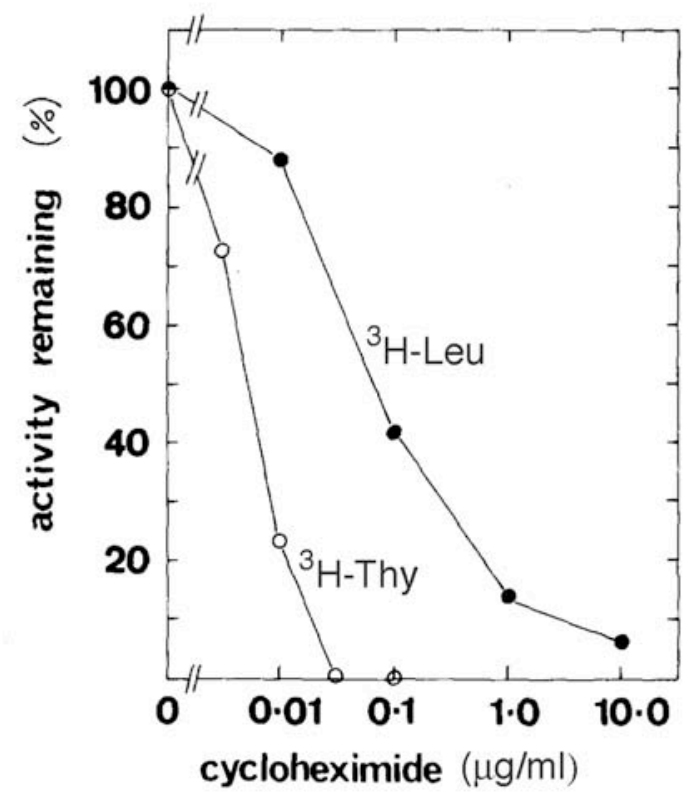

Figure 1. Inhibition of protein synthesis by cycloheximide and its effect on the initiation of DNA synthesis in mouse 3T3 cells. Quiescent cells were stimulated with serum in the presence of the indicated concentrations of cycloheximide. Cultures were pulse-labelled for protein synthesis with ${ }^{3} \mathrm{H}$-leucine at 2 hours $(0)$ and continuously labelled with ${ }^{3} \mathrm{H}$-thymidine for 24 hours $(0)$. Ordinate: radioactivity incorporated as percentage of control (no cycloheximide) (Brooks, 1977).

In the absence of pyrophosphate, supranormal concentrations of either $\mathrm{Ca}^{2+}$ or orthophosphate caused graded increases in DNA synthesis (Rubin and Sanui, 1977). The effect of supranormal $\mathrm{Ca}^{2+}$ depended on orthophosphate and vice versa. The stimulation was associated with increasing turbidity of the medium, consistent with the involvement of a precipitate interacting with the cell membrane. Several other non-physiological, non-specific treatments could initiate the coordinate response, although most of them could not maintain multiple rounds of cell multiplication. One exception was the woundhealing experiment, which allowed continued cell multiplication until the cleared strip was completely covered with cells. The sum total of these experiments indicates that perturbation of the cell membrane, which could be brought about by specific or non-specific means, is involved in growth stimulation, and raises the question of what intracellular response(s) or 
second messenger(s) mediate the diverse reactions of the coordinate response.

Variations of intracellular $\mathrm{K}^{+}, \mathrm{Na}^{+}$or $\mathrm{Ca}^{+}$failed to reproduce the coordinate response in a balanced manner (Moscatelli et al., 1979) nor did changes in the $\mathrm{pH}$ of the medium (Rubin, 2005). Intracellular $\mathrm{Mg}^{2+}$ was considered a logical candidate as a second messenger for regulating the coordinate response to membrane perturbation because it is required for every phosphoryl transfer in the cell. Drastic reduction in $\mathrm{Mg}^{2+}$ concentration in the medium reduced the number of cells in the S-period of the cell cycle, but the effect was erratic (Rubin, 1975a). Phosphorylated compounds that bind $\mathrm{Mg}^{2+}$ were more reproducible, especially inorganic pyrophosphate in concentrations that exceeded the concentration of $\mathrm{Mg}^{2+}$ in the medium. Unlike the very low concentrations of pyrophosphate that stimulated mouse fibroblasts via a precipitate with $\mathrm{Ca}^{2+}$ (Bowen-Pope and Rubin, 1983), the inhibition of fibroblast growth was most efficient in low concentrations of $\mathrm{Ca}^{2+}$ in which there was no precipitate. $\mathrm{Mg}^{2+}$ deprivation, further lowered by its binding with pyrophosphate, suppressed all elements of the coordinate response in a balanced manner (Rubin, 1975a).

\section{Changes in total and free $\mathrm{Mg}^{2+}$ after stimulation of cells with mitogens}

The major cellular cations were measured in confluent chick embryo fibroblasts 16 hours after a fresh change of medium with insulin substituting for serum (Sanui and Rubin, 1978). There was more than a 16-fold increase in the overall rate of DNA synthesis in the optimal concentration of insulin, with a $22 \%$ increase in total $\mathrm{Mg}^{2+}$ of the cells as measured by atomic absorption spectrophotometry. There was no change in total cell $\mathrm{Ca}^{2+}$, negligible change in $\mathrm{Na}^{+}$and a $14 \%$ increase in $\mathrm{K}^{+}$, an element that plays an adjunct role to $\mathrm{Mg}^{2+}$ in the synthesis of protein in cell-free systems (Schreier and Staehelin, 1973). In an experiment with a contact -inhibited and serumlimited line of mouse fibroblasts, the addition of fresh medium containing serum induced a 15-fold increase of DNA synthesis at 17 hours, associated with a $15 \%$ increase in total $\mathrm{Mg}^{2+}$ (Sanui and Rubin, 1982). There was only a marginal increase in $\mathrm{K}^{+}$, and decreases in $\mathrm{Na}^{+}$and $\mathrm{Ca}^{2+}$. These results were consistent with a central role for $\mathrm{Mg}^{2+}$ in supporting cell proliferation.

It seemed likely to us that intracellular free $\mathrm{Mg}^{2+}$ combined with ATP $^{4-}$ is its regulatory form, possibly made available by cation exchange with intracellular $\mathrm{K}^{+}$that releases bound $\mathrm{Mg}^{2+}$ from the inner surface of the cell membrane (Rubin, 1976; Sanui and Rubin, 1978). The free $\mathrm{Mg}^{2+}$ would combine with ATP $^{4-}$ to form MgATP $^{2-}$, which would stimulate phosphoryl transfer reactions throughout the cell, and reduce the inhibitory KATP $^{3-}$ form. However, we did not have an efficient means at the time of measuring free $\mathrm{Mg}^{2+}$ in the cell. The development of a $\mathrm{Mg}^{2+}$ sensitive fluorescent dye, mag-fura-2, made it possible to quantify intracellular free $\mathrm{Mg}^{2+}$ (Murphy et al., 1989; Raju et al., 1989). It was used to monitor free $\mathrm{Mg}^{2+}$ in a quiescent, confluent line of mouse fibroblasts stimulated by insulin or by insulin combined with epidermal growth factor (Ishijima et al., 1991). The combination of the two growth factors led to a significant increase of intracellular free $\mathrm{Mg}^{2+}$ from a basal level of $0.22 \mathrm{mM}$ to $0.29 \mathrm{mM}$ at 30 minutes, and to $0.35 \mathrm{mM}$ at 60 minutes. After 60 minutes the free $\mathrm{Mg}^{2+}$ levels seemed to decline, but the measurements were no longer reliable because of leakage of the dye. Epidermal growth factor produced a 50-fold increase in DNA synthesis when added to a serum-starved line of differentiated myocytes (Grubbs, 1991). Free $\mathrm{Mg}^{2+}$ increased after a 5- minute lag period, rising gradually from an initial $0.32 \mathrm{mM}$ to as high as 1.4 $\mathrm{mM}$ at 20 minutes, and then levelled off. There was no change in the cellular $\mathrm{pH}$ or free $\mathrm{Ca}^{2+}$ during the 20-minute period. The dose-response to epidermal growth factor was the same for free $\mathrm{Mg}^{2+}$ and the synthesis of DNA, strengthening the case for the dependence of the proliferative response on the rise of free $\mathrm{Mg}^{2+}$.

\section{Correlations between cellular $\mathrm{Mg}^{2+}$, protein synthesis and the onset of DNA synthesis}

A series of experiments was carried out in which external $\mathrm{Mg}^{2+}$ was varied over a wide range (1-48 $\mathrm{mM}$ ) in a medium containing only $0.02 \mathrm{Ca}^{2+}$ (Rubin et al 1979). The low $\mathrm{Ca}^{2+}$ concentration increased the permeability of the cells for entry of $\mathrm{Mg}^{2+}$. Intracellular $\mathrm{Mg}^{2+}$ content and the rate of protein synthesis were measured at 3 and 17 hours and the rate of DNA synthesis at 17 hours. 
The results showed that the intracellular $\mathrm{Mg}^{2+}$ concentration increased as the external $\mathrm{Mg}^{2+}$ concentration was increased, but the rate of protein synthesis at 3 hours peaked at $20 \mathrm{mM}$ external $\mathrm{Mg}^{2+}$ concentration, and declined with higher (30-48 mM) $\mathrm{Mg}^{2+}$ concentrations (Figure $2 \mathrm{~A}, \mathrm{~B})$. The rate of DNA synthesis at 17 hours peaked at the same $\mathrm{Mg}^{2+}$ concentration as protein synthesis at 3 hours (Figure 2A), consistent with the dependence of the onset of the S-period on the rate of protein synthesis during the preceding G1-period.

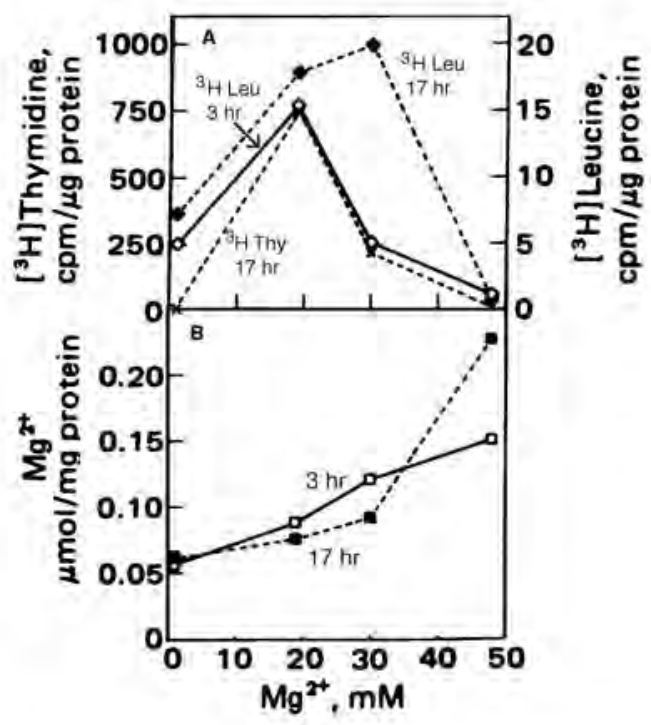

Figure 2. Effects of extracellular $\mathrm{Mg}^{2+}$ on intracellular $\mathrm{Mg}^{2+}$ and on protein and DNA synthesis. Quiescent mouse 3T3 cells were stimulated with $10 \%$ dialyzed serum in $\mathrm{Ca}^{2+}$ deprived medium with varying concentrations of $\mathrm{Mg}^{2+}$. At 3 hours some of the cultures were A) labelled with ${ }^{3} \mathrm{H}$-leucine; and at 17 hours with ${ }^{3} \mathrm{H}$ leucine or ${ }^{3} \mathrm{H}$-thymidine; B) processed for measurement of intracellular $\mathrm{Mg}^{2+}$ at 3 and 17 hours (Rubin et al., 1979).

Protein synthesis at 17 hours showed a stimulation at $30 \mathrm{mM}$ external $\mathrm{Mg}^{2+}$ which had first inhibited protein synthesis at 3 hours, and consequently reduced DNA synthesis at 17 hours (Figure 2A). The increased protein synthesis at 17 hours was associated with a decrease of cellular $\mathrm{Mg}^{2+}$ from its inhibitory level at 3 hours to a physiological stimulatory level at 17 hours (Figure $2 \mathrm{~A}, \mathrm{~B})$, indicating the cells were able to reduce $\mathrm{Mg}^{2+}$ content to functional levels. If the external
$\mathrm{Mg}^{2+}$ levels were carried to even higher external levels (48 $\mathrm{mM})$, the cellular $\mathrm{Mg}^{2+}$ rose to very high levels at 17 hours and both protein and DNA synthesis remained close to zero, indicating toxic damage of the cells. The overall results provided strong evidence of $\mathrm{Mg}^{2+}$ as an indirect regulator of the onset of DNA synthesis through its direct effect on the rate of preceding protein synthesis. It should be recalled however that the free $\mathrm{Mg}^{2+}$ level is an indicator of the fraction of ATP ${ }^{4-}$ that is complexed to $\mathrm{Mg}^{2+}$ as $\mathrm{MgATP}^{2-}$ which is the true regulator (Rubin, 2005).

Figure 3 plots the rate of protein synthesis at 3 hours against the intracellular concentration of $\mathrm{Mg}^{2+}$, showing the sharp increase in protein synthesis with small increases of intracellular $\mathrm{Mg}^{2+}$ in the physiological range $(0.05-0.08$ $\mu \mathrm{mole} / \mathrm{mg}$ protein). At high concentrations of $\mathrm{Mg}^{2+}$, protein synthesis is reduced both in cultured cells and in cell free systems (Schreier and Staehelin, 1973; and Figure 4). The same concentration dependence is exhibited by DNA synthesis in cells at 17 hours (Rubin et al., 1978, and Figure 5), which is consistent with the dependence of DNA synthesis on prior protein synthesis. The increase of protein synthesis with ascending $\mathrm{Mg}^{2+}$ at physiological intracellular levels has to be maintained through the G-1 period in order to get the full response of DNA

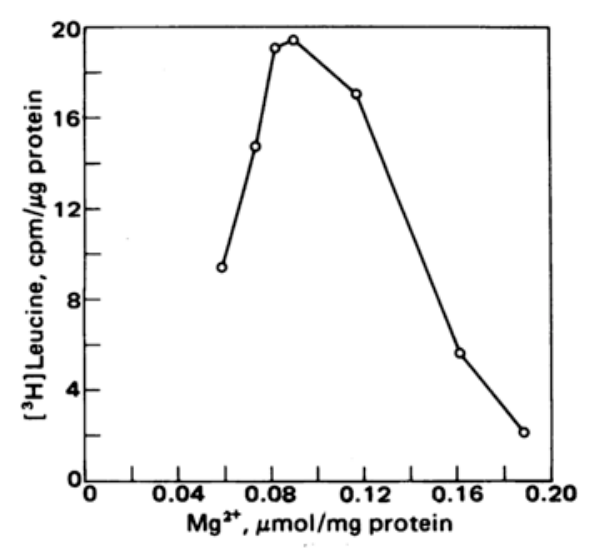

Figure 3. Relationship between intracellular $\mathrm{Mg}^{2+}$ and protein synthesis. Quiescent mouse 3T3 cultures were stimulated with $10 \%$ dialyzed serum in $\mathrm{Ca}^{2+}$-deprived medium and varying concentrations of $\mathrm{Mg}^{2+}$. At 3 hours some of the cultures were labelled with ${ }^{3} \mathrm{H}$-leucine, or processed for measurement of intracellular $\mathrm{Mg}^{2+}$ (Rubin et al., 1979). 


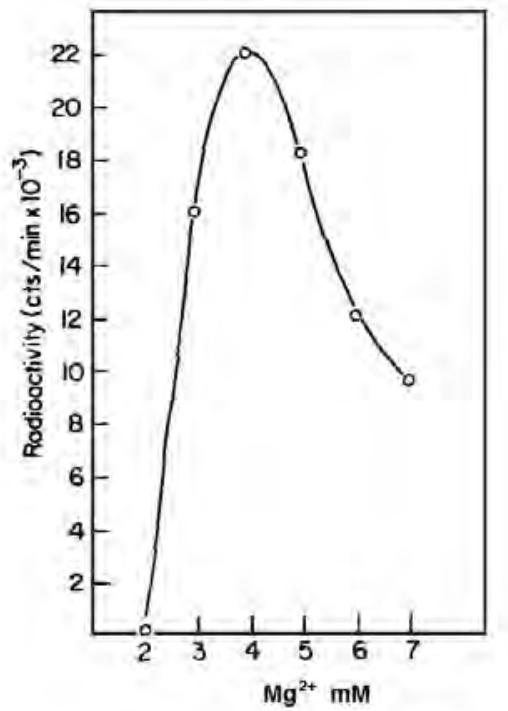

Figure 4. $\mathrm{Mg}^{2+}$ dependency of globin synthesis by mouse liver polysomes in a cell-free system, with labelling by ${ }^{14} \mathrm{C}$-leucine. After correction for chelation of $\mathrm{Mg}^{2+}$ by ATP and GTP, the effective $\mathrm{Mg}^{2+}$ concentration is $1.4 \mathrm{mM}$ lower than that indicated (Schreier and Staehelin, 1973). The correction does not take into account that $32 \%$ of cytosolic $\mathrm{Mg}^{2+}$ is bound to low affinity, nondiffusible sites (Corkey et al., 1986). Note the steep increase in globin synthesis with $\mathrm{Mg}^{2+}$ at lower levels, closer to an estimated intracellular free $\mathrm{Mg}^{2+}$, if account is taken of all the low affinity binding sites.

synthesis. The high concentration of total $\mathrm{Mg}^{2+}$ in cells, and the low affinity of $\mathrm{Mg}^{2+}$ binding sites, results in rather large changes of free $\mathrm{Mg}^{2+}$ with small variations in total cell $\mathrm{Mg}^{2+}$. This makes $\mathrm{Mg}^{2+}$ an unlikely short-term regulatory molecule like $\mathrm{Ca}^{2+}$, but long term change in total $\mathrm{Mg}^{2+}$, as exhibited in mitogen stimulation, may have profound effects on metabolic regulation given the sensitivity of free $\mathrm{Mg}^{2+}$ to alterations in total $\mathrm{Mg}^{2+}$ (Corkey et al., 1986).

\section{Response of protein synthesis in frog oocytes to injection of $\mathrm{Mg}^{2+}$ resembles that of stimulation by gonadotropin}

Frog oocytes are large enough (850-950 micrometres in diameter) to allow microinjection of materials, and to measure cations and protein synthesis in single cells (Horowitz and Lau, 1988). When stimulated by gonadotropin these quiescent cells begin to synthesize protein that drives their

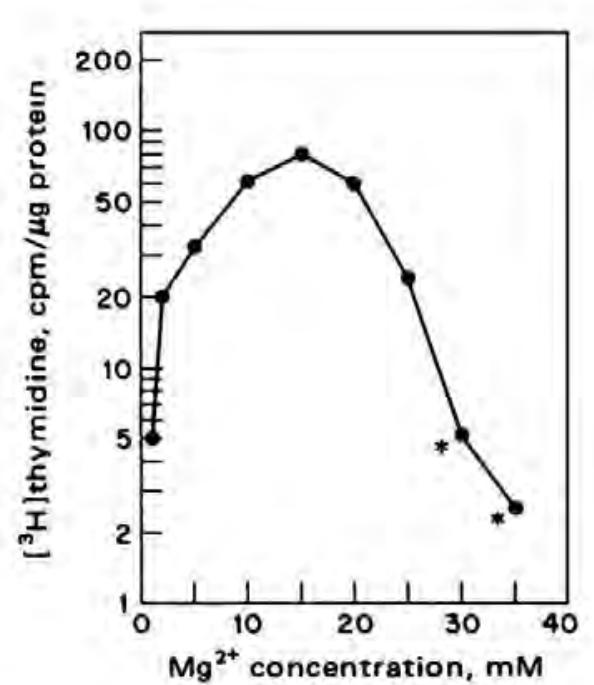

Figure 5. DNA synthesis as a function of extracellular $\mathrm{Mg}^{2+}$. Quiescent mouse 3T3 cells were stimulated with $10 \%$ dialyzed serum in $\mathrm{Ca}^{2+}$ deprived medium with varying concentrations of $\mathrm{Mg}^{2+}$. At 17 hours the cultures were labelled with ${ }^{3} \mathrm{H}$-thymidine. The asterisks associated with the two highest concentrations of $\mathrm{Mg}^{2+}$ in the medium and greatly reduced incorporation in DNA indicate many foci of degenerated cells (Rubin et al., 1978).

growth and maturation into eggs. Exposing oocytes in saline to gonadotropin that acts via cell surface receptors increases cytoplasmic $\mathrm{K}^{+}$ activity and protein synthesis for at least 10 days (Lau et al., 1988). These effects are mimicked by microinjection of $\mathrm{K}^{+}$into oocytes immersed in paraffin oil to prevent contributions from external sources. The results suggested that $\mathrm{K}^{+}$ activity is critical to activate and perpetuate protein synthesis.

Further analysis of the results showed that there was a greater effect of $\mathrm{K}^{+}$on translation in the oocytes than expected from its effect on cell-free translation (Horowitz and Tluczek, 1989). Also, injection of $\mathrm{Na}^{+}$into the oocytes stimulated protein synthesis to the same extent as $\mathrm{K}^{+}$, despite the fact that $\mathrm{Na}^{+}$has no effect on cell-free translation systems. These results indicated that $\mathrm{Na}^{+}$and $\mathrm{K}^{+}$compete with each other for binding to high affinity anions such as phosphate groups 
on membranes. $\mathrm{Ca}^{2+}$ was ruled out as the displaced cation, but $\mathrm{Mg}^{2+}$ stimulated protein synthesis at much lower and narrower concentrations than did $\mathrm{K}^{+}$. It was concluded that $\mathrm{Mg}^{2+}$, presumably as MgATP ${ }^{2-}$, is the effector directly controlling translation rates in oocytes. There was also evidence that stimulation of the oocytes by gonadotropin, which was necessarily done in saline medium containing $\mathrm{Mg}^{2+}$, added extracellular $\mathrm{Mg}^{2+}$ to that contributed by intracellular cation exchange (Horowitz and Tluczek, 1989).

\section{Role of $\mathrm{Mg}^{2+}$ in regulating uridine uptake}

The uptake rate of uridine into the acid soluble fraction of the cytoplasm increases several fold within a few minutes after stimulating cells with serum (Bowen-Pope and Rubin, 1977), and is sensitive to the concentration of $\mathrm{Mg}^{2+}$ in the medium (Rubin, 1976). External uridine is not required for growth of the cells, and its increased uptake does not require protein synthesis (Bowen-Pope and Rubin, 1977). The regulation of uridine uptake by $\mathrm{Mg}^{2+}$ occurs at its phosphorylation, which is the same step at which serum modulates uridine uptake (Vidair and Rubin, 1981).

Phosphorylation of uridine is catalysed by uridine kinase, which responds to $\mathrm{Mg}^{2+}$ in cell-free systems in a manner similar to that exhibited by uridine uptake in living cells. Unlike protein synthesis, uridine uptake is not inhibited by high concentrations of $\mathrm{Mg}^{2+}$ in the cells, which is consistent with its simpler dependence on initial phosphorylation vs the many steps at which net protein synthesis may be affected by $\mathrm{Mg}^{2+}$ (Bowen-Pope et al., 1979). Thymidine uptake does not respond to mitogenic stimulation, nor does it require $\mathrm{Mg}^{2+}$ at the concentrations required by uridine (Vidair and Rubin, 1981). This is presumably because the $\mathrm{Km}$ of uridine kinase for $\mathrm{Mg}^{2+}$ is higher than that of thymidine kinase and within the normal range of intracellular $\mathrm{Mg}^{2+}$ variation (Vidair and Rubin, 2005). The divalent cation ionophore A23187 facilitates the manipulation of intracellular $\mathrm{Mg}^{2+}$ concentration without increasing the generalized permeability of the cell. In high concentrations of extracellular $\mathrm{Mg}^{2+}$, the ionophore-treated cells take up uridine in the absence of protein growth factors at the same high rate as in their presence. The results support the thesis that increased uridine uptake in cells treated with growth factors is determined by a membrane-activated increase in intracellular free $\mathrm{Mg}^{2+}$.

\section{Molecular studies of $\mathrm{Mg}^{2+}$ regulation in lymphocytes}

TRPM7 is a membrane protein with intrinsic ion channel and protein kinase domains (Schmitz et al., 2003). An established line of chicken lymphocytes deficient in TRPM7 entered a quiescent phase when cultured in standard concentrations $(0.4 \mathrm{mM})$ of $\mathrm{Mg}^{2+}$ similar to that of lymphocytes in the organism. Raising the $\mathrm{Mg}^{2+}$ concentration in the medium to $15 \mathrm{mM}$ resulted in an increase in both the viability and proliferation of the cells, implying increased protein accumulation accompanying progress through the cell cycle. This activation indicated that TRPM7 has an essential role in regulating physiological concentrations of $\mathrm{Mg}^{2+}$ by an uptake pathway with functional coupling between its channel and kinase domains. TRPM7 channels are required for $\mathrm{PI} 3-\mathrm{K}$ function as a central regulator of growth in lymphocytes (Sahni and Scharenberg, 2008), as had been hypothesized from studies of $\mathrm{Mg}^{2+}$ regulation in fibroblasts (Rubin, 2005). The PI 3-K pathway leads through a protein kinase cascade to mTOR kinase which is a central regulator of protein synthesis (Schmelzle and Hall, 2000). mTOR accomplishes this role by phosphorylating serines and threonines on two proteins which drive further steps that initiate translation (Terada et al., 1994; Sahni and Scharenberg, 2008). The mTOR phosphorylations have a high $\mathrm{Km}$ for $\mathrm{MgATP}^{2-}$, which is in the physiological range of free $\mathrm{Mg}^{2+}$. Total $\mathrm{Mg}^{2+}$ in the TRPM7-deficient lymphocytes in standard medium underwent a significant decrease, but no change in free $\mathrm{Mg}^{2+}$ was detected, which contrasts with the increase in free $\mathrm{Mg}^{2+}$ in mitogen-stimulated fibroblasts (Ishijima et al., 1991) and in myocytes (Grubbs, 1991).

A number of the known properties of quiescent, ex-vivo lymphocytes were also measured in the TRPM7-deficient lymphocytes in standard medium containing $0.4 \mathrm{mM} \mathrm{Mg}^{2+}$ (Sahni et al., 2010). The DNA content and size of the two cell sources were similar, and suggested that TRPM7 and $\mathrm{Mg}^{2+}$ are involved in the activation of normal quiescent lymphocytes. The TRPM7-deficient 
lymphocytes increased in size and entered rapid proliferation when the concentration of $\mathrm{Mg}^{2+}$ in the medium was increased. The key cell regulator p27 was elevated in both normal, quiescent lymphocytes and in TRPM7-deficient cells in standard medium. A reduction in RNA, storeoperated $\mathrm{Ca}^{2+}$ entry and altered energy metabolism was found in the quiescent TRPM7deficient cells and in normal quiescent lymphocytes. The addition of excess $\mathrm{Mg}^{2+}$ to the deficient cells altered all these processes in a characteristic manner of activation of normal lymphocytes, just as it activated the coordinate response of fibroblasts in $\mathrm{Ca}^{2+}$-deficient medium (Rubin et al., 1979). The implication of these and foregoing results is that cytosolic free $\mathrm{Mg}^{2+}$ and MgATP $^{2-}$ play a central role in the regulation of all cell types and stages of development across the animal kingdom.

\section{Loss of $\mathrm{Mg}^{2+}$ regulation in neoplastically transformed cells}

Neoplastically transformed cells have the capacity to proliferate in lower concentrations of serum and to higher saturation densities than non-transformed cells (Rubin, 1981). Cell transformation causes a selective loss of the regulatory role of $\mathrm{Mg}^{2+}$ in cellular multiplication (McKeehan and Ham, 1978). Deprivation of $\mathrm{Mg}^{2+}$ caused transformed cells to flatten and to assume a regularly patterned, non-overlapping relationship to one another (Rubin et al., 1981). After 3 days of $\mathrm{Mg}^{2+}$ deprivation, the rate of DNA synthesis became highly dependent on both serum concentration and population density, thus resembling the growth behaviour of nontransformed cells. The capacity of the normalized transformed cells to produce colonies in agar in normal $\mathrm{Mg}^{2+}$ is greatly reduced after 7 days of $\mathrm{Mg}^{2+}$ deprivation in monolayer culture (Rubin and Chu, 1982).

The $\mathrm{Ca}^{2+}$ content of transformed cells is only onethird that of non-transformed cells, but it increases to that of the non-transformed cells in $\mathrm{Mg}^{2+}$-deprived medium (Rubin et al., 1981). The normalization of these parameters in $\mathrm{Mg}^{2+}$ deprived transformed cells is reversed a few days after their subculture in $\mathrm{Mg}^{2+}$-adequate medium. The results support the suggestion that a defect in $\mathrm{Mg}^{2+}$ regulation of the multiplication of transformed cells is a basic feature of neoplastic transformation (McKeehan and Ham, 1978) which could be exerted at the level of protein synthesis (Rubin, 2005; Sahni and Scharenberg, 2008). Nothing has been established about the cause of the postulated loss of $\mathrm{Mg}^{2+}$ control in transformed cells but one possibility has to do with their actively moving surface membranes (Abercrombie and Ambrose, 1958), which could result in lowering of the binding of $\mathrm{Mg}^{2+}$ to anionic groups on their internal surface, and its release into the cytosol (Rubin, 2005). This, of course, would shift the question to what causes the excessive membrane activity, which gets into the somatic cell genetics of neoplastic transformation, and is beyond the scope of this article.

\section{Conclusions}

There were several lines of thought that led to the suspicion that $\mathrm{Mg}^{2+}$ plays a central role in regulation of growth and replication. Among them were the observations that a variety of unrelated and non-specific substances could initiate at least one round of DNA synthesis and cell replication (Rubin, 1975b; Rubin and Sanui, 1977). Another was that a number of reactions were activated by mitogenic substances that were essential for cell replication and others that were not, but all responded directly to the mitogens hours before the synthesis of DNA was initiated (Bowen-Pope and Rubin, 1977). These early responses were considered part of a coordinate response and drove a search for a second messenger that could regulate all members of that response. That search was pursued by depriving confluent, contact-inhibited cultures of each extracellular candidate in turn, and determining the capacity of its removal to inhibit both the early responses and later increases in DNA synthesis of freshly stimulated cultures (Rubin, 1976; Rubin et al., 1979). The most significant early response of the cells for controlling later DNA synthesis was the synthesis of proteins, and the only measured cation that correlated quantitatively with both reactions was total intracellular $\mathrm{Mg}^{2+}$ concentration produced by variation in extracellular $\mathrm{Mg}^{2+}$. In order to reliably produce a change of intracellular $\mathrm{Mg}^{2+}$ over a wide range, it was necessary to drastically lower the concentration of $\mathrm{Ca}^{2+}$ in the medium while varying the extracellular concentration of $\mathrm{Mg}^{2+}$. The deprivation of $\mathrm{Ca}^{2+}$ increased the 
permeability of the cells for $\mathrm{Mg}^{2+}$ as well as for other small molecules. It was concluded that the concentration of free $\mathrm{Mg}^{2+}$ in the cells determined the level of the coordinate response, and most significantly of protein synthesis.

This conclusion was in part based on small increases in total $\mathrm{Mg}^{2+}$ assuming that there were accompanying increases in free $\mathrm{Mg}^{2+}$. Supporting evidence for this conclusion was provided by experiments in Xenopus oocytes which allowed direct injection and measurement of free $\mathrm{Mg}^{2+}$ (Horowitz and Tluczek, 1989). However, there was a failure to demonstrate an increase in free $\mathrm{Mg}^{2+}$ by indicator measurements in activated lymphocytes (Rink et al., 1982), despite the reported increase in total $\mathrm{Mg}^{2+}$ (Sahni et al., 2010). It should be noted that although $\mathrm{Mg}^{2+}$ is a co-factor in all phosphoryl transfers in cells, the actual regulatory substrate is $\mathrm{MgATP}^{2-}$ in which the $\mathrm{Mg}^{2+}$ is chelated by $\mathrm{ATP}^{4-}$, and therefore would not be registered by indicators of free $\mathrm{Mg}^{2+}$.

The PI 3-K pathway is essential for the regulation of protein synthesis (Richardson et al., 2004). It leads through a protein kinase cascade to mTOR kinase, which is considered a central regulator of protein synthesis (Schmelzle and Hall, 2000). mTOR accomplishes this role by phosphorylating serines and threonines on two protein substrates which regulate the initiation of translation, mainly of ribosomal proteins and elongation factors (Terada et al., 1994). The mTOR phosphorylations have a high $\mathrm{Km}$ for $\mathrm{MgATP}^{2-}$ (Dennis et al., 2001; Jaeschke et al., 2004). It may be significant for the coordination of protein synthesis with energy production that the kinases

\section{References}

Abercrombie M, Ambrose EJ (1958) Interference microscope studies of cell contacts in tissue culture. Exp Cell Res 15:332-45.

Bowen-Pope DF, Rubin H (1977) Magnesium and calcium effects on uptake of hexoses and uridine by chick embryo fibroblasts. Proc Natl Acad Sci USA 74:1585-9. of carbohydrate metabolism which phosphorylate low molecular substrates have a Km for MgATP ${ }^{2-}$ similar to that of mTOR (Edelman et al., 1987; Garner and Rosett, 1973). Hence MgATP ${ }^{2-}$ may have as important a role in regulating energy production as it does in regulating protein and DNA synthesis.

One aspect of $\mathrm{Mg}^{2+}$ regulation that needs more investigation is the putative role of the loss of its function in cell transformation (McKeehan and Ham, 1978; Rubin et al., 1981). A major target for mTOR phosphorylation is the eukaryotic initiation factor $4 \mathrm{E}$ (elF4E). Enhanced phosphorylation of eIF4E up-regulates several proteins implicated in tumorigenesis (Furic et al., 2010). Since mTOR is itself regulated by MgATP ${ }^{2-}$ (Rubin, 2005; Sahni and Scharenberg, 2008), the loss of regulation by $\mathrm{Mg}^{2+}$ in tumorigenesis, if confirmed, should lead to increased activity of elF4E and could account for its frequent involvement in human cancer (Hay, 2010). Hence, the measurement of free $\mathrm{Mg}^{2+}$ in neoplastic transformation would be a logical next step.

\section{Acknowledgements}

I am grateful to Dorothy M. Rubin for editing the manuscript. The meticulous preparation of material and measurements of cations by the late Dr. Hisashi Sanui in cells and media by atomic absorption spectrophometry lent great accuracy and reliability to the results. The work of graduate students Dan Bowen-Pope, Dave Moscatelli, Mark Terasaki and Charlie Vidair contributed much in their day to an understanding of the role of $\mathrm{Mg}^{2+}$ in the coordinate response of cells to mitogenic stimuli.

Bowen-Pope DF, Vidair C, Sanui H, Rubin H (1979) Separate roles for calcium and magnesium in their synergistic effect on uridine uptake by cultured cells. Proc Natl Acad Sci USA 76:1308-12.

Bowen-Pope DF, Rubin H (1983) Growth stimulatory precipitates of $\mathrm{Ca}^{2+}$ and pyrophosphate. J Cell Physiol 117:51-61. 
Brooks RF (1977) Continuous protein synthesis is required to maintain the probability of entry into $S$ phase. Cell 12:311-7.

Corkey BE, Duszynski J, Rich TL, Matschinsky B, Williamson JR (1986) Regulation of free and bound magnesium in rat hepatocytes and isolated mitochondria. J Biol Chem 261:2567-74.

Dennis PB, Jaeschke A, Saitoh M, Fowler B, Kozma SC, Thomas G (2001) Mammalian TOR: a homeostatic ATP sensor. Science 294:1102-5.

Edelman AM, Blumenthal DK, Krebs EG (1987) Protein serine/threonine kinases. Ann Rev Biochem 56:567-93.

Furic L, Rong L, Larsson O, Koumakpayi IH, Yoshida K, Brueschke A, Petroulakis E, Bobichaud N, Pollak M, Gaboury LA, Pandolfi PP, Saad F, Sonenberg N (2010) elF4E phosphorylation promotes tumorigenesis and is associated with prostate cancer progression. Proc Natl Acad Sci USA 107:14134-9.

Garner P, Rosett T (1973) The influence of $\mathrm{Mg}^{2+}$ /adenine nucleotide ratios and absolute concentration of $\mathrm{Mg}^{2+}$ /adenine nucleotide on the observed velocity of some kinase reactions. FEBS Lett 34:243-6.

Grubbs RD (1991) Effect of epidermal growth factor on $\mathrm{Mg}^{2+}$ homeostasis in $\mathrm{BC} 3 \mathrm{H}-1$ myocytes. Am J Physiol 260:C1158-C64.

Hay N (2010) Mnk earmarks elF4E for cancer therapy. Proc Natl Acad Sci USA 107:13975-6.

Horowitz SB, Lau Y-T (1988) A function that relates protein synthetic rates to potassium activity in vivo. $J$ Cellular Physiol 135:425-34.

Horowitz SB, Tluczek LJM (1989) Gonadotropin stimulates oocyte translation by increasing magnesium activity through intracellular potassium-magnesium exchange. Proc Natl Acad Sci USA 86:9652-6.

Ishijima S, Sonoda T, Tatibana M (1991) Mitogeninduced early increase in cytosolic free $\mathrm{Mg}^{2+}$ concentration in single Swiss 3 T3 fibroblasts. Am J Physiol 261:C1074-C80.

Jaeschke A, Dennis PB, Thomas G (2004) mTOR: a mediator of intracellular homeostasis. In: TOR, Target of Rapamycin (Thomas G, Sabatini DM, Hall MN, eds), Vol. 279, Berlin: Springer Verlag, 283-98.

Lau Y-T, Yassin RR, Horowitz SB (1988) Potassium salt microinjection into Xenopus oocytes mimics gonadotropin treatment. Science 240:1231-323.
McKeehan W, Ham RG (1978) Calcium and magnesium ions and the regulation of multiplication in normal and transformed cells. Nature 275:756-8.

Moscatelli D, Sanui H, Rubin H (1979) Effects of depletion of $\mathrm{K}^{+}, \mathrm{Na}^{+}$, or $\mathrm{Ca}^{2+}$, on DNA synthesis and cell cation content in chick embryo fibroblasts. J Cell Physiol 101:117-28.

Murphy E, Freudenrich CC, Levy LA, London RE (1989) Monitoring cytosolic free magnesium in cultured chicken heart cells by use of the fluorescent indicator Furaptra. Proc Natl Acad Sci USA 86:2981-4.

Raju B, Murphy E, Levy LA, Hall RD, London RE (1989) A fluorescent indicator for measuring cytosolic free magnesium. Am J Physiol 256:C540-C8.

Richardson CJ, Schalm SS, Blenis J (2004) PI3-kinase and TOR: PIKTORing cell growth. Seminars in Dev Biol 15:147-59.

Rink TJ, Tsien RY, Pozzan T (1982) Cytoplasmic pH and free $\mathrm{Mg}^{2+}$ in lymphocytes. J Cell Biol 95:189-96.

Rubin H (1975a) Central role for magnesium in coordinate control of metabolism and growth in animal cells. Proc Natl Acad Sci USA 72:3551-5.

Rubin H (1975b) Nonspecific nature of the stimulus to DNA synthesis in cultures of chick embryo cell. Proc Natl Acad Sci USA 72:1676-80.

Rubin H, Steiner R (1975) Reversible alteration in the mitotic cycle of chick embryo cells in various states of growth regulation. J Cell Physiol 85:261-70.

Rubin H (1976) Magnesium deprivation reproduces the coordinate effects of serum removal or cortisol addition on transport and metabolism in chick embryo fibroblasts. J Cell Physiol 89:613-26.

Rubin H, Sanui H (1977) Complexes of inorganic pyrophosphate, orthophosphate and calcium as stimulants of 3 T3 cells multiplication. Proc Natl Acad Sci USA 74:5025-30.

Rubin H, Terasaki M, Sanui H (1978) Magnesium reverses inhibitory effects of calcium deprivation on coordinate response of 3T3 cells to serum. Proc Natl Acad Sci USA 75:4379-83.

Rubin H, Terasaki M, Sanui H (1979) Major intracellular cations and growth control: Correspondence among magnesium content, protein synthesis, and the onset of DNA synthesis in Balb/c 3T3 cells. Proc Natl Acad Sci USA 76:3917-21. 
Rubin H (1981) Growth regulation, reverse transformation, and adaptability of 3T3 cells in decreased $\mathrm{Mg}^{2+}$ concentration. Proc Natl Acad Sci USA 78:328-32.

Rubin H, Vidair C, Sanui H (1981) Restoration of normal appearance, growth behavior and calcium content to transformed 3 T3 cells by magnesium deprivation. Proc Natl Acad Sci USA 78:2350-4.

Rubin H, Chu B (1982) Self-normalization of highly transformed 3T3 cells through maximized contact interaction. Proc Natl Acad Sci USA 79:1903-7.

Rubin $\mathrm{H}$ (2005) Central roles of $\mathrm{Mg}^{2+}$ and of $\mathrm{MgATP}^{2-}$ in the regulation of protein synthesis and cell proliferation: significance for neoplastic transformation. Adv Cancer Res 93:1-58.

Sahni J, Scharenberg AM (2008) TRPM7 ion channels are required for sustained phosphoinositide 3-kinase signaling in lymphocytes. Cell Metabolism 8:84-93.

Sahni J, Tamura R, Sweet I, Scharenberg AM (2010) TRPM7 regulates quiescent/proliferative metabolic transitions in lymphocytes. Cell Cycle 9:3565-74.

Sanui H, Rubin H (1978) Membrane bound and cellular cationic changes associated with insulin stimulation of cultured cells. J Cell Physiol 96:265-78.
Sanui H, Rubin H (1982) Changes of intracellular and externally bound cations accompanying serum stimulation of mouse Balb/c3T3 cells. Exp Cell Res 139:15-25.

Sanui H, Rubin H (1984) lonic changes associated with lead stimulation of DNA synthesis in Balb/c3T3 cells. Biological Trace Element Research 6:289-307.

Schmelzle T, Hall MN (2000) TOR, a central controller of cell growth. Cell 103:253-62.

Schmitz C, Perraud A-L, Johnson CO, Inabe K, Smith MK, Penner R, Kurosaki T, Fleig A, Scharenberg A (2003) Regulation of vertebrate cellular $\mathrm{Mg}^{2+}$ homeostasis by TRPM7. Cell 114:191-200.

Schreier MH, Staehelin T (1973) Initiation of mammalian protein synthesis: the importance of ribosome and initiation factor quality for the efficiency of in vitro systems. J Mol Biol 73:329-49.

Terada N, Patel HR, Takase K, Kohno K, Nairns AC (1994) Rapamycin selectively inhibits translation of mRNAs encoding elongation factors and ribosomal proteins. Proc Natl Acad Sci USA 91:11477-81.

Vidair C, Rubin $\mathrm{H}$ (1981) Evaluation of $\mathrm{Mg}^{2+}$ as an intracellular regulator of uridine uptake. J Cell Physiol 108:317-25.

Vidair C, Rubin $\mathrm{H}$ (2005) $\mathrm{Mg}^{2+}$ as activator of uridine phosphorylation and other cellular responses to growth factors. Proc Natl Acad Sci USA 102:662-6. 


\title{
Magnesium and the Yin-Yang interplay in apoptosis
}

\author{
Valentina Trapani, Lucia Mastrototaro and Federica I. Wolf \\ Istituto di Patologia Generale e Centro di Ricerche Oncologiche Giovanni XXIII, Università Cattolica del Sacro Cuore, Rome, Italy. \\ $\triangle$ fwolf@rm.unicatt.it
}

\begin{abstract}
Apoptosis is a distinctive feature in the physiology of the developing brain, but also a key event in pathological conditions of the adult brain. The basic mechanisms executing cell death by apoptosis are conserved among different tissues and in different conditions, while the initiating event(s) may be more specific. Magnesium appears to be an important player in the process, though it might exert opposite actions depending on extra/intracellular availability. Extracellular magnesium deficiency induces apoptosis, mainly through increased oxidative stress, while intracellular magnesium mobilization from intracellular stores and consequent increase of cytosolic free magnesium seem to act in the effector phase. The molecular mechanism and the physio-pathological meaning of these findings await further characterization. The issue is even more complex in the context of the brain, where many concurring factors may determine a pro- or anti-apoptotic environment. A deeper understanding of the yin-yang role of magnesium in apoptosis may cast light on the basic processes that regulate cell fate, and consequently may open up novel opportunities for a successful therapeutic intervention for all the pathological conditions where excessive and undue apoptosis takes place.
\end{abstract}

\section{Cell death by apoptosis}

Multicellular organisms maintain their homeostasis thanks to a tightly regulated mode of cell death. Programmed cell death, or apoptosis, is an internally controlled suicide program consisting in a stereotyped sequence of biochemical and morphological changes that allow the cell to die without adversely affecting its neighbours, i.e. without causing inflammation. As such, apoptosis is distinct from necrosis, the traumatic form of cell death whereby massive disruption of the surrounding tissue takes place. A cell may be doomed to die either by apoptosis or necrosis depending on the intensity and duration of the stimulus, the rapidity of the death process, and the extent of ATP depletion suffered by the cell.

Apoptosis occurs in numerous physiological, adaptive and pathological events: 1) during development; 2) as a homeostatic mechanism to maintain cell populations in proliferating tissues; 3) as a defence mechanism such as in immune reactions; 4) following cell injury from various agents; and 5) in aging. There is general agreement that this form of cell death is an energy-dependent cascade of molecular events leading to cell shrinkage with maintenance of organelle integrity; long-recognized features of apoptosis are protein cleavage and cross-linking, chromatin condensation, DNA fragmentation and, ultimately, phagocytic recognition and engulfment of the dying cell. The process of apoptosis is triggered by a diverse range of cell signals, which may originate either extracellularly (i.e. death receptor ligation with their cognate ligands, extrinsic pathway) or intracellularly (e.g. DNA damage or organelle dysfunction, intrinsic pathway).

The apoptotic cascade consists of three parts: first, cells process the receptor-mediated death signal (initiation phase, somewhat more cellspecific), then get ready to implement apoptosis (effector phase, more conserved among different tissues) and finally commit suicide (degradation phase). During the initiation phase, the receptormediated death signal activates an intracellular cascade of events including: 1) activation of initiator caspases (caspase-8, for example), that can act early in the cell death process before, or independently of, mitochondrial changes; 2) increase in levels of oxyradicals and $\mathrm{Ca}^{2+} ; 3$ ) transcription and translocation of pro-apoptotic $\mathrm{Bcl}-2$ family members (Bax and Bad) to the mitochondrial membrane. The execution phase involves: 1) increased mitochondrial $\mathrm{Ca}^{2+}$ and oxyradical levels; 2) opening of permeability 
transition pores (PTP) in the mitochondrial membrane; 3) release of cytochrome $c$ and many other apoptosis-inducing factors from the mitochondrial matrix into the cytosol; 4) formation of a molecular complex (the apoptosome) between cytochrome $c$ and apoptotic protease-activating factor 1 (Apaf-1), that recruits and activates pro-caspase-9. Activated caspase-9, in turn, activates caspase- 3 , which begins the degradation phase: proteins are cleaved by various caspases and packed by transglutaminases, while nucleic acids are cleaved by activated $\mathrm{Ca}^{2+} / \mathrm{Mg}^{2+}$ endonucleases, which results in the formation of apoptotic bodies and subsequent phagocytosis by elicited macrophages or neighbouring cells. It is interesting to underline the role of mitochondria in triggering apoptosis. Whether or not a direct injury jeopardizing the efficiency of these energy producing organelles occurs, mitochondria play a pivotal role in the cell death decision, as apoptotic factors are released by active mechanisms, which can be unrelated to mitochondrial damage (Tait and Green, 2010).

\section{Role of Magnesium in Apoptosis}

In the attempt to review the role of magnesium in apoptosis, one should first of all keep in mind that the effects of extracellular magnesium availability may differ from those due to intracellular magnesium fluxes or mobilization. In fact, decreased extracellular magnesium availability does not necessarily reflect an intracellular depletion, as demonstrated by the fact that in vivo serum magnesium levels are not a reliable marker of pathological hypomagnesemia. Sensitivity to extracellular magnesium availability is highly tissue-specific, and can be translated in molecular terms by considering the expression and activity of magnesium-specific cation channels, such as the transient receptor potential melastatin (TRPM) - 6 and -7 channels, which, by modulating transmembrane cation fluxes, regulate the intracellular ion concentration (Schlingmann et al., 2007). Furthermore, intracellular magnesium is tightly buffered to meet specific metabolic requirements. As discussed elsewhere in this book, large changes in total cell magnesium occur with little or no change in cytosolic free magnesium, suggesting that the changes in total magnesium are due to changes in bound or sequestered magnesium (Murphy, 2000). ATP is the most important intracellular magnesium buffer. Following massive ATP hydrolysis intracellular free magnesium transiently increases and is subsequently released into the extracellular environment. As a consequence, in a dying cell an increase in ionized magnesium occurs. Therefore, caution should be used also when investigating the role of intracellular magnesium as a player in the apoptotic program, in order to discriminate coincidental from causative events.

In conclusion, whether considering extracellular magnesium availability or intracellular magnesium concentration, the story is more complicated than it appears at first glance. We will attempt to discuss the issue by reviewing the latest available data in view of the complex cellular physiology of magnesium.

\section{Extracellular magnesium and apoptosis}

Several important aspects of magnesium biochemistry and physiology point to a possible role for this cation in the apoptotic process. As discussed in other chapters of this book, magnesium is a key modulator of cell proliferation and metabolism and, most importantly, magnesium availability appears to affect the occurrence of oxidative stress. Many studies are available in the literature on the subject, but the most convincing ones investigate the effects of hypomagnesemia in vivo. In these circumstances, however, other factors, including inflammation, cytokine production and activation of phagocyte oxidative burst, concur to what is referred to as the prooxidant effect of hypomagnesemia (Mazur et al., 2007). Induction of apoptosis mediated by oxidative stress following magnesium deprivation has been documented in several tissues. Accelerated thymus involution, a classical example of apoptosis, was found in magnesiumdeficient rats (Malpuech-Brugère et al., 1999). Dietary magnesium deficiency also induced apoptosis in cardiovascular tissues (Altura et al., 2009; Tejero-Taldo et al., 2007) and in the liver (Martin et al., 2003; Martin et al., 2008). In addition to the induction of reactive oxygen species, low magnesium availability could in principle trigger apoptosis by affecting DNA structure. This occurs not only by promoting oxidative DNA damage, but also by impairing DNA repair mechanisms, since magnesium is required for several crucial DNA repair enzyme 
activities, e.g. endonucleases, ligases, topoisomerases (Hartwig, 2001).

\section{Intracellular magnesium and apoptosis}

As to intracellular events, several in vitro studies have suggested a promoting role for magnesium in apoptosis. An early increase in intracellular magnesium seems to follow both extrinsic and intrinsic induction of apoptosis (Patel et al., 1994; Chien et al., 1999; Zhang et al., 2005). The most straightforward explanation is that the increase in intracellular magnesium concentration is necessary for stimulating the activity of $\mathrm{Ca}^{2+}$ / $\mathrm{Mg}^{2+}$ dependent endonucleases, which perform the apoptotic event par excellence, i.e. nucleosomal DNA fragmentation. Interestingly, however, the source of intracellular magnesium was hypothesized to be in the mitochondria (Chien et al., 1999), which is particularly appealing, as it is well established that mitochondria play a central role in the onset of the apoptotic program. Other lines of evidence point to the same direction. While the opening of the permeability transition pores appears to be dispensable for the release of cytochrome $c$, this is not the case for the presence of magnesium, which instead seems an absolute requirement (Eskes et al., 1998; Kim et al., 2000). Another study (Salvi et al., 2004) indicated that an apoptotic compound, gliotoxin, can specifically activate a magnesium efflux system from mitochondria in conditions of preserved mitochondrial integrity (i.e. high membrane potential, no swelling and retention of other ions). Most importantly, it has been shown that mitochondria act in fact as magnesium stores, and that $\mathrm{Mg}^{2+}$ release can occur following $\mathrm{Ca}^{2+}$ release and prior to ATP hydrolysis (Kubota et al., 2005). It is noteworthy that the same Authors have recently demonstrated that glutamate administration to rat hippocampal neurons triggers the same pathway, whereby $\mathrm{Ca}^{2+}$ accumulation in the mitochondria is required for $\mathrm{Mg}^{2+}$ release from the organelles (Shindo et al., 2010). The presence of a specific mitochondrial channel for magnesium, Mrs2, (Kolisek et al., 2003) corroborates these findings. As the activity of Mrs2 is dependent from membrane potential, it can be speculated that stored magnesium might be released through the channel upon depolarization. Intriguingly, in a recent paper Mrs2 expression has been associated with resistance to drug-induced apoptosis in cancer cells (Chen et al., 2009): by upregulating magnesium uptake into mitochondria, Mrs2 might counteract the increase in cytosolic magnesium that seems to be necessary for the execution of the apoptotic program via the mitochondrial pathway (Wolf and Trapani, 2009). In conclusion, the latest findings seem to concur to suggest a finer involvement of magnesium in the apoptotic cascade: not just a biochemical factor, but rather a crucial control element in life vs death decisions (see Table 1 for a summary). At present, these are only attractive speculations; most importantly, it has to be clarified whether the increase in intracellular magnesium occurring following an apoptotic stimulus is just a coincidental event (for example, due to mitochondrial depolarization) or rather a causative determinant in the downstream signalling cascade.

Table 1: Magnesium and apoptosis

\begin{tabular}{|c|c|}
\hline EXTRACELLULAR & INTRACELLULAR \\
\hline DECREASED MAGNESIUM & INCREASED MAGNESIUM \\
Impaired trophic signalling factor & Mitochondrial damage \\
Increased oxidative stress & Mitochondrial dysfunction \\
Increased DNA damage & Ca $^{2+}$ signals \\
Decreased DNA repair & Excitotoxicity \\
N & $\sqrt{ }$ \\
EXTRINSIC AND INTRINSIC PATHWAY & INTRINSIC PATHWAY
\end{tabular}




\section{Neuronal Apoptosis}

In contrast to the rapid turnover of cells in proliferative/renewing tissues, neurons commonly survive for the entire lifetime of the organism this enduring nature of neurons is necessary for maintaining the function of those cells within neuronal circuits. However, during development of the central and peripheral nervous systems, many neurons undergo apoptosis during a time window that coincides with the process of synaptogenesis (Oppenheim, 1991). Initial overproduction of neurons, followed by death of some, is probably an adaptive process that provides enough neurons to form nerve cell circuits that are precisely matched to their functional specifications. However, the persistence of neurons throughout life to preserve brain function implies that a considerable evolutionary pressure was placed on the development of mechanisms that guarded against neuronal death and/or promoted neuronal survival and plasticity.

\section{Trophic factors}

Like all cells, neuronal survival requires trophic support. Viktor Hamburger and Rita LeviMontalcini described in a seminal paper that the survival of developing neurons is directly related to the availability of their innervating targets (Hamburger and Levi-Montalcini, 1949). This laid the foundation for the neurotrophin hypothesis, which proposed that immature neurons compete for target-derived trophic factors that are in limited supply. Neurotrophins, which include nerve growth factor (NGF), brain-derived neurotrophic factor (BDNF), and neurotrophins 3 and 4/5 (Lewin and Barde, 1996), generally activate and ligate the Trk receptors (TrkA, TrkB and $\operatorname{TrkC}$ ), which are cell-surface receptors with intrinsic tyrosine kinase activity. They can autophosphorylate; for instance, after the binding of NGF to TrkA, the receptor phosphorylates several tyrosine residues within its own cytoplasmic tail. These phosphotyrosines in turn serve as docking sites for other molecules such as phospholipase $\mathrm{Cg}$, phosphoinositide 3kinase $(\mathrm{PI}(3) \mathrm{K})$ and adaptor proteins such as Shc, and these signal transduction molecules coordinate neuronal survival through the Akt and MAP kinase signalling pathways (Hennigan et al., 2007). Several neurotrophic factors and cytokines use a survival pathway involving the transcription factor NF-KB (Mattson and Camandola, 2000).
The neurotrophin hypothesis predicts correctly that neuronal survival requires a positive survival signal. It does not, however, provide a concrete hypothesis as to how neurons die in the absence of trophic support; it was assumed that neurons die simply of passive starvation. In 1988, using cultured sympathetic neurons as a model system, Johnson and colleagues showed that inhibition of RNA and protein synthesis blocked sympathetic neuronal cell death induced by nerve growth factor (NGF) deprivation (Martin et al., 1988), providing the first tangible evidence that neurons might actually actively instigate their own demise.

\section{Glutamate receptors}

Lack of neurotrophic support is undeniably the best-studied signal that may trigger apoptosis during development of the nervous system. However, most neurons in the mammalian central nervous system possess receptors for another trigger of apoptosis, glutamate.

Glutamate is a major excitatory neurotransmitter with a crucial role in neural development, synaptic plasticity, and learning and memory under physiological conditions (Riedel et al., 2003). Glutamate receptors are classified into several classes. In this context, we will go into some detail only for N-methyl-D-aspartate (NMDA) receptors. NMDA receptors are tetraheteromeric ligand-gated ion channels that open upon the binding of glutamate. Magnesium binds the channel pore in a voltage-dependent manner; thus, at normal physiological resting membrane potential, the NMDA receptor is blocked by $\mathrm{Mg}^{2+}$. Synaptic release of glutamate causes $\mathrm{Na}^{+}$influx through $\alpha$-amino-3-hydroxy-5-methyl-4-isoxazolepropionate (AMPA) receptors in the postsynaptic cell, resulting in partial membrane depolarization sufficient to lift the $\mathrm{Mg}^{2+}$ block and activate the channel. The activated NMDA receptor is permeable to $\mathrm{Na}^{+}$but, crucially, also to $\mathrm{Ca}^{2+}$. This $\mathrm{Ca}^{2+}$ influx mediates most of the physiological effects of NMDA receptor activity, leading to postsynaptic depolarization and action potential in the postsynaptic neuron.

Physiological levels of synaptic NMDA receptor activity are essential for neuronal survival (Hetman and Kharebava, 2006). However, regulation of glutamatergic neurotransmission is critical, as improper management of glutamate levels and glutamate receptor activity may impair 
not only its signalling properties, but can lead to cell death. The concept of excitotoxicity was first proposed by John Olney in 1969 as a toxic effect of excessive or prolonged activation of receptors by excitatory amino acids (Olney, 1969). Although the molecular pathways involved in excitotoxicity are still not fully understood at the present, there is a wealth of evidence suggesting that overstimulation of glutamate receptors produces multiple adverse effects including impairment of intracellular calcium homeostasis, dysfunction of mitochondria and endoplasmic reticulum, increase in nitric oxide (NO) production and free radicals, persistent activation of proteases and kinases, increases in expression of pro-death transcription factors and immediate early genes (IEGs), ultimately leading to apoptosis (Wang and Qin, 2010). Excitotoxicity might mediate neuronal damage in various neurological disorders including ischemia and traumatic brain injury (Arundine and Tymianski, 2004) and neurodegenerative diseases (Lipton and Rosenberg, 1994; Rego and Oliveira, 2003); it has also been implicated in neonatal brain injury (Johnston, 2005). NMDA receptors are important mediators of glutamateinduced excitotoxicity, as calcium entering through over-activated NMDA receptors results in more cell death as opposed to calcium entering through non-NMDA glutamate receptors or voltage-gated calcium channels (Cristofanilli and Akopian, 2006), which maybe due in part to their high permeability to $\mathrm{Ca}^{2+}$ and incomplete desensitization.

Thus, responses to NMDA receptor activity follow a classical hormetic dose-response curve: both too much and too little can be harmful (Hardingham, 2009).

\section{Pathological stimuli}

Another trigger of neuronal death is increased oxidative stress, whereby free radicals (such as the superoxide anion radical and the hydroxyl radical) damage cellular lipids, proteins and nucleic acids by disrupting chemical bonds in those molecules. Metabolic stress, in which levels of glucose, oxygen and other molecules required for ATP (energy) production are decreased, and environmental toxins may also initiate neuronal apoptosis (Mattson, 2000). These stimuli are mostly involved in aging and pathological conditions, such as acute or chronic neurodegenerative diseases. The classical and most studied pathway of oxidation-induced apoptosis consists in cell-damage triggered phosphorylation of p53 and transcription of pro-apoptotic factors like Bid or Bad leading to mitochondrialdependent activation of caspase 9 .

The genetic and environmental factors that trigger neuronal apoptosis may be different in various physiological and pathological settings and can vary from those active in other tissues, but most of the subsequent biochemical events that execute the cell death process are highly conserved and shared with all other cell types. Thus, the key components of the apoptosis program in neurons, like that of other cell types, are Apaf-1 and proteins in the $\mathrm{Bcl}-2$ and caspase families. Nevertheless, different types of neurons, and neurons at different developmental stages, express different combinations of $\mathrm{Bcl}-2$ and caspase family members, which is one way of providing the specificity of regulation (Yuan and Yankner, 2000).

\section{Tissue specific factors}

An alternative way to control cell death or survival is through the expression of tissuespecific proteins that affect signal transduction reactions and may be either pro-apoptotic or prosurvival.

The prostate apoptosis response- 4 (Par-4) protein was identified as being upregulated in prostate tumour cells undergoing apoptosis, but is now known to be essential in developmental and pathological neuronal death (Guo et al., 1998). Levels of Par-4 increase rapidly in response to various apoptotic stimuli through enhanced translation of Par-4 messenger RNA. Par-4 acts at an early stage of the apoptotic cascade prior to caspase activation and mitochondrial dysfunction, by a mechanism that may involve inhibition of the antiapoptotic transcription factor NF-k $\beta$ and suppression of $\mathrm{Bcl}-2$ expression and/or function (Mattson et al., 1999).

Rai is an Shc-related adaptor protein whose expression is exclusively restricted to the nervous system. It exerts a prosurvival function in neuronal cells by activating the PI3K/Akt signalling pathway (Pelicci et al., 2002).

A neuron-specific splice variant of the variable subunit $B \beta$ was characterized, which is induced 
upon neuronal differentiation, targets protein phosphatase $2 \mathrm{~A}$ to mitochondria and accelerates neuronal cell apoptosis after survival factor deprivation (Dagda et al., 2003).

Lately, a brain-specific isoform of mitochondrial apoptosis-inducing factor, AIF2, has been isolated (Hangen et al., 2010). AIF2 dimerizes with AIF1 and has a stronger membrane anchorage. Therefore it is conceivable the neuron-specific AIF2 may have been 'designed' to be retained in mitochondria and to minimize its potential neurotoxic activity.

In conclusion, although mature neurons are among the most long-lived cell types in mammals, immature neurons die in large numbers and the regulation of apoptosis has a major role in sculpting the developing brain. Furthermore, not only is apoptosis important in regulating neuronal development, but it might also be a cardinal feature in the adult brain in several pathological conditions that will be detailed in the section on neurodegenerative diseases.

\section{Role of Magnesium in Neuronal Apoptosis}

Deciphering the role of magnesium in the apoptotic process is further complicated in the nervous system, as the blood brain barrier may restrain serum magnesium availability within the CNS. Indeed, this has been advocated as the cause for the failure of clinical trials assessing magnesium therapy in acute and chronic brain injury (Vink et al., 2009).

A significant part of the literature on the effects of magnesium on apoptosis in the brain is related to perinatal brain, as magnesium is often used as a treatment for pre-eclampsia/eclampsia and preterm labour. The basis for its use as a tocolytic is due to its observed effects in reducing myometrial contractility through extra- and intracellular mechanisms of action (Fomin et al., 2006). However, the use of magnesium sulphate as a drug in obstetrical medicine for both mother and fetus is highly controversial. A recent metaanalysis of all trials has recommended the use of magnesium for neuroprotection in the preterm fetus, as it was both safe and effective (Doyle et al., 2009). Nevertheless, some caution is mandatory, as several reports point to detrimental effects of magnesium for the fetal brain, depending on the dose (Mittendorf et al., 2006; Dribben et al., 2009), on the period of neurodevelopment when the exposure occurred (Dribben et al., 2009) or on the level of stress experienced (Krueger et al., 2001). In addition, some papers report a pro-apoptotic action of magnesium in placental tissues (Black et al., 2001; Gude et al., 2000). The major hurdle to establishing protective or deleterious effects of tocolytic magnesium to the developing brain is that magnesium's effects on preterm infant's brains, and the mechanisms of those effects, are not understood.

As discussed earlier, neuronal apoptosis can be triggered by three main mechanisms: 1) lack of growth factors; 2) overstimulation of glutamate receptors; and 3) oxidative stress. Magnesium could play a (different) role in each of these signalling pathways.

First, most growth factors work through receptor tyrosine kinases (RTKs) which require two magnesium ions for maximal activity. Kinases downstream of these RTKs, such as PI -kinase and the anti-apoptotic kinase Akt require magnesium both for their activation and activity. Therefore, extracellular magnesium could modulate the effect of growth factors on CNS cells, or activate their second messenger systems directly, ultimately affecting viability, proliferation, and apoptosis in these cells.

Secondly, under conditions of normal membrane polarization magnesium is known to block the NMDA glutamate receptor and prevent ion flow through the channel. Therefore, in principle, magnesium should protect against excitotoxicityinduced apoptosis. Nevertheless, as already discussed, normal physiological patterns of NMDA receptor activity promote neuroprotection against both apoptotic and excitotoxic insults; vice versa, NMDA receptor blockade can promote neuronal death outright or render neurons vulnerable to secondary trauma (Hardingham, 2009). Indeed, most NMDA antagonists have failed miserably as neuroprotective agents in clinical trials, in large part because of intolerable side effects (Lipton, 2006). Moreover, it has been shown that various drugs that inhibit neuronal activity, including NMDA antagonists, trigger widespread apoptotic neurodegeneration in the 
developing brain and cause long-term neurobehavioral deficits; in the infant rodent brain, peak sensitivity to this effect is in the P3 to P7 period (Ikonomidou et al., 1999). Thus, although other mechanisms may play a role, it seems likely that the neuroapoptogenic action demonstrated for magnesium in some studies could be mediated by its action at NMDA receptors.

Third, as mentioned earlier, magnesium deprivation may induce or exacerbate oxidative stress, another trigger of neuronal death, especially in pathological conditions. In this regard, magnesium supplementation might well have a neuroprotective action.

\section{Apoptosis in neurodegenerative diseases}

As already mentioned, neuronal death by apoptosis is a distinctive physiological feature of the developing brain; once synaptogenesis is over, the remaining neurons will usually survive throughout life to preserve brain function. Unfortunately, however, many people experience excessive death of specific neuronal subpopulations as a result of chronic diseases including Alzheimer's disease, Parkinson's disease, Huntington's disease and amyotrophic lateral sclerosis. Neuronal death may also occur as a consequence of acute conditions, such as stroke or traumatic brain and spinal cord injury. For each of these disorders, apoptosis has been implicated as the main form of cell death, though it is very difficult to demonstrate it for both technical and ethical reasons. Much of the evidence supporting an apoptotic mode of neuronal death comes from studies of animal and cell-culture models of neurodegenerative disorders (Mattson, 2000).

Physiological apoptosis in the developing brain and pathological apoptosis in the adult brain share similar molecular mechanisms in the effector phase, but may differ in the initiation. Whereas trophic factor withdrawal has a prominent role in apoptosis during development, toxic insults resulting from biochemical or genetic accidents seem to be the triggering event in neurodegenerative disorders. An emerging theme is the toxicity of abnormal protein structures or aggregates (Yuan and Yankner, 2000). Indeed, a defining feature of Alzheimer's disease is accumulation of amyloid plaques formed by aggregates of amyloid- $\beta$ peptide $(A \beta)$. Parkinson's disease is characterized by the appearance of $\alpha$-synuclein oligomers. Huntington's disease is caused by expansions of a trinucleotide (CAG) sequence in the huntingtin gene producing a protein containing increased polyglutamine repeats. The neuropathological signature of amyotrophic lateral sclerosis (ALS) is the presence of ubiquitinated inclusions immunoreactive for the proteins TDP-43 and/or FUS/TLS in the cytoplasm of motor neurons. Moreover, mutations in specific genes may predispose to neuronal degeneration, e.g. presenilin in Alzheimer's disease, parkin in Parkinson's disease, huntingtin in Huntington's disease, and $\mathrm{Cu} / \mathrm{Zn}$ superoxide dismutase (SOD) in ALS (please refer to the dedicated chapters in this book for further details).

The identification of specific genetic and environmental factors responsible for chronic neurodegenerative disorders has bolstered evidence for a shared pathway of neuronal apoptosis involving oxidative stress, perturbed calcium homeostasis and mitochondrial dysfunction ultimately converging on caspase activation (Zündorf and Reiser, 2010; Gibson et al., 2010).

In Alzheimer's disease, toxic forms of $A \beta$ may induce $\mathrm{Ca}^{2+}$ influx into neurons by formation of an oligomeric pore in the plasma membrane, thereby rendering neurons vulnerable to excitotoxicity and apoptosis (Supnet and Bezprozvanny, 2010). $A \beta$ also generates hydrogen peroxide and hydroxyl radicals, producing membrane lipid peroxidation and consequently membrane depolarization and excitotoxicity through NMDA receptor channels and voltagegated $\mathrm{Ca}^{2+}$ channels (Sultana and Butterfield, 2010; Butterfield et al., 2010). In addition, $A \beta$ accumulates in mitochondria, impairs the activity of complexes III and IV of the respiratory chain, causes elevated cytoplasmic $\mathrm{Ca}^{2+}$ levels and oxidative stress, and reduces ATP synthesis, further increasing $\mathrm{Ca}^{2+}$ overload and oxidative stress (Moreira et al., 2010).

Mitochondrial dysfunction is a defect occurring early in the pathogenesis of both sporadic and familial Parkinson's disease. Mitochondrial association of $\alpha$-synuclein in cells was linked to impairment of respiratory complex I activity, 
oxidative modification of mitochondrial proteins, and increased levels of $\mathrm{Ca}^{2+}$ and nitric oxide (Navarro and Boveris, 2009).

Mitochondrial $\mathrm{Ca}^{2+}$ overload is also a decisive commitment step for Huntington's disease. Mutant huntingtin facilitates opening of the PTP and directly impairs the mitochondrial function. Furthermore, huntingtin forms a ternary complex with other proteins, which causes $\mathrm{Ca}^{2+}$ release from the ER and renders neurons more sensitive to $\mathrm{Ca}^{2+}$-mediated cellular dysfunction (Damiano et al., 2010).

The mutations in SOD responsible for ALS do not decrease antioxidant activity of the enzyme, but result in the gain of an adverse pro-apoptotic activity that may involve increased peroxidase activity. Through interactions with hydrogen peroxide or superoxide anion, the mutant enzyme may induce oxidative damage to membranes and disturbances in mitochondrial function that make neurons vulnerable to excitotoxic apoptosis (Vucic and Kiernan, 2009). Also, stroke and trauma, and the consequent brain ischemia, initiate biochemical and molecular events involving many of the same neurodegenerative cascades that occur in the chronic neurodegenerative diseases described above, namely excitotoxicity, calcium overload and oxidative stress (Arundine and Tymianski, 2004).

In conclusion, despite the concurrence of diverse genetic and environmental factors for acute and chronic neurodegenerative disorders, such conditions possess a common denominator as to the ultimate mechanisms executing neuronal death, which are identical to those involved in normal brain development and all converge to mitochondrial dysfunction. On one hand, these findings testify the evolutionary importance of conserving fundamental processes. On the other hand, they call for further research to understand the basic mechanisms and the key regulators of neuronal fate, as novel opportunities for targeted therapeutic intervention may arise.

\section{Role of magnesium in neurodegenerative diseases}

As detailed above, there is increasing evidence that neuronal death by apoptosis is associated with both acute and chronic neurodegenerative disorders. Interestingly, brain free magnesium levels have been shown to decline in a number of such pathologies (Vink et al., 2009). As a consequence, a considerable research effort has been directed toward establishing the mechanisms of such decline and the potential for magnesium administration as a neuroprotective agent.

Brain magnesium decline is a ubiquitous feature of traumatic brain injury and is associated with the development of motor and cognitive deficits. Experimentally, parenteral administration of magnesium up to $12 \mathrm{~h}$ post-trauma restores brain magnesium homeostasis and profoundly improves both motor and cognitive outcome. Although the mechanism of action is unclear, magnesium has been shown to attenuate a variety of secondary injury factors, including brain edema, cerebral vasospasms, glutamate excitotoxicity, calcium-mediated events, lipid peroxidation, mitochondrial permeability transition, and apoptosis. Disappointingly, magnesium therapy has failed in clinical trials. Increase in brain free magnesium concentration seems to be essential to confer neuroprotection, and intravenous magnesium administration only marginally increases CSF magnesium concentration, which suggests that the integrity of the bloodbrain barrier and the regulation of magnesium in the cerebrospinal fluid are largely maintained following acute brain injury and limit magnesium bioavailability in the brain (Vink and Nimmo, 2009).

Magnesium therapy has also been described in the clinical stroke literature, with similar negative results, though magnesium was shown to be beneficial in a subgroup of patients with noncortical, or lacunar, strokes (Ginsberg, 2008). Magnesium may protect via multiple mechanisms, including NMDA receptor blockade, inhibition of excitatory neurotransmitter release, blockade of calcium channels, as well as vascular smooth muscle relaxation, and may be more bioavailable, as it is well known that the blood brain barrier around infarcted tissue is highly permeable, thus potentially facilitating local magnesium entry to the injured tissues.

Intriguingly, the cation channel TRPM7, which is crucial for $\mathrm{Mg}^{2+}$ homeostasis and cell survival (Schmitz et al., 2003), seems to be a critical 
mediator of anoxic cell death. It appears that TRPM7 gating lies downstream of NMDA receptor-mediated NO free-radical production. In this paradigm, excitotoxicity is likely the initiating signal in a cell death cascade that involves nNOS activation with subsequent peroxynitrite production, which in turn activates TRPM7 channels. Calcium influx through TRPM7 then creates a positive feedback loop of ROS production, which eventually kills the cell (Aarts and Tymianski, 2005). TRPM7 suppression made neurons resistant to ischemic death after brain ischemia and preserved neuronal morphology and function; also, it prevented ischemia-induced deficits in long-term potentiation and preserved performance in memory tasks (Sun et al., 2009). The role of the magnesium-specific channel TRPM6 in these processes is currently unclear, but, given its localization in the brain, its permeability to $\mathrm{Mg}^{2+}$ and its functional interaction with TRPM7, it may be involved in neuronal death as well (Cook et al., 2009). At present, the role of TRPM6/7 channels in magnesium transport seems less important than their facilitation of other cation fluxes, but it cannot be ruled out, especially considering the known involvement of magnesium in excitotoxicity and oxidative stress, as discussed earlier.

With respect to chronic brain degeneration, the first connection between magnesium and three apparently dissimilar neurodegenerative disorders (ALS, Parkinson's and Alzheimer's diseases) came from the observation that those conditions occurred at extraordinarily high rates in geographically separate foci among three genetically different, homogenous western Pacific populations (Durlach et al., 1997). Intensive research conducted over the years led to the identification of two candidate environmental factors: 1 ) severely low levels of $\mathrm{Ca}^{2+}$ and $\mathrm{Mg}^{2+}$ in the soil and drinking water coupled with abnormal mineral metabolism; and 2 ) the putative neurotoxin $\beta$-methylamino-Lalanine (L-BMAA), derived from the cycad plant, a traditional food source in Guam. Experimental findings supported this hypothesis: animals fed diets that mimic the mineral composition in the disease foci environment showed signs of neuronal damage; of the various combinations of $\mathrm{Ca}^{2+}$ and $\mathrm{Mg}^{2+}$ contents tested, exposure to low
$\mathrm{Mg}^{2+}$ (one-fifth of the normal level) was more deleterious, causing significant loss of dopaminergic neurons in the substantia nigra (Oyanagi et al., 2006). Furthermore, motor neurons are selectively more vulnerable to LBMAA toxicity, and these toxic effects are mediated through $\left[\mathrm{Ca}^{2+}\right]_{i}$ rises via $\mathrm{Ca}-\mathrm{A} / \mathrm{K}$ channels and other pathways, and subsequent reactive oxygen species generation (Rao et al., 2006).

In the hyperendemic foci in the Western Pacific, the unique mineral composition of the environment creates a condition of increased oxidative stress because low $\mathrm{Mg}^{2+}$ levels cause lipid peroxidation while transition metals act as redox catalysts causing increased ROS production. Therefore, the genes whose function is most likely to be affected are ion channels expressed in the CNS and modulated by oxidative stress. TRPM7 and TRPM2 fulfil these conditions; their functional properties are interconnected with calcium and magnesium homeostasis, oxidative stress, mitochondrial dysfunction, and immune mechanisms, all principal suspects in neurodegeneration (Hermosura and Garruto, 2007). Indeed, variants of both genes have been found in Guamanian neurodegenerative disorders and may contribute to their pathogenesis (Hermosura et al., 2008; Hermosura et al., 2005).

Despite the evidence implicating magnesium in the aetiology of neurodegenerative diseases, the therapeutic effects of magnesium supplementation are not well documented. Magnesium administration proved beneficial in an in vitro model of Parkinson's disease, where in particular magnesium concentration seems critical in the onset of the disease (Hashimoto et al., 2008), but there is still much to be done to fully appreciate the therapeutic potential of magnesium.

\section{Conclusion}

An extensive and up-to-date review of the literature shows that drawing a clear-cut conclusion on the role of magnesium in neuroapoptosis is not an easy task. Magnesium may play pleiotropic roles in different stages of 

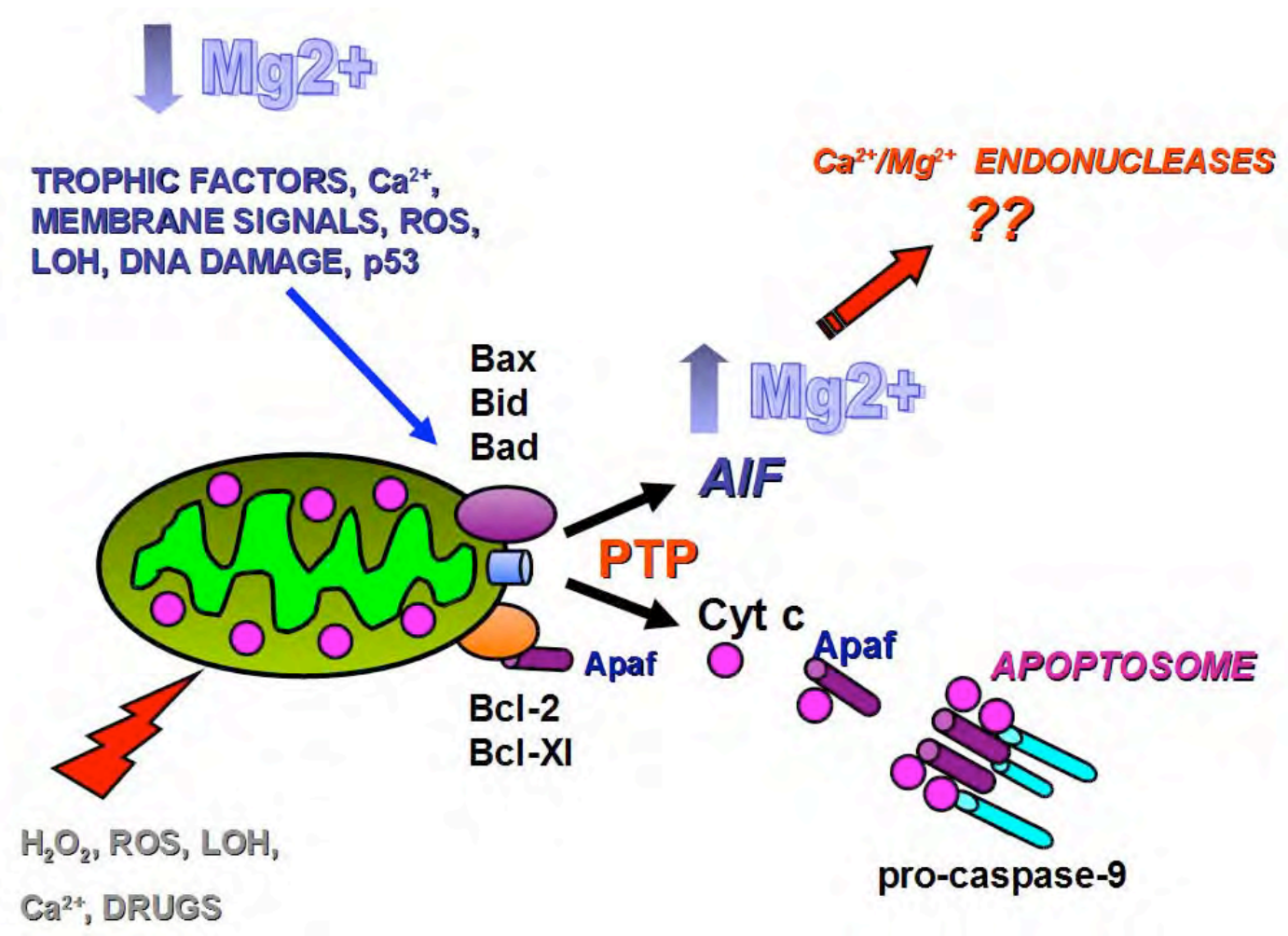

Figure 1. Potential points of interaction between magnesium and apoptotic factors.

the apoptotic process, that range from modulating growth factor- and $\mathrm{Ca}^{2+}$-dependent signal transduction to affecting oxidative stressrelated reactions. From this point of view, increased extracellular magnesium availability should in principle be considered antiapoptogenic. On the other hand, an increase in cytosolic free magnesium, which seems to be released from mitochondrial stores, appears to accompany the intrinsic apoptotic process. The pathophysiological meaning of this occurrence in the context of apoptosis induction/execution awaits to be defined (see Figure 1 for a summary).

In a more physiological basis, magnesium availability in the brain should be considered within a more complex and interconnected picture, where other conditions, such as developmental stage, inflammation, acute or chronic injuries, blood brain barrier integrity, and so on, may concur in determining a pro or antiapoptotic environment.

We believe that further investigation into the role of magnesium in the molecular mechanisms of apoptosis is warranted for a deeper understanding of the basic processes that regulate cell fate, and ultimately for a successful therapeutic intervention when these processes go awry.

\section{Acknowledgments}

Supported by the Italian Ministry of Education, University and Research PRIN 2007ZT39FN and Linea D1 2004-2009. 


\section{References}

Aarts MM, and Tymianski M (2005) TRPM7 and ischemic CNS injury. Neuroscientist 11:116-23.

Altura BM, Shah NC, Jiang XC, Li Z, Perez-Albela JL, Sica AC, Altura BT (2009) Short-term magnesium deficiency results in decreased levels of serum sphingomyelin, lipid peroxidation, and apoptosis in cardiovascular tissues. Am J Physiol Heart Circ Physiol 297:H86-92.

Arundine M, Tymianski M (2004) Molecular mechanisms of glutamate-dependent neurodegeneration in ischemia and traumatic brain injury. Cell Mol Life Sci 61:657-68.

Black S, Yu H, Lee J, Sachchithananthan M, Medcalf RL (2001) Physiologic concentrations of magnesium and placental apoptosis: prevention by antioxidants. Obstet Gynecol 98:319-24.

Butterfield DA, Bader Lange ML, Sultana R (2010) Involvements of the lipid peroxidation product, $\mathrm{HNE}$, in the pathogenesis and progression of Alzheimer's disease. Biochim Biophys Acta 1801(8):924-9.

Chen Y, Wei X, Yan P, Han Y, Sun S, Wu K, Fan D (2009) Human mitochondrial Mrs2 protein promotes multidrug resistance in gastric cancer cells by regulating $\mathrm{p} 27$, cyclin D1 expression and cytochrome C release. Cancer Biol Ther 8:607-14.

Chien MM, Zahradka KE, Newell MK, Freed JH (1999) Fas-induced $B$ cell apoptosis requires an increase in free cytosolic magnesium as an early event. J Biol Chem 274:7059-66.

Cook NL, van den Heuvel C, Vink R (2009) Are the transient receptor potential melastatin (TRPM) channels important in magnesium homeostasis following traumatic brain injury? Magnes Res 22:22534.

Cristofanilli M, Akopian A (2006) Calcium channel and glutamate receptor activities regulate actin organization in salamander retinal neurons. J Physiol 575:543-54

Dagda RK, Zaucha JA, Wadzinski BE, Strack S (2003) A developmentally regulated, neuron-specific splice variant of the variable subunit Bbeta targets protein phosphatase $2 \mathrm{~A}$ to mitochondria and modulates apoptosis. J Biol Chem 278:24976-85.

Damiano M, Galvan L, Déglon N, Brouillet E (2010) Mitochondria in Huntington's disease. Biochim Biophys Acta 1802:52-61.
Deshmukh M, Johnson EM (1997) Programmed cell death in neurons: focus on the pathway of nerve growth factor deprivation-induced death of sympathetic neurons. Mol Pharmacol 51:897-906.

Doyle LW, Crowther CA, Middleton P, Marret S, Rouse D (2009) Magnesium sulphate for women at risk of preterm birth for neuroprotection of the fetus. Cochrane Database Syst Rev (1):CD004661.

Durlach J, Bac P, Durlach V, Durlach A, Bara M, GuietBara A (1997) Are age-related neurodegenerative diseases linked with various types of magnesium depletion? Magnes Res 10:339-53.

Eskes R, Antonsson B, Osen-Sand A, Montessuit S, Richter C, Sadoul R, Mazzei G, Nichols A, Martinou JC (1998) Bax-induced cytochrome $C$ release from mitochondria is independent of the permeability transition pore but highly dependent on $\mathrm{Mg}^{2+}$ ions. J Cell Biol 143:217-24.

Fomin VP, Gibbs SG, Vanam R, Morimiya A, Hurd WW (2006) Effect of magnesium sulfate on contractile force and intracellular calcium concentration in pregnant human myometrium. Am J Obstet Gynecol 194:1384-90.

Gibson GE, Starkov A, Blass JP, Ratan RR, Beal MF (2010) Cause and consequence: mitochondrial dysfunction initiates and propagates neuronal dysfunction, neuronal death and behavioral abnormalities in age-associated neurodegenerative diseases. Biochim Biophys Acta 1802:122-34.

Ginsberg MD (2008) Neuroprotection for ischemic stroke: past, present and future. Neuropharmacology 55:363-89.

Gude NM, Stevenson JL, Moses EK, King RG (2000) Magnesium regulates hypoxia-stimulated apoptosis in the human placenta. Clin Sci (Lond) 98:375-80.

Guo Q, Fu W, Xie J, Luo H, Sells SF, Geddes JW, Bondada V, Rangnekar VM, Mattson MP (1998) Par-4 is a mediator of neuronal degeneration associated with the pathogenesis of Alzheimer disease. Nature Med 4:957-62.

Hamburger V, Levi-Montalcini R (1949) Proliferation, differentiation and degeneration in the spinal ganglia of the chick embryo under normal and experimental conditions. J Exp Zool 111:457-502. 
Hangen E, De Zio D, Bordi M, Zhu C, Dessen P, Caffin F, Lachkar S, Perfettini JL, Lazar V, Benard J, Fimia GM, Piacentini $M$, Harper $F$, Pierron $G$, Vicencio JM, Bénit $P$, de Andrade $A$, Höglinger $G$, Culmsee $C$, Rustin $P$, Blomgren K, Cecconi F, Kroemer G, Modjtahedi N (2010) A brain-specific isoform of mitochondrial apoptosis-inducing factor: AIF2. Cell Death Differ 17:1155-66.

Hardingham GE (2009) Coupling of the NMDA receptor to neuroprotective and neurodestructive events. Biochem Soc Trans 37(Pt 6):1147-60.

Hartwig A (2001) Role of magnesium in genomic stability. Mutat Res 475:113-21.

Hashimoto T, Nishi K, Nagasao J, Tsuji S, Oyanagi K (2008) Magnesium exerts both preventive and ameliorating effects in an in vitro rat Parkinson disease model involving 1-methyl-4-phenylpyridinium (MPP+) toxicity in dopaminergic neurons. Brain Res 1197:14351.

Hennigan A, O'Callaghan RM, Kelly AM (2007) Neurotrophins and their receptors: roles in plasticity, neurodegeneration and neuroprotection. Biochem Soc Trans 35(Pt 2):424-7.

Hermosura MC, Cui AM, Go RC, Davenport B, Shetler CM, Heizer JW, Schmitz C, Mocz G, Garruto RM, Perraud AL (2008) Altered functional properties of a TRPM2 variant in Guamanian ALS and PD. Proc Natl Acad Sci USA 105:18029-34.

Hermosura MC, Garruto RM (2007) TRPM7 and TRPM2-Candidate susceptibility genes for Western Pacific ALS and PD? Biochim Biophys Acta 1772:82235.

Hermosura MC, Nayakanti H, Dorovkov MV, Calderon FR, Ryazanov AG, Haymer DS, Garruto RM (2005) A TRPM7 variant shows altered sensitivity to magnesium that may contribute to the pathogenesis of two Guamanian neurodegenerative disorders. Proc Natl Acad Sci USA 102:11510-5

Hetman M, Kharebava G (2006) Survival signaling pathways activated by NMDA receptors. Curr Top Med Chem 6:787-99.

Ikonomidou C, Bosch F, Miksa M, Bittigau P, Vockler J, Dikranian K, Tenkova TI, Stefovska V, Turski L, Olney JW (1999) Blockade of NMDA receptors and apoptotic neurodegeneration in the developing brain. Science 283:70-74.

Johnston MV (2005) Excitotoxicity in perinatal brain injury. Brain Pathol 15:234-40.
Kim TH, Zhao Y, Barber MJ, Kuharsky DK, Yin XM (2000) Bid-induced cytochrome $c$ release is mediated by a pathway independent of mitochondrial permeability transition pore and Bax. J Biol Chem 275:39474-81.

Kolisek M, Zsurka G, Samaj J, Weghuber J, Schweyen RJ, Schweigel M (2003) Mrs2p is an essential component of the major electrophoretic $\mathrm{Mg}^{2+}$ influx system in mitochondria. EMBO J 22:1235-44.

Krueger RC Jr, Santore MT, Dawson G, Schwartz NB (2001) Increased extracellular magnesium modulates proliferation in fetal neural cells in culture. Brain Res Dev Brain Res 127:99-109.

Kubota T, Shindo Y, Tokuno K, Komatsu H, Ogawa H, Kudo S, Kitamura Y, Suzuki K, Oka K (2005) Mitochondria are intracellular magnesium stores: investigation by simultaneous fluorescent imagings in PC12 cells. Biochim Biophys Acta 1744:19-28.

Lewin GR, Barde YA (1996) Physiology of the neurotrophins. Annu Rev Neurosci 19:289-317.

Lipton SA (2006) Paradigm shift in neuroprotection by NMDA receptor blockade: memantine and beyond. Nat Rev Drug Discov 5:160-70.

Lipton SA, Rosenberg PA (1994) Excitatory amino acids as a final common pathway for neurologic disorders. $N$ Engl J Med 330:613-22.

Malpuech-Brugère $C$, Nowacki W, Gueux E, Kuryszko J, Rock E, Rayssiguier Y, Mazur A (1999) Accelerated thymus involution in magnesium-deficient rats is related to enhanced apoptosis and sensitivity to oxidative stress. Br J Nutr 81:405-11.

Martin DP, Schmidt RE, Di Stefano PS, Lowry OH, Carter JG, Johnson EM Jr (1988) Inhibitors of protein synthesis and RNA synthesis prevent neuronal death caused by nerve growth factor deprivation. J Cell Biol 106:829-844.

Martin H, Richert L, Berthelot A (2003) Magnesium deficiency induces apoptosis in primary cultures of rat hepatocytes. J Nutr 133:2505-11.

Martin $\mathrm{H}$, Uring-Lambert $\mathrm{B}$, Adrian $\mathrm{M}$, Lahlou A, Bonet $A$, Demougeot $C$, Devaux $S$, Laurant $P$, Richert $L$, Berthelot A (2008) Effects of long-term dietary intake of magnesium on oxidative stress, apoptosis and ageing in rat liver. Magnes Res 21:124-30.

Mattson MP (2000) Apoptosis in neurodegenerative disorders. Nat Rev Cell Mol Biol 1:120-9. 
Mattson MP, Camandola S (2000) NF-kB in neurodegenerative disorders. J Clin Invest 61:134-9.

Mattson MP, Duan W, Chan SL, Camandola S (1999) Par-4: an emerging pivotal player in neuronal apoptosis and neurodegenerative disorders. $\mathrm{J} \mathrm{Mol}$ Neurosci 13:17-30.

Mazur A, Maier JA, Rock E, Gueux E, Nowacki W, Rayssiguier $Y$ (2007) Magnesium and the inflammatory response: potential physiopathological implications. Arch Biochem Biophys 458:48-56.

Mittendorf R, Dammann O, Lee KS (2006) Brain lesions in newborns exposed to high-dose magnesium sulfate during preterm labor. J Perinatol 26:57-63.

Moreira PI, Carvalho C, Zhu X, Smith MA, Perry G (2010) Mitochondrial dysfunction is a trigger of Alzheimer's disease pathophysiology. Biochim Biophys Acta 1802:2-10.

Murphy E (2000) Mysteries of magnesium homeostasis. Circ Res 86:245-8.

Navarro A, Boveris A (2009) Brain mitochondrial dysfunction and oxidative damage in Parkinson's disease. J Bioenerg Biomembr 41:517-21.

Olney JW (1969) Brain lesions, obesity, and other disturbances in mice treated with monosodium glutamate. Science 164:719-721.

Oppenheim RW (1991) Cell death during development of the nervous system. Annu Rev Neurosci 14:453-501.

Oyanagi K, Kawakami E, Kikuchi-Horie K, Ohara K, Ogata K, Takahama S, Wada M, Kihira T, Yasui M (2006) Magnesium deficiency over generations in rats with special references to the pathogenesis of the Parkinsonism-dementia complex and amyotrophic lateral sclerosis of Guam. Neuropathology 26:115-28.

Patel T, Bronk SF, Gores GJ (1994) Increases of intracellular magnesium promote glycodeoxycholateinduced apoptosis in rat hepatocytes. J Clin Invest 94:2183-92.

Pelicci G, Troglio F, Bodini A, Melillo RM, Pettirossi V, Coda L, De Giuseppe A, Santoro M, Pelicci PG (2002) The neuron-specific Rai (ShcC) adaptor protein inhibits apoptosis by coupling Ret to the phosphatidylinositol 3-kinase/Akt signaling pathway. Mol Cell Biol 22:735163.

Rao SD, Banack SA, Cox PA, Weiss JH (2006) BMAA selectively injures motor neurons via AMPA/kainate receptor activation. Exp Neurol 201:244-52.
Rego AC, Oliveira CR (2003) Mitochondrial dysfunction and reactive oxygen species in excitotoxicity and apoptosis: implications for the pathogenesis of neurodegenerative diseases. Neurochem Res 28:156374.

Riedel G, Platt B, Micheau J (2003) Glutamate receptor function in learning and memory. Behav Brain Res 140:1-47.

Salvi M, Bozac A, Toninello A (2004) Gliotoxin induces $\mathrm{Mg}^{2+}$ efflux from intact brain mitochondria. Neurochem Int 45:759-64.

Schlingmann KP, Waldegger S, Konrad M, Chubanov V, Gudermann T (2007) TRPM6 and TRPM7--Gatekeepers of human magnesium metabolism. Biochim Biophys Acta 1772:813-21.

Schmitz C, Perraud AL, Johnson CO, Inabe K, Smith MK, Penner R, Kurosaki T, Fleig A, Scharenberg AM (2003)

Regulation of vertebrate cellular $\mathrm{Mg}^{2+}$ homeostasis by TRPM7. Cell 114:191-200.

Shindo Y, Fujimoto A, Hotta K, Suzuki K, Oka K (2010) Glutamate-induced calcium increase mediates magnesium release from mitochondria in rat hippocampal neurons. J Neurosci Res 88:3125-32.

Sultana R, Butterfield DA (2010) Role of oxidative stress in the progression of Alzheimer's disease. J Alzheimers Dis 19:341-53.

Sun HS, Jackson MF, Martin LJ, Jansen K, Teves L, Cui $\mathrm{H}$, Kiyonaka S, Mori Y, Jones M, Forder JP, Golde TE, Orser BA, Macdonald JF, Tymianski M (2009) Suppression of hippocampal TRPM7 protein prevents delayed neuronal death in brain ischemia. Nat Neurosci 12:1300-7.

Supnet C, Bezprozvanny I (2010) The dysregulation of intracellular calcium in Alzheimer disease. Cell Calcium 47:183-9.

Tait SW, Green DR (2010) Mitochondria and cell death: outer membrane permeabilization and beyond. Nat Rev Mol Cell Biol 11:621-32.

Tejero-Taldo MI, Chmielinska JJ, Weglicki WB (2007) Chronic dietary $\mathrm{Mg}^{2+}$ deficiency induces cardiac apoptosis in the rat heart. Magnes Res 20:208-12.

Vink R, Cook NL, van den Heuvel C (2009) Magnesium in acute and chronic brain injury: an update. Magnes Res 22:158S-62S.

Vink R, Nimmo AJ (2009) Multifunctional drugs for head injury. Neurotherapeutics 6:28-42. 
Vucic S, Kiernan MC (2009) Pathophysiology of neurodegeneration in familial amyotrophic lateral sclerosis. Curr Mol Med 9:255-72.

Wang Y, Qin ZH (2010) Molecular and cellular mechanisms of excitotoxic neuronal death. Apoptosis 15:1382-402.

Wolf Fl, Trapani V (2009) Multidrug resistance phenotypes and MRS2 mitochondrial magnesium channel: two players from one stemness? Cancer Biol Ther 8:615-7.

Yuan J, Yankner BA (2000) Apoptosis in the nervous system. Nature 407:802-9.
Zhang XY, Bai DC, Wu YJ, Li WG, Liu NF (2005)

Proanthocyanidin from grape seeds enhances antitumor effect of doxorubicin both in vitro and in vivo. Pharmazie 60:533-8.

Zhang XY, Li WG, Wu YJ, Bai DC, Liu NF (2005) Proanthocyanidin from grape seeds enhances doxorubicin-induced antitumor effect and reverses drug resistance in doxorubicin-resistant K562/DOX cells. Can J Physiol Pharmacol 83:309-18.

Zündorf G, Reiser G (2010) Calcium dysregulation and homeostasis of neural calcium in the molecular mechanisms of neurodegenerative diseases provide multiple targets for neuroprotection. Antioxid Redox Signa 14:1275-88. 


\title{
Brain free magnesium homeostasis as a target for reducing cognitive aging
}

\author{
Jean-Marie Billard
}

Université Paris Descartes, Faculté de Médecine René Descartes, Centre de Psychiatrie et Neurosciences, UMR 894, Paris, France. $\bowtie$ jean-marie.billard@inserm.fr

\begin{abstract}
In the general deterioration of physiological functions that takes place in aging, the prevalence of cognitive impairments, and particularly of those related to learning and memory, makes these deficits a major concern of public health. Although the exact nature of cellular and molecular substrates underlying learning and memory still remains an open issue for the neurobiologist, the current hypothesis assumes that it is determined by the capacity of brain neuronal networks to express short- and long-term changes in synaptic strength. Accordingly, the capacity of functional plasticity is impaired in the brain of aged memory-deficient animals. Short-term changes in synaptic transmission closely depend on transmitter release and neuronal excitability while long-term modifications are mainly related to the activation of the N-methyl-D-aspartate receptor (NMDA-R), a subtype of glutamate receptors. Because transmitter release, neuronal excitability and NMDA-R activation are modulated by magnesium $\left(\mathrm{Mg}^{2+}\right)$, a change in brain $\mathrm{Mg}^{2+}$ homeostasis could affect synaptic strength and plasticity in neuronal networks and consequently could alter memory capacities. In addition, alteration of brain $\mathrm{Mg}^{2+}$ levels could be regarded as a possible mechanism contributing to cognitive aging. According to these postulates, long-term increase in $\mathrm{Mg}^{2+}$ levels facilitates the conversion of synapses to a plastic state while learning and memory capacities are enhanced in adult animals fed with a diet enriched in $\mathrm{Mg}^{2+}$-L-threonate, a treatment that significantly elevates brain $\mathrm{Mg}^{2+}$ levels. Because $\mathrm{Mg}^{2+}$-L-threonate also improves learning and memory in aged animals, the regulation of brain $\mathrm{Mg}^{2+}$ homeostasis may therefore be regarded as a relevant target for the development of new pharmacological strategies aimed at minimizing cognitive aging.
\end{abstract}

\section{Introduction}

Alterations in brain anatomy and physiology are frequent features that gradually take place in the course of aging, to finally impair cognitive functions, such as learning and memory. In the last decades, extensive behavioural experiments performed in animal models of aging confirm that the efficient learning and memory in young individuals is slowed down with age, while forgetfulness is accelerated (Barnes and McNaughton, 1985; Gallagher and Rapp, 1997; Lanahan et al., 1997; Norris and Foster, 1999; Ward et al., 1999), and, like in humans, deficits concern several forms of memory, including spatial, associative and long-term memory (Clayton et al., 2002; Gruart et al., 2008; Houston et al., 1999; Rosenzweig and Barnes, 2003; Sykova et al., 2002; Winocur and Moscovitch, 1990; Zornetzer et al., 1982). Neuronal or synaptic loss is unlikely to significantly account for the senescent-associated cognitive deficits (Eriksen et al., 2009; Geinisman et al., 2004; Luebke et al., 2010; Morrison and Hof, 2007; Rapp and Gallagher, 1996) and a wealth of data now rather indicate that changes in functional properties within neuronal networks are mainly concerned (Billard 2006; Burke and Barnes, 2006; Craik and Bialystok, 2006; Disterhoft et al., 1994; Driscoll et al., 2003; Erickson and Barnes, 2003; Foster, 2007; Grady and Craik, 2000; Hsu et al., 2002; Kelly et al., 2006; Lister and Barnes, 2009; Magnusson, 1998; Mora et al., 2007; Sykov et al., 1998; Toescu and Verkhratsky, 2004). In particular, studies of long-lasting modifications of glutamatergic neurotransmission, such as longterm potentiation (LTP) and long-term depression (LTD) of synaptic strength, now considered as functional substrates of memory encoding (Bear and Malenka, 1994; Bliss, 1990; Collingridge and Bliss, 1995; Eichenbaum, 1996; Izquierdo, 1991; Kim and Linden, 2007; Lisman and Mclntyre, 
2001; Martin et al., 2000; Teyler and DiScenna, 1987), show substantial changes with age (Barnes, 2003; Billard, 2006; Burke and Barnes, 2006; Foster, 2006; Norris et al., 1996). Among the different mechanisms that may account for these age-associated impairments of synaptic plasticity (Foster, 2007; Rosenzweig and Barnes 2003), the activation of the N-methyl-D-aspartate subtype of glutamate receptors (NMDA-R) has received particular attention. Indeed, these receptors are pivotal for the regulation of synaptic strength, by means of their high permeability to calcium $\left(\mathrm{Ca}^{2+}\right)$, which triggers the activation of specific intracellular protein kinases and phosphatases (Wang et al., 1997). Although it is obvious that NMDA-R activation is impaired in aging (Barnes et al., 1997; Junjaud et al., 2006; Potier et al., 2000), all of the underlying mechanisms have yet to be definitely characterized and it remains to be determined to what extent they are involved in age-related deficits in synaptic plasticity and of memory capacities.

Among the different mechanisms possibly involved in cognitive aging is a change in ion homeostasis (Roberts, 1999) since transmitter release, cellular excitability and expression of synaptic plasticity closely depend on ion flux across neuronal membranes. Although $\mathrm{Ca}^{2+}$ regulation has initially gathered the largest interest in aging studies (Thibault et al., 2007; Toescu et al., 2004; Verkhratsky and Toescu, 1998), the role of magnesium $\left(\mathrm{Mg}^{2+}\right)$, which is found in a relative large concentration in the central nervous system (CNS) (Chutkow, 1974; Poenaru et al., 1997), is now much more considered. Indeed, aging is a risk factor for $\mathrm{Mg}^{2+}$ deficit (Wakimoto and Block, 2001) [for a review see (Durlach et al., 1998)] and brain $\mathrm{Mg}^{2+}$ levels are significantly reduced in ageassociated neurodegenerative diseases (Andrasi et al., 2000; Andrasi et al., 2005; Basun et al., 1991). On the other hand, the well-known regulation of NMDA-R activation by $\mathrm{Mg}^{2+}$ (Mayer and Westbrook, 1987; Nowak et al., 1984) and the fact that altered $\mathrm{Mg}^{2+}$ levels impair memory functions (Bardgett et al., 2005; Bardgett et al., 2007; Landfield and Morgan, 1984), strongly suggest that a change in $\mathrm{Mg}^{2+}$ homeostasis could contribute to the physiopathology of cognitive aging.

After reviewing the different roles of $\mathrm{Mg}^{2+}$ in the regulation of synaptic mechanisms at glutamat- ergic synapses, the present report will consider whether $\mathrm{Mg}^{2+}$ could be involved in deficits of these mechanisms that occur in the aging brain, and, finally, recent data will be presented suggesting $\mathrm{Mg}^{2+}$ as a relevant dietary component that could help in reducing age-associated memory impairments.

\section{The impact of brain $\mathrm{Mg}^{2+}$ levels on synaptic mechanisms contributing to cognitive functions}

Although $\mathrm{Mg}^{2+}$ is the second most abundant intracellular mineral after potassium and is present at large amount in the cerebrospinal fluid of both rodents $(0.8 \mathrm{mM}$ ) (Chutkow, 1974) and humans (1.0 to $1.2 \mathrm{mM}$ ) (Basun et al., 1991; Kapaki et al., 1989), its role on neural activity and synaptic plasticity has been much less considered compared to other divalent cations such as calcium $\left(\mathrm{Ca}^{2+}\right)$. This is rather surprising considering that $\mathrm{Mg}^{2+}$ is a cofactor for more than 300 enzymes and also tightly interacts with phospholipids and nucleic acids (Hofmann et al., 2000; Wolf and Cittadini, 2003), suggesting that the mineral should be able to modulate brain activity on a broad scale. Initially, the role of $\mathrm{Mg}^{2+}$ has mainly been evaluated in vitro by lowering extracellular levels $\left(\left[\mathrm{Mg}^{2+}\right]_{\mathrm{e}}\right)$, a procedure that increases spontaneous firing rate of neurons through membrane depolarization (Furukawa et al., 2009; Stone et al., 1992). This decrease in $\left[\mathrm{Mg}^{2+}\right]_{\mathrm{e}}$ can lead to paroxysmal events in slice preparations from both animals and humans, which resemble abnormal activities occurring during sustained seizures in vivo (Armand et al., 1998; Jones and Heinemann, 1988; Stanton et al., 1987). Using high performance liquid chromatography, quantification of basal efflux of amino acids indicates that only levels of glutamate, the neurotransmitter involved in most of excitatory synapses in the CNS, are significantly enhanced in low $\left[\mathrm{Mg}^{2+}\right]_{\mathrm{e}}$ medium (Furukawa et al., 2009; Smith et al., 1989). These data point out a first contribution of $\mathrm{Mg}^{2+}$ regarding the activity of excitatory synapses in the brain, that is, the regulation of the probability of transmitter release (Figure 1, left), as initially characterized at neuromuscular junctions (Kelly and Robbins, 1983; Kuno and Takahashi, 1986). But several lines of evidence show that beside this ubiquitous control on the probability of glutamate release at presynaptic terminals, $\mathrm{Mg}^{2+}$ is also able to influence 


\section{Effects of short-term elevation of external magnesium}

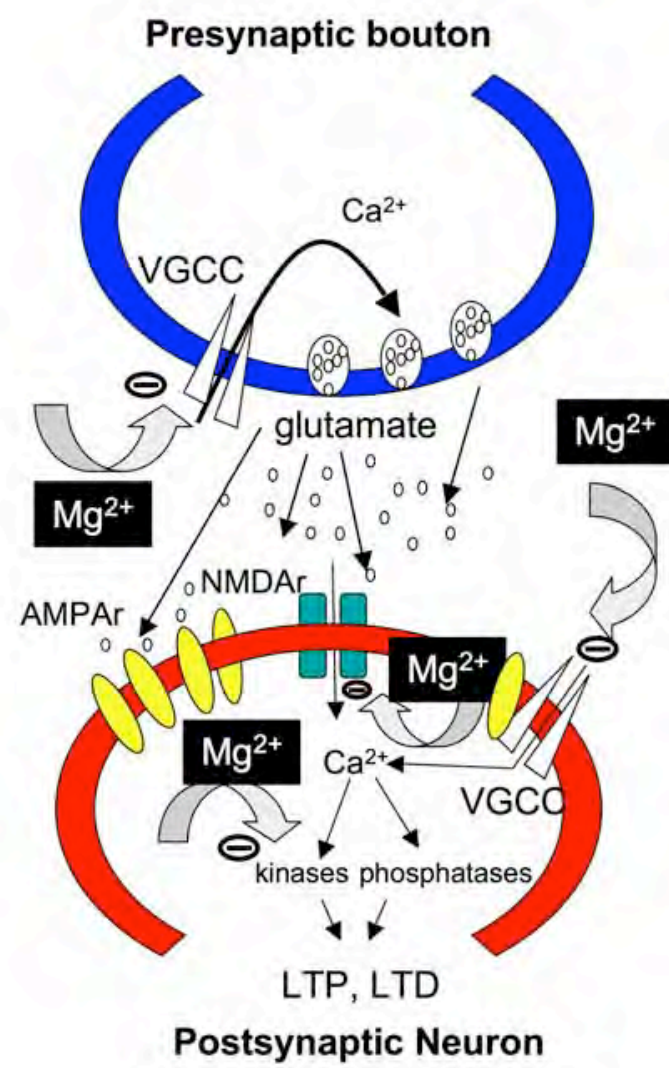

\section{Effects of long-term elevation of external magnesium}

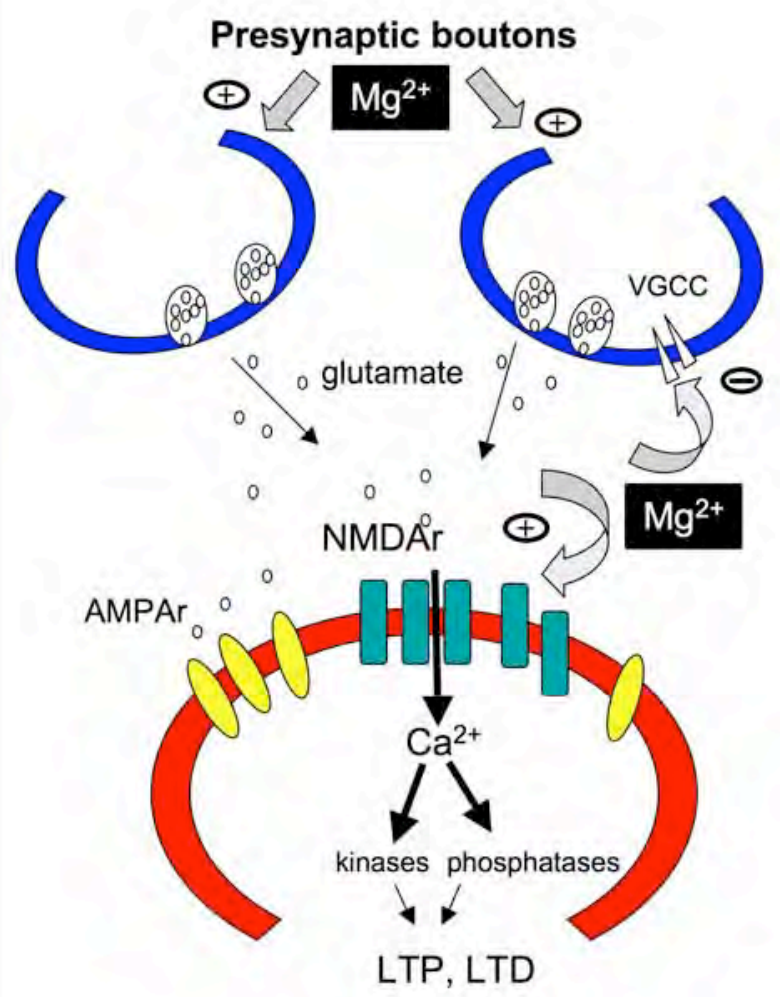

Postsynaptic Neuron

Figure 1. Schematic representation of the positive and negative effects of short-term (left) and long-term (right) elevation of brain $\mathrm{Mg}^{2}$ levels on neurotransmission and synaptic plasticity at an excitatory glutamatergic synapse. AMPAr: $\alpha$-amino-3-hydroxy-5-methyl-4-isoxazolepropionic acid receptor; NMDAr: N-methylD-aspartate receptor, VGCC: voltage-gated calcium channels.

synaptic activity by acting at postsynaptic level (Figure 1, left). For instance, frequency potentiation (FP), which represents an increase in synaptic strength rapidly developing during repetitive activation of glutamatergic afferent fibres (Anderson and Lomo, 1966; Andreasen and Lambert, 1998), is greater after elevating $\left[\mathrm{Mg}^{2+}\right]_{\mathrm{e}}$ (Landfield and Morgan, 1984). This is mainly due to the ability of $\mathrm{Mg}^{2+}$ to reduce the calciumdependent post-burst after hyper-polarization (AHP) of membrane potential, which is normally induced in depolarized neurons to limit excessive firing (Hotson and Prince, 1980; Lorenzon and Foehring, 1992; Madison and Nicoll, 1984). AHPs are reversibly reduced by the acquisition of learning-dependent behavioural tasks such as trace eye blink conditioning or spatial water maze (Moyer et al., 2000; Thompson et al., 1996). Thus, by controlling the initial postsynaptic depolarization through the regulation of AHP amplitude and duration, $\mathrm{Mg}^{2+}$ is able to modulate synaptic strength and to alter cognitive abilities (Disterhoft and Oh, 2006).

Although there is no doubt that $\mathrm{Mg}^{2+}$ may be considered as firmly involved in the regulation of synaptic activity through the pre- and postsynaptic mechanisms described above, an additional and essential role for the mineral has emerged at the end of the 1980s from electrophysiological studies demonstrating that $\mathrm{Mg}^{2+}$ inhibits currents through channels associated with the N-methyl-D-aspartate subtype of glutamate receptors (NMDA-R) by directly blocking the ion pore (Bekkers and Stevens, 1993; Jahr and Stevens, 1990; Mayer and Westbrook, 1987; Nowak et al., 1984). A transient rise in $\left[\mathrm{Mg}^{2+}\right]_{\mathrm{e}}$ within physiological range, i.e. from 0.8 to 1.2 
$\mathrm{mM}$, rapidly reduces the current amplitude mediated by NMDA-R by more than $60 \%$ in cultured neurons clamped at potentials below -50 $\mathrm{mV}$ while further increase in $\left[\mathrm{Mg}^{2+}\right]_{\mathrm{e}}$ is significantly less effective (Slutsky et al., 2004). Importantly, this depression effect does not occur at depolarized potentials, revealing a voltage dependency for the block of NMDA-R by $\mathrm{Mg}^{2+}$, which underlies the pivotal role of NMDA-R in the induction of synaptic plasticity such as long-term potentiation (LTP) and long-term depression (LTD). Indeed, following high-frequency activation of presynaptic terminals, the glutamate-induced depolarization of membrane potential releases NMDA-R from $\mathrm{Mg}^{2+}$ block allowing $\mathrm{Ca}^{2+}$ to enter the cells (Figure 1, left). As a consequence, activation of specific kinases or phosphatases is triggered, thus modifying synaptic strength (Wang et al., 1997). Several lines of evidence show effects of transient changes in $\mathrm{Mg}^{2+}$ levels on the induction of synaptic plasticity. For instance, a rapid rise in $\left[\mathrm{Mg}^{2+}\right]_{\mathrm{e}}$ selectively antagonizes LTP by reducing the depolarizationinduced $\mathrm{Ca}^{2+}$ influx (Dunwiddie and Lynch, 1979; Malenka et al., 1992; Malenka and Nicoll, 1993). Interestedly, LTP is also suppressed in slices bathed with a $\mathrm{Mg}^{2+}$-free medium (Frankiewicz and Parsons, 1999; Jouvenceau et al., 2002), a loss that is independent of NMDA-R activation but rather due to changes in signalling cascades in post-synaptic neurons that remain to be characterized (Hsu et al., 2000). At intracellular level, $\mathrm{Mg}^{2+}$ also regulates the activity of $\mathrm{Ca}^{2+}$ dependent protein kinases governing NMDAdependent LTP. For instance, it controls the subcellular localization of protein kinase C (PKC), which closely determines the function of the enzyme (Tanimura et al., 2002) and stimulates the dephosphorylation and deactivation of Calmodulin Kinase II (CaMKII) (Easom et al., 1998).

Despite much of data underlining that a transient increase in $\mathrm{Mg}^{2+}$ levels unables synapses to remain highly plastic, an unexpected opposite result has been reported in vitro after studying the effects of long-term elevation of the mineral (Figure 1, right). Indeed, when $\left[\mathrm{Mg}^{2+}\right]_{\mathrm{e}}$ is increased within physiological range in neuronal cultures for more than several hours, NMDA-R-mediated currents are enhanced and the expression of LTP significantly facilitated (Slutsky et al., 2004). In the same conditions, synaptic strength is not modified following a single action potential but increased after a burst of inputs. These long-term facilitation effects of $\mathrm{Mg}^{2+}$ on neurotransmission and synaptic plasticity also occur in vivo since they are found in young rats fed with a diet enriched in $\mathrm{Mg}^{2+}$-L-threonate $\left(\mathrm{Mg}^{2+}-\mathrm{T}\right)$, a new highly bioavailable compound that enhances loading of $\mathrm{Mg}^{2+}$ into the brain (Slutsky et al., 2010). The increase in the density of functional presynaptic boutons (Slutsky et al., 2010) coupled with the weaker glutamate release at synapses found after chronic $\mathrm{Mg}^{2+}$ elevation (Slutsky et al., 2004) indicate that long-lasting changes in $\mathrm{Mg}^{2+}$ homeostasis are able to modify the pattern of synaptic assemblies within neuronal networks, from a limited number of synapses with high probability of release to a larger density with low probability of release (Figure 1, right). In addition, both the increase in $\left[\mathrm{Mg}^{2+}\right]_{\mathrm{e}}$ in vitro and the elevation of brain $\mathrm{Mg}^{2+}$ in vivo up-regulate the expression of NR2B-containing NMDA-R (Slutsky et al., 2004; Slutsky et al., 2010). This increase, proposed to counterbalance the higher blockade of NMDA-R opening associated with chronic elevation of $\left[\mathrm{Mg}^{2+}\right]_{\mathrm{e}}$, contributes to the greater capacity of synapses to be highly plastic.

Because the pattern and strength of synaptic transmission are widely believed to code memory traces (Bear and Malenka, 1994; Bliss, 1990; Collingridge and Bliss, 1995; Eichenbaum, 1996; Izquierdo, 1991; Kim and Linden, 2007; Lisman and McIntyre, 2001; Martin et al., 2000; Neves et al., 2008; Teyler and DiScenna, 1987), their susceptibility to short- and long-term changes in $\mathrm{Mg}^{2+}$ homeostasis described above predict that cognitive abilities would also be modulated by altering $\mathrm{Mg}^{2+}$ levels. Accordingly, $\mathrm{Mg}^{2+}$ deficiency impairs fear-conditioning (Bardgett et al., 2005; Bardgett et al., 2007) while chronically elevating plasma $\mathrm{Mg}^{2+}$ over several days improves reversal learning in the hippocampus-dependent T-maze task (Landfield and Morgan, 1984). However, whether brain $\mathrm{Mg}^{2+}$ levels are really altered in these studies has been questioned considering that $\mathrm{Mg}^{2+}$ loading into the brain is tightly regulated by active transport processes that maintain a concentration gradient between the cerebrospinal fluid (CSF) and the plasma. In fact, $\mathrm{Mg}^{2+}$ levels are poorly affected in the brain after long-lasting increase in plasma $\mathrm{Mg}^{2+}$ induced by intravenous injection of $\mathrm{MgSO}_{4}$ both in animals and humans (Kim et al., 1996; McKee et al., 2005). 
Nevertheless, this rigorous control of brain $\mathrm{Mg}^{2+}$ levels has recently been overcome using the highly bioavailable compound $\mathrm{Mg}^{2+}-\mathrm{T}$, which increases $\mathrm{Mg}^{2+}$ concentrations in CSF by at least $15 \%$ (Slutsky et al., 2010). Behavioural characterization of the $\mathrm{Mg}^{2+}$-T-treated rats indicates significant improvements of learning abilities, working memory as well as short- and long-term memory compared to control animals (Slutsky et al., 2010), confirming that even moderate, changes in brain $\mathrm{Mg}^{2+}$ homeostasis are capable of altering cognitive performances.

\section{The impact of brain $\mathrm{Mg}^{2+}$ levels on impaired synaptic mechanisms underlying cognitive aging}

Although a link between $\mathrm{Mg}^{2+}$ deficiency and cellular senescence has been proposed for the agerelated deterioration of a large range of physiological functions (Killilea and Maier, 2008), a causal effect on deficits of cognitive functions is still questioned. Even aging is thought of as a general risk factor for $\mathrm{Mg}^{2+}$ deficit (Durlach et al., 1993; Wakimoto and Block, 2001). In fact, $\mathrm{Mg}^{2+}$ concentrations determined in various brain structures only slightly decrease in healthy aging (Morita et al., 2001; Takahashi et al., 2001). However, since even a very small disturbance of $\left[\mathrm{Mg}^{2+}\right]_{\mathrm{e}}$ is able to substantially modify synaptic assemblies supporting cognitive performances as reported above, it may be postulated that agerelated changes in $\mathrm{Mg}^{2+}$ levels, even of very weak amplitude, could contribute to the physiopathology of cognitive aging.

Regarding the regulation of transmitter release at presynaptic terminals, the increase in basal release of glutamate determined in the brain of aged rats and monkeys in vivo (Massieu and Tapia, 1997; Quintero et al., 2007) suggests a possible role for $\mathrm{Mg}^{2+}$. According to this postulate, spontaneous miniature end-plate potentials increase in amplitude at neuromuscular junctions in aging, that has been shown to reflect an impaired regulation of transmission release by $\mathrm{Mg}^{2+}$ (Kelly and Robbins, 1983). Nevertheless, whether a similar effect of $\mathrm{Mg}^{2+}$ also occurs at central synapses still remains to be demonstrated.

Age-related decrease in neuronal excitability is well documented, indicating that the amplitude and duration of AHPs are enhanced with age
(Disterhoft and Oh, 2006; Disterhoft and Oh, 2007; Power et al., 2002; Thibault et al., 2007). AHP amplitude inversely correlates with both acquisition and probe performance in learning behaviours among aged animals (Tombaugh et al., 2005) and pharmacological treatments that rescue the age-related alteration of AHPs, also minimize memory deficits (Oh et al., 1999; Weiss et al., 2000). These data strongly suggest that a decrease in neuronal excitability is a potent mechanism contributing to the physiopathology of cognitive aging (Disterhoft and Oh, 2006). Although the involvement of $\mathrm{Mg}^{2+}$ in this functional deficit has not yet been formally demonstrated, some indirect experimental data suggest that this is probably the case. Frequency potentiation, which is negatively correlated to AHP magnitude (Thibault et al., 2001), is reduced by age (Diana et al., 1994; Landfield and Lynch, 1977; Landfield et al., 1986; Thibault et al., 2001) and this alteration is prevented by elevating $\left[\mathrm{Mg}^{2+}\right]_{\mathrm{e}}$ (Landfield and Morgan, 1984; Landfield et al., 1986). From these results, it may be hypothesized that the age-related facilitation of $\mathrm{Ca}^{2+}$ conductances supporting the increase in AHPs is not only due to a greater density of $\mathrm{Ca}^{2+}$ channels on neuronal membranes (Campbell et al., 1996; Thibault and Landfield, 1996; Thibault et al., 2001; Veng et al., 2003) but also to some extent, to a weaker competition between cations following $\mathrm{Mg}^{2+}$ depletion.

Regarding the expression of long-lasting synaptic plasticity, extensive electrophysiological studies report age-related deficits of both LTP and LTD in aged memory-impaired animals that reflects a shift in $\mathrm{Ca}^{2+}$ sources, with a weaker role for NMDA-R, and an increased contribution of voltagegated $\mathrm{Ca}^{2+}$ channels and intracellular stores with different kinetic properties (Foster and Kumar, 2002; Gant et al., 2006; Junjaud et al., 2006; Kumar and Foster, 2005; Shankar et al., 1998; Thibault et al., 2007). NMDA-R activation is impaired in aged animals (Barnes et al., 1997; Burke and Barnes, 2010; Clayton et al., 2002; Eckles-Smith et al., 2000; Fontan-Lozano et al., 2007; Kollen et al., 2010; Magnusson, 1998; Ontl et al., 2004; Potier et al., 2000), not because of a reduced receptor density but rather to changes in pharmacological properties of the glutamatergic receptor (Billard and Rouaud, 2007; Junjaud et al., 2006; Kollen et al., 2010; Kuehl-Kovarik et al., 2003; Mothet et al., 2006; Turpin et al., 2009). 
Among the possible mechanisms affecting NMDA-R activation in aging, a role for $\mathrm{Mg}^{2+}$ has been evaluated. No significant alteration of NMDA-R susceptibility to $\mathrm{Mg}^{2+}$ block occurs during aging since the percent decrease in NMDA-Rmediated synaptic potentials is comparable in young and aged animals after transient $\left[\mathrm{Mg}^{2+}\right]_{\mathrm{e}}$ elevation (Barnes et al., 1997; Potier et al., 2000). Also altering $\left[\mathrm{Mg}^{2+}\right]_{\mathrm{e}}$ affects NMDA-R-dependent LTP as well as short-term potentiation (STP) in a similar way in the two groups of animals indicating that a change in $\mathrm{Mg}^{2+}$ block of NMDA- $\mathrm{R}$ is unlikely to contribute to the age-related impairment of synaptic plasticity (Potier et al., 2000). However, a role for $\mathrm{Mg}^{2+}$ in LTP deficits occurring in aged animals does not definitively be discarded. For instance, a weaker activation of NMDA-R by its co-agonist $D$-serine has been shown to underlie LTP impairment in aged rodents, that is due to a weaker production of the amino acid by its synthesizing enzyme serine racemase (SR) (Junjaud et al., 2006; Mothet et al., 2006; Potier et al., 2010; Turpin et al., 2009). Because SR activity is potently stimulated by $\mathrm{Mg}^{2+}$, which increases 5- to 10-fold the rate of racemization of $L-$ to $D$-serine, an impaired activation of SR by $\mathrm{Mg}^{2+}$ may therefore be possibly regarded as a potent mechanism linking lower brain $\mathrm{Mg}^{2+}$ levels to deficits of NMDA-R activation and related synaptic plasticity in aging.

Regarding the effects of long-lasting elevation of $\left[\mathrm{Mg}^{2+}\right]_{\mathrm{e}}$, functional improvements also occur at synapses of the aging brain (Slutsky et al., 2010). The density of synaptophysin- and synaptobrevinimmunostained puncta is decreased in the hippocampal dentate gyrus of aged animals whereas synaptic loss in the stratum radiatum of CA1 subfield is less pronounced and even remains controversial (Burke and Barnes, 2006; Geinisman et al., 2004; Smith et al., 2000). However, in aged $\mathrm{Mg}^{2+}-\mathrm{T}$ treated rats, the number of functional synaptic connections is significantly increased in both hippocampal areas compared to aged controls (Slutsky et al., 2010). As in young animals, a treatment with $\mathrm{Mg}^{2+}-\mathrm{T}$ also up-regulates NR2Bcontaining NMDA-R, thus improving LTP expression in slices from aged animals (Slutsky et al., 2010). Interestingly, both the increase in functional synaptic connections and the facilitated induction of synaptic plasticity (Figure 2A) (see (Slutsky et al., 2010)). In addition, it is worth noting in these aged treated animals that
A

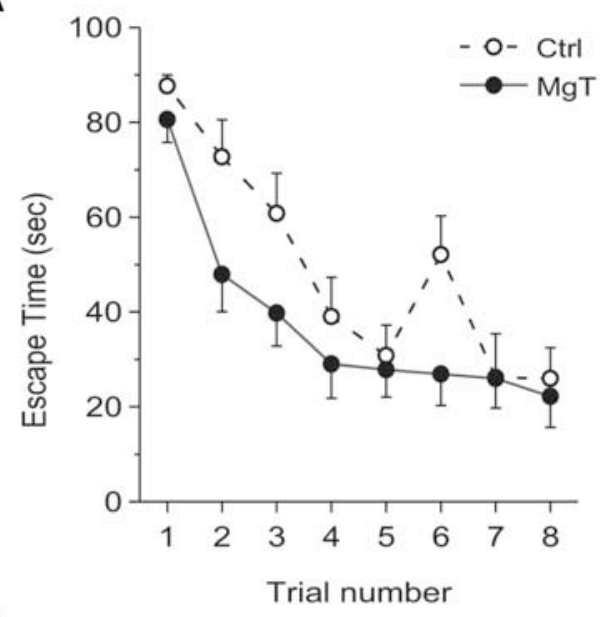

B

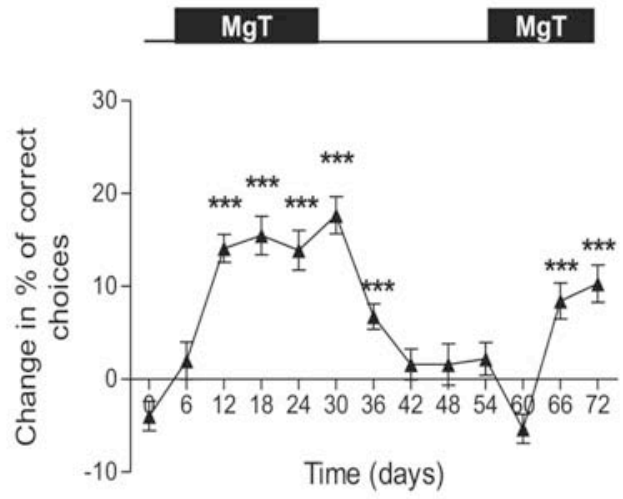

Figure 2. Elevation of brain $\mathrm{Mg}^{2}$ levels by $\mathrm{Mg}^{2+}-\mathrm{T}$ improves memory in aged rats. (A). $\mathrm{Mg}^{2+}-\mathrm{T}$ aged treated rats show shorter escape time in the water maze task indicating faster learning. (B). Time course of the reversible benefit effect of $\mathrm{Mg}^{2+}-\mathrm{T}$ treatment on spatial working memory in aged rats. Modified from (Slutsky et al., 2010).

the control level after the end of $\mathrm{Mg}^{2+}-\mathrm{T}$ supplementation and the time course of this decrease matches that of the reinstallation of impairment in memory scores (Figure 2B).

\section{Other possible routes involving brain $\mathrm{Mg}^{2+}$ in cognitive aging}

The basal forebrain cholinergic complex including the medial septum/diagonal band, the substantia innominata, and the nucleus basalis of Meynert of Broca, represents a second major excitatory pathway of the CNS (Dutar et al., 1995; McKinney et al., 1983; Mesulam et al., 1983; Mesulam et al., 1984; Woolf et al., 1984). During the past fifty years, many experiments were directed at testing 
whether this cholinergic pathway is involved in aspects of cognition and a significant role in learning and memory has been firmly debated (see (McKinney and Jacksonville, 2005) for a review). Electrically-induced release of acetylcholine generates slow and long-lasting excitatory postsynaptic potentials involving the activation of muscarinic receptors (Dutar and Nicoll, 1988; Misgeld et al., 1989; Muller and Misgeld, 1986). These responses are impaired in aging (Lippa et al., 1985; Potier et al., 1993; Segal, 1982; Shen and Barnes, 1996; Taylor and Griffith, 1993), that reflects a decreased transmitter release from cholinergic terminals (Aubert et al., 1995; Birthelmer et al., 2003; Scali et al., 1994; Takei et al., 1989; Vannucchi et al., 1997) but also a weaker activation of postsynaptic receptors (Calhoun et al., 2004; Lippa et al., 1985; Potier et al., 1992; Potier et al., 1993; Segal, 1982; Vannucchi et al., 1997). However, it is worth noting that the degree of memory deficit does not appear to closely correlate with changes in cholinergic synaptic activity (Calhoun et al., 2004; Potier et al., 1993; Shen and Barnes, 1996), suggesting that acetylcholine should not play a critical role in cognitive aging as initially proposed (Bartus et al., 1982; Bartus et al., 1985; Pepeu, 1988).

In addition to reducing acetylcholine release (Jope, 1981; Vickroy and Schneider, 1991), $\mathrm{Mg}^{2+}$ also acts on postsynaptic muscarinic receptors through several distinct mechanisms. First, $\mathrm{Mg}^{2+}$ is able to bind to the allosteric region of the $\mathrm{M} 2$ subtype of receptors, decreasing the inhibitory effects of some modulators on the dissociation of orthosteric ligands (Burgmer et al., 1998). Second, $\mathrm{Mg}^{2+}$ reduces a non-selective cationic conductance activated by muscarinic agonists

\section{References}

Anderson P, Lomo T (1966) Mode of activation of hippocampal pyramidal cells by excitatory synapses on dendrites. Exp Brain Res 2:247-60.

Andrasi E, Igaz S, Molnar Z, Mako S (2000) Disturbances of magnesium concentrations in various brain areas in Alzheimer's disease. Magnes Res 13:189-96.
(Guerineau et al., 1995), and, finally, the cation is necessary for activating G-proteins coupled to muscarinic receptors (Cladman and Chidiac, 2002; Shiozaki and Haga, 1992; Zhang et al., 2004). Regarding these multiple interactions, it is obvious that changes in $\mathrm{Mg}^{2+}$ levels are likely to contribute to the alteration of cholinergic neurotransmission that occurs in aging. Although direct evidence is still lacking, several data of the literature argue for the possibility that changes in brain $\mathrm{Mg}^{2+}$ may contribute to the age-associated impairment of acetylcholine-dependent synaptic activity. For instance, hypomagnesia significantly weakens responses of cortical neurons to iontophoretically applied acetylcholine (El-Beheiry and Puil, 1990) while the high-affinity binding at the muscarinic receptor in Alzheimer's disease is closely regulated by $\mathrm{Mg}^{2+}$ (Ladner and Lee, 1999).

\section{Conclusion}

Although it is obvious that additional studies are necessary to unravel the mechanisms connecting brain $\mathrm{Mg}^{2+}$ homeostasis and cognitive aging, experimental data has progressively accumulated showing that even moderate changes in the concentration of the mineral are able to significantly affect the assembly and functionality of neuronal networks involved in cognition. Recently, the World Health Organization reached consensus that in a majority of the world's population, the dietary $\mathrm{Mg}^{2+}$ intake is lower than recommended, especially in the aging population (see also (Ford and Mokdad, 2003; Galan, 1997). Based on the results summarized in the present review, there is no doubt that elevating $\mathrm{Mg}^{2+}$ levels in the brain of the elderly could represent a promising strategy to minimize or even prevent cognitive deficits that take place with age.

Andrasi E, Pali N, Molnar Z, Kosel S (2005) Brain aluminum, magnesium and phosphorus contents of control and Alzheimer-diseased patients. J Alzheimers Dis 7:273-84.

Andreasen M, Lambert JD (1998) Factors determining the efficacy of distal excitatory synapses in rat hippocampal CA1 pyramidal neurones. J Physiol 507 (Pt 2):441-62. 
Armand V, Louvel J, Pumain R, Heinemann U (1998) Effects of new valproate derivatives on epileptiform discharges induced by pentylenetetrazole or low $\mathrm{Mg} 2+$ in rat entorhinal cortex-hippocampus slices. Epilepsy Res 32:345-55.

Aubert I, Rowe W, Meaney MJ, Gauthier S, Quirion R (1995) Cholinergic markers in aged cognitively impaired Long-Evans rats. Neuroscience 67:277-92.

Bardgett ME, Schultheis PJ, McGill DL, Richmond RE, Wagge JR (2005) Magnesium deficiency impairs fear conditioning in mice. Brain Res 1038:100-6.

Bardgett ME, Schultheis PJ, Muzny A, Riddle MD, Wagge JR (2007) Magnesium deficiency reduces fearinduced conditional lick suppression in mice. Magnes Res 20:58-65.

Barnes CA, McNaughton BL (1985) An age comparison of the rates of acquisition and forgetting of spatial information in relation to long-term enhancement of hippocampal synapses. Behav Neurosci 99:1040-8.

Barnes CA, Rao G, Shen J (1997) Age-related decrease in the $\mathrm{N}$-methyl-D-aspartateR-mediated excitatory postsynaptic potential in hippocampal region CA1. Neurobiol Aging 18:445-52.

Barnes CA (2003) Long-term potentiation and the ageing brain. Philos Trans $R$ Soc Lond B Biol Sci 358:765-72.

Bartus RT, Dean RL, 3rd, Beer B, Lippa AS (1982) The cholinergic hypothesis of geriatric memory dysfunction. Science 217:408-14.

Bartus RT, Dean RL, Pontecorvo MJ, Flicker C (1985) The cholinergic hypothesis: a historical overview, current perspective, and future directions. Ann $N Y$ Acad Sci 444:332-58.

Basun H, Forssell LG, Wetterberg L, Winblad B (1991) Metals and trace elements in plasma and cerebrospinal fluid in normal aging and Alzheimer's disease. J Neural Transm Park Dis Dement Sect 3:23158.

Bear MF, Malenka RC (1994) Synaptic plasticity: LTP and LTD. Curr Opin Neurobiol 4:389-99.

Bekkers JM, Stevens CF (1993) NMDA receptors at excitatory synapses in the hippocampus: test of a theory of magnesium block. Neurosci Lett 156:73-7.
Billard JM, Rouaud E (2007) Deficit of NMDA receptor activation in CA1 hippocampal area of aged rats is rescued by D-cycloserine. Eur J Neurosci 25:2260-8.

Birthelmer A, Stemmelin J, Jackisch R, Cassel JC (2003) Presynaptic modulation of acetylcholine, noradrenaline, and serotonin release in the hippocampus of aged rats with various levels of memory impairments. Brain Res Bull 60:283-96.

Bliss TV (1990) Long-term potentiation. Science 249:973.

Burgmer U, Schulz U, Trankle C, Mohr K (1998) Interaction of $\mathrm{Mg} 2+$ with the allosteric site of muscarinic M2 receptors. Naunyn Schmiedebergs Arch Pharmacol 357:363-70.

Burke SN, Barnes CA (2006) Neural plasticity in the ageing brain. Nat Rev Neurosci 7:30-40.

Burke SN, Barnes CA (2010) Senescent synapses and hippocampal circuit dynamics. Trends Neurosci 33:153-61.

Calhoun ME, Mao Y, Roberts JA, Rapp PR (2004) Reduction in hippocampal cholinergic innervation is unrelated to recognition memory impairment in aged rhesus monkeys. J Comp Neurol 475:238-46.

Campbell LW, Hao SY, Thibault O, Blalock EM, Landfield PW (1996) Aging changes in voltage-gated calcium currents in hippocampal CA1 neurons. $J$ Neurosci 16:6286-95.

Chutkow JG (1974) Metabolism of magnesium in central nervous system. Relationship between concentrations of magnesium in cerebrospinal fluid and brain in magnesium deficiency. Neurology 24:7807.

Cladman W, Chidiac P (2002) Characterization and comparison of RGS2 and RGS4 as GTPase-activating proteins for $\mathrm{m} 2$ muscarinic receptor-stimulated $\mathrm{G}(\mathrm{i})$. Mol Pharmacol 62:654-9.

Clayton DA, Mesches MH, Alvarez E, Bickford PC, Browning MD (2002) A hippocampal NR2B deficit can mimic age-related changes in long-term potentiation and spatial learning in the Fischer 344 rat. $J$ Neurosci 22:3628-37.

Collingridge GL, Bliss TV (1995) Memories of NMDA receptors and LTP. Trends Neurosci 18:54-6. 
Craik FI, Bialystok E (2006) Cognition through the lifespan: mechanisms of change. Trends Cogn Sci 10:131-8.

Diana G, Scotti de Carolis A, Frank C, Domenici MR, Sagratella S (1994) Selective reduction of hippocampal dentate frequency potentiation in aged rats with impaired place learning. Brain Res Bull 35:107-11.

Disterhoft JF, Moyer JR, Jr., Thompson LT (1994) The calcium rationale in aging and Alzheimer's disease. Evidence from an animal model of normal aging. Ann N Y Acad Sci 747:382-406.

Disterhoft JF, Oh MM (2006) Learning, aging and intrinsic neuronal plasticity. Trends Neurosci 29:58799.

Disterhoft JF, Oh MM (2007) Alterations in intrinsic neuronal excitability during normal aging. Aging Cell 6:327-36.

Driscoll I, Hamilton DA, Petropoulos H, Yeo RA, Brooks WM, Baumgartner RN, Sutherland RJ (2003) The aging hippocampus: cognitive, biochemical and structural findings. Cereb Cortex 13:1344-51.

Dunwiddie TV, Lynch G (1979) The relationship between extracellular calcium concentrations and the induction of hippocampal long-term potentiation. Brain Res 169:103-10.

Durlach J, Durlach V, Bac P, Rayssiguier Y, Bara M, Guiet-Bara A (1993) Magnesium and ageing. II. Clinical data: aetiological mechanisms and pathophysiological consequences of magnesium deficit in the elderly. Magnes Res 6:379-94.

Durlach J, Bac P, Durlach V, Rayssiguier Y, Bara M, Guiet-Bara A (1998) Magnesium status and ageing: an update. Magnes Res 11:25-42.

Dutar P, Nicoll RA (1988) Classification of muscarinic responses in hippocampus in terms of receptor subtypes and second-messenger systems: electrophysiological studies in vitro. J Neurosci 8:4214-24.

Dutar P, Bassant MH, Senut MC, Lamour Y (1995) The septohippocampal pathway: structure and function of a central cholinergic system. Physiol Rev 75:393-427.

Easom RA, Tarpley JL, Filler NR, Bhatt H (1998) Dephosphorylation and deactivation of $\mathrm{Ca} 2+$ / calmodulin-dependent protein kinase II in betaTC3cells is mediated by $\mathrm{Mg} 2+-$ and okadaic-acid-sensitive protein phosphatases. Biochem J 329 ( Pt 2):283-8.
Eckles-Smith K, Clayton D, Bickford P, Browning MD (2000) Caloric restriction prevents age-related deficits in LTP and in NMDA receptor expression. Brain Res Mol Brain Res 78:154-62.

Eichenbaum H (1996) Learning from LTP: a comment on recent attempts to identify cellular and molecular mechanisms of memory. Learn Mem 3:61-73.

el-Beheiry H, Puil E (1990) Effects of hypomagnesia on transmitter actions in neocortical slices. $\mathrm{Br} J$ Pharmacol 101:1006-10.

Erickson CA, Barnes CA (2003) The neurobiology of memory changes in normal aging. Exp Gerontol 38:619.

Eriksen N, Stark AK, Pakkenberg B (2009) Age and Parkinson's disease-related neuronal death in the substantia nigra pars compacta. J Neural Transm Suppl:203-13.

Fontan-Lozano A, Saez-Cassanelli JL, Inda MC, de los Santos-Arteaga M, Sierra-Dominguez SA, Lopez-Lluch G, Delgado-Garcia JM, Carrion AM (2007) Caloric restriction increases learning consolidation and facilitates synaptic plasticity through mechanisms dependent on NR2B subunits of the NMDA receptor. J Neurosci 27:10185-95.

Ford ES, Mokdad AH (2003) Dietary magnesium intake in a national sample of US adults. J Nutr 133:2879-82.

Foster TC, Kumar A (2002) Calcium dysregulation in the aging brain. Neuroscientist 8:297-301.

Foster TC (2006) Biological markers of age-related memory deficits: treatment of senescent physiology. CNS Drugs 20:153-66.

Foster TC (2007) Calcium homeostasis and modulation of synaptic plasticity in the aged brain. Aging Cell 6:319-25.

Frankiewicz T, Parsons CG (1999) Memantine restores long term potentiation impaired by tonic $\mathrm{N}$-methyl-Daspartate (NMDA) receptor activation following reduction of $\mathrm{Mg} 2+$ in hippocampal slices. Neuropharmacology 38:1253-9.

Furukawa Y, Kasai N, Torimitsu K (2009) Effect of Mg2+ on neural activity of rat cortical and hippocampal neurons in vitro. Magnes Res 22:174S-81S.

Galan p, Preziosi P, Durlach V, Valeix P, Ribas L, Bouzid D, Favier A, Hercberg S (1997) Dietary magnesium intake in a French adult population. Magnes Res 10:321-28. 
Gallagher M, Rapp PR (1997) The use of animal models to study the effects of aging on cognition. Annu Rev Psychol 48:339-70.

Gant JC, Sama MM, Landfield PW, Thibault O (2006) Early and simultaneous emergence of multiple hippocampal biomarkers of aging is mediated by $\mathrm{Ca} 2+-$ induced Ca2+ release. J Neurosci 26:3482-90.

Geinisman Y, Ganeshina O, Yoshida R, Berry RW, Disterhoft JF, Gallagher M (2004) Aging, spatial learning, and total synapse number in the rat CA1 stratum radiatum. Neurobiol Aging 25:407-16.

Grady CL, Craik FI (2000) Changes in memory processing with age. Curr Opin Neurobiol 10:224-31.

Gruart A, Lopez-Ramos JC, Munoz MD, Delgado-Garcia JM (2008) Aged wild-type and APP, PS1, and APP + PS1 mice present similar deficits in associative learning and synaptic plasticity independent of amyloid load. Neurobiol Dis 30:439-50.

Guerineau NC, Bossu JL, Gahwiler BH, Gerber U (1995) Activation of a nonselective cationic conductance by metabotropic glutamatergic and muscarinic agonists in CA3 pyramidal neurons of the rat hippocampus. $J$ Neurosci 15:4395-407.

Hofmann K, Tomiuk S, Wolff G, Stoffel W (2000) Cloning and characterization of the mammalian brainspecific, Mg2+-dependent neutral sphingomyelinase. Proc Natl Acad Sci U S A 97:5895-900.

Hotson JR, Prince DA (1980) A calcium-activated hyperpolarization follows repetitive firing in hippocampal neurons. J Neurophysiol 43:409-19.

Houston FP, Stevenson GD, McNaughton BL, Barnes CA (1999) Effects of age on the generalization and incubation of memory in the F344 rat. Learn Mem 6:111-9.

Hsu KS, Ho WC, Huang CC, Tsai JJ (2000) Transient removal of extracellular $\mathrm{Mg}(2+)$ elicits persistent suppression of LTP at hippocampal CA1 synapses via PKC activation. J Neurophysiol 84:1279-88.

Hsu KS, Huang CC, Liang YC, Wu HM, Chen YL, Lo SW, Ho WC (2002) Alterations in the balance of protein kinase and phosphatase activities and age-related impairments of synaptic transmission and long-term potentiation. Hippocampus 12:787-802.

Izquierdo I (1991) Role of NMDA receptors in memory. Trends Pharmacol Sci 12:128-9.
Jahr CE, Stevens CF (1990) Voltage dependence of NMDA-activated macroscopic conductances predicted by single-channel kinetics. J Neurosci 10:3178-82.

Jones RS, Heinemann U (1988) Synaptic and intrinsic responses of medical entorhinal cortical cells in normal and magnesium-free medium in vitro. $J$ Neurophysiol 59:1476-96.

Jope RS (1981) Acetylcholine turnover and compartmentation in rat brain synaptosomes. $J$ Neurochem 36:1712-21.

Jouvenceau A, Potier B, Poindessous-Jazat F, Dutar P, Slama A, Epelbaum J, Billard JM (2002) Decrease in calbindin content significantly alters LTP but not NMDA receptor and calcium channel properties. Neuropharmacology 42:444-58.

Junjaud G, Rouaud E, Turpin F, Mothet JP, Billard JM (2006) Age-related effects of the neuromodulator Dserine on neurotransmission and synaptic potentiation in the CA1 hippocampal area of the rat. J Neurochem 98:1159-66.

Kapaki E, Segditsa J, Papageorgiou C (1989) Zinc, copper and magnesium concentration in serum and CSF of patients with neurological disorders. Acta Neurol Scand 79:373-8.

Kelly KM, Nadon NL, Morrison JH, Thibault O, Barnes CA, Blalock EM (2006) The neurobiology of aging. Epilepsy Res 68 Suppl 1:S5-20.

Kelly SS, Robbins N (1983) Progression of age changes in synaptic transmission at mouse neuromuscular junctions. J Physiol 343:375-83.

Killilea DW, Maier JA (2008) A connection between magnesium deficiency and aging: new insights from cellular studies. Magnes Res 21:77-82.

Kim SJ, Linden DJ (2007) Ubiquitous plasticity and memory storage. Neuron 56:582-92.

Kim YJ, McFarlane C, Warner DS, Baker MT, Choi WW, Dexter $F$ (1996) The effects of plasma and brain magnesium concentrations on lidocaine-induced seizures in the rat. Anesth Analg 83:1223-8.

Kollen $M$, Stephan A, Faivre-Bauman A, Loudes $C$, Sinet PM, Alliot J, Billard JM, Epelbaum J, Dutar P, Jouvenceau A (2010) Preserved memory capacities in aged Lou/C/Jall rats. Neurobiol Aging 31:129-42. 
Kuehl-Kovarik MC, Partin KM, Magnusson KR (2003) Acute dissociation for analyses of NMDA receptor function in cortical neurons during aging. J Neurosci Methods 129:11-7.

Kumar A, Foster TC (2005) Intracellular calcium stores contribute to increased susceptibility to LTD induction during aging. Brain Res 1031:125-8.

Kuno M, Takahashi T (1986) Effects of calcium and magnesium on transmitter release at la synapses of rat spinal motoneurones in vitro. J Physiol 376:543-53.

Ladner CJ, Lee JM (1999) Reduced high-affinity agonist binding at the $\mathrm{M}(1)$ muscarinic receptor in Alzheimer's disease brain: differential sensitivity to agonists and divalent cations. Exp Neurol 158:451-8.

Lanahan A, Lyford G, Stevenson GS, Worley PF, Barnes CA (1997) Selective alteration of long-term potentiation-induced transcriptional response in hippocampus of aged, memory-impaired rats. J Neurosci 17:2876-85.

Landfield PW, Lynch G (1977) Impaired monosynaptic potentiation in in vitro hippocampal slices from aged, memory-deficient rats. J Gerontol 32:523-33.

Landfield PW, Morgan GA (1984) Chronically elevating plasma Mg2+ improves hippocampal frequency potentiation and reversal learning in aged and young rats. Brain Res 322:167-71.

Landfield PW, Pitler TA, Applegate MD (1986) The effects of high $\mathrm{Mg} 2+-$ to-Ca2+ ratios on frequency potentiation in hippocampal slices of young and aged rats. J Neurophysiol 56:797-811.

Lippa AS, Loullis CC, Rotrosen J, Cordasco DM, Critchett DJ, Joseph JA (1985) Conformational changes in muscarinic receptors may produce diminished cholinergic neurotransmission and memory deficits in aged rats. Neurobiol Aging 6:317-23.

Lisman JE, McIntyre CC (2001) Synaptic plasticity: a molecular memory switch. Curr Biol 11:R788-91.

Lister JP, Barnes CA (2009) Neurobiological changes in the hippocampus during normative aging. Arch Neurol 66:829-33.

Lorenzon NM, Foehring RC (1992) Relationship between repetitive firing and afterhyperpolarizations in human neocortical neurons. J Neurophysiol 67:35063.
Luebke J, Barbas H, Peters A (2010) Effects of normal aging on prefrontal area 46 in the rhesus monkey. Brain Res Rev 62:212-32.

Madison DV, Nicoll RA (1984) Control of the repetitive discharge of rat CA 1 pyramidal neurones in vitro. $J$ Physiol 354:319-31.

Magnusson KR (1998) The aging of the NMDA receptor complex. Front Biosci 3:e70-80.

Malenka RC, Lancaster B, Zucker RS (1992) Temporal limits on the rise in postsynaptic calcium required for the induction of long-term potentiation. Neuron 9:121-8

Malenka RC, Nicoll RA (1993) NMDA-receptordependent synaptic plasticity: multiple forms and mechanisms. Trends Neurosci 16:521-7.

Martin SJ, Grimwood PD, Morris RG (2000) Synaptic plasticity and memory: an evaluation of the hypothesis. Annu Rev Neurosci 23:649-711.

Massieu L, Tapia R (1997) Glutamate uptake impairment and neuronal damage in young and aged rats in vivo. J Neurochem 69:1151-60.

Mayer ML, Westbrook GL (1987) Permeation and block of $\mathrm{N}$-methyl-D-aspartic acid receptor channels by divalent cations in mouse cultured central neurones. J Physiol 394:501-27.

McKee JA, Brewer RP, Macy GE, Borel CO, Reynolds JD, Warner DS (2005) Magnesium neuroprotection is limited in humans with acute brain injury. Neurocrit Care 2:342-51.

McKinney M, Coyle JT, Hedreen JC (1983) Topographic analysis of the innervation of the rat neocortex and hippocampus by the basal forebrain cholinergic system. J Comp Neurol 217:103-21.

McKinney M, Jacksonville MC (2005) Brain cholinergic vulnerability: relevance to behavior and disease. Biochem Pharmacol 70:1115-24.

Mesulam MM, Mufson EJ, Wainer BH, Levey AI (1983) Central cholinergic pathways in the rat: an overview based on an alternative nomenclature (Ch1-Ch6). Neuroscience 10:1185-201.

Mesulam MM, Mufson EJ, Levey AI, Wainer BH (1984) Atlas of cholinergic neurons in the forebrain and upper brainstem of the macaque based on monoclonal choline acetyltransferase immunohistochemistry and acetylcholinesterase histochemistry. Neuroscience 12:669-86. 
Misgeld U, Muller W, Polder HR (1989) Potentiation and suppression by eserine of muscarinic synaptic transmission in the guinea-pig hippocampal slice. $J$ Physiol 409:191-206.

Mora F, Segovia G, del Arco A (2007) Aging, plasticity and environmental enrichment: structural changes and neurotransmitter dynamics in several areas of the brain. Brain Res Rev 55:78-88.

Morita A, Abdireyim D, Kimura M, Itokawa Y (2001) The effect of aging on the mineral status of female SAMP1 and SAMR1. Biol Trace Elem Res 80:53-65.

Morrison JH, Hof PR (2007) Life and death of neurons in the aging cerebral cortex. Int Rev Neurobiol 81:4157.

Mothet JP, Rouaud E, Sinet PM, Potier B, Jouvenceau A, Dutar P, Videau C, Epelbaum J, Billard JM (2006) A critical role for the glial-derived neuromodulator $D$ serine in the age-related deficits of cellular mechanisms of learning and memory. Aging Cell 5:267-74.

Moyer JR, Jr., Power JM, Thompson LT, Disterhoft JF (2000) Increased excitability of aged rabbit CA1 neurons after trace eyeblink conditioning. J Neurosci 20:5476-82.

Muller W, Misgeld U (1986) Slow cholinergic excitation of guinea pig hippocampal neurons is mediated by two muscarinic receptor subtypes. Neurosci Lett 67:10712.

Neves G, Cooke SF, Bliss TV (2008) Synaptic plasticity, memory and the hippocampus: a neural network approach to causality. Nat Rev Neurosci 9:65-75.

Norris CM, Korol DL, Foster TC (1996) Increased susceptibility to induction of long-term depression and long-term potentiation reversal during aging. J Neurosci 16:5382-92.

Norris CM, Foster TC (1999) MK-801 improves retention in aged rats: implications for altered neural plasticity in age-related memory deficits. Neurobiol Learn Mem 71:194-206.

Nowak L, Bregestovski P, Ascher P, Herbet A, Prochiantz A (1984) Magnesium gates glutamateactivated channels in mouse central neurones. Nature 307:462-5.

Oh MM, Power JM, Thompson LT, Moriearty PL, Disterhoft JF (1999) Metrifonate increases neuronal excitability in CA1 pyramidal neurons from both young and aging rabbit hippocampus. J Neurosci 19:1814-23.
Ontl T, Xing Y, Bai L, Kennedy E, Nelson S, Wakeman M, Magnusson K (2004) Development and aging of Nmethyl-D-aspartate receptor expression in the prefrontal/frontal cortex of mice. Neuroscience 123:467-79.

Pepeu G (1988) Acetylcholine and brain aging. Pharmacol Res Commun 20:91-7.

Poenaru S, Manicom R, Rouhani S, Aymard P, Bajenaru $O$, Rayssiguier $Y$, Emmanouillidis E, Gueux E, Nkanga N, Durlach J, Dall'ava J (1997) Stability of brain content of magnesium in experimental hypomagnesemia. Brain Res 769:329-32.

Potier B, Rascol O, Jazat F, Lamour Y, Dutar P (1992) Alterations in the properties of hippocampal pyramidal neurons in the aged rat. Neuroscience 48:793-806.

Potier B, Lamour Y, Dutar P (1993) Age-related alterations in the properties of hippocampal pyramidal neurons among rat strains. Neurobiol Aging 14:17-25.

Potier B, Poindessous-Jazat F, Dutar P, Billard JM (2000) NMDA receptor activation in the aged rat hippocampus. Exp Gerontol 35:1185-99.

Potier B, Turpin FR, Sinet PM, Rouaud E, Mothet JP, Videau C, Epelbaum J, Dutar P, Billard JM (2010) Contribution of the d-Serine-Dependent Pathway to the Cellular Mechanisms Underlying Cognitive Aging. Front Aging Neurosci 2:1.

Power JM, Wu WW, Sametsky E, Oh MM, Disterhoft JF (2002) Age-related enhancement of the slow outward calcium-activated potassium current in hippocampal CA1 pyramidal neurons in vitro. J Neurosci 22:7234-43.

Quintero JE, Day BK, Zhang Z, Grondin R, Stephens ML, Huettl P, Pomerleau F, Gash DM, Gerhardt GA (2007) Amperometric measures of age-related changes in glutamate regulation in the cortex of rhesus monkeys. Exp Neurol 208:238-46.

Rapp PR, Gallagher M (1996) Preserved neuron number in the hippocampus of aged rats with spatial learning deficits. Proc Natl Acad Sci U S A 93:9926-30.

Roberts EL, Jr. (1999) Using hippocampal slices to study how aging alters ion regulation in brain tissue. Methods 18:150-9.

Rosenzweig ES, Barnes CA (2003) Impact of aging on hippocampal function: plasticity, network dynamics, and cognition. Prog Neurobiol 69:143-79. 
Scali C, Casamenti F, Pazzagli M, Bartolini L, Pepeu G (1994) Nerve growth factor increases extracellular acetylcholine levels in the parietal cortex and hippocampus of aged rats and restores object recognition. Neurosci Lett 170:117-20.

Segal M (1982) Changes in neurotransmitter actions in the aged rat hippocampus. Neurobiol Aging 3:121-4.

Shankar S, Teyler TJ, Robbins N (1998) Aging differentially alters forms of long-term potentiation in rat hippocampal area CA1. J Neurophysiol 79:334-41.

Shen J, Barnes CA (1996) Age-related decrease in cholinergic synaptic transmission in three hippocampal subfields. Neurobiol Aging 17:439-51.

Shiozaki K, Haga T (1992) Effects of magnesium ion on the interaction of atrial muscarinic acetylcholine receptors and GTP-binding regulatory proteins. Biochemistry 31:10634-42.

Slutsky I, Sadeghpour S, Li B, Liu G (2004)

Enhancement of synaptic plasticity through chronically reduced $\mathrm{Ca} 2+$ flux during uncorrelated activity. Neuron 44:835-49.

Slutsky I, Abumaria N, Wu LJ, Huang C, Zhang L, Li B, Zhao X, Govindarajan A, Zhao MG, Zhuo M, Tonegawa S, Liu G (2010) Enhancement of learning and memory by elevating brain magnesium. Neuron 65:165-77.

Smith DA, Connick JH, Stone TW (1989) Effect of changing extracellular levels of magnesium on spontaneous activity and glutamate release in the mouse neocortical slice. Br J Pharmacol 97:475-82.

Smith TD, Adams MM, Gallagher M, Morrison JH, Rapp PR (2000) Circuit-specific alterations in hippocampal synaptophysin immunoreactivity predict spatial learning impairment in aged rats. J Neurosci 20:658793.

Stanton PK, Jones RS, Mody I, Heinemann U (1987) Epileptiform activity induced by lowering extracellular [Mg2+] in combined hippocampal-entorhinal cortex slices: modulation by receptors for norepinephrine and N-methyl-D-aspartate. Epilepsy Res 1:53-62.

Stone TW, Bartrup JT, Brooks PA, Connick JH, Smith DA (1992) Interactions of adenosine and magnesium on neuronal excitability and transmitter sensitivity in the hippocampal slice. Epilepsy Res Suppl 8:237-42.

Sykova E, Mazel T, Simonova Z (1998) Diffusion constraints and neuron-glia interaction during aging. Exp Gerontol 33:837-51.
Sykova E, Mazel T, Hasenohrl RU, Harvey AR, Simonova Z, Mulders WH, Huston JP (2002) Learning deficits in aged rats related to decrease in extracellular volume and loss of diffusion anisotropy in hippocampus. Hippocampus 12:269-79.

Takahashi S, Takahashi I, Sato H, Kubota Y, Yoshida S, Muramatsu Y (2001) Age-related changes in the concentrations of major and trace elements in the brain of rats and mice. Biol Trace Elem Res 80:145-58.

Takei N, Nihonmatsu I, Kawamura H (1989) Agerelated decline of acetylcholine release evoked by depolarizing stimulation. Neurosci Lett 101:182-6.

Tanimura A, Nezu A, Morita T, Hashimoto N, Tojyo $\mathrm{Y}$ (2002) Interplay between calcium, diacylglycerol, and phosphorylation in the spatial and temporal regulation of PKCalpha-GFP. J Biol Chem 277:29054-62.

Taylor L, Griffith WH (1993) Age-related decline in cholinergic synaptic transmission in hippocampus. Neurobiol Aging 14:509-15.

Teyler TJ, DiScenna P (1987) Long-term potentiation. Annu Rev Neurosci 10:131-61.

Thibault O, Landfield PW (1996) Increase in single Ltype calcium channels in hippocampal neurons during aging. Science 272:1017-20.

Thibault O, Hadley R, Landfield PW (2001) Elevated postsynaptic [Ca2+]i and L-type calcium channel activity in aged hippocampal neurons: relationship to impaired synaptic plasticity. J Neurosci 21:9744-56.

Thibault O, Gant JC, Landfield PW (2007) Expansion of the calcium hypothesis of brain aging and Alzheimer's disease: minding the store. Aging Cell 6:307-17.

Thompson LT, Moyer JR, Jr., Disterhoft JF (1996) Trace eyeblink conditioning in rabbits demonstrates heterogeneity of learning ability both between and within age groups. Neurobiol Aging 17:619-29.

Toescu EC, Verkhratsky A (2004) Ca2+ and mitochondria as substrates for deficits in synaptic plasticity in normal brain ageing. J Cell Mol Med 8:18190.

Toescu EC, Verkhratsky A, Landfield PW (2004) Ca2+ regulation and gene expression in normal brain aging. Trends Neurosci 27:614-20.

Tombaugh GC, Rowe WB, Rose GM (2005) The slow afterhyperpolarization in hippocampal CA1 neurons covaries with spatial learning ability in aged Fisher 344 rats. J Neurosci 25:2609-16. 
Turpin FR, Potier B, Dulong JR, Sinet PM, Alliot J, Oliet SH, Dutar P, Epelbaum J, Mothet JP, Billard JM (2009) Reduced serine racemase expression contributes to age-related deficits in hippocampal cognitive function. Neurobiol Aging 32:1495-504.

Vannucchi MG, Scali C, Kopf SR, Pepeu G, Casamenti F (1997) Selective muscarinic antagonists differentially affect in vivo acetylcholine release and memory performances of young and aged rats. Neuroscience 79:837-46.

Veng LM, Mesches MH, Browning MD (2003) Agerelated working memory impairment is correlated with increases in the L-type calcium channel protein alpha1D (Cav1.3) in area CA1 of the hippocampus and both are ameliorated by chronic nimodipine treatment. Brain Res Mol Brain Res 110:193-202.

Verkhratsky A, Toescu EC (1998) Calcium and neuronal ageing. Trends Neurosci 21:2-7.

Vickroy TW, Schneider CJ (1991) Characterization of divalent cation-induced $[3 \mathrm{H}]$ acetylcholine release from EGTA-treated rat hippocampal synaptosomes. Neurochem Res 16:1175-85.

Wakimoto P, Block G (2001) Dietary intake, dietary patterns, and changes with age: an epidemiological perspective. J Gerontol A Biol Sci Med Sci 56 Spec No 2:65-80.

Wang JH, Ko GY, Kelly PT (1997) Cellular and molecular bases of memory: synaptic and neuronal plasticity. J Clin Neurophysiol 14:264-93.
Ward MT, Stoelzel CR, Markus EJ (1999) Hippocampal dysfunction during aging II: deficits on the radial-arm maze. Neurobiol Aging 20:373-80.

Weiss C, Preston AR, Oh MM, Schwarz RD, Welty D, Disterhoft JF (2000) The M1 muscarinic agonist Cl1017 facilitates trace eyeblink conditioning in aging rabbits and increases the excitability of CA1 pyramidal neurons. J Neurosci 20:783-90.

Winocur G, Moscovitch M (1990) Hippocampal and prefrontal cortex contributions to learning and memory: analysis of lesion and aging effects on maze learning in rats. Behav Neurosci 104:544-51.

Wolf FI, Cittadini A (2003) Chemistry and biochemistry of magnesium. Mol Aspects Med 24:3-9.

Woolf NJ, Eckenstein F, Butcher LL (1984) Cholinergic systems in the rat brain: I. projections to the limbic telencephalon. Brain Res Bull 13:751-84.

Zhang Q, Okamura M, Guo ZD, Niwa S, Haga T (2004) Effects of partial agonists and $\mathrm{Mg} 2+$ ions on the interaction of $\mathrm{M} 2$ muscarinic acetylcholine receptor and $\mathrm{G}$ protein Galpha i1 subunit in the M2-Galpha i1 fusion protein. J Biochem 135:589-96.

Zornetzer SF, Thompson R, Rogers J (1982) Rapid forgetting in aged rats. Behav Neural Biol 36:49-60. 


\section{Section 2}

Magnesium in Neurological Diseases 



\title{
The role of magnesium therapy in learning and memory
}

\author{
Michael R. Hoane
}

Restorative Neuroscience Laboratory, Brain and Cognitive Science Program, Department of Psychology, Southern Illinois University, Carbondale, IL, USA.

$\triangle$ mhoane@siu.edu

\begin{abstract}
The old saying "you are what you eat" is becoming increasingly important in the field of neuroscience these days. There is mounting evidence that nutritional factors are beginning to play a major role in cognitive status, or cognitive wellbeing. One of these emerging factors is magnesium $\left(\mathrm{Mg}^{2+}\right)$. Although the physiological investigation of $\mathrm{Mg}^{2+}$ has a long history, its role in cognitive function is just starting to emerge. The focus of this chapter is to review the available literature on the effects of $\mathrm{Mg}^{2+}$ on cognitive function in the healthy and diseased/injured brain. In addition, data from our laboratory will be presented that has investigated the effects of $\mathrm{Mg}^{2+}$ manipulation on learning and memory tasks in rodents, as well as the ability of $\mathrm{Mg}^{2+}$ therapy to improve cognitive performance in the damaged brain.
\end{abstract}

\section{Introduction}

The purpose of this chapter is to provide a review of the literature on the role of $\mathrm{Mg}^{2+}$ in cognitive function. Although the research on this topic is generally sparse, there is an accumulating body of evidence suggesting that $\mathrm{Mg}^{2+}$ is vitally important. The role that micronutrients play in maintaining and promoting cognitive ability and neural plasticity has started to receive a good deal of attention. A recent paper has provided an excellent review on how various nutrients promote cognitive performance and neural plasticity (Gómez-Pinilla, 2008). Although this review does not specifically discuss the role of $\mathrm{Mg}^{2+}$, it is clear that this is a rapidly evolving area of research. Another review does address the role of $\mathrm{Mg}^{2+}$ and its relationship with other micronutrients on cognitive function and performance. It especially highlights the important role that $\mathrm{Mg}^{2+}$ has in interacting with other micronutrients (Huskisson et al., 2007). Recent evidence also suggests that $\mathrm{Mg}^{2+}$ plays an important role in age-related deficits in neurotransmitter release, neuronal excitability and synaptic plasticity related to cognition (Billard, 2006), and these topics will be reviewed in Chapter 6.

Additionally, there is ample evidence that $\mathrm{Mg}^{2+}$ plays an important role in the pathophysiological processes following traumatic brain injury (TBI) and that $\mathrm{Mg}^{2+}$ therapy is effective in promoting functional recovery in a variety of animal models (Hoane and Barth, 2001; Hoane, 2004; Sen and Gulati, 2010; van den Heuvel and Vink, 2004; Vink et al., 2009). Specifically, this chapter will focus on reviewing the literature on $\mathrm{Mg}^{2+}$ and cognitive function and review a series of studies conducted in our laboratory that have investigated the ability of $\mathrm{Mg}^{2+}$ therapy to alter cognitive function and to improve cognitive recovery following focal and traumatic brain injuries in the rat.

\section{Introduction to rodent cognitive assessment}

The Morris water maze (MWM) is a standard task for measuring cognitive/spatial performance in rodents. This task uses a water-filled tank with a submerged escape platform and many different aspects of memory can be assessed (Hoane et al., 2003; Hoane, 2005; Hoane et al., 2009; Kaufman et al., 2010; Lindner, 1997; Lindner et al., 1998; Quigley et al., 2009). Briefly, a reference memory trial consists of placing the rat into the water at one of 4 randomly chosen start locations. A computer-assisted video tracking system is used to measure the swim latency and distance to the submerged escape platform. On trials designed to measure working memory the escape platform is relocated to a new position in the tank every day. The first trial (in the new location) is considered an information trial and the subsequent 3 trials are test trials and are averaged to form the dependent variable. Additional paradigms can be used that 
incorporate various mazes or dividers that are placed into the tank that can test other aspects of learning and memory in the rodent.

Additionally, the Barnes spatial maze, a dry version of the MWM, can also be used to assess cognitive/spatial performance (Vink et al., 2003). Rats are placed on an elevated $1.2 \mathrm{~m}$ circular tabletop with 19 holes cut around the periphery. The rat learns the location of the hole that leads to an escape tunnel. The latency to find the correct hole is then analysed. Operant chambers (Skinner boxes) can be used to assess appetitive conditioning of learning ratios under various conditions. In general, rats are shaped to barpress for a reinforcer and then are shifted to more complicated reward paradigms. Thus, there are many ways in which rodent cognitive assessments can be made and many of these have been utilized to examine the role of $\mathrm{Mg}^{2+}$ in cognition.

\section{Role of $\mathrm{Mg}^{2+}$ in health and cognition}

The importance of $\mathrm{Mg}^{2+}$ in normal cellular functioning has been well documented, as has its importance in the pathophysiology following injury. Previously, several reviews have been written that address these issues (Hoane and Barth, 2001; Hoane, 2004; van den Heuvel and Vink, 2004) so only a brief synopsis will be provided in this paper. $\mathrm{Mg}^{2+}$ is involved in many critical cellular processes including cellular respiration, protein synthesis, membrane stability and regulation of vascular tone. A more detailed discussion on the physiology of $\mathrm{Mg}^{2+}$ is presented in several chapters within Section 1 of this book.

Although the main focus of this chapter is on animal models, there are some interesting human studies that have examined the role of $\mathrm{Mg}^{2+}$ in cognitive ability. A recent study examined the correlation between levels of several trace minerals (iron, $\mathrm{Mg}^{2+}$, potassium and zinc) in the hair of adolescent girls and their academic record. Although care must be taken with the interpretation of correlational studies of this nature, there was evidence that some trace minerals correlated more highly with increased academic performance. Specifically, it was found that $\mathrm{Mg}^{2+}$ and zinc demonstrated a strong positive correlation with academic performance (Wang et al., 2008). Furthermore, a recent case report of a patient presenting with anorexia nervosa and Wernicke-Korsakoff syndrome was found to have a low serum $\mathrm{Mg}^{2+}$ level (Saad et al., 2010). Although the main cause of this condition was thiamine deficiency, a major adjunct factor was believed to be $\mathrm{Mg}^{2+}$ deficiency. The results from this case study are supported by a review article on micronutrients and cognitive performance that details the interrelationships between $\mathrm{Mg}^{2+}$ and other micronutrients such as the B-group vitamins and how deficiencies in these nutrients interact to produce cognitive deficits (Huskisson et al., 2007). Thus, there is an expanding literature that suggests that $\mathrm{Mg}^{2+}$ status plays an important role in cognitive performance.

A unique condition in which $\mathrm{Mg}^{2+}$ has been implicated is TBI. McIntosh and colleagues have shown that $\mathrm{Mg}^{2+}$ homeostasis is disrupted following CNS injury (McIntosh et al., 1988; Vink et al., 1988). Fluid percussion injury (FPI) produced a rapid and severe decline in intra- and extracellular $\mathrm{Mg}^{2+}$ levels, which correlated significantly with the severity of the behavioural deficits observed following injury (Mclntosh et al., 1988; Vink et al., 1988). Additional research on the role of $\mathrm{Mg}^{2+}$ and neurological diseases will be presented in Section 2 of this book, while $\mathrm{Mg}^{2+}$ in cognitive function following TBI is addressed later in this chapter. These findings suggest that $\mathrm{Mg}^{2+}$ plays an important role in normal physiology and in the pathophysiological events that occur following injury to the nervous system.

\section{Recent laboratory data}

\section{$\mathrm{Mg}^{2+}$ therapy and learning}

From a purely pharmacological standpoint, administration of $\mathrm{Mg}^{2+}$ immediately prior to the acquisition of a learning task should have a detrimental effect on that task given the noncompetitive antagonistic properties of $\mathrm{Mg}^{2+}$ at the NMDA receptor. It has been shown that administration of other NMDA antagonists such as MK-801 and PCP have been shown to disrupt spatial learning in rodents (Kesner et al., 1993; McLamb et al., 1990; Murray and Ridley, 1997); however, in some cases a facilitative effect can be shown (Pussinen and Sirvio, 1999). In order to examine the biological activity of $\mathrm{Mg}^{2+}$ administration on the acquisition of learning, a $\mathrm{MgCl}_{2}$ solution was administered $(1 \mathrm{mmol} / \mathrm{kg}$ or 2 
$\mathrm{mmol} / \mathrm{kg}$, i.p.) prior to the acquisition of learning a reference memory task in the MWM (Hoane, 2007). Intact rats received daily injections of $\mathrm{MgCl}_{2}, 30$ min prior to the start of their MWM session and were tested for 5 consecutive days for 4 trials per day with an intertrial interval (ITI) of $15 \mathrm{~min}$. The reference memory acquisition paradigm was used in the MWM. In this paradigm, the submerged escape platform stayed in the same location for every trial and the animals were released from 1 of 4 different starting points in the maze. As can be seen in Figure 1 , the vehicle-treated $(0.9 \%$ saline, i.p.) group showed steady acquisition of the task. In comparison, the $\mathrm{MgCl}_{2}$-treated animals showed a more varied response. The initial acquisition of the $2 \mathrm{mmol}$ group was slightly lengthened on day 1 compared to the other groups. On day 4, both groups of $\mathrm{MgCl}_{2}$-treated animals started to show a lengthening of their escape latencies compared to vehicle-treated animals. On the last 2 test days the comparison of swim latencies between the 2 $\mathrm{mmol}$ and saline group was significantly different $(p<0.01)$ indicating a possible learning impairment on the task.

Thus, it appears that daily dosing with the higher dose of $\mathrm{MgCl}_{2}$ produced a significant degree of amnesia after 3 days. Unfortunately, testing was terminated after 5 days so it is unknown if this effect would have persisted or worsened with additional testing. It should be noted that, in general, the NMDA antagonists that work at the PCP site on the NMDA receptor (i.e., MK-801 and $\mathrm{PCP}$ ) seem to have a greater amnesic effect than $\mathrm{MgCl}_{2}$. This may raise some concerns for continued dosing regimens of $\mathrm{Mg}^{2+}$ therapy lasting more than a couple days. However, a longer window of time between administrations and testing may reveal different findings, because this effect is perceived to be state dependent. Thus, given these behavioural results it is clear that systemic injections of $\mathrm{MgCl}_{2}$ did indeed exert behavioural effects in uninjured animals with an intact blood-brain barrier (BBB), and therefore give support to the ability of $\mathrm{MgCl}_{2}$ to cross the BBB. This finding supports earlier research to this fact (Hallack et al., 1992).

We have also recently investigated the effect of dietary $\mathrm{Mg}^{2+}$ deficiency on learning acquisition in the MWM. Rats were placed on either a standard laboratory diet or a commercially available $\mathrm{Mg}^{2+}$ -

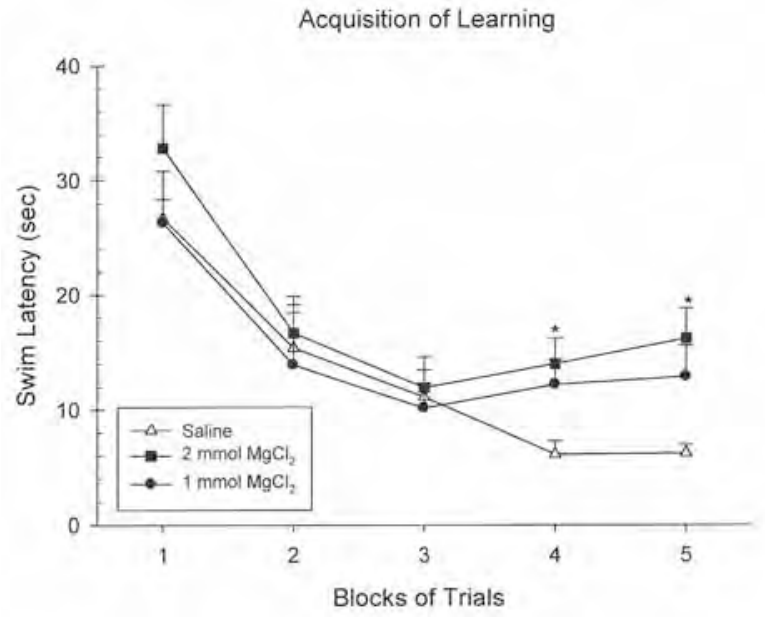

Figure 1. The effects of repeated pre-testing administration of $\mathrm{MgCl}_{2}$ ( 1 or $2 \mathrm{mmol} / \mathrm{kg}$ ) on the acquisition of a reference memory task in the MWM. Plotted are the mean ( \pm SEM) swim latencies to find the submerged escape platform. The $2 \mathrm{mmol}$ dose of $\mathrm{MgCl}_{2}$ significantly impaired swim latencies starting on the $4^{\text {th }}$ day of testing ( $^{*}$ $=p<0.05)$.

deficient diet for 14 days. Peripheral blood collections were performed for determination of serum $\mathrm{Mg}^{2+}$ levels and all animals were placed back on to the standard diet. The serum analysis indicated a significant loss in serum $\mathrm{Mg}^{2+}$ in the deficiency group $(p<0.05)$. One week later, animals were tested for the acquisition of a reference memory task over 4 days ( 4 trials per day, $15 \mathrm{~min}$ ITI). As can be seen in Figure $2 \mathrm{~A}$, the 14 days of $\mathrm{Mg}^{2+}$ deficiency significantly impaired the initial acquisition of the task on the first day $(p<0.05)$. The animals were then switched to a more challenging working memory assessment in the MWM. As can be seen in Figure 2B, the animals that experienced $\mathrm{Mg}^{2+}$ deficiency showed impaired learning on the task $(p<0.05)$. Additionally, animals were trained to acquire a fixed ration (FR-10) schedule of reinforcement in an operant chamber. The total number of bar presses is shown in Figure 2C. The higher number of responses results in a greater number of reinforcers being delivered and can be equated to the learning of the reinforcement schedule. Animals on the $\mathrm{Mg}^{2+}$-deficient diet responded at a significantly lower level $(p<0.05)$ than those animals on the $\mathrm{Mg}^{2+}$-normal diet, suggesting a learning impairment. A somewhat similar finding has been shown in mice exposed to $\mathrm{M} \mathrm{Mg}^{2+}$-deficient 

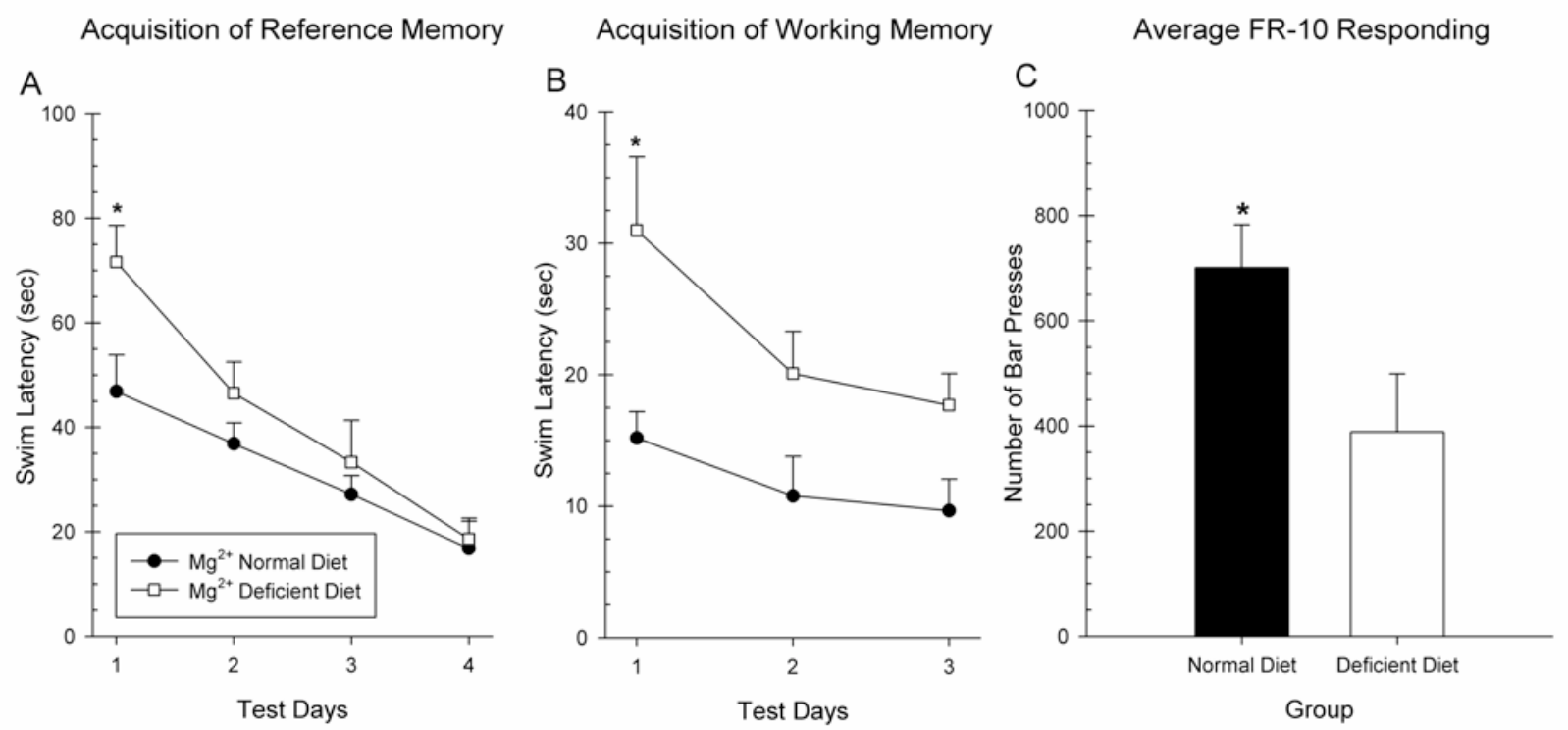

Figure 2. The effects of dietary restriction of $\mathrm{Mg}^{2+}$ on cognitive performance in the MWM. Plotted are the mean swim latencies ( \pm SEM) on the acquisition of reference memory task (A), working memory (B), and the acquisition of a FR-10 reinforcement schedule in an operant chamber (C). A two-week period of dietary $\mathrm{Mg}^{2+}$ deficiency, followed by reinstatement back to a $\mathrm{Mg}^{2+}$-normal diet resulted in significant impairments in cognitive performance on all measures $(*=p<0.05)$.

diet (Bardgett et al., 2005; Bardgett et al., 2007). In this study, 10 days of $\mathrm{Mg}^{2+}$ deficiency resulted in impaired learning of a conditioned fear task. However, maze performance in the MWM was not impaired. Thus, these data suggest that even temporary $\mathrm{Mg}^{2+}$ deficiency may interfere with learning.

A newly formulated form of $\mathrm{Mg}^{2+}$, magnesium-Lthreonate $(\mathrm{MgT})$ has been shown to increase cerebrospinal fluid levels of $\mathrm{Mg}^{2+}$ to a much greater degree than other $\mathrm{Mg}^{2+}$ preparations (e.g. $\mathrm{MgCl}_{2}$ ) (Slutsky et al., 2010). Administration of MgT has also been shown to result in an enhancement of various forms of cognitive/ learning ability in rodents, including working memory. This effect was also evident in aged rats following which cognitive abilities were generally reduced. The beneficial behavioural effects were thought to be caused by beneficial changes in synaptic plasticity within the hippocampus (Slutsky et al., 2010).

\section{Effects of $\mathrm{Mg}^{2+}$ therapy on cognitive function following brain injury}

Several studies have investigated the ability of $\mathrm{Mg}^{2+}$ to improve acute cognitive function in animal models of TBI. It has been shown that administration of $\mathrm{MgCl}_{2}$ following $\mathrm{FPI}$ improved cognitive outcome by reducing memory loss following injury (Smith et al., 1993). However, administration of $\mathrm{MgCl}_{2}$ failed to improve the acquisition of a reference memory task in the MWM following injury (Bareyre et al., 1999). It has also been recently shown that administration of $\mathrm{MgSO}_{4}(250 \mu \mathrm{mol} / \mathrm{kg}$, i.v.), $30 \mathrm{~min}$ following diffuse axonal injury improved recovery of a spatial memory task on the Barnes maze (Vink et al., 2003). In addition, administration of an intravenous solution of $\mathrm{MgCl}_{2}(150 \mu \mathrm{mol})$ prior to FPI prevented the occurrence of injury-induced impairments on working and reference memory tasks in a radial maze (Enomoto et al., 2005).

There have been very few attempts to examine the ability of $\mathrm{Mg}^{2+}$ therapy to resolve long-term cognitive dysfunction. A recent study has shown severe cognitive deficits in the acquisition of a reference memory task in the MWM when tested 8 months post-FPI (Browne et al., 2004). The administration of $\mathrm{MgSO}_{4}(125 \mu \mathrm{mol}$, i.v.) or NPS 1506 (an NMDA antagonist) failed to improve the acquisition of the reference memory task compared to vehicle controls. However, it was 
found that $\mathrm{MgSO}_{4}$ did reduce the amount of ipsilateral hippocampal cell loss. Thus, preservation of the hippocampus failed to result in significant cognitive improvement (Browne et al., 2004). Given the limited number of studies and their mixed results, it is important to further investigate the effect of $\mathrm{Mg}^{2+}$ therapy on the recovery of cognitive function following injury.

\section{Examination of $\mathrm{Mg}^{2+}$ therapy following focal injury}

In our laboratory we have examined recovery of cognitive function in a bilateral focal cortical ablation model. Rats were given small $\left(4 \mathrm{~mm}^{2}\right)$ electrolytic lesions aimed at the bilateral anterior medial cortex (bAMC) of the frontal lobe (Hoane et al., 2003). Administration of $\mathrm{Mg}^{2+}$ therapy occurred $15 \mathrm{~min}$ following injury with rats receiving either injections of $\mathrm{MgCl}_{2}$ (1 or 2 $\mathrm{mmol} / \mathrm{kg}$, i.p.) or saline $(1 \mathrm{ml} / \mathrm{kg})$. This regimen was repeated again 24 and $72 \mathrm{hrs}$ later, so that each rat received 3 injections within the first 72 hrs following injury. Behavioural testing began 5 days after injury and included the assessment of cognitive function. The MWM was used to investigate the acquisition of reference and working memory. In addition, the MWM tank was also used to examine spatial ability using a delayed matching-to-sample (DMTS) task, a very sensitive measure of spatial working memory.

As can be seen in Figure $3 A$ \& 3B, the results of the behavioural testing indicated that $b A M C$ lesions produced severe deficits in cognitive function on both the reference and working memory tasks in the MWM. $\mathrm{Mg}^{2+}$ therapy with either the 1 or $2 \mathrm{mmol}$ dose did not significantly facilitate the acquisition of reference memory in the MWM. However, the mean swim latencies for both $\mathrm{MgCl}_{2}$ groups were greatly improved compared to saline in the last 2 blocks of trials. Although $\mathrm{Mg}^{2+}$ therapy did not demonstrate a statistically significant improvement on reference memory performance in the present study, it did improve working memory performance in the MWM. Administration of the $2 \mathrm{mmol}$ dose of $\mathrm{MgCl}_{2}$ significantly reduced the working memory deficit compared to saline treatment on the first 2 days of testing. The $1 \mathrm{mmol}$ dose of $\mathrm{MgCl}_{2}$ also reduced the working memory impairments on the first day of testing and significantly improved working memory performance on day 2 of testing. Severe cognitive impairments were also seen on the DMTS spatial memory test following injury. The $2 \mathrm{mmol}$ dose of $\mathrm{MgCl}_{2}$ significantly reduced the number of trials needed to reach the criterion of $80 \%$ correct choices compared to salinetreated rats, while the $1 \mathrm{mmol}$ dose of $\mathrm{MgCl}_{2}$ did not, although the number of trials was greatly reduced. Thus, $\mathrm{Mg}^{2+}$ therapy was effective in a task and dose-dependent manner in this study.

\section{$\mathrm{Mg}^{2+}$ therapy in the traumatically injured brain}

To examine the ability of $\mathrm{Mg}^{2+}$ therapy following $T B I$, groups of rats were prepared with a cortical contusion injury $(\mathrm{CCl})$ or sham procedure and then assigned to either $\mathrm{MgCl}_{2}(1.0 \mathrm{mmol} / \mathrm{kg}$, i.p.) or saline treatment conditions (Hoane, 2005). $\mathrm{Mg}^{2+}$ therapy was administered $15 \mathrm{~min}$ and $24 \mathrm{hr}$ following injury. Rats were then examined for cognitive/spatial performance in the MWM, investigating the acquisition of reference and working memory. Administration of $\mathrm{MgCl}_{2}$ following $\mathrm{CCl}$ significantly reduced some of the behavioural impairments observed following injury (see Figure 3C \& 3D). The acquisition of reference memory in the MWM was significantly improved compared to saline-treated rats. In contrast, $\mathrm{MgCl}_{2}$ did not improve working memory performance.

In a second study, the ability of $\mathrm{Mg}^{2+}$ therapy to improve cognitive/spatial performance following unilateral $\mathrm{CCl}$ was examined. Groups of rats were given unilateral $\mathrm{CCls}$ or sham surgeries of the left sensorimotor motor/frontal cortex. One hr following injury, rats were administered $1 \mathrm{mmol}$ $/ \mathrm{kg} \mathrm{MgCl} 2$ or saline. They were then tested for their ability to acquire a reference memory task in the MWM on 4 consecutive days (4 trials/day) starting 11 days after $\mathrm{CCl}$. Their working memory performance was measured on days 16 and 17. It was found that the single $1 \mathrm{mmol} / \mathrm{kg}$ dose of $\mathrm{MgCl}_{2}$ effectively facilitated the acquisition of the reference memory task compared to treatment with saline (see Figure 4). In a similar manner, the working memory performance was greatly enhanced following $\mathrm{CCl}$ in the $\mathrm{Mg}^{2+}$-treated rats compared to the saline-treated rats. In fact, the working memory performance of the $\mathrm{Mg}^{2+}$ treated rats could not be distinguished from the sham controls on either day of working memory testing (see working memory graph in Figure 4). $\mathrm{Mg}^{2+}$ treatment appeared to have prevented the occurrence of the working memory deficit following unilateral frontal injury. 

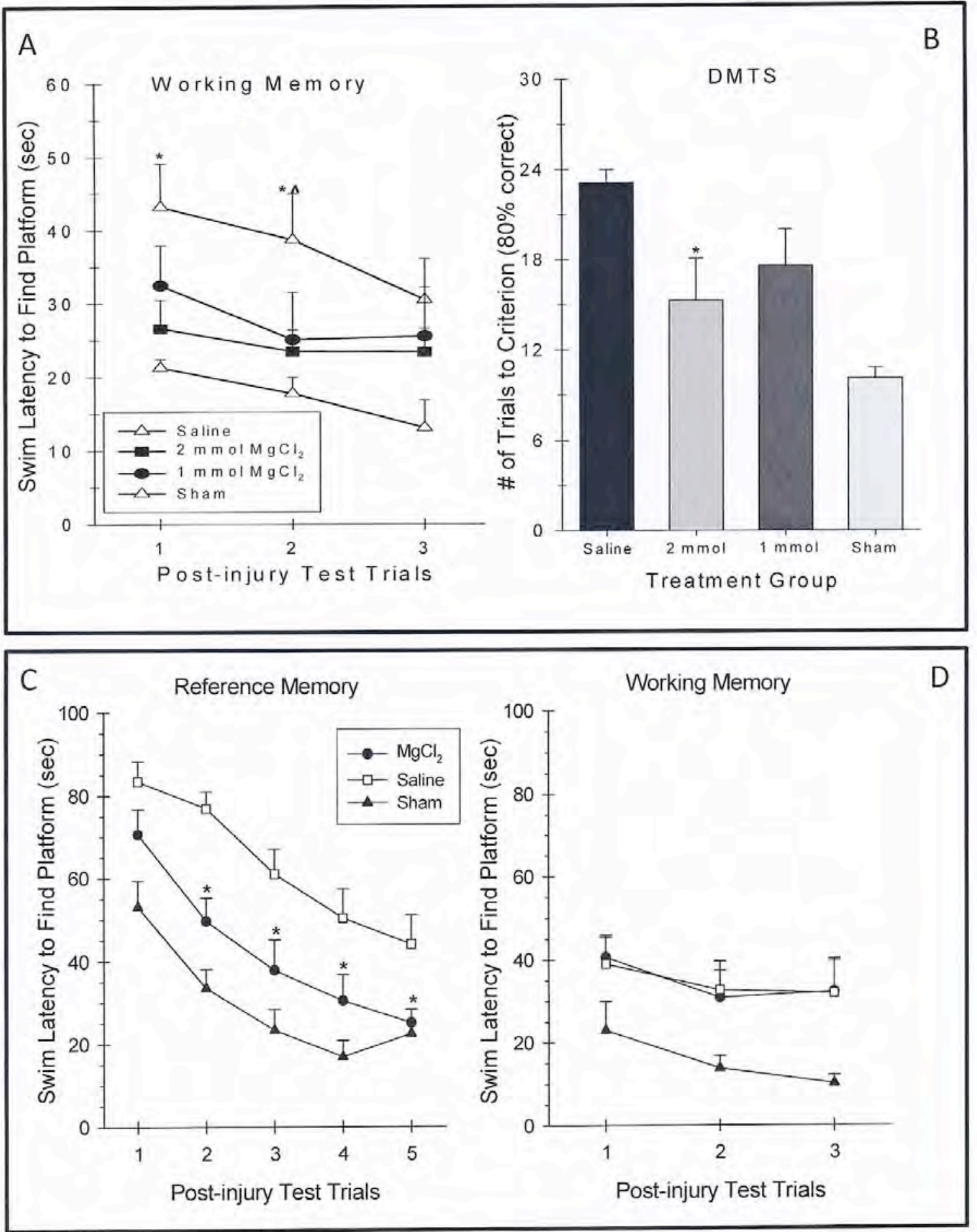

Figure 3. The effects of a regimen of $\mathrm{MgCl}_{2}$ (1 or $2 \mathrm{mmol} / \mathrm{kg}$ ) administered following bAMC focal lesions on cognitive performance in the MWM. Plotted are the mean swim latencies ( \pm SEM) during the acquisition phase of a working memory task (A) and the mean number of trials to criterion in the DMTS test (B) is presented. There was a dose and task-dependent effect on recovery. The $2 \mathrm{mmol}$ dose provided the greatest improvement on both measures of working memory ( ${ }^{*}=p<0.05$ comparing $2 \mathrm{mmol} \mathrm{MgCl}_{2}$ to saline; ${ }^{\wedge}=p<0.05$ comparing $1 \mathrm{mmol} \mathrm{MgCl}_{2}$ to saline). Adapted from Hoane et al., (2003). The effects of a regimen of $\mathrm{MgCl}_{2}(1 \mathrm{mmol} / \mathrm{kg})$ administered $15 \mathrm{~min}$ following bilateral $\mathrm{CCl}$ of the frontal cortex. Plotted are the mean swim latencies (+SEM) on the acquisition of reference memory (C) and working memory (D) tasks in the MWM. Administration of $\mathrm{MgCl}_{2}$ significantly improved the acquisition of a reference memory task, but not working memory following $\mathrm{CCl}(*=p<0.05)$. Adapted from Hoane et al., (2005). 

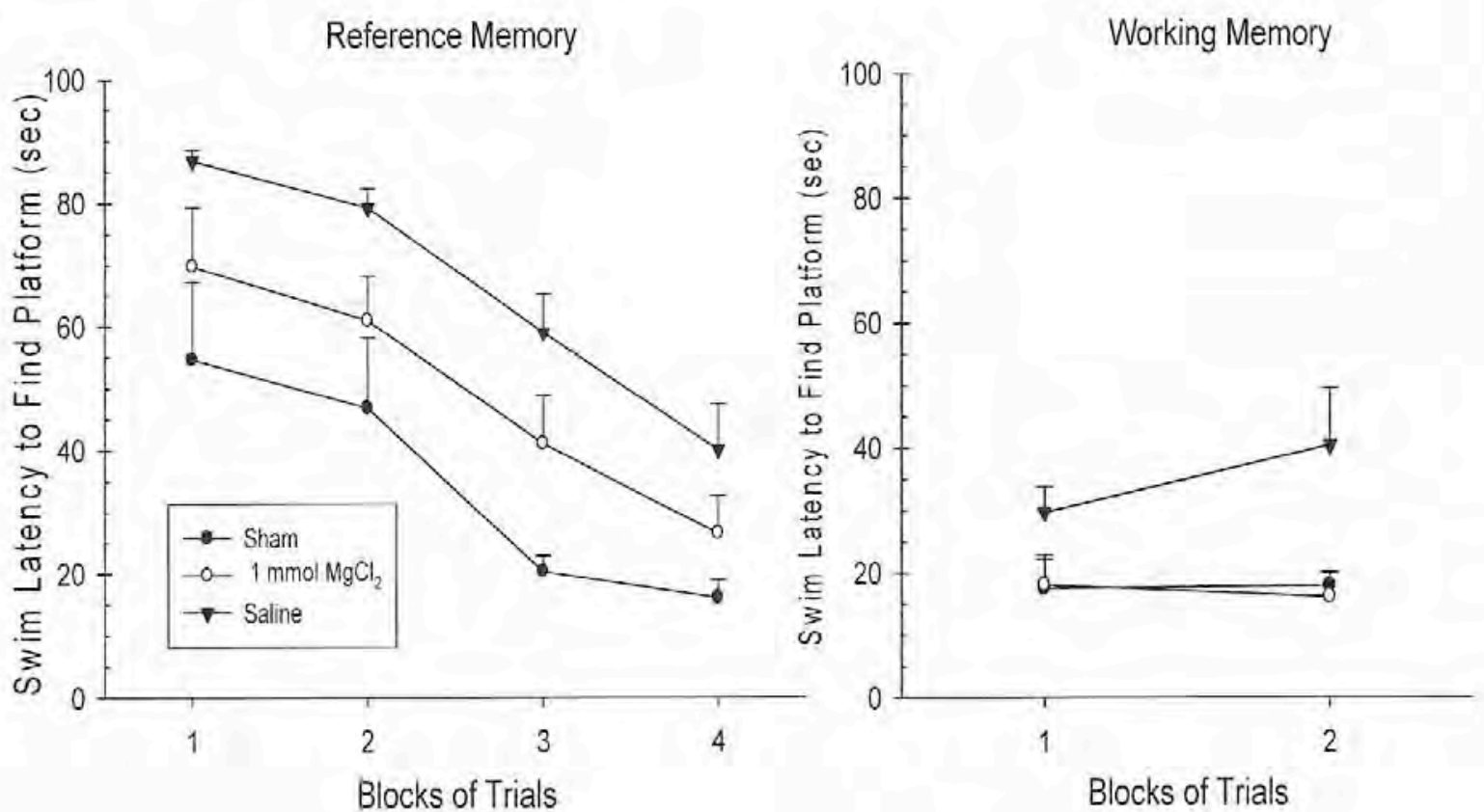

Figure 4. The effects of $\mathrm{MgCl}_{2}(1 \mathrm{mmol} / \mathrm{kg})$ administration $1 \mathrm{hr}$ following unilateral $\mathrm{CCl}$ of the sensorimotor/frontal cortex. Plotted are the mean swim latencies ( $\pm \mathrm{SEM}$ ) on the acquisition of reference memory and working memory tasks in the MWM. Administration of $\mathrm{MgCl}_{2}$ significantly improved the acquisition of a reference memory task and working memory following CCl. Adapted from Hoane (2007).

\section{Discussion}

The results of these studies have demonstrated a wide range of conditions in which $\mathrm{Mg}^{2+}$ therapy regulates cognitive function. It was first shown that daily injections of $\mathrm{MgCl}_{2}$ administered 30 mins prior to training on the task worsened acquisition of the reference memory task. It was also demonstrated that a two-week regimen of dietary $\mathrm{Mg}^{2+}$ deficiency impaired learning on 3 different cognitive tasks. This finding is especially interesting because the animals had been placed back onto a normal laboratory diet prior to the assessment phase of the study.

$\mathrm{Mg}^{2+}$ therapy in several models of cortical ablation and TBI have demonstrated positive effects on cognitive recovery. However, these effects occurred in a task and dose-dependent manner. Following focal ablation of the bAMC, $\mathrm{MgCl}_{2}$ improved working memory performance on several measures and slightly improved reference memory performance. The $\mathrm{CCl}$ studies performed in our laboratory have shown that $\mathrm{MgCl}_{2}$ administration following injury improved cognitive performance in a task-dependent manner. Previous studies that have examined the ability of $\mathrm{Mg}^{2+}$ therapy to improve cognitive performance following injury have shown mixed results. For example, administration of $\mathrm{MgCl}_{2}$ following FPI has been shown to improve cognitive outcome by reducing memory loss in the MWM (Smith et al., 1993), but administration of $\mathrm{MgCl}_{2}$ failed to improve the acquisition of a reference memory task in the MWM following injury (Bareyre et al., 1999). Thus, in a similar manner the current series of studies has shown similar mixed results. That is, significant effects were seen in some cases and non-significant effects were seen in others. However, in general we saw significant improvements in cognitive function by $\mathrm{MgCl}_{2}$ in each of our studies. The discrepant results mainly varied based on dose and task-dependent properties of the studies.

From a mechanistic standpoint, $\mathrm{Mg}^{2+}$ therapy has multiple routes by which it can disrupt the pathophysiological processes that occur following injury and enhance cognitive recovery. In addition to offsetting injury-induced $\mathrm{Mg}^{2+}$ 
depletion (McIntosh et al., 1988; Vink et al., 1988) and preventing excitotoxic neuronal death (Nowak et al., 1984) mediated by the NMDA receptor, $\mathrm{Mg}^{2+}$ has been shown to have several other effects. For instance, administration of $\mathrm{MgSO}_{4}$ has been shown to limit the generation of injury-induced edema following closed-head injury (Feldman et al., 1996) and it has been more recently shown that $\mathrm{MgSO}_{4}(30 \mathrm{mg} / \mathrm{kg}$ ) reduced aquaporin-4 immunoreactivity, thus contributing to edema reduction following injury (Ghabriel et al., 2006). Administration of $\mathrm{MgCl}_{2}$ has also been shown to reduce the expression of p53 mRNA, a gene associated with the induction of cell death, following lateral FPI (Muir et al., 1999). In this study it was found that $750 \mu \mathrm{mol} / \mathrm{kg}$ of $\mathrm{MgCl}_{2}$ reduced the expression of p53 mRNA in the injured cortex compared to saline-treated controls (Muir et al., 1999). High concentrations of $\mathrm{Mg}^{2+}(3 \mathrm{mM})$ have been shown to inhibit lipid peroxidation (Regan et al., 1998). Regardless of the mechanisms of action, the data presented in this review has shown that $\mathrm{MgCl}_{2}$ has strong biological activity, appears to cross the $\mathrm{BBB}$, and can improve cognitive performance following cortical ablation or TBI.

\section{Conclusion}

The studies presented in this book chapter have demonstrated a wide range of activities for $\mathrm{Mg}^{2+}$ therapy in relationship to cognitive function in

\section{References}

Barbagallo M, Dominguez LJ (2010) Magnesium and aging. Curr Pharm Des 16:832-9.

Bardgett ME, Schultheis PJ, McGill DL, Richmond RE, Wagge JR (2005) Magnesium deficiency impairs fear conditioning in mice. Brain Res 1038:100-6.

Bardgett ME, Schultheis PJ, Muzny A, Riddle MD, Wagge JR (2007) Magnesium deficiency reduces fearinduced conditional lick suppression in mice. Magnes Res 20:58-65.

Bareyre FM, Saatman KE, Helfaer MA, Sinson GP, Weisser JD, Brown AL, Mclntosh TK (1999) Alterations in ionized and total blood magnesium after the rodent. Daily injections of $\mathrm{MgCl}_{2}$ prior to the acquisition of a learning task blocked the acquisition of a reference memory task and dietary deficiency of $\mathrm{Mg}^{2+}$ impaired learning on a number of different tasks. Using the damaged brain as a model to examine the ability of $\mathrm{Mg}^{2+}$ therapy to improve cognitive performance also demonstrated significant advantages with the therapy. Thus, it does appear that $\mathrm{Mg}^{2+}$ status and therapy have significant effects on cognitive performance in the brain and that further research is warranted. In addition, an accumulating body of research suggests that $\mathrm{Mg}^{2+}$ also plays an important role in human cognitive performance. Given the reported rates of magnesium deficiency in humans it is likely that this could impair cognitive performance (Barbagallo and Dominguez, 2010; Elin, 2010). In addition, the most intriguing factor related to $\mathrm{Mg}^{2+}$ therapy for cognitive wellbeing may reside in the use of $\mathrm{Mg}^{2+}$ as a co-therapy with other vital nutrients, which may produce the strongest effects.

\section{Acknowledgements}

A special thanks is given to Alicia Swan for critical reading of an earlier draft of this chapter. Partial support provided by ARRA funds from NINDS grant NS045647-04 experimental traumatic brain injury: Relationship to neurobehavioral outcome and neuroprotective efficacy of magnesium chloride. J Neurochem 73:27180.

Billard JM (2006) Ageing, hippocampal synaptic activity and magnesium. Magnes Res 19:199-215.

Browne KD, Leoni MJ, Iwata A, Chen XH, Smith DH (2004) Acute treatment with MgSO4 attenuates longterm hippocampal tissue loss after brain trauma in the rat. J Neurosci Res 77:878-83.

Elin RJ (2010) Assessment of magnesium status for diagnosis and therapy. Magnes Res. 23:194-8. 
Enomoto T, Osugi T, Satoh H, Mclntosh TK, Nabeshima $T$ (2005) Pre-Injury magnesium treatment prevents traumatic brain injury-induced hippocampal ERK activation, neuronal loss, and cognitive dysfunction in the radial-arm maze test. J Neurotrauma 22:783-92.

Feldman Z, Gurevitch B, Artru AA, Oppenheim A, Shohami E, Reichenthal E, Shapira Y (1996) Effect of magnesium given 1 hour after head trauma on brain edema and neurological outcome. J Neurosurg 85:1317.

Ghabriel MN, Thomas A, Vink R (2006) Magnesium restores altered aquaporin-4 immunoreactivity following traumatic brain injury to a pre-injury state. Acta Neurochir Suppl 96:402-6.

Gómez-Pinilla F (2008) Brain foods: The effects of nutrients on brain function. Nat Rev Neurosci 9:56878.

Hallack M, Berman RF, Ortemkauf SM, Evans MI, Cotton DB (1992) Peripheral magnesium sulfate enters the brain and increases the threshold for hippocampal seizures in rats. Am J Obstet Gynecol 167, 1605-10.

Hoane MR (2004) Magnesium therapy and recovery of function in experimental models of brain injury and neurodegenerative disease. Clin Calcium 14:65-70.

Hoane MR (2005) Treatment with magnesium improves reference memory but not working memory while reducing GFAP expression following traumatic brain injury. Restor Neurol Neurosci 23:67-77.

Hoane MR (2007) Assessment of cognitive function following magnesium therapy in the traumatically injured brain. Magnes Res 20:229-36.

Hoane MR, Barth TM (2001) The behavioral and anatomical effects of $\mathrm{MgCl}_{2}$ therapy in an electrolytic lesion model of cortical injury in the rat. Magnes Res 14:51-63.

Hoane MR, Kaufman NA, Vitek MP, McKenna SE (2009) COG1410 improves cognitive performance and reduces cortical neuronal loss in the traumatically injured brain. J Neurotrauma 26:1-10.

Hoane MR, Knotts AA, Akstulewicz SL, Aquilano M, Means LW (2003) The behavioral effects of magnesium therapy on recovery of function following bilateral anterior medial cortex lesions in the rat. Brain Res Bull 60:105-14.

Hoane MR, Wolyniak J, Akstulewicz SL (2005) Administration of riboflavin improves behavioral outcome and reduces edema formation and GFAP expression following traumatic brain injury. $J$ Neurotrauma 22:1112-22.

Huskisson E, Maggini S, Ruf M (2007) The influence of micronutrients on cognitive function and performance. J Int Med Res 35:1-19.

Kaufman NA, Beare JE, Tan AA, Vitek MP, McKenna SE, Hoane MR (2010) COG1410, an apolipoprotein Ebased peptide, improves cognitive performance and reduces cortical loss following moderate fluid percussion injury in the rat. Behav Brain Res 214:395401.

Kesner RP, Dakis M, Bolland BL (1993) Phencyclidine disrupts long- but not short-term memory within a spatial learning task. Psychopharmacology 111:85-90.

Lindner MD (1997) Reliability, distribution, and validity of age-related cognitive deficits in the Morris water maze. Neurobiol Learn Mem 68:203-20.

Lindner MD, Plone MA, Cain CK, Frydel BR, Francis JM, Emerich DF, Sutton RL (1998) Dissociable long-term cognitive deficits after frontal versus sensorimotor cortical contusions. J Neurotrauma 15:199-216.

McIntosh TK, Faden Al, Yamakami I, Vink R (1988) Magnesium deficiency exacerbates and pretreatment improves outcome following traumatic brain injury in rats: ${ }^{31} \mathrm{P}$ magnetic resonance spectroscopy and behavioral studies. J Neurotrauma 5:17-31.

McLamb RL, Williams LR, Nanry KP, Wilson WA, Tilson HA (1990) MK-801 impedes the acquisition of a spatial memory task in rats. Pharmacol Biochem Behav 37:415.

Muir JK, Raghupathi R, Emery DL, Bareyre FM, Mclntosh TK (1999) Postinjury magnesium treatment attenuates traumatic brain injury-induced cortical induction of p53 mRNA in rats. Exp Neurol 159:584-93.

Murray TK, Ridley RM (1997) The effect of dizocilpine (MK-801) on conditional discrimination learning in the rat. Behav Pharmacol 8:383-8.

Nowak L, Bregestovski P, Ascher P, Herbert A, Prochiantz A (1984) Magnesium gates glutamate activated channels in mouse central neurones. Nature 307:462-5.

Pussinen R, Sirvio J (1999) Effects of D-cycloserine, a positive modulator of $\mathrm{N}$-methyl-D-aspartate receptors, and ST 587, a putative alpha-1 adrenergic agonist, individually and in combination, on the non-delayed and delayed foraging behaviour of rats assessed in the radial arm maze. J Psychopharmacol 13:171-9. 
Quigley A, Tan AA, Hoane MR (2009) The effects of hypertonic saline and nicotinamide on sensorimotor and cognitive function following cortical contusion injury in the rat. Brain Res 1304:138-48.

Regan RF, Jasper E, Guo Y, Panter SS (1998) The effect of magnesium on oxidative neuronal injury in vitro. $J$ Neurochem 70:77-85.

\section{Saad L, Silva L, Banzato C, Dantas C, Garcia C (2010)} Anorexia nervosa and Wernicke-Korsakoff syndrome: a case report. J Med Case Reports 4:1-5.

Sen AP, Gulati A (2010) Use of magnesium in traumatic brain injury. Neurotherapeutics 7:91-9.

Slutsky I, Abumaria N, Wu LJ, Huang C, Zhang L, Li B, Zhao X, Govindarajan A, Zhao MG, Zhuo M, Tonegawa S, Liu G (2010) Enhancement of learning and memory by elevating brain magnesium. Neuron 65:165-77.

Smith DH, Okiyama K, Gennarelli TA, Mclntosh TK (1993) Magnesium and ketamine attenuate cognitive dysfunction following experimental brain injury. Neurosci Lett 157:211-4.
Van Den Heuvel C, Vink R (2004) The role of magnesium in traumatic brain injury. Clin Calcium 14:9-14.

Vink R, Cook NL, van de Heuvel C (2009) Magnesium in acute and chronic brain injury: an update. Magnes Res 22:158S-62S.

Vink R, McIntosh TK, Demediuk P, Weiner MW, Faden Al (1988) Decline in intracellular free $\mathrm{Mg}^{2+}$ is associated with irreversible tissue injury after brain trauma. J Biol Chem 263:757-61.

Vink R, O'Connor CA, Nimmo AJ, Heath DL (2003) Magnesium attenuates persistent functional deficits following diffuse traumatic brain injury in rats. Neurosci Lett 336:41-4.

Wang C-T, Li Y, Wang F-J, Shi Y-M, Lee B-T (2008) Correlation between iron, magnesium, potassium and zinc content in adolescent girl's hair and their academic records. Chang Gung Med J 31:358-62. 


\title{
Magnesium in headache
}

\author{
Lisa A. Yablon ${ }^{\varpi}$ and Alexander Mauskop
}

The New York Headache Center, 30 East 76th Street, New York, NY 10021, USA.

$\square$ drmauskop@nyheadache.com

\begin{abstract}
Magnesium's role in migraine pathogenesis is well-described, with deficiencies known to promote cortical spreading depression, alter nociceptive processing and neurotransmitter release, and encourage the hyperaggregation of platelets, all major elements of migraine development. Research on magnesium has found it to be a potentially well-tolerated, safe and inexpensive option for migraine prevention, while it may also be effective as an acute treatment option for headaches including migraines, tensiontype headaches and cluster headaches, particularly in certain patient subsets. This chapter will review the various aspects of migraine in which magnesium plays a part, as well as numerous studies on the use of magnesium in both headache prophylaxis and in the acute treatment of headaches, offering recommendations in its use in clinical practice.
\end{abstract}

\section{Magnesium in the Body}

Magnesium (Mg), the second most abundant intracellular divalent cation, is a cofactor of many enzymes and is involved in a plethora of cellular functions. It plays a central role in both glucose metabolism and in ATP function. Over 300 enzymes require the presence of magnesium ions for their catalytic action, including all enzymes utilizing or synthesizing ATP, or those that use other nucleotides to synthesize DNA and RNA. ATP exists in cells as a chelate of ATP and a magnesium ion. Because of the important interaction between phosphate and magnesium ions, magnesium ions are essential to the basic nucleic acid chemistry of life, and thus are essential to all cells of all known living organisms.

Magnesium is involved in the formation of phospholipids and the insertion of proteins into the phospholipid membrane, and is therefore critical to membrane stabilization (Durlach et al., 1987). It also contributes to contraction of the cytoskeleton at the myoneural junction, playing a vital role in the function of both skeletal, cardiac and other smooth muscles.

Magnesium is absorbed in the gastrointestinal tract, via intestinal epithelial channels in the ileum as well as by the renal system's thick ascending limb, distal tubule, and loop of Henle of the nephron (Wagner, 2007). It facilitates calcium absorption via the thick ascending limb, and the absorption of both ions is regulated by the parathyroid hormone (PTH) secreting cells of the parathyroid gland (Bapty et al., 1998). The calcium/magnesium sensing receptor within the parathyroid gland regulates absorption of both ions by detecting their levels in ionized form, and then controlling PTH secretion, thereby maintaining calcium homeostasis (Brown et al., 1993). Dietarily, absorption is affected by protein intake as well as phosphate, phytate and fat. Absorbed dietary magnesium is largely excreted through the urine, although most iatrogenically administered oral magnesium is excreted through the faeces.

Adult human bodies contain approximately 24 grams of magnesium, with $67 \%$ located in the skeleton, $31 \%$ intracellularly $(20 \%$ in skeletal muscle), and only 1-2\% extracellularly. Of this amount, one half is ionized, and $25-30 \%$ is protein bound. As a result, levels found on routine serum testing, which only reflects that magnesium found in the extracellular space, is not representative of true total body magnesium stores (Moe, 2008).

Serum levels are typically $0.7-1.0 \mathrm{mmol} / \mathrm{L}$ or $1.8-$ $2.4 \mathrm{mEq} / \mathrm{L}$. Serum magnesium levels may appear normal even in cases of underlying intracellular deficiency, and true hypomagnesemia is common, possibly due to decreased intake or 
absorption, increased loss via the urine or diarrhea, or genetic factors (Henrotte, 1982).

\section{Primary and Secondary Hypomagnesemia}

Familial hypomagnesemia with secondary hypocalcemia has been studied in various kindreds, and heredity has been found to be $X$ linked in some families, and autosomal recessive in others (Walder et al., 1997). There are currently more than 30 known mutations in the TRPM6 gene that are associated with familial hypomagnesemia and hypocalcemia. Another hereditary form of hypomagnesemia, tubular hypomagnesemia/hypokalemia with hypocalciuria (Gitelman's syndrome), is hypothesized to be due to two different types of genetic transmission, one autosomal recessive and one autosomal dominant with high phenotype variability (Bettinelli et al., 1995).

A population study in Germany found the prevalence of serum hypomagnesemia to be $14.5 \%$, with even higher frequencies in females (Schimatschek and Rempis, 2001). Additionally, chronic disease is associated with hypomagnesemia, including diabetes, asthma, cardiovascular disease, sickle cell anaemia, preeclampsia and eclampsia (Laires et al., 2004). 10$20 \%$ of hospitalized patients are deficient in magnesium, and up to $65 \%$ of patients in intensive care units are hypomagnesemic. Alcoholism is also associated with inadequate magnesium levels, in part due to poor nutrition (Bohmer and Mathiesen, 1982). It has also been implicated in patients with end-stage renal disease suffering from hemodialysis headache (Goksel et al., 2006), and is often seen in conjunction with electrolyte abnormalities including hypokalemia, hyponatremia, hypocalcemia and hypophosphatemia (Whang et al., 1985). A number of medications such as diuretics, aminoglycosides and digoxin are associated with hypomagnesemia, and patients with refractory hypocalcemia and hypokalemia should be evaluated for hypomagnesemia (Innerarity, 2000).

\section{Magnesium Imbalances}

Clinical symptoms of hypomagnesemia include hallucinations, depression, delirium, lethargy, weakness, paresthesias, tremors, premenstrual syndrome, cold extremities, leg or foot cramps, seizures, ventricular arrhythmias and congestive heart failure (Douban et al., 1996). However, since total body stores are not accurately represented by serum levels, routine blood testing and even erythrocyte $\mathrm{Mg}$ concentrations may reveal normal levels, particularly in patients with low free (ionized) magnesium levels. Urinary fractional excretion or the oral magnesium load test can estimate the total body magnesium status. Intravenous magnesium loading tests are likely the most accurate and practical assessment, whereby total excretion of urinary magnesium is calculated over a 24 hour period, following administration of a loading dose; a retention of $20 \%$ or more indicates deficiency (Arnaud, 2008).

Hypermagnesemia, on the other hand, is a rare condition due to the nephron's rapid response to increased levels. It usually develops only in people with kidney failure who are given magnesium salts or who take drugs that contain magnesium such as laxatives or antacids. Clinical symptoms include nausea, muscle weakness, lethargy, confusion, hypotension and arrhythmias. In mild cases, withdrawing magnesium supplementation is often sufficient. In more severe cases, intravenous calcium gluconate and diuretics or dialysis may be required.

\section{Magnesium Levels in Migraineurs}

For many decades, it was postulated that magnesium deficiency played a role in migraine pathogenesis. However, the lack of simple and reliable measures of magnesium levels prevented further research to prove this theory. While low serum, cerebrospinal fluid and cerebral tissue levels of magnesium have been found to be low in patients with migraine (Jain et al., 1985; Ramadan et al., 1989; Schoenen et al., 1991), these results have been inconsistent, with both normal and low levels detected in the same tissues of some patients. The variability of results may be due to the need to measure ionized magnesium as a true reflection of magnesium metabolism, and the development of an ion selective electrode for ionized magnesium in whole blood, serum, plasma and aqueous samples has made an accurate and rapid measurement of ionized magnesium levels possible (Altura et al., 1992). 
A study measuring ionized magnesium levels in 40 patients during an acute migraine attack found that $50 \%$ had levels below $0.54 \mathrm{mmol} / \mathrm{l}$ (normal adult range 0.54-0.65 mmol/l) (Mauskop et al., 1995), with all subjects having total serum magnesium levels within normal limits. Intravenous administration of $1 \mathrm{~g}$ of magnesium sulfate was most effective in those with low ionized magnesium, with $86 \%$ of patients reporting sustained pain relief over 24 hours in those found to have low serum ionized magnesium, while this was the case in only $16 \%$ of patients with normal levels. This finding was extended to patients with various headache types, including migraine without aura, cluster head-ache, chronic migraine and chronic tension type headache (Mauskop et al., 1996), with most patients demonstrating low ionized magnesium levels. In addition, high serum ionized calcium to magnesium ratios were found in all headache types except for in those patients with chronic tension-type headaches. Based on these findings, it has been suggested that tension type headache may possibly be discriminated from chronic migraine based on serum ionized magnesium levels (Mauskop et al., 1994).

\section{Migraine Pathogenesis}

Migraine is the most common form of disabling primary headache that afflicts patients, affecting approximately $12 \%$ of Western populations (Lipton et al., 2007). It is clear that there is genetic transmission of the disorder, although specific genes for most forms of migraine have not yet been identified. Additionally, although the exact etiology remains to be defined, current theories centre on hyperexcitability of the cortex and trigeminovascular complex. In migraineurs, headache triggers stimulate the release of neuropeptides from the trigeminovascular neurons, including calcitonin gene-related peptide and substance P. Additionally, stimulation of the trigeminal ganglion increases cerebral blood flow due to the release of vasoactive intestinal peptide by the facial nerve (Goadsby and Macdonald, 1985). The vasodilation is accompanied by mast cell degranulation, blood vessel edema and increased vascular permeability, resulting in meningeal neurogenic inflammation.

The trigeminal nerve transmits this information to the brainstem trigeminal nucleus caudalis, and then on to the thalamic nuclei and the cortex, where the pain is ultimately perceived (Moskowitz, 1984). Other structures, including the periaqueductal gray matter and the locus coeruleus and the dorsal raphe nuclei, modulate pain transmission and therefore its perception (Martin and Behbehani, 2001).

Recent research has elucidated the aura phase of migraine, which affects up to $5 \%$ of the adult population (Agostini and Aliprandi, 2006). Migraine aura is the presentation of characteristic neurological symptoms usually developing prior to the onset of the painful phase of a migraine headache, believed to be due to a phenomenon known as cortical spreading depression (CSD). CSD was originally described by Leao (1944) and is an intense depolarization of neuronal and glial membranes, with alterations in membrane resistance and ion flow. There is subsequent massive release of glutamate and potassium as well as an increase in intracellular sodium and calcium. This results in a strong wave of depolarization that spreads across contiguous neuronal tissue. It can be triggered by depolarization of a small region of brain tissue or by direct application of excitatory amino acids, and activation of the N-methyl-D aspartate (NMDA) receptor can evoke CSD (Gorji et al., 2001). There are characteristic alterations in cerebral blood flow, with an initial brief oligemia followed by a profound hyperemia, and a mild, long-lasting oligemia (Otori et al., 2003). Precisely how the aura phase of a migraine evolves into the painful phase remains unknown, and it has been theorized that it is due to the action of a number of inflammatory proteins including calcitonin gene-related peptide (CGRP), nitric oxide and vasoactive peptide (Goadsby et al., 1990), which feed into the trigeminal nerve and generate pial artery dilation and CSD, and, ultimately, headache.

Using phosphorus nuclear magnetic resonance spectroscopy (MRS), low levels of magnesium have been found in the cerebral tissue of some migraineurs both during attacks and interictally (Ramadan et al., 1989). Another study utilized the same technology to assess the brain cytosolic free magnesium concentration and free energy released by the hydrolysis of adenosine triphosphate, an index of cellular bioenergetics in both migraineurs and patients with cluster 
headaches (Lodi et al., 2001). Cytosolic free magnesium and the free energy released by the reaction of ATP hydrolysis were significantly reduced in the occipital lobes of patients with all types of migraine as well as in cluster headache patients. The authors of this study took these results to lend support to their hypothesis that the reduction of free magnesium in tissue with mitochondrial dysfunction is due to a bioenergetics deficit, as magnesium is essential for mitochondrial membrane stability and the coupling of oxidative phosphorylation. Phosphorus nuclear MRS demonstrated reduced magnesium in the occipital cortices of patients with hemiplegic migraine, with decreases correlating with the severity of neurological complaints (Boska et al., 2002). Interestingly, this study also found increased magnesium in the brains of patients with migraine without aura, which the authors attributed to a possible decrease in intracellular potassium, which may occur in neuronal tissue prone to hyper-excitability.

Various hypotheses abound as to the reason behind the hypomagnesemia observed in migraineurs. Some suggest that during a migraine headache, excessive amounts of magnesium are excreted due to stress. Others propose that stress triggers excretion of magnesium, with secondary hypomagnesemia causing a migraine (Durlach, 1976). During attacks as well as interictally, magnesium levels in both serum and saliva are decreased (Gallai et al., 1992), perhaps an indicator of low cerebral magnesium levels and therefore a decreased threshold for migraine development (Ramadan et al., 1989). Interictal studies on intracellular and serum levels in patients with migraines and tension-type headaches have shown inconsistent results. However a study of migraineurs utilizing the magnesium load test found that after loading with magnesium lactate, there was retention of the magnesium, suggesting a systemic deficiency. Further, interictal levels of erythrocyte magnesium are lower in both adult (Schoenen et al., 1991) and juvenile migraineurs with and without aura (Soriani et al., 1995). In support of these studies, one in which migraineurs with low erythrocyte magnesium levels as well as decreased ionized lymphoycyte magnesium levels were given mineral water containing magnesium over a two-week period. Both erythrocyte and lymphocyte magnesium levels rose (Thomas et al., 2000). These suggest that assays of erythrocyte magnesium may be a useful and easily available tool to assess magnesium deficiency in migraineurs.

\section{Magnesium in Migraine Pathogenesis}

Magnesium is believed to be involved in a number of the aspects of migraine pathophysiology, and deficiency has been linked to cortical spreading depression (Mody et al., 1987), platelet aggregation (Baudouin-Legros et al., 1986), release of substance $P$ (Weglicki and Phillips, 1992), neurotransmitter release (Coan and Collingridge, 1985) and vasoconstriction (Altura and Altura, 1982).

NMDA receptors are associated with nociception and the resulting neuroplastic changes in the trigeminal nociceptive neurons, as well as with regulation of cerebral blood flow (Foster and Fagg, 1987). Magnesium ions may block the NMDA receptor, thereby preventing calcium ions from moving intracellularly, and stopping the calcium's effects on neurons and cerebral vasculature (Coan and Collingridge, 1985). Decreased magnesium levels therefore facilitate the NMDA receptor, increasing its effects on CSD, as well as the effect of glutamate on the NMDA receptor. The NMDA receptor has been shown to have a part in both the initiation and spread of cortical depression (Ferrari, 1992). Magnesium has been shown to block CSD induced by glutamate, and CSD is more easily initiated with decreased magnesium levels (Mody et al., 1987).

Nitric oxide (NO) plays a role as a synaptic modulator, affecting nociceptive processing (Meller and Gebhart, 1993) in addition to its involvement in the regulation of blood flow both intracranially and extracranially. It augments the NMDA receptor-evoked currents, thereby facilitating glutaminergic transmission (Choi and Lipton, 2000), which, as previously discussed, can be inhibited by magnesium. Production of NO can be inhibited by decreased magnesium levels.

CGRP, a neuropeptide, is released from activated trigeminal sensory nerves, is involved in the dilation of intracranial blood vessels and may also increase nociceptive transmission in the brainstem and spinal cord. It is therefore believed to play a central part in the development of 
migraines. A positive correlation has been demonstrated between migraines and serum CGRP levels, and after the pain of a migraine subsides, levels are observed to return to normal (Coderre et al., 1993). It has hence been hypothesized that inhibition of the release of CGRP either centrally or from the trigeminal nerve, may inhibit intracranial vasodilation thereby aborting migraine attacks. CGRP antagonists lack vasoconstrictive properties and have an advantage over current acute migraine treatment, triptans, which are contraindicated in patients with cardiovascular risk factors due to their vasoconstrictive effects.

Circulating CGRP levels have been shown to be decreased by the administration of magnesium in patients with primary Raynaud's phenomenon (PRP). A study followed CGRP levels in 12 women with PRP and 12 controls before and after the administration of intravenous magnesium sulfate. While there was no significant difference between baseline circulating CGRP in the two groups, following the infusion of magnesium sulfate there was a significant reduction in CGRP levels in the women with PRP only as well as a significant increase in RBC magnesium levels in the women with PRP but not in the control subjects (Myrdal et al., 1994).

Serotonin, released from platelets during migraines, promotes cerebral vasoconstriction as well as triggering nausea and vomiting. Cerebral vascular muscle serotonin receptors may develop increased affinity if serum ionized magnesium falls and the ratio of serum ionized calcium to magnesium increases. This may lead to further cerebral vasoconstriction and facilitates the release of serotonin from neuronal storage sites (Altura and Turlapaty, 1982). Pretreatment with magnesium has been shown to reduce serotonininduced vasoconstriction (Goldstein and Zsoter, 1978).

\section{Oral Magnesium Supplementation}

A double-blind placebo-controlled study was performed in which 24 women with menstruallyrelated migraine were given supplementation with $360 \mathrm{mg}$ of magnesium pyrrolidone carboxylic acid, divided into 3 divided daily doses taken from ovulation to the first day of menstrual flow (Facchinetti et al., 1991). The treatment was well tolerated, with only one patient dropping out of the study due to side effects (magnesiuminduced diarrhoea), and significant reductions were observed in number of days of headache, total pain index and in Menstrual Distress Questionnaire score.

A larger double-blind, placebo-controlled, randomized study on 81 migraineurs receiving $600 \mathrm{mg}$ of trimagnesium dicitrate taken once daily, showed attack frequency reduction of $41.6 \%$ in the magnesium group and $15.8 \%$ in controls. Three patients dropped out of the study due to side effects, $18.6 \%$ of patients complained of diarrhoea and $4.7 \%$ of gastric irritation (Peikert et al., 1996).

A third placebo-controlled double-blind trial showed no effect of magnesium on migraine (Pfaffenrath et al., 1996), possibly attributable to the use of poorly-absorbed magnesium salt, as almost half of the treatment group complained of diarrhoea.

A recent double-blind placebo-controlled, randomized study investigated the effects of 600 $\mathrm{mg}$ of magnesium citrate supplementation per day in patients with migraine without aura. A combination of clinical assessments, visualevoked potentials (VEPs) and brain single-photon emission computerized tomography (SPECT) were performed to assess neurogenic and vascular mechanisms of action (Koseoglu et al., 2008). Supplementation was associated with significant decrease in migraine attack frequency and severity, as well as decreased P1 amplitude on VEPs, and increased cortical blood flow to the inferolateral temporal, inferofrontal and insular regions seen on SPECT. The authors suggested that magnesium may interfere with both neurogenic and vascular mechanisms of migraine and may hence be an effective prophylactic treatment. Other studies using SPECT have shown conflicting results (Ramadan et al., 1991; Ferrari et al., 1995; Olesen et al., 1982).

Women with menstrually-related migraine (MRM) may be particularly prone to developing magnesium deficiency. A prospective study involving 270 women, 61 of whom had MRM, showed that $45 \%$ had ionized magnesium deficiency during MRM attacks, 15\% during nonmenstrual attacks, $14 \%$ during menstruation 
without migraine and $15 \%$ between menstruation without migraine (Mauskop et al., 2002). Serum ionized calcium/magnesium levels were elevated in MRM, with normal ionized calcium levels.

Magnesium deficiency has also been found in paediatric migraine, with decreased levels of serum, RBC and mononuclear blood cell magnesium found in paediatric migraineurs with or without aura compared with patients with tension type headache and controls (Soriani et al., 1995; Mazzotta et al., 1999). Magnesium supplementation may be a safe and welltolerated option for migraine prophylaxis in the prevention of paediatric migraine. However, while a double-blind placebo-controlled, randomized trial of patients aged 3 to 17 years found a statistically significant downward trend in headache frequency in those treated with magnesium oxide, the difference in the slopes of the two lines was not statistically significant (Wang et al., 2003). It could therefore not be determined if oral magnesium was superior to placebo in preventing frequent migraines in children and adolescents.

While oral magnesium is generally well tolerated, the most prominent side-effect is diarrhoea. Magnesium toxicity leads to loss of deep tendon reflexes as well as generalized muscle weakness. Severe toxicity can manifest as cardiac muscle weakness, respiratory paralysis and death, and patients with renal disease are at greater risk for developing toxicity. Although the diarrhoea itself may prevent the development of toxicity, patients should be cautioned regarding excessive intake.

\section{Intravenous Magnesium}

Studies examining the use of intravenous magnesium in the treatment of acute migraine have been conflicting. A study on 40 patients with acute migraine attacks showed an $85 \%$ correlation between levels of serum ionized magnesium (measured during an attack) and clinical response to $1 \mathrm{~g}$ of intravenous magnesium sulphate (Mauskop et al., 1995). Although the study was neither double-blinded nor placebocontrolled, both researchers and subjects were blinded to ionized magnesium levels. A further study on various headache types found $1 \mathrm{~g}$ of magnesium sulphate to provide rapid relief in patients with low serum ionized magnesium levels (Mauskop et al., 1996).

A single-blind placebo-controlled, randomized trial involved 30 patients with migraines who were randomized to receive either magnesium sulphate $1 \mathrm{~g}$ or placebo (Demirkaya et al., 2001). After 30 minutes, patients in the placebo group who had ongoing pain, nausea or vomiting were given magnesium sulphate $1 \mathrm{~g}$. Treatment was superior to placebo in terms of both response rate $(100 \%$ for magnesium sulphate vs $7 \%$ for placebo) and pain-free rate $(87 \%$ for magnesium sulphate vs $0 \%$ for placebo) and those treated did not experience headache recurrence within 24 hours. $87 \%$ complained of flushing or a burning sensation in the face and neck.

A double-blind placebo-controlled, randomized study evaluated the efficacy of magnesium sulphate $1 \mathrm{~g}$ on the pain and associated symptoms of migraine with and without aura (Bigal et al., 2002). In subjects with migraine without aura, although there was a significant decrease in the intensity of photophobia and phonophobia, no significant differences were observed in pain relief or nausea. However subjects with migraine with aura had significant improvement in pain and all associated symptoms.

An emergency room-based double-blind placebocontrolled, randomized study of 44 subjects with acute migraines tested a combination of magnesium sulphate $2 \mathrm{~g}$ and metoclopramide $20 \mathrm{mg}$ versus metoclopramide $20 \mathrm{mg}$ alone at 15 minute intervals for up to 3 doses, or until pain relief occurred (Corbo et al., 2001). Pain intensity was recorded using a standard visual analogue scale (VAS). Although both groups experienced more than $50 \mathrm{~mm}$ improvement in the VAS score, improvement was smaller in the magnesium group, both comparing VAS score improvements and evaluating normal functional status. The authors suggested that magnesium may diminish the efficacy of metoclopramide in decreasing migraine pain.

A study involving 22 patients with cluster headaches who were treated with magnesium sulphate $1 \mathrm{~g}$ found that $41 \%$ of patients reported 'meaningful relief' (defined as complete cessation 
of attacks or relief for more than 3 days) after treatment (Mauskop et al., 1995).

\section{Conclusion}

Magnesium is central to numerous physiological functions, and the role it plays in the various aspects of migraine pathogenesis is well described. Although some studies have shown an association between migraines and magnesium deficiency, it is difficult to assess this with routine blood testing and serum magnesium levels are a poor reflection of body stores of the cation. Therefore, treatment should be based on clinical suspicion, with both oral and intravenous magnesium available as simple, safe, inexpensive

\section{References}

Agostini E, Aliprandi A (2006) The complications of migraine with aura. Neurol Sci 27(Suppl 2): S91-5.

Altura BT, Altura BM (1982) The role of magnesium in etiology of strokes and cerebral vasospasm.

Magnesium 1:277-91.

Altura BM, Turlapaty PDMV (1982) Withdrawal of magnesium enhances coronary atrerial spasm produced by vasoactive agents. Br J Pharmacol 77:649-59.

Altura BT, Shirley TL, Young CC, Dell'Ofrano K, Hiti J, Welsh R, Yeh Q, Barbour RL, Altura BM (1994) Characterization of a new ion selective electrode for ionized magnesium in whole blood, plasma, serum and aqueous samples. Scan J Clin Lab Invest 54 (Suppl. 217):21-36.

Altura BT, Shirley T, Young CC, Dell-Ofrano K, Handwerker SM, Altura BM (1992) A new method for the rapid determination of ionized $\mathrm{Mg}^{2+}$ in whole blood, serum and plasma. Meth Find Exp Clin Pharmacol 14:297-304.

Arnaud MJ (2008) Update on the assessment of magnesium status. Br J Nutr 99 (Suppl 3):S24-S36.

Bapty BW, Dai L, Ritchie G, Jirik F, Canaff L, Hendy GN, Quamme GA (1998) Activation of $\mathrm{Mg}^{2+} \mathrm{Ca}^{2+}$ sensing inhibits hormone-stimulated $\mathrm{Mg}^{2+}$ uptake in mouse distal convoluted tubule cells. Am J Physiol 275:F35360. and well-tolerated options for the management of migraines. In patients with symptoms suggestive of hypomagnesemia such as premenstrual syndrome, cold extremities and foot or leg cramps, we suggest daily magnesium supplementation with $400 \mathrm{mg}$ of chelated magnesium, magnesium oxide or slow-release magnesium. While some patients may require doses of up to $1000 \mathrm{mg}$, diarrhoea and abdominal pain may be limiting factors. Intravenous magnesium may be used in patients who are unable to tolerate or absorb oral magnesium or who are non-compliant with daily dosing. It may also be used for the treatment of acute migraines, or as a monthly prophylactic infusion, often administered premenstrually.

Baudouin-Legros N, Dard B, Guicheney P (1986) Hyperactivity of platelets from spontaneously hypertensive rats. Role of external magnesium. Hypertension 8:694-9.

Bettinelli A, Bianchetti MG, Borella P, Volpini E, Metta MG, Basilico E, Selicorni A, Bargellini A, Grassi MR (1995) Genetic heterogeneity in tubular hypomagnesemia-hypokalemia with hypocalciuria (Gitelman's syndrome). Kidney Int 47:547-51.

Bigal ME, Bordini CA, Tepper SJ, Speciali JG (2002) Intravenous magnesium sulphate in the acute treatment of migraine without aura and migraine with aura. A randomized, double-blind, placebo-controlled study. Cephalalgia 22:345-53.

Bohmer T, Mathiesen B (1982) Magnesium deficiency in chronic alcoholic patients uncovered by an intravenous loading test. Scand J Clin Lab Invest 42:633-6.

Boska MD, Welch KMA, Barker PB, Nelson JA, Schultz L (2002) Contrasts in cortical magnesium, phospholipid and energy metabolism between migraine syndromes. Neurology 58:1227-33.

Brown EM, Gamba G, Riccardi D, Lombardi M, Butters R, Kifor O, Sun A, Hediger MA, Lytton J, Hebert SC (1993) Cloning and characterization of an extracellular $\mathrm{Ca}^{2+}$-sensing receptor from bovine parathyroid. Nature 366:575-80. 
Choi YB, Lipton SA (2000) Redox modulation of the NMDA receptor. Sal Mol Life Sci 57:1535-41.

Coan EJ, Collingridge GL (1985) Magnesium ions block an N-methyl-D-aspartate-mediated component of synaptic transmission in rat hippocampus. Neurosci Lett 53:21-6.

Coderre TI, Katz J, Faccerino AL, Melzack R (1993) Contribution of central neuroplasticity to pathological pain: review of clinical and experimental evidence. Pain 52:259-85.

Corbo J, Esses D, Bijur PE, lannaccone R, Gallagher EJ (2001) Randomized clinical trial of intraveous magnesium sulfate as an adjunctive medication for emergency department treatment of migraine headache. Ann Emerg Med 38:621-7.

Demirkaya S, Vural O, Dora B, Topcuoglu MA (2001) Efficacy of intravenous magnesium sulfate in the treatment of acute migraine attacks. Headache 41:171-7.

Douban S, Brodsky MA, Whang DD, Whang R (1996) Significance of magnesium in congestive heart failure. Am Heart J Sep 132:664-71.

Durlach J (1976) Neurological manifestations of magnesium imbalance. In: Handbook of Clinical Neurology (Vinken PJ, Bruyn GW, eds), Amsterdam, Holland, pp 454-579.

Durlach J, Poenaru S Rouhani S, Bara, M, Guiet-Bara, A (1987) The control of central neuronal hyperexcitability in magnesium deficiency. In: Nutrients and Brain Function (Essman WB, ed) Karger, Basel, Switzerland, pp 48-71.

Fachianetti F, Sances G, Borella P, Genazzeni AR, Nappi $G$ (1991) Magnesium prophylaxis of menstrual migraine: effects on intracellular magnesium. Headache 31:298-301.

Ferrari MD (1992) Biochemistry of migraine. Pathol Biol (Paris) 40:287-92.

Ferrari MD, Haan J, Blokland JA, Arndt JW, Minnee P, Zwinderman AH, Pauwels EK, Saxena PR (1995) Cerebral blood flow during migraine attacks without aura and effect of sumatriptan. Arch Neurol 52:135-9.

Foster AC, Fagg GE (1987) Neurobiology. Taking apart NMDA receptors. Nature 329:395-96.

Gallai V, Sarchielli P, Coata G, Firenze C, Morucci P, Abbritti G (1992) Serum and salivary magnesium levels in migraine: Results in a group of juvenile patients. Headache 32:132-53.

Goadsby PJ, Edvinsson L, Elkman R (1990) Vasoactive peptide release in the extracerebral circulation of humans during migraine headache. Ann Neurol 28:183-7.

Goadsby PJ, Macdonald GJ (1985) Extracranial vasodilatation mediated by VIP (Vasoactive Polypeptide). Brain Res 329:285-8.

Goksel BK, Torun D, Karaca S, Karatas M, Tan M, Sezgin N, Benli S, Sezer S, Ozdemir, N (2006) Is low blood magnesium level associated with hemodialysis headache? Headache 46:40-5.

Goldstein S, Zsoter TT (1978) The effect of magnesium on the response of smooth muscle to 5hydroxytryptamine. Br J Pharmacol 62:507-14.

Gorji A, Scheller D, Straub H, Tegtmeier F, Kohling R, Hohling JM, Tuxhorn I, Ebner A, Wolf P, Werner Panneck H, Oppel F, Speckmann EJ (2001) Spreading depression in human neocortical slices. Brain Res 906:74-83.

Henrotte JG (1982) Genetic regulation of red blood cell magnesium content and major histocompatibility complex. Magnesium 1:69-80.

Innerarity S (2000) Hypomagnesemia in acute and chronic illness. Crit Care Nurs 23:1-19.

Jain AC, Sethi NC, Balbar PK (1985) A clinical electroencephalographic and trace element study with special reference to zinc, copper and magnesium in serum and cerebrospinal fluid (CSF) in cases of migraine. J Neurol Suppl 232:161.

Koseoglu E, Talashoglu A, Gonul AS, Kula M (2008) The effects of magnesium prophylaxis in migraine without aura. Mag Res 21:101-8.

Laires MJ, Monteiro CP, Bicho M (2004) Role of cellular magnesium in health and human disease. Front Biosci 1:262-76.

Leao AAP (1944) Spreading depression of activity in cerebral cortex. J Neurophysiol 7:159-390.

Lipton RB, Bigal ME, Diamon M. AMPP Advisory Group (2007) Migraine prevalence, disease burden, and the need for preventative therapy. Neurology 68:343-9. 
Lodi R, lotti S, Cortelli P, Pierangeli G, Cevoli S, Clementi V, Soriani S, Montagna P, Barbiroli B (2001) Deficient energy metabolism is associated with low free magnesium in the brains of patients with migraine and cluster headache. Brain Res Bull 54:437-41.

Martin VT, Behbehani MM (2001) Toward a rational understanding of migraine trigger factors. Med Clin North Am 85:911-41.

Mauskop A, Altura BT, Altura BM (2002) Serum ionized magnesium levels and serum ionized calcium/ionized magnesium ratios in women with menstrual migraine. Headache 42:242-8.

Mauskop A, Altura BT, Cracco RQ, Altura BM (1994) Chronic daily headache - one disease or two? Diagnostic role of serum ionized magnesium. Cephalalgia 14:24-8.

Mauskop A, Altura BT, Cracco RQ, Altura BM (1996) Intravenous magnesium sulfate rapidly alleviates headaches of various types. Headache 36:156-60.

Mauskop A, Altura BT, Cracco RQ, Altura BM (1995) Intravenous magnesium sulphate relieves cluster headaches in patients with low serum ionized magnesium levels. Headache 35:597-600.

Mauskop A, Altura BT, Cracco RQ, Altura BM (1995) Intravenous magnesium sulphate relieves migraine attacks in patients with low serum ionized magnesium levels: a pilot study. Clin Sci 89:633-6.

Mazzotta G, Strachielli P, Alberti A, Gallai V (1999) Intracellular $\mathrm{Mg}^{2+}$ concentration and electromyographical ischemic test in juvenile headache. Cephalalgia 19:802-9.

Meller ST, Gebhart GF (1993) Nitric oxide (NO) and nociceptive processing in the spinal cord. Pain 52:12736.

Mody I, Lambert JD, Heinemann U (1987) Low extracellular magnesium induces epileptiform activity and spreading depression in rat hippocampal slices. $J$ Neurophysiol 57:869-88.

Moe SM (2008) Disorders involving calcium, phosphorous and magnesium. Prim Care 35:215-37.

Moskowitz MA (1984) The neurobiology of vascular head pain. Ann Neurol 16:157-68.

Myrdal U, Leppert J, Edvinsson L, Ekman R, Hedner T, Nilsson H, Ringqvist I (1994) Magnesium sulphate infusion decreases circulating calcitonin gene-related peptide (CGRP) in women with primary Raynaud's phenomenon. Clin Physiol 14:539-46.

Olesen J, Laurtizen M, Tfelt-Hansen P, Herriksen L, Larsen B (1982) Spreading cerebral oligemia in classical and normal cerebral blood flow in common migraine. Headache 22:242-8.

Otori T, Greenberg JH and Welsh FA (2003) Cortical spreading depression causes a long-lasting decrease in cerebral blood flow and induces tolerance to permanent focal ischemia in the rat brain. $J$ Cereb Blood Flow Metab 23:43-50.

Peikert A, Wilmzig C, Kohne-Volland R (1996)

Prophylaxis of migraine with oral magnesium: results from a prospective, multi-center, placebo-controlled and double-blind randomized study. Cephalalgia 16:257-63.

Pfaffenrath V, Wessley P, Meyer C, Isler HR, Evers S, Grotemeyer KH, Tanieri Z, Soyka D, Gobel H, Fisher M (1996) Magnesium in the prophylaxis of migraine: a double-bind, placebo-controlled study. Cephalalgia 16:436-40.

Ramadan NM, Halvorsan $\mathrm{H}$, Vande-Linde A, Levine SR, Helpern JA, Welch KMA (1989) Low brain magnesium in migraine. Headache 29:590-3.

Ramadan NM, Levine SR, Welch KMA (1991) Interictal cerebral blood flow asymmetries in migraine with aura. Cephalalgia 11(Suppl):42-3.

Schimatschek HF, Rempis R (2001) Prevalence of hypomagnesemia in an unselected German population of 16,000 individuals. Magnes Res 14:283-90.

Schoenen J, Sianard-Gainko J, Lenaerts M (1991) Blood magnesium levels in migraine. Cephalalgia 11:97-9.

Soriani S, Arnaldi C, De Carlo L, Arcudi D, Mazzotta D, Battistella P, Sartori S, Abbasciano V (1995) Serum and red blood cell magnesium levels in juvenile migraine patients. Headache 35:14-6.

Thomas J, Millot JM, Sebille S, Delabroise AM, Thomas E, Manfait M, Arnaud MJ (2000) Free and total magnesium in lymphocytes of migraine patients effects of magnesium-rich mineral water intake. Clin Chim Ecta 295:64-75.

Wagner CA (2007) Disorders of renal magnesium handling explain renal magnesium transport. J Nephrol 20:507-10. 
Walder RY, Shalev H, Brennan TMH, Carmi R, Elbeduor K, Scott DA, Hanauer A, Mark AL, Patil S, Stone EM, Sheffield VC (1997) Familial Hypomagnesemia Maps to Chromosome 9q, not to the X Chromosome: Genetic Linkage Mapping and Analysis of a Balanced

Translocation Breakpoint. Hum Mol Genet 6:1491-7.

Wang F, Van den Eeden SK, Ackerson LM, Salk SE, Reince RH, Elin RJ (2003). Oral magnesium oxide prophylaxis of frequent migrainous headache in children: a randomized, double-blind, placebocontrolled trial. Headache 43:601-10.
Weglicki WB, Phillips TM (1992) Pathobiology of magnesium deficiency: a cytokine/neurogenic inflammation hypothesis. Am J Physiol 263:R734-7.

Whang R, Oei TO, Watanabe A (1985) Frequency of hypomagnesemia in hospitalized patients receiving digitalis. Arch Intern Med 145:655-6. 


\title{
The role of magnesium in edema and blood brain barrier disruption
}

\author{
Mehmet Kaya ${ }^{\bowtie}$ and Bulent Ahishali \\ Departments of Physiology and of Histology and Embryology, Istanbul Faculty of Medicine, Istanbul University, Capa 34093 \\ Istanbul, Turkey. \\ $\triangle$ mehkaya@istanbul.edu.tr
}

\begin{abstract}
The blood-brain barrier (BBB) is constituted primarily of brain capillary endothelial cells and is a prerequisite for the maintenance of brain homeostasis that is essential for optimal brain function. However, a variety of pathological conditions, such as sepsis, multiple sclerosis and epilepsia disrupt the BBB integrity and lead to the development of brain edema. Ionized magnesium $\left(\mathrm{Mg}^{2+}\right)$ is a crucial cofactor that plays an essential role within the cell and regulates a variety of biochemical reactions. Changes in intra- and extracellular $\mathrm{Mg}^{2+}$ concentrations influence the functions of cells and tissues. A growing body of evidence suggests that $\mathrm{Mg}^{2+}$ plays a pivotal role in ameliorating BBB disruption via a number of mechanisms during certain neurological diseases. Systemic delivery of $\mathrm{Mg}^{2+}$ may constitute an alternative approach in the future, both to improve BBB integrity and to decrease brain edema in the course of a variety of diseases involving brain tissue.
\end{abstract}

\section{Introduction}

\section{Blood-Brain Barrier}

The blood-brain barrier (BBB) is composed mainly of brain capillary endothelial cells and represents a dynamic structure that regulates the trafficking of molecules between blood and brain tissue. The passage of many circulating substances from the capillary bed into the brain parenchyma is tightly controlled by physical and enzymatic barriers provided by the endothelial cells of capillaries in the brain parenchyma (Abbott et al., 2010; Cardoso et al., 2010). In this way, the BBB is equipped with regulating means that enable the maintenance of neuronal homeostasis. In addition, the BBB harbours transport mechanisms that provide bidirectional control of exchange of nutrients, electrolytes and neurotoxins, and thus establishes an optimum milieu that is strictly essential to neuronal survival. Although the BBB appears to possess a static structure, it has the ability to adapt readily to sudden changes.

About $95 \%$ of the microvessels in the brain display BBB properties, and almost every neuron is estimated to be nourished by a distinct capillary vessel (Pardridge, 2005). When regulating its own activities, the mature BBB receives support from at least three different cell types: 1) pericytes which share the same basement membrane with endothelial cells; 2) astrocytes which envelope
$99 \%$ of the abluminal face of endothelial cells; and 3) neurons (Guo and Lo, 2009; Correale and Villa, 2009). Nevertheless, under normal conditions, BBB function is regulated primarily by capillary endothelial cells (Fisher, 2009; Abbott et al., 2010).

In brain tissue, the barrier-type endothelial cells have a continuous basal membrane and do not exhibit fenestrations. These cells contain many mitochondria, but harbour very few caveola (pinocytotic vesicles) in their luminal surfaces. Tight junctions (TJs) between adjacent brain capillary endothelial cells possess occludin and claudin proteins that serve to preserve junctional integrity. Meanwhile, zonula occludens (ZO)-1 and ZO-2, cingulin, cadherin, cathenin, vinculin and actin constitute accessory proteins that aid in the assembly of TJs.

Passive diffusion across the BBB mainly depends on the lipid solubility and molecular weight of molecules. Lipophilic substances with molecular weights less than 400-600 Da can pass readily into the brain tissue by passive diffusion. In the normally functioning BBB, oxygen, carbon dioxide, nicotine, ethanol, lipid-soluble substances such as heroin, and amphiphilic drugs (containing both hydrophobic and hydrophilic moieties) are carried by this route. In addition, a number of molecules are transported across the 
BBB by other mechanisms including carrier- and receptor-mediated transport, adsorptive-mediated endocytosis and fluid phase-mediated endocytosis (Loscher and Potschka, 2005; Pardridge, 2007). However, many pathological conditions alter the functional and structural characteristics of the BBB impairing the maintenance of neural homeostasis.

\section{Magnesium Physiology}

Magnesium ion $\left(\mathrm{Mg}^{2+}\right)$, the most abundant divalent cation in living cells, exists not only in the intracellular compartment but also in circulation and cerebrospinal fluid (CSF). Serum $\mathrm{Mg}^{2+}$ concentration normally ranges between 1.7-2.3 $\mathrm{mEq} / \mathrm{L}$ in humans and may decrease during several pathological conditions (Romani and Scarpa, 2000; Musso, 2009). Cell membranes have a particularly low permeability to $\mathrm{Mg}^{2+}$ and hence the ion moves slowly between intracellular and interstitial compartments. Under physiological conditions, the $\mathrm{Mg}^{2+}$ concentration within the cell is maintained in a relatively narrow range between 0.5 and $1 \mathrm{mM}$ (Dai and Quamme, 1991; Romani and Scarpa, 2000). $\mathrm{Mg}^{2+}$ acts as a regulatory cation at the systemic and cellular levels, and participates in almost all anabolic and catabolic processes in the body. It plays a fundamental role in a wide range of cellular events, biochemical reactions and physiological functions, by activating over 325 enzyme systems, including those involved in ATP synthesis, carbohydrate metabolism, $\mathrm{K}^{+}$and $\mathrm{Ca}^{2+}$ transport, cell proliferation and membrane stability and function (Grubbs and Maguire, 1987; Saris et al., 2000; Wolf and Trapani, 2008; Barbagallo and Dominguez, 2010).

During normal physiological processes, $\mathrm{Mg}^{2+}$ works as a voltage dependent antagonist and a noncompetitive inhibitor of the N-methyl-Daspartic acid (NMDA) receptors and ion channels in the brain. Although pharmacokinetic and pharmacodynamic studies in rats have shown $\mathrm{Mg}^{2+}$ entry into the brain upon systemic treatment (Hallak et al., 1992; Hoane, 2007), no concomitant rise of $\mathrm{Mg}^{2+}$ in CSF was noted following parenteral administration in humans with brain insults (McKee et al., 2005).

A reduction of $\mathrm{Mg}^{2+}$ level within the cell in pathological states can be considered as an injury factor in the brain and may lead to serious biological and metabolic dysfunction. Decline in intracellular free $\mathrm{Mg}^{2+}$ concentration reduces ATP synthesis, and utilization in the maintenance of ion gradients via the $\mathrm{Na}^{+}-\mathrm{K}^{+}$ATPase (Grubbs and Maguire, 1987). Also, the reduction in $\mathrm{Mg}^{2+}$ concentration within the cell results in an impairment of membrane stability by promoting free radical production (Ebel and Gunther, 1980; Bara and Guiet-Bara, 1984). Among the other events associated with $\mathrm{Mg}^{2+}$ deficiency in brain are opening of $\mathrm{Ca}^{2+}$ channels, cellular entry of $\mathrm{Ca}^{2+}$, release of certain neurotransmitters, activation of NMDA receptors, membrane oxidation and activation of nuclear factor kappa $\mathrm{B}\left(\mathrm{NF}_{\mathrm{K}} \mathrm{B}\right)$ (Weglicki et al., 1994; Altura et al., 2003; Billard, 2006; Rayssiguier et al., 2010). On the other hand, beneficial effects of magnesium supplementation have been shown in experimental models, and a variety of mechanistic pathways have been put forward including decrease in intracellular $\mathrm{Ca}^{2+}$ concentration, increase in antioxidant capacity and induction of endothelial cell proliferation (Kaya et al., 2001; 2004; Esen et al., 2005; Euser et al., 2008; Wolf et al., 2008; 2009).

\section{Brain edema and magnesium}

Two major types of brain edema, termed cytotoxic and vasogenic edema, were described by Klatzo in the late 1960s, and later two more types were added to the classification (Klatzo, 1967; Marmarou, 2004; Nag et al., 2009). Cytotoxic brain edema is characterized by sustained intracellular water accumulation, causing cellular injury in the absence of BBB damage and involving mainly astrocytes. On the other hand, vasogenic edema results in extracellular water accumulation in brain parenchyma through BBB disruption. The other types of edema are interstitial edema, which is observed in patients with hydrocephalus, and osmotic edema caused by imbalances of osmotically active substances, promoting water influx into cells.

Energy depletion followed by a failure of the $\mathrm{Na}^{+}-$ $\mathrm{K}^{+}$ATPase plays a major role in the pathogenesis of cytotoxic brain edema, and increased uptake of $\mathrm{Na}^{+}$into the cell cannot be equilibrated by the defective pump. Under certain pathological conditions, such as traumatic brain injury (TBI), cerebral ischemia and acute hypertension, the 
brain edema, which is initially cytotoxic, acquires a vasogenic character in the following stages. Brain edema caused by trauma has also been proposed to be mainly vasogenic in nature due to the opening of TJs in the BBB (Unterberg et al., 2004). Magnesium supplementation has been reported to decrease regional brain tissue water content and attenuate brain edema formation after experimental TBI (Okiyama et al., 1995; Feldman et al., 1996). In addition, magnesium treatment protects the blood-spinal cord barrier, improves clinical recovery, and preserves normal spinal cord ultrastructure in experimental spinal cord injury in rats (Kaptanoglu et al., 2003).

Aquaporin (AQP)-4, a bidirectional transmembrane water channel expressed mainly in astrocytes and to a lesser extent in barrier type of endothelial cells and pial membranes, may play a crucial role in the pathogenesis of cytotoxic and vasogenic brain edema and aggravation/resolution of ischemic and traumatic brain edema (AmiryMoghaddam et al., 2003; Zador et al., 2009). Experimental studies focusing on the treatment of brain edema showed beneficial effects of magnesium administered in combination with various pharmacological drugs in animal models (Royo et al., 2003; Sen and Gulati, 2010). Upregulation of AQP-4 in brain injury leads to an increase in brain water content, resulting in brain edema (Taniguchi et al., 2000; Papadopoulos and Verkman, 2005) and treatment with magnesium causes the down-regulation of AQP-4 (Ghabriel et al., 2006) and thereby attenuates brain edema (Okiyama et al., 1995).

It has been reported that $\mathrm{Mg}^{2+}$ exerts neuroprotective effects in an anoxic insult by improving the recovery of synaptic transmission and blocking the loss of protein kinase C (PKC) (Libien et al., 2005). These data are mechanistically consistent with the observation that the treatment of the astrocytes with a PKC activator caused a rapid decrease in AQP-4 mRNA and that this effect was inhibited by a specific PKC inhibitor (Nakahama et al., 1999). Among the other mechanisms put forward for the beneficial effects of $\mathrm{Mg}^{2+}$ in decreasing brain edema are restriction of the opening of paracellular pathways through $\mathrm{Ca}^{2+}$ antagonism, alleviation of the oxidative stress, and prevention of hypertensive encephalopathy through reduction in cerebral perfusion pressure (Belfort et al.,
2008; Euser and Cipolla 2009). $\mathrm{Mg}^{2+}$ has also been shown to reduce brain edema and protect brain morphology in experimental cold-injury by inhibition of lipid peroxidation (Turkoglu et al., 2008).

\section{Blood-brain barrier and magnesium}

$\mathrm{Mg}^{2+}$ is slowly transported across the BBB into the brain by transporters and exchangers located in endothelial cell membranes, including the $\mathrm{Na}^{+} / \mathrm{Mg}^{2+}$ exchanger, the $\mathrm{Mg}^{2+} / \mathrm{Ca}^{2+}$ exchanger and cation channels. Following systemic administration, regional increases in $\mathrm{Mg}^{2+}$ has been detected in the cerebral cortex and hippocampus in rats with an intact BBB (Hallak et al., 1992; Touyz, 2008). In both physiological and pathological conditions, $\mathrm{Mg}^{2+}$ can directly influence BBB properties. Low $\mathrm{Mg}^{2+}$ concentration in the circulation is associated with increase in endothelial permeability, decrease in vasodilator capacity and an increase in the production of vasoconstrictor substances, cytokines and oxidative products (Touyz, 2003; Maier et al., 2004). In pathological conditions with BBB impairment, $\mathrm{Mg}^{2+}$ passes into the extracellular compartment of the brain in significantly higher concentrations and plays an important role in the pathophysiological processes that follow BBB disruption. An elevation in free $\mathrm{Mg}^{2+}$ concentration in capillary endothelial cells increases the proliferation of endothelial cells, restores the cell's ability to generate and utilize ATP for cellular repair mechanisms, and improves disrupted BBB integrity in a variety of insults. In addition to the stimulatory effect of $\mathrm{Mg}^{2+}$ on endothelial cell migration and proliferation, the observation that high $\mathrm{Mg}^{2+}$ concentration facilitates the re-endothelialization of vascular injuries may also provide new insights into the role of $\mathrm{Mg}^{2+}$ in angiogenesis (Maier et al., 2004).

A decrease in $\mathrm{Mg}^{2+}$ level in the microcirculation of the cortical structures causes a rapid and progressive damage to microvessels, leading to focal haemorrhages and brain edema (Altura et al., 1991). Increasing brain bioavailability of parenterally administered magnesium by artificial BBB disruption has been considered as a necessary step in assessing the therapeutic benefits of magnesium supplementation after TBI (Sen and Gulati, 2010). Magnesium therapy has been shown to be effective in a variety of animal 
models of experimental BBB disruption. Accumulated data indicate that magnesium administration improves functional outcome following BBB disruption and decreases brain edema (Okiyama et al., 1995; Feldman et al., 1996; Kaya et al., 2004; Esen et al., 2005; Euser et al., 2008). Treatment with magnesium and MK801 (dizocilpine), a noncompetitive NMDA receptor antagonist, either alone or in combination, can reduce brain edema development and help to restore BBB permeability after experimental diffuse brain injury (Feng et al., 2004; Imer et al., 2009).

One of the major mechanisms responsible for the pharmacological action of $\mathrm{Mg}^{2+}$ is blockage of NMDA or alpha-amino-3-hydroxy-5-methyl isoxazole-4-proprionic acid (AMPA) channels/ receptors in cerebral vascular system as well as brain parenchyma (Huang et al., 1994). The observation that blockade of NMDA or AMPA receptors could attenuate $B B B$ disruption in focal cerebral ischemia suggest that ionotropic glutamate receptors are involved, at least partly, in BBB disruption (Liu et al., 2010). Magnesium can modulate hypoxic-ischemic events in the cerebral cortex by blocking the action of local putative excitatory amino acid neurotransmitters and consequently, high extracellular $\mathrm{Mg}^{2+}$ has been shown to be effective in blocking the pathophysiological mechanisms of rupture and spasm in the brain microvasculature (Huang et al., 1994; Chaon et al., 2006). Other possible mechanisms of action of $\mathrm{Mg}^{2+}$ in regulating vascular function involve its antioxidant, antiinflammatory, and growth regulatory properties via which burden of oxidative stress and inflammation in the endothelial cells of microvessels are attenuated (Weglicki et al., 1996; Mazur et al., 2007). In addition, treatment of human endothelial cells with magnesium has induced reduction of cellular pro-oxidant levels and diminished the release of pro-inflammatory cytokines (Wolf et al., 2008).

Although the effects of magnesium on BBB characteristics and the formation of brain edema in various pathophysiological conditions has not been thoroughly elucidated, the studies mentioned above suggest that magnesium provides protective effects on BBB integrity and reduces brain edema by more than one mechanism (Figure 1). Yet, further studies are still needed to more accurately assess the role of magnesium in the $\mathrm{BBB}$ response to various insults in humans and animals.

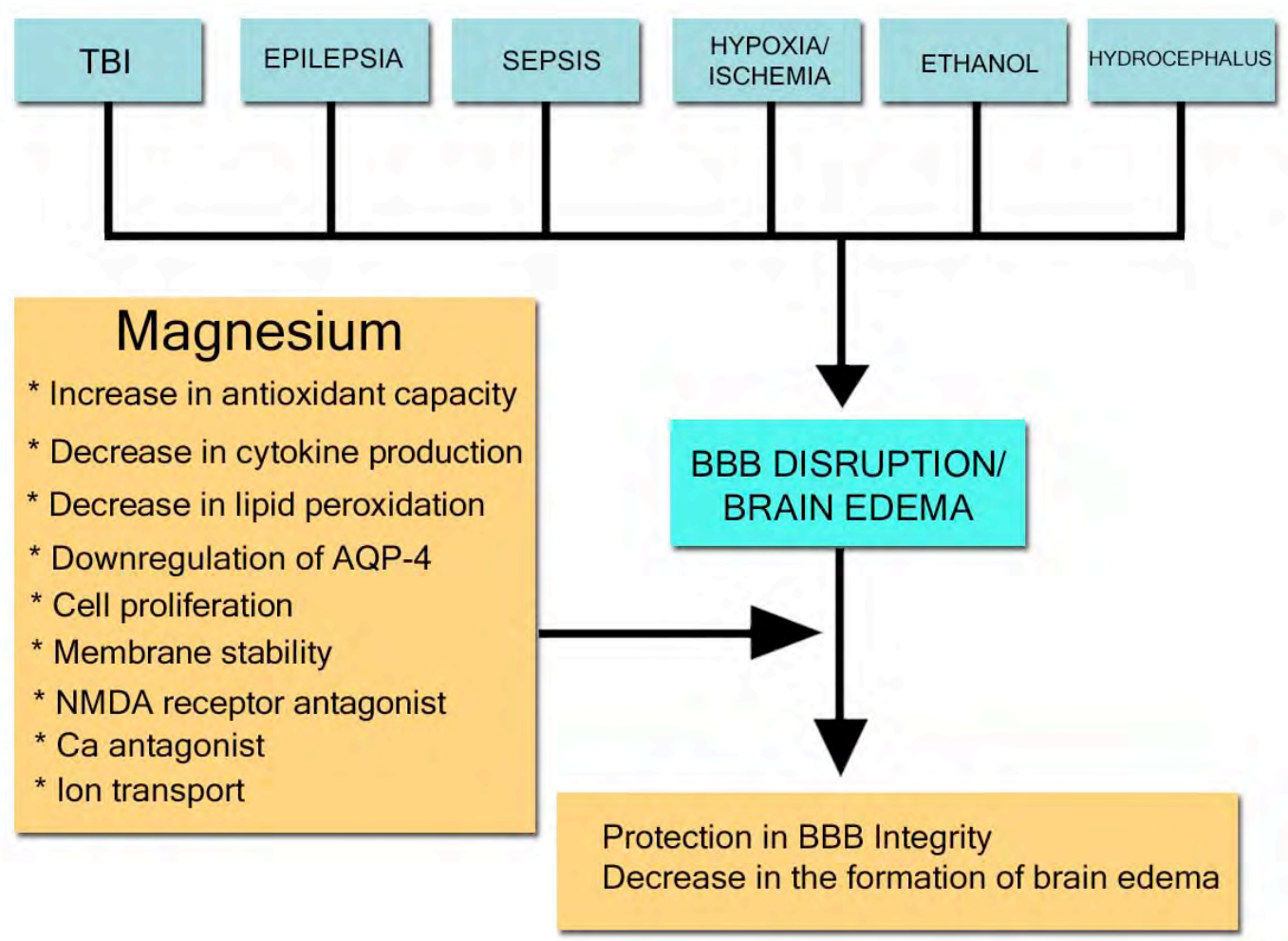

Figure 1. Effects of magnesium on BBB integrity. 


\section{Blood-brain barrier, magnesium and traumatic brain injury}

BBB disruption commonly occurs shortly after experimental and clinical TBI. Brain intracellular and extracellular $\mathrm{Mg}^{2+}$ concentrations, as well as serum $\mathrm{Mg}^{2+}$ levels, are decreased following central nervous system injury and a decline of $\mathrm{Mg}^{2+}$ concentration in brain can further increase the severity of BBB disruption and be a critical factor in the development of irreversible tissue damage (Vink et al., 1987; Vink and Cernak, 2000; Vink et al., 2009). Reduction in brain intracellular free $\mathrm{Mg}^{2+}$ is also associated with brain intracellular acidosis and a concomitant reduction of brain energy stores (Vink et al., 1988; Altura et al., 1995).

Magnesium salts, such as magnesium sulphate $\left(\mathrm{MgSO}_{4}\right)$ or magnesium chloride were shown to penetrate the BBB and cause enhancement of brain intracellular free $\mathrm{Mg}^{2+}$ concentration following TBI (Heath and Vink, 1999). Accumulated data from animal models indicates that administration of magnesium salts into the circulation or extracellular brain compartments can provide an effective therapy for TBI (Vink and Cernak, 2000; Saatman et al., 2001; Hoane, 2007) by improving $\mathrm{BBB}$ integrity and decreasing brain edema (Esen et al., 2003). In contrast to the animal studies mentioned above, McKee and colleagues (McKee et al., 2005) described the functional characteristics of the BBB in patients with TBI by using $\mathrm{MgSO}_{4}$ infusions initiated at an average of 5 days after injury. The authors showed that the increased serum $\mathrm{Mg}^{2+}$ concentrations yielded only a marginal increase of total and ionized $\mathrm{Mg}^{2+}$ in CSF and they concluded that the regulation of magnesium by the constituents of the BBB remains largely intact following brain injuries. Meanwhile, in a doubleblinded trial conducted to check the validity of animal data in humans, and to explore whether magnesium infusion initiated within 8 hours of major head injury and continued for 5 days would decrease mortality and improve the functional outcome in head-injured patients, it was reported that there was no clinical suggestion of a beneficial effect of the magnesium regimen in these patients (Temkin et al., 2007).

Based on the above-mentioned data, it can be concluded that although there has not been a consensus between animal and human studies regarding the efficiency of magnesium administration in $\mathrm{TBI}$, it can be effective in the recovery of BBB damage at least in animal models.

\section{Blood-brain barrier, magnesium and seizures}

Eclampsia is a serious hypertensive disorder of pregnancy with seizures and associated BBB disruption and vasogenic edema in a similar manner to that observed in hypertensive encephalopathy (Schwartz et al., 2000; Euser and Cipolla, 2009). In animal models, magnesium treatment has been shown to contribute to the protection of the BBB during eclampsia, to decrease the increased $B B B$ permeability, and to prevent the development of brain edema in certain experimental settings, including acute hypertension and hypoglycemiainduced seizures (Kaya et al., 2001 and 2004; Euser et al., 2008). The above-mentioned beneficial effects of magnesium on BBB integrity may be related to its ability to scavenge free radicals. The antioxidant action of magnesium has been reported in two recent studies (Ariza et al., 2005; Turkoglu et al., 2008), suggesting that it could protect against free radical surge associated with epileptic seizures. However, the exact mechanism/s of action of magnesium treatment in the improvement of BBB integrity in eclampsia still remains to be elucidated.

\section{Blood-brain barrier, magnesium and sepsis}

Magnesium supplementation is one of the experimental methods and pharmacological approaches developed for the treatment of BBB disruption and brain edema caused by septic encephalopathy. The impairment of BBB integrity during sepsis has been shown in several studies, and magnesium administration in the early stages of sepsis reduced BBB permeability and brain edema (Papadopoulos et al., 2005; Esen et al., 2005). Magnesium deficiency leads to an elevation in plasma inflammatory cytokines and excessive production of free radicals, and aggravates endotoxic shock (Weglicki et al., 1994; Matsui et al., 2007). Treatment with magnesium decreases the concentration of inflammatory cytokines and free radicals and increases antioxidant capacity and survival rate in rats (Salem et al., 1995). Finally, alterations in endothelial cells, which are protagonists in the 
vascular changes during inflammation, are reversible upon magnesium supplementation (Mazur et al., 2007). Although the abovementioned studies suggest that magnesium is involved in the protection of the $\mathrm{BBB}$ and brain edema in sepsis, the literature data is quite limited at present and additional studies are needed to explain the pathophysiologic mechanisms involved in this protection.

\section{Blood-brain barrier, magnesium and brain hypoxia/ischemia}

Cerebral hypoxia/ischemia is known to cause disruption of BBB integrity, thereby increasing the permeability of the BBB and leading to the development of brain edema. Extracellular $\mathrm{Mg}^{2+}$ concentration has been shown to significantly decrease to approximately $60 \%$ of basal values in the ipsilateral cortex in hypoxia-ischemia (Lee et al., 2002). Meanwhile, magnesium deficient rats are more susceptible to cerebral hypoxia/ ischemia than rats fed with a normal or high magnesium diet (Demougeot et al., 2004). Magnesium administration significantly attenuates the hypoxia-induced increase in reactive oxygen species and contributes to the repair of the disrupted BBB in hypoxia/ischemia (Ravishankar et al., 2001; Goñi-de-Cerio et al., 2009). The protection of the BBB by magnesium in hypoxic conditions could be multifactorial and, in addition to the above mentioned effects, may involve other factors such as decrease in the production of cytokines, increase in antioxidative products and blockade of NMDA or AMPA channels/ receptors. There is at present only limited knowledge about the role of magnesium on BBB integrity and brain edema in hypoxia/ischemia and future research is needed to determine the possible mechanisms of magnesium supplementation in improving the functions of BBB.

\section{Blood-brain barrier, magnesium and ethanol}

Acute and chronic ethanol treatment gives rise to significant increases in BBB permeability to a variety of molecules that do not normally cross the BBB. Increase in free radical concentration in ethanol-treated endothelial cells leads to phosphorylation of TJ proteins, activation of paracellular pathway and thus disruption of the BBB (Haorah et al., 2005). A number of studies have provided evidence for the alterations in serum $\mathrm{Mg}^{2+}$ levels in acute and chronic ethanol treatments. A single dose of ethanol (2 g/ kg) injected to mice significantly decreased total $\mathrm{Mg}^{2+}$ concentration in serum (Papierkowski et al., 1998). Furthermore, acute or chronic alcohol consumption impairs $\mathrm{Mg}^{2+}$ transport and homeostasis at the capillary level in the brain (Romani, 2008). However, little is known about whether any improvement in BBB integrity can be achieved by magnesium treatment in acute or chronic ethanol intake. Therefore, this lack of basic knowledge compounds the difficulty we face when interpreting the importance of efforts of increasing serum $\mathrm{Mg}^{2+}$ levels under these conditions.

\section{Blood-brain barrier, magnesium and hydrocephalus}

Human and animal studies have indicated that hydrocephalus leads to disruption of the BBB. In a study involving a total of 21 patients with normalpressure hydrocephalus, a slight plasma-like protein pattern has been demonstrated in CSF in $38 \%$ of the patients prior to surgical intervention, indicating BBB dysfunction (Wikkels $\varnothing$ and Blomstrand, 1982). There is only one study in the current literature that evaluates the effects of magnesium in hydrocephalus, and a mild protection against brain damage was shown using $\mathrm{MgSO}_{4}$ therapy in a rat model of childhood-onset hydrocephalus (Khan et al., 2003). Further studies are necessary to increase our understanding of the effects of deficiency or supplementation of magnesium on the functional and structural characteristics of the BBB during hydrocephalus in both animal and human studies.

\section{Conclusion}

It is clear from the discussion above that magnesium plays a variety of essential roles within the cell by modulating the activity of more than 325 enzymes as a cofactor. These enzymes are important for the survival of various cell types including endothelial cells of the BBB. Meanwhile, magnesium deficiency leads to or worsens a variety of central nervous system pathologies by increasing inflammatory cytokines and reactive oxygen species and disturbing the activity of transporters in neurons, astrocytes, pericytes and capillary endothelial cells, which together constitute the neurovascular unit of the brain. 
Besides, the alterations in the $\mathrm{Mg}^{2+}$ concentration in intra- and extracellular fluids are associated with development or aggravation of BBB disruption and brain edema in various clinical disorders and experimental settings. On the other hand, magnesium supplementation can play multiple roles in protecting BBB integrity and improving brain edema. However, owing to the availability of limited knowledge, it is hard to

\section{References}

Abbott NJ, Patabendige AA, Dolman DE, Yusof SR, Begley DJ (2010) Structure and function of the bloodbrain barrier. Neurobiol Dis 37:13-25.

Altura BM, Gebrewold A, Huang QF, Altura BT (1991) Deficits in brain-CSF magnesium result in cerebrovasospasm and rupture of cerebral microvessels: Possible relation to stroke. Clin Res 39:394A.

Altura BM, Gebrewold A, Altura BT, Gupta RK (1995) Role of brain [Mg2+]i in alcohol-induced hemorrhagic stroke in a rat model: a 31P-NMR in vivo study. Alcohol 12(2):131-6.

Altura BM, Gebrewold A, Zhang A, Altura BT (2003) Low extracellular magnesium ions induce lipid peroxidation and activation of nuclear factor-kappa B in canine cerebral vascular smooth muscle: possible relation to traumatic brain injury and strokes. Neurosci Lett 341:189-92.

Amiry-Moghaddam M, Otsuka T, Hurn PD, Traystman RJ, Haug FM, Froehner SC, Adams ME, Neely JD, Agre P, Ottersen OP, Bhardwaj A (2003) An alphasyntrophin-dependent pool of AQP4 in astroglial endfeet confers bidirectional water flow between blood and brain. Proc Natl Acad Sci USA 100:2106-11.

Ariza AC, Bobadilla N, Fernandez C, Munoz-Fuentes RM, Larrea F, Halhali A (2005) Effects of magnesium sulfate on lipid peroxidation and blood pressure regulators in pre-eclampsia. Clin Biochem 38:128-33.

Bara M, Guiet-Bara A (1984) Potassium, magnesium and membranes. Magnesium 3:212-25.

Barbagallo M, Dominguez LJ (2010) Magnesium and aging. Curr Pharm Des 16:832-39. come to a full understanding of the highly specific actions of magnesium on BBB integrity and brain edema in the course of a variety of pathophysiologies involving the BBB. For this reason, research in this field should continue in order to provide a thorough explanation of the impact of magnesium on the BBB, brain edema and related pathologies.

Billard JM (2006) Ageing, hippocampal synaptic activity and magnesium. Magnes Res 19:199-215.

Belfort M, Allred J, Dildy G (2008) Magnesium sulfate decreases cerebral perfusion pressure in preeclampsia. Hypertens Pregnancy 27:315-27.

Cardoso FL, Brites D, Brito MA (2010) Looking at the blood-brain barrier: molecular anatomy and possible investigation approaches. Brain Res Rev 64:328-63.

Chaon A, Lisott E, Eblen-Zajjur A (2006) Magnesium sulphate reduces cell volume in physiological conditions but not in the cytotoxic oedema during global brain ischemia. Brain Injury 20:1087-91.

Correale J, Villa A (2009) Cellular Elements of the Blood-Brain Barrier. Neurochem Res 34:2067-77.

Dai LJ, Quamme G A (1991) Intracellular Mg2+ and magnesium depletion in isolated renal thick ascending limb cells. J Clin Invest 88:1255-64.

Demougeot C, Bobillier-Chaumont S, Mossiat C, Marie C, Berthelot A (2004) Effect of diets with different magnesium content in ischemic stroke rats. Neurosci Lett 362:17-20.

Ebel H, Gunther T (1980) Magnesium metabolism: a review. J Clin Chem Clin Biochem 18:257-70.

Esen F, Erdem T, Aktan D, Kalaycı R, Cakar N, Kaya M, Telci $L$ (2003) Effects of magnesium administration on brain edema and blood brain barrier breakdown after experimental traumatic brain injury in rats. $J$ Neurosurg Anesthesiol 15:119-25. 
Esen F, Erdem T, Aktan D, Orhan M, Kaya M, Eraksoy $\mathrm{H}$, Cakar N, Telci L (2005) Effect of magnesium sulfate administration on blood-brain barrier in a rat model of intraperitoneal sepsis: A randomized controlled experimental study. Critical Care 9:R18-23.

Euser AG, Bullinger L, Cipolla MJ (2008) Magnesium sulphate treatment decreases blood brain barrier permeability during acute hypertension in pregnant rats. Exp Physiol 93:254-61.

Euser AG, Cipolla MJ (2009) Magnesium Sulfate for the Treatment of Eclampsia. Stroke 40:1169-75.

Feldman Z, Gurevitch B, Artru AA, Oppenheim A, Shohami E, Reichenthal E, Shapira Y (1996) Effect of magnesium given 1 hour after head trauma on brain edema and neurological outcome. J Neurosurg 85:1317.

Feng DF, Zhu ZA, Lu YC (2004) Effect of magnesium on traumatic brain edema in rats. Chin J Traumatol 7:14852.

Fisher M (2009) Pericyte signaling in the neurovascular unit. Stroke 40:S13-S15.

Ghabriel MN, Thomas A, Vink R (2006) Magnesium restores altered aquaporin-4 immunoreactivity following traumatic brain injury to a pre-injury state. Acta Neurochir Suppl 96:402-6.

Goñi-de-Cerio F, Alvarez A, Alvarez FJ, Rey-Santano MC, Alonso-Alconada D, Mielgo VE, Gastiasoro E, Hilario E (2009) MgSO4 treatment preserves the ischemia-induced reduction in S-100 protein without modification of the expression of endothelial tight junction molecules. Histol Histopathol 24(9):1129-38.

Grubbs RD, Maguire ME (1987) Magnesium as a regulatory cation: criteria and evaluation. Magnes 6:113-27.

Guo S, Lo EH (2009) Dysfunctional cell-cell signaling in the neurovascular unit as a paradigm for central nervous system disease. Stroke 40(3):S4-7.

Hallak M, Berman RF, Irtenkauf SM, Evans MI, Cotton DB (1992) Peripheral magnesium sulfate enters the brain and increases the threshold for hippocampal seizures in rats. Am J Obstet Gynecol 167:1605-10.

Haorah J, Knipe B, Leibhart J, Ghorpade A, Persidsky Y (2005) Alcohol-induced oxidative stress in brain endothelial cells causes blood-brain barrier dysfunction. J Leukoc Biol 78(6):1223-32.
Heath DL, Vink R (1999) Improved motor outcome in response to magnesium therapy received up to 24 hours after traumatic diffuse axonal brain injury. $J$ Neurosurg 90:504-9.

Hoane MR (2007) Assessment of cognitive function following magnesium therapy in the traumatically injured brain. Magnes Res 20:229-36.

Huang Q F, Gebrewold A, Zhang A, Altura BT, Altura BM (1994) Role of excitatory amino acids in regulation of rat pial microvasculature. Am J Physiol Regul Integr Comp Physiol 266:R158-63.

Imer M, Omay B, Uzunkol A, Erdem T, Sabanci PA, Karasu A, Albayrak SB, Sencer A, Hepgul K, Kaya M (2009) Effect of magnesium, MK-801 and combination of magnesium and MK-801 on blood-brain barrier permeability and brain edema after experimental traumatic diffuse brain injury. Neurol Res 31:977-81.

Kaptanoglu E, Beskonakli E, Okutan O, Selcuk Surucu $H$, Taskin $Y(2003)$ Effect of magnesium sulphate in experimental spinal cord injury: evaluation and ultrastructural findings and early clinical results. J Clin Neurosci 10:329-34.

Kaya M, Küçük M, Bulut Kalayci R, Cimen V, Gürses C, Elmas I, Arican N (2001) Magnesium sulfate attenuates increased blood-brain barrier permeability during insulin-induced hypoglycemia in rats. Can J Physiol Pharmacol 79:793-8.

Kaya M, Gulturk S, Elmas I, Arican N, Kocyildiz ZC, Kucuk M, Yorulmaz H, Sivas A. (2004) The effects of magnesium sulfate on blood-brain barrier disruption caused by intracarotid injection of hyperosmolar mannitol in rats. Life Sci 76:201-12.

Khan OH, Enno T, Del Bigio MR (2003) Magnesium sulfate therapy is of mild benefit to young rats with kaolin-induced hydrocephalus. Pediatr Res 53:970-6.

Klatzo I (1967) Presidental address: neuropathological aspects of brain edema. J Neuropathol Exp Neurol 26:1-14.

Lee MS, Wu YS, Yang DY, Lee JB, Cheng FC (2002) Significantly decreased extracellular magnesium in brains of gerbils subjected to cerebral ischemia. Clin Chim Acta 318:121-5.

Libien J, Sacktor TC, Kass IS (2005) Magnesium blocks the loss of protein kinase $C$, leads to a transient translocation of PKC (alpha) and PKC (epsilon), and improves recovery after anoxia in rat hippocampal slices. Brain Res Mol Brain Res 136:104-11. 
Liu X, Hunter C, Weiss HR, Chi OZ (2010) Effects of blockade of ionotropic glutamate receptors on bloodbrain barrier disruption in focal cerebral ischemia. Neurol Sci 31:699-703.

Löscher W, Potschka H (2005) Blood-brain barrier active efflux transporters: ATP-binding cassette gene family. NeuroRx 2:86-98.

Maier JA, Bernardini D, Rayssiguier Y, Mazur A (2004) High concentrations of magnesiummodulate vascular endothelial cell behaviour in vitro. Biochim Biophys Acta 1689:6-12.

Marmarou A (2004) The pathophysiology of brain edema and elevated intracranial pressure. Cleveland Clin J Med 71:S6-8.

Matsui T, Kobayashi $\mathrm{H}$, Hirai S, Kawachi $\mathrm{H}$, Yano $\mathrm{H}$ (2007) Magnesium deficiency stimulated mRNA expression of tumor necrosis factor-a in skeletal muscle of rats. Nutrition Research 27: 66-8.

Mazur A, Maier JA, Rock E, Gueux E, Nowacki W, Rayssiguier Y (2007) Magnesium and the inflammatory response: potential physiopathological implications. Arch Biochem Biophys 458:48-56.

Mckee JA, Brewer RP, Macy GE, Phillips-Bute B, Campbell KA, Borel CO, Reynolds JD, Warner DS (2005) Analysis of the brain bioavailability of peripherally administered magnesium sulfate: a study in humans with acute brain injury undergoing prolonged induced hypermagnesemia. Crit Care Med 33:661-6.

Musso CG (2009) Magnesium metabolism in health and disease. Int Urol Nephrol 41:357-62.

Nag S, Manias JL, Stewart DJ (2009) Pathology and new players in the pathogenesis of brain edema. Acta Neuropathol 118(2):197-217.

Nakahama K, Nagano M, Fujioka A, Shinoda K, Sasaki H (1999) Effect of TPA on aquaporin 4 mRNA expression in cultured rat astrocytes. Glia 25:240-6.

Okiyama K, Smith DH, Gennarelli TA, Simon RP, Leach $M$, Mclntosh TK. (1995) The sodium channel blocker and glutamate release inhibitor BW1003C87 and magnesium attenuate regional cerebral edema following experimental brain injury in the rat. $J$ Neurochem 64:802-9.

Papadopoulos MC, Verkman AS (2005) Aquaporin-4 gene disruption in mice reduces brain swelling and mortality in pneumococcal meningitis. J Biol Chem 280:13906-12.
Papierkowski A, Pasternak K (1998) The effect of a single dose of morphine and ethanol on magnesium level in blood serum and tissues in mice. Magnes Res 11:85-9.

Pardridge WM (2005) Molecular biology of the bloodbrain-barrier. Mol Biotechnol 30:57-70.

Pardridge WM (2007) Blood-brain-barrier delivery. Drug Discov Today 12:54-61.

Rayssiguier Y, Libako P, Nowacki W, Rock E (2010) Magnesium deficiency and metabolic syndrome: stress and inflammation may reflect calcium activation. Magnes Res 23(2):73-80.

Ravishankar S, Ashraf QM, Fritz K, Mishra OP, Delivoria-Papadopoulos M (2001) Expression of Bax and $\mathrm{Bcl}-2$ proteins during hypoxia in cerebral cortical neuronal nuclei of newborn piglets: effect of administration of magnesium sulfate. Brain Res 901:23-9.

Romani AM (2008) Magnesium homeostasis and alcohol consumption. Magnes Res 21:197-204.

Romani AM, Scarpa A (2000) Regulation of cellular magnesium. Frontiers in Bioscience 5:720-34.

Royo NC, Shimizu S, Schouten JW, Stover JF, Mclntosh TK (2003) Pharmacology of traumatic brain injury. Curr Opin Pharmacol 3:27-32.

Saatman KE, Bareyre FM, Grady MS, McIntosh TK (2001) Acute cytoskeletal alterations and cell death induced by experimental brain injury are attenuated by magnesium treatment and exacerbated by magnesium deficiency. J Neuropathol Exp Neurol 60:183-94.

Salem M, Kasinski N, Munoz R, Chernow B (1995) Progressive magnesium deficiency increases mortality from endotoxin challenge: protective effects of acute magnesium replacement therapy. Crit Care Med 23:108-18.

Saris NL, Mervaala E, Karppanen H, Khawaja JA, Lewenstam A (2000) Magnesium: an update on physiological, clinical and analytical aspects. Clin Chim Acta 294:1-26.

Schwartz RB, Feske SK, Polak JF, DeGirolami U, laia A, Beckner KM, Bravo SM, Klufas RA, Chai RY, Repke JT (2000) Preeclampsia-eclampsia: Clinical and neuroradiographic correlates and insights into the pathogenesis of hypertensive encephalopathy. Radiology 217:371-6. 
Sen AP, Gulati A (2010) Use of magnesium in traumatic brain injury. Neurotherapeutics 7:91-9.

Taniguchi M, Yamashita T, Kumura E, Tamatani M, Kobayashi A, Yokawa T, Maruno M, Kato A, Ohnishi T, Kohmura E, Tohyama M, Yoshimine T (2000) Induction of aquaporin-4 water channel mRNA after focal cerebral ischemia in rat. Mol Brain Res 78:131-7.

Temkin NR, Anderson GD, Winn HR, Ellenbogen RG, Britz GW, Schuster J, Lucas T, Newell DW, Mansfield PN, Machamer JE, Barber J, Dikmen SS (2007) Magnesium sulfate for neuroprotection after traumatic brain injury: a randomised controlled trial. Lancet Neurol 6:29-38.

Touyz RM (2003) Role of magnesium in the pathogenesis of hypertension. Mol Aspects Med 24:107-36.

Touyz RM (2008) Transient receptor potential melastatin 6 and 7 channels, magnesium transport, and vascular biology: implications in hypertension. Am J Physiol Heart Circ Physiol 294:H1103-8.

Turkoglu OF, Eroglu H, Okutan O, Tun MK, Bodur E, Sargon MF, Oner L, Beskonakli E (2008) A comparative study of treatment for brain edema Magnesium sulphate versus dexamethasone sodium phosphate. $J$ Clin Neurosci 15:60-5.

Unterberg AW, Stover J, Kress B, Kiening KL (2004) Edema and brain trauma. Neuroscience 129:1021-9.

Vink R, Mclntosh T K, Demediuk P, Faden Al (1987) Decrease in total and free magnesium concentration following traumatic brain injury in rats. Biochem Biophys Res Commun 149:594-9.

Vink R, McIntosh TK, Demediuk P, Weiner MW, Faden Al (1988) Decline in intracellular free $\mathrm{Mg}^{2+}$ is associated with irreversible tissue injury after brain trauma. J Biol Chem 263:757-61.
Vink R, Cernak I (2000) Regulation of intracellular free magnesium central nervous system injury. Front Biosci 1:656-65.

Vink R, Cook NL, van den Heuvel C (2009) Magnesium in acute and chronic brain injury: an update. Magnes Res 22:158S-162S.

Weglicki WB, Phillips TM, Mak IT, Cassidy MM, Dickens BF, Stafford R, Kramer JH (1994) Cytokines, neuropeptides, and reperfusion injury during magnesium deficiency. Ann N Y Acad Sci 723:246-57.

Weglicki WB, Mak, IT, Kramer JH, Dickens BF, Cassidy MM, Stafford RE, Phillips TM (1996) Role of free radicals and substance $P$ in magnesium deficiency. Cardiovasc Res 31:677-82.

Wikkels $\varnothing$ C, Blomstrand C (1982) Cerebrospinal fluid proteins and cells in normal-pressure hydrocephalus. $J$ Neurol 228:171-80.

Wolf FI, Trapani V, Simonacci M, Ferré S, Maier JAM (2008) Magnesium deficiency and endothelial dysfunction: is oxidative stress involved? Magnes Res 21:58-64.

Wolf Fl, Trapani V (2008) Cell (patho) physiology of magnesium. Clin Sci 114:27-35.

Wolf FI, Trapani V, Simonacci M, Boninsegna A, Mazur A, Maier JA (2009) Magnesium deficiency affects mammary epithelial cell proliferation: involvement of oxidative stress. Nutr Cancer 61:131-6.

Zador Z, Stiver S, Wang V, Manley GT (2009) Role of aquaporin-4 in cerebral edema and stroke. In: Aquaporins- Handbook of experimental pharmacology 190 (Beitz E, eds), Springer, Heidelberg, pp 159-70. 


\title{
Magnesium and hearing loss
}

\author{
Isabelle Sendowski, ${ }^{1, \bigotimes}$ Xavier Holy, ${ }^{2}$ Florent Raffin ${ }^{1}$ and Yves Cazals ${ }^{3}$ \\ ${ }^{1}$ Institut de Recherche Biomédicale des Armées, Antenne de la Tronche, La Tronche, France. \\ ${ }^{2}$ Institut de Recherche Biomédicale des Armées, Antenne de Brétigny, Brétigny-sur-Orge, France. \\ ${ }^{3}$ UMR6231, Université Paul Cézanne, Marseille, France. \\ $\triangle$ Isendowski@crssa.net
}

\begin{abstract}
Hearing loss is a major public health problem with a large number of causes. Among them, noise-induced hearing loss, drug ototoxicity and sudden sensorineural hearing loss have been proven to result, in part, from metabolic disorders. Metabolic disorders have multiple origins such as ionic, ischemic, excitotoxic and production of cochlear free radicals causing cell death, via necrosis or apoptosis. The efficacy of magnesium, administered either to prevent or to treat hearing damage, has been demonstrated in several studies in animals and in humans, particularly in noise-induced hearing loss. The exact mechanism by which $\mathrm{Mg}^{2+}$ acts is not fully known. Different hypotheses exist including calcium antagonism, vasodilatation, antioxidant and anti-NMDA properties. Because it is a relatively safe and well-known treatment, magnesium therapy, alone or in association, could be of a great interest to improve auditory recovery.
\end{abstract}

\section{Introduction}

According to the World Health Organization, 278 million people worldwide have moderate to profound hearing loss in both ears. Besides pathologies of unknown origin, such as sudden hearing loss and Meniere's disease, hearing loss has so many known causes; it would be arduous to list them all. Briefly, it can be due to the aging process, exposure to loud noise, certain medications, infections, head or ear trauma, congenital or hereditary factors, diseases, as well as a number of other causes. In noise-induced hearing loss (NIHL), sudden sensorineural hearing loss (SSHL) or iatrogenic hearing loss (ototoxicity), sensory cell death involves metabolic processes. Pharmacological intervention to prevent or ameliorate the evolution of these hearing impairments, as well as susceptibility factors, have been studied for a number of years in animal models or in humans. Among them, magnesium seems to play an important role. Magnesium therapy is well documented because it is usually prescribed in other pathologies. Its side effects and contraindications are few and it is cheap. Magnesium, which easily crosses the hematocochlear barrier, presents neuroprotective and vasodilatory effects, and is able to limit cochlear damage. These observations have led to many investigations, the aim of them being to evaluate the pertinence of magnesium administration in prevention or treatment in such a hearing impairment. This article presents some arguments that emphasize the interest of magnesium therapy in some forms of hearing loss.

\section{How can we hear?}

Sound waves are transmitted via the bones of the middle ear to the fluid environment of the inner ear, where the sensory organ is in the cochlea. The human cochlea is a $30-35 \mathrm{~mm}$ long coiled tube containing three parallel chambers (Figure $1 \mathrm{~A})$ : the scala vestibuli and the scala tympani, which both contain perilymph, and the scala media, which contains endolymph. In contrast to perilymph, which is similar to cerebrospinal fluid, endolymph contains a large amount of potassium (154 mM) maintained by the cells of the stria vascularis. The organ of Corti (Figure 1B) is composed of supporting cells and hair cells. Of all the sensory organs, the organ of Corti functions with the smallest number of sensory receptor cells. The human cochlea only contains about 15,000 hair cells, including approximately 3,000 inner hair cells (IHCS) and 12,000 outer hair cells (OHCs). The IHCs form one row (Figure 2A) and are the primary sensory receptors. OHCs are organized into three (and sometimes four) rows 
A

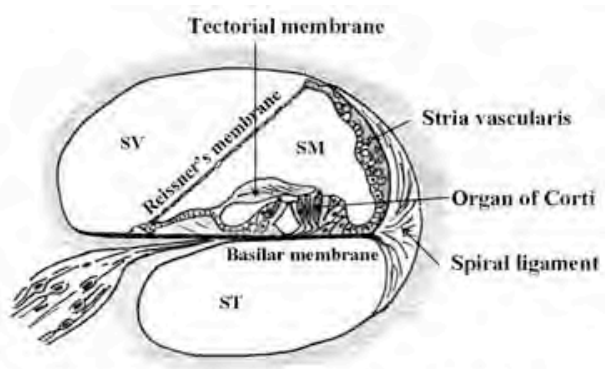

SV; Seala vestibuli; ST: Seala tyminani; SM: Scala media

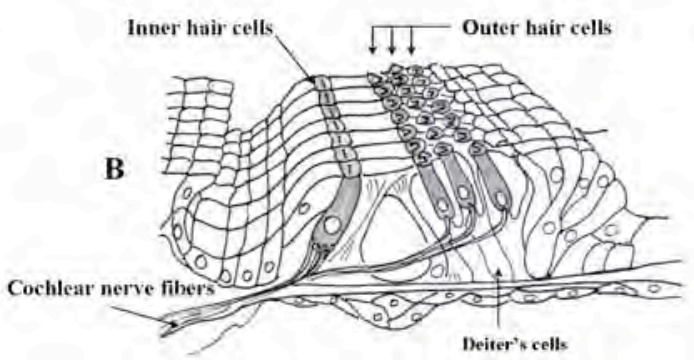

Figure 1. Schematic representation of the inner ear. A: section of the cochlea; B: organ of Corti.

along the outer edge of the organ of Corti. The longer stereocilia of the $\mathrm{OHCs}$ are connected to the tectorial membrane.

In the hearing process, sound reaches the inner ear and the basilar membrane supporting the hair cells is displaced. When the hair bundle is deflected, transduction channels are opened and $\mathrm{K}^{+}$enters the hair cells. The depolarisation evoked by this transduction current activates voltagegated $\mathrm{Ca}^{2+}$ channels and $\mathrm{Ca}^{2+}$ influx. In IHCs, increased intracellular $\mathrm{Ca}^{2+}$ causes mobilization of synaptic vesicles and exocytotic release of glutamate at the base of the hair cells. Glutamate release modulates the activity of the auditory nerve fibres by activating specific receptors, among them the glutamate ionotropic receptors (AMPA, NMDA and Kainite) and the metabotropic receptors. The $\mathrm{OHCs}$ respond quite differently to changes in membrane potential. Their membranes include a protein (prestin), which alters conformation with the membrane potential and forces cell length changes at acoustic frequencies. This mechanism is thought to amplify and tune the mechanical responses of the basilar membrane. This sensory system is so efficient that, near auditory threshold, a sound vibration of less than 0.1 nanometres is detected, corresponding to a stereocilia displacement 10,000 times lower than its diameter (about one micrometre). However, exposure to loud noise can weaken the system.

\section{Magnesium and noise-induced hearing loss (NIHL)}

Excessive noise is the predominant cause of permanent sensorineural hearing loss. Reports from WHO (2004) state that "worldwide, $16 \%$ of the disabling hearing loss in adults is attributed to occupational noise, ranging from $7 \%$ to $21 \%$ in the various subregions". At least 30 million people in the United States encounter hazardous levels of noise at work, particularly in jobs such as construction, mining, agriculture, manufacturing, transportation and in the military. Moreover, the incidence of NIHL continues to grow, partly due to the growing popularity of portable music players with highly efficient headphones (Zhao et al., 2010). The hearing loss can be caused by a single exposure to very loud sounds (impulse noise) or by repeated exposure to louder sounds over an extended period. It may be temporary (temporary threshold shifts, TTS) or permanent (permanent threshold shifts, PTS). It is frequently associated with tinnitus.

The mechanisms of damage are of dual origin, mechanical and metabolic. Mechanical damage is immediately developed when the movements of the basilar membrane are excessive, thus inducing detachment of the hairs of the tectorial membrane, disconnection of the interciliary bridges (Figure 2D), or even rupture of membranes. Modern research has provided new insights into the biological mechanisms of NIHL such as free radical production (oxidative stress), glutamatergic excitotoxicity, and ionic and ischemic disorders. They are responsible for delayed hair cell death by necrosis and apoptosis.

\section{Oxidative stress}

It has been demonstrated that an increase in reactive oxygen and nitrogen species (ROS and RNS, respectively) is involved in noise trauma (see Henderson et al., 2006 for review). The superoxide radical anion, nitric oxide (NO) and its redox-related forms, in conjunction with an imbalance of antioxidant defences, have been demonstrated to play a significant role in NIHL as they largely participate in cellular mechanisms that underlie hair cell death. ROS ototoxicity is believed to be associated with deleterious cellular 

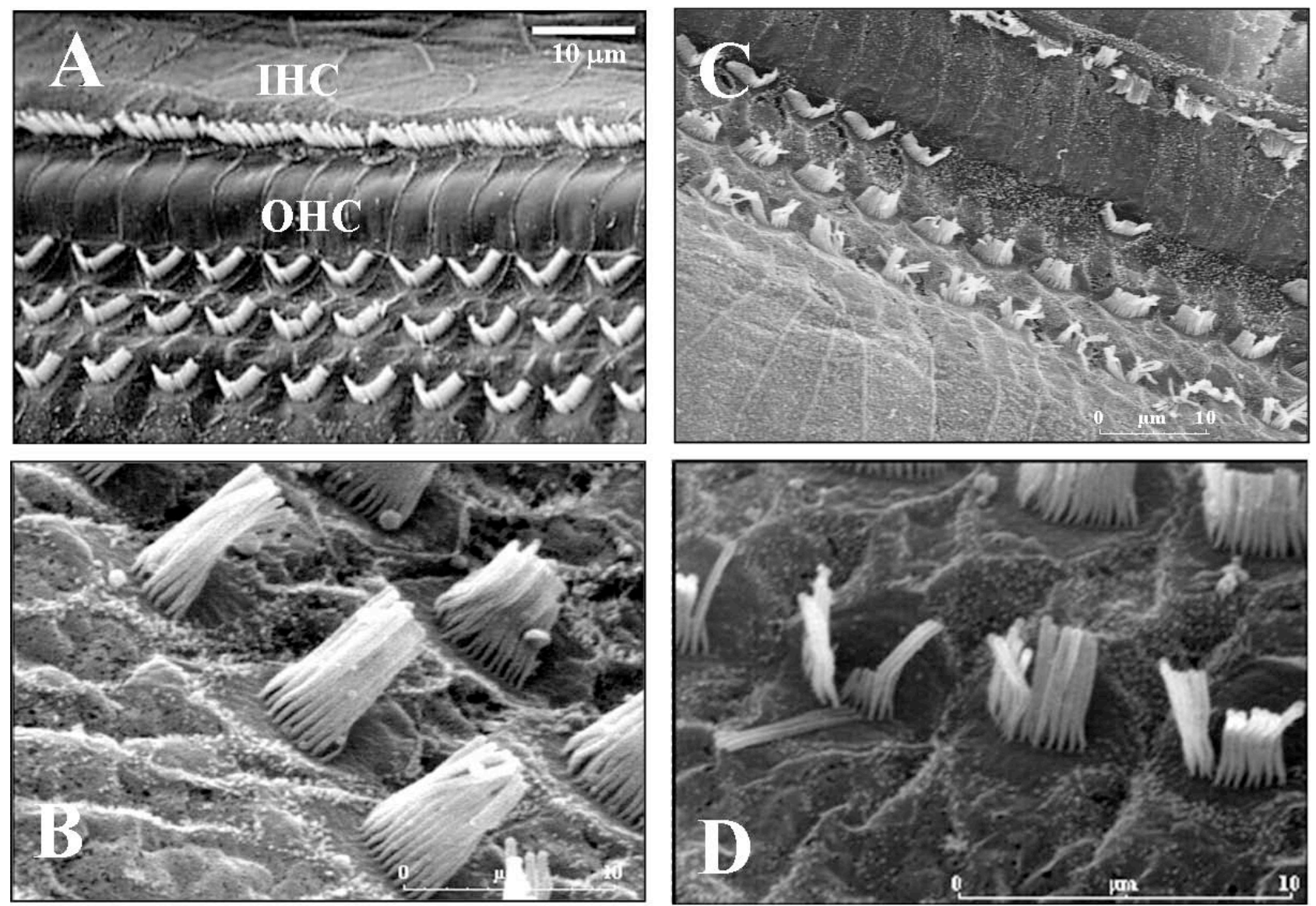

Figure 2. A, B: normal aspect of the hair cells and their stereocilia, observed with scanning electron microscopy. $\mathrm{C}$ and $\mathrm{D}$ : Organ of Corti after traumatizing noise exposure: $\mathrm{OHCs}$ are partially missing (C). The stereocilia of hair cells are abnormally bent with broken interciliary bridges (D).

effects at multiple sites, including lipid peroxidation, DNA strand breaks, alteration in carbohydrate and protein structures, and a triggering of cell death gene expression, leading to necrosis or apoptosis. Oxidants are also initiators of intracellular cell death signalling pathways that may lead to apoptosis. The noiseinduced ROS formation may occur with a delay of 7-10 days following exposure to noise (Yamashita et al., 2004).

\section{Excitotoxicity}

Excitotoxicity is a phenomenon of biochemical events, triggered by the interaction of excitatory amino acids with ion channel-bound receptor complexes, that can lead to cell death. During high-level noise exposure, the IHCs are highly active, leading to the release of large amounts of glutamate into the synapses with the auditory fibres. The level of glutamate in the synapses can overstimulate the glutamate receptors, especially the NMDA receptors, and result in high intracellular $\mathrm{Ca}^{2+}$ levels. The increase in cytosolic $\mathrm{Ca}^{2+}$ concentration is not only caused by an influx of extracellular $\mathrm{Ca}^{2+}$, but also by the release of calcium ions from intracellular stores such as the endoplasmic reticulum or mitochondria. Dendritic swelling and vacuolization are a result of excessive postsynaptic ion influx into the VIIIth nerve terminals at the inner hair cell synapse (Pujol et al., 1990).

\section{lonic disorders}

High-level noise stimulation results in a massive entrance of potassium through the apical channels of the stereocilia. Moreover, mechanical damage with ruptures of the membranes lining the endolymphatic spaces, could involve an excessive influx of $\mathrm{K}^{+}$. This increased $\mathrm{K}^{+}$ concentration may be toxic for the hair cells (Zenner, 1986). The consecutive increase in intracellular $\mathrm{Ca}^{2+}$ over-activates a series of enzymes including phospholipases, proteases, and endonucleases. The result is membrane 
breakdown, depolymerization of microtubules and disruption of protein synthesis.

\section{Ischemic process}

Unlike most tissues in which increased metabolism increases blood flow to provide additional oxygen to stressed cells, the cochlea shows reduced blood vessel diameter and red blood cell velocity (Quirk and Seidman, 1995) and decreased blood flow (Thorne and Nuttal, 1987) post-noise. This noise-induced vasoconstriction is a direct consequence of noise-induced formation of 8-isoprostane- $F_{2 a}$, a vasoactive by-product of free radicals (Miller et al., 2003). Cochlear ischemia is aggravated in animals with low $\mathrm{Mg}^{2+}$ content (Scheibe et al., 2000a).

These different mechanisms can lead to cell death, which may be more or less rapid, due to necrosis or apoptosis (Yang et al., 2004). Recent studies have revealed that these two types of cell death exist following exposure to intense noise and that apoptosis appears extremely rapidly after the noise stress. Apoptosis has been shown to be the primary cell death pathway in the first day following noise exposure (Hu et al., 2002; 2006).

Contrary to birds or reptiles, a mammal's auditory hair cells do not regenerate if they are destroyed. Thus, these new metabolic insights bring hope for possible prevention or treatment to limit oxidative stress, ischemia and the apoptosis cascade. A large number of treatments have been tested over the last few years (see Le Prell et al., 2007a; Shibata and Raphael, 2010 for reviews). Among them, magnesium has aroused some interest.

Over a number of years, researchers and clinicians have demonstrated the influence of magnesium in the susceptibility to recover following acoustic trauma. NIHL of guinea pigs was found to increase with decreasing $\mathrm{Mg}^{2+}$ content of the drinking water while the $\mathrm{Mg}^{2+}$ content of the food was low and constant. In Mgdeficient guinea pigs, the hearing threshold shift after 10 days of continuous noise exposure was negatively correlated to the $\mathrm{Mg}^{2+}$ content of the perilymph (Ising et al., 1982). Coherent results were obtained in rats (Joachims et al., 1983). Similarly, electrocochleographic measurements of the auditory threshold shifts induced by impulse noises (Devrière et al., 1991) showed that Mg-deficient animals are slightly more susceptible to this type of noise. This susceptibility is less pronounced than after a long duration exposure to a continuous noise, as observed in the previous experiments (Ising et al., 1982). After exposure to continuous noise, up to $75 \%$ of the variance of PTS in guinea pigs could be explained by the level of perilymph $\mathrm{Mg}^{2+}$ (Vormann and Günther, 1993). The increased susceptibility to NIHL with $\mathrm{Mg}^{2+}$ deficit has not only been demonstrated in animal experiments. In a retrospective study in humans, subjective thresholds across frequencies of 3,4 and $5 \mathrm{kHz}$ were negatively correlated with serum magnesium (Joachims et al., 1987). This finding was the first indication that magnesium status in humans may be one of the factors determining variations in sensitivity to noise-induced hearing loss. Günther et al., (1989) reported that NIHL observed in 24 air force pilots was negatively correlated to serum $\mathrm{Mg}^{2+}$ concentration. However, Walden et al., (2000), exploring the susceptibility of soldiers to NIHL, failed to demonstrate any correlation between audiometric index and body magnesium.

Because $\mathrm{Mg}^{2+}$ deficiency increases the susceptibility to NIHL, several studies have been conducted in animals or in humans to point out the possible prophylactic efficacy of magnesium. Joachims et al., (1983) observed that guinea pigs with physiologically high $\mathrm{Mg}^{2+}$ levels, when exposed to a single shot impulse or a series of impulses, had significantly smaller threshold increases as compared to physiologically low $\mathrm{Mg}^{2+}$ animals. Scheibe et al., (2000b) showed that oral magnesium supplementation significantly reduces TTS and PTS in guinea pigs subjected to a series of impulses. The mean PTS was found to correlate negatively with the total $\mathrm{Mg}^{2+}$ concentration of perilymph and plasma. Conversely, they did not observe any significant effect on PTS following exposure to a gunshot noise. More recently, Attias et al., (2003) explored the activity of the outer hair cells in guinea pigs by means of otoacoustic emission after impulse noise exposure. In animals supplemented with $\mathrm{Mg}^{2+}$, the thresholds were less significantly affected by noise exposure and the audition recovery was faster. In humans, preventive administration of magnesium has also been shown to be effective in noise-related 
hearing loss. Attias et al., (1994) tested the prophylactic effect of magnesium in human subjects exposed to hazardous noise. The study was carried out in 300 young, normal-hearing recruits who underwent 2 months of basic military training. This training included repeated exposure to high levels of impulse noises while using earplugs. The subjects received an additional drink daily containing either $167 \mathrm{mg}$ magnesium aspartate or a placebo. The NIHL was significantly more frequent and more severe in the placebo group (28.5\%) than in the magnesium group (11.2\%). Moreover, the severity of the NIHL was negatively correlated to the magnesium content of red and mononuclear cells. This prophylactic effect in humans was confirmed by Attias et al., (2004) for temporary threshold shifts. Subjects were exposed to a traumatizing noise over $10 \mathrm{~min}$ in order to produce TTS without PTS. Compared to a placebo, the preventive oral intake of magnesium (122 $\mathrm{mg} \mathrm{Mg}^{2+}$ aspartate during 10 days) provided significant protection against TTS. A negative correlation between the blood magnesium levels and the TTS was also noted.

Whilst magnesium is well known as a preventive treatment for sound trauma, it may also be useful therapeutically. In animals submitted to a series of sound impulses, the systemic administration of magnesium significantly reduced hearing loss after 7 days (Scheibe et al., 2001; 2002). This treatment was more effective if it was quickly instigated. Threshold shift reduction 7 days after acoustic trauma in guinea pigs was confirmed using distortion product otoacoustic emissions (DPOAE, a non invasive technique to test OHC function)(Haupt et al., 2003). In another study (Sendowski et al., 2006) it has been shown that a 7-day post-trauma magnesium treatment reduced auditory threshold shift measured seven days after gunshot noise exposure. However, this improvement was temporary, suggesting that it could be potentially beneficial to prolong the magnesium administration. This hypothesis has since been confirmed (Abaamrane et al., 2009). The latter study compared 4 post-NIHL treatments - a 7-day magnesium treatment, a 1month magnesium treatment, conventional therapy with methylprednisolone, or a placebo. It was demonstrated that 3 months after an impulse noise trauma, the 1-month $\mathrm{Mg}$ treatment preserved more hair cells from death
(Figure 3), but this preservation was found to be partial. These observations suggest that post$\mathrm{NIHL}$ treatments can be improved if continued beyond the period of one week.

Another track of research concerns combination of different therapies, including magnesium. Treatment with a combination of vitamins A, C, E and magnesium, initiated 1 hour before noise exposure, produced a compelling reduction in NIHL and cell death, while effects of either the antioxidant agents (vitamin A, C, or E) or magnesium alone were small and not statistically reliable (Le Prell et al., 2007b). Thus, the combination of magnesium with other agents could also be of great interest, and suggests that they act at different and complementary levels of the cellular death process.

\section{Magnesium and ototoxicity}

Various pharmaceuticals are known to impair the auditory system. The best-known substances are the aminoglycoside antibiotics and cisplatin. Both cause high frequency sensorineural hearing loss, which is usually permanent and associated with loss of outer hair cells in the basal turn of the cochlea. Aminoglycoside antibiotics (such as gentamicin, neomycin and kanamycin) are used to treat bacteria not responsive to conventional antibiotics. Their clinical use is limited by toxic side effects that include cochlear toxicity, vestibular toxicity and nephrotoxicity. Aminoglycoside antibiotics possibly cause formation of free radicals by forming complexes with iron, which is vital for normal mitochondrial function. Free radicals can rapidly react with cell constituents, including cell membranes and DNA. The resulting oxidative stress can trigger apoptotic cell death. The intrinsic apoptosis pathway is the major pathway induced by aminoglycosides in the cochlea (Rybak and Withworth, 2005).

Vormann and Günther (1991) demonstrated that $\mathrm{Mg}$ deficiency in growing rats aggravated the ototoxic effects of the aminoglycoside antibiotic, gentamicin. Administration of gentamicin to $\mathrm{Mg}^{2+}$ deficient pregnant rats from day 16-20 of gestation produced hearing threshold shifts in the maternal rats as well in their offspring (Vormann and Günther 1991). These data suggest that $\mathrm{Mg}^{2+}$ deficiency is a relevant predisposing 

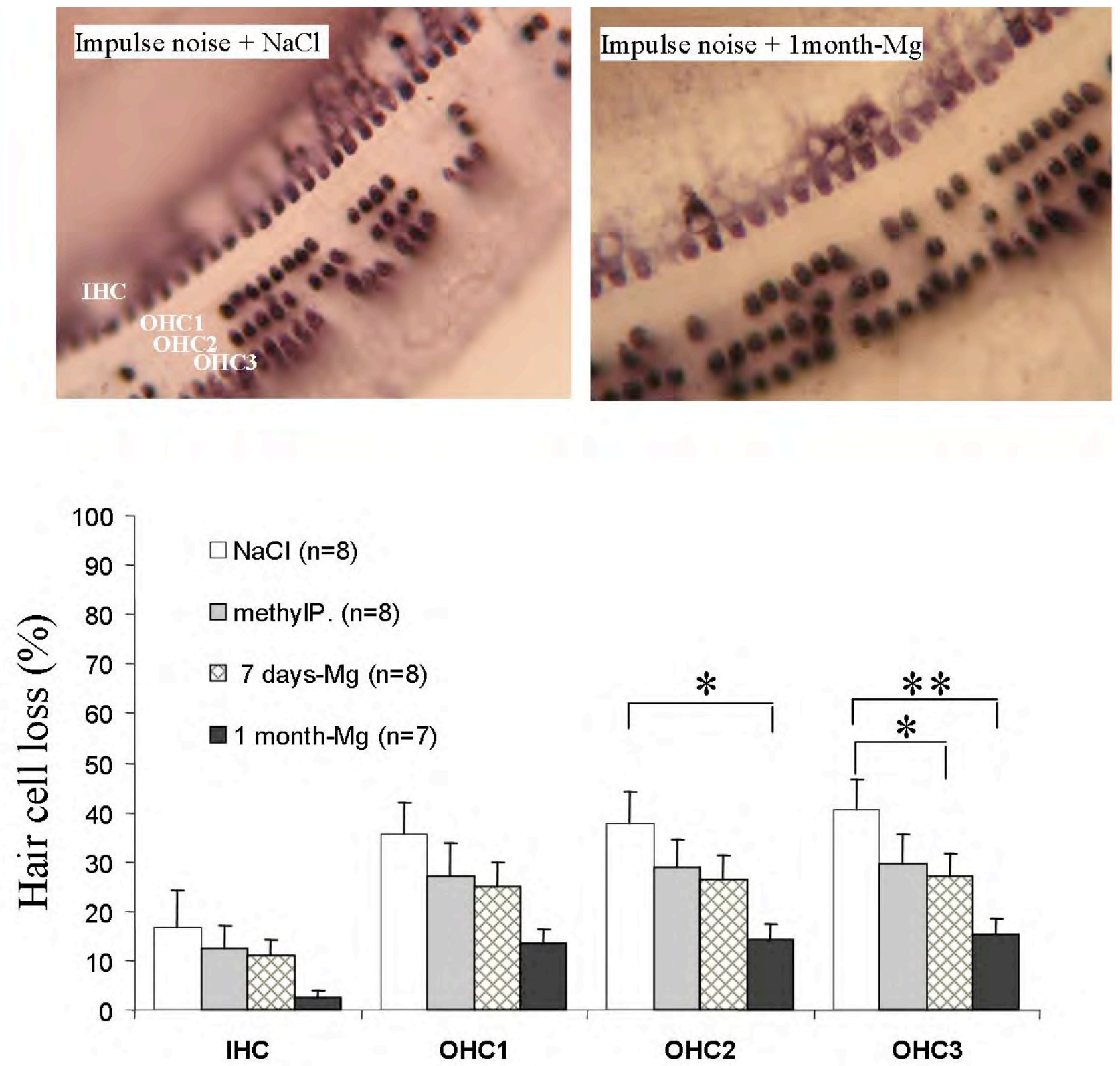

Figure 3. Global hair cells loss in guinea pigs, 3 months after an impulse noise exposure, for 4 treatments groups: placebo $(\mathrm{NaCl})$, a 7-day or 1-month magnesium treatment, and conventional therapy with methylprednisolone. (* $p<0.05 ;{ }^{* *} p<0.01$ ) (after Abaamrane et al., 2009).

risk factor for the development of ototoxicity induced by some pharmaceuticals. Recently, it has been shown, in the zebrafish model, that extracellular magnesium or calcium ions modulate hair cell death from neomycin and gentamycin, but exert their protective effects through different mechanisms (Coffin et al., 2009). In mammals, preliminary injection of magnesium solution, by means of electrophoresis, reduced the ototoxic effect of kanamicin (Spasov et al., 1999).
Other well-known ototoxic treatments are platinum compounds. Cisplatin is a frequently used chemotherapeutic agent in the treatment of many types of neoplasias, especially of the head and neck (Rybak and Withworth, 2005). The mechanism of antineoplastic action is associated with the selective and persistent inhibition of the deoxyribonucleic acid synthesis (Williams and Whitehouse, 1979). Its side effects include ototoxicity, kidney toxicity, medullar suppression and gastrointestinal disorders. The nephrotoxic 
effect of cisplatin is severe damage in the renal proximal cells within the thick ascending loop of Henle, manifested by hypomagnesemia. These types of toxicity can impact treatment, as they reduce the chemotherapy dosage, frequency and duration for many patients. Some audiometric studies have reported elevated hearing threshold in $75-100 \%$ of patients treated with cisplatin (McKeage, 1995).

The auditory lesions seem to result from free radical-induced damage to many tissues (Kopke et al., 1997; Rybak et al., 1995; Dehne et al., 2001). Oxygen reactive species are generated in the cochlea after exposure to cisplatin (Clerici and Yang, 1996) and such oxidative stress can cause cochlear cell death by apoptosis secondary to the activation of caspase-3 (Garcia-Berrocal et al., 2007; De Freitas et al., 2009). Inner ear cell apoptosis can be triggered by the formation of complexes between cisplatin and the DNA of the damaged cell, preventing the progression of the cell cycle. Cevette et al., (2000) observed a significant increase in otoacoustic emission amplitude (which provides frequency-specific information about $\mathrm{OHC}$ function) in two patients undergoing cisplatin treatment supplemented with magnesium. The authors of this study concluded that $\mathrm{Mg}^{2+}$ might be a promising agent against cisplatin ototoxicity. However, the study of Sahin et al., (2006) showed that a $\mathrm{Mg}^{2+}$ rich diet prevented the severe hypomagnesemia that cisplatin causes in guinea pigs, but failed to provide any protection against its ototoxic effect.

\section{Magnesium and sudden sensorineural hearing loss}

The US National Institute for Deafness and Communication Disorders defines SSHL as the idiopathic loss of hearing of at least $30 \mathrm{~dB}$ over at least 3 contiguous test frequencies occurring within 3 days. Estimates of the overall incidence of SSHL range from 5 to 20 per 100000 persons per year (Conlin and Parnes, 2007). Many etiologic causes of SSHL have been proposed, but many remain unconfirmed. In the majority of patients $(71 \%)$, the aetiology remains idiopathic. A wide range of disorders might lead to the development of SSHL. The theorized major causative factors can be broken down into several categories: 1) viral infection (13\%); 2) vascular impairment; 3) immune-mediated mechanisms; 4) inner ear abnormalities; and 5) CNS abnormalities, including tumours, trauma, haemorrhage, infarction and other pathologies (Chau et al., 2010). The lack of understanding of the mechanism of SSHL has rendered the development of a specific treatment difficult, and currently, empirical guidelines are used. Because of the spontaneous recovery rates of $32 \%$ to $70 \%$ (Colin and Parnes, 2010), some otolaryngologists choose not to treat SSHL. However the most common approach to treatment of SSHL is with systemic steroids in moderate doses. Nageris et al., (2004) evaluated the efficiency of two treatments after SSHL in humans. The first one consisted of corticosteroid and magnesium, and the second one (control) in corticosteroid and placebo. They observed that more patients treated with magnesium experienced hearing improvement, and at a larger magnitude, than control subjects. Gordin and colleagues (2002) reported a significantly greater recovery rate among patients treated with magnesium and carbogen vs patients treated with carbogen alone.

\section{How does magnesium therapy work?}

Magnesium plays an essential role in the regulation of most cellular functions. However, it is recognized that magnesium status in humans is often deficient. In the recent French sU.VI.MAX study (the "supplementation en vitamines et mineraux antioxidants" study) of 5,448 subjects, it was shown that $77 \%$ of women and $72 \%$ of men had dietary magnesium intakes lower than the recommended dietary allowance (Galan et al., 1997). The deficiency is increased by chronic stress, loud noise exposure or chemotherapy with platinum compounds (Galland, 1991; Mocci et al., 2001, Sahin et al., 2006). Scheibe et al., (1999) were the first to study the correlation between the plasma, perilymph and cerebrospinal magnesium contents in the same animal. Contrary to the blood brain barrier, the blood perilymph barrier is not able to concentrate $\mathrm{Mg}^{2+}$ taken up from plasma. Also, the perilymph $\mathrm{Mg}^{2+}$ concentration correlates well with the plasma level, and a $\mathrm{Mg}^{2+}$ deficiency affects perilymph content.

The exact manner by which $\mathrm{Mg}^{2+}$ affects the susceptibility to hearing loss is still unknown, but several mechanisms could be evoked. By its 
nature, magnesium is a calcium antagonist and therefore blocks the excessive release of calcium, both in the hair cells and in the cochlear vasculature, limiting cell energy depletion and inducing the vasodilatation of arterioles. Through these mechanisms, magnesium could limit ischemia induced by acoustic trauma. Haupt and Scheibe (2002) demonstrated that cochlear blood flow as well as perilymphatic $\mathrm{pO}_{2}$ levels were significantly increased in noise-traumatized animals that received magnesium supplements. Not only does magnesium combat ischemia, but it is also thought to prevent cell damage caused by hypoxia. Konig et al., (2003) noted a protective effect of magnesium on hypoxia-induced hair cell loss in vitro. In other models, high magnesium concentration has been shown to attenuate the hypoxia/ischemia-induced disruption of the mitochondrial membrane potential, a critical event in triggering cell death (Sharikabad et al., 2001). This mechanism is supported by the observation that an increase in the extracellular magnesium concentration led to a decrease in hypoxia induced apoptosis by maintaining the normal ratio of $\mathrm{Bax}$ to $\mathrm{Bcl}-2$ proteins involved in determining the survival of cells or their death (Ravishankar, 2001).

Another potential mechanism explaining the $\mathrm{Mg}^{2+}$ efficiency in NIHL or in drug ototoxicity, involves free radical production. Two mechanisms have been suggested (Garcia et al., 1998). It may directly inhibit free radical production, or it may facilitate scavenging of free radicals. Afanas'ev et al., (1995) showed that $\mathrm{Mg}^{2+}$ inhibits reduced NADP oxidase, an enzyme that produces superoxide radical. However, despite the protective effect of magnesium against oxidative stress, as demonstrated in different models (Sharikabad et al., 2001, Bede et al., 2008), no data are currently available about the cochlea.

Lastly, $\mathrm{Mg}^{2+}$ could act on glutamate excitotoxicity, especially in NIHL. Magnesium apparently enhances the survival capability of the cochlear afferents, reducing the effect of glutamateinduced inner hair cell damage (Ehrenberger and
Felix, 1995). Magnesium is able to modulate the opening of $\mathrm{Na}^{+} / \mathrm{Ca}^{++}$channels of the NMDA receptors (Mayer et al., 1984). The blockade of the NMDA receptors by $\mathrm{Mg}^{2+}$ is voltagedependent, but extracellular $\mathrm{Mg}^{2+}$ behaves as a non-competitive NMDA antagonist, without the side effects presented by the other noncompetitive NMDA antagonists. In the hearing process, if $\mathrm{Mg}^{2+}$ is low, an excess of $\mathrm{Ca}^{2+}$ could enter hair cells. In turn, more glutamate would then be produced in response to this $\mathrm{Ca}^{2+}$ influx. Increased glutamate would also greatly increase the activity of the NMDA receptor, which is also operating with low magnesium. With the double insult of high glutamate and low $\mathrm{Mg}^{2+}$, a flood of $\mathrm{Ca}^{2+}$ could go through the NMDA channels into the nerve cell, and the energetic system could be compromised.

\section{Conclusion}

Hearing loss is a significant clinical issue. We now know many of the molecular pathways leading to apoptotic cell death that are triggered by noise and other environmentally mediated traumas such as aminoglycoside antibiotics and chemotherapeutics. There is increasing evidence for their similarity, since free radical formation and apoptotic cascades have been implicated in all. Interventions can be directed at preventing initial ROS formation, maintaining cochlear blood flow or blocking apoptosis. A large number of therapeutics, including magnesium, have been tested. Magnesium, by its neuroprotective and vasodilatory effects, has the potency to prevent as well as to limit hearing loss, particularly after noise exposure or sudden sensorineural hearing loss. Magnesium therapy at the recommended dosage appears to be safe with few contraindications (such as severe renal failure). In contrast to other therapeutic agents (like corticosteroids), it easily crosses the perilymph blood barrier and reaches the organ of Corti. The majority of studies have shown that magnesium is partly effective. Using magnesium in combination with other agents could improve recovery after hearing loss. 


\section{References}

Abaamrane L, Raffin F, Gal M, Avan P, Sendowski I (2009) Long-term administration of magnesium after acoustic trauma caused by gunshot noise in guinea pigs. Hear Res 247:137-45.

Afanas'ev I, Suslova T, Cheremisina Z, Abramova N, Korkina L (1995) Study of antioxidant properties of metal aspartates. Analyst 120:859-62.

Attias J, Bresloff I, Haupt H, Scheibe F, Ising H (2003) Preventing noise induced otoacoustic emission loss by increasing magnesium (Mg2+) intake in guinea pigs. J Basic Clin Physiol Pharmacol 14:119-36.

Attias J, Sapir S, Bresloff I, Reshef-Haran I, Ising H (2004) Reduction in noise-induced temporary threshold shift in humans following oral magnesium intake. Clin Otolaryngol 29:635-41.

Attias J, Weisz G, Almog S, Shahar A, Wiener M, Joachims Z, Netzer A, Ising H (1994) Oral magnesium intake reduces permanent hearing loss induced by noise exposure. Am J Otol 15:26-32.

Bede O, Ngy D, Suranyi A, Horvath I, Szlavik M, Gyurkovits K (2008) Effects of magnesium supplementation on the glutathione redox system in atopic asthmatic children. Inflamm Res 57:279-86.

Cevette MJ, Drew D, Webb TM, Marion MS (2000) Cisplatin ototoxicity, incresed DPOAE amplitudes, and magnesium deficiency. Distorsion product otoacoustic emissions. J Am Acad Audiol 11:323-9.

Chau J, Lin J, Atashband S, Irvine R, Westerberg B (2010) Systematic review of the evidence fort he etiology of adult sudden sensorineural hearing loss. Laryngoscope 120:1011-21.

Clerici W, Yang L (1996) Direct effects of intraperilymphatic reactive oxygen species generation on cochlear function. Hear Res 101:14-22.

Coffin AB, Reinhart KE, Owens KN, Raible DW, Rubel EW (2009) Extracellular divalent cations modulate aminoglycoside-induced hair cell death in the zebrafish lateral line. Hear Res 253:42-51.

Conlin E, Parnes $L$ (2007) Treatment of sudden sensorineural hearing loss: A systematic review. Arch Otolaryngol Head Neck Surg 133:573-81.

De Freitas MR, Figueiredo AA, Brito GA, Leitao RF, Carvalho JV, Gomes RM, Ribeiro A (2009) The role of apoptosis in cisplatin-induced ototoxicity in rats. Braz J Otolaryngol 75:745-52.
Dehne N, Lautermann J, Petrat F, Rauen U, De Groot H (2001) Cisplatin Ototoxicity: involvement of iron and enhanced formation of superoxide anion radicals. Toxicol Appl Pharmacol 174:27-34.

Devrière F, Ising H, Dancer A (1991) Déficits auditifs provoqués par l'exposition à des bruits impulsionnels chez des cobayes carencés ou enrichis en magnésium. J Acoustique 4:363-6.

Ehrenberger K, Felix D (1995) Receptor pharmacological models for inner ear therapies with emphasis on glutamate receptors: a survey. Acta Otolaryngol (Stockh) 115:236-40.

Galan P, Preziosi P, Durlach V, Valeix P, Ribas L, Bouzid D, Favier A, Hercberg S (1997) Dietary magnesium intake in a French adult population. Magnes Res 10:321-8.

Galland L (1991) Magnesium, stress and neuropsychiatric disorders. Magnes Trace Elem 10:287-301.

Garcia L, Dejong S, Martin S, Smith R, Buettner G, Kerber $R$ (1998) Magnesium reduces free radicals in an in vivo coronary occlusion-reperfusion model. J Am Coll Cardiol 32:536-9.

García-Berrocal JR, Nevado J, Ramírez-Camacho R, Sanz R, González-García JA, Sánchez-Rodríguez C, Cantos B, España P, Verdaguer JM, Trinidad Cabezas A (2007) The anticancer drug cisplatin induces an intrinsic apoptotic pathway inside the inner ear. $\mathrm{Br} \mathrm{J}$ Pharmacol 152:1012-20.

Gordin A, Goldenberg D, Golz A, Netzer A, Joachims H (2002) Magnesium: a new therapy for idiopathic sudden sensorineural hearing loss. Otol Neurotol 23:447-51.

Günther T, Ising H, Joachims Z (1989) Biochemical mechanisms affecting susceptibility to noise-induced hearing loss. Am J Otol 10:36-41.

Haupt H, Scheibe F (2002) Preventive magnesium supplement protects the inner ear against noiseinduced impairment of blood flow and oxygenation in the guinea pig. Magnes Res 15:17-25.

Haupt H, Scheibe F, Mazurek B (2003) Therapeutic efficacy of magnesium in acoustic trauma in the guinea pig. Otorhinolaryngol Relat Spec 65:134-9. 
Henderson D, Bielefeld EC, Harris KC, Hu BH (2006) The role of oxidative stress in noise-induced hearing loss. Ear and Hearing 27:1-19.

Hu BH, Henderson D, Nicotera TM (2002) Involvement of apoptosis in progression of cochlear lesion following exposure to intense noise. Hear Res 166:62-71.

Hu BH, Henderson D, Nicotera T (2006) Extremely rapid induction of outer hair cell apoptosis in the chinchilla cochlea following exposure to impulse noise. Hear Res 211:16-25.

Ising $\mathrm{H}$, Handrock $\mathrm{M}$, Gunther $\mathrm{T}$, Fischer $\mathrm{R}$, Dombrowski M (1982) Increased noise trauma in guinea pigs through magnesium deficiency. Arch Otorhinolaryngol 236:139-46.

Joachims Z, Babisch W, Ising H, Guenther T, Handrock $M$ (1983) Dependence of noise-induced hearing loss upon perilymph magnesium concentration. J Acoust Soc Am 74:104-8.

Joachims Z, Ising H, Gunther T (1987) Noise-induced hearing loss in humans as a function of serum $\mathrm{Mg}$ concentration. Magnes Bull 9:130-1.

Konig O, Winter E, Fuchs J, Haupt H, Mazurek B, Weber N, Gross J (2003) Protective effect of magnesium and MK 801 on hypoxia-induced hair cell loss in new-born rat cochlea. Magnes Res 16:98-105.

Kopke RD, Liu W, Gabaizadeh R, Jacono A, Feghali J, Spray D (1997) Use of organotypic cultures of Corti's organ to study the protective effects of antioxidant molecules on cisplatin-induced damage of auditory hair cells. Am J Otolaryngol 18:559-71.

Le Prell CG, Hughes LF, Miller JM (2007b) Free radical scavengers vitamins $A, C$, and $E$ plus magnesium reduce noise trauma. Free Radic Biol Med 42:1454-63.

Le Prell CG, Yamashita D, Minami SB, Yamasoba T, Miller JM (2007a) Mechanisms of noise-induced hearing loss indicate multiple methods of prevention. Hear Res 226:22-43.

Mayer ML, Westbrook GL, Guthrie P (1984) Voltage dependent block by magnesium of NMDA response in spinal cord neurons. Nature 309:261-3.

McKeage MJ (1995) Comparative adverse effects of platinium drugs. Drug Safety 13:228-244.

Miller JM, Brown JN, Schacht J (2003) 8-isoprostagladin $\mathrm{F}$ (2alpha), a product noise of exposure, reduces inner ear blood flow. Audiol Neurootol 8:20721.
Mocci F, Canalis P, Tomasi PA, Casu F, Pettinato S (2001) The effect of noise on serum and urinary magnesium and catecholamines in humans. Occup Med 51:56-61.

Nageris B, Ulanovski D, Attias J (2004) Magnesium treatment for sudden hearing loss. Ann Otol Laryngol 113:672-5.

Pujol R, Puel JL, Gervais d'Aldin C, Eybalin M (1990) Physiopathology of the glutamatergic synapses in the cochlea. Acta Oto-Laryngol 113:330-4.

Quirk WS, Seidman MD (1995) Cochlear vascular changes in response to loud noise. Am J Otol 16:322-5.

Ravishankar S, Ashraf Q, Fritz K, Mishra O, DelivoriaPapadopoulos M (2001) Expression of Bax and Bcl-2 proteins during hypoxia in cerebral cortical neuronal nuclei of newborn piglets: effect of administration of magnesium sulphate. Brain Res 901:23-9.

Rybak LP, Ravi R, Somani SM (1995) Mechanism of protection by diethyldithiocarbamate against cisplatin ototoxicity: antioxidant system. Fundam Appl Toxicol 26:293-300.

Rybak LP, Withworth CA (2005) Ototoxicity: therapeutic opportunities. Drug Discovery Today 10:1313-21.

Sahin AA, Oysu CC, Yilmaz HB, Topak M, Kulekci M, Okar I (2006) Effect of oral magnesium supplementation on cisplatin ototoxicity. $J$ of Otolaryngol 35:112-6.

Scheibe F, Haupt H, Ising H (1999) Total magnesium concentrations of perilymph, cerebrospinal fluid and blood in guinea pigs fed different magnesiumcontaining diets. Eur Arch Otorhinolaryngol 256:215-9.

Scheibe F, Haupt $H$, Ising $H$ (2000b) Preventive effect of magnesium supplement on noise-induced hearing loss in the guinea pig. Eur Arch Otorhinolaryngol 257:10-6.

Scheibe F, Haupt $H$, Ising $H$, Cherny L (2002) Therapeutic effect of parenteral magnesium on noiseinduced hearing loss in guinea pig. Magnes Res 15:2736.

Scheibe F, Haupt H, Mazurek B, Konig O (2001) Therapeutic effect of magnesium on noise-induced hearing loss. Noise \& Health 3:79-84. 
Scheibe F, Haupt H, Vlastos GA (2000a) Preventive magnesium supplement reduces ischemia-induced hearing loss and blood viscosity in the guinea pig. Eur Arch Otorhinolaryngol 257:355-61.

Sendowski I, Raffin F, Braillon-Cros A (2006) Therapeutic efficacy of Magnesium after acoustic trauma caused by gunshot noise in guinea pigs. Acta Oto-Laryngol 126:122-9.

Sharikabad M, Ostbye K, Lyberg T, Brors O (2001) Effect of extracellular Mg2+ on ROS and Ca2+ accumulation during reoxygenation of rat cardiomyocytes. Am J Phyiol Heart Circ Physiol 280:H344-53.

Shibata SB, Raphael Y (2010) Future approaches for inner ear protection and repair. J Commun Disord 43:295-310.

Spasov AA, Lobzov MS, Sanzharovskaia NK, Kozhevnikova EV, Kuzubova EA (1999) The effect of polikatan on the ototoxic action of kanamycin. Eksp Klin Farmakol 62:65-6.

Thorne RP, Nuttal AL (1987) Laser Doppler measurements of cochlear blood flow during loud sound exposure in guinea pigs. Hear Res 27:1-10.

Vormann J, Günther T (1993) Influence of magnesium on drug-and noise-induced inner ear damage. Animal studies. Schriften Ver Wasser Boden Lufthyg 88:491502.
Vormann J, Günther T (1991) The role of magnesium and zinc in determining vulnerability of the auditory system to salicylate and gentamicin. Arch Compl Env Stud 3:75-82.

Walden BE, Henselman LW, Morris ER (2000) The role of magnesium in the susceptibility of soldiers to noiseinduced hearing loss. J Acoust Soc Am 108:453-6.

World Health Organization (2004) Occupational noise: assessing the burden of disease from work-related hearing impairment at national and local levels. World Health Organization, Series Number 9, Geneva.

Williams CJ, Whitehouse JM (1979) Cis-paltinum: a new anticancer agent. Br Med J 23:1689-91.

Yamashita D, Jiang H, Schacht J, Miller J (2004) Delayed production of free radicals following noise exposure. Brain Res 1019:201-9.

Yang W, Henderson D, Hu BH, Nicotera T (2004) Quantitative analysis of apoptotic and necrotic outer hair cells after exposure to different levels of continuous noise. Hear Res 196:69-76.

Zenner H (1986) K+-induced motility and depolarization of cochlear hair cells. Direct evidence for a new pathophysiological mechanism in Meniere's disease. Arch Otorhinolaryngol 243:108-11.

Zhao F, Manchaiah VK, French D, Price SM (2010) Music exposure and hearing disorders: an overview. Int J Audiol 49:54-64. 



\title{
The role of magnesium in pain
}

\author{
Hyo-Seok Na, Jung-Hee Ryu and Sang-Hwan Do \\ Department of Anesthesiology and Pain Medicine, Seoul National University Bundang Hospital, Seongnam, South Korea. \\ \shdo@snu.ac.kr
}

\begin{abstract}
Magnesium plays an important role in the prevention of central sensitization and in the attenuation of established pain hypersensitivity, and its main mode of action appears to involve its voltage-gated antagonist action at N-methyl-D-aspartate (NMDA) receptors. Given the putative function of the NMDA receptor in pain transduction, magnesium has been investigated in various clinical conditions associated with acute or chronic pain. The parenteral administration of magnesium, via an intravenous, intrathecal, or epidural route, may reduce pain, and anaesthetic and analgesic requirements during the intra- and post-operative periods. The beneficial effects of magnesium treatment have also been demonstrated in patients suffering from neuropathic pain, such as in those with malignancy-related neurologic symptoms, postherpetic neuralgia, diabetic neuropathy, and chemotherapy-induced peripheral neuropathy. In addition, magnesium therapy has been shown to be effective in alleviating dysmenorrhea, headaches, and acute migraine attacks. Magnesium is playing an evolving role in pain management, but a more thorough understanding of the mechanisms underlying its antinociceptive action and additional clinical studies are required to clarify its role as an analgesic adjuvant.
\end{abstract}

\section{Introduction}

The research interest in NMDA receptors has led to an examination of the interactions between NMDA receptors and the induction and maintenance of central sensitization after nociceptive stimuli (Woolf and Thompson, 1991). Ketamine and magnesium are representative NMDA receptor antagonists, and in particular, magnesium can regulate calcium access into cells by antagonizing the NMDA receptor (Paoletti and Neyton, 2007), which has encouraged investigations on the use of magnesium as an analgesic adjuvant. Recent studies have proposed a role for NMDA receptor antagonists in the management of postoperative pain and in other acute and chronic pain conditions. This chapter describes the pharmacologic basis of pain relief by the magnesium ion, and surveys various clinical studies that have examined the antinociceptive effects of magnesium.

\section{Mechanism of magnesium as an analgesic adjuvant}

Although magnesium has no direct analgesic effect, it inhibits calcium ions entering cells by blocking NMDA receptors, which causes an antinociceptive effect. Furthermore, this antinociceptive effect is related to its prevention of central sensitization caused by peripheral tissue injury (Woolf and Thompson, 1991). Central sensitization is the result of the enhancement of neuronal properties in nociceptive pathways of the central nervous system, and is triggered by repetitive nociceptive afferent inputs, which manifests as a prolonged reduction in the pain threshold. Central sensitization produces pain hypersensitivity, such as wind-up or long-term potentiation of pain, that is, it causes pain even when peripheral stimuli are not intense and continues to cause pain after the initiating stimuli have disappeared (Latremoliere and Woolf, 2009; Woolf, 1983; Woolf and Salter, 2000).

Increased intracellular calcium levels seem to play a major role in the initiation of central sensitization (Pockett, 1995; Woolf and Chong, 1993), and the build-up of intracellular calcium is associated with various receptors on postsynaptic neurons of the spinal dorsal horn, such as, NMDA, $\alpha$-amino-3-hydroxy-5-methyl-4-isoxazole propionate (AMPA), kainate, and glutamate receptors (Latremoliere and Woolf, 2009). Of these receptors, NMDA receptor activation has been demonstrated to be essential for initiating and maintaining central sensitization. 
The NMDA receptor is a membrane ion channel expressed in the central nervous system. It is a tetramer composed of four different subunits, that is, two NR1 and two NR2 (Dingledine et al., 1999). NMDA receptors regulate the cellular inflows of $\mathrm{Na}^{+}$and $\mathrm{Ca}^{2+}$, and the outflow of $\mathrm{K}^{+}$. This voltage-dependent ion channel is blocked non-competitively in the resting state by the magnesium ion and by ketamine (phencyclidine site blockade), MK-801, memantine, and others (Felsby et al., 1996; Paoletti and Neyton, 2007) (Fig. 1). On the other hand, the NMDA receptor channel is opened by membrane depolarization induced by the sustained release of glutamate and neuropeptides, which include substance $P$ and calcitonin gene-related peptide (CGRP) (Baranauskas and Nistri, 1998; Mayer et al., 1984).

Extracellular magnesium blocks the NMDA receptor in a voltage-dependent manner (Mayer et al., 1984), and thus, can prevent the establishment of central sensitization and abolish existing hypersensitivity. Other noncompetitive or competitive NMDA receptor antagonists, such as MK801 and D-CPP, also prevent and reverse the hyperexcitability of neurons produced by nociceptive afferent inputs (Ma and Woolf, 1995; Woolf and Thompson, 1991).

\section{Perioperative pain}

Many authors have investigated the adjuvant role of magnesium in the context of intra- and postoperative analgesia. Magnesium has been shown to be effective for treating intra- and postoperative pain and for blunting autonomic, somatic, and endocrine reflexes to noxious stimuli (Kara et al., 2002; Koinig et al., 1998; Levaux et al., 2003). Usually, magnesium is administered as an i.v. $30-50 \mathrm{mg} / \mathrm{kg}$ bolus of magnesium sulphate as a loading dose, and maintained at $6-20 \mathrm{mg} / \mathrm{kg} / \mathrm{h}$ by continuous infusion until the end of surgery (Koinig et al., 1998; Ryu et al., 2008; Ryu et al., 2009; WilderSmith et al., 1997), or for 4 hours after the initial bolus (Seyhan et al., 2006).

Tramer et al., (1996) conducted the first prospective, randomized study on the effect of magnesium on analgesic requirements, pain, comfort, and quality of sleep during the immediate postoperative period. They showed

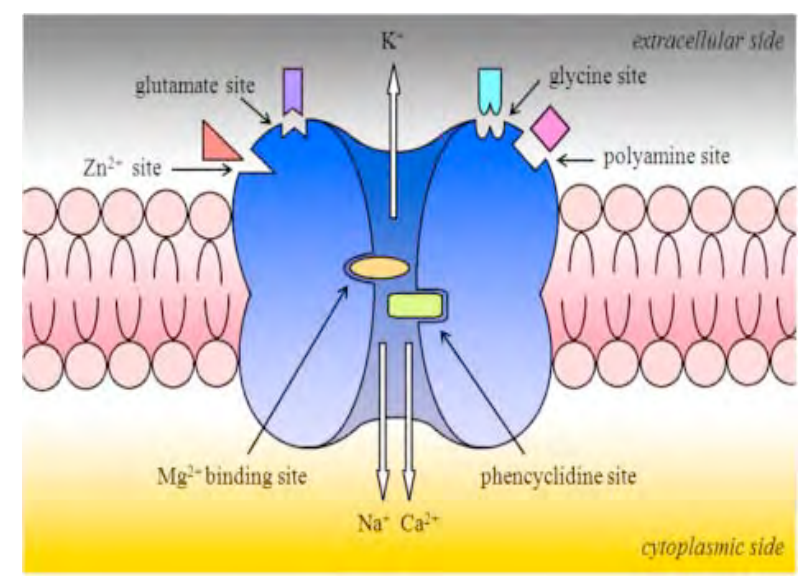

Figure 1. Scheme of the NMDA receptor and its ligand binding sites. The NMDA receptor is composed of four subunits (two NR1 and two NR2). In the extracellular region, there are agonist-binding sites for glutamate and glycine, co-agonists for the efficient opening of the ion channel. The ion channel includes the binding sites for the pore block, one of which is for the $\mathrm{Mg}^{2+}$ and the other is for ketamine, MK-801, memantine, and so on.

that magnesium sulphate reduces analgesic requirements and discomfort, and improves quality of sleep during the postoperative period, and that it does not cause any adverse effect at $48 \mathrm{~h}$ after surgery. Oguzhan et al., (2008) studied the effect of a magnesium sulphate infusion on postoperative requirements for opioids, intraoperative muscle relaxant, inhalational anaesthetic consumption, and post-operative pain during and after lumbar disc surgery. Their results suggested that intraoperative magnesium administration significantly reduced intraoperative muscle relaxant and opioid requirements, and also reduced postoperative pain and opioid use.

When used during a variety of surgeries, magnesium was also found to reduce the need for intraoperative anaesthetics and muscle relaxants and to reduce the amount of morphine required to treat postoperative pain. Ryu et al., (2009) compared remifentanil and magnesium during middle ear surgery in terms of postoperative pain and other complications. In this study, magnesium or remifentanil combined with sevoflurane provided adequate hypotensive anaesthesia, but patients in the magnesium group experienced a more comfortable postoperative course with better analgesia, less 
shivering, and less postoperative nausea and vomiting (PONV). Furthermore, the amount of sevoflurane required to maintain surgical anaesthesia was significantly lower in the magnesium group than in the remifentanil group (Ryu et al., 2009).

The use of magnesium as an analgesic adjunct has also been found to be beneficial in patients on total intravenous anaesthesia (TIVA). Significant reductions in intraoperative propofol, atracurium, and postoperative morphine consumption were observed in patients undergoing gynaecological surgery (Seyhan et al., 2006). In another study on gynaecologic patients receiving TIVA, post-operative pain scores, cumulative analgesic consumption, and shivering incidents were significantly lower in patients treated with magnesium, to the extent that the authors concluded magnesium sulphate during TIVA improved the quality of postoperative analgesia (Ryu et al., 2008). Furthermore, Choi et al., (2002) found that intravenous magnesium sulphate reduced propofol infusion requirements during the maintenance of propofol-nitrous oxide anaesthesia in patients undergoing total abdominal hysterectomy.

The usefulness of adjunctive magnesium for postoperative pain control has also been examined in the context of regional anaesthesia. Recent studies have suggested that magnesium may play a beneficial role in spinal anaesthesia. For example, magnesium was found to prevent the induction of central sensitization by peripheral nociceptive stimulation at a spinal site of action, by blocking NMDA receptors in a voltage-dependent fashion (Woolf and Thompson, 1991). Utilizing the same mechanism, the addition of small doses of magnesium sulphate to local anaesthetics for spinal anaesthesia enhances the duration of anaesthesia and reduces postoperative analgesic requirements and the incidence of side effects of high doses of local anaesthetics and opioids. In animal studies, the intrathecal co-administration of magnesium sulphate during spinal anaesthesia was found to significantly potentiate morphine analgesia in normal rats and in a surgical model of mechanical allodynia (Kroin et al., 2000). In another experimental model, intrathecal magnesium sulphate produced a state of spinal anaesthesia and general sedation in rats that lasted for around $1 \mathrm{~h}$, and at $6 \mathrm{~h}$ post-injection, animals recovered and showed no evidence of neurotoxicity (Bahar et al., 1996). Clinical studies have also shown that intrathecal magnesium sulphate added to fentanyl prolongs analgesia without increasing side effects during labour analgesia (Buvanendran et al., 2002; Ozalevli et al., 2005). Furthermore, the i.v. infusion of magnesium sulphate during spinal anaesthesia was found to improve postoperative analgesia and reduce the cumulative consumption of analgesics after total hip replacement arthroplasty (Hwang et al., 2010). Postoperative adjunctive i.v. magnesium infusion was also found to increase time to analgesic need and to reduce total analgesia consumption after spinal anaesthesia (Apan et al., 2004).

Comparatively little is known of the effect of epidural magnesium sulphate, whereas intrathecal magnesium sulphate has been investigated on many occasions. Caudal epidural anaesthesia with lidocaine plus magnesium sulphate was found to produce analgesia of longer duration than lidocaine plus distilled water in cattle (Dehghani and Bigham, 2009). Arcioni et al., (2007) compared intrathecal, epidural, combined intrathecal and epidural magnesium sulphate, and spinal anaesthesia alone (controls) in patients undergoing orthopaedic surgery to investigate whether intrathecal and/or epidural magnesium sulphate could reduce postoperative analgesic requirements. The results obtained suggested that combined intrathecal and epidural magnesium sulphate significantly reduced postoperative analgesic requirements (Arcioni et al., 2007).

However, not all investigations have reported postoperative analgesic effects for magnesium sulphate. For example, perioperative i.v. magnesium infusion was not found to reduce postoperative pain or analgesic consumption in patients undergoing abdominal hysterectomy (Ko et al., 2001) or caesarean delivery (Paech et al., 2006). Furthermore, in a recent report issued by Tramer and Glynn (2007), pretreatment with magnesium sulphate was found to have no effect on postoperative pain or analgesic requirements over the first three postoperative days in patients undergoing ambulatory ilioinguinal hernia repair or varicose vein surgery. However, in this study, a single dose $(4 \mathrm{~g})$ of intravenous magnesium 
sulphate was used instead of a loading dose plus continuous infusion.

Although some debate exists concerning the role of magnesium sulphate as an analgesic adjuvant, the consensus is that magnesium sulphate acts to support a neuromuscular blockade. Magnesium acts as a calcium channel blocker at presynaptic nerve terminals and reduces acetylcholine release at the motor endplate (Fisher, 1999). This diminishes muscle fibre excitability and reduces end plate potential amplitudes, which leads to the potentiation of a neuromuscular blockade by nondepolarizing neuromuscular blockers (Fisher, 1999). Some authors have focused on the direct effect of magnesium on neuromuscular blockade (Fuchs-Buder et al., 1995; Fuchs-Buder and Tassonyi, 1996; Kussman et al., 1997; Ross and Baker, 1996; Telci et al., 2002), whereas others concluded that a perioperative adjuvant magnesium infusion enhances neuromuscular blockade (Lee and Kwon 2009; Oguzhan et al., 2008; Ryu et al., 2008; Ryu et al., 2009; Seyhan et al., 2006).

Controlled hypotension is sometimes required during surgery to maintain a bloodless operative field, and investigations on perioperative magnesium infusion for the control of hypotension during middle ear surgery decreased the incidences of postoperative nausea and vomiting (PONV) (Ryu et al., 2008; 2009), which could have been due to lower sevoflurane consumption (Apfel et al., 2002; Ryu et al., 2009) rather than any antiemetic effect of the magnesium. Nevertheless, since PONV is one of the most common and distressing complications after surgery, this effect is interesting, because it could be used to benefit, for example, patients undergoing ambulatory surgery. Perioperative i.v. magnesium infusion has another advantageous effect, in that it decreases the incidence of shivering by up to $70-90 \%$ (Ryu et al., 2008; Ryu et al., 2009; Tramer and Glynn, 2007). Shivering is one of the leading causes of postoperative discomfort (Alfonsi, 2001), and increases oxygen consumption (Alfonsi, 2001). Thus, the prevention of shivering is one of the most obvious benefits of magnesium sulphate administration in surgical patients.

When mixed with local anaesthetic, magnesium may also show beneficial effects in intravenous regional anaesthesia. In one study, magnesium was added to lidocaine for intravenous regional anaesthesia, and was found to improve quality of anaesthesia and analgesia, specifically, sensory and motor block onset times were shorter and postoperative analgesia was better with magnesium (Turan et al., 2005). However, in this study, recovery after intravenous regional anaesthesia was prolonged in the magnesium group (Turan et al., 2005). In contrast, i.v. magnesium infusion during general anaesthesia has not been found to delay recovery from anaesthesia in most investigations (Lee and Kwon, 2009; Ozcan et al., 2007; Ryu et al., 2008). Another consideration during magnesium administration is that magnesium may cause cardiovascular depression by acting as a calcium channel blocker. The consequent inhibition of catecholamine release lowers plasma epinephrine and norepinephrine concentrations after endotracheal intubation, and therefore reduces hypertensive responses during anaesthesia induction (James et al., 1989). Accordingly, magnesium should be used with caution in hypovolemic patients and in those with limited cardiac capacity.

\section{Other pain conditions}

Numerous clinical studies have found that magnesium has beneficial effects in patients suffering from neuropathic pain, dysmenorrhea, tension headache, acute migraine attack, and others. These effects are considered to be due to blockage of the NMDA receptor, attenuation of central sensitization, and muscle relaxing effects.

\section{Neuropathic pain}

Neuropathic pain is caused by damage to or by the dysfunction of a component of the peripheral or central nervous system (Backonja, 2003). Thus, the causes of neuropathic pain are various and include spinal cord injury, multiple sclerosis, diabetic neuropathy, radiation injury, complications of chemotherapy, a malignancyrelated neurologic symptom, amongst others. Its common features include burning, a cold sensation, numbness, itching, and a shooting or abnormal sensation called dysesthesia in a continuous or episodic manner. The mechanism responsible for neuropathic pain remains unknown, but peripheral or central sensitization is a prime candidate (Bridges et al., 2001; Ossipov 
et al., 2000). Neuropathic pain is extremely difficult to treat, and is only partially relieved in some $40-60 \%$ of patients. Favoured pharmacologic treatments are antidepressants, anticonvulsants, opioids, and NMDA receptor antagonists (O'Connor and Dworkin, 2009). Magnesium has been shown to have beneficial effects on neuropathic pain in some clinical and animal studies. When magnesium sulphate (bolus doses of $500 \mathrm{mg}$ or $1 \mathrm{~g}$ ) was given intravenously to patients with uncontrolled neuropathic pain due to cancer infiltration, pain intensity and pain relief scores improved (Crosby et al., 2000). In another study, magnesium chloride (bolus 0.16 $\mathrm{mmol} / \mathrm{kg}$ over 10 minutes followed by continuous infusion at $0.16 \mathrm{mmol} / \mathrm{kg} / \mathrm{h}$ ) reduced protracted pain and regions of allodynia in patients suffering from peripheral neuropathic pain (Felsby et al., 1996). In addition, in an animal model of neuropathic pain induced hyperalgesia subsided after intraperitoneal magnesium therapy (total 150 or $200 \mathrm{mg} / \mathrm{kg}$ for 5 days) (Begon et al., 2000).

\section{Postherpetic neuralgia}

Postherpetic neuralgia (PHN), a type of neuropathic pain, is characterized by chronic persistent pain after an acute herpes zoster attack (Rowbotham and Petersen, 2001). Typically, PHN causes a burning, aching, sharp, and lancinating pain that occurs in a continuous or paroxysmal pattern, and is often accompanied by hyperalgesia and allodynia. Brill et al., (2002) showed that magnesium can relieve hyperalgesia and allodynia in PHN. When they administered i.v. magnesium sulphate at $30 \mathrm{mg} / \mathrm{kg}$ over $30 \mathrm{~min}$ to patients with intractable PHN, pain was either relieved completely or diminished (Brill et al., 2002).

\section{Diabetic neuropathy}

Diabetes mellitus is the most common etiologic factor of peripheral neuropathy, and diabetic neuropathy is often expressed as numbness, a tingling sensation, and hypersensitivity to pain. These symptoms are limited to the fingers and toes during early disease, and then spread to the proximal extremities to produce a characteristic glove and stocking pattern (Head, 2006). Conventional treatments include antidepressants, anticonvulsants, lidocaine patches, opioid or nonopioids analgesics, and various alternative treatments (Head, 2006). Recently, dietary magnesium supplementation has been at the focus of attention, and oral doses of magnesium sulphate have been found to be effective at reducing thermal pain thresholds in rats. However, appropriate treatment dosages and durations have not been established (Hasanein et al., 2006). De Leeuw et al., (2004) also demonstrated that long-term magnesium supplementation (300 mg daily for 5 years) can favourably influence the progression of diabetic polyneuropathy.

\section{Chemotherapy-induced peripheral neuropathy}

CIPN is one of the most common side effects in cancer patients administered many chemotherapeutic agents, such as cisplatin, 5fluorouracil, vinca alkaloids, taxoids, and etoposide (Head, 2006; Hildebrand, 2006; Wolf et al., 2008). The symptoms and signs of CIPN may resolve completely or partially, but are sometimes irreversible (Kannarkat et al., 2007). Because the pathophysiology of CIPN has not been fully elucidated, various agents have been used to prevent or treat CIPN. Recently, it was reported that a $\mathrm{Ca}^{2+} / \mathrm{Mg}^{2+}$ infusion before and after oxaliplatin could prevent the development of CIPN (Gamelin et al., 2004), but the overall benefit of this is uncertain because of the different treatment efficacies of oxaliplatin and $\mathrm{Ca}^{2+} / \mathrm{Mg}^{2+}$ infusion combinations (Hochster et al., 2007). Furthermore, oxaliplatin metabolites are chelated by calcium and magnesium, and this could explain the observed neuroprotective effect. However, Bujalska et al., (2009) found that pretreatment with magnesium (30 $\mathrm{mg} / \mathrm{kg}$, i.p.) enhanced the analgesic effects of opioids in a vincristine-induced neuropathy model, and reemphasized the antagonistic effect that magnesium has on NMDA receptors.

\section{Dysmenorrhea}

Dysmenorrhea refers to a gynaecological medical complaint characterized by severe uterine cramps associated with menstruation. Dysmenorrhea may precede or co-occur with menstruation, and causes a dull, nauseating, colicky to sharp or cramping pain. The overproduction of uterine prostaglandins or vasopressin, which stimulates myometrial contraction of the uterus, has been identified as a potential contributory factor (Stromberg et al., 1984; Woolf and Chong, 1993). When dysmenorrhea is severe enough to restrict daily activities, nonsteroidal anti-inflammatory drugs 
or oral contraceptive pills are required to reduce uterine contraction, and thus, relieve the pain (Harel, 2008). The benefits and safety of magnesium for the treatment of primary dysmenorrhea have also been reviewed (Bettendorf et al., 2008; Proctor and Murphy 2001), but more research is required to verify its efficacy. Briefly, oral magnesium on the day before menstruation and during the first and second days of the menstrual cycle for six consecutive cycles, was found to have a therapeutic effect on dysmenorrhea (FontanaKlaiber and Hogg, 1990). However, the optimum treatment regimen has yet to be established and the mechanism responsible has not been determined. Nevertheless, it has been hypothesized that magnesium inhibits the biosynthesis of PGF2- $\alpha$, which alleviates myometrial contraction and muscle relaxation by competing with calcium for entry into myometrial cells through voltage-gated channels (Seifert et al., 1989; Zahradnik and Breckwoldt, 1988).

\section{Tension headache}

Tension headaches are characterized by a diffuse, gradual, and pressure-like aching pain bilaterally over the top sides of the cranium (Altura and Altura, 2001). Its prevalence has been reported to range from around $10 \%$ to nearly $90 \%$

\section{References}

Alfonsi P (2001) Postanaesthetic shivering: epidemiology, pathophysiology, and approaches to prevention and management. Drugs 61:2193-205.

Altura BM, Altura BT (2001) Tension headaches and muscle tension: is there a role for magnesium? Med Hypotheses 57:705-13.

Apan A, Buyukkocak U, Ozcan S, Sari E, Basar H (2004) Postoperative magnesium sulphate infusion reduces analgesic requirements in spinal anaesthesia. Eur J Anaesthesiol 21:766-9.

Apfel CC, Kranke P, Katz MH, Goepfert C, Papenfuss T, Rauch S, Heineck R, Greim CA, Roewer N (2002) Volatile anaesthetics may be the main cause of early but not delayed postoperative vomiting: a randomized controlled trial of factorial design. Br J Anaesth 88:65968.
(Rasmussen et al., 1991; Robbins and Lipton, 2010; Russell et al., 2006). Although the exact aetiology of tension headaches is unknown, several pathophysiologies have been suggested, such as, muscle tension (Jensen and Rasmussen, 1996; Langemark and Olesen 1987), increased myofascial pain sensitivity (Pfaffenrath et al., 1998), platelet aggregation (Mishima et al., 1997), and others. Furthermore, in one study, it was shown that the magnesium ion levels of platelets were lower in patients with a tension headache (Mishima et al., 1997), and that magnesium supplementation appeared to ameliorate headaches, including the tension headaches (Mauskop et al., 1996; Thomas et al., 1994).

\section{Conclusion}

The postoperative analgesic adjuvant role of magnesium and its use as an analgesic therapy for the treatment of acute or chronic pain have been suggested for decades. Its antinociceptive effect has been suggested to be due to the blocking of NMDA receptors, and thus, the prevention of central sensitization. More consistent and convincing evidence is required before magnesium can be viewed as an effective adjuvant pain treatment.
Arcioni R, Palmisani S, Tigano S, Santorsola C, Sauli V, Romano S, Mercieri M, Masciangelo R, De Blasi RA, Pinto G (2007) Combined intrathecal and epidural magnesium sulfate supplementation of spinal anesthesia to reduce post-operative analgesic requirements: a prospective, randomized, doubleblind, controlled trial in patients undergoing major orthopedic surgery. Acta Anaesthesiol Scand 51:482-9.

Backonja MM (2003) Defining neuropathic pain. Anesth Analg 97:785-90.

Bahar M, Chanimov M, Grinspun E, Koifman I, Cohen ML (1996) Spinal anaesthesia induced by intrathecal magnesium sulphate. Anaesthesia 51:627-33.

Baranauskas G, Nistri A (1998) Sensitization of pain pathways in the spinal cord: cellular mechanisms. Prog Neurobiol 54:349-65. 
Begon S, Pickering G, Eschalier A, Dubray C (2000) Magnesium and MK-801 have a similar effect in two experimental models of neuropathic pain. Brain Res 887:436-9.

Bettendorf B, Shay S, Tu F (2008) Dysmenorrhea: contemporary perspectives. Obstet Gynecol Surv 63:597-603.

Bridges D, Thompson SW, Rice AS (2001) Mechanisms of neuropathic pain. Br J Anaesth 87:12-26.

Brill S, Sedgwick PM, Hamann W, Di Vadi PP (2002) Efficacy of intravenous magnesium in neuropathic pain. Br J Anaesth 89:711-4.

Bujalska M, Makulska-Nowak H, Gumulka SW (2009) Magnesium ions and opioid agonists in vincristineinduced neuropathy. Pharmacol Rep 61:1096-104.

Buvanendran A, McCarthy RJ, Kroin JS, Leong W, Perry $\mathrm{P}$, Tuman KJ (2002) Intrathecal magnesium prolongs fentanyl analgesia: a prospective, randomized, controlled trial. Anesth Analg 95:661-6.

Choi JC, Yoon KB, Um DJ, Kim C, Kim JS, Lee SG (2002) Intravenous magnesium sulfate administration reduces propofol infusion requirements during maintenance of propofol-N2O anesthesia. Part I: comparing propofol requirements according to hemodynamic responses. Part II: comparing bispectral index in control and magnesium groups.

Anesthesiology 97:1137-41.

Crosby V, Wilcock A, Corcoran R (2000) The safety and efficacy of a single dose (500 mg or $1 \mathrm{~g}$ ) of intravenous magnesium sulfate in neuropathic pain poorly responsive to strong opioid analgesics in patients with cancer. J Pain Symptom Manage 19:35-9.

De Leeuw I, Engelen W, De Block C, Van Gaal L (2004) Long term magnesium supplementation influences favourably the natural evolution of neuropathy in Mgdepleted type 1 diabetic patients (T1dm). Magnes Res 17:109-14.

Dehghani SN, Bigham AS (2009) Comparison of caudal epidural anesthesia by use of lidocaine versus a lidocaine-magnesium sulfate combination in cattle. Am J Vet Res 70:194-7.

Dingledine R, Borges K, Bowie D, Traynelis SF (1999) The glutamate receptor ion channels. Pharmacol Rev 51:7-61.
Felsby S, Nielsen J, Arendt-Nielsen L, Jensen TS (1996) NMDA receptor blockade in chronic neuropathic pain: a comparison of ketamine and magnesium chloride. Pain 64:283-91.

Fisher DM (1999) Clinical pharmacology of neuromuscular blocking agents. Am J Health Syst Pharm 56:S4-9.

Fontana-Klaiber H, Hogg B (1990) Therapeutic effects of magnesium in dysmenorrhea. Schweiz Rundsch Med Prax 79:491-4.

Fuchs-Buder T, Wilder-Smith OH, Borgeat A, Tassonyi E (1995) Interaction of magnesium sulphate with vecuronium-induced neuromuscular block. $\mathrm{Br} \mathrm{J}$ Anaesth 74:405-9.

Fuchs-Buder T, Tassonyi E (1996) Magnesium sulphate enhances residual neuromuscular block induced by vecuronium. Br J Anaesth 76:565-6.

Gamelin L, Boisdron-Celle M, Delva R, Guerin-Meyer V, Ifrah N, Morel A, Gamelin E (2004) Prevention of oxaliplatin-related neurotoxicity by calcium and magnesium infusions: a retrospective study of 161 patients receiving oxaliplatin combined with 5Fluorouracil and leucovorin for advanced colorectal cancer. Clin Cancer Res 10:4055-61.

Harel Z (2008) Dysmenorrhea in adolescents and young adults: from pathophysiology to pharmacological treatments and management strategies. Expert Opin Pharmacother 9:2661-72.

Hasanein P, Parviz M, Keshavarz M, Javanmardi K, Mansoori M, Soltani N (2006) Oral magnesium administration prevents thermal hyperalgesia induced by diabetes in rats. Diabetes Res Clin Pract 73:17-22.

Head KA (2006) Peripheral neuropathy: pathogenic mechanisms and alternative therapies. Altern Med Rev 11:294-329.

Hildebrand J (2006) Neurological complications of cancer chemotherapy. Curr Opin Oncol 18:321-4.

Hochster HS, Grothey A, Childs BH (2007) Use of calcium and magnesium salts to reduce oxaliplatinrelated neurotoxicity. J Clin Oncol 25:4028-9.

Hwang JY, Na HS, Jeon YT, Ro YJ, Kim CS, Do SH (2010) I.V. infusion of magnesium sulphate during spinal anaesthesia improves postoperative analgesia. $\mathrm{Br} \mathrm{J}$ Anaesth 104:89-93. 
James MF, Beer RE, Esser JD (1989) Intravenous magnesium sulfate inhibits catecholamine release associated with tracheal intubation. Anesth Analg 68:772-6.

Jensen R, Rasmussen BK (1996) Muscular disorders in tension-type headache. Cephalalgia 16:97-103.

Kannarkat G, Lasher EE, Schiff D (2007) Neurologic complications of chemotherapy agents. Curr Opin Neurol 20:719-25.

Kara H, Sahin N, Ulusan V, Aydogdu T (2002) Magnesium infusion reduces perioperative pain. Eur J Anaesthesiol 19:52-6.

Ko SH, Lim HR, Kim DC, Han YJ, Choe H, Song HS (2001) Magnesium sulfate does not reduce postoperative analgesic requirements. Anesthesiology 95:640-6.

Koinig $\mathrm{H}$, Wallner $\mathrm{T}$, Marhofer $\mathrm{P}$, Andel $\mathrm{H}$, Horauf $\mathrm{K}$, Mayer N (1998) Magnesium sulfate reduces intra- and postoperative analgesic requirements. Anesth Analg 87:206-10.

Kroin JS, McCarthy RJ, Von Roenn N, Schwab B, Tuman KJ, Ivankovich AD (2000) Magnesium sulfate potentiates morphine antinociception at the spinal level. Anesth Analg 90:913-7.

Kussman B, Shorten G, Uppington J, Comunale ME (1997) Administration of magnesium sulphate before rocuronium: effects on speed of onset and duration of neuromuscular block. Br J Anaesth 79:122-4.

Langemark M, Olesen J (1987) Pericranial tenderness in tension headache. A blind, controlled study. Cephalalgia 7:249-55.

Latremoliere A, Woolf CJ (2009) Central sensitization: a generator of pain hypersensitivity by central neural plasticity. J Pain 10:895-926.

Lee DH, Kwon IC (2009) Magnesium sulphate has beneficial effects as an adjuvant during general anaesthesia for Caesarean section. Br J Anaesth 103:861-6.

Levaux C, Bonhomme V, Dewandre PY, Brichant JF, Hans P (2003) Effect of intra-operative magnesium sulphate on pain relief and patient comfort after major lumbar orthopaedic surgery. Anaesthesia 58:131-5.

Ma QP, Woolf CJ (1995) Noxious stimuli induce an Nmethyl-D-aspartate receptor-dependent hypersensitivity of the flexion withdrawal reflex to touch: implications for the treatment of mechanical allodynia. Pain 61:383-90.
Mauskop A, Altura BT, Cracco RQ, Altura BM (1996) Intravenous magnesium sulfate rapidly alleviates headaches of various types. Headache 36:154-60.

Mayer ML, Westbrook GL, Guthrie PB (1984) Voltagedependent block by $\mathrm{Mg} 2+$ of NMDA responses in spinal cord neurones. Nature 309:261-3.

Mishima K, Takeshima T, Shimomura T, Okada H, Kitano A, Takahashi K, Nakashima K (1997) Platelet ionized magnesium, cyclic AMP, and cyclic GMP levels in migraine and tension-type headache. Headache 37:561-4.

O'Connor AB, Dworkin RH (2009) Treatment of neuropathic pain: an overview of recent guidelines. Am J Med 122:S22-32.

Oguzhan N, Gunday I, Turan A (2008) Effect of magnesium sulfate infusion on sevoflurane consumption, hemodynamics, and perioperative opioid consumption in lumbar disc surgery. J Opioid Manag 4:105-10.

Ossipov MH, Lai J, Malan TP, Jr., Porreca F (2000) Spinal and supraspinal mechanisms of neuropathic pain. Ann N Y Acad Sci 909:12-24.

Ozalevli M, Cetin TO, Unlugenc H, Guler T, Isik G (2005) The effect of adding intrathecal magnesium sulphate to bupivacaine-fentanyl spinal anaesthesia. Acta Anaesthesiol Scand 49:1514-9.

Ozcan PE, Tugrul S, Senturk NM, Uludag E, Cakar N, Telci L, Esen $F$ (2007) Role of magnesium sulfate in postoperative pain management for patients undergoing thoracotomy. J Cardiothorac Vasc Anesth 21:827-31.

Paech MJ, Magann EF, Doherty DA, Verity LJ, Newnham JP (2006) Does magnesium sulfate reduce the short- and long-term requirements for pain relief after caesarean delivery? A double-blind placebocontrolled trial. Am J Obstet Gynecol 194:1596-602.

Paoletti P, Neyton J (2007) NMDA receptor subunits: function and pharmacology. Curr Opin Pharmacol 7:39-47.

Pfaffenrath V, Brune K, Diener HC, Gerber WD, Gobel $H$ (1998) The treatment of tension type headache. Headache 38:725-6.

Pockett S (1995) Spinal cord synaptic plasticity and chronic pain. Anesth Analg 80:173-9. 
Proctor ML, Murphy PA (2001) Herbal and dietary therapies for primary and secondary dysmenorrhoea. Cochrane Database Syst Rev:CD002124.

Rasmussen BK, Jensen R, Schroll M, Olesen J (1991) Epidemiology of headache in a general population--a prevalence study. J Clin Epidemiol 44:1147-57.

Robbins MS, Lipton RB (2010) The epidemiology of primary headache disorders. Semin Neurol 30:107-19.

Ross RM, Baker T (1996) An effect of magnesium on neuromuscular function in parturients. $J$ Clin Anesth 8:202-4.

Rowbotham MC, Petersen KL (2001) Zoster-associated pain and neural dysfunction. Pain 93:1-5.

Russell MB, Levi N, Saltyte-Benth J, Fenger K (2006) Tension-type headache in adolescents and adults: a population based study of 33,764 twins. Eur J Epidemiol 21:153-60.

Ryu JH, Kang MH, Park KS, Do SH (2008) Effects of magnesium sulphate on intraoperative anaesthetic requirements and postoperative analgesia in gynaecology patients receiving total intravenous anaesthesia. Br J Anaesth 100:397-403.

Ryu JH, Sohn IS, Do SH (2009) Controlled hypotension for middle ear surgery: a comparison between remifentanil and magnesium sulphate. Br J Anaesth 103:490-5.

Seifert B, Wagler P, Dartsch S, Schmidt U, Nieder J (1989) Magnesium--a new therapeutic alternative in primary dysmenorrhea. Zentralbl Gynakol 111:755-60.

Seyhan TO, Tugrul M, Sungur MO, Kayacan S, Telci L, Pembeci K, Akpir K (2006) Effects of three different dose regimens of magnesium on propofol requirements, haemodynamic variables and postoperative pain relief in gynaecological surgery. $\mathrm{Br}$ J Anaesth 96:247-52.

Stromberg P, Akerlund M, Forsling ML, Granstrom E, Kindahl H (1984) Vasopressin and prostaglandins in premenstrual pain and primary dysmenorrhea. Acta Obstet Gynecol Scand 63:533-8.

Telci L, Esen F, Akcora D, Erden T, Canbolat AT, Akpir K (2002) Evaluation of effects of magnesium sulphate in reducing intraoperative anaesthetic requirements. $\mathrm{Br} \mathrm{J}$ Anaesth 89:594-8.
Thomas J, Tomb E, Thomas E, Faure G (1994) Migraine treatment by oral magnesium intake and correction of the irritation of buccofacial and cervical muscles as a side effect of mandibular imbalance. Magnes Res 7:123-7.

Tramer MR, Schneider J, Marti RA, Rifat K (1996) Role of magnesium sulfate in postoperative analgesia. Anesthesiology 84:340-7.

Tramer MR, Glynn CJ (2007) An evaluation of a single dose of magnesium to supplement analgesia after ambulatory surgery: randomized controlled trial. Anesth Analg 104:1374-9.

Turan A, Memis D, Karamanlioglu B, Guler T, Pamukcu $Z$ (2005) Intravenous regional anesthesia using lidocaine and magnesium. Anesth Analg 100:1189-92.

Wilder-Smith CH, Knopfli R, Wilder-Smith OH (1997) Perioperative magnesium infusion and postoperative pain. Acta Anaesthesiol Scand 41:1023-7.

Wolf S, Barton D, Kottschade L, Grothey A, Loprinzi C (2008) Chemotherapy-induced peripheral neuropathy: prevention and treatment strategies. Eur J Cancer 44:1507-15.

Woolf CJ (1983) Evidence for a central component of post-injury pain hypersensitivity. Nature 306:686-8.

Woolf CJ, Thompson SW (1991) The induction and maintenance of central sensitization is dependent on $\mathrm{N}$-methyl-D-aspartic acid receptor activation; implications for the treatment of post-injury pain hypersensitivity states. Pain 44:293-9.

Woolf CJ, Chong MS (1993) Preemptive analgesia-treating postoperative pain by preventing the establishment of central sensitization. Anesth Analg 77:362-79.

Woolf CJ, Salter MW (2000) Neuronal plasticity: increasing the gain in pain. Science 288:1765-9.

Zahradnik HP, Breckwoldt M (1988) Drug therapy of dysmenorrhea. Gynakologe 21:58-62. 



\title{
The role of magnesium in CNS injury
}

\author{
Naomi L. Cook, Frances Corrigan and Corinna van den Heuvel ${ }^{凶}$
}

Discipline of Anatomy and Pathology \& Adelaide Centre for Neuroscience Research, School of Medical Sciences, The University of Adelaide, Adelaide, South Australia 5005, Australia.

$\triangle$ corinna.vandenheuvel@adelaide.edu.au

\begin{abstract}
Traumatic injury to the central nervous system (CNS) is a leading cause of morbidity and mortality, and represents a significant public health issue. Despite intensive research, no effective neuroprotective therapy exists, and survivors of CNS injury, including traumatic brain injury (TBI) and spinal cord injury $(\mathrm{SCl})$, can be left with severe disabilities that require long-term rehabilitation. Much of the damage that occurs after $\mathrm{TBI}$ and $\mathrm{SCl}$ develops over time with the primary injury initiating a secondary injury cascade made up of deleterious biochemical and pathophysiological reactions. This delayed development of secondary injury provides a vital opportunity for therapeutic intervention and considerable effort is currently being directed toward identifying these injury factors and developing interventions that may potentially prevent their actions. Magnesium $\left(\mathrm{Mg}^{2+}\right)$ decline has been identified as playing a key role in the secondary injury process, in part because of its central role in the regulation of a large number of known injury factors and that its decline is associated with the development of motor and cognitive deficits. $\mathrm{Mg}^{2+}$ administration has been extensively investigated both preclinically in $\mathrm{TBI}$ and $\mathrm{SCl}$ and clinically as a neuroprotectant in TBI with varied success. This chapter focuses on the role of $\mathrm{Mg}^{2+}$ in TBI and $\mathrm{SCl}$ pathophysiology, with particular emphasis on $\mathrm{Mg}^{2+}$ as a potential therapeutic agent.
\end{abstract}

\section{Introduction}

A decline in $\mathrm{Mg}^{2+}$ following TBI was first identified using in vivo magnetic resonance spectroscopy (Vink et al., 1988) and further declines have subsequently been reported by numerous laboratories following a variety of insults to the CNS including TBI, SCl, stroke and drug intoxication. It has been observed that a traumatic insult to the CNS results in a decrease in the intracellular free $\mathrm{Mg}^{2+}$ concentration of between $40-60 \%$, while the total tissue $\mathrm{Mg}^{2+}$ falls by between $10-$ $15 \%$. This $\mathrm{Mg}^{2+}$ decline is confined to the site of injury and the decrease is associated with the development of neurological dysfunction. Treatment with $\mathrm{Mg}^{2+}$ has been shown to attenuate the neurological changes experimentally following TBI and $\mathrm{SCl}$, however, clinical studies of $\mathrm{Mg}^{2+}$ as a neuroprotective agent show conflicting results (Dhandapani et al., 2008; Temkin et al., 2007).

\section{Traumatic CNS injury}

Injuries to the CNS are a significant problem, with TBI the leading cause of death and disability in people under the age of 40 years (Fleminger and
Ponsford, 2005). In the USA, approximately 1.5 million people are affected by TBI annually. Of these, 230,000 people are hospitalized and survive, 80,000 people develop a TBI-related disability, and 50,000 people die (Pitkänen et al., 2005). In addition, SCl affects around 200,000 people in the USA with 10,000 new cases each year (Anonymous, 2008; Buechner et al., 2000) with the cost of caring for patients with $\mathrm{SCl}$ in the US estimated to be $\$ 9.73$ billion (Weaver et al., 2000). Since no effective treatment is available, survivors of $\mathrm{TBI}$ and $\mathrm{SCl}$ may be left with debilitating neurological deficits that confer social, financial and emotional burdens and adversely affect their quality of life.

The neurological damage resulting from traumatic injuries to the CNS is due to both direct, immediate biomechanical processes (the primary injury) and indirect, delayed (secondary) injury mechanisms (Morales et al., 2005) and the neuronal death in $\mathrm{TBI}$ and $\mathrm{SCl}$ is likely to involve both necrotic and apoptotic pathways (Liou et al., 2003). The primary event is irreversible and in TBI comprises contusions, lacerations, tissue shearing and stretching of nerve fibres (Gentile and 
McIntosh, 1993), whereas the most common primary injuries to the spinal cord result from fracture/dislocation or crush injury to the vertebral column. In contrast, secondary injury consists of a series of complex biochemical changes that are triggered by the primary event and evolve over the minutes to days and even months after the insult (Vink and Van Den Heuvel, 2010). A number of secondary injury factors have been identified, including disruption to the blood-brain barrier (BBB), edema, ischemia, hypertension, inflammation, excitotoxicity, oxidative stress and $\mathrm{Mg}^{2+}$ decline, all of which can be deleterious to neuronal cells (Barone and Kilgore, 2006; Cormio et al., 1997; Gentile and Mclntosh 1993; Golding, 2002).

\section{Secondary injury factors}

As stated above, secondary injury involves series of complex biochemical changes that are triggered by the primary event and may continue for days to weeks after the insult (Roth and Farls 2000). To provide an example of the secondary injury cascade, shearing of nerve fibres at the time of insult results in massive ion fluxes across cell membranes, loss of membrane potential and rapid release of neurotransmitters from damaged neurons. This may result in excitotoxicity, which can evoke an inflammatory response, thereby stimulating further processes (such as the changes described above), eventually leading to cell death (Vink and Van Den Heuvel, 2004). Secondary injury is associated with significant morbidity and mortality following traumatic CNS injury (Gentile and Mclntosh, 1993), but given that it manifests over time, this provides an opportunity to administer a pharmacological agent to impede or prevent further injury and thus improve outcome. Several injury processes relevant to traumatic CNS injury will now be discussed including diffuse axonal injury (DAl), edema, oxidative stress, mitochondrial dysfunction, inflammation and lastly significant discussion will be given to magnesium decline.

\section{Diffuse Axonal Injury}

DAI is a principal cause of coma following TBI, and significantly contributes to TBI-related morbidity and mortality (Li and Feng, 2009). Classically, DAI was categorized as a primary injury, whereby axons are torn and rupture at the time of insult (Adams et al., 1982). However, more recent evidence suggests that DAI is triggered at the time of injury by inertial forces and may evolve over a period of hours to days, progressing from focal disruptions to axons to impaired axonal transport, axolemmal swelling and, ultimately, axonal disconnection (Büki and Povlishock, 2006; Mazzeo et al., 2009; Stone et al., 2004). DAl is generally diagnosed at post-mortem by immunostaining for $\beta$-amyloid precursor protein (APP) (Gentleman et al., 1993), although recent imaging advances may allow its detection at an earlier stage (Li and Feng, 2009).

\section{Edema}

Cerebral edema is defined as an abnormal accumulation of fluid within the brain parenchyma, resulting in a volumetric enlargement (swelling) of brain tissue (Heo et al., 2005). Swollen tissues may exert harmful effects by increasing pressure in surrounding tissues, leading to ischemia and additional edema (Simard et al., 2007). Swelling has particularly deleterious consequences in the brain, due to the fact that the volume of the intracranial cavity is fixed (Ayata and Ropper, 2002). The resulting rise in intracranial pressure (ICP) with cerebral edema formation is a potentially lethal process (Kimelberg, 1995). Indeed, cerebral edema plays an important role in the outcome of TBI victims: uncontrolled brain swelling with the accompanying rise in ICP is the leading cause of death in TBI patients (Marmarou et al., 2000).

Cerebral edema is classified as cytotoxic and vasogenic, depending upon whether or not BBB permeability is increased (Verlooy and Van Reempts, 2005). The BBB is composed of a monolayer of endothelial cells forming tight junctions that prevent the direct interaction of the peripheral circulation with the CNS (Palmer, 2010). Cytotoxic edema refers to the movement of water, $\mathrm{Na}^{+}$and $\mathrm{Cl}^{-}$from the extracellular to the intracellular space, which results in cell swelling and predisposes cells to oncotic cell death. However, cytotoxic edema does not involve increases in BBB permeability, nor does it contribute to the actual net increase in brain water (Ayata and Ropper, 2002; Simard et al., 2007). Conversely, vasogenic edema is associated with the degradation of tight junctions between endothelial cells of the BBB (Heo et al., 2005). Consequently, the BBB becomes more permeable to macromolecules and permits the movement of 
fluid from capillaries to the extravascular compartment (Heo et al., 2005; Simard et al., 2007); hence vasogenic edema causes a net increase in brain water content (Ayata and Ropper, 2002). In TBI, the increase in BBB permeability may occur by mechanical injury, autodestructive mediators or both (Unterberg et al., 2004). However, despite the potentially lethal consequences of edema in $\mathrm{TBI}$, there is currently no effective therapy to prevent its formation or progression. Treatments such as mannitol, corticosteroids, barbiturates, induction of hypothermia, drainage of the cerebrospinal fluid (CSF) and surgical decompression (Ayata and Ropper, 2002; Clausen and Bullock, 2001) have had limited success in managing rises in ICP (Vink and Van Den Heuvel, 2004).

Neurogenic inflammation is a local inflammatory reaction of neurons in response to infection, toxins or trauma, which is characterized by vasodilation, plasma extravasation and edema (Black, 2002; Ro et al., 2005; Turner et al., 2006). Studies from our laboratory (Nimmo et al., 2004; Vink et al., 2003) have shown that neurogenic inflammation may be involved in the formation of vasogenic edema and the functional deficits following diffuse $\mathrm{TBI}$ in rats, and that the neuropeptide, substance $P(S P)$, is a potent initiator of neurogenic inflammation. Further research from our laboratory (Donkin et al., 2009) has demonstrated that a SP receptor antagonist inhibits BBB breakdown and edema formation, and significantly improves long-term functional outcome and motor deficits resulting from TBI. These important results may provide a novel pharmacological treatment for TBI.

\section{Oxidative Stress}

Reactive oxygen species (ROS) comprise a range of chemical entities, including hydroxyl radicals, peroxynitrite, superoxide anions, hydrogen peroxide, nitric oxide and singlet oxygen (Chong et al., 2005; Ellis, 2007). ROS are highly reactive molecules, possessing one or more unpaired electrons in their outer orbits (Halliwell, 1992). While some ROS are normal by-products of cellular metabolism (Yu, 1994), their overproduction can lead to cell injury and tissue damage via reactions with proteins, lipids and nucleic acids, and cellular activation by ROS induces signalling cascades that lead to rises in intracellular $\mathrm{Ca}^{2+}$ concentrations (Alexandrova and Bochev, 2005; Massullo et al., 2006). When ROS are generated in excess of endogenous antioxidant mechanisms, the result is oxidative stress (Finkel and Holbrook, 2000).

Brain tissue is highly susceptible to damage by oxidative stress for a number of reasons. The brain has a high rate of oxygen metabolism, which increases the likelihood of excess ROS production (Chong et al., 2005). Furthermore, it contains high levels of polyunsaturated fatty acids, which are particularly vulnerable to damage by free radicals (Halliwell, 1992). Critically, the brain has relatively low antioxidant and repair capacities compared with other organs (Vink and Nimmo, 2009). All of these factors render brain tissue extremely sensitive to increases in ROS, and oxidative stress is indeed relevant to TBI. Studies demonstrate that the production of ROS is enhanced following TBI, and this is accompanied by an impairment of antioxidant defences (Ansari et al., 2008; Lima et al., 2008). TBI-induced oxidative stress can lead to cytoskeletal damage, mitochondrial dysfunction and altered signal transduction, which can be deleterious to neuronal and vascular cells (Ansari et al., 2008; Chong et al., 2005).

\section{Mitochondrial Dysfunction}

Mitochondria are organelles that are present in all cells and play a critical role in regulating cellular energy production via the electron transport chain (Bayir and Kagan, 2008; Mazzeo et al., 2009). Consequently, it would be expected that disruption to mitochondrial function would have serious adverse consequences to cells, particularly those with a high-energy demand, such as neurons. Indeed, mitochondrial dysfunction is an important factor in TBI pathophysiology that can lead to energy depletion, free radical release and apoptosis, or programmed cell death (Vink and Nimmo, 2009). In experimental TBI, alterations in mitochondrial function have been reported within hours of injury and may persist for days (Robertson, 2004). Furthermore, in clinical TBI studies, patients with profound mitochondrial dysfunction have a poor outcome (Signoretti et al., 2008).

As discussed, traumatic CNS injury leads to conditions of oxidative stress. Oxidative stress directly contributes to mitochondrial dysfunction, 
promoting the release of cytochrome $\mathrm{c}$ from the inner membrane of the mitochondrion, which triggers an apoptotic cascade involving caspases and other pro-apoptotic proteins (Bayir and Kagan, 2008; Chong et al., 2005). Mitochondria also constitute an important intracellular source of ROS, which are produced during the synthesis of ATP (Kowaltowski et al., 2009). During states of electron transport chain dysregulation, as may occur following TBI, there is an increase in the production of ROS (Bayir and Kagan, 2008). Mitochondria have also been implicated in glutamate-mediated excitotoxicity, by participating in the release of glutamate, $\mathrm{Ca}^{2+}$ sequestration and the generation of ROS (Nicholls and Budd, 2000).

\section{Inflammation}

The inflammatory response represents another important secondary injury process of traumatic CNS injury. Injury-induced inflammation may be beneficial in the short term, by removing cellular debris or promoting regeneration, however, in excess, inflammatory responses can have severe deleterious effects that lead to neurodegeneration (Maas et al., 2008; Vink et al., 2009). Inflammation can occur within the neural tissue, where activated microglia and astrocytes release cytokines, chemokines and ROS. There is also evidence that the complement system is activated following TBI (Stahel et al., 1998). Furthermore, disruption to the BBB in response to brain insult is associated with the transmigration of leukocytes to the area of damage, which themselves produce cytokines (Jain, 2008). Indeed, levels of the proinflammatory cytokines, tumour necrosis factor (TNF)- $\alpha$ and interleukin (IL)-1 $\beta$ are elevated following experimental TBI (Fan et al., 1995; Fan et al., 1996), and may act synergistically to exacerbate the neuroinflammatory response and excitotoxicity (Morganti-Kossmann et al., 2007). On the other hand, anti-inflammatory cytokines, such as IL-10 and transforming growth factor (TGF)- $\beta$, may exert neuroprotective effects in TBI (Knoblach and Faden, 1998) by suppressing the actions of TNF- $\alpha$ and IL-1 $\beta$ (Benveniste et al., 1995). Finally, IL-6 may act either to promote or inhibit inflammation in different situations, exemplifying the complexity of the neuroinflammatory pathways associated with TBI (Morganti-Kossmann et al., 2007).

\section{$\mathrm{Mg}^{2+}$ depletion following traumatic CNS injury}

$\mathrm{Mg}^{2+}$ depletion has been observed both experimentally within the brain of animals and clinically in the blood of humans following traumatic CNS injuries. This depletion is an important secondary injury factor that has been associated with poor neurological outcome in both humans and experimental animals. A significant decline in serum ionized $\mathrm{Mg}^{2+}$ levels was measured in TBI patients, with the magnitude of $\mathrm{Mg}^{2+}$ decline linked to the severity of TBI (Kahraman et al., 2003). Similarly $\mathrm{Mg}^{2+}$ levels decline following experimental $\mathrm{SCl}$ in the acute phase, with levels significantly lower for up to 3 days following injury, before gradually returning to sham levels (Chanimov et al., 2006; Lemke et al., 1987). Like TBI, the degree of reduction is also dependent on injury severity (Lemke and Faden, 1990) suggesting a drop in $\mathrm{Mg}^{2+}$ levels plays a central role in the secondary injury cascade. Indeed, following TBI in rats the decrease in brain intracellular free $\mathrm{Mg}^{2+}$ concentration observed following injury was correlated with the development of neurological deficits (Heath and Vink, 1996; Vink et al., 1988). In a study investigating the consequences of $\mathrm{Mg}^{2+}$ deficiency prior to TBI in rats, the $\mathrm{Mg}^{2+}$-deficient group was shown to have significantly greater cortical cell loss compared to the vehicle group, as well as cytoskeletal alterations in cortical and hippocampal neurons (Saatman et al., 2001).

\section{Mechanisms of $\mathrm{Mg}^{2+}$ neuroprotection}

Given the significance of $\mathrm{Mg}^{2+}$ decline with respect to neurological outcome following CNS injury, $\mathrm{Mg}^{2+}$ has been investigated as a potential neuroprotective agent for CNS injury. $\mathrm{Mg}^{2+}$ represents an attractive interventional therapy due to its low cost, ease of use, low risk of adverse effects and its ability to penetrate the BBB (Sacco et al., 2007). Furthermore, it is able to modulate many processes relevant to both TBI and $\mathrm{SCl}$.

$\mathrm{Mg}^{2+}$ is crucial to many diverse biological processes and is an essential co-factor required for the function of numerous enzymes (Ebel and Günther, 1980). Given its vital physiological roles, a decline in $\mathrm{Mg}^{2+}$ concentration is likely to have an adverse effect on cellular functioning, the 
maintenance of membrane potential and the capacity for cells to undergo repair (Vink and Cernak, 2000). $\mathrm{Mg}^{2+}$ also blocks $\mathrm{N}$-methyl-Daspartate receptors (NMDAR), thereby regulating $\mathrm{Ca}^{2+}$ influx (Sen and Gulati, 2010), with a decline in $\mathrm{Mg}^{2+}$ exacerbating excitotoxicity post-injury. In the absence of injury, $\mathrm{Mg}^{2+}$ deficiency impairs antioxidant defences (Blache et al., 2006), with $\mathrm{Mg}^{2+}$ inhibiting the production of reactive oxygen species (Blache et al., 2006), reducing lipid peroxidation (Regan et al., 1998; Suzer et al., 1999), and ultimately decreasing oxidative stress in TBI patients (Cernak et al., 2000). In addition $\mathrm{Mg}^{2+}$ has a positive effect on cerebral energy state, preserving mitochondrial membrane potential with an improvement in oxidative phosphorylation and a decrease in lactic acid production when administered post-injury (Feldman et al., 1996; Van Den Heuvel and Vink, 2004). The acute inflammatory response is also modulated by $\mathrm{Mg}^{2+}$, with neutrophil invasion reduced following $\mathrm{SCl}$ in animals treated with $\mathrm{MgSO}_{4}$ (Gok et al., 2007). This correlates with studies that show that $\mathrm{Mg}^{2+}$ deficiency evokes an inflammatory response (Malpuech-Brugère et al., 2000), including the release of pro-inflammatory cytokines and SP (Mazur et al., 2006).

\section{$\mathrm{Mg}^{2+}$ and Substance $\mathrm{P}$}

Within one week of $\mathrm{Mg}^{2+}$ deficiency, plasma SP concentration was elevated (Weglicki and Phillips, 1992) and this was associated with the develop-ment of neurogenic inflammation (Weglicki et al., 1994). This is relevant to TBI, as SP expression increases following experimental TBI (Donkin et al., 2009), with administration of SP receptor antagonists increasing levels of free $\mathrm{Mg}^{2+}$ in the brain, indicating that the decline in $\mathrm{Mg}^{2+}$ could be mediated, in part, by elevations in SP (Vink et al., 2004).

SP has a number of neuroinflammatory roles, including stimulating the release of histamine and serotonin (5HT) from mast cells, which can compromise the BBB along with nitric oxide (NO) and bradykinin. It is also a potent initiator of neurogenic inflammation (vasodilation, plasma protein extravasation and edema) (Harrison and Geppetti, 2001) with the resulting disruption to the $B B B$ resulting in the entry of toxins, metal ions and inflammatory cells into the brain (see Figure 1). Furthermore, SP stimulates immune cells to release pro-inflammatory cytokines, including TNF- $\alpha$ and several interleukin family members (IL-1 $\beta, 1 \mathrm{~L}-2$ and II- 6 ) resulting in further inflammation (Delgado et al., 2003). Additionally, NK-1R activation stimulates second messenger pathways that increase $\mathrm{Ca}^{2+}$ entry to neurons (Suh and Hille, 2005; Yang et al., 2003). Since SP also activates NMDAR (Wu et al., 2004), $\mathrm{Ca}^{2+}$ influx through these channels may lead to excitotoxicity and the production of free radicals such as peroxynitrite, which can be highly toxic to cellular macromolecules. This $\mathrm{Ca}^{2+}$ influx is facilitated by low $\mathrm{Mg}^{2+}$ levels, as blockade of NMDAR is reduced and members of the transient receptor potential melastatin (TRPM) family may be activated allowing further $\mathrm{Ca}^{2+}$ entry (Schmitz et al., 2003). The induction of this secondary injury cascade by SP correlates with results which show increased SP is deleterious following TBI, as it is associated with the development of cerebral edema and poorer neurological outcome (Donkin et al., 2009). Taken together, SP release and the associated $\mathrm{Mg}^{2+}$ decline following TBI can result in neuroinflammation, BBB disruption, excitotoxicity and oxidative stress, with restoring $\mathrm{Mg}^{2+}$ homeostasis a potential target to limit these secondary injury factors and thus cell death.

\section{$\mathrm{Mg}^{2+}$ and TRPM7 channels}

TRPM7 is one of the few mammalian $\mathrm{Mg}^{2+}$ transporters described to date, playing an important role in regulating $\mathrm{Mg}^{2+}$ entry into cells. It is ubiquitously expressed and consists of an ion channel fused to a protein kinase domain (Runnels et al., 2001), with the channel permeable to a number of divalent cations including $\mathrm{Mg}^{2+}$ (Monteilh-Zoller et al., 2003). Of note TRPM7 has been implicated in the anoxic death of neuronal cells (Aarts et al., 2003), and activation of TRPM7 during ischemia has been proposed as a key factor contributing to excitotoxicity and other deleterious processes (MacDonald et al., 2006). We have recently found changes in TRPM7 channel expression following experimental TBI in rats (Cook et al., unpublished), suggesting a role for this protein in traumatic CNS injury.

TRPM7 has been suggested to mediate cell death via a positive feedback loop whereby $\mathrm{Ca}^{2+}$ entry into cells as a result of injury causes the production of free radicals, which activate TRPM7, leading to further $\mathrm{Ca}^{2+}$ influx and additional free radical production (Aarts and 


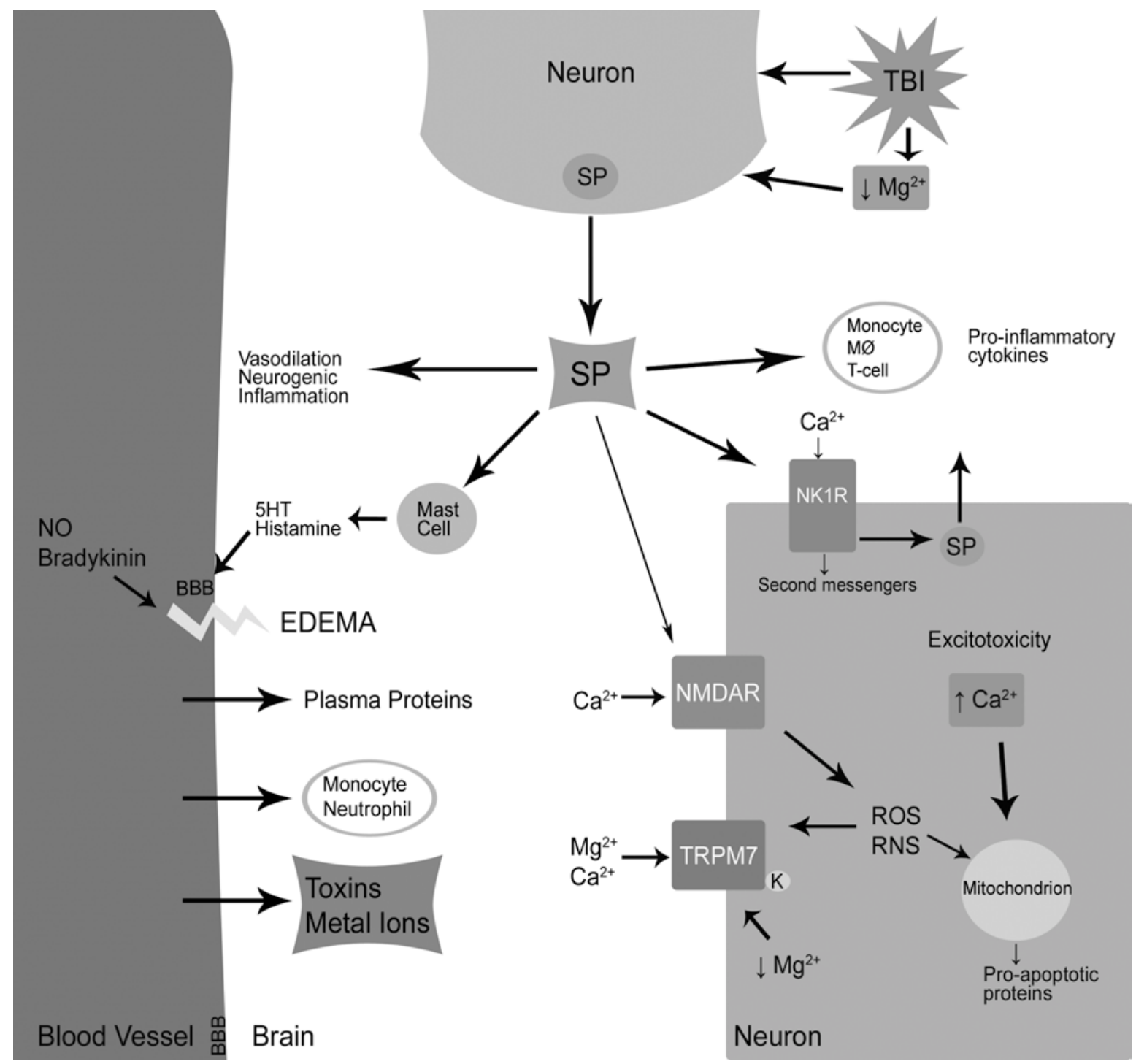

Figure 1. Potential mechanisms of neuronal damage resulting from $\mathrm{Mg}^{2+}$ depletion and SP release in TBI.

Tymianski, 2005). Importantly, a decline in intracellular $\mathrm{Mg}^{2+}$ concentration, such as that observed following $\mathrm{TBI}$ and $\mathrm{SCl}$, can lead to the generation of reactive oxygen and nitrogen species (Altura et al., 2003), which could further activate TRPM7, thereby exacerbating inflammation, oxidative stress and cell death pathways (see Figure 1). Given that $\mathrm{Mg}^{2+}$ levels remain suppressed for several days following injury (Heath and Vink 1996), this is potentially a critical and persistent pathway leading to cell death after $\mathrm{TBI}$ and $\mathrm{SCl}$. However, additional studies are required to determine whether TRPM7 directly mediates cell death in traumatic CNS injury, and if changes in TRPM7 expression following TBI are linked to the observed decline in $\mathrm{Mg}^{2+}$ levels.

\section{$\mathrm{Mg}^{2+}$ administration in traumatic CNS studies}

\section{Experimental TBI Studies}

A number of studies have investigated whether this attenuation of secondary injury factors by restoring $\mathrm{Mg}^{2+}$ homeostasis after $\mathrm{TBI}$ is effective in improving neurological and functional outcome. Substantial evidence from studies of experimental TBI suggests that $\mathrm{Mg}^{2+}$ administration may be beneficial following head injury. $\mathrm{Mg}^{2+}$ significantly reduced cortical cell loss (Saatman et al., 2001) and motor deficits (Bareyre et al., 1999) in injured rats compared to vehicle controls. A number of other studies have also demonstrated a neuroprotective effect of $\mathrm{Mg}^{2+}$ following experimental TBI (Browne et al., 
2004; Fromm et al., 2004; McIntosh et al., 1989; Turner et al., 2004). $\mathrm{Mg}^{2+}$ treatment following TBI in rats has been shown to reduce apoptosis and the expression of p53-related (apoptosisinducing) proteins (Lee et al., 2004) as well as modulating levels of $\mathrm{Bax}$ and $\mathrm{Bcl}-2$, which are also regulators of apoptosis (Ravishankar et al., 2001).

Although $\mathrm{Mg}^{2+}$ has been shown to improve neurological outcome when administered up to 24 hours after injury (Hoane and Barth, 2002), the best results have been achieved when the therapeutic window is restricted to 12 hours (Vink and Cernak, 2000). $\mathrm{Mg}^{2+}$ decline has been shown to persist for at least 4 days after injury (Heath and Vink, 1996), however, the concentration of $\mathrm{Mg}^{2+}$ in CNS injury has been shown never to fall below $0.2 \mathrm{mM}$ (Vink et al., 1988). Thus, it is likely that the length of time for which free $\mathrm{Mg}^{2+}$ concentration is reduced, rather than the magnitude of decline, is the parameter that influences neurological outcome (Vink and Cernak, 2000). In addition to the positive effect seen with respect to motor performance, $\mathrm{Mg}^{2+}$ also significantly improves cognitive function following TBI (reviewed in (Hoane, 2007)).

\section{Experimental SCI Studies}

In experimental $\mathrm{SCl}$, administration of $\mathrm{Mg}^{2+}$ has been found to have beneficial effects when administered to rats using both the clip compression (Ditor et al., 2007; Suzer et al., 1999) and weight drop models of SCl (Gok et al., 2007; Kaptanoglu et al., 2003a; Kaptanoglu et al., 2003b; Kwon et al., 2009) as well as when administered to rabbits following $\mathrm{SCl}$ (Ozdemir et al., 2005). The beneficial effect of $\mathrm{Mg}^{2+}$ include decreases in membrane damage (Kaptanoglu et al., 2003a; Kaptanoglu et al., 2003b), normalization of energy balance (Ozdemir et al., 2005), protection against axonal damage (Suzer et al., 1999), preservation of the BBB (Kaptanoglu et al., 2003a; Kaptanoglu et al., 2003b) and reduction in apoptosis as seen by a reduction in activated caspase-3 (Solaroglu et al., 2005). However, unlike $\mathrm{TBI}$, the clinical window for administration of $\mathrm{Mg}^{2+}$ in $\mathrm{SCl}$ appears only to extend to 8 hours, with no improvement in outcome seen in animals treated at later time points (Kwon et al., 2009; Wiseman et al., 2009). In contrast, earlier treatment leads to a decrease in lesion size with an associated significant improve-ment in motor outcome seen early, at
24 hours following injury (Kaptanoglu et al., 2003a; Kaptanoglu et al., 2003b; Suzer et al., 1999), as well as later, at 4 weeks post-injury (Wiseman et al., 2009).

Despite this evidence supporting a potential therapeutic role of $\mathrm{Mg}^{2+}$ in $\mathrm{SCl}$, the neuroprotective effects were only demonstrated following extremely high doses of $\mathrm{MgSO}_{4}(600$ $\mathrm{mg} / \mathrm{kg}$ ) and smaller doses were less effective at providing significant histological or functional benefit (Kaptanoglu et al., 2003a; Kaptanoglu et al., 2003b; Suzer et al., 1999). Such a bolus dose of $600 \mathrm{mg} / \mathrm{kg}$ far exceeds the $2-4 \mathrm{~g}$ bolus dose given to patients with emergencies such a preeclampsia or cardiac arrest (Gowda and Khan, 2004; Leeman and Fontaine 2008). Given the high doses necessary to see these clinical improvements, further studies investigated whether combining $\mathrm{Mg}^{2+}$ with polyethylene glycol (PEG), a hydrophilic polymer used as an excipient for many pharmacological agents, would reduce the dosage needed. Indeed, by treating animals with $60 \mathrm{mg} / \mathrm{kg}$ of $\mathrm{MgSO}_{4}$ in a PEG formulation, lesion volume was reduced to a greater extent than seen in animals treated with $\mathrm{MgSO}_{4}$ or PEG alone, with only the $\mathrm{MgSO}_{4}$ in PEG leading to a significant improvement of motor outcome at 6 weeks post-injury (Kwon et al., 2009). Similarly with a higher dose of $\mathrm{Mg}^{2+}(300 \mathrm{mg} / \mathrm{kg})$, only the combination of $\mathrm{MgSO}_{4}$ in PEG led to a significant reduction in lesion volume, tissue sparing at the site of injury and modest BBB improvements at 6 weeks post injury (Ditor et al., 2007). The ability to reduce the necessary dosage for treating $\mathrm{SCl}$ by placing $\mathrm{Mg}^{2+}$ in PEG makes it more likely that a clinical translation can be made, with the dosage optimized so that beneficial effects are seen with a comparable amount of $\mathrm{Mg}^{2+}$ administered to that shown to be safe in patients suffering from cardiac arrest and pre-eclampsia (Kwon et al., 2009).

\section{Clinical translation}

Although $\mathrm{Mg}^{2+}$ is a multifactorial pharmacological intervention with a proven safety record in previous clinical studies, it has yet to be used clinically to treat $\mathrm{SCl}$. Interestingly, experimental data suggests that the $\mathrm{Mg}^{2+}$ in PEG formulation is more effective than methylprednisolone, the only pharmacological treatment currently available clinically for $\mathrm{SCl}$ (Bracken and Holford, 2002). This formulation reduced lesion volume by $67 \%$ 
compared to $34 \%$ following $\mathrm{SCl}$, with an associated significant improvement in motor outcome at 6 weeks post-injury in the animals treated with $\mathrm{Mg}^{2+}$ compared to the methylprednisolone group (Kwon et al., 2009). This is important as treatment with methylprednisolone remains controversial with only modest improvements noted in motor and sensory outcome of patients treated within 8 hours (Bracken and Holford, 2002), which are offset by an increase in complications such as severe sepsis and pneumonia (Hurlbert, 2006). This highlights the importance of finding new treatments for $\mathrm{SCl}$, with the $\mathrm{Mg}^{2+} / \mathrm{PEG}$ formulation soon poised to be translated into human $\mathrm{SCl}$ clinical trials (as reviewed by (Kwon et al., 2010).

Despite the positive results obtained using $\mathrm{Mg}^{2+}$ as a therapy for TBI in animal models, a recent Phase III clinical trial (Temkin et al., 2007) found that $\mathrm{MgSO}_{4}$ given to patients for 5 days after injury was not neuroprotective and possibly even had a negative effect on outcome. Given that all patients (including the control group) received $\mathrm{Mg}^{2+}$ to restore depleted serum levels to normal, it is unclear whether this restoration of serum $\mathrm{Mg}^{2+}$ level was sufficient to confer positive effect in all patients irrespective of the treatment group. This result was in marked contrast to another clinical trial (Dhandapani et al., 2008) which reported that acute $\mathrm{Mg}^{2+}$ administration (less than 24 hours) resulted in significant improvements in neurological outcome, postoperative brain swelling and 1-month mortality.

\section{References}

Aarts $M$, lihara $K$, Wei $W-L$, Xiong $Z$, -G., Arundine $M$, Cerwinski W, MacDonald JF, Tymianski M (2003) A key role for TRPM7 channels in anoxic neuronal death. Cell 115:863-77.

Aarts MM, Tymianski M (2005) TRPMs and neuronal cell death. Pflugers Arch 451:243-9.

Adams JH, Graham DI, Murray LS, Scott G (1982) Diffuse axonal injury due to nonmissile head injury in humans: an analysis of 45 cases. Ann Neurol 12:55763.
The differences in trial results could be attributed to possible limitations in central $\mathrm{Mg}^{2+}$ transport (McKee et al., 2005), and the recently described $\mathrm{Mg}^{2+}$ transporters from the TRPM family have been suggested to be involved in this process (Cook et al., 2009). However, as described in this review, further investigation is warranted to elucidate the exact role of these channels in the pathophysiology of TBI and SCI.

\section{Conclusions}

Secondary injury factors involve numerous pathological processes that account for significant morbidity and mortality following traumatic CNS injury. Deficits in intracellular $\mathrm{Mg}^{2+}$ concentration occurring following $\mathrm{TBI}$ and $\mathrm{SCl}$ are associated with poor neurological outcome, but despite promising experimental studies, $\mathrm{Mg}^{2+}$ has not been proven clinically effective. While it is unlikely that targeting a single factor will significantly improve neurological outcome, $\mathrm{Mg}^{2+}$ may still be a useful neuroprotective agent as part of a combination treatment regime that targets multiple components of the secondary injury cascade.

\section{Acknowledgments}

Supported, in part, by the Neurosurgical Research Foundation of Australia and a National Health and Medical Research Council postgraduate research award (NLC).
Alexandrova ML, Bochev PG (2005) Oxidative stress during the chronic phase after stroke. Free Rad Biol Med 39:297-316.

Altura BM, Gebrewold A, Zhang A, Altura BT (2003) Low extracellular magnesium ions induce lipid peroxidation and activation of nuclear factor-kappa $B$ in canine cerebral vascular smooth muscle: possible relation to traumatic brain injury and strokes. Neurosci Lett 341:189-92.

Anonymous (2008) Spinal cord injury Facts and figures at a glance. J Spinal Cord Med 31:357-8. 
Ansari MA, Roberts KN, Scheff SW (2008) Oxidative stress and modification of synaptic proteins in hippocampus after traumatic brain injury. Free Rad Biol Med 45:443-52.

Ayata C, Ropper AH (2002) Ischaemic brain oedema. J Clin Neurosci 9:113-24.

Bareyre FM, Saatman KE, Helfaer MA, Sinson G, Weisser JD, Brown AL, Mclntosh TK (1999) Alterations in ionized and total blood magnesium after experimental traumatic brain injury: relationship to neurobehavioral outcome and neuroprotective efficacy of magnesium chloride. J Neurochem 73:27180.

Barone FC, Kilgore KS (2006) Role of inflammation and cellular stress in brain injury and central nervous system diseases. Clin Neurosci Res 6:329-56.

Bayir H, Kagan VE (2008) Bench-to-bedside review: mitochondrial injury, oxidative stressand apoptosis there is nothing more practical than a good theory. Crit Care 12:206.

Benveniste EN, Tang LP, Law RM (1995) Differential regulation of astrocyte TNF-a expression by the cytokines TGF-b, IL-6 and IL-10. Int J Dev Neurosci 13:341-9.

Blache D, Devaux S, Joubert O, Loreau N, Schneider M, Durand $P$, Prost $M$, Guame V, Adrian M, Laurant $P$, Berthelot A (2006) Long-term magnesium-deficient diet shows relationships between blood pressure, inflammation and oxidant stress defense in aging rats. Free Radic Biol Med 41:277-84.

Black PH (2002) Stress and the inflammatory response: a review of neurogenic inflammation. Brain Beh Immun 16:622-53.

Bracken MB, Holford TR (2002) Neurological and functional status 1 year after acute spinal cord injury: estimates of functional recovery in National Acute Spinal Cord Injury Study II from results modeled in National Acute Spinal Cord Injury Study III. J Neurosurg 96:259-66.

Browne KD, Leoni MJ, Iwata A, Chen X-H, Smith DH (2004) Acute treatment with $\mathrm{MgSO}_{4}$ attenuates longterm hippocampal tissue loss after brain trauma in the rat. J Neurosci Res 77:878-83.

Buechner JS, Speare MC, Fontes J (2000) Hospitalizations for spinal cord injuries, 1994-1998. Med Health R I 83:92-3.
Buki A, Povlishock JT (2006) All roads lead to disconnection? - Traumatic axonal injury revisited. Acta Neurochir 148:181-93.

Cernak I, Savic VJ, Kotur J, Prokic V, Veljovic M, Grbovic D (2000) Characterization of plasma magnesium concentration and oxidative stress following graded traumatic brain injury in humans. $J$ Neurotrauma 17:53-68.

Chanimov M, Berman S, Gofman V, Weissgarten Y, Averbukh Z, Cohen ML, Vitin A, Bahar M (2006) Total cell associated electrolyte homeostasis in rat spinal cord cells following apparently irreversible injury. Med Sci Monit 12:BR63-7.

Chong ZZ, Li F, Maiese K (2005) Oxidative stress in the brain: novel cellular targets that govern survival during neurodegenerative disease. Prog Neurobiol 75:207-46.

Clausen T, Bullock R (2001) Medical treatment and neuroprotection in traumatic brain injury. Curr Pharm Design 7:1517-32.

Cook NL, Van Den Heuvel C, Vink R (2009) Are the transient receptor potential melastatin (TRPM) channels important in magnesium homeostasis following traumatic brain injury? Magnes Res 22:22534.

Cormio M, Robertson CS, Narayan RK (1997) Secondary insults to the injured brain. J Clin Neurosci 4:132-48.

Delgado AV, McManus AT, Chambers JP (2003) Production of tumor necrosis factor-alpha, interleukin 1-beta, and interleukin 6 by rat leukocyte subpopulations after exposure to substance P. Neuropeptides 37:355-61.

Dhandapani SS, Gupta A, Vivekanandhan S, Sharma BS, Mahapatra AK (2008) Randomized controlled trial of magnesium sulphate in severe closed traumatic brain injury. Ind J Neurotrauma 5:27-33.

Ditor DS, John SM, Roy J, Marx JC, Kittmer C, Weaver LC (2007) Effects of polyethylene glycol and magnesium sulfate administration on clinically relevant neurological outcomes after spinal cord injury in the rat. J Neurosci Res 85:1458-67.

Donkin JJ, Nimmo AJ, Cernak I, Blumbergs PC, Vink R (2009) Substance $P$ is associated with the development of brain edema and functional deficits after traumatic brain injury. $J$ Cereb Blood Flow Metab 29:1388-98. 
Ebel H, Günther T (1980) Magnesium metabolism: a review. J Clin Chem Clin Biochem 18:257-70.

Ellis EM (2007) Reactive carbonyls and oxidative stress: potential for therapeutic intervention. Pharmacol \& Therapeutics 115:13-24.

Fan L, Young PR, Barone FC, Feuerstein GZ, Smith DH, Mclntosh TK (1995) Experimental brain injury induces expression of interleukin-1b mRNA in the rat brain. Mol Brain Res 30:125-30.

Fan L, Young PR, Barone FC, Feuerstein GZ, Smith DH, Mclntosh TK (1996) Experimental brain injury induces differential expression of tumor necrosis factor-a mRNA in the CNS. Mol Brain Res 36:287-91.

Feldman Z, Gurevitch B, Artru AA, Oppenheim A, Shohami E, Reichenthal E, Shapira Y (1996) Effect of magnesium given 1 hour after head trauma on brain edema and neurological outcome. J Neurosurg 85:1317.

Finkel T, Holbrook NJ (2000) Oxidants, oxidative stress and the biology of ageing. Nature 408:239-47.

Fleminger S, Ponsford J (2005) Long term outcome after traumatic brain injury. Br Med J 331:1419-20.

Fromm L, Heath DL, Vink R, Nimmo AJ (2004) Magnesium attenuates post-traumatic depression/anxiety following diffuse traumatic brain injury in rats. J Am Coll Nutr 23:529S-33S.

Gentile NT, Mclntosh TK (1993) Antagonists of excitatory amino acids and endogenous opioid peptides in the treatment of experimental central nervous system injury. Ann Emerg Med 22:1028-34.

Gentleman SM, Nash MJ, Sweeting CS, Graham DI, Roberts GW (1993) B-Amyloid precursor protein (B$A P P)$ as a marker for axonal injury after head injury. Neurosci Lett 160:139-44.

Gok B, Okutan O, Beskonakli E, Kilinc K (2007) Effects of magnesium sulphate following spinal cord injury in rats. Chin J Physiol 50:93-7.

Golding EM (2002) Sequelae following traumatic brain injury: the cerebrovascular perspective. Brain Res Rev 38:377-88.

Gowda RM, Khan IA (2004) Magnesium in treatment of acute myocardial infarction. Int J Cardiol 96:467-9.

Halliwell B (1992) Reactive oxygen species and the central nervous system. J Neurochem 59:1609-23.
Harrison S, Geppetti P (2001) Substance P. Int J Biochem Cell Biol 33:555-76.

Heath DL, Vink R (1996) Traumatic brain axonal injury produces sustained decline in intracellular free magnesium concentration. Brain Res 738:150-3.

Heo JH, Han SW, Lee SK (2005) Free radicals as triggers of brain edema formation after stroke. Free Rad Biol Med 39:51-70.

Hoane MR (2007) Assessment of cognitive function following magnesium therapy in the traumatically injured brain. Magnes Res 20:229-36.

Hoane MR, Barth TM (2002) The window of opportunity for administration of magnesium therapy following focal brain injury is $24 \mathrm{~h}$ but is task dependent in the rat. Physiol Behav 76:271-80.

Hurlbert RJ (2006) Strategies of medical intervention in the management of acute spinal cord injury. Spine (Phila Pa 1976) 31:S16-21.

Jain KK (2008) Neuroprotection in traumatic brain injury. Drug Discovery Today 13:1082-9.

Kahraman S, Ozgurtas T, Kayalt H, Atabey C, Kutluay T, Timurkaynak E (2003) Monitoring of serum ionized magnesium in neurosurgical intensive care unit: preliminary results. Clin Chim Acta 334:211-5.

Kaptanoglu E, Beskonakli E, Okutan O, Selcuk Surucu $H$, Taskin $Y$ (2003a) Effect of magnesium sulphate in experimental spinal cord injury: evaluation with ultrastructural findings and early clinical results. J Clin Neurosci 10:329-34.

Kaptanoglu E, Beskonakli E, Solaroglu I, Kilinc A, Taskin $Y(2003 b)$ Magnesium sulfate treatment in experimental spinal cord injury: emphasis on vascular changes and early clinical results. Neurosurg Rev 26:283-7.

Kimelberg HK (1995) Current concepts of brain edema. J Neurosurg 83:1051-9.

Knoblach SM, Faden AI (1998) Interleukin-10 improves outcome and alters proinflammatory cytokine expression after experimental traumatic brain injury. Exp Neurol 153:143-51.

Kowaltowski AJ, de Souza-Pinto NC, Castilho RF, Vercesi AE (2009) Mitochondria and reactive oxygen species. Free Rad Biol Med 47:333-43. 
Kwon BK, Okon E, Hillyer J, Mann C, Baptiste D, Weaver LC, Fehlings MG, Tetzlaff W (2010) A systematic review of non-invasive pharmacologic neuroprotective treatments for acute spinal cord injury. J Neurotrauma 27:1-44.

Kwon BK, Roy J, Lee JH, Okon E, Zhang H, Marx JC, Kindy MS (2009) Magnesium chloride in a polyethylene glycol formulation as a neuroprotective therapy for acute spinal cord injury: preclinical refinement and optimization. J Neurotrauma 26:137993.

Lee JS, Han YM, Yoo DS, Choi SJ, Choi BH, Kim JH, Kim YH, Huh PW, Ko YJ, Rha HK, Cho KS, Kim DS (2004) A molecular basis for the efficacy of magnesium treatment following traumatic brain injury. $J$ Neurotrauma 21:549-61.

Leeman L, Fontaine P (2008) Hypertensive disorders of pregnancy. Am Fam Physician 78:93-100.

Lemke M, Demediuk P, McIntosh TK, Vink R, Faden Al (1987) Alterations in tissue $\mathrm{Mg++}, \mathrm{Na}+$ and spinal cord edema following impact trauma in rats. Biochem Biophys Res Commun 147:1170-5.

Lemke M, Faden Al (1990) Edema development and ion changes in rat spinal cord after impact trauma: injury dose-response studies. J Neurotrauma 7:41-54.

Li X-Y, Feng D-F (2009) Diffuse axonal injury: Novel insights into detection and treatment. J Clin Neurosci 16:614-9.

Lima FD, Souza MA, Furian AF, Rambo LM, Ribeiro LR, Martignoni FV, Hoffmann MS, Fighera MR, Royes LFF, Oliveria MS, de Mello CF (2008) Na+,K+-ATPase activity impairment after experimental traumatic brain injury: relationship to spatial learning deficits and oxidative stress. Behav Brain Res 193:306-10.

Liou AKF, Clark RS, Henshall DC, Yin X-M, Chen J (2003) To die or not to die for neurons in ischemia, traumatic brain injury and epilepsy: a review on the stressactivated signaling pathways and apoptotic pathways. Prog Neurobiol 69:103-42.

Maas AIR, Stocchetti N, Bullock R (2008) Moderate and severe traumatic brain injury in adults. Lancet Neurol 7:728-41.

MacDonald JF, Xiong Z-G, Jackson MF (2006) Paradox of $\mathrm{Ca}^{2+}$ signaling, cell death and stroke. Trends Neurosci 29:75-81.
Malpuech-Brugère $C$, Nowacki $W$, Daveau $M$, Gueux $E$, Linard C, Rock E, Lebreton J-P, Mazur A, Rayssiguier Y (2000) Inflammatory response following acute magnesium deficiency in the rat. Biochim Biophys Acta 1501:91-8.

Marmarou A, Fatouros PP, Barzo P, Portella G, Yoshihara M, Tsuji O, Yamamoto T, Laine F, Signoretti S, Ward JD, Bullock R, Young HF (2000) Contribution of edema and cerebral blood volume to traumatic brain swelling in head-injured patients. J Neurosurg 93:18393.

Massullo P, Sumoza-Toledo A, Bhagat H, PartidaSánchez S (2006) TRPM channels, calcium and redox sensors during innate immune responses. Sem Cell Dev Biol 17:654-66.

Mazur A, Maier JAM, Rock E, Gueux E, Nowacki W, Rayssiguier $Y$ (2006) Magnesium and the inflammatory response: potential physiopathological implications. Arch Biochem Biophys 458:48-56.

Mazzeo AT, Beat A, Singh A, Bullock R (2009) The role of mitochondrial transition pore, and its modulation, in traumatic brain injury and delayed neurodegeneration after TBI. Exp Neurol 218:363-70.

McIntosh TK, Vink R, Yamakami I, Faden Al (1989) Magnesium protects against neurological deficit after brain injury. Brain Res 482:252-60.

McKee JA, Brewer RP, Macy GE, Borel CO, Reynolds JD, Warner DS (2005) Magnesium neuroprotection is limited in humans with acute brain injury. Neurocrit Care 2:342-51.

Monteilh-Zoller MK, Hermosura MC, Nadler MJS, Scharenberg AM, Penner R, Fleig A (2003) TRPM7 provides an ion channel mechanism for cellular entry of trace metal ions. J Gen Physiol 121:49-60.

Morales DM, Marklund N, Lebold D, Thompson HJ, Pitkänen A, Maxwell WL, Longhi L, Laurer $H$, Maegele M, Neugebauer E, Graham DI, Stocchetti N, Mclntosh TK (2005) Experimental models of traumatic brain injury: do we really need to build a better mousetrap? Neuroscience 136:971-89.

Morganti-Kossmann MC, Satgunaseelan L, Bye N, Kossmann T (2007) Modulation of immune response by head injury. Injury 38:1392-400.

Nicholls DG, Budd SL (2000) Mitochondria and neuronal survival. Physiol Rev 80:315-60. 
Nimmo AJ, Cernak I, Heath DL, Hu X, Bennett CJ, Vink R (2004) Neurogenic inflammation is associated with development of edema and functional deficits following traumatic brain injury in rats. Neuropeptides 38:40-7.

Ozdemir M, Cengiz SL, Gurbilek M, Ogun TC, Ustan ME (2005) Effects of magnesium sulphate on spinal cord tissue lactate and malondialdehyde levels after spinal cord trauma. Magnes Res 18:170-4.

Palmer AM (2010) The role of the blood-CNS barrier in CNS disorders and their treatment. Neurobiol Dis 37:312.

Pitkänen A, Longhi L, Marklund N, Morales DM, McIntosh TK (2005) Neurodegeneration and neuroprotective strategies after traumatic brain injury. Drug Discov Today 2:409-18.

Ravishankar S, Ashraf QM, Fritz K, Mishra OP, Delivoria-Papadopoulos M (2001) Expression of Bax and $\mathrm{Bcl}-2$ proteins during hypoxia in cerebral cortical neuronal nuclei of newborn piglets: effect of administration of magnesium sulfate. Brain Res 901:23-9.

Regan RF, Jasper E, Guo Y, Panter SS (1998) The effect of magnesium on oxidative neuronal injury in vitro. $J$ Neurochem 70:77-85.

Ro JY, Zhang Y, Nies M (2005) Substance P does not play a critical role in neurogenic inflammation in the rat masseter muscle. Brain Res Rev 1047:38-44.

Robertson CL (2004) Mitochondrial dysfunction contributes to cell death following traumatic brain injury in adult and immature animals. J Bioenerg Biomemb 36:363-8.

Roth P, Farls K (2000) Pathophysiology of traumatic brain injury. Crit Care Nurs Quart 23:14-25.

Runnels LW, Yue L, Clapham DE (2001) TRP-PLIK, a bifunctional protein with kinase and ion channel activities. Science 291:1043-7.

Saatman KE, Bareyre FM, Grady MS, McIntosh TK (2001) Acute cytoskeletal alterations and cell death induced by experimental brain injury are attenuated by magnesium treatment and exacerbated by magnesium deficiency. J Neuropathol Exp Neurol 60:183-94.

Sacco RL, Chong JY, Prabhakaran S, Elkind MSV (2007) Experimental treatments for acute ischaemic stroke. Lancet 369:331-41.
Schmitz C, Perraud A-L, Johnson CO, Inabe K, Smith MK, Penner R, Kurosaki T, Fleig A, Scharenberg AM (2003) Regulation of vertebrate cellular $\mathrm{Mg}^{2+}$ homeostasis by TRPM7. Cell 114:191-200.

Sen AP, Gulati A (2010) Use of magnesium in traumatic brain injury. Neurotherapeutics 7:91-9.

Signoretti S, Marmarou A, Aygok GA, Fatouros PP, Portella G, Bullock RM (2008) Assessment of mitochondrial impairment in traumatic brain injury using high-resolution proton magnetic resonance spectroscopy. J Neurosurg 108:42-52.

Simard JM, Kent TA, Chen M, Tarasov KV, Gerzanich V (2007) Brain oedema in focal ischaemia: molecular pathophysiology and theoretical implications. Lancet Neurol 6:258-68.

Solaroglu I, Kaptanoglu E, Okutan O, Beskonakli E, Attar A, Kilinc K (2005) Magnesium sulfate treatment decreases caspase-3 activity after experimental spinal cord injury in rats. Surg Neurol 64 Suppl 2:S17-21.

Stahel PF, Morganti-Kossmann MC, Kossmann T (1998) The role of the complement system in traumatic brain injury. Brain Res Rev 27:243-56.

Stone JR, Okonkwo DO, Dialo AO, Rubin DG, Mutlu LK, Povlishock JT, Helm GA (2004) Impaied axonal transport and altered axolemmal permeability occur in distinct populations of damaged axons following traumatic brain injury. Exp Neurol 190:59-69.

Suh BC, Hille B (2005) Regulation of ion channels by phosphatidylinositol 4,5-bisphosphate. Curr Opin Neurobiol 15:370-8.

Suzer T, Coskun E, Islekel H, Tahta K (1999) Neuroprotective effect of magnesium on lipid peroxidation and axonal function after experimental spinal cord injury. Spinal Cord 37:480-4.

Temkin NR, Anderson GD, Winn HR, Ellenbogen RG, Britz GW, Schuster J, Lucas T, Newell DW, Mansfield PN, Machamer JE, Barber J, Dikmen SS (2007) Magnesium sulfate for neuroprotection after traumatic brain injury: a randomised controlled trial. Lancet Neurol 6:29-38.

Turner RJ, Blumbergs PC, Sims NR, Helps SC, Rodgers KM, Vink R (2006) Increased substance $P$ immunoreactivity and edema formation following reversible ischemic stroke. Acta Neurochir 596:263-6. 
Turner RJ, DaSilva KW, O'Connor C, Van Den Heuvel C, Vink R (2004) Magnesium gluconate offers no more protection than magnesium sulphate following diffuse traumatic brain injury in rats. J Am Coll Nutr 23:541S$4 \mathrm{~S}$.

Unterberg AW, Stover J, Kress B, Kiening KL (2004) Edema and brain trauma. Neuroscience 129:1021-9.

Van Den Heuvel C, Vink R (2004) The role of magnesium in traumatic brain injury. Clin Calcium 14:1187-92.

Verlooy J, Van Reempts J (2005) The blood-brain barrier in trauma, stroke and edema. Int Congr Series 1277:227-34.

Vink R, Cernak I (2000) Regulation of intracellular free magnesium in central nervous system injury. Front Biosci 5:d656-65.

Vink R, Cook NL, van den Heuvel C (2009) Magnesium in acute and chronic brain injury: an update. Magnes Res 22:158S-62S.

Vink R, Donkin JJ, Cruz MI, Nimmo AJ, Cernak I (2004) A substance $P$ antagonist increases brain intracellular free magnesium concentration after diffuse traumatic brain injury in rats. J Am Coll Nutr 23:538S-40S.

Vink R, Mclntosh TK, Demediuk P, Weiner MW, Faden Al (1988) Decline in intracellular free $\mathrm{Mg}^{2+}$ is associated with irreversible tissue injury after brain trauma. J Biol Chem 263:757-61.

Vink R, Nimmo AJ (2009) Multifunctional drugs for head injury. Neurotherapeutics 6:28-42.

Vink R, Van Den Heuvel C (2004) Recent advances in the development of multifactorial therapies for the treatment of traumatic brain injury. Exp Opin Investig Drugs 13:1263-74.
Vink R, Van Den Heuvel C (2010) Substance P antagonists as a therapeutic approach to improving outcome following traumatic brain injury. Neurotherapeutics 7:74-80.

Vink R, Young A, Bennett CJ, Hu X, O'Connor C, Cernak I, Nimmo AJ (2003) Neuropeptide release influences brain edema formation after diffuse traumatic brain injury. Acta Neurochir S86:257-60.

Weaver FM, Hammond MC, Guihan M, Hendricks RD (2000) Department of Veterans Affairs Quality Enhancement Research Initiative for spinal cord injury. Med Care 38:182-91.

Weglicki WB, Mak IT, Phillips TM (1994) Blockade of cardiac inflammation in $\mathrm{Mg}^{2+}$ deficiency by substance $P$ receptor inhibition. Circ Res 74:1009-13.

Weglicki WB, Phillips TM (1992) Pathobiology of magnesium deficiency: a cytokine/neurogenic inflammation hypothesis. Am J Physiol Regul Integr Comp Physiol 263:734-7.

Wiseman DB, Dailey AT, Lundin D, Zhou J, Lipson A, Falicov A, Shaffrey $\mathrm{Cl}$ (2009) Magnesium efficacy in a rat spinal cord injury model. J Neurosurg Spine 10:30814.

Wu ZZ, Guan BC, Li ZW, Yang Q, Liu CJ, Chen JG (2004) Sustained potentiation by substance $P$ of NMDAactivated current in rat primary sensory neurons. Brain Res 1010:117-26.

Yang YL, Yao KH, Li ZW (2003) Similarities of SP-, NKAand NKB-induced currents in rat dorsal root ganglion neurons. Brain Res 991:18-25.

Yu BP (1994) Cellular defenses against damage from reactive oxygen species. Physiol Rev 74:139-62. 



\title{
The use of magnesium in experimental cerebral ischemia
}

\author{
Bruno P. Meloni, ${ }^{\bowtie y m ~ C a m p b e l l ~ a n d ~ N e v i l l e ~ W . ~ K n u c k e y ~}$
}

Centre for Neuromuscular and Neurological Disorders, University of Western Australia, and the Australian Neuromuscular Research Institute, Department of Neurosurgery, Sir Charles Gairdner Hospital, Nedlands, WA, 6009, Australia.

$\bowtie$ meloni@cyllene.uwa.edu.au

\begin{abstract}
The rationale for trialing magnesium as a neuroprotective agent following cerebral ischemia has been based both on its role in maintaining brain tissue homeostasis and on its known cellular actions that are likely to counteract damaging ischemic processes. A number of studies using animal models of cerebral ischemia, seizure, perinatal hypoxia-ischemia, subarachnoid hemorrhage and traumatic brain injury have reported positive outcomes with magnesium therapy. However, scrutiny of the animal cerebral ischemia data shows that about $46 \%$ of studies have not shown a neuroprotective effect. Furthermore, the IMAGES clinical trial found magnesium to be largely ineffective in treating strokes. In this review, we present the majority of published cerebral ischemia animal studies (focal and global) that have used magnesium as a neuroprotective therapy, and discuss the possible reasons for the inconsistent results. Our examination suggests that, in the majority of experiments, post-ischemic hypothermia has probably been a confounding factor in producing the positive outcomes. In addition, experimental design has not always been appropriate with respect to magnesium dosage, and to the time and route of magnesium administration. Moreover, data from our own laboratory indicates that magnesium is only neuroprotective when combined with post-ischemic hypothermia. Finally, additional information regarding the efficacy of magnesium as a stroke treatment will be available on completion of the FASTMag trial, but in the meantime the neuroprotective potential of magnesium should be explored when combined with post-ischemic hypothermia, and potentially with other agents, in cerebral ischemia models.
\end{abstract}

\section{Introduction}

More than any other organ, the brain is dependent on the aerobic metabolism of glucose for energy, making it exceedingly sensitive to ischemic disease. Inadequate supply of oxygenated blood leads to a rapidly developing energy crisis that, within minutes, results in the death of cells comprising the neurovascular unit. Restoration of blood supply, or the presence of collateral circulation, may preserve some tissue only for it to die over the hours or days that follow an ischemic event. Such delayed death may occur from one or more of a range of pathological processes; glutamate excitotoxicity, calcium dysregulation and overload, apoptosis, autophagy, inflammation, oxygen free radical generation and edema have all been implicated. Consequently, interruption of these processes presents an opportunity for therapeutic intervention to protect the damaged cells within the neurovascular unit after cerebral ischemia. Many therapies have been found to offer neuroprotection in experimental models of cerebral ischemia, magnesium among them.

Since magnesium is important in many normal cellular processes such as protein synthesis, energy metabolism, maintenance of ionic gradients and vascular smooth muscle tone, abnormalities in serum and CNS magnesium status before, during and after cerebral ischemia can be expected to have an effect on neurological outcome. Indeed, clinical and experimental studies have shown that subjects with low serum or CSF magnesium have worsened neurological outcomes following brain ischemia and trauma (Lampl et al., 1998; McIntosh et al., 1988; Vink et al., 1988). There is also ample evidence demonstrating that there are marked changes in intracellular and extracellular free magnesium concentrations in brain tissue following ischemic and traumatic insults (Helpern et al., 1993; Vande Linde and Chopp, 1991; Vink et al., 1996; Lee et al., 2002). As such, maintaining normal serum magnesium concentration should be considered a 
part of standard supportive care in such patients. On top of that, however, the $\mathrm{Mg}^{2+}$ cation has properties that may specifically counteract a number of the damaging process associated with cerebral ischemia. In particular, magnesium can reduce synaptic glutamate release (Nowak et al., 1984), decrease calcium influx via voltage gated calcium channels (Iseri and French, 1984), stabilise mitochondria (Kowaltowski et al., 1998; $\mathrm{Xu}$ et al., 2002), increase cerebral blood flow (Chi et al., 1990) and reduce free radical production (Garcia et al., 1998).

For these reasons, restoration of magnesium homeostasis and exploration of the potential positive effects in the ischemic brain have prompted the investigation of magnesium as a neuroprotective agent following such disorders as traumatic brain injury (Heath and Vink, 1996; Vink et al., 1988), seizure (Cotton et al., 1993), subarachnoid hemorrhage (Van den Bergh et al., 2002) and cerebral ischemia (Muir, 1998). Here we overview the experimental animal studies that have to date tested magnesium as a neuroprotectant in the major animal models of cerebral ischemia, specifically focal (ischemic stroke) and global (cardiac arrest) brain ischemia models.

This review will present experimental findings from these studies, offer potential explanations for conflicting or inconsistent results and consider the future application of magnesium in cerebral ischemia.

\section{Efficacy of magnesium treatment in models of focal cerebral ischemia}

The results from 16 studies using magnesium in animal models of focal cerebral ischemia are summarized in Table 1. As can be seen in this table, there is considerable variability in study design (stroke model, animal species used, magnesium dose, route and timing of administration, outcome measures), which precludes direct comparison of outcomes. There were 5 studies using permanent focal ischemia models, 9 using transient models and single studies using an embolic and an endothelin-1 (lacunar stroke) model. As a broad summary, 10 of the 16 studies reported a neuroprotective effect for magnesium based on infarct volume reduction. Magnesium treatment in the endothelin-1 model did not reduce infarct volume, but did improve some behavioral outcomes. Thus, there is slightly better than equivocal support for the claim that magnesium has a neuroprotective effect in these models.

\section{Magnesium in Permanent Focal Cerebral Ischemia}

In the 5 studies using permanent middle cerebral artery occlusion (MCAO), 3 reported positive outcomes with magnesium treatment. Two of the studies, 1 using rats (Izumi et al., 1991) and 1 using mice (Roffe et al., 1996), were otherwise similar in their experimental procedures, yet reported contrasting results. Izumi et al., (1991) observed both a neuroprotective effect and a hyperglycemia associated with administration of magnesium chloride; if the hyperglycemia was managed with insulin, there was a further reduction of infarct volume. In contrast, Roffe et al., (1996) found no neuroprotection, and in fact reported that magnesium chloride treatment increased edema in the infarcted hemispheres. While increased edema was not evident in animals treated with magnesium and insulin, the combined treatment did not reduce infarct volume.

Two other studies (Lee et al., 1999; Chung et al., 2004), which were similar with respect to magnesium dose and timing of administration, but different in animal species used and route of administration, reported positive findings, while a study from our laboratory was negative (Campbell et al., 2008a). Among these, our study was the only trial to use a dosing regimen (IV loading dose and 24 hour infusion) similar to the IMAGES human stroke trial. Furthermore, we paid careful attention to body temperatures postsurgery to ensure that animals did not become hypothermic during the recovery period or thereafter. The possibility of post-ischemic hypothermia having confounded these experimental results, especially in the earlier animal studies, will be discussed in a later section.

It is of interest that Lee et al., (1999) commented that, in unpublished experiments, if the intraarterial route of magnesium administration was changed to an intravenous route, magnesium was no longer protective. 
TABLE 1. Summary of animal studies using magnesium following focal cerebral ischemia

\begin{tabular}{|c|c|c|c|c|c|c|c|}
\hline Reference & Animal model & Mg salt & Route & Magnesium dose & Time of treatment & Post-ischemic temperature monitoring & Neuroprotection/Outcome \\
\hline \multicolumn{8}{|l|}{ Permanent Model } \\
\hline Izumi et al., 1991 & pMCAO, rat & $\mathrm{MgCl}_{2}$ & IP & $1 \mathrm{mmol} / \mathrm{kg} \times 2$ & Immediately \& $1 \mathrm{~h}$ after MCAO & Measured $1.5,4,24 \& 48$ h post MCAO & Yes; reduced infarct volume at $48 \mathrm{~h}$ \\
\hline Roffe et al., 1996 & pMCAO, mouse & $\mathrm{MgCl}_{2}$ & IP & $1 \mathrm{mmol} / \mathrm{kg} \times 2$ & Immediately \& $1 \mathrm{~h}$ after MCAO & No & No; based on infarct volume at $24 \mathrm{~h}$ \\
\hline Lee et al., 1999 & pMCAO, rat & $\mathrm{MgSO}_{4}$ & IA & $0.75 \mathrm{mmol} / \mathrm{kg}$ & 10min before MCAO & No & Yes; reduced infarct volume at $24 \mathrm{~h}^{\#}$ \\
\hline Chung et al., 2004 & pMCAO, gerbil & $\mathrm{MgSO}_{4}$ & IP & $0.75 \mathrm{mmol} / \mathrm{kg}$ & 10min before MCAO & No & Yes; reduced infarct volume at $24 \mathrm{~h}$ \\
\hline Campbell et al., 2008a & pMCAO, rat & $\begin{array}{l}\mathrm{MgSO}_{4} . \\
7 \mathrm{H}_{2} \mathrm{O}\end{array}$ & IV & $\begin{array}{l}0.36+0.12 \mathrm{mmol} / \mathrm{kg} / \mathrm{h} \text { for } \\
24 \mathrm{~h}\end{array}$ & $2 \mathrm{~h}$ after MCAO & Yes, actively monitored \& controlled & No; based on infarct volume at $48 \mathrm{~h}$ \\
\hline \multicolumn{8}{|l|}{ Transient Model } \\
\hline Marinov et al., 1996 & 2 or $1.5 \mathrm{~h} \mathrm{tMCAO}$, rat & $\mathrm{MgSO}_{4}$ & IA & 0.25 or $0.75 \mathrm{mmol} / \mathrm{kg}$ & Immediately before MCAO & Monitored for $45 \mathrm{~min}$ after ischemia & Yes; reduced infarct volume at $24 \mathrm{~h}$ \\
\hline $\begin{array}{l}\text { Schmid-Elsaesser et al., } \\
1999\end{array}$ & 1.5h tMCAO, rat & $\mathrm{MgCl}_{2}$ & IV & $1 \mathrm{mmol} / \mathrm{kg} \times 2$ & $\begin{array}{l}\text { Before MCAO \& at time of } \\
\text { reperfusion }\end{array}$ & $\begin{array}{l}\text { Monitored for } 1 \mathrm{~h} \text { after ischaemia \& rats } \\
\text { housed in warm cages for } 8 \mathrm{~h}\end{array}$ & $\begin{array}{l}\text { No; }(25 \% \text { reduction infarct volume } \\
\text { at } 7 \text { days) }\end{array}$ \\
\hline Kinoshita et al., 2001 & $2 \mathrm{~h} \mathrm{tMCAO}$, rat & $\mathrm{MgSO}_{4}$ & IV & $0.21 \mathrm{mmol} / \mathrm{kg}$ over $2 \mathrm{~h}$ & During MCAO & No & Yes; reduced infarct volume at $24 \mathrm{~h}$ \\
\hline Lin et al., 2002 & $1 \mathrm{~h} \mathrm{tMCAO}$, gerbil & $\mathrm{MgSO}_{4}$ & IP & $0.75 \mathrm{mmol} / \mathrm{kg}$ & $30 \mathrm{~min}$ before ischaemia & No & Yes; reduced infarct volume at $24 \mathrm{~h}$ \\
\hline $\begin{array}{l}\text { Westermaier et al., } \\
2003\end{array}$ & 1.5h tMCAO, rat & $\mathrm{MgSO}_{4}$ & $I V \& I A$ & $0.75 \mathrm{mmol} / \mathrm{kg}$ & Immediately before MCAO & As for Schmid-Elsaesser et al., 1999 & $\begin{array}{l}\text { No; ( } 25 \% \text { reduction) based on } \\
\text { infarct volume at } 7 \text { days }\end{array}$ \\
\hline \multirow[t]{2}{*}{ Zhu et al., 2004a } & $\begin{array}{l}\text { 1. } 45 \mathrm{~min} \mathrm{tMCAO} \text {, } \\
\text { rat }\end{array}$ & $\begin{array}{l}\mathrm{MgSO}_{4} . \\
7 \mathrm{H}_{2} \mathrm{O}\end{array}$ & IV & $\begin{array}{l}0.18,0.36 \text { or } \\
0.72 \mathrm{mmol} / \mathrm{kg}\end{array}$ & Immediately before MCAO & Monitoring \& maintained for $6 \mathrm{~h}$ & No; based on infarct volume at $24 \mathrm{~h}$ \\
\hline & 2. $2 \mathrm{~h} \mathrm{tMCAO}$, rat & & IA & 0.37 or $0.74 \mathrm{mmol} / \mathrm{kg}$ & Immediately before MCAO & No & No; based on infarct volume at $72 \mathrm{~h}$ \\
\hline Lee et al., 2005 & 1.5h tMCAO, rat & $\mathrm{MgSO}_{4}$ & IA & $0.75 \mathrm{mmol} / \mathrm{kg}$ & At time of reperfusion & $\begin{array}{l}\text { Yes warm environment } 4 \mathrm{~h} \text { post } \\
\text { reperfusion. Less hyperthermia in } \mathrm{Mg} \\
\text { treated }\end{array}$ & $\begin{array}{l}\text { Yes; reduced infarct volume at } 72 \mathrm{~h} \\
\& \text { improve functional outcomes }\end{array}$ \\
\hline $\begin{array}{l}\text { Westermaier et al., } \\
2005\end{array}$ & 1.5h tMCAO, rat & $\mathrm{MgSO}_{4}$ & IV & $\begin{array}{l}0.75 \text { or } 2 \times 1 \mathrm{mmol} / \mathrm{kg} \text { or } 1 \\
\mathrm{mmol} / \mathrm{kg}+0.5 \mathrm{mmol} / \mathrm{kg} / \mathrm{h} \\
\text { for } \approx 2.5 \mathrm{~h}\end{array}$ & Immediately before MCAO & As for Schmid-Elsaesser et al., (1999) & $\begin{array}{l}\text { Yes; based on infarct volume at } 7 \\
\text { days. Note: } 31 \% \text { reduction for } 0.75 \\
\mathrm{mmol} / \mathrm{kg} \text { dose was not significant }\end{array}$ \\
\hline Campbell et al., 2008b & $45 \mathrm{~min} \mathrm{tMCAO}$, rat & $\begin{array}{l}\mathrm{MgSO}_{4} . \\
7 \mathrm{H}_{2} \mathrm{O}\end{array}$ & IV & 0.36 or $0.72 \mathrm{mmol} / \mathrm{kg}$ & Immediately before MCAO & $\begin{array}{l}\text { No, rats mildly hypothermic for } 1 \text { - } 4 \mathrm{~h} \\
\text { post surgery }\end{array}$ & Yes; for $0.36 \mathrm{mmol}$ at $24 \mathrm{~h}$ \\
\hline \multicolumn{8}{|l|}{ Other Models } \\
\hline Yang et al., 2000 & Embolic, rat & $\mathrm{MgSO}_{4}$ & IV & $0.75 \mathrm{mmol} / \mathrm{kg}$ & 2,6 or $8 \mathrm{~h}$ after ischaemia & No & Yes; reduced infarct volume at $72 \mathrm{~h}$ \\
\hline Lecrux et al., 2008 & ET-1 injection, rat & $\mathrm{MgSO}_{4}$ & SC & $\begin{array}{l}2.5 \mathrm{mmol} / \mathrm{kg}+ \\
1.66 \mathrm{mmol} / \mathrm{kg} \times 4\end{array}$ & $\begin{array}{l}\text { 30min before ET-1 injection \& } \\
\text { every h for } 4 \mathrm{~h}\end{array}$ & No mentioned & $\begin{array}{l}\text { No infarct reduction, but improved } \\
\text { functional outcomes at day } 3 \& 10\end{array}$ \\
\hline IMAGES trial, 2004* & Human stroke & $\mathrm{MgSO}_{4}$ & IV & $\begin{array}{l}0.2+0.034 \mathrm{mmol} / \mathrm{kg} / \mathrm{h} \text { for } \\
24 \mathrm{~h}\end{array}$ & Within $12 \mathrm{~h}$ & Normal patient care & $\begin{array}{l}\text { No based on neurological outcome, } \\
\text { except in lacunar stroke patients }\end{array}$ \\
\hline
\end{tabular}

\#Authors mentioned (unpublished data) that same dose administered IV was ineffective. ${ }^{*}$ IMAGES trial has been included for references purposes 
TABLE 2. Summary of animal studies using magnesium following global cerebral ischemia

\begin{tabular}{|c|c|c|c|c|c|c|c|}
\hline Reference & Animal model & Mg salt & Route & Magnesium dose & Time of treatment & $\begin{array}{l}\text { Post-ischemic temperature } \\
\text { monitoring }\end{array}$ & Neuroprotection/outcome \\
\hline Blair et al., 1989 & 10min: $2 \mathrm{VO}$ rat & $\mathrm{MgCl}_{2}$ & IV & $5.0 \mathrm{mmol} / \mathrm{kg}$ & Immediately before ischemia & No & $\begin{array}{l}\text { No; Mg exacerbated CA1 injury due to } \\
\text { elevated plasma glucose levels }\end{array}$ \\
\hline Tsuda et al., 1991 & 20min: 4VO rat & $\mathrm{MgCl}_{2}$ & $\begin{array}{l}\text { IC: CA1 } \\
\text { region }\end{array}$ & $1 \mu \mathrm{l}$ of $50 \mathrm{mM}$ & $\begin{array}{l}10 \mathrm{~min} \text { before, } 0,2,12,24 \text { or } \\
48 \mathrm{~h} \text { after ischemia }\end{array}$ & No & $\begin{array}{l}\text { Yes; at } 10 \mathrm{~min} \text { before } \& 0,2,12,24 \mathrm{~h} \\
\text { after ischemia; based on CA1 injury }\end{array}$ \\
\hline Okawa, 1992 & $\begin{array}{l}\text { 18min: aorta occlusion } \\
\text { dog }\end{array}$ & $\mathrm{MgSO}_{4}$ & IV & $\begin{array}{l}0.66 \mathrm{mmol} / \mathrm{kg}+ \\
0.33 \mathrm{mmol} / \mathrm{kg} / \mathrm{h} \text { for } 3 \mathrm{~h}+ \\
0.083 \mathrm{mmol} / \mathrm{kg} / \mathrm{h} \text { for } 45 \mathrm{~h}\end{array}$ & Immediately after ischemia & No & Yes; based on neurological outcome \\
\hline Sirin et al., 1998 & 15min: 4VO rat & $\mathrm{MgSO}_{4}$ & SC & $5 \mathrm{mmol} / \mathrm{kg}$ & $48 \mathrm{~h}$ before ischemia & No & $\begin{array}{l}\text { Yes; reduced CA1 injury \& improved } \\
\text { neurological outcomes }\end{array}$ \\
\hline \multirow[t]{2}{*}{ Milani et al., 1999} & 15min: 4VO rat & $\mathrm{MgCl}_{2}$ & SC & $\begin{array}{l}2.5 \mathrm{mmol} / \mathrm{kg} \times 4 \\
5.0 \mathrm{mmol} / \mathrm{kg} \times 4 \\
7.5 \mathrm{mmol} / \mathrm{kg} \times 4\end{array}$ & $\begin{array}{l}1,2,24 \& 48 \mathrm{~h} \text { after ischemia } \\
\text { for } 2.5,5 \& 7.5 \mathrm{mmol} / \mathrm{kg} \text { dose }\end{array}$ & $\begin{array}{l}\text { First few hours after ischemia rats } \\
\text { housed at } 30^{\circ} \mathrm{C}\end{array}$ & No; based on CA1 \& subiculum injury \\
\hline & & & & $5.0 \mathrm{mmol} / \mathrm{kg} \mathrm{x1}$ & $\begin{array}{l}2 \mathrm{~h} \text { after ischemia for } 5 \\
\mathrm{mmol} / \mathrm{kg} \text { dose }\end{array}$ & & \\
\hline \multirow[t]{2}{*}{ Miles et al., 2001} & $8 \mathrm{~min}: 2 \mathrm{VO}$ rat & $\begin{array}{l}\mathrm{MgSO}_{4} \\
7 \mathrm{H}_{2} \mathrm{O}\end{array}$ & IV & $\begin{array}{l}0.36 \mathrm{mmol} / \mathrm{kg}+0.06,0.12 \\
0.24 \text { or } 0.48 \mathrm{mmol} / \mathrm{kg} / \mathrm{h} \text { for } \\
48 \mathrm{~h}\end{array}$ & $\begin{array}{l}\text { Before, } 4,8,12 \text { or } 24 \mathrm{~h} \text { after } \\
\text { ischemia }\end{array}$ & No & $\begin{array}{l}\text { Yes; reduced CA1 injury when } 0.12 \\
\mathrm{mmol} / \mathrm{kg} / \mathrm{h} \text { infusion dose used }\end{array}$ \\
\hline & & $\mathrm{MgCl}_{2}$ & & $\begin{array}{l}0.36 \mathrm{mmol} / \mathrm{kg}+0.12 \\
\mathrm{mmol} / \mathrm{kg} / \mathrm{h} \text { for } 48 \mathrm{~h}\end{array}$ & Before or $8 \mathrm{~h}$ after ischemia & No & $\begin{array}{l}\text { Yes; before ischemia. No; at } 8 \mathrm{~h} \text { after } \\
\text { ischemia; based on CA1 injury }\end{array}$ \\
\hline Zhou et al., 2003 & 10min: 2VO gerbil & $\mathrm{MgSO}_{4}$ & IP & $16.6 \mathrm{mmol} / \mathrm{kg}$ & $30 \mathrm{~min}$ before ischemia & No & Yes; based on TUNEL staining \\
\hline Zhu et al., 2004b & $8 \mathrm{~min}: 2 \mathrm{VO}$ rat & $\begin{array}{l}\mathrm{MgSO}_{4} \\
7 \mathrm{H}_{2} \mathrm{O}\end{array}$ & IV & $\begin{array}{l}0.36 \mathrm{mmol} / \mathrm{kg}+ \\
0.06 \text { or } 0.12 \text { or } 0.24 \\
\mathrm{mmol} / \mathrm{kg} / \mathrm{h} \text { for } 48 \mathrm{~h}\end{array}$ & Before ischemia & $\begin{array}{l}\text { Maintained normothermic or self- } \\
\text { regulated }\end{array}$ & $\begin{array}{l}\text { No when animals maintained } \\
\text { normothermic; based on CA1 injury } \\
\text { Yes when combined with } \\
\text { hypothermia }\end{array}$ \\
\hline Zhu et al., 2005 & $8 \mathrm{~min}: 2 \mathrm{VO}$ rat & $\begin{array}{l}\mathrm{MgSO}_{4} \\
7 \mathrm{H}_{2} \mathrm{O}\end{array}$ & IV & $\begin{array}{l}0.36 \mathrm{mmol} / \mathrm{kg}+ \\
0.12 \mathrm{mmol} / \mathrm{kg} / \mathrm{h} \text { for } 48 \mathrm{~h}\end{array}$ & $\begin{array}{l}\text { Immediately before or } 2 \mathrm{~h} \text { after } \\
\text { ischemia }\end{array}$ & $\begin{array}{l}\text { Maintained normothermic or } \\
\text { hypothermic }\end{array}$ & $\begin{array}{l}\text { No; based on CA1 injury in } \\
\text { normothermic rats } \\
\text { Yes when combined with hypothermia }\end{array}$ \\
\hline
\end{tabular}




\section{Magnesium in Transient Focal Cerebral Ischemia} In the 10 studies using transient focal ischemia, 6 reported positive outcomes with magnesium treatment. As in the permanent focal ischemia studies, some of the trials, while similar in key respects, reported contrasting results. For example, Marinov et al., (1996) reported positive findings when either of 2 doses of magnesium sulphate $(0.75$ or $0.25 \mathrm{mmol} / \mathrm{kg})$ were administered intra-arterially before 2 or 1.5 hours of MCAO in rats. In contrast, Zhu et al., (2004a) reported negative findings in trials consisting of different doses of magnesium sulphate $(0.18$, $0.36,0.72$ or $0.74 \mathrm{mmol} / \mathrm{kg}$ ) administered either intra-arterially or intravenously before 45 minutes or 2 hours of MCAO in rats. In a subsequent experiment from the Zhu laboratory (Campbell et al., 2008b), rats administered 0.36 $\mathrm{mmol} / \mathrm{kg}$ magnesium intravenously before $\mathrm{MCAO}$, and allowed to self-regulate their body temperatures post-ischemia, experienced up to 4 hours of mild hypothermia and had significantly reduced striatal infarct volumes.

Similarly, Lee et al., (2005) observed a modest, magnesium-induced hypothermic effect in that magnesium treatment attenuated the postischemic hyperthermia normally observed in their focal model. When administered immediately after reperfusion, magnesium reduced infarct volumes and improved behavioral outcomes in rats. They ruled out magnesium's hypothermic effect as being responsible for the neuroprotection by inducing the same level of hypothermia in a separate group of animals, showing that these animals did not have either reduced infarct volumes or improved behavioral outcomes.

The Schmid-Elsaesser laboratory has been exploring the efficacy of magnesium following cerebral ischemia, alone and in combination with other treatments (e.g., tirilazad, hypothermia), dating back to 1999 (Schmid-Elsaesser et al., 1999; Zausinger et al., 2003ab; Westermaier et al., 2003; 2005). To summarize their several studies, they have shown that when magnesium is administered before, during or after MCAO it results in infarct volume reductions ranging from $25-42 \%$, with reductions of $>31 \%$ being statistically significant. After surgery, they routinely monitored animals' body temperatures for 1 hour after reperfusion and kept them in a warm environment for the first eight hours (though without further monitoring) to minimize the possibility of hypothermia (Robert SchmidElsaesser, personal communication).

\section{Other Focal Cerebral Ischemia Models}

Yang et al., (2000) assessed the neuroprotective efficacy of intravenously administered magnesium at different time points following ischemia $(2,6$ or 8 hours) using an embolic stroke model in rats. They found that magnesium treatment administered 2 or 6 hours, but not 8 hours, after ischemia significantly reduced infarct volumes. In addition, magnesium treatment appeared to improve animal survival and neurological outcome. While animals' body temperatures were monitored during surgical recovery, it was not reported what measures were taken to control their temperatures or to ensure they did not become hypothermic until the 72-hour trial end-point.

To assess the effects of magnesium on white matter injury associated with lacunar stroke, Lecrux et al., (2008) injected the vasoconstrictive peptide endothelin-1 into the internal capsule in rats (the study was prompted by post-hoc analysis of the IMAGES stroke trial data, which revealed that magnesium provided a small, but significant benefit in lacunar stroke patients (IMAGES, 2004)). In this study, relatively high subcutaneous doses of magnesium sulphate were given, which increased magnesium serum levels nearly four fold and reduced blood pressure. The treatment did not influence infarct volumes, but motor functional impairments were reduced in magnesium treated animals when assessed at 3 and 10 days.

\section{Efficacy of magnesium treatment in models of global (forebrain) cerebral ischemia}

The results from 9 studies using magnesium in models of global cerebral ischemia are summarized in Table 2. As in the focal ischemia studies, there is considerable variability in study design and protocols. At the histological level, 4 of the studies found a neuroprotective effect, 2 studies did not, while 2 studies reported a positive outcome only when magnesium treatment was combined with post-ischemic hypothermia. One study, using an aortic occlusion model in dogs, reported improved neurological outcomes with magnesium treatment. Again, this is moderate 
support for the contention that magnesium is neuroprotective in these models.

In the first study, by Blair et al., (1989), intravenous magnesium chloride appeared in fact to exacerbate CA1 neuronal injury. In this study, the magnesium dose was relatively high (5 $\mathrm{mmol} / \mathrm{kg}$ ), and was considered responsible for elevating serum glucose levels from $150 \mathrm{mg} / \mathrm{dl}$ to $220 \mathrm{mg} / \mathrm{dl}$, and thus contributing to increased CA1 neuronal loss. When hyperglycemia was controlled by the simultaneous administration of magnesium and insulin, CA1 injury was no different to saline treated controls. As a measure to ensure the delivery of magnesium to the target tissue, Tsuda et al., (1991) administered magnesium chloride ( $1 \mu \mathrm{l} ; 50 \mathrm{mM}$ solution) directly to the CA1 sector of the hippocampus at 10 minutes before ischemia or at $0,2,12,24$ or 48 hours after ischemia. A neuroprotective effect was observed in CA1 neurons at all time points except 48 hours post-ischemia. A lower dose of magnesium chloride $(1 \mu \mathrm{l} ; 10 \mathrm{mM})$ administered at the 24 hour time point also showed a neuroprotective trend, but was not significant at the $P<0.01$ level. Animal body temperatures were not monitored during recovery, so a potential confounding factor is that the animals might have become hypothermic during this period. The potential for hypothermia will also have been compounded since the animals were re-anaesthetized in those groups receiving magnesium post-ischemia.

While the implications of post-ischemic hypothermia will be discussed in more detail later, it should be emphasized that 3 studies (Milani et al., 1999; Zhu et al., 2004b; 2005) in which measures were taken to avoid postischemic hypothermia, including 2 from our laboratory, found no significant neuroprotection (CA1 neuronal survival) despite starting magnesium treatment before or early after cerebral ischemia and using multiple and continuous dosing regimens. Furthermore, in the 2 studies from our laboratory (Zhu et al., 2004b; 2005), when either spontaneous or controlled mild hypothermia occurred during treatment, a neuroprotective effect was observed. In the remaining studies (Okawa et al., 1992; Sirin et al., 1998; Miles et al., 2001; Zhou et al., 2003) no measures were reported to have been taken to avoid post-ischemic hypothermia, and all of these reported positive histological or behavioral outcomes.

\section{Reasons for inconsistent results with magnesium following cerebral ischemia}

On the evidence from these studies, to the question of whether magnesium is neuroprotective following cerebral ischemia, the answer must be: sometimes. A second question arises, then, as to what factor or factors determine when magnesium will be neuroprotective, and when it will not. Taking into account the differences in study design (species; ischemia model; post-surgical temperature control; dosage, route and time of magnesium administration) we propose several explanations that may reasonably account for the inconsistencies in the reported data.

\section{Confounding effects of post-ischemic hypothermia}

As previously reported (Meloni et al., 2006) there is reason to believe that the discrepant results found in these studies most likely can be attributed to the confounding effects of postischemic hypothermia. In the magnesium studies described in Tables 1 and 2, the animals were invariably maintained normothermic during ischemia, but in most cases there is no mention of body temperature monitoring or maintenance during recovery from surgery and in the postischemic period. This is crucial because it is now accepted and documented that post-surgical hypothermia commonly occurs in these models of cerebral ischemia, and it has been shown that post-ischemic hypothermia has confounded the results of previous studies (Buchan and Pulsinelli, 1990; Corbett et al., 1990; Welsh et al., 1990; Dietrich et al., 1995; Behringer et al., 2002). This is especially relevant in magnesium studies since there is also evidence that magnesium itself has hypothermia inducing properties (Zweifler et al., 2004; Wadhwa et al., 2005). Therefore, in those studies that did not actively maintain animals normothermic after ischemia, the occurrence of spontaneous hypothermia cannot be ruled out, and in fact is highly likely.

Further than that, direct evidence of the synergistic effects of mild hypothermia with magnesium has been generated from several studies in our laboratory. In an early global 
ischemia study (Miles et al., 2001) we did not monitor or control for animal body temperatures after ischemia; in this study we observed a neuroprotective effect. In subsequent studies (Zhu et al., 2004b; 2005), however, using the same model and magnesium treatment, but using controlled normothermic conditions after ischemia, we did not see a neuroprotective effect. Moreover, we observed that if body temperature was only monitored without being controlled, the animals did become mildly hypothermic during surgical recovery, and magnesium treatment in these animals did significantly reduce CA1 neuronal death. Note that control animals also became hypothermic to a similar degree, yet they did not show neuroprotection.

In subsequent experiments, when we compared magnesium efficacy in normothermic animals and in animals rendered mildly hypothermic $\left(35^{\circ} \mathrm{C}\right)$ for 6 hours immediately after global ischemia, we observed that treatment with magnesium and mild hypothermia together increased CA1 neuronal survival. Importantly, no neuroprotection was observed either in normothermic animals receiving magnesium or in animals rendered hypothermic for 6 hours post-ischemia. We have also obtained similar findings with focal ischemia experiments. For example, we have demonstrated that following focal ischemia, magnesium treatment in normothermic animals does not result in reduced infarct volumes. In contrast, if magnesium treatment is combined with a period of mild hypothermia (spontaneous or controlled) reduced brain infarcts are observed (Campbell et al., 2008ab). In our controlled hypothermia experiments using a permanent focal model (Campbell et al., 2008b) we did not observe a protective effect with mild hypothermia alone.

Taken together, these experiments illustrate two important points. The first is that ischemic control animals, though they might experience some level of hypothermia, are unlikely to show any evidence of neuroprotection. The second is that the combination of magnesium and mild hypothermia does not appear to act in an additive way, but rather synergistically to unmask a neuroprotective effect. These findings highlight the necessity to maintain post-ischemic animal body temperatures in drug evaluation studies and, since in the majority of magnesium/cerebral ischemia studies this was not reported to have been done, there is reason to question the validity of the results.

\section{Dosage, route and time of magnesium administration}

It is a matter of speculation as to the degree to which magnesium dosage and route of administration is another contributing factor for the discrepant findings, in large part due to the fact that the optimal dose of magnesium remains unknown.

As a rule of thumb, early experimental and clinical studies have aimed to achieve serum concentrations of about double the baseline (i.e., to increase serum magnesium from $\approx 0.8$ to $\approx 1.5$ $\mathrm{mmol} / \mathrm{l})$, which seems reasonable as magnesium at this level appears to be safe and well tolerated (Muir and Lees, 1995; 1998). However, data from our laboratory indicate that magnesium doses that double baseline serum levels may be too high. For example, magnesium doses that resulted in serum magnesium levels $>1.4 \mathrm{mmol} / \mathrm{L}$ (e.g., using an IV loading dose of $0.36 \mathrm{mmol} / \mathrm{kg}$, followed by an infusion of 0.24 or 0.48 $\mathrm{mmol} / \mathrm{kg} / \mathrm{h}$ ) provided no or minimal CA1 protection. On the other hand, a lower dose $(0.36$ $\mathrm{mmol} / \mathrm{kg} \mathrm{IV}$, then $0.12 \mathrm{mmol} / \mathrm{kg} / \mathrm{h}$ ), which resulted in serum magnesium from 1 - 1.2 $\mathrm{mmol} / \mathrm{l}$, provided marked CA1 survival following global ischemia (note that in these experiments the animals' body temperatures were not monitored during the post-ischemia period; Miles et al., 2001). In addition, we have shown that the higher loading dose of $0.72 \mathrm{mmol} / \mathrm{kg}$, when given before focal ischemia (in animals that were maintained normothermic in the 6 hour postischemic period), produced a trend towards increased infarct volume.

The time of administration will also have a marked influence on the potential neuroprotective effect of any intervention. Most of the studies here administered magnesium before or immediately after induction of cerebral ischemia; hence, increased magnesium levels were present in serum, and possibly the brain, at the time of ischemia. Post-ischemic treatments with magnesium, following both focal and global cerebral ischemia, have, however, produced positive outcomes, with treatment as late as 6 
hours after focal ischemia (Yang et al., 2000) and 24 hours after global ischemia showing a benefit (Tsuda et al., 1991). Even allowing that hypothermia has likely confounded some outcomes, it is encouraging to note that delayed treatment with magnesium can be effective.

While different routes of administration (intravenous, intra-arterial, intraperitoneal, subcutaneous, intracranial) have been used to deliver magnesium, it is difficult to comment on how this may have influenced outcomes in the absence of measures such as the serum and CSF concentrations achieved. Again of particular interest is the positive study using intracranial delivery (Tsuda et al., 1991), which reported efficacy even at 24 hours post-ischemia. By bypassing the blood brain barrier, intracranial delivery would guarantee increased brain magnesium and thus maximize its effects.

\section{CSF magnesium levels and magnesium salt}

Only 1 cerebral ischemia study has examined the extent to which exogenously administered magnesium penetrates into either brain or CSF. This study, by Okawa et al., (1992), used an IV loading dose $(0.664 \mathrm{mmol} / \mathrm{kg})$ followed by an infusion of magnesium sulphate $\quad 0.332$ $\mathrm{mmol} / \mathrm{kg} / \mathrm{h}$ for $3 \mathrm{~h}$, then $0.083 \mathrm{mmol} / \mathrm{kg} / \mathrm{h}$ for 45 h) in dogs subjected to global cerebral ischemia. They showed a significant increase in CSF magnesium lasting from 20 minutes to 7 days after treatment. Interestingly, dogs not subjected to global ischemia, but treated with the same magnesium regimen, did not show significantly elevated CSF magnesium. In a study using rats not subjected to cerebral ischemia, Sjöström and Wester (1990) showed elevated brain and CSF magnesium shortly after IV administration of 2.0 $\mathrm{mmol} / \mathrm{kg}$ magnesium chloride. The Okawa et al., (1992) study suggests that exogenously administered magnesium may have increased access to the brain following cerebral ischemia, making it likely that in cerebral ischemia experiments magnesium has been present in the target tissue, and thus able to exert both vascular and neural effects.

Magnesium sulphate and magnesium chloride have both been used in models of cerebral ischemia, with both positive and negative results, yet no studies have directly compared their efficacy. The only study that has assessed both magnesium salts was an early global ischemia study from our laboratory (Miles et al., 2001). Our data appeared to favour greater efficacy for magnesium sulphate, but unfortunately the trials with the two magnesium salts were not performed concurrently, and once again there is the likelihood of post-ischemic hypothermia confounding the results. Interestingly, in our experiments animals treated with magnesium chloride did not become hyperglycaemic (unpublished observation), which is in contrast to two earlier studies (Blair et al., 1989; Izumi et al., 1991). A possible contributing factor to the hyperglycaemia reported by Blair et al., (1989) and Izumi et al., (1991) is that they found magnesium treatment was associated with hypothermia, which can reduce insulin secretion (Polderman, 2009) predisposing the animals to hyper-glycaemia.

In summary, the discrepancies in the outcomes of these studies are explicable in light of several unanswered questions that still surround magnesium treatment for cerebral ischemia. On the available evidence, it seems fair to say that magnesium does indeed have neuroprotective properties when a suitable dose is given to achieve moderately increased (above normal) levels in the target tissue in conjunction with a mild degree of hypothermia.

\section{Future use of magnesium following stroke and cerebral ischemia}

As it stands, in light of the failure of the IMAGES stroke trial to find a significant treatment effect with magnesium (IMAGES, 2004), the future use of magnesium for the treatment of stroke, and possibly other forms of cerebral ischemia, will depend heavily on the outcome of the FAST-MAG Phase 3 trial (Saver et al., 2004, Stroke Trials Directory, 2010). This trial is currently underway, and is assessing whether field administration of magnesium within 2 hours of stroke onset improves clinical outcomes. In essence, the FASTMAG trial will address whether the long delay to magnesium treatment in the IMAGES trial (enrolment up to 12 hours after the onset of stroke) was the principal reason for its ineffectiveness. However, based on our own assessment of magnesium under normothermic conditions, we predict that if FAST-MAG patients 
are maintained normothermic this trial will also show little or no benefit. As discussed, the evidence suggests that in order for magnesium to produce a positive outcome after stroke/cerebral ischemia it needs to be combined with mild hypothermia (Meloni et al., 2009). Fortunately, hypothermia induction may only require a reduction in body temperature of $1-2^{\circ} \mathrm{C}$ involving basic cooling measures, which may be achievable in conscious patients. It is anticipated that future cerebral ischemia experimental studies will play a vital role in assessing magnesium in combination with hypothermia, and potentially with other agents as well, with successful outcomes guiding the design of clinical trials.

\section{Conclusion}

There is evidence from several models and using a number of treatment regimens that magnesium has neuroprotective properties that can reduce brain damage and neurological deficits after cerebral ischemia. Given that hypomagnesaemia is known to occur after stroke, magnesium supplementation has a place in the normal supportive care of such patients. Beyond that, the question remains as to whether, and by how much, supplementation should aim to increase, rather than just restore, serum magnesium levels. The FAST-MAG trial may provide part of the answer, though probably without being definitive.

\section{References}

Behringer W, Safar P, Kentner R, Wu X, Kagan VE, Radovsky A, Clark RSB, Kochanek PM, Subramanian $M$, Tyurin VA, Tyurin YY, Tisherman SA (2002) Antioxidant tempol enhances hypothermic cerebral preservation during prolonged cardiac arrest in dogs. J Cereb Blood Flow Metab 22:105-17.

Blair JL, Warner DS, Todd MM (1989) Effects of elevated plasma magnesium versus calcium on cerebral ischemic injury in rats. Stroke 20:507-12.

Buchan A, Pulsinelli WA (1990) Hypothermia but not $\mathrm{N}$-methyl-D-aspartate antagonist, MK-801, attenuates neuronal damage in gerbils subjected to transient global ischemia. J Neurosci 10:311-16.
The rationale for trialing magnesium as a neuroprotective agent following stroke has been based on its role in maintaining normal brain functions, on its known cellular actions that are anticipated to counteract damaging ischemic processes and subsequently on positive experimental data from animal models of cerebral ischemia. However, close scrutiny of the animal data shows that about $46 \%$ of studies have not shown a neuroprotective effect, and that the majority, if not all, of the positive studies were potentially confounded by post-ischemic hypothermia. In addition, experimental design has not always been well-founded with respect to magnesium dosage, and to the time and route of magnesium administration. Moreover, recent animal studies under controlled post-ischemic conditions indicate that magnesium is only neuroprotective when combined with hypothermia. Finally, additional information regarding the efficacy of magnesium as a stroke treatment will be available on completion of the FAST-MAG trial, but in the meantime the neuroprotective potential of magnesium remains a valid subject for investigation in cerebral ischemia models, with emphasis on its combination with post-ischemic hypothermia.

\section{Acknowledgments}

The authors would like to thank William Gow for compiling the reference list.

Campbell K, Meloni BP, Knuckey NW (2008a)

Combined magnesium and mild hypothermia $\left(35^{\circ} \mathrm{C}\right)$ treatment reduces infarct volumes after permanent middle cerebral artery occlusion in the rat at 2 and 4, but not 6 hours. Brain Res 1230:258-64.

Campbell K, Meloni BP, Zhu H, Knuckey NW (2008b) Magnesium treatment and spontaneous mild hypothermia after transient focal cerebral ischemia in the rat. Brain Res Bull 77:320-2.

Chi OZ, Pollak P, Weiss HR (1990) Effects of magnesium sulfate and nifedipine on regional cerebral blood flow during middle cerebral artery ligation in the rat. Arch Int Pharmacodyn Ther 304:196-205. 
Chung SY, Lin JY, Lin MC, Liu HM, Wang MF, Chung FC (2004) Synergistic efficacy of magnesium sulfate and FK506 on cerebral ischemia-induced infarct volume in gerbil. Med Sci Monit 10:105-8.

Corbett D, Evans S, Thomas C, Wang D, Jonas AR (1990) MK-801 reduced cerebral ischemic injury by inducing hypothermia. Brain Res 514:300-4.

Cotton DB, Hallak M, Janusz C, Irtenkauf SM, Berman RF (1993) Central anticonvulsant effects of magnesium sulfate on N-methyl-D-aspartate-induced seizures. Am J Obstet Gynecol 168:974-8.

Dietrich WD, Lin B, Globus MY-T, Green EJ, Ginsberg MD, Busto R (1995) Effect of delayed MK-801 (dizocilpine) treatment with or without immediate postischemic hypothermia on chronic neuronal survival after global forebrain ischemia in rats. $J$ Cereb Blood Flow Metab 15:960-8.

Garcia LA, Dejong SC, Martin SM, Smith RS, Buettner GR, Kerber RE (1998) Magnesium reduces free radicals in an in vivo coronary occlusion-reperfusion model. J Am Coll Cardiol 32:536-9.

Heath DL, Vink R (1996) Traumatic brain axonal injury produces sustained decline in intracellular free magnesium concentration. Brain Res 738:150-3.

Helpern JA, Vande Linde AMQ, Welch KMA, Levine SR, Schultz LR, Ordidge RJ Halvorson HR, Hugg JW (1993) Acute elevation and recovery of intracellular $\left[\mathrm{Mg}^{2+}\right]$ following human focal cerebral ischemia. Neurology 43:1577-81.

IMAGES (2004) Magnesium for acute stroke (Intravenous Magnesium Efficacy in Stroke trial): randomized controlled trial. Lancet 363:439-45.

Iseri LT, French JH (1984) Magnesium: nature's physiologic calcium blocker. Am Heart J 108:188-93.

Izumi Y, Roussel S, Pinard E, Seylaz J (1991) Reduction of infarct volume by magnesium after middle cerebral artery occlusion in rats. J Cereb Blood Flow Metab 11:1025-30.

Kinoshita Y, Ueyama T, Senba E, Terada T, Nakai K, Itakura T (2001) Expression of c-fos, heat shock protein 70, neurotrophins, and cyclooxygenase-2 mRNA in response to focal cerebral ischemia/reperfusion in rats and their modification by magnesium sulfate. J Neurotrauma 18:435-45.

Kowaltowski AJ, Naia-da-Silva ES, Castilho RF, Vercesi $\mathrm{AE}$ (1998) $\mathrm{Ca}^{2+}$-stimulated mitochondrial reactive oxygen species generation and permeability transition are inhibited by dibucaine or $\mathrm{Mg}^{2+}$. Arch Biochem Biophys 359:77-81.

Lampl Y, Geva D, Gilad R, Eshel Y, Ronen L, SarovaPinhas I (1998) Cerebrospinal fluid magnesium level as a prognostic factor in ischaemic stroke. $J$ Neurol 245:584-8.

Lecrux C, McCabe C, Weir CJ, Gallagher L, Mullin J, Touzani O, Muir KW, Lees KR, Macrae IM (2008) Effects of magnesium treatment in a model of internal capsule lesion in spontaneously hypertensive rats. Stroke 39:448-54.

Lee EJ, Ayoub IA, Harris FB, Hassan M, Ogilvy CS, Maynard KI (1999) Mexiletine and magnesium independently, but not combined, protect against permanent focal cerebral ischemia in Wistar rats. $J$ Neurosci Res 58:442-8.

Lee EJ, Lee MY, Chang GL, Chen LH, Hu YL, Chen TY, Wu TS (2005) Delayed treatment with magnesium: reduction of brain infarction and improvement of electrophysiological recovery following transient focal cerebral ischemia in rats. J Neurosurg 102:1085-93.

Lee M-S, Wu YS, Yang DY, Lee JB, Cheng FC (2002) Significantly decreased extracellular magnesium in brains of gerbils subjected to cerebral ischemia. Clin Chim Acta 318:121-5.

Lin J-Y, Chung S-Y, Lin MC, Cheng FC (2002) Effects of magnesium sulfate on energy metabolites and glutamate in the cortex during focal cerebral ischemia and reperfusion in the gerbil monitored by a dualprobe microdialysis technique. Life Sci 71:803-11.

Marinov MB, Harbaugh KS, Hoopes PJ, Pikus HJ, Harbaugh RE (1996) Neuroprotective effects of preischemia intraarterial magnesium sulfate in reversible focal cerebral ischemia. J Neurosurgery $85: 117-24$.

McIntosh TK, Faden Al, Yamakami I, Vink R (1988) Magnesium deficiency exacerbates and pre-treatment improves outcome following traumatic brain injury in rats: ${ }^{31} \mathrm{P}$ magnetic resonance spectroscopy and behavioral studies. J Neurotrauma 5:17-30.

Meloni BP, Campbell K, Zhu H, Knuckey NW (2009) In search of clinical neuroprotection after brain ischemia: the case for mild hypothermia $\left(35^{\circ} \mathrm{C}\right)$ and magnesium. Stroke 40:2236-40.

Meloni BP, Zhu H, Knuckey NW (2006) Is magnesium neuroprotective following global and focal cerebral ischaemia? A review of published studies. Magnes Res 19:123-37. 
Milani H, Lepri ER, Giordani F, Favero-Filho LA (1999) Magnesium chloride alone or in combination with diazepam fails to prevent hippocampal damage following transient forebrain ischemia. Braz J Med Biol Res 32:1285-93.

Miles AN, Majda BT, Meloni BP, Knuckey NW (2001) Postischemic intravenous administration of magnesium sulfate inhibits hippocampal CA1 neuronal death after transient global ischemia in rats. Neurosurgery 49:1443-51.

Muir KW (1998) New experimental and clinical data on the efficacy of pharmacological magnesium infusions in cerebral infarcts. Magnes Res 11:43-56.

Muir KW, Lees KR (1995) A randomised, double blind, placebo-controlled pilot trial of intravenous magnesium sulphate in acute stroke. Stroke 26:11838.

Muir KW, Lees KR (1998) Dose optimization of intravenous magnesium sulphate after acute stroke. Stroke 29:918-23.

Nowak L, Bregestovski P, Ascher $\mathrm{P}$, Herbet A, Prochiantz A (1984) Magnesium gates glutamateactivated channels in mouse central neurones. Nature 307:462-5.

Okawa M (1992) Effects of magnesium sulfate on brain damage by complete global brain ischemia (Japanese). Masui (Jap J Anesthesiol) 41:341-55.

Polderman KS (2009) Mechanisms of action, physiological effects, and complications of Hypothermia. Crit Care Med 37 (Suppl.): S186 -S202.

Roffe C, Thomas L, Fotheringham A, Davies I (1996) The effect of magnesium on infarct size and oedema after middle cerebral artery occlusion. Cerebrovasc Dis 6 (Supplement 2):42 (Abstract).

Saver JL, Kidwell C, Eckstein M, Starkmam S (2004) Pre-hospital neuroprotective therapy for acute stroke: results of the field administration of stroke therapymagnesium (FAST-MAG) pilot trial. Stroke 35, e106-8.

Schmid-Elsaesser R, Zausinger S, Hungerhuber E, Baethmann A, Reulen HJ (1999) Neuroprotective effects of combination therapy with tirilazad and magnesium in rats subjected to reversible focal cerebral ischemia. Neurosurgery 44:163-72.

Sirin BH, Coskun E, Yilik L, Ortac R, Sirin H, Tetik C (1998) Neuroprotective effects of preischemia subcutaneous magnesium sulfate in transient cerebral ischemia. Eur J Cardiothorac Surg 14:82-8.
Sjöström LG, Wester PO (1990) Accumulation of magnesium in rat brain after intravenously induced hypermagnesemia. Cerebrovasc Dis 5: 241 (Abstract).

Stroke Trials Directory (2010). www.stroke.org/. Tsuda T, Kogure K, Nishioka K, Watanabe T (1991) $\mathrm{Mg}^{2+}$ administered up to twenty-four hours following reperfusion prevents ischemic damage of the CA1 neurons in the rat hippocampus. Neuroscience 44:33541.

Van den Bergh WM, Zuur JK, Kamerling NA, Van Asseldonk JT, Rinkel GJ, Tulleken CA, Nicolay K (2002) Role of magnesium in the reduction of ischemic depolarisation and lesion volume after experimental subarachnoid hemorrhage. J Neurosurg 97:416-22.

Vande Linde AMQ, Chopp M (1991) Chronic changes in brain $\mathrm{Mg}^{2+}$ concentration after forebrain ischemia in the rat. Metab Brain Dis 6:199-206.

Vink R, Heath DL, Mclntosh TK (1996) Acute and prolonged alterations in brain free magnesium following fluid percussion-induced brain trauma in rats. J Neurochem 66:2477-83.

Vink R, Mclntosh TK, Demediuk P, Weiner MW, Faden Al (1988) Decline in intracellular free $\mathrm{Mg}^{2+}$ is associated with irreversible tissue injury after brain trauma. J Biol Chem 263:757-61.

Wadhwa A, Sengupta P, Durrani J, Akca O, Lenhardt R, Sessler DI, Doufas AG (2005) Magnesium sulphate only slightly reduces the shivering threshold in humans. $\mathrm{Br} J$ Anaesth 94:756-62.

Welsh FA, Sims RE, Harris VA (1990) Mild hypothermia prevents ischemic injury in gerbil hippocampus. $J$ Cereb Blood Flow Metab 10:557-63.

Westermaier T, Hungerhuber E, Zausinger S, Baethmann A, Schmid-Elsaesser R (2003) Neuroprotective efficacy of intra-arterial and intravenous magnesium sulfate in a rat model of transient focal cerebral ischemia. Acta Neurochir 145:393-9.

Westermaier T, Zausinger S, Baethmann A, SchmidElsaesser R (2005) Dose finding of intravenous magnesium sulphate in transient focal cerebral ischemia in rats. Acta Neurochir 147:525-32.

Xu M, Dai W, Deng X (2002) Effects of magnesium sulfate on brain mitochondrial respiratory function in rats after experimental traumatic brain injury. Chin J Traumatol 5:361-4. 
Yang Y, Li Q, Ahmad F, Shuaib A (2000) Survival and histological evaluation of therapeutic window of postischemia treatment with magnesium sulfate in embolic stroke model of rat. Neurosci Lett 285:119-22.

Zausinger S, Schöller K, Plesnila N, Schmid-Elsaesser R (2003a) Combination drug therapy and mild hypothermia after transient focal cerebral ischemia in rats. Stroke 34:2246-51

Zausinger S, Westermaier T, Plesnila N, Steiger HJ, Schmid-Elsaesser R (2003b) Neuroprotection in transient focal cerebral ischemia by combination drug therapy and mild hypothermia: comparison with customary therapeutic regimen. Stroke 34:1526-32.

Zhou H, Ma Y, Zhou Y, Liu Z, Wang K, Cheng G (2003) Effects of magnesium sulfate on neuron apoptosis and expression of caspase-3, bax and bcl-2 after cerebral ischemia-reperfusion injury. Chin Med J 116:1532-34.
Zhu H, Martin RL, Meloni BP, Oltvolgyi C, Moore S, Majda BT, Knuckey NW (2004a) Magnesium sulfate fails to reduce infarct volume following transient focal ischemia in rats. Neurosci Res 49:347-53.

Zhu H, Meloni BP, Bojarski C, Knuckey MW, Knuckey NW (2005) Post-ischemic modest hypothermia $\left(35^{\circ} \mathrm{C}\right)$ combined with intravenous magnesium is more effective at reducing CA1 death than either treatment used alone following global cerebral ischemia in rats. Exp Neurol 193:361-8.

Zhu H, Meloni BP, Moore SR, Majda BT, Knuckey NW (2004b) Intravenous administration of magnesium is only neuroprotective following transient global ischemia when present with post-ischemic mild hypothermia. Brain Res 1014:53-60.

Zweifler RM, Voorhees ME, Mahmood MA, Parnell M (2004) Magnesium sulfate increases the rate of hypothermia via surface cooling and improves comfort. Stroke 35:2331-4. 


\title{
Magnesium in subarachnoid haemorrhage
}

\author{
Walter M. van den Bergh \\ Department of Intensive Care, Academic Medical Centre, Amsterdam, The Netherlands. \\ $\triangle$ w.m.vandenbergh@amc.nl
}

\begin{abstract}
Subarachnoid haemorrhage (SAH) caused by a ruptured aneurysm accounts for only $5 \%$ of strokes, but occurs at a fairly young age and carries a worse prognosis. Delayed cerebral ischemia is an important cause of death and dependence after aneurysmal SAH. The current mainstay of preventing delayed cerebral ischemia is nimodipine and maintenance of normovolemia, but even with this strategy delayed cerebral ischemia occurs in a considerable proportion of patients. Magnesium is an inexpensive, easily available neuroprotective agent that reduces cerebral vasospasm and infarct volume after experimental $\mathrm{SAH}$. In a meta-analysis of all randomized clinical trials, magnesium shows a tendency to reduce the occurrence of delayed cerebral ischemia and poor outcome after SAH, but the question if magnesium is advantageous in SAH patients is still in abeyance. Currently a large phase III trial aiming for $\mathbf{1 2 0 0}$ patients is being conducted that will hopefully provide definite evidence whether magnesium treatment is beneficial in subarachnoid haemorrhage patients.
\end{abstract}

\section{Subarachnoid haemorrhage}

Subarachnoid haemorrhage (SAH) caused by a ruptured aneurysm accounts for only $5 \%$ of strokes, but occurs at a fairly young age and carries a worse prognosis than other types of stroke (van Gijn et al., 2007). The cardinal feature is a history of unusually severe headache that started suddenly, but patients frequent deteriorate into unconsciousness shortly after onset. On admission two-thirds of all patients have depressed consciousness, of whom half are in coma (Brilstra et al., 2000). The school of Hippocrates already described the clinical picture of a SAH: "When persons in good health are suddenly seized with pains in the head, and straightway are laid down speechless, and breathe with stertor, they die in seven days, unless fever comes on" (Hippocrates et al., 1527; Clarke, 1963). Attila the Hun is suspected to have had a SAH as he died suddenly after severe headache developed during sexual intercourse (Babinski, 1893).

The incidence of SAH in most populations is 6-7 per 100000 person-years (Linn et al., 1996). About one in eight patients die before reaching the hospital. The in-hospital case fatality is about one-third (Nieuwkamp et al., 2009). Of patients who survive the SAH approximately one-third remain dependent. Because of the young age
SAH occurs, and its poor prognosis, the loss of productive life years from $\mathrm{SAH}$ is as large as that from ischemic stroke, the most frequent subtype of stroke.

The presence of an aneurysm was first demonstrated by an angiograph of the carotid artery by Moniz in 1933 (Moniz, 1933), 6 years after he had introduced this technique (Moniz, 1927). Aneurysms arise at sites of arterial branching, usually at the base of the brain, either on the circle of Willis itself or at a nearby branching point. Most intracranial aneurysms will never rupture. Risk factors for subarachnoid haemorrhage are hypertension, smoking, and excessive alcohol intake, all of which more-or-less double the risk (Feigin et al., 2005).

Diagnosis is made by CT brain scanning. Recurrent bleeding is the most imminent danger; a first aim is therefore occlusion of the aneurysm. Endovascular obliteration by means of platinum spirals (coiling) is the preferred mode of treatment, but some patients require a direct neurosurgical approach (clipping) (Molyneux et al., 2002).

\section{Delayed cerebral ischemia}

For those patients who survive the first 24 hours after the haemorrhage, delayed cerebral 
ischemia (DCI) is consistently the leading cause of death and disability, adversely affecting more than one in five of all patients who have suffered SAH and survived (Dorsch, 1995; Roos et al., 2000).

Unlike thrombo-embolic stroke, cerebral ischemia after SAH has a gradual onset and often involves more than the territory of a single cerebral artery or one of its branches. The clinical manifestations evolve gradually, over several hours, and consist of hemispheric focal deficits, a reduction in the level of consciousness, or both. It is mainly a diagnosis of exclusion, when clinical deterioration occurs and hydrocephalus, recurrent bleeding, hypoxia, and metabolic abnormalities have been ruled out. The peak frequency of cerebral ischemia is from 5-14 days after SAH. The time course for delayed cerebral ischemia parallels that of angiographic vasospasm, but arterial narrowing - a complex process in itself - is neither a necessary nor a sufficient condition. Although about $70 \%$ of patients may develop arterial narrowing, only 20$30 \%$ will manifest neurological deficits (Weir et al., 1999; Rabinstein et al., 2004).

Nimodipine is a dihydropyridine calcium channel blocker that selectively blocks the voltagedependent L-type calcium channel. Nimodipine is accepted as beneficial in preventing $\mathrm{DCl}$ and subsequent poor outcome when given orally 6 times $(60 \mathrm{mg})$ for 21 days after the haemorrhage (Dorhout Mees et al., 2007b). The initial thought was that nimodipine could prevent or ameliorate cerebral vasospasm, but nimodipine does not influence large vessel diameter and its pivotal method of action must be explained otherwise.

Cerebral vasospasm, although often used as a synonym, apparently is a too simplistic explanation for delayed cerebral ischemia. Research, however, has focused on the mechanisms involved in the development of cerebral vasospasm as a means by which cerebral ischemia can be prevented. The precise underlying pathogenic mechanisms remain obscure, but it seems that endothelial mechanisms provide the most prominent contribution to this process, and there is growing evidence that the constituents of a subarachnoid blood clot, especially oxyhaemoglobin, a product of haemoglobin breakdown, seem to be the principle initiating factor (Pluta et al., 1998; Dumont et al., 2003; Kolias et al., 2009).

The current mainstay of preventing and treating $\mathrm{DCl}$ include neuroprotection with nimodipine and maintenance of normovolemia, but even with this strategy $\mathrm{DCl}$ still occurs in up to $30 \%$ of patients and improvement in clinical outcome has been modest (Hop et al., 1997; Brilstra et al., 2000; Roos et al., 2000; Dorhout Mees et al., 2008). Reducing the consequences of ischemia through neuroprotection may therefore improve outcome after SAH. DCl after SAH is a unique pathophysiological process because the timing and development of potential cerebral ischemia can be predicted. The interval of 4 or more days between the bleeding and the onset of ischemia provides an opportunity for preventive treatment.

\section{Magnesium and cerebral ischemia: opportunities and concerns}

Magnesium is a popular drug for acute treatment of ischemia because of its ease of use, low cost, and favourable side-effect profile. In several experimental models of cerebral ischemia, a significant neuroprotective effect of magnesium has been demonstrated with reported infarct reduction of 25-61\% (Izumi et al., 1991; Marinov et al., 1996; Muir, 1998). Putative modes of action include inhibition of the presynaptic release of the excitatory amino acid glutamate, and blockade of the postsynaptic NMDA glutamate receptor and voltage dependent calcium channels (van den Bergh et al., 2004b). Magnesium reduces the production of endothelin and completely attenuates the vasoconstrictive effect of endothelin, possibly by inhibiting calcium channel-mediated, smooth-muscle contraction (Kemp et al., 1999; Berthon et al., 2003). Furthermore, magnesium increases cardiac contractility, which may improve cerebral perfusion in dysautoregulated brain tissue.

However, acute ischemic stroke has not the quality of cerebral vasospasm-associated $\mathrm{DCl}$ in SAH that occurs mostly more than 4 days after the initial bleeding, allowing the administration of a treatment before ischemia develops. Delayed treatment might explain why magnesium was ineffective in ischemic stroke in the IMAGES study (Muir et al., 2004). Again a precedent exists 
for nimodipine, which was ineffective when tested in ischemic stroke. A study of acute administration of magnesium in stroke (FASTMAG) is underway (Saver et al., 2004).

Another concern about the suitability of magnesium to protect the ischemic brain is that regulation of cerebrospinal fluid magnesium is largely maintained following acute brain injury and limits the brain bioavailability of magnesium sulphate. Current dose regimens may only marginally increase concentrations of magnesium in CSF in brain-injured humans (Mckee et al., 2005), with this modest increase occurring in the range of $10-19 \%$. However, experimental evidence suggests that this modest elevation may be sufficient for neuroprotection. In a study using ${ }^{31} \mathrm{P}-\mathrm{MR}$ spectroscopy (MRS) to investigate intracellular brain free magnesium in 37 patients with good-grade aneurysmal SAH, patients were randomized for magnesium therapy and compared (Wong et al., 2009). Magnesium treatment after aneurysmal SAH produced a small (+13\%; mean difference $0.018 \pm 0.007 \mathrm{mM}$ ), but significant elevation of intracellular free magnesium. In a randomized controlled trial where magnesium therapy was compared with nimodipine, CSF magnesium concentration was non-statistically higher in the magnesium group $(1.19 \pm 0.20$ vs $1.14 \pm 0.21 ; p=0.26)$ (SchmidElsaesser et al., 2006).

The negative effect of magnesium on blood pressure may also have an impact on results. Currently, vasopressors are often used to prevent hypotension in SAH patients, and $\mathrm{DCl}$ is often treated with induced hypertension with the aim to increase cerebral perfusion. However, the lowering effect of magnesium on blood pressure is limited and far less than that of nimodipine.

Finally, there is a dose related association between magnesium sulphate administration and hypocalcaemia, but symptomatic hypocalcaemia is rare and it does not result in an increased risk of poor outcome (van den Bergh et al., 2008b). There is no basis for routine supplementation of calcium in patients with hypocalcaemia when treated with magnesium. Of course, supplementation might be considered in individual patients to prevent isolated clinical symptoms of hypocalcaemia such as muscle weakness or cardiac arrhythmias.

\section{Hypomagnesemia in subarachnoid haemorrhage}

In observational clinical studies, the vast majority of SAH patients develop hypomagnesaemia at some time within three weeks after the haemorrhage (Warren et al., 1993; van den Bergh et al., 2003). Low magnesium serum levels are frequently present at admission and are thus most likely caused by intracellular shift of magnesium ions. Intracellular $\mathrm{Mg}^{2+}$ levels are indeed increased in SAH. However, $90 \%$ of the intracellular $\mathrm{Mg}^{2+}$ is complexed with ATP, and the increase of intracellular $\mathrm{Mg}^{2+}$ during ischemia may also be the result of the release of $\mathrm{Mg}^{2+}$ from this complex. ATP binds with $\mathrm{Mg}^{2+}$ with an associate constant of 4 , while binding affinity with ADP is about 2 times smaller. The cytosolic and mitochondrial $\mathrm{Mg}^{2+}$ concentration will increase in cells with a poor energy state and less ATP (Jung et al., 1990; Saris et al., 2000; Wong et al., 2009). The increase of intracellular $\mathrm{Mg}^{2+}$ is even less than might be expected from ATP utilization, probably because of a disappearance of $\mathrm{Mg}^{2+}$ by binding to other cell components. Hypomagnesaemia that developed later on in the course might also be caused by renal loss as very often is the case in critically ill patients.

The diminished availability and subsequent decreased extracellular $\mathrm{Mg}^{2+}$ after $\mathrm{SAH}$ results in significantly increased intracellular free $\mathrm{Ca}^{2+}$ in cerebral vascular muscle cells. This may cause cerebral microvascular constriction, followed by a proinflammatory response, inducing vascular smooth muscle, endothelial and neuronal cell damage (Barbour et al., 2002). Furthermore, hypomagnesaemia results in a reduced endothelial NO release by which means hypomagnesaemia can induce vasoconstriction (Pearson et al., 1998; Shechter, 2000). All the above gives rise to the concept of a causal relationship between the decreased availability of magnesium and the development of vasospasmassociated $\mathrm{DCl}$. When testing this hypothesis in 107 prospectively studied consecutive patients, we found that hypomagnesaemia during day 4 and 10 after the haemorrhage increased the risk for $\mathrm{DCl}$ three fold (van den Bergh et al., 2003). Apart from $\mathrm{DCl}$, in a study in 62 patients, more than one-third had hypomagnesaemia at admission which was independently related to cardiac dysrhythmias, especially a long PR interval and a shorter QTc interval (van den Bergh et al., 2004a). 
This link with cardiac complications might be another reason why hypomagnesaemia may be related to poor outcome (van der Bilt et al., 2009).

\section{Magnesium treatment in subarachnoid haemorrhage}

\section{Animal studies}

Magnesium has a vasorelaxing effect in oxyhaemoglobin-induced vasospasm and it ameliorates cerebral vasospasm in experimental SAH (Miura, 1988; Ram et al., 1991; Pyne et al., 2001), although no effect of magnesium sulphate on the angiographic diameter of large cerebral vessels in a monkey model of SAH could be noted (Macdonald et al., 2004). Neither does magnesium infusion decrease middle cerebral artery blood flow velocities in patients with $\mathrm{DCl}$ (van den Bergh, unpublished data).

Magnesium induces a dose-dependent vasodilatation, reduces cerebrovascular tone, increases CBF and protects the metabolism (Seelig et al., 1983; Altura and Altura, 1984; Torregrosa et al., 1994) However, in a human study in 6 patients, magnesium sulphate infusion did not increase cerebral blood volume and cerebral blood flow compared with pretreatment levels, or compared with 6 control patients (Wong et al., 2010a).

We have demonstrated in an experimental model that the duration of ischemic depolarizations after SAH is substantially reduced after pretreatment with magnesium sulphate (van den Bergh et al., 2002). Magnesium also postpones anoxic depolarization (van der $\mathrm{Hel}$ et al., 1998). This maintenance of the membrane potential may, at least partly, explain the neuroprotective properties of magnesium in SAH. We have shown in a rat model of SAH that pre-treatment with magnesium sulphate reduces acute cerebral lesion volume with more than $60 \%$ (van den Bergh et al., 2002).

\section{Large randomized controlled trials}

In 2005, the "Magnesium and Acetylsalicylic acid in Subarachnoid Haemorrhage" (MASH-I) trial was published, in which 283 patients between November 2000 and January 2004 were randomized to placebo or a continuous infusion of $64 \mathrm{mmol} / \mathrm{l} /$ day of magnesium sulphate starting within 4 days of SAH and continuing 14 days after occlusion of the aneurysm (van den Bergh et al., 2005). The results showed a trend towards less cerebral ischemia in patients allocated to magnesium and improved overall outcome, with a $35 \%$ reduction in $\mathrm{DCl}$ (RR 0.65; $95 \% \mathrm{Cl} 0.40-$ 1.05 ) and a $23 \%$ reduction in poor outcomes (RR $0.77 ; 0.54-1.09)$. The mean magnesium level during treatment was $1.47 \pm 0.32 \mathrm{mmol} / \mathrm{L}$, and side effects were mild and sparse (van Norden et al., 2005). A post-hoc analysis suggested that the treatment effect of magnesium is larger after endovascular occlusion than after neurosurgical clipping, which underlines the conclusion that further trials are not at risk of being underpowered if the proportion of endovascular treated patients increases (van den Bergh et al., 2009).

In 2010, the results of the Intravenous Magnesium Sulphate in Aneurysmal Subarachnoid Haemorrhage (IMASH) trial were published (Wong et al., 2010b). IMASH was a phase III randomized, clinical, international multicentre trial that evaluated the effect of magnesium sulphate on the clinical outcome of 327 patients with aneurysmal SAH, of which $90 \%$ were recruited in Hong Kong and China and the remaining 10\% in Australia from 2002 to 2008. After randomization, $20 \mathrm{mmol}$ of magnesium sulphate was given over 30 minutes, followed by infusion of $80 \mathrm{mmol} / \mathrm{day}$, or equivalent volume of saline, within $48 \mathrm{~h}$ after onset of symptoms and continued for up to 14 days from the day of haemorrhage. Mean time from ictus to start of study drug infusion was $32 \pm 15$ (SD) hours. More than $90 \%$ of the patients completed at least 10 days of study drug infusion. The randomization was single-blinded. The study aimed for a plasma magnesium concentration in the treatment group of twice the serum baseline level, but below 2.5 $\mathrm{mmol} / \mathrm{L}$. Average serum magnesium concentrations in the treatment group were 1.67 $\pm 0.27 \mathrm{mmol} / \mathrm{L}$ compared with $0.91 \pm 0.16$ $\mathrm{mmol} / \mathrm{L}$ in controls.

The proportion of patients with a 6-month favourable outcome, defined as an extended Glasgow Outcome Scale of 5 to 8, were similar for both treatment groups (OR 1.0; $95 \% \mathrm{Cl} 0.7-1.6$ ). Also in the secondary outcome analyses, which included incidence of clinical vasospasm, Barthel Index, modified Rankin score, modified National 
Institute of Health Stroke Score, and MCA velocities as measured by transcranial Doppler, there were no significant differences between the 2 groups.

Although the study was underpowered, the results do not support a significant clinical benefit of magnesium therapy in SAH. Possible explanations for the differences in results between MASH-1 and IMASH are the assumed predominant Asian origin of recruited patients and the time window of administration; too late or too short. In the MASH-1 study the median start of treatment was 28 hours, and it was continued for at least 2 weeks. On the other hand, the achieved serum magnesium concentration might have been too high, as in the treatment group higher magnesium concentrations were associated with a worse outcome (Wong et al., 2010c). Furthermore, the average serum magnesium concentration of $0.91 \pm 0.16$ $\mathrm{mmol} / \mathrm{L}$ in the control group was quite high and could have diluted the results.

\section{Is there an optimal serum magnesium concentration for neuroprotection?}

In animal models of stroke, evidence was found for a dose-response effect, with optimal serum magnesium concentrations of approximately 1.40 to $1.50 \mathrm{mmol} / \mathrm{l}$ (Marinov et al., 1996; Yang et al., 2000; Miles et al., 2001).

The mean serum magnesium level of 1.47 $\mathrm{mmol} / \mathrm{L}$ achieved in the MASH-1 study was close to the proposed optimal serum magnesium level for achieving maximal neuroprotection. In a substudy performed in patients with a serum magnesium concentration $\geq 1.10 \mathrm{mmol} / \mathrm{L}$, we found no linear relationship between serum magnesium levels and risk reduction for $\mathrm{DCl}$ (Dorhout Mees et al., 2007a). Magnesium sulphate therapy results in a stable risk reduction of $\mathrm{DCl}$ over a broad range of achieved serum magnesium concentrations, and strict titration of the dosage therefore does not seem necessary, although concentrations below $1.28 \mathrm{mmol} / \mathrm{l}$ could decrease the effect on DCI. In contrast, no such relationship was observed for poor outcome. Risks tended to be higher in patients with magnesium concentrations above the 75th percentile $(1.62 \mathrm{mmol} / \mathrm{L})$ with an OR for poor outcome of $4.9(95 \% \mathrm{Cl} 1.2$ to 19.7$)$ in patients with a serum concentration above $1.62 \mathrm{mmol} / \mathrm{L}$ compared to the lowest quartile (1.10-1.28 $\mathrm{mmol} / \mathrm{L})$.

The aim of the IMASH study to achieve a plasma magnesium concentration of twice the serum baseline level resulted in an average serum magnesium concentration in the treatment group of $1.67 \pm 0.27 \mathrm{mmol} / \mathrm{L}$, which might have had a negative effect on clinical outcome. Of course, an optimal neuroprotective concentration of serum magnesium can only be validated if current large trials show that magnesium therapy is effective.

\section{Systematic review and meta-analysis}

We assessed in a systematic review whether magnesium decreases the occurrence of secondary ischemia and poor outcome after aneurysmal SAH. We sought to identify all unconfounded clinical controlled trials with magnesium in patients with aneurysmal SAH. Trials were identified in the Stroke Group Trials Register of the Cochrane Library, PUBMED and MEDLINE. Outcome measures were $\mathrm{DCl}$ and poor outcome. An estimate of the treatment effect across trials was calculated with the MantelHaenszel method according to the intention-totreat principle.

Between 2002 and 2010, four non-randomized and eight randomized controlled trials on magnesium were published, all but one comparing magnesium therapy with placebo in addition to nimodipine. The exception was a small randomized controlled trial in 104 patients that found similar outcomes in patients treated with nimodipine versus patients treated with magnesium (Schmid-Elsaesser et al., 2006). When the four non-randomized studies are combined, 118 patients with magnesium therapy were compared with a historical control group of 138 patients (Chia et al., 2002; Prevedello et al., 2006; Stippler et al., 2006; Friedlich et al., 2009). When the results are pooled, it all boils down to the conclusion that a trend towards less symptomatic vasospasm was found in favour of the treatment group and, if provided, a positive effect with a less extent on outcome.

Seven trials totalling 954 patients were eligible for the meta-analyses [Table 1] (Veyna et al., 2002; van den Bergh et al., 2005; Wong et al., 2006; Muroi et al., 2008; Westermaier et al., 2010; Akdemir et al., 2010; Wong et al., 2010b). 
Table 1: Characteristics and results of randomized control trials of magnesium in aneurysmal subarachnoid haemorrhage.

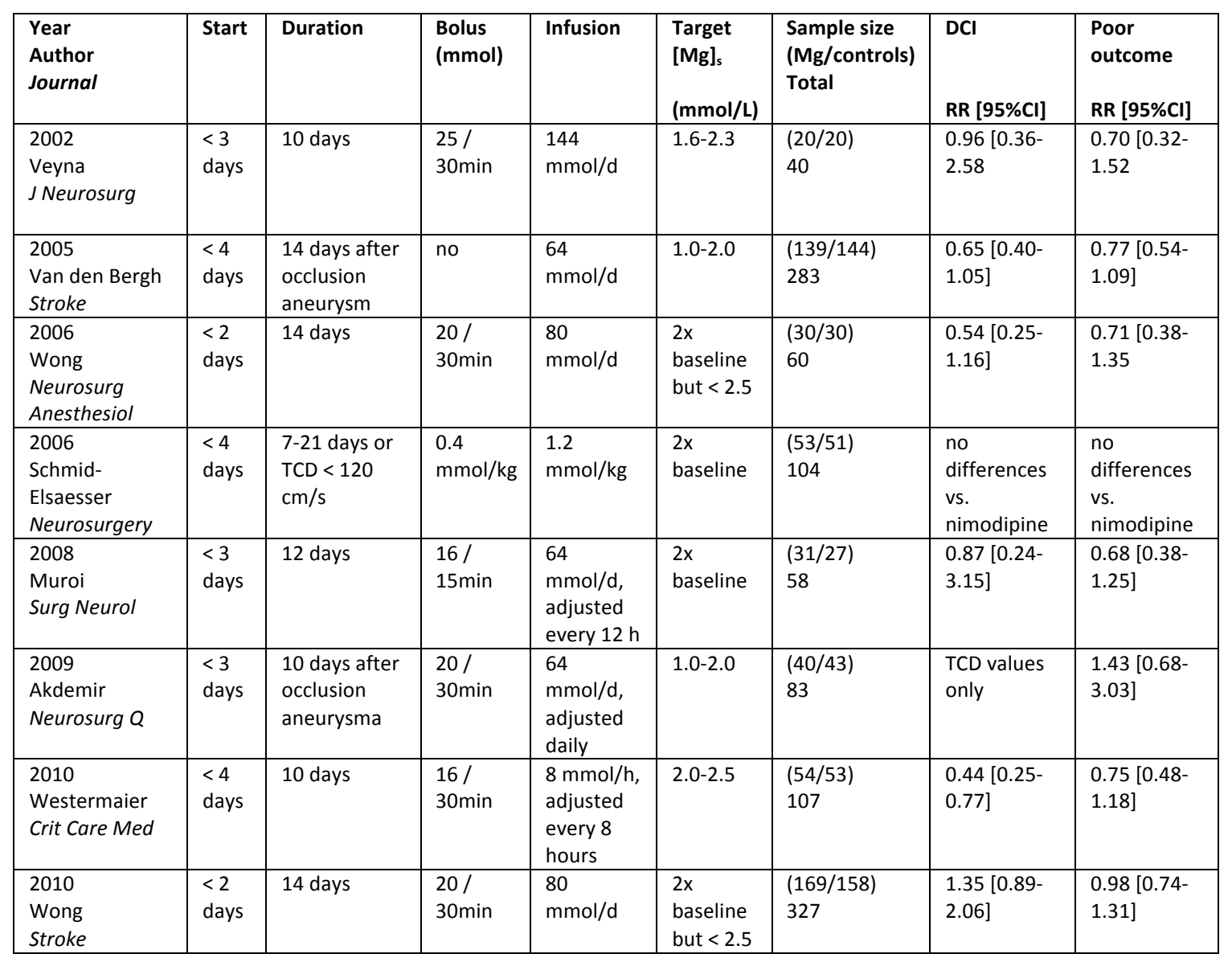

All used magnesium sulphate in addition to nimodipine. The overall relative risk for $\mathrm{DCl}$ was 0.86 (95\% Cl 0.67-1.11) (Figure 1), and for poor outcome 0.89 (95\% Cl 0.78-1.02) (Figure 2).

\section{Headache}

In patients with $\mathrm{SAH}$, headache typically is severe, usually lasts for 1-2 weeks and often requires treatment with opioids. Opioids, however, have a wide range of potential side effects, which may have a negative effect on patient comfort and functional outcome. Blocking the NMDA receptor is thought to be involved in pain modulation by preventing the induction of central pain sensitization. Several studies have reported that magnesium reduces perioperative pain or analgesic requirements. Headache relief would be an important additional effect of magnesium because this would reduce the need of opioids with their potentially harmful side effects. In a sub-study of the MASH-1 trial we found that magnesium treatment was associated with less severe headache and less frequent use of opioids (Dorhout Mees et al., 2010). These data imply that intravenous magnesium therapy, besides a supposed beneficial effect on outcome, also provides pain relief for SAH patients.

\section{On-going phase 3 clinical controlled trials}

There is currently one on-going phase 3 clinical controlled trial evaluating the effect of magnesium in SAH. The Magnesium in Aneurysmal Subarachnoid Haemorrhage (MASHII) study is a phase III randomized, clinical, international multicentre trial that studies the effect of magnesium sulphate after aneurysmal SAH [ISRCTN68742385] (van der Bilt et al., 2009). Study medication $(64 \mathrm{mmol} /$ day magnesium sulphate or placebo) is given via continuous infusion within 4 days and until 20 days after the haemorrhage. Outcome is determined with the modified Rankin scale three months after the haemorrhage. Analysis will be according to the intention-to-treat principle. So far, in August 2010, 
Figure 1. Studies with magnesium in addition to nimodipine - effect on delayed cerebral ischemia.

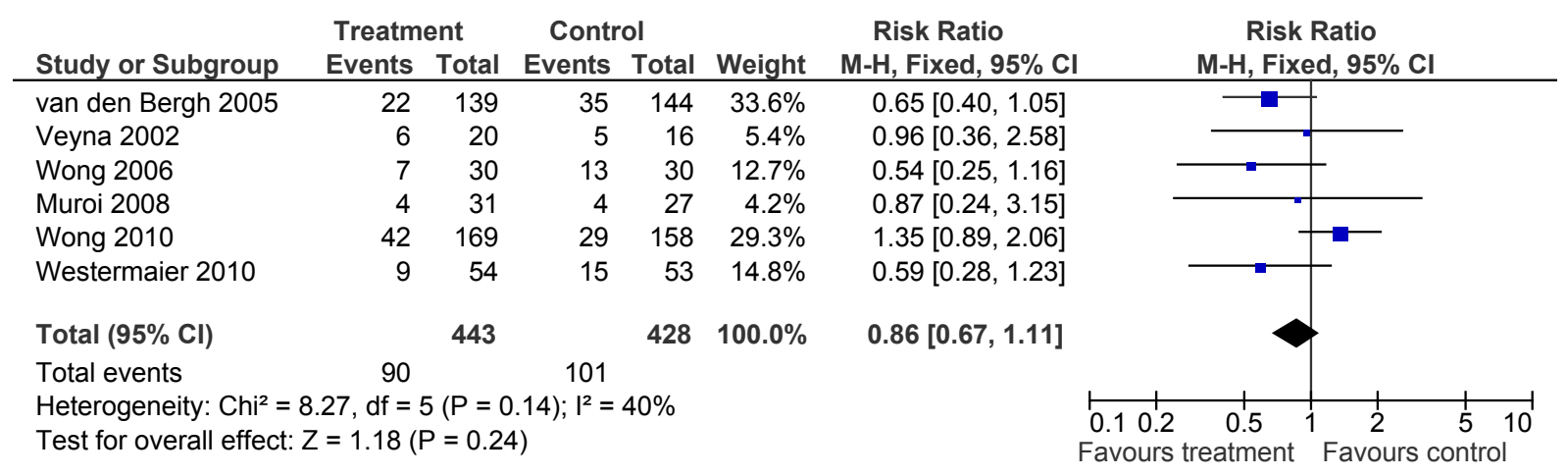

Figure 2. Studies with magnesium in addition to nimodipine - effect on poor outcome.

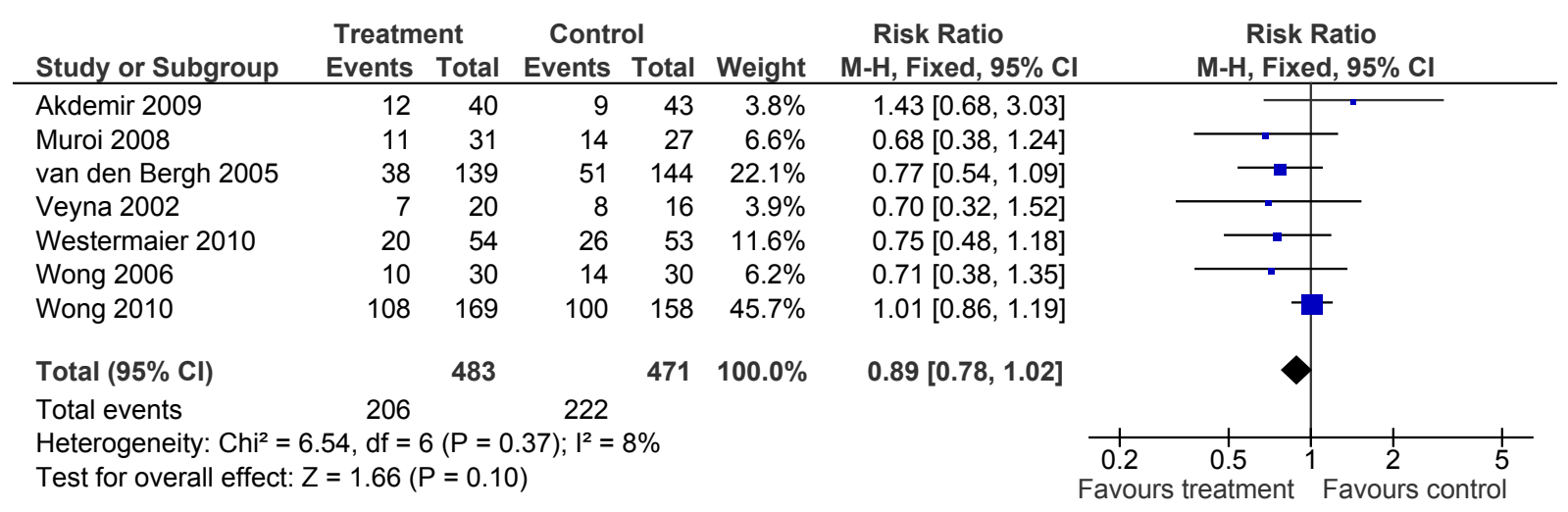

over 1000 patients have been included in 6 Dutch, 1 UK, and 1 Chilean hospital. Based on the results of the MASH-1 study sample size, calculations indicate that 1200 patients are needed to give a statistically significant result (with $\alpha=5 \%$ and a power of $80 \%$ ). We aim to include these patients before the end of 2011. A first interim analysis was performed after 300 patients and the second and last interim analysis was performed after recruitment and follow-up of 750 patients. At both interim analyses, the Data Monitoring Committee gave unanimous advice to continue the study.

\section{Conclusion}

In conclusion, magnesium is a promising agent to prevent the occurrence of delayed cerebral ischemia and to improve outcome in patients with SAH. Hence, at present, the evidence is insufficient and larger randomized controlled trials are needed to determine the safety and efficacy of magnesium sulphate infusion before it can be recommended for patients with aneurysmal SAH. Currently, a large phase III trial is being conducted that will hopefully provide definite evidence whether magnesium treatment is beneficial in SAH patients.

In the meantime, it is probably wise to maintain serum magnesium in the high-normal range, e.g. 0.8-1.0 $\mathrm{mmol} / \mathrm{l}$, after SAH to prevent the hypomagnesaemia associated neurological and cardiac complications. Magnesium therapy can be considered as a safe alternative for morphine in the treatment of headache. In case magnesium therapy is considered as a remedy to improve outcome, target serum magnesium levels are probably around $1.4 \mathrm{mmol} / \mathrm{l}$ and better not exceed $1.6 \mathrm{mmol} / \mathrm{l}$. 


\section{Acknowledgements}

The Netherlands Heart Foundation (grants 99.107 and 2005B016) and the Dutch Brain Foundation (grants 8F00(2).26 and 15F07(2)) are gratefully acknowledged for their financial support for some of the studies mentioned in this overview. Dr Sanne Dorhout Mees is gratefully acknowledged for her assistance with the meta-analysis.

\section{References}

Akdemir H, Kulaksizoglu EO, Tucer B, Menku A, Postalci L, Gunaldi O (2010) Magnesium sulphate therapy for cerebral vasospasm after aneurysmal subarachnoid haemorrhage. Neurosurg Q 19:35-9.

Altura BT, Altura BM (1984) Interactions of Mg and K on cerebral vessels--aspects in view of stroke. Review of present status and new findings. Magnesium 3:195211.

Babinski J (1893) Contractures organique et hystérique. Bull Soc Méd Hôp 3:327-43.

Barbour RL, Gebrewold A, Altura BT, Altura BM (2002) Optical spectroscopy and prevention of deleterious cerebral vascular effects of ethanol by magnesium ions. Eur J Pharmacol 447:79-86.

Berthon N, Laurant P, Fellmann D, Berthelot A (2003) Effect of magnesium on mRNA expression and production of endothelin-1 in DOCA-salt hypertensive rats. J Cardiovasc Pharmacol 42:24-31.

Brilstra EH, Rinkel GJ, Algra A, van Gijn J (2000) Rebleeding, secondary ischemia, and timing of operation in patients with subarachnoid haemorrhage. Neurology 55:1656-60.

Chia RY, Hughes RS, Morgan MK (2002) Magnesium: a useful adjunct in the prevention of cerebral vasospasm following aneurysmal subarachnoid haemorrhage. J Clin Neurosci 9:279-81.

Clarke E (1963) Apoplexy in Hippocratic writings. Bull Hist Med 37:301.

Dorhout Mees SM, Bertens D, van der Worp HB, Rinkel GJ, van den Bergh WM (2010) Magnesium and headache after aneurysmal subarachnoid haemorrhage. J Neurol Neurosurg Psychiatry 81:490-3.

Dorhout Mees SM, Rinkel GJ, Feigin VL, Algra A, van den Bergh WM, Vermeulen M, van Gijn J (2008) Calcium antagonists for aneurysmal subarachnoid hemorrhage. Stroke 39:514-5.
Dorhout Mees SM, van den Bergh WM, Algra A, Rinkel GJ (2007a) Achieved serum magnesium concentrations and occurrence of delayed cerebral ischaemia and poor outcome in aneurysmal subarachnoid haemorrhage. J Neurol Neurosurg Psychiatry 78:72931.

Dorhout Mees SM, van den Bergh WM, Algra A, Rinkel GJ (2007b) Antiplatelet therapy for aneurysmal subarachnoid haemorrhage. Cochrane Database Syst Rev CD006184.

Dorsch NW (1995) Cerebral arterial spasm - a clinical review. BrJ Neurosurg 9:403-12.

Dumont AS, Dumont RJ, Chow MM, Lin CL, Calisaneller T, Ley KF, Kassell NF, Lee KS (2003) Cerebral vasospasm after subarachnoid haemorrhage: putative role of inflammation. Neurosurgery 53:123-33.

Feigin VL, Rinkel GJ, Lawes CM, Algra A, Bennett DA, van GJ, Anderson CS (2005) Risk factors for subarachnoid haemorrhage: an updated systematic review of epidemiological studies. Stroke 36:2773-80.

Friedlich D, Agner C, Boulos AS, Mesfin F, Feustel P, Bernardini GL, Popp AJ (2009) Retrospective analysis of parenteral magnesium sulphate administration in decreased incidence of clinical and neuroradiological cerebral vasospasm: a single center experience. Neurol Res 31:621-5.

Hippocrates:, Leonicenus N, Laurentianus L (1527) (trans.) Aphorismi, cum Galeni Commentariis; Praedictiones, cum Galeni Commentariis, Paris: Simon Sylvius.

Hop JW, Rinkel GJ, Algra A, van Gijn J (1997) Casefatality rates and functional outcome after subarachnoid haemorrhage: a systematic review. Stroke 28:660-4.

Izumi Y, Roussel S, Pinard E, Seylaz J (1991) Reduction of infarct volume by magnesium after middle cerebral artery occlusion in rats. $J$ Cereb Blood Flow Metab 11:1025-30. 
Jung DW, Apel L, Brierley GP (1990) Matrix free Mg2+ changes with metabolic state in isolated heart mitochondria. Biochemistry 29:4121-8.

Kemp PA, Gardiner SM, March JE, Rubin PC, Bennett T (1999) Assessment of the effects of endothelin-1 and magnesium sulphate on regional blood flows in conscious rats, by the coloured microsphere reference technique. Br J Pharmacol 126:621-6.

Kolias AG, Sen J, Belli A (2009) Pathogenesis of cerebral vasospasm following aneurysmal subarachnoid haemorrhage: putative mechanisms and novel approaches. J Neurosci Res 87:1-11.

Linn FH, Rinkel GJ, Algra A, van Gijn J (1996) Incidence of subarachnoid haemorrhage: role of region, year, and rate of computed tomography: a meta-analysis. Stroke 27:625-9.

Macdonald RL, Curry DJ, Aihara Y, Zhang ZD, Jahromi BS, Yassari R (2004) Magnesium and experimental vasospasm. J Neurosurg 100:106-10.

Marinov MB, Harbaugh KS, Hoopes PJ, Pikus HJ, Harbaugh RE (1996) Neuroprotective effects of preischemia intraarterial magnesium sulphate in reversible focal cerebral ischemia. J Neurosurg 85:11724.

McKee JA, Brewer RP, Macy GE, Phillips-Bute B, Campbell KA, Borel CO, Reynolds JD, Warner DS (2005) Analysis of the brain bioavailability of peripherally administered magnesium sulphate: A study in humans with acute brain injury undergoing prolonged induced hypermagnesemia. Crit Care Med 33:661-6.

Miles AN, Majda BT, Meloni BP, Knuckey NW (2001) Postischemic intravenous administration of magnesium sulphate inhibits hippocampal CA1 neuronal death after transient global ischemia in rats. Neurosurgery 49:1443-50.

Miura K (1988) [Changes in Mg++ concentration of CSF after subarachnoid haemorrhage and Mg++--effects on the contractions of bovine cerebral artery]. No Shinkei Geka 16:1251-9.

Molyneux A, Kerr R, Stratton I, Sandercock P, Clarke M, Shrimpton J, Holman R (2002) International Subarachnoid Aneurysm Trial (ISAT) of neurosurgical clipping versus endovascular coiling in 2143 patients with ruptured intracranial aneurysms: a randomised trial. Lancet 360:1267-74.

Moniz E (1927) L'encéphalographie artérielle, son importance dans la localisation des tumeurs cérébrales. Rev Neurol (Paris) 48:72-90.
Moniz E (1933) Anévrysme intra-cranien de la carotide interne droite rendu visible par l'artériographie cérébrale. Rev Oto-Neuro-Ophthal 11:198-203.

Muir KW (1998) New experimental and clinical data on the efficacy of pharmacological magnesium infusions in cerebral infarcts. Magnes Res 11:43-56.

Muir KW, Lees KR, Ford I, Davis S (2004) Magnesium for acute stroke (Intravenous Magnesium Efficacy in Stroke trial): randomised controlled trial. Lancet 363:439-45.

Muroi C, Terzic A, Fortunati M, Yonekawa Y, Keller E (2008) Magnesium sulfate in the management of patients with aneurysmal subarachnoid haemorrhage: a randomized, placebo-controlled, dose-adapted trial. Surg Neurol 69:33-9.

Nieuwkamp DJ, Setz LE, Algra A, Linn FH, de Rooij NK, Rinkel GJ (2009) Changes in case fatality of aneurysmal subarachnoid haemorrhage over time, according to age, sex, and region: a meta-analysis. Lancet Neurol 8:635-42.

Pearson PJ, Evora PR, Seccombe JF, Schaff HV (1998) Hypomagnesemia inhibits nitric oxide release from coronary endothelium: protective role of magnesium infusion after cardiac operations. Ann Thorac Surg 65:967-72.

Pluta RM, Afshar JK, Boock RJ, Oldfield EH (1998) Temporal changes in perivascular concentrations of oxyhemoglobin, deoxyhemoglobin, and methemoglobin after subarachnoid haemorrhage. $J$ Neurosurg 88:557-61.

Prevedello DM, Cordeiro JG, de Morais AL, Saucedo NS, Jr., Chen IB, Araujo JC (2006) Magnesium sulfate: role as possible attenuating factor in vasospasm morbidity. Surg Neurol 65 Suppl 1:S1.

Pyne GJ, Cadoux-Hudson TA, Clark JF (2001) Magnesium protection against in vitro cerebral vasospasm after subarachnoid haemorrhage. $\mathrm{Br} \mathrm{J}$ Neurosurg 15:409-15.

Rabinstein AA, Friedman JA, Weigand SD, McClelland RL, Fulgham JR, Manno EM, Atkinson JL, Wijdicks EF (2004) Predictors of cerebral infarction in aneurysmal subarachnoid haemorrhage. Stroke 35:1862-6.

Ram Z, Sadeh M, Shacked I, Sahar A, Hadani M (1991) Magnesium sulfate reverses experimental delayed cerebral vasospasm after subarachnoid haemorrhage in rats. Stroke 22:922-7. 
Roos YB, de Haan RJ, Beenen LF, Groen RJ, Albrecht KW, Vermeulen M (2000) Complications and outcome in patients with aneurysmal subarachnoid haemorrhage: a prospective hospital based cohort study in the Netherlands. J Neurol Neurosurg Psychiatry 68:337-41.

Saris NE, Mervaala E, Karppanen H, Khawaja JA, Lewenstam A (2000) Magnesium. An update on physiological, clinical and analytical aspects. Clin Chim Acta 294:1-26.

Saver JL, Kidwell C, Eckstein M, Starkman S (2004) Prehospital neuroprotective therapy for acute stroke: results of the Field Administration of Stroke TherapyMagnesium (FAST-MAG) pilot trial. Stroke 35:e106-8.

Schmid-Elsaesser R, Kunz M, Zausinger S, Prueckner S, Briegel J, Steiger HJ (2006) Intravenous magnesium versus nimodipine in the treatment of patients with aneurysmal subarachnoid haemorrhage: a randomized study. Neurosurgery 58:1054-65.

Seelig JM, Wei EP, Kontos HA, Choi SC, Becker DP (1983) Effect of changes in magnesium ion concentration on cat cerebral arterioles. Am J Physiol 245:H22-6.

Shechter M (2000) The role of magnesium as antithrombotic therapy. Wien Med Wochenschr 150:343-7.

Stippler M, Crago E, Levy El, Kerr ME, Yonas H, Horowitz MB, Kassam A (2006) Magnesium infusion for vasospasm prophylaxis after subarachnoid haemorrhage. J Neurosurg 105:723-9.

Torregrosa G, Perales AJ, Salom JB, Miranda FJ, Barbera MD, Alborch E (1994) Different effects of $\mathrm{Mg} 2+$ on endothelin-1- and 5-hydroxytryptamineelicited responses in goat cerebrovascular bed. J Cardiovasc Pharmacol 23:1004-10.

van den Bergh WM, Algra A, Rinkel GJ (2004a) Electrocardiographic abnormalities and serum magnesium in patients with subarachnoid haemorrhage. Stroke 35:644-8.

van den Bergh WM, Algra A, Rinkel GJ (2009) Magnesium and aspirin treatment in patients with subarachnoid haemorrhage: comparison of effects after endovascular and neurosurgical aneurysm occlusion. J Neurol 256:213-6.

van den Bergh WM, Algra A, van der Sprenkel JW, Tulleken CA, Rinkel GJ (2003) Hypomagnesemia after aneurysmal subarachnoid haemorrhage. Neurosurgery 52:276-82. van den Bergh WM, Algra A, van Kooten F, Dirven CM, van Gijn J, Vermeulen M, Rinkel GJ (2005) Magnesium sulfate in aneurysmal subarachnoid haemorrhage: a randomized controlled trial. Stroke 36:1011-5.

van den Bergh WM, Dijkhuizen RM, Rinkel GJ (2004b) Potentials of magnesium treatment in subarachnoid haemorrhage. Magnes Res 17:301-13.

van den Bergh WM, Van de Water JM, Hoff RG, Algra A, Rinkel GJ (2008b) Calcium homeostasis during magnesium treatment in aneurysmal subarachnoid hemorrhage. Neurocrit Care 8:413-7.

van den Bergh WM, Zuur JK, Kamerling NA, van Asseldonk JT, Rinkel GJ, Tulleken CA, Nicolay K (2002) Role of magnesium in the reduction of ischemic depolarization and lesion volume after experimental subarachnoid haemorrhage. J Neurosurg 97:416-22.

van der Bilt I, Hasan D, Vandertop WP, Wilde AA, Algra A, Visser FC, Rinkel GJ (2009) Impact of cardiac complications on outcome after aneurysmal subarachnoid haemorrhage: a meta-analysis. Neurology 72:635-42.

van der Hel WS, van den Bergh WM, Nicolay K, Tulleken KA, Dijkhuizen RM (1998) Suppression of cortical spreading depressions after magnesium treatment in the rat. Neuroreport 9:2179-82.

van Gijn J, Kerr RS, Rinkel GJ (2007) Subarachnoid haemorrhage. Lancet 369:306-18.

van Norden AG, van den Bergh WM, Rinkel GJ (2005) Dose evaluation for long-term magnesium treatment in aneurysmal subarachnoid haemorrhage. J Clin Pharm Ther 30:439-42.

Veyna RS, Seyfried D, Burke DG, Zimmerman C, Mlynarek M, Nichols V, Marrocco A, Thomas AJ, Mitsias PD, Malik GM (2002) Magnesium sulfate therapy after aneurysmal subarachnoid haemorrhage. J Neurosurg 96:510-14.

Warren BB, Muizelaar JP, Choi SC (1993) Magnesium's role in cerebral vasospasm and outcome in patients with aneurysmal subarachnoid haemorrhage, In: Cerebral Vasospasm (Findlay JM, ed), pp 401-404.

Weir B, Macdonald RL, Stoodley M (1999) Etiology of cerebral vasospasm. Acta Neurochir Suppl (Wien) 72:27-46. 
Westermaier T, Stetter C, Vince GH, Pham M, Tejon JP, Eriskat J, Kunze E, Matthies C, Ernestus RI, Solymosi L, Roosen K (2010) Prophylactic intravenous magnesium sulfate for treatment of aneurysmal subarachnoid haemorrhage: a randomized, placebo-controlled, clinical study. Crit Care Med 38:1284-90.

Wong GK, Chan MT, Boet R, Poon WS, Gin T (2006) Intravenous magnesium sulfate after aneurysmal subarachnoid haemorrhage: a prospective randomized pilot study. J Neurosurg Anesthesiol 18:142-8.

Wong GK, Kwok R, Tang K, Yeung D, Ahuja A, King AD, Poon WS (2010a) Effects of magnesium sulfate infusion on cerebral perfusion in patients after aneurysmal SAH. Acta Neurochir Suppl 106:133-5.

Wong GK, Poon WS, Chan MT, Boet R, Gin T, Ng SC, Zee BC (2010b) Intravenous magnesium sulphate for aneurysmal subarachnoid haemorrhage (IMASH): a randomized, double-blinded, placebo-controlled, multicenter phase III trial. Stroke 41:921-6.
Wong GK, Poon WS, Chan MT, Boet R, Gin T, Ng SC, Zee BC (2010c) Plasma magnesium concentrations and clinical outcomes in aneurysmal subarachnoid haemorrhage patients: post hoc analysis of intravenous magnesium sulphate for aneurysmal subarachnoid haemorrhage trial. Stroke 41:1841-14.

Wong GK, Yeung DK, Ahuja AT, King AD, Lam CW, Chan MT, Gin T, Poon WS (2009) Intracellular free magnesium of brain and cerebral phosphoruscontaining metabolites after subarachnoid haemorrhage and hypermagnesemic treatment: a (31)P-magnetic resonance spectroscopy study. J Neurosurg 113:763-9.

Yang Y, Li Q, Ahmad F, Shuaib A (2000) Survival and histological evaluation of therapeutic window of postischemia treatment with magnesium sulfate in embolic stroke model of rat. Neurosci Lett 285:119-22. 



\title{
Magnesium in clinical stroke
}

\author{
Jeffrey L. Saver ${ }^{\bowtie}$ and Sidney Starkman \\ UCLA Stroke Center and Department of Neurology, University of California, Los Angeles, CA 90095, USA. \\ $\triangle$ jsaver@ucla.edu
}

\begin{abstract}
Stroke is the second leading cause of death and a leading cause of disability worldwide. Unfortunately, current therapies for acute ischemic stroke are of extremely limited effectiveness. The ideal neuroprotective agent for stroke would be inexpensive, readily available, easy to administer and have no significant adverse side effects. Intravenous magnesium sulphate offers promise as just such an agent. In clinical trials to date, administration of magnesium sulphate has shown favourable trends toward neuroprotection in resuscitated cardiac arrest patients, in cardiac bypass surgery and carotid endarterectomy. In acute stroke, eight pilot clinical trials of magnesium sulphate have shown good safety, and the subset of pilot trials with randomized controls have shown favourable effects on clinical outcome point estimates. The first phase 3 trial of magnesium sulphate for focal stroke was the Intravenous Magnesium Efficacy in Stroke (IMAGES) Trial. Hampered by late administration of the study agent, IMAGES overall produced a neutral result, but exploratory analyses identified early (under 3 hour), lacunar stroke, haemorrhagic stroke, and severely hypertensive patients as having a potential differential beneficial response to magnesium that deserved further investigation. The Field Administration of Stroke Therapy - Magnesium (FAST-MAG) trial has been specifically designed to test magnesium in the hyperacute time window by initiating therapy in the prehospital setting.
\end{abstract}

\section{Introduction}

Stroke is the second leading cause of death and a leading cause of disability worldwide (Lopez et al., 2006). More than 5.3 million individuals die from a stroke each year, and many more survive with permanent incapacities. One in six individuals worldwide will suffer a stroke in their lifetime (Kaste et al., 2010). In Western nations, ischemic cerebral infarctions account for about $80 \%$ of strokes, intracerebral haemorrhages $15 \%$, and subarachnoid haemorrhages $4 \%$.

Unfortunately, current therapies for acute ischemic stroke are of extremely limited effectiveness. To date, the only United States Food and Drug Administration (FDA)-approved pharmacologic treatment for acute ischemic stroke remains intravenous tissue plasminogen activator (tPA), a thrombolytic agent which must be administered within 3 hours of symptom onset, and only after neuroimaging has ruled out intracerebral haemorrhage (Adams et al., 2007). tPA carries significant bleeding risks, is costly, and is not being used in most acute stroke patients. Current estimates are that only $3-8 \%$ of acute ischemic stroke patients in the US receive IPA
(California Acute Stroke Pilot Registry (CASPR) Investigators, 2005; Reeves et al., 2005). The only other pharmacologic agent of proven utility in acute ischemic stroke is aspirin, which confers only minimal benefit, helping only one of every 77 patients treated (Sandercock et al., 2008). Mechanical embolectomy devices have been developed and approved for the technical indication of removing acute thrombi from the cerebral vasculature, but have not yet been demonstrated in a randomized clinical trial to improve patient outcome and are employed in only a tiny fraction of stroke patients nationally (Smith et al., 2008; The Penumbra Pivotal Stroke Trial Investigators, 2009). New, effective, widely applicable treatments for acute ischemic stroke are desperately needed.

\section{Magnesium sulphate: a highly promising} neuroprotective therapy for stroke

When focal occlusions disrupt blood flow to the brain, a cascade of molecular events producing cell injury ensues (Schaller and Graf, 2004; Obrenovitch, 1995). Cell death proceeds rapidly in the infarct core, where blood flow is most drastically curtailed, but more slowly in the 
ischemic penumbra, where blood flow is variably reduced and molecular elaboration of neuronal injury may proceed over 3-12 hours or more. Central molecular events in the ischemic cascade include accumulation of intracellular calcium, release of excitatory amino acid neurotransmitters, generation of oxygen free radicals, nitric oxide formation, and the release of cytokines by infiltrating polymorphonuclear leukocytes. These and additional events afford numerous targets for pharmacologic blockade. A multitude of neuroprotective drugs interfering with various pathways of ischemic injury substantially reduce infarct volume in focal stroke animal models when administered 10-120 minutes after ischemia onset, including excitatory amino acid antagonists, oxygen free radical scavengers, and voltage sensitive calcium channel blockers (Green and Shuaib, 2006).

The ideal neuroprotective agent for stroke would be inexpensive, readily available, easy to administer and have no significant adverse side effects. An agent demonstrated to be safe and potentially beneficial in both ischemic and haemorrhagic stroke would have the added benefit of potentially earlier administration prior to obtaining a head CT or brain MRI scan. Intravenous magnesium sulphate offers promise as just such an agent.

Laboratory studies have identified multiple neuronal and vascular effects of magnesium that likely contribute to the potent neuroprotective effects observed in stroke models (Muir, 2000). In a wide variety of in vitro systems and animal models, magnesium has been shown to produce cerebral arteriolar vasodilation and increased cerebral blood flow, inhibit presynaptic release of excitatory neurotransmitters, noncompetitively block the N-methyl-D-aspartate (NMDA) receptor, presynaptically potentiate adenosine, block voltage-gated calcium channels, suppress cortical spreading depression and anoxic depolarizations, relax vascular smooth muscle resulting in vasodilation of large and small vascular beds and increased cerebral blood flow, antagonize endothelin-1 and other vasoconstrictors, and replete an underlying and/or ischemia-induced Mg deficient state. Magnesium ions cross the intact blood-brain barrier efficaciously so that intravenous magnesium sulphate significantly raises cerebrospinal fluid and brain extracellular fluid magnesium to supraphysiologic levels (Thurnau et al., 1987; Wester et al., 1984). The brain penetration of magnesium may increase even further in ischemic regions where the blood-brain barrier is disrupted (Sjostrom and Wester, 1994). Magnesium sulphate is neuroprotective in preclinical models of cerebral and spinal cord ischemia, excitotoxic injury, and head trauma. Magnesium (Mg) is economical, widely available, simple to administer and has a long established safety and tolerability profile in myocardial infarction and eclampsia, as well as in initial human focal stroke studies. Unlike most synthetic neuroprotective compounds, parenteral magnesium has no major adverse effects in doses that achieve serum levels in the range of preclinical neuroprotective concentrations.

Magnesium is neuroprotective in rodent models of reversible and permanent focal cerebral ischemia. In contrast to commercially-supported neuroprotective compounds, magnesium has not been the subject of a systematic preclinical development program. However, academic investigations have demonstrated benefits in diverse models and laboratories, satisfying a key criterion of stroke drug development guidelines often not met by commercially-supported agents (Stroke Therapy Academic Industry Roundtable (STAIR), 1999). At least nine preclinical studies (eight reported) have examined the effect of systemic magnesium sulphate upon final infarct size in animal focal ischemic stroke models (Muir, 2000 ; 2001). Eight of the nine demonstrated substantial decreases in infarct size in treated animals, with reductions ranging from $26-61 \%$ in unconfounded studies. A recent study demonstrated synergistic effects of magnesium when combined with antioxidant and hypothermic therapies in a rat MCAO stroke model, suggesting that magnesium is a promising first agent upon which to build a combination neuroprotection regimen (Scholler et al., 2004).

\section{Clinical trials of magnesium sulphate in diverse settings of acute cerebral ischemia}

Uniquely among neuroprotective agents being developed for focal stroke, magnesium sulphate has shown signals of potential efficacy in numerous controlled clinical trials in closely allied settings of acute human cerebral ischemia, 
including 1) delayed cerebral infarction after subarachnoid haemorrhage, 2) newborn hypoxicischemic injury, 3) global cerebral ischemia after cardiac arrest, and 4) ischemic brain injury during cardiac and cerebrovascular surgical procedures. For detailed review of the first setting, the reader is referred to Chapter 14 in this volume. We here briefly survey the clinical trial experience with the latter two conditions.

\section{Global cerebral ischemia after cardiac arrest}

Paramedic, field-administration of magnesium sulphate showed favourable trends toward neuroprotection in resuscitated cardiac arrest patients in the Brain-Cardiopulmonary Resuscitation (B-CPR) trial (Longstreth et al., 2002). The B-CPR trial tested magnesium sulphate and diazepam in a $2 \times 2$ factorial, placebo-controlled design. Paramedic personnel injected study medications to out-of-hospital cardiac arrest patients immediately following return of spontaneous circulation. Three hundred patients were randomized to intravenous magnesium sulphate $(2 \mathrm{~g})$ or matching placebo, and then to diazepam (10 mg) or matching placebo. The pre-specified primary outcome measure was awakening, defined as having comprehensible speech or following commands. The study was powered to detect only a substantial treatment effect - absolute difference in the primary endpoint of $20 \%$. The magnesium sulphate group showed a trend toward efficacy, with $46.7 \%$ awakening among those who received only magnesium versus $37.3 \%$ among those who received only placebo (risk difference $9.3 \%, \mathrm{Cl}-6.4$ to $25.1 \%$ ). In contrast, the diazepam group showed no evidence of treatment effect. No adverse effects of prehospital administration of magnesium sulphate therapy were noted

\section{Brain ischemic during surgical procedures}

The operative setting uniquely allows the administration of neuroprotective agents to occur prior to the onset of ischemia, when benefit will be greatest. Individual randomized controlled trials have suggested benefits of magnesium sulphate for neuroprotection in cardiac bypass surgery and carotid endarterectomy.

In on-pump, coronary artery bypass graft surgery, neuropsychological dysfunction occurs in up to $50-80 \%$ of patients at the time of discharge, related in part to intra-operative brain ischemia. The Cleveland Clinic trial randomized 350 patients undergoing elective cardiac bypass surgery to magnesium sulphate or placebo during the operation and for the first 24 hours postoperatively. Patients received a 2 g loading dose and subsequent titration of a continuous infusion to maintain serum magnesium levels at twice normal. Magnesium exerted a benefit on early cognitive function. On the study's primary endpoint of neurologic decline or neurologic death at 96 hours post-operatively, magnesium patients outperformed placebo $(p=0.01)$ (Bhudia et al., 2006). By 3 months post-operatively, there were no performance differences between groups, possibly reflecting a test ceiling effect as placebo patients improved.

In carotid endarterectomy, cerebral ischemia due to intraoperative hypoperfusion and microembolic events produces clinical deficits evident on detailed neurocognitive testing. Standardized neuropsychometric evaluation has demonstrated cognitive decline in up to $25 \%$ of patients undergoing carotid surgery. The Columbia University trial randomly assigned 108 patients undergoing carotid surgery to placebo infusion or one of three magnesium sulphate dose tiers (Mack et al., 2009). The highest magnesium dose tier was a $4 \mathrm{~g}$ loading dose followed by $16 \mathrm{~g}$ over the next 24 hours. On a neuropsychological test battery the day after surgery, patients treated with intravenous magnesium infusion demonstrated less postoperative neurocognitive impairment than those treated with placebo (OR $0.27,95 \% \mathrm{Cl} 0.10-0.74, \mathrm{p}=0.01$ ).

\section{Pilot clinical trials of magnesium sulfate in focal stroke}

There have been eight pilot clinical trials of magnesium sulphate in acute stroke, exploring dose optimization, feasibility, safety, and signals of efficacy. Among these, 6 were randomized and 2 uncontrolled. All showed good safety with each randomized controlled trial (RCT) showing favourable effects on clinical outcome point estimates (Wester et al., 1984; Muir and Lees, 1995; Muir and Lees, 1998; Galeas et al., 1998; Bradford et al., 1998; Lampl et al., 2001; Saver et al., 2002; Muir et al., 2004). A meta-analysis of the four phase 2 RCTs with combinable data (total of 162 patients) found a favourable clinical 
outcome trend, with late disability or death seen in $44.3 \%$ of magnesium sulphate patients compared with $52.7 \%$ of placebo patients (OR 0.67, 95\% Cl, 0.35-1.26) (Muir, 2001).

The pilot trial that optimized a dose regimen for subsequent phase 3, pivotal trials was the dose ranging study reported by Muir and Lees (1998). They sought a regimen that efficiently and safely achieved a doubling of the serum magnesium concentration. This target was selected as serum concentrations of $3.48 \mathrm{mEq} / \mathrm{L}$ and above had been neuroprotective in preclinical models of focal cerebral ischemia, and doubling of serum concentrations was known to be efficacious in the prophylaxis and treatment of seizures in preeclamptic and eclamptic women (the normal range for serum magnesium is $1.4-1.7 \mathrm{mEq} / \mathrm{L}$ ). They randomized 25 patients to placebo or one of three magnesium sulphate dose schedules: loading infusions of 8,12 , or $16 \mathrm{mmol}$, all followed by a maintenance infusion of $65 \mathrm{mmol}$ over 24 hours. All dose tiers were found safe, with no major effects on heart rate, blood pressure, blood glucose, or other physiologic variables. Serum magnesium concentrations rose to target levels most rapidly in the highest loading infusion group and were maintained in all groups for at least 24 hours. The highest loading dose infusion regimen was therefore identified as safe and most efficient, and taken forward to later phase clinical trials.

A distinctive pilot trial was undertaken to demonstrate the feasibility and safety of having paramedics initiate magnesium sulphate in the field, prior to hospital arrival. Magnesium sulphate's properties make it a particularly attractive agent to study in the prehospital setting (Table 1). In the Field Administration of Stroke Therapy - Magnesium (FAST-MAG) Pilot Trial, the primary prespecified feasibility trial endpoint was reduction in time interval from paramedic arrival on scene to start of study agent infusion, compared with a historical control group of ambulance-arriving patients enrolled in standard post-hospital arrival fashion in acute neuroprotective trials (Saver et al., 2004). Twenty patients were enrolled, all receiving active therapy of a $4 \mathrm{~g}$ loading dose initiated by paramedics in the field, followed by a $16 \mathrm{~g}$ maintenance infusion administered upon hospital arrival. Substantially more rapid initiation of neuroprotective therapy was found with field administration versus historic controls, with the mean interval from paramedic arrival on scene to initiation of study drug being 23 minutes in fieldinitiation patients vs 141 minutes in hospitalinitiated patients $(p<0.0001)$. Experimental neuroprotective therapy was started 2 hours earlier as a result of the strategy of prehospital initiation of therapy.

The pharmacologic efficacy of field administration in the FAST-MAG Pilot Trial was assessed by measuring the serum magnesium level 10 minutes after completion of the bolus loading dose. In the 19 patients completing the loading dose, post bolus serum magnesium levels were mean $3.6 \mathrm{mEq} / \mathrm{L}$ (range 2.4-5),

Table 1. Properties of Magnesium Sulphate Advantageous for Prehospital Study

\begin{tabular}{|lll|}
\hline \multicolumn{1}{|c|}{ Magnesium Sulphate Properties } & & \multicolumn{1}{c|}{ Import for Prehospital Trial } \\
\hline Stable at room temperature & $\bullet$ & Can be stored in rescue vehicles \\
\hline No major antithrombotic effects & $\bullet$ & Can be used in ischemic and haemorrhagic stroke \\
& - & May be started in ambulance prior to CT or MR scan \\
\hline $\begin{array}{l}\text { Loading intravenous dose quickly } \\
\text { achieves target blood levels }\end{array}$ & $\bullet$ & $\begin{array}{l}\text { Paramedics can efficiently initiate through standard } \\
\text { field intravenous line }\end{array}$ \\
\hline $\begin{array}{l}\text { Well-established safety record in } \\
\text { clinical practice }\end{array}$ & $\bullet$ & Extensive monitoring not required \\
\hline $\begin{array}{l}\text { Neuroprotective in multiple stroke } \\
\text { models }\end{array}$ & $\bullet$ & Promising agent for efficacy study \\
\hline
\end{tabular}


demonstrating successful achievement of target supraphysiologic, neuroprotective levels of magnesium sulphate in all patients completing the loading dose. These results of paramedic loading dose administration were comparable to those of standard in hospital loading dose administration in the dose-optimization clinical trial of Muir and Lees (1998).

Safety and exploratory efficacy endpoints in the FAST-MAG Pilot trial were encouraging. No drug related serious major adverse event was observed. Although conclusions derived from an open-label, small pilot trial must be extremely guarded, clinical efficacy suggested potential beneficial treatment effects. Dramatic early recovery, defined as complete recovery or improvement by 10 or more points on the National Institute of Health Stroke Scale (NIHSS) by 24 hours, occurred in $27 \%$ of cerebral infarct patients. This response compares quite favourably with rates of dramatic early recovery noted in the NINDS-tPA Trial 1 placebo group (12\%) and approximates the treatment effect of tPA in yielding dramatic early recovery in the NINDS-tPA Trial 1 (27\%) (Haley et al., 1997).

\section{IMAGES phase 3 trial}

The first phase 3 trial of magnesium sulphate for focal stroke was the Intravenous Magnesium Efficacy in Stroke (IMAGES) trial (Muir et al., 2004). Funded by the British Medical Research Council, IMAGES was an international trial that enrolled 2589 patients within 12 hours of onset to receive $4 \mathrm{~g}$ magnesium sulphate bolus followed by $16.25 \mathrm{~g}$ magnesium sulphate maintenance infusion over 24 hours or matched placebo.

The performance of IMAGES demonstrated a worldwide consensus that magnesium sulphate in focal human stroke shows robust promise and merits phase 3 trial testing. However, the design of IMAGES had a critical drawback common among trials of its era: a late enrollment window. IMAGES permitted enrollment of patients up to 12 hours after symptom onset, well after ischemic injury has already reached completion in many patients. Additionally, IMAGES required that a patient's deficit be persistent for at least one hour before enrollment could occur, precluding agent initiation within the first 60 minutes of onset in the trial.
The IMAGES trial overall had a neutral result, but trial data suggest that the nonpositive outcome may have been due to late administration of study agent. The primary efficacy endpoint in IMAGES was death and disability at 3 months, identified by the joint binary outcome of outcome of a Barthel score $<95$ and a modified Rankin score $>1$. On this endpoint, the point estimate favouring magnesium did not reach statistical significance $(\mathrm{OR} 0.95,95 \% \mathrm{Cl} 0.80$ 1.13). However, the vast majority of patients in IMAGES were enrolled late after stroke onset. Median time to treatment was 7 hours. The single hour with a plurality of enrolled patients was hour 12 . Only 71 patients (3.1\% of the entire IMAGES cohort) were enrolled within 3 hours of onset, only 16 patients (0.6\%) were enrolled within 2 hours, and no patients were enrolled within 1 hour.

IMAGES generally confirmed the safety of magnesium sulphate administration in acute stroke. No statistically significant differences were noted in time to first serious adverse event or to mortality. Nominal differences in mortality did not reach statistical significance (OR 1.22, $95 \% \mathrm{Cl}$ 0.98-1.53) and no individual causes of death were in excess in the magnesium group. Mild cardiovascular effects of magnesium were observed, as expected. Magnesium slightly reduced the blood pressure during the period of infusion, on average by 4 points systolic and 3 points diastolic. This reduction disappeared by 24 hous after the end of the infusion. Heart rate was minimally reduced at 12 hours into the infusion, but not at other time points. No increase in clinically reported hypotension, cardiac failure, cardiac conduction block, or flushing was reported $\mathrm{n}$ the magnesium group.

Exploratory analyses in IMAGES identified four patient subgroups with potential differential beneficial response to magnesium that deserve further investigation: 1) lacunar stroke, 2) haemorrhagic stroke, 3) severely hypertensive patients, and 4) early, under 3 hour, treated patients. Among 765 patients with lacunar syndromes, unfavourable outcomes were less common in the treatment group, OR 0.70 (0.530.92) (Aslanyan et al., 2007). This observation is intriguing in light of in vitro models indicating that magnesium has biological protective activities in white matter ischemia (Lecrux et al., 
2008). Among 1193 patients with initial blood pressures higher than the trial median, unfavourable outcomes also were less common in treated patients (OR 0.78, 0.61-0.99) (Muir et al., 2004). The optimal treatment of elevated blood pressure in acute stroke remains uncertain; it is possible that the slight antihypertensive action of magnesium sulphate was beneficial in these patients, in addition to neuroprotective actions. While ischemic stroke patients were the primary target of IMAGES, 7\% of enrolled patients had primary supratentorial intracerebral haemorrhages. Among these 168 patients, the OR for an unfavorable outcome was nominally lower among magnesium-treated patients: 0.84 (0.41-1.74) (Muir et al., 2004). Primary haemorrhage patients are another group among whom mild antihypertensive effects, as well as neuroprotective effects, could be beneficial.

Few patients were enrolled in IMAGES in the first three hours after stroke onset, the time period when acute stroke treatments are most effective; however, among these patients, results were quite encouraging. There are not enough under 1 or under 2 hour patients to permit analysis of these subgroups, but 79 patients $(3.3 \%$ of the study cohort) were enrolled within 3 hours. On the IMAGES primary endpoint, the global odds ratio comparing magnesium vs placebo for a favorable death or disability outcome was 0.66 (95\% Cl 0.25-1.70)(Muir et al., 2004). Considering the modified Rankin component of the IMAGES primary endpoint, a non-disabled outcome (mRS 0-1) was achieved in $45.9 \%$ of magnesium patients vs $33.3 \%$ of placebo patients, a $12.6 \%$ absolute difference. Mortality observations in the IMAGES under 3 hour cohort were also favourable, with death observed in $26 \%$ of placebo vs $19 \%$ of magnesium patients (OR 0.65 , $95 \% \mathrm{Cl} 0.23$ - 1.92). With the small numbers of patients, these results do not reach statistical significance, but do represent a suggestive signal of potential efficacy of magnesium within very early time windows.

\section{The key variable of time and the prehospital strategy for magnesium sulphate}

In the past several decades, more than 100 neuroprotective agents have been tested in randomized controlled clinical trials in acute ischemic stroke (Kidwell et al., 2001; Hong et al.,
2011). Several agents have shown promising findings in safety and preliminary efficacy phase 2 trials. However, no agent has been shown unequivocally to be beneficial in definitive phase 3 trials and none has been approved by the FDA as indicated for ischemic stroke. These results have led many investigators to ask the question, "Why do neuroprotective drugs work in animals but not humans?" (DeGraba and Pettigrew, 2000). Leading basic and clinial neuroscientists have identified 4 key design defects of past human clinical trials of neuroprotective clinical trials: 1) failure to administer study agents at neuroprotective doses in humans because of limiting side effects; 2) failure to select patients for trials who will respond to the known mechanisms of action of study drug; 3 ) failure to employ sample sizes large enough to detect modest benefits of study agent; and 4) failure to treat patients early enough after stroke onset (DeGraba and Pettigrew, 2000; Stroke Therapy Academic Industry Roundtable II (Stairll) 2001; Gorelick, 2000; De Keyser et al., 1999; Fisher, 2003; Ovbiagele et al., 2003; Wahlgren and Ahmed, 2004; Muir and Teal, 2005).

The NIH Field Administration of Stroke Therapy Magnesium (FAST-MAG) trial was designed to remedy each of these past difficulties. The trial employs magnesium sulphate at doses known to be both well tolerated and to yield serum levels neuroprotective in animal models. The trial tests an agent likely to be beneficial for both gray and white matter ischemic injury, based on vasodilating effects in both superficial and deep circulations and cytoprotective effects in both gray and white matter. The trial is adequately powered to detect even a modest treatment effect, and will be larger than $95 \%$ of all previous phase 3 neuroprotective trials. Most importantly, the FAST-MAG trial is designed to address the crucial factor of delayed time to treatment which has hindered all past human clinical trials of neuroprotective drugs.

Rodent and nonhuman primate experimental studies suggest the duration of the therapeutic window within which neuroprotective intervention can ameliorate bioenergetic failure in the ischemic penumbra is very brief, generally less than 2-3 hours. Most animal studies of neuroprotective agents initiate therapy within 160 minutes after ischemia onset. For magnesium 
sulphate specifically, a strong time constraint upon potential benefit was found in a rodent middle cerebral artery occlsuion model. Treating with magnesium sulphate at 2 hours after occlusion reduced infarct size by $61 \%$, while treatment at 6 hours reduced infarct size by $48 \%$ and treatment at 8 hours reduced infarct size by 19\% (Yang et al., 2000).

However, although it is in the first 2 hours of onset that neuroprotective agents in general and magnesium sulphate in particular are most beneficial in focal animal stroke models, no prior human clinical neuroprotective agent trial has enrolled any substantial cohort of patients in this time window (Kidwell et al., 2001; Ferguson et al., 2004). Early neuroprotective trials permitted initiation of therapy at lengthy intervals after symptom onset, up to 48 hours, well after the critical period for stabilizing the penumbral region had closed. More recent trials have utilized shorter entry windows, but continue to treat few patients within the first 1 or 2 hours. Validating methods to safely initiate experimental neuroprotective therapies more quickly is critical if the dramatic benefits of neuroprotective agents evident in the laboratory are to be achieved in human stroke victims. This perspective is supported by the pooled analysis of the first 6 major intravenous tPA trials (Brott et al., 2002). Due to the powerful effect of time to treatment upon benefit of tPA, the sample sizes required to demonstrate treatment benefit escalate rapidly with increasing time window. A trial enrolling patients in the 0-90 minute epoch would require a sample size of only 90 to demonstrate benefit, a 91-180 minute trial would require a sample size of 528 , a $181-270$ minute trial, 816 , and a $271-360$ minute trial a sample size of 4007 .

Enrolling patients in the field in acute neuroprotective trials is a highly promising approach to the challenge of testing neuroprotective agents in hyperacute time epochs. The FAST-MAG trial is the first phase 3 focal stroke trial in the field, but recent trials in both cardiac and other brain disorders demonstrate the feasibility and advantages of the prehospital treatment approach. In the analogous setting of acute myocardial ischemia, randomized clinical trials have shown that paramedic prehospital diagnosis and initiation of defibrillation and thrombolytic therapy reduces time to treatment start and improves clinical outcome (Bozik et al., 2001). In status epilepticus, the Prehospital Treatment of Status Epilepticus (PHTSE) trial showed that paramedic initiation of anticonvulsants in the field is safe, reliable, and yields better clinical outcomes than standard inhospital therapy (Alldredge et al., 2001). In global brain ischemia due to cardiac arrest, the Melbourne hypothermia trial demonstrated a benefit of paramedic-initiated, whole body cooling in resuscitated out of hospital cardiac arrest patients (Bernard et al., 2002)

Prehospital trials of treatments for focal stroke face several design challenges, including accurate identification of stroke patients, rating of pretreatment stroke severity, elicitation of informed consent in the field prior to hospital arrival, and randomization to appropriate treatment arm. The Field Administration of Stroke Therapy - Magnesium (FAST-MAG) investigators developed several novel methods to address these obstacles, first in a pilot, 20 patient feasibility trial (Saver et al., 2004) and then in an ongoing, pivotal, 1700 patient phase 3 trial.

- Stroke identification - LAPSS: Paramedics identify stroke patients using the Los Angeles Prehospital Stroke Screen (LAPSS), an 8 item diagnostic inventory that takes 1-2 minutes to perform, is well validated, and is a standard part of ambulance personnel training worldwide.

- Stroke severity rating - LAMS: The Los Angeles Motor Scale (LAMS) is a 0-5 point rating of motor deficit severity that is derived directly from the face, arm, and grip weakness exam section of the LAPSS. When performing the LAPSS, paramedics also automatically perform the LAMS. Though simple and rapid, the LAMS is a useful assessment of stroke deficit severity, correlating well with concurrently measured 13 item NIH Stroke Scale (NIHSS) scores, and predicting final three month disability, activity of daily living, and neurologic deficit outcomes nearly as well as the full NIHSS (Llanes et al., 2004).

- Informed consent elicitation - cellular telemedicine: Most recent prehospital treatment trials have been conducted for conditions that render patients incompetent 
to provide consent, such as cardiac arrest, under regulations permitting waiver of explicit consent in emergency circumstances. In acute stroke, however, many patients retain decision-making capacity and require fully informed consent. Having paramedics elicit consent has the drawbacks of having nonstroke experts answering subject queries and of diverting paramedic attention from prehospital duties. In the cellular telemedicine enrolment system developed for FAST-MAG, rescue vehicles carry written informed consent forms and cellular phones that permit instant connection to on-call enrolling physician-investigators (Sanossian et al., 2009). The physician-investigator discusses the trial by phone with the patient or their legally authorized representative while paramedics perform prehospital care duties unimpeded.

- Pre-encounter randomization: Keep It Simple and Straightforward (KISS) is an important principle in prehospital research. In the potentially chaotic prehospital setting, complex randomization schemes and multiple drug source bins have a high risk of leading to administration of unintended treatment regimens. To promote reliable and rapid administration of study agent, the randomized phase 3 FAST-MAG trial employs a pre-encounter randomization system. Each ambulance is stocked with only one blinded study kit at a time. When the study kit is used in a patient, the ambulance is restocked within 24 hours with the next kit in that ambulance's permuted block treatment schedule. When given approval to start study procedures by the enrolling physicianinvestigator, paramedics can quickly and unambiguously proceed with administering the sole, "pre-randomized" kit available in the vehicle.

Using these techniques, the FAST-MAG Phase 3 Trial during its initial years of performance has already enrolled more patients in the hyperacute, under 2 hour period than all prior acute stroke treatment trials combined. By late January of 2011, 1190 patients had been enrolled, with a median time interval from last known well to start of study infusion of 46 minutes (Saver et al., 2011). Fully, 869 patients (73\%) were enrolled within the first hour after onset, and another 286
(24\%) in the second hour. In this initial cohort, patient demographics are typical for the stroke population, with average age 69 and $58 \%$ of the population male. Stroke mimicking conditions have been enrolled at a low rate, $3 \%$, below the $5 \%$ allotted in trial sample size projections. The enrolling event was acute cerebral ischemia in $73 \%$ of patients and intracerebral haemorrhage in $24 \%$. These preliminary observation findings indicate that the FAST-MAG trial is accomplishing several innovations in stroke clinical trials, including being: 1) the first prehospital randomized stroke trial; 2) the first treatment trial to enrol a substantial cohort of patients in first 60 minutes after onset (the "golden hour"); and 3) the first trial testing neuroprotective therapy delivery in advance of recanalization therapy, potentially permitting more brain tissue to still be salvageable when reperfusion is achieved.

Commercial sponsors have been reluctant to conduct paramedic administered trials of neuroprotective agents because of perceived regulatory hurdles and because of the lack of established phase 3 trial methodology in the prehospital setting. Successful completion of the FAST-MAG trial may blaze a trail for future neuroprotective field trials, both governmental and commercial, and could lead to a resurgence of research activity in neuroprotection for stroke.

Acute stroke investigators have suggested the desirability of paramedic treatment of acute stroke in the field (Alberts, 1997; The European Ad Hoc Consensus Group, 1998; Rajajee and Saver, 2005). A vision of future stroke care widely shared in the stroke research community is: 1) paramedic administration of a group of complementary neuroprotective agents in the field to protect the brain during transfer and extend the time window for recanalization therapy; 2) definitive thrombolysis or other revascularization procedure on hospital arrival; and 3) in-hospital administration of a second group of neuroprotective agents specifically active against reperfusion injury (Table 2). The FAST-MAG Trial constitutes the first major test of the prehospital element of this stroke treatment strategy. The need for a prehospital neuroprotective agent has become even more urgent with the dissemination of designated Stroke Center systems throughout the United States (Schwamm 
Table 2. Vision for Future Comprehensive Acute Ischemic Stroke Care

\begin{tabular}{|c|c|c|c|c|}
\hline Stage & Typical Setting & Diagnostic Steps & $\begin{array}{l}\text { Treatment } \\
\text { Strategy }\end{array}$ & Treatment Components \\
\hline $\begin{array}{l}\text { Hyperacute } \\
\text { (0-2 hours) }\end{array}$ & Prehospital and ED & Clinical screens & $\begin{array}{l}\text { Stabilize } \\
\text { penumbra }\end{array}$ & $\begin{array}{l}\text { Neuroprotective } \\
\text { cocktail, e.g. } \\
\text { magnesium }\end{array}$ \\
\hline $\begin{array}{l}\text { Early Acute } \\
\text { (1-4.5 hours) }\end{array}$ & $\begin{array}{l}\text { Primary Stroke } \\
\text { Center ED + ICU }\end{array}$ & Rapid CT & $\begin{array}{l}\text { Reperfuse } \\
\text { rapidly }\end{array}$ & - IV thrombolysis \\
\hline $\begin{array}{l}\text { Later Acute } \\
\text { (2-10 hours) }\end{array}$ & $\begin{array}{l}\text { Comprehensive } \\
\text { Stroke Center }\end{array}$ & $\begin{array}{l}\text { Multimodal MRI } \\
\text { or } \mathrm{CT}\end{array}$ & $\begin{array}{l}\text { Reperfuse } \\
\text { definitively }\end{array}$ & $\begin{array}{ll}\text { - } & \text { Endovascular } \\
\text { - } & \text { Recanalization } \\
\text { cocktail }\end{array}$ \\
\hline Early Subacute & Stroke Unit & $\begin{array}{l}\text { Complete work- } \\
\text { up }\end{array}$ & $\begin{array}{l}\text { Deter } \\
\text { reocclusion, } \\
\text { recurrence }\end{array}$ & $\begin{array}{ll}\text { - } & \text { Antithrombotics } \\
\text { - } & \text { Secondary } \\
& \text { prevention } \\
\end{array}$ \\
\hline Subacute & Rehabilitation Unit & Functional MRI & $\begin{array}{l}\text { Enhance } \\
\text { neurorepair }\end{array}$ & $\begin{array}{ll}\text { - } & \text { Nerve growth } \\
\text { factors } \\
\text { - } & \text { Stem cells } \\
\text { Transcranial } \\
\text { magnetic } \\
\text { stimulation } \\
\text { Neural prosthetics } \\
\text { (brain-machine } \\
\text { interface) }\end{array}$ \\
\hline
\end{tabular}

et al., 2005). At the end of 2010, 18 states and additional counties, encompassing $52 \%$ of the American populace, had adopted regulations requiring routing of ambulances directly to designated Stroke Centers (Song and Saver, 2011). Identification of a proven prehospital treatment agent would help mitigate the impact of the resulting slight increase in average prehospital transport time for stroke patients.

\section{References}

Adams HP, Jr., del Zoppo G, Alberts MJ, et al., (2007) Guidelines for the early management of adults with ischemic stroke: a guideline from the American Heart Association/American Stroke Association Stroke Council, Clinical Cardiology Council, Cardiovascular Radiology and Intervention Council, and the Atherosclerotic Peripheral Vascular Disease and
Paramedics reach, within 2 hours of onset, 20$50 \%$ of the 600,000 ischemic stroke patients in the United States each year. Positive results in the FAST-MAG clinical trial would mean that 120,000 - 300,000 Americans suffering an acute stroke each year could potentially benefit from treatment with magnesium sulfate therapy. This would represent a tremendous advance in reducing the nationwide, and worldwide, burden of stroke.
Quality of Care Outcomes in Research Interdisciplinary Working Groups: the American Academy of Neurology affirms the value of this guideline as an educational tool for neurologists. Stroke 38:1655-711.

Alberts MJ (1997) Hyperacute stroke therapy with tissue plasminogen activator. Am J Cardiol 80(4C):29D34D. 
Alldredge BK, Gelb AM, Isaacs SM, Corry MD, Allen F, Ulrich S, Gottwald MD, O'Neil N, Neuhaus JM, Segal MR, Lowenstein DH (2001) A Comparison of Lorazepam, Diazepam, and Placebo for the Treatment of Out-of-Hospital Status Epilepticus. N Engl J Med 345:631-7.

Aslanyan S, Weir CJ, Muir KW, Lees KR (2007) Magnesium for treatment of acute lacunar stroke syndromes: further analysis of the IMAGES trial. Stroke 38:1269-73.

Bernard SA, Gray TW, Buist MD, Jones BM, Silvester W, Gutteridge G, Smith K (2002) Treatment of comatose survivors of out-of-hospital cardiac arrest with induced hypothermia. N Engl J Med 346:557-63.

Bhudia SK, Cosgrove DM, Naugle RI, Rajeswaran J, Lam BK, Walton E, Petrich J, Palumbo RC, Gillinov AM, Apperson-Hansen C, Blackstone EH (2006)

Magnesium as a neuroprotectant in cardiac surgery: a randomized clinical trial. J Thorac Cardiovasc Surg 131:853-61.

Bozik M, Bogousslavsky J, Fayad P, et al (2001) POST010: Efficacy and safety of MaxiPost in patients with acute stroke. Neurology 56(suppl 3):A76.

Bradford APJ, Muir KW, Lees KR, IMAGES Study Group (1998) IMAGES pilot study of intravenous magnesium in acute stroke. Cerebrovasc Dis 8:86A.

Brott TG, Kaste M, Albers GW (2002) Analysis of the NINDS, ECASS I and ECASS II, and Atlantis data sets. In: 27th International Stroke Conference, San Antonio, TX.

California Acute Stroke Pilot Registry (CASPR) Investigators (2005) Prioritizing interventions to improve rates of thrombolysis for ischemic stroke. Neurology 64:654-9.

De Keyser J, Sulter G, Luiten PG (1999) Clinical trials with neuroprotective drugs in acute ischaemic stroke: are we doing the right thing? Trends Neurosci 22:53540.

DeGraba TJ, Pettigrew LC (2000) Why do neuroprotective drugs work in animals but not humans? Neurol Clin 18:475-93.

Ferguson KN, Kidwell CS, Starkman S, Saver JL (2004) Hyperacute treatment initiation in neuroprotective agent stroke trials. J Stroke Cerebrovas Dis 13:109-12.

Fisher M, Ratan R (2003) New perspectives on developing acute stroke therapy. Ann Neurol 53:10-20.
Galeas T, Ziogas G, Valotasiou B (1998) The role of magnesium (Mg)--a natural calcium (Ca) antagonist in the Ca channels of the platelets--in the treatment of acute ischaemic stroke, In: Consensus Conference on Stroke, Edinburgh, UK: Royal College of Physicians of Edinburgh, p. 15.

Gorelick PB (2000) Neuroprotection in acute ischaemic stroke: a tale of for whom the bell tolls? Lancet 355:1925-6.

Green AR, Shuaib A (2006) Therapeutic strategies for the treatment of stroke. Drug Discovery Today 11:68193.

Haley EC, Jr., Lewandowski C, Tilley BC (1997) Myths regarding the NINDS rt-PA Stroke Trial: setting the record straight. Ann Emerg Med 30:676-82.

Hong KS, Lee SJ, Hao Q, Liebeskind DS, Saver JL (2011) Acute Stroke Trials in the 1st decade of the 21th Century. Stroke 42:e314.

Kaste M, Norrving B (2010) From the World Stroke Day to the World Stroke Campaign: one in six: act now! Int J Stroke 5:342-3.

Kidwell CS, Liebeskind DS, Starkman S, Saver JL (2001) Trends in acute ischemic stroke trials through the 20th century. Stroke 32:1349-59.

Lampl Y, Gilad R, Geva D, Eshel Y, Sadeh M (2001) Intravenous administration of magnesium sulfate in acute stroke: a randomized double-blind study. Clin Neuropharmacol 24:11-5.

Lecrux C, McCabe C, Weir CJ, Gallagher L, Mullin J, Touzani O, Muir KW, Lees KR, Macrae IM (2008) Effects of Magnesium Treatment in a Model of Internal Capsule Lesion in Spontaneously Hypertensive Rats. Stroke 39:448-54.

Llanes JN, Kidwell CS, Starkman S, Leary MC, Eckstein M, Saver JL (2004) The Los Angeles Motor Scale (LAMS): a new measure to characterize stroke severity in the field. Prehosp Emerg Care 8:46-50.

Longstreth WT, Jr., Fahrenbruch CE, Olsufka M, Walsh TR, Copass MK, Cobb LA (2002) Randomized clinical trial of magnesium, diazepam, or both after out-ofhospital cardiac arrest. Neurology 59:506-14.

Lopez AD, Mathers CD, Ezzati M, Jamison DT, Murray CJ (2006) Global and regional burden of disease and risk factors, 2001: systematic analysis of population health data. Lancet 367:1747-57. 
Mack WJ, Kellner CP, Sahlein DH, Ducruet AF, Kim GH, Mocco J, Zurica J, Komotar RJ, Haque R, Sciacca R, Quest DO, Solomon RA, Connolly ES, Heyer EJ (2009) Intraoperative magnesium infusion during carotid endarterectomy: a double-blind placebo-controlled trial. J Neurosurg 110:961-7.

Muir KW (2000) Therapeutic potential of magnesium in the treatment of acute stroke. J Stroke Cerebrovasc Dis 9:257-67.

Muir KW (2001) Magnesium for neuroprotection in ischaemic stroke: rationale for use and evidence of effectiveness. CNS Drugs 15:921-30.

Muir KW (2001) Systematic review of clinical trials of magnesium for acute stroke. Stroke 31:379A.

Muir KW, Lees KR (1995) A randomised, double-blind, placebo-controlled pilot trial of intravenous magnesium sulfate in acute stroke. Stroke 26:1183-8.

Muir KW, Lees KR (1998) Dose optimization of intravenous magnesium sulfate after acute stroke. Stroke 29:918-23.

Muir KW, Lees KR, Ford I, Davis S (2004) Magnesium for acute stroke (Intravenous Magnesium Efficacy in Stroke trial): randomised controlled trial. Lancet 363:439-45.

Muir KW, Teal PA (2005) Why have neuro-protectants failed?: lessons learned from stroke trials. J Neurol 252:1011-20.

Obrenovitch TP (1995) The ischaemic penumbra twenty years on. Cerebrovasc Brain Metab Reviews 7:297-323.

Ovbiagele B, Kidwell CS, Starkman S, Saver JL (2003) Neuroprotective agents for the treatment of acute ischemic stroke. Curr Neurol Neurosci Rep 3:9-20.

Rajajee V, Saver J (2005) Prehospital care of the acute stroke patient. Tech Vasc Interv Radiol 8:74-80.

Reeves MJ, Arora S, Broderick JP, et al (2005) Acute stroke care in the US: results from 4 pilot prototypes of the Paul Coverdell National Acute Stroke Registry. Stroke 36:1232-40.

Sandercock PA, Counsell C, Gubitz GJ, Tseng MC (2008) Antiplatelet therapy for acute ischaemic stroke. Cochrane Database Syst Rev CD000029.

Sanossian N, Starkman S, Liebeskind DS, Ali LK, Restrepo L, Hamilton S, Conwit R, Saver JL, FAST-MAG Trial Investigators (2009) Simultaneous ring voice-
over-Internet phone system enables rapid physician elicitation of explicit informed consent in prehospital stroke treatment trials. Cerebrovasc Dis 28:539-44.

Saver J, Eckstein M, Stratton S, et al., (2011) Field Administration of Stroke Therapy - Magnesium (FASTMAG) Phase 3 Trial, In: International Stroke Conference, Los Angeles, CA.

Saver JL, Kidwell C, Eckstein M, Starkman S (2004) Prehospital neuroprotective therapy for acute stroke: results of the Field Administration of Stroke TherapyMagnesium (FAST-MAG) pilot trial. Stroke 35:e106-8.

Saver JL, Kidwell CS, Leary MC, et al., (2002) Results of the Field Administration of Stroke Treatment Magnesium (FAST-MAG) pilot trial: a study of prehospital neuroprotective therapy. Stroke 33:363-4.

Schaller B, Graf R (2004) Cerebral ischemia and reperfusion: the pathophysiologic concept as a basis for clinical therapy. J Cereb Blood Flow Metab 24:35171.

Scholler K, Zausinger S, Baethmann A, SchmidElsaesser R (2004) Neuroprotection in ischemic stroke-combination drug therapy and mild hypothermia in a rat model of permanent focal cerebral ischemia. Brain Research 1023:272-8.

Schwamm LH, Pancioli A, Acker JE, 3rd, et al., (2005) Recommendations for the establishment of stroke systems of care: recommendations from the American Stroke Association's Task Force on the Development of Stroke Systems. Stroke 36:690-703.

Sjostrom LG, Wester P (1994) Systematically induced hypermagnesemia increases the bioavailability of magnesium in rat brain. Soc Neurosci Abstr 20:181.

Smith WS, Sung G, Saver J, et al (2008) Mechanical Thrombectomy for Acute Ischemic Stroke: Final Results of the Multi MERCI Trial. Stroke 39:1205-12.

Song S, Saver J (2011) Growth of Regional Stroke Systems of Care in the United States in the First Decade of the 21st Century. Stroke 42:e340.

Stroke Therapy Academic Industry Roundtable (STAIR) (1999) Recommendations for standards regarding preclinical neuroprotective and restorative drug development. Stroke 30:2752-8.

Stroke Therapy Academic Industry Roundtable II (STAIR-II) (2001) Recommendations for clinical trial evaluation of acute stroke therapies. Stroke 32:1598606. 
The European Ad Hoc Consensus Group (1998) Neuroprotection as initial therapy in acute stroke. Cerebrovasc Dis 8:59-72.

The Penumbra Pivotal Stroke Trial Investigators (2009) The penumbra pivotal stroke trial: safety and effectiveness of a new generation of mechanical devices for clot removal in intracranial large vessel occlusive disease. Stroke 40:2761-8.

Thurnau GR, Kemp DB, Jarvis A (1987) Cerebrospinal fluid levels of magnesium in patients with preeclampsia after treatment with intravenous magnesium sulfate. Am J Obstet Gynecol 1987:1435-8.
Wahlgren N, Ahmed N (2004) Neuroprotection in cerebral ischaemia: facts and fancies--the need for new approaches. Cerebrovasc Dis 17 (Suppl 1):153-66.

Wester PO, Asplund K, Eriksson W, Hagg E, Lithner F, Strand $T$ (1984) Infusion of magnesium in patients with acute brain infarction. Acta Neurol Scand 70:143.

Yang Y, Li Q, Ahmad F, Shuaib A (2000) Survival and histological evaluation of therapeutic window of postischemia treatment with magnesium sulfate in embolic stroke model of rat. Neurosci Lett 285:119-22. 


\title{
Magnesium and cancer: more questions than answers
}

\author{
Marzia Leidi, ${ }^{1}$ Federica Wolf $^{2}$ and Jeanette A.M. Maier ${ }^{1, \bigotimes}$ \\ ${ }^{1}$ Università di Milano, Dipartimento di Scienze Cliniche Luigi Sacco, 20157 Milano, Italy. \\ ${ }^{2}$ Università Cattolica del Sacro Cuore, Istituto di Patologia generale e Centro di Ricerche Oncologiche Giovanni XXIII, Facoltà di \\ Medicina "A. Gemelli", 00168 Roma, Italy. \\ $\bowtie$ jeanette.maier@unimi.it
}

\begin{abstract}
The relationship between $\mathrm{Mg}$ and cancer is still a puzzle to disentangle. The knowledge derived from preclinical studies reveals a complex scenario in which low magnesium has both anti- and pro-tumour effects, such as inhibition of tumour growth at its primary site and facilitation of tumour implantation at its metastatic sites. In different cell types, neoplastic transformation dramatically disrupts the controlled and coordinated fluctuations of intracellular magnesium, an event that offers selective advantages to the cells. It is difficult to translate the lesson learnt from experimental models to humans. Based on epidemiological studies, $\mathrm{Mg}$ deficiency seems to be linked to increased risk of some types of cancers. The demonstration of an impairment of magnesium homeostasis in oncologic patients further complicates the field. We need more translational and clinical data to draw firm conclusions about the contribution of magnesium to tumours.
\end{abstract}

\section{Introduction}

Although cancer mortality has declined in the last two decades, particularly in western countries (Jemal et al., 2010), cancer exacts a very high toll as a leading cause of death all over the world. Great strides have been made to disclose the molecular bases of neoplasia, but how to prevent or cure cancer still remains a largely unanswered question.

There is general agreement about the environmental origin of cancer. It has been estimated that more than two-thirds of human cancers can be prevented by changes in lifestyle. Statistical and epidemiological data point to diet as responsible for about 35\% of human cancer mortality (Doll and Peto, 1981). While many dietary constituents transform normal cells into malignant ones in vitro and stimulate the development, growth and spread of tumours in vivo, evidence from population and laboratory studies indicate that many microconstituents inhibit, retard or reverse tumorigenesis (Manson, 2003). There is general agreement about the inverse correlation between regular consumption of fruit, cereals and vegetables and the risk of cancer. Edible plant matter contains many beneficial microconstituents, vitamins and minerals. In particular, magnesium (Mg), which is predominantly obtained by eating unprocessed grains and green leafy vegetables, is an essential micronutrient implicated in a large array of regulatory, metabolic and structural activities (Wolf and Trapani, 2008). Evidence suggests that the occidental diet is relatively deficient in $\mathrm{Mg}$ because of a preference for calorie-rich, micronutrient-poor foods, low Mg content in water and soil, and processing of many food items (Ford and Mokdad, 2003). Inadequate dietary intake of $\mathrm{Mg}$ is not the only cause of $\mathrm{Mg}$ deficiency. $\mathrm{Mg}$ homeostasis is very tightly tuned in the intestine and the renal tubules through a complex network of transporters, some of which have recently been defined at the molecular level (Quamme, 2010). Consequently, Mg deficiency accompanies chronic gastrointestinal and renal diseases, therapies with some classes of diuretics or anticancer drugs, and also complicates diabetes mellitus. In addition, it is common in alcoholics and in the elderly (Ford and Mokdad, 2003).

Considerable debate remains about the role of $\mathrm{Mg}$ in human tumours, partly due to the lack of reliable and selective analytical procedures to measure Mg. However, there has been a recent resurgence of interest in the relationship between $\mathrm{Mg}$ and tumours both in experimental 
and in clinical oncology, leading to the question whether $\mathrm{Mg}$ is $\mathrm{Dr}$ Jekyll or Mr Hyde in tumour biology and clinics.

\section{Low magnesium and cancer: insights from human studies}

Although not conclusive, a clinical study indicated a potential benefit of $\mathrm{Mg}$ deficiency in patients with malignant tumours (Parsons et al., 1974). These results have been sustained by the fact that $\mathrm{Mg}$ is required in all the steps involved in cell growth, from receptor-mediated intracellular signalling and transphosphorylation reactions to gene transcription, protein synthesis, DNA duplication and cell division (Wolf and Trapani, 2008). Another study showed that the serum level of $\mathrm{Mg}$ is frequently decreased in patients with solid tumours, independent of therapies, and that this decrease correlated to the stage of malignancy (Sartori et al., 1992). This finding seems to be due to the avidity of the tumours for $\mathrm{Mg}$, which behave as $\mathrm{Mg}$ traps. Also, therapies influence $\mathrm{Mg}$ homeostasis. It has been shown that serum $\mathrm{Mg}$ decreases at the end of the first week of radiotherapy (Cohen and Kitzes, 1985) as well as after treatment with different chemotherapeutics that induce $\mathrm{Mg}$ waste. Indeed, cisplatin is nephrotoxic (Yao et al., 2007), and cetuximab, a monoclonal antibody against the Epidermal Growth Factor (EGF) receptor, reversibly and specifically inhibits $\mathrm{Mg}$ reabsorption in the renal distal convoluted tubule (Muallem and Moe, 2007). An obvious question is whether radiation- or drug-induced hypomagnesemia amplifies the effect of DNA damaging cancer treatments by acting as a chemo- and radiosensitizer. Recently, decreased serum $\mathrm{Mg}$ has been suggested to contribute to the therapeutic effects of cetuximab in patients with colon carcinoma (Vincenzi et al., 2008). However, it is still controversial whether to supplement severely hypomagnesemic oncologic patients with Mg or not (Wolf et al., 2009).

Several epidemiological studies have provided evidence that a correlation exists between dietary $\mathrm{Mg}$ and various types of cancer. A high content of $\mathrm{Mg}$ in drinking water seems to protect from liver and oesophageal cancer (Tukiendorf and Rybak, 2004; Yang et al., 2002). In addition, $\mathrm{Mg}$ content in drinking water was inversely correlated with death by breast, prostate, and ovarian cancers, whereas no correlation existed for other tumours (Yang et al., 2000a; 2000b; 2002; Chiu et al., 2004). In particular, a high serum calcium Ca:Mg ratio has been suggested as a novel risk factor which increases the development of postmenopausal breast cancer (Sahmoun and Singh, 2010), the most commonly diagnosed cancer among women in North America and Europe, with a mortality that ranks second only to lung cancer (Jemal et al., 2010). Indeed, in western populations, and even more in postmenopausal women who are taking $\mathrm{Ca}$ supplements to prevent osteoporosis, a high $\mathrm{Ca}: \mathrm{Mg}$ intake is rather common and determines a negative $\mathrm{Mg}$ balance, since the two minerals compete for the same transporters in almost all tissues. $\mathrm{Ca}$ and $\mathrm{Mg}$ have equally important roles in regulating cell growth (McKeehan and Ham, 1978) and $\mathrm{Mg}$ is considered the natural $\mathrm{Ca}$ antagonist. It is therefore feasible that alterations in Ca:Mg ratio might lead to dysregulated cell functions. The ratio of $\mathrm{Ca}: \mathrm{Mg}$ is also to be considered in relation to colon cancer, one of the three big killers, together with breast and lung cancer, in western society. Ca has been indicated as a chemopreventive agent for colon cancer (Lamprecht and Lipkin, 2003).

It should also be pointed that Mg might have a role in colon cancer prevention. Indeed, large epidemiological studies in Sweden, in the Netherlands, in the USA and in Taiwan have demonstrated an association between low intake of $\mathrm{Mg}$ and the risk of colon cancer (Larsson et al., 2005; Folsom et al., 2006; van den Brandt et al., 2007; Chiu et al., 2010) and a large populationbased prospective study in Japan showed a significant inverse correlation between dietary intake of $\mathrm{Mg}$ and colon cancer in men, but not in women ( $\mathrm{Ma}$ et al., 2010). Intriguingly, the association between low intake of $\mathrm{Mg}$ and colon cancer seems to be due, at least in part, to the increased formation of $\mathrm{N}$-nitroso compounds, most of which are potent carcinogens (Chiu et al., 2010). That $\mathrm{Mg}$ has a role in colon neoplasia is supported by the association of adenomatous and hyperplastic polyps with a genetic polymorphism of Transient Receptor Potential Melastatin (TRPM)-7 (Dai et al., 2007), a ubiquitous ion channel with a central role in $\mathrm{Mg}$ uptake and homeostasis (Schmitz et al., 2003). Indeed, the subjects carrying the Thr1482-lle variant allele are at high risk of $\mathrm{Mg}$ deficiency 
and, in turn, of colorectal neoplasia (Dai et al., 2007). While total Mg consumption has been linked to a significant lower risk of colorectal adenoma and hyperplastic polyps (Dai et al., 2007), unequivocal evidence that $\mathrm{Mg}$ reduces the risk of cancer will only be obtained by demonstrating that $\mathrm{Mg}$ supplementation prevents colorectal cancer.

The role of $\mathrm{Mg}$ in lung cancer is rather controversial. The first, case-control study correlated low dietary Mg with poor DNA repair capacity and increased lung cancer risk both in men and women (Mahabir et al., 2008). This link was more evident in the elderly, current smokers, drinkers and in those with a late-stage disease. Apart from its role in maintaining genomic stability, the authors proposed several additional mechanisms by which $\mathrm{Mg}$ might have protected against lung cancer, i.e. by regulating cell multiplication and protecting against oxidative stress, invariably associated with $\mathrm{Mg}$ deficiency (Guerrero-Romero and Rodriguez-Moràn, 2006). Unexpectedly, these results were not supported by a recent prospective analysis which showed that dietary $\mathrm{Mg}$ intake slightly increased lung cancer risk (Mahabir et al., 2010). These contrasting data could result from recall bias. In addition, residual confounders may account for weak associations because smoking is a very strong risk factor for lung cancer, and diet might not be perfectly measured. More studies of dietary $\mathrm{Mg}$ and lung cancer risk are required to carefully consider measurement error issues and confounders such as smoking.

A last topic to consider is the implication of inflammation in the initiation and development of cancer in $\mathrm{Mg}$ deficient individuals. The inflammation-cancer connection is a wellestablished paradigm (Colotta et al., 2009) and low Mg status has been clearly linked to increased inflammatory stress in humans (Nielsen, 2010). While in some context, such as lymphomas, inflammation exerts an anti-neoplastic role, cancer-related inflammation is involved in the early and late steps of the most common solid tumours. Indeed, inflammatory mediators induce genetic instability and contribute to the proliferation of tumour cells, promote invasion, metastasis and angiogenesis as well as an impaired response to therapies (Colotta et al., 2009).
In summary, Mg deficiency seems to be linked to an increased risk of some types of cancers in humans, but population studies are rather complicated to interpret. Too many issues need to be considered - genotype, risk factors, interactions among different nutrients and, in particular, the $\mathrm{Mg}: \mathrm{Ca}$ ratio, the degree of the inflammatory response, etc - to unravel the role of low $\mathrm{Mg}$ in the complex, multistep process of human cancerogenesis.

\section{Low magnesium and cancer: highlights from animal models.}

Two main questions have been addressed in animal models: 1) whether a relationship exists between $\mathrm{Mg}$ and the sensitivity to various carcinogens; and 2) whether primary tumour growth and metastasis are affected by $\mathrm{Mg}$ deficiency. Several studies indicate that $\mathrm{Mg}$ exerts a protective effect in the early phases of chemical cancerogenesis. $\mathrm{Mg}$ prevents lead- and nickel-induced lung tumours in mice (Poirier et al., 1984), inhibits nickel-induced carcinogenesis in the rat kidney (Kasprzak et al., 1994) and protects against 3-methyl-cholantrene induced fibrosarcomas in rats (Patiroğlu et al., 1997). Analogous with data in humans, $\mathrm{Mg}$ acts as a protective agent in colorectal cancer by inhibiting c-myc expression and ornithine decarboxylase activity in the mucosal epithelium of the intestine (Mori et al., 1997). Whether Mg acts directly or by controlling inflammation is an issue that has not received adequate consideration until now. However, it should be pointed out that the inflammatory response in $\mathrm{Mg}$ deficient rodents is very accentuated (Mazur et al., 2007) and, as mentioned above, inflammation markedly contributes to cancer.

Back to the second question, i.e. whether $\mathrm{Mg}$ deficiency impacts on tumour growth in vivo, a study showed that dietary $\mathrm{Mg}$ deprivation inhibited tumour growth in rats by limiting glutathione (GSH) synthesis (Mills et al., 1986), for which $\mathrm{Mg}$ is an obligatory cofactor. More recently, in mice subcutaneously injected with Lewis lung carcinoma, mammary adenocarcinoma and colon carcinoma cells, a low $\mathrm{Mg}$ containing diet was shown to inhibit primary tumour growth, an effect that was promptly reversed by re-introducing $\mathrm{Mg}$ in the diet (Nasulewicz et al., 2004). Surprisingly, Mg 
deficient mice developed far more lung metastases than controls. Some cellular and molecular aspects were investigated to explain how a low Mg diet inhibited the development of primary tumours while enhancing metastatization. Regarding primary tumours, it is noteworthy that $\mathrm{Mg}$ deficiency directly inhibited tumour cell growth by downregulating cyclin B and D3, crucial for the progression through the cell cycle, and by upregulating p21, p27 and Jumonji, all involved in braking cell proliferation (Nasulewicz et al., 2004; Maier et al., 2007). Mg deficiency also affects tumour development indirectly, because it promotes DNA oxidative damage (Maier et al., 2007) and impairs angiogenesis, one of the hallmarks of cancer (Hanahan and Weinberg, 2000). Indeed, Mgdeficient mice develop tumours that are significantly less vascularized than controls (Maier et al., 2007). The angiostatic effect of low $\mathrm{Mg}$ can be ascribed to two different mechanisms. First, low $\mathrm{Mg}$ inhibits endothelial growth and migration, pivotal steps in the formation of new vessels (Bernardini et al., 2005). Second, it has recently been shown that $\mathrm{Mg}$ deficiency suppresses hypoxia-inducing factor (HIF)- $1 \alpha$ activity in paraganglion cells (Torii et al., 2009). HIF1 $\alpha$ is fundamental to initiate the cell response to hypoxia and, therefore, the release of angiogenic factors. If this finding will also be confirmed in in vivo models, it might be involved in impairing angiogenesis upon $\mathrm{Mg}$ deficiency. In vitro, the suppression of HIF- $1 \alpha$ activity is due to the upregulation of IPAS, which acts as a dominant negative inhibitor by preventing HIF-1 $\alpha$ DNA binding. Intriguingly, IPAS upregulation is caused by the activation of NFKB, the transcription factor orchestrating the inflammatory response and also implicated in tumorigenesis (Karin et al., 2006). Inflammation, which is very intense in Mg deficient mice (Mazur et al., 2007), might impact both on the growth of primary tumours and on metastases. Indeed, inflammatory mediators induce genetic instability (Colotta et al., 2009) and might synergize with low $\mathrm{Mg}$ in causing mutations thus allowing the generation of highly aggressive cells. Tumour Necrosis Factor (TNF) $\alpha$, the target of NFKB and prototypical pro-inflammatory cytokine, is increased in the serum of $\mathrm{Mg}$ deficient rodents (Mazur et al., 2007). In spite of its name, TNFa has several marked pro-tumoural functions, because it facilitates the epithelial to mesenchymal transition, it enhances tumour invasion and recruits leukocytes within the tumour, thus amplifying the inflammatory process (Drutskaya et al., 2010). Moreover, TNF $\alpha$ contributes to immune suppression (Drutskaya et al., 2010). Together with TNF $\alpha$, interleukins (IL) 1 and 6, both induced under $\mathrm{Mg}$ deprivation, augment the capacity of cancer cells to metastatize (Royuela et al., 2008). TNF $\alpha$ and IL1s also upregulate endothelial adhesion molecules in lung capillaries, thus facilitating the tethering of metastatic cells to the vessel wall and their subsequent transmigration and colonization in the adjacent tissues. In addition, $\mathrm{Mg}$ is an absolute requirement for the function of the metastasis-suppressor gene product NM23-H1 (Ma et al., 2008). NM23-H1 knockout mice showed accelerated metastasis (Boissan et al., 2005). It is therefore possible that low $\mathrm{Mg}$ availability impairs NM23-H1 anti-metastatic activity in mice.

Briefly, in rodents, $\mathrm{Mg}$ deficiency seems to participate both in early (initiation) and in late (progression) phases of tumorigenesis. The following scenario is feasible. Low Mg promotes oxidative stress and inflammation which generate genetic instability and, therefore, increases the risk of mutations. Since $\mathrm{Mg}$ is an essential cofactor in almost all enzymatic systems involved in DNA synthesis and repair, under low $\mathrm{Mg}$ availability mutations may become permanent thus generating the so-called "initiated" cell. The persistence of oxidative stress and inflammation together might generate further mutations, which render the cell immortal and self sufficient in terms of proliferation. Some cells might also acquire an invasive, metastatic phenotype and colonize distant organs (Figure 1).

\section{Magnesium and cancer: lessons from cultured cells}

Even though studies on cultured cells can only focus on some isolated aspects of the complex events implicated in the development of tumours, they have produced an extraordinary body of knowledge. The field of $\mathrm{Mg}$ research in cancer makes no exception. 


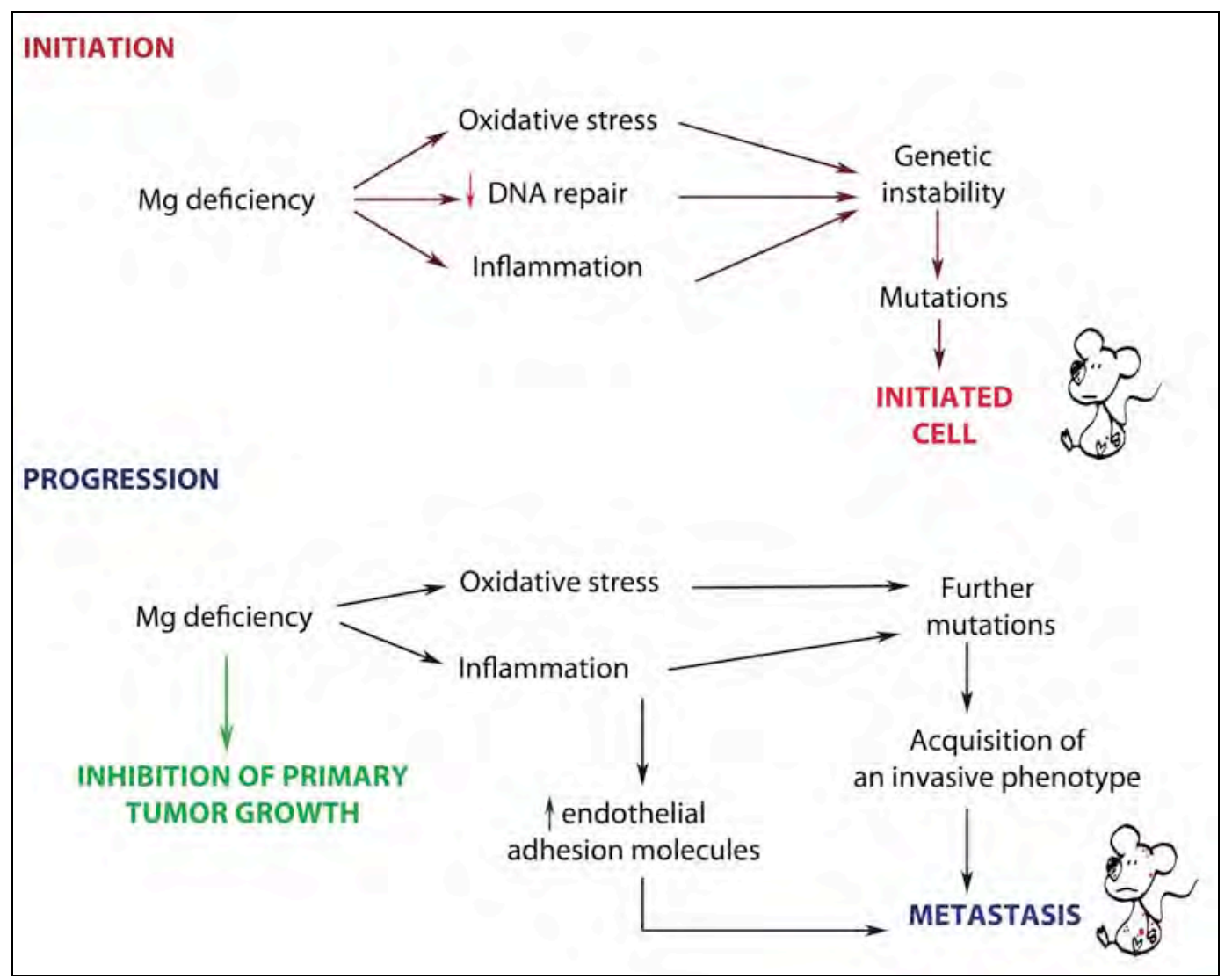

Figure 1. A low $\mathrm{Mg}$ content in the diet might influence both early and late steps of tumorigenesis in mice. Upper panel: By inducing oxidative stress and inflammation and by inhibiting DNA repair enzyme, low $\mathrm{Mg}$ enhances the risk of DNA damage and, therefore, might synergize with carcinogens giving rise to an initiated cell, which is potentially capable of originating a tumour. Lower panel: Once the tumour has developed, low Mg facilitates metastasis by further damaging DNA and by inducing inflammatory events, in the face of an inhibition of primary tumour growth.

\section{The impact of extracellular Mg on tumour cells}

Based on the results obtained in humans and in animal models, the effects of low extracellular $\mathrm{Mg}$ on tumour cells has been investigated. While normal diploid cells are very sensitive to low extracellular $\mathrm{Mg}$ and are rapidly growth retarded, neoplastic cells are rather refractory to $\mathrm{Mg}$ deprivation (Wolf et al., 2009). Indeed, tumour cells require longer times and much lower concentrations of extracellular $\mathrm{Mg}$ than normal cells to slow their proliferation rate (Wolf, 2004; Maier et al., 2007). Another peculiarity of tumour cells is their avidity for $\mathrm{Mg}$, which accumulates within the cells even when extracellular $\mathrm{Mg}$ concentrations are low. The molecular mechanisms involved have not been completely disclosed, but it is known that Na-dependent $\mathrm{Mg}$ extrusion is impaired in tumour cells and contributes to elevate intracellular Mg (Wolf et al., 2007). In addition, TRPM7 is overexpressed in breast tumours and in neoplastic cultured cells (Guilbert et al., 2009), and this feature may well account for the avidity of cancer cells for extracellular $\mathrm{Mg}$. Also nuclear $\mathrm{Mg}$ content is higher in neoplastic than in normal cells (Lukacs et al., 1996). Considering that $\mathrm{Mg}$ acts a second messenger and activates a vast array of enzymes, it is noteworthy that not only does intracellular $\mathrm{Mg}$ increase, but it is also differently distributed in transformed vs normal cells.

\section{The role of intracellular $\mathrm{Mg}$ in tumour cells}

A very simple question is then raised: does intracellular $\mathrm{Mg}$ impact on different aspects of the neoplastic phenotype? Before addressing this issue, it is useful to rapidly review the latest hypotheses proposed to explain the contribution of $\mathrm{Mg}$ to normal cell proliferation. Since $\mathrm{Mg}$ participates in all the major metabolic processes as well as to redox reactions, it is no surprise that 


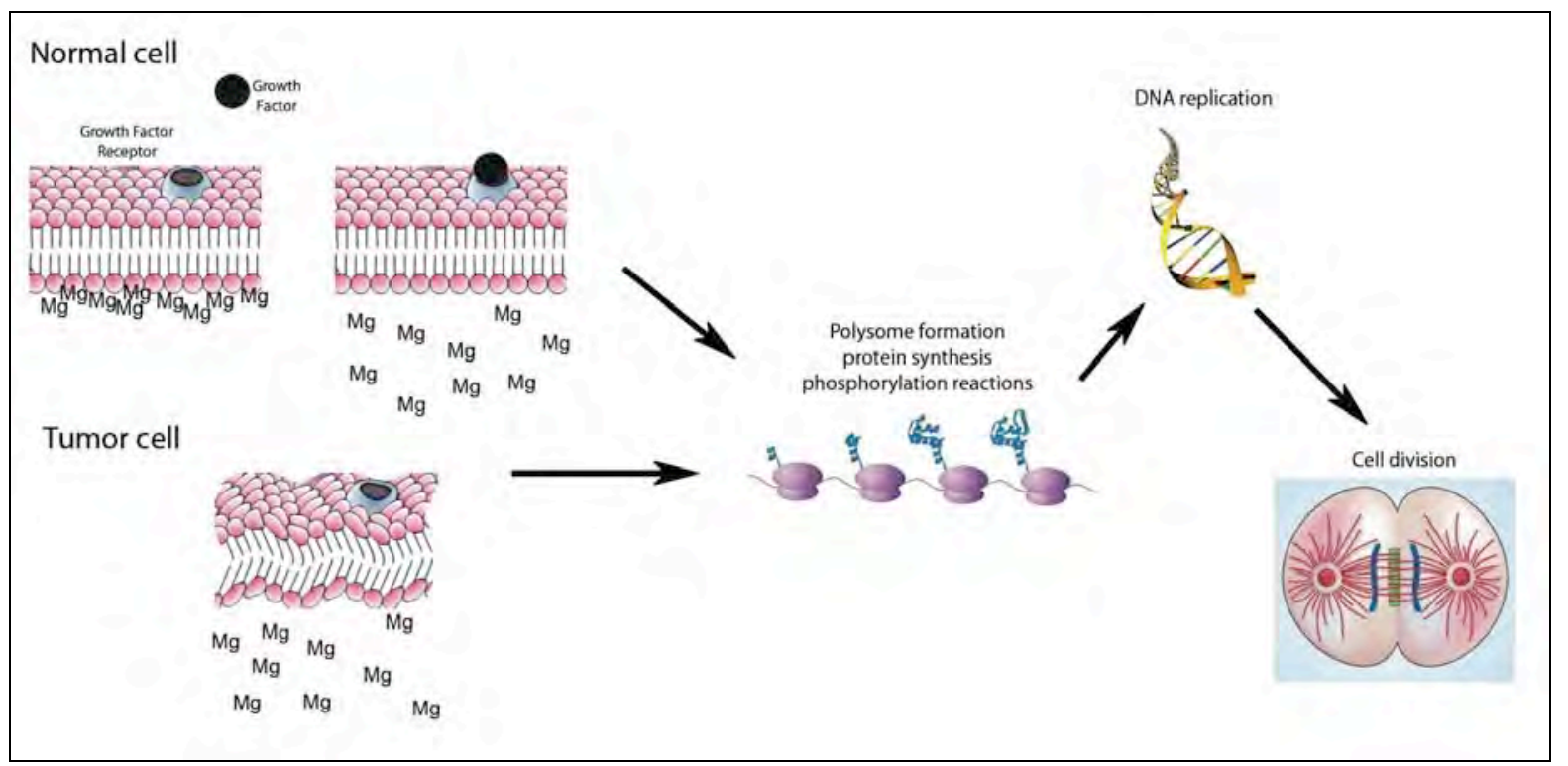

Figure 2. Intracellular $\mathrm{Mg}$ controls cell proliferation. In normal cells, the binding of a growth factor to its receptor determines membrane perturbations which decrease $\mathrm{Mg}$ binding affinity for negatively charged membrane sites, thus increasing intracellular $\mathrm{Mg}$ levels. Increased intracellular $\mathrm{Mg}$ promotes polysome formation, protein synthesis, DNA synthesis and ultimately cell division (according to Rubin, 2007). In neoplastic cells, the membranes are altered and differently organized. These modifications decrease the binding affinity for $\mathrm{Mg}$, which therefore increases intracellular $\mathrm{Mg}$, promoting protein and DNA synthesis.

it has a direct role in controlling cell survival and growth. Cell survival is strictly related to attachment to the extracellular matrix. Adhesion and consequent spreading are mediated by diverse molecules, most notably by integrins which require divalent cations such as $\mathrm{Ca}$ and $\mathrm{Mg}$ to function (Rouslathi and Pierschbacher, 1987). Once the cells adhere and spread, they are alive, metabolically active and ready to respond to growth factors. According to a recently proposed model (Rubin, 2007), intracellular Mg levels vary in quiescent vs growth factor-activated, proliferating cells. In quiescent cells, cytosolic $\mathrm{Mg}$ stabilizes the plasma membrane and subcellular organelles by complexing with negatively charged phospholipids (Suh and Halle, 2007), while nuclear $\mathrm{Mg}$ stabilizes the double helix and retains the chromatin structure (Figure 2). Binding of growth factors to their cognate receptors perturbs the membranes and, consequently, decreases $\mathrm{Mg}$ binding affinity for the membrane, thus resulting in increased focal concentrations of intracellular Mg (Rubin, 2007). Also Mg influx from the extracellular milieu contributes to increase total intracellular $\mathrm{Mg}$ in response to growth factors. In particular, EGF regulates $\mathrm{Mg}$ transepithelial transport by increasing TRPM6 activity and surface expression (Thebault et al., 2009). Intracellular Mg then behaves as a second messenger and regulates a wide variety of reactions ultimately leading to cell division.

Neoplastic transformation dramatically disrupts these controlled and coordinated fluctuations of intracellular Mg (Figure 2). Membrane perturbations that are typically associated with the neoplastic phenotype determine the release of intracellularly bound Mg. Accordingly, a seminal study showed a significant increase of intracellular Mg in human brain tumours by 31P-NMR (Taylor et al., 1991).

Having high intracellular $\mathrm{Mg}$ might represent a selective advantage for the transformed cells. $\mathrm{Mg}$ forms complexes with ATP, ADP and GTP, being necessary for the activity of enzymes implicated in the transfer of phosphate groups such as glucokinase, phosphofructokinase, phosphoglycerate-kinase and pyruvate kinase (Gunther, 2008), enzymes of glycolysis, which is known to be the preferential pathway utilized by neoplastic cells to produce energy despite the presence of oxygen. This issue has recently been reappraised. The expression of TRPM7 and, therefore, the 
capability of the cells to take up $\mathrm{Mg}$ has been associated with the transition from a quiescent to a proliferative metabolic state in which it is aerobic glycolysis that generates the energy necessary for cell growth (Sahni et al., 2010).

$\mathrm{Mg}$ also complexes with DNA polymerase, ribonucleases, adenylcyclase, phosphodiesterases, guanylate-cyclase, ATPases and GTPases, being therefore implicated in the metabolism of nucleic acids and proteins and in signal transduction. In addition, Mg activates telomerase (Lue, 1999), a ribonucleoprotein that is responsible for maintaining the terminal repeats of telomeres and conferring limitless replicative potential. It is because of the reactivation of telomerase activity that transformed cells become immortal (Hanahan and Weinberg, 2000).

Intriguingly, the nuclear Ser/Thr phosphatase PPM1D (also known as WIP1), which is overexpressed in human primary breast, gastric, pancreatic, ovarian and neuroblastoma tumours, requires $\mathrm{Mg}$ for its activity. PPM1D is involved in the regulation of several essential signalling pathways that are implicated in cancer pathogenesis (Le Guezennec and Bulavin, 2010). The oncogenic properties of PPM1D were originally thought to stem from its ability to dephosphorylate and, consequently, inactivate the p53 tumour suppressor gene. However, recent studies have shown that PPM1D also targets other key stress response kinases that function in DNA damage response and repair. In addition, PPM1D complements several oncogenes, such as Ras, Myc, and HER-2/neu, for cellular trans-formation both in vitro and in vivo. Therefore, a rise in intracellular Mg might result in a permanent activation of PPM1D and potently contribute to several steps of tumour development.

A last issue needs consideration. Tumours should be regarded as complex tissues in which normal cell types within the stroma serve as active collaborators in cancer progression. Many of the growth signals driving the proliferation and invasion of carcinoma cells originate from the stromal cell components of the tumour mass. Indeed, low $\mathrm{Mg}$ modulates the functions of normal cells present in the tumour microenvironment. In particular, endothelial cells cultured in low $\mathrm{Mg}$ release higher amounts of metalloproteases and growth factors (Ferrè et al., 2010). Similar results were obtained in cultured human fibroblasts (Maier et al., unpublished results). In addition, low $\mathrm{Mg}$ promotes endothelial and fibroblast senescence (Killilea and Maier, 2008) and senescent cells can modify the tissue environment in a way that synergizes with oncogenic mutations to promote the progression of cancers (Campisi, 2003). On the other hand, low Mg impairs the acquisition of an angiogenic phenotype in vitro. Yet, the question about the role of $\mathrm{Mg}$ deficiency in cancer remains: good or bad?

\section{A synthetic view}

How do we reconcile this wealth of information? Although some results are controversial, it is possible to conclude that most of the data available point to low $\mathrm{Mg}$ as a contributing factor to tumorigenesis. $\mathrm{Mg}$ deficiency increases oxidative stress, which causes DNA damage, and impairs DNA repair mechanisms. It therefore may lead to mutations that produce oncogenes with dominant gain of function, and tumour suppressor genes with recessive loss of function (Hanahan and Weinberg, 2000). Upon transformation, the cells lose the capability to regulate $\mathrm{Mg}$ homeostasis. $\mathrm{Mg}$ also accumulates in the cell when $\mathrm{Mg}$ availability is low, its distribution is altered and is not subject to coordinated fluctuations in response to various stimuli. Low extracellular $\mathrm{Mg}$ also affects normal stromal cells that can release growth factors and proteases, thus potentiating tumour growth and development. In addition, in vivo low $\mathrm{Mg}$ activates an inflammatory response, which has many tumour-promoting effects.

\section{Big questions}

The connection between $\mathrm{Mg}$ and cancer is rather strong, but several questions remain. First, it might be asked whether $\mathrm{Mg}$ deficiency is sufficient for the development of cancer. Indeed, low $\mathrm{Mg}$ increases the levels of reactive oxygen species, which mutate DNA and, therefore, generate genetic instability. Another candidate as a potential carcinogen in $\mathrm{Mg}$ deficiency is inflammation. However, it is more likely that a low $\mathrm{Mg}$ state only contributes to tumorigenesis by synergizing with many different factors. 
A second aspect that is often underevaluated is the effect on the tumour of an aberrant Ca:Mg ratio that is inevitably created in $\mathrm{Mg}$ deficiency. Even though $\mathrm{Ca}$ and $\mathrm{Mg}$ have become the gold standard when discussing nutritional supplements, the balance between the two cations needs to be carefully evaluated both in vitro and in vivo.

A third question arises about whether outcomes in the cancer mouse model can predict a real human disease mechanism. Mg deficiency retards primary tumour growth but enhances metastases in mice. It would be relevant to consider the balance between cancer-promoting and inhibiting responses by low $\mathrm{Mg}$ in humans also.

The last big question is whether knowledge about the connection between low $\mathrm{Mg}$ and cancer can be translated into useful approaches to prevent and treat cancer. Most of the data available suggest that $\mathrm{Mg}$ could be a chemo-preventive agent. Therefore, correcting $\mathrm{Mg}$ intake might represent an effective and low cost preventive measure to reduce cancer risk. The picture is different in oncologic patients, because hypomagnesemia might be beneficial since it sensitizes neoplastic cells to radiation or

\section{References}

Bernardini D, Nasulewicz A, Mazur A, Maier JA (2005) Magnesium and microvascular endothelial cells: a role in inflammation and angiogenesis. Frontiers Biosci 10: 1177-82.

Boissan M, Wendum D, Arnaud-Dabernat S, Munier A, Debray M, Lascu I, Daniel JY, Lacombe ML (2005) Increased lung metastasis in transgenic NM23Null/SV40 mice with hepatocellular carcinoma. J Natl Cancer Inst 97:836-45.

Campisi J (2003) Cancer and aging: rival demons? Nat Rev Cancer 3:339-49.

Chiu HF, Chang CC, Yang CY (2004) Calcium and Magnesium in drinking water and the risk of death from ovarian cancer. Magnes Res 17:28-34.

Chiu HF, Tsai SS, Wu TN, Yang CY (2010) Colon cancer and the content of nitrate and magnesium in drinking water. Magnes Res 23:81-9. chemotherapeutics. Therefore the "hamletic" doubt remains: to treat or not to treat hypomagnesemic oncologic patients?

\section{Conclusion}

Future research should focus on elucidating how $\mathrm{Mg}$ is involved in preventing cancer by modulating crucial events in signal transduction pathways and the cell cycle. Hints might derive from nutrigenomics, i.e. the use of highthroughput genomic methods in nutritional studies, which can provide information about the cross-talk between $\mathrm{Mg}$ and genes. The next step should lead to translation of this basic research into nutritional preventive protocols for human tumours, an approach that will involve many scientific disciplines. The challenge is great, but the reward might be enormous.

\section{Acknowledgements}

This work is supported by MIUR-PRIN 2007 grant number 2007ZT39FN.

Cohen L, Kitzes R (1985) Early radiation induced proctosigmoiditis responds to magnesium therapy. Magnesium 4:16-9.

Colotta F, Allavena P, Sica A, Garlanda C, Mantovani A (2009) Cancer-related inflammation, the seventh hallmark of cancer: link to genetic instability. Carcinogenesis 30:1073-81.

Dai Q, Shrubsole J, Ness RM, Schlundt D, Cai Q, Smalley WE, Li M, Shyr Y, Zheng W (2007) The relation of magnesium and calcium intakes and a genetic polymorphism in the magnesium transporter to colorectal neoplasia risk. Am J Clin Nutr 86:743-51.

Doll R, Peto R (1981) The causes of cancer: quantitative estimates of avoidable risks of cancer in the US today. J Natl Cancer Inst 66:1191-308.

Drutskaya MS, Efimov GA, Kruglov AA, Kuprash DV, Nedospasov SA (2010) Tumor necrosis factor, lymphotoxin and cancer. IUBMB Life 62:283-9. 
Ferrè S, Baldoli E, Leidi M, Maier JA (2010) Magnesium deficiency promotes a pro-atherogenic phenotype in cultured human endothelial cells via activation of NFkB. Biochim Biophys Acta 1802:952-8.

Folsom AR, Hong CP (2006) Magnesium intake and reduced risk of colon cancer in a prospective study of women. Am J Epidemiol 163:232-5.

Ford ES, Mokdad AH (2003) Dietary magnesium intake in a national sample of US adults. J Nutr 133:2879-82.

Guerrero-Romero F, Rodríguez-Morán M (2006) Hypomagnesemia, oxidative stress, inflammation, and metabolic syndrome. Diabetes Metab Res Rev 22:4716.

Guilbert A, Gautier M, Dhennin-Duthille I, Haren N, Sevestre H, Ouadid-Ahidouch H (2009) Evidence that TRPM7 is required for breast cancer cell proliferation. Am J Physiol Cell Physiol 297:C493-502.

Günther T (2008) Comment on the number of magnesium activated enzymes. Magnes Res 21:185-7.

Hanahan D, Weinberg RA (2000) The hallmarks of cancer. Cell 100:57-70.

Jemal A, Siegel R, Xu J, Ward E (2010) Cancer Statistics, 2010. CA Cancer J Clin 60:277-300.

Karin M, Cao Y, Greten FR, Li ZW (2002) NF-kB in cancer: from innocent bystander to major culprit. Nat Rev Cancer 2:301-10.

Kasprzak KS, Diwan BA, Rice JM (1994) Iron accelerates while magnesium inhibits nickel-induced carcinogenesis in the rat kidney. Toxicology 90:129-40.

Killilea DW, Maier JA (2008) A connection between magnesium deficiency and aging: new insights from cellular studies. Magnes Res 21:77-82.

Lamprecht SA, Lipkin M (2003) Chemoprevention of colon cancer by calcium, vitamin D and folate: molecular mechanisms. Nat Rev Cancer 3:601-13.

Larsson SC, Bergkvist L, Wolk A (2005) Magnesium intake in relation to risk of colon cancer in women. JAMA 293:86-9.

Le Guezennec X, Bulavin DV (2010) WIP1 phosphatase at the crossroads of cancer and aging. Trends Biochem Sci 35:109-14.

Lue NF (1999) Sequence-specific and conformationdependent binding of yeast telomerase RNA to single- stranded telomeric DNA. Nucleic Acids Res 1999 27:2560-7.

Lukács GL, Zs-Nagy I, Steiber J, Györi F, Balázs G (1996) Relative intranuclear magnesium and phosphorus contents in normal and tumor cells of the human thyroid gland as revealed by energy-dispersive X-ray microanalysis. Scanning Microsc 10:1191-200.

Ma E, Sasazuki S, Inoue M, Iwasaki M, Sawada N, Takachi R, Tsugane S (2010) High dietary intake of magnesium may decrease risk of colorectal cancer in Japanese men. J Nutr 140:779-85.

Ma W, Chen J, Xue X, Wang Z, Liu H, Wang T, Bai Y, Tang SC, Zhou Q (2008) Alteration in gene expression profile and biological behavior in human lung cancer cell line NL9980 by MN23-H1 gene silencing. Biochem Biophys Res Commun 371:425-30.

Mahabir S, Forman MR, Dong YQ, Park Y, Hollenbeck A, Schatzkin A (2010) Mineral intake and lung cancer risk in the NIH-American Association of Retired Persons Diet and Health study. Cancer Epidemiol Biomarkers Prev 19:1976-83.

Mahabir S, Wei Q, Barrera SL, Dong YQ, Etzel CJ, Spitz MR, Forman MR (2008) Dietary magnesium and DNA repair capacity as risk factors for lung cancer. Carcinogenesis 29:949-56.

Maier JA, Nasulewicz-Goldeman A, Simonacci M, Boninsegna A, Mazur A, Wolf Fl (2007) Insights into the mechanisms involved in magnesium-dependent inhibition of primary tumour growth. Nutr Cancer 59:192-8.

Manson MM (2003) Cancer prevention - the potential for diet to modulate molecular signalling. Trend $\mathrm{Mol}$ Med 9:11-8.

Mazur A, Maier JA, Rock E, Gueux E, Nowacki W, Rayssiguier $Y$ (2007) Magnesium and the inflammatory response: potential physiopathological implications. Arch Biochem Biophys 458:48-56.

Mc Keehan, Ham RG (1978) Ca and Mg ions and the regulation of multiplication in normal and transformed cells. Nature 275:756-8.

Mills BJ, Lindeman RD, Lang CA (1986) Magnesium deficiency inhibits biosynthesis of blood glutathione and tumor growth in the rat. Proc Soc Exp Biol Med 181:326-32. 
Mori H, Tanaka T, Sugie S, Yoshimi N, Kawamori T, Hirose Y, Ohnishi M (1997) Chemopreventium by naturally occuring and sinthetic agents in oral, liver and large bowel carcinogenesis. J Cell Biochem Suppl 27:35-41.

Muallem S, Moe OW (2007) When EGF is offside, magnesium is wasted. J Clin Invest 117:2086-9.

Nasulewicz A, Wietrzyk J, Wolf FI, Dzimira S, Madej J, Maier JA, Rayssiguier Y, Mazur A, Opolski A (2004) Magnesium Deficiency Inhibits Primary Tumor Growth But Favors Metastasis In Mice. Biochim Biophys ActaMol Basis Dis 1739:26-32.

Nielsen FH (2010) Magnesium, inflammation, and obesity in chronic disease. Nutr Rev 68:333-40.

Parson FM,Edwards GF, Anderson CK, Ahmad S, Clark PB, Hetherington C, Young GA (1974) Regression of malignant tumours in potassium and magnesium depletion induced by diet and haemodialysis. Lancet 16:243-4.

Patiroğlu T, Sahin G, Kontaş O, Uzüm K, Saraymen R (1997) Protective effect of magnesium supplementation on experimental 3-methyl cholanthrene-induced fibrosarcoma and changes in tissue magnesium distribution during carcinogenesis in rats. Biol Trace Elem Res 56:179-85.

Poirier LA, Theiss JC, Arnold LJ, Shimkin MB (1984) Inhibition by magnesium and calcium acetates of lead subacetate- and nickel acetate-induced lung tumours in strain A mice. Cancer Res 44:1520-2.

Quamme GA (2010) Molecular identification of ancient and modern mammalian magnesium transporters. Am J Physiol Cell Physiol 298:C407-29.

Royuela $M$, Rodríguez-Berriguete $G$, Fraile $B$, Paniagua R (2008) TNF-alpha/IL-1/NF-kappaB transduction pathway in human cancer prostate. Histol Histopathol 23:1279-90.

Rubin H (2007) The logic of the Membrane, Magnesium, Mitosis (MMM) model for the regulation of animal cell proliferation. Arch Biochem Biophys 458: 16-23.

Ruoslahti E, Pierschbacher MD (1987) New perspectives in cell adhesion: RGD and integrins. Science 238:491-7.

Sahmoun AE, Singh BB (2010) Does a higher ratio of serum calcium to magnesium increase the risk for postmenopausal breast cancer? Med Hypotheses 75:315-8.
Sahni J, Tamura R, Sweer IR, Scharenberg AM (2010) TRPM7 regulates quiescent/proliferative metabolic transitions in lymphocytes. Cell Cycle 9:3565-74.

Sartori S, Nielsen I, Tassinari D, Mazzotta D,Vecchiatti G, Sero A, Abbasciano V (1992) Serum and erythrocyte magnesium concentrations in solid tumours: relationship with stage and malignancy. Magnes Res 5:189-92.

Schmitz C, Perraud AL, Johnson CO, Inabe K, Smith MK, Penner R, Kurosaki T, Fleig A, Scharenberg AM (2003) Regulation of vertebrate cellular Mg homeostasis by TRPM7. Cell 114:191-200.

Suh BC, Halle B (2007) Electrostatic interaction of internal Mg with membrane PIP2 seen with KCNQ K+ channel. J General Physiol 130:241-56.

Taylor JS, Vigneron DB, Murphy-Boesch J, Nelson SJ, Kessler HB, Coia L, Curran W, Brown TR (1991) Free magnesium levels in normal human brain and brain tumors: 31P chemical-shift imaging measurements at 1.5 T. Proc Natl Acad Sci USA 88:6810-14.

Thebault S, Alexander RT, Tiel Groenestege WM, Hoenderop JG, Bindels RJ (2009) EGF increases TRPM6 activity and surface expression. J Am Soc Nephrol 20: 78-85.

Torii S, Kobayashi K, Takahashi M, Katahira K, Goryo K, Matsushita N, Yasumoto K, Fujii-Kuriyama Y, Sogawa K (2009) Magnesium deficiency causes loss of response to intermittent hypoxia in paraganglion cells. $J$ Biol Chem 284:19077-89.

Tukiendorf A, Rybak Z (2004) New data on ecological analysis of possible relationship between magnesium in drinking water and liver cancer. Magnes Res 17:4652.

van den Brandt PA, Smits KM, Goldbohm RA, Weijenberg MP (2007) Magnesium intake and colon cancer risk in the Netherlands Cohort Study. Br J Cancer 96:510-13.

Vincenzi B, Santini D, Galluzzo S, Russo A, Fulfaro F, SillettaM, Battistoni F, Rocci L, Zobel BB, Adamo V, Dicuonzo G, Tonini G. (2008) Early magnesium reduction in advanced colorectal cancer patients treated with cetuximab plus irinotecan as predictive factor of efficacy and outcome. Clin Cancer Res 14:4219-24.

Wolf Fl, Cittadini AR, Maier JA (2009) Magnesium and tumours: ally or foe? Cancer Treatment Rev 35:378-82. 
Wolf Fl, Maier JA, Nasulewicz A, Feillet-Coudray C, Mazur A, Simonacci M, Cittadini A (2007) Magnesium and neoplasia: from carcinogenesis to tumour growth and progression or treatment. Arch Biochem Biophys 458:24-32.

Wolf Fl, Trapani V (2008) Cell (patho)physiology of magnesium. Clin Sci 114:27-35.

Wolf FI. TRPM7: Channeling the Future of Cellular Magnesium Homeostasis? (2004) Sci STKE 233:pe23.

Yang CY, Chiu HF, Cheng MF, Hsu TY, Cheng MF, Wu TN (2000a) Calcium and Magnesium in drinking water and the risk of death from breast cancer. J Toxicol Environ Health A 60:231-41.
Yang CY, Chiu HF, Tsai SS, Cheng MF, Lin MC, Sung FC (2000b) Calcium and Magnesium in drinking water and the risk of death from prostate cancer. J Toxicol Environ Health A 60:17-26.

Yang CY, Chiu HF, Tsai SS, Wu TN, Chang CC (2002) Calcium and Magnesium in drinking water and the risk of death from esophageal cancer. Magnes Res 15:21522.

Yao X, Panichpisal K, Kurtzman N, Nugent K (2007) Cisplatin nephrotoxicity: a review. Am J Med Sci 334:115-24. 



\title{
Magnesium in Parkinson's disease: an update in clinical and basic aspects
}

\author{
Kiyomitsu Oyanagi ${ }^{\bowtie}$ and Tomoyo Hashimoto
}

Department of Brain Disease Research, Shinshu University School of Medicine, Matsumoto, Nagano 390-8621, Japan. $\triangle$ k123ysm@shinshu-u.ac.jp

\begin{abstract}
Magnesium ( $\mathrm{Mg}$ ) is essential for cell functions such as transport of calcium and potassium ions, and modulates signal transduction, energy metabolism, and cell proliferation. Several studies elucidated a reduced concentration of $\mathrm{Mg}$ in patients with Parkinson's disease (PD), and experimentally, severe loss of dopaminergic neurons exclusively in the substantia nigra in 1-year-old rats that had been subjected to continuously low Mg intake (one-fifth of the normal level) over generations. A study conducted by the authors revealed a significant and striking effect of $\mathrm{Mg}$ to prevent neurite and neuron pathology, and also to ameliorate neurite pathology in a rat Parkinson disease (PD) model involving culture of ventral mesencephalic-striatal cells with 1-methyl-4-phenylpyridinium $\left(\mathrm{MPP}^{+}\right) . \mathrm{Mg}$ is expected to prevent and ameliorate Parkinson's disease in cases where it would be able to cross into the brain in a suitable way.
\end{abstract}

\section{Introduction}

Parkinson's disease (PD) is a neurodegenerative disease occurring in middle-aged and aged humans characterized by clinical symptoms including tremor and rigidity (Parkinson, 1817). It has been reported that almost $90 \%$ of the patients are sporadic and $10 \%$ are familial. Sporadic PD shows neuropathological features involving the appearance of Lewy bodies (Lewy, 1912; Tretiakoff, 1919) and loss of neurons in the substantia nigra (Figures 1 and 2) and substantia innominata. After establishment of the disease as an entity, it was revealed that dopaminergic neurons in the ventral tegmental area, noradrenergic neurons in the locus coeruleus and motor vagal nucleus, serotonergic neurons in the dorsal raphe nucleus, and neurons in the sympathetic ganglia and visceral autonomic nervous system are involved in the disease with neuronal loss and presence of Lewy bodies (Jellinger, 1999). In the present manuscript, the authors review the role of magnesium $(\mathrm{Mg})$ in the pathogenesis and pathomechanisms in clinical and basic aspects of PD.

\section{Mg in Parkinson's disease and related diseases}

Uitti et al., (1989) analysed four brain regions (frontal cortex, caudate nucleus, substantia nigra and cerebellum) for concentrations of 24 metals
(Ag, Al, As, B, Be, Ca, Cd, Co, Cr, Cu, Fe, K, Pb, Mg, $\mathrm{Mn}, \mathrm{Mo}, \mathrm{Na}, \mathrm{Ni}, \mathrm{P}, \mathrm{Se}, \mathrm{Ti}, \mathrm{V}, \mathrm{W}, \mathrm{Zn}$ ) by atomic absorption and atomic emission spectroscopy in brains of 9 patients with PD, 15 patients with other chronic neurological diseases and 12 subjects of controls. The results were that brains of PD and parkinsonism secondary to neurofibrillary tangle disease showed lower concentrations of $\mathrm{Mg}$ in the caudate nucleus and copper in the substantia nigra than control brains. Barbiloni et al., (1999) performed in vivo phosphorus magnetic resonance spectroscopy on the occipital lobes of 13 patients with PD, 15 patients with multiple system atrophy and 16 age-matched healthy subjects. They reported that patients with PD showed significantly increased contents of inorganic phosphate $(\mathrm{Pi})$, decreased cytosolic free $\left[\mathrm{Mg}^{2+}\right]$, and unchanged phosphocreatine and $\mathrm{pH}$. Bocca et al., (2006) examined concentrations of $\mathrm{Ca}, \mathrm{Cu}, \mathrm{Fe}, \mathrm{Mg}, \mathrm{Si}$ and $\mathrm{Zn}$ by inductively coupled plasma atomic emission spectrometry (ICP-AES) in blood, urine and cerebrospinal fluid (CSF) of 91 PD patients and 18 controls. They concluded that $\mathrm{Mg}$ concentration in CSF of PD patients decreased with the duration and severity of the disease.

It has been proposed that $\mathrm{Mg}$ deficiency is involved in the pathogenesis of parkinsonismdementia complex (PDC) and amyotrophic lateral 


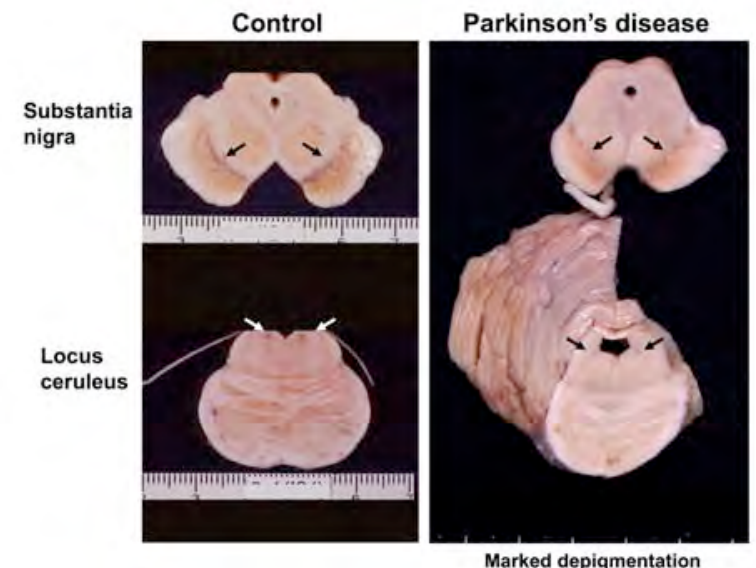

Marked depigmentation

Figure 1. Midbrain and upper pons. The substantia nigra and locus coeruleus in the patient with Parkinson's disease show marked depigmentation as compared with those of controls.

sclerosis (ALS) in the Chamorro population on Guam, which is a member of the Mariana Islands in the western Pacific Ocean, as well as in the Kii peninsula of Japan and in West New Guinea (Yase 1978, Garruto et al., 1984). PDC is a disease entity that was established by Hirano et al., (1961a; 1961b) that affects the neurons in the substantia nigra, brainstem, and temporal and frontal cortex. The disease is characterized by the presence of neurofibrillary tangles in the remaining neurons, and disease-specific granular hazy inclusions in the astrocytes (Oyanagi et al., 1997; Oyanagi, 2005), tau-positive fine granules in the cerebral white matter (Yamazaki et al., 2005), and widespread TDP-43-immunopositive inclusions (Hasegawa et al., 2007). Patients exhibit parkinsonism and dementia, and usually die within about 5 years from infectious diseases (Hirano et al., 1961a; 1961b; Chen and Chase, 1985). ALS is a motor neuron disease affecting the Betz cells in the cerebral cortex, and facial and hypoglossal nuclei in the brainstem and anterior horn cells in the spinal cord, and usually patients die of respiratory failure within 5 years after the onset.

\section{Possible pathomechanisms in Parkinson's disease}

\section{Mitochondrial damage and oxidative stress}

Increased expression of 4-hydroxy-2-nonenal (HNE) (Yoritaka et al., 1996), decreased activity of mitochondrial complex I and a decreased amount

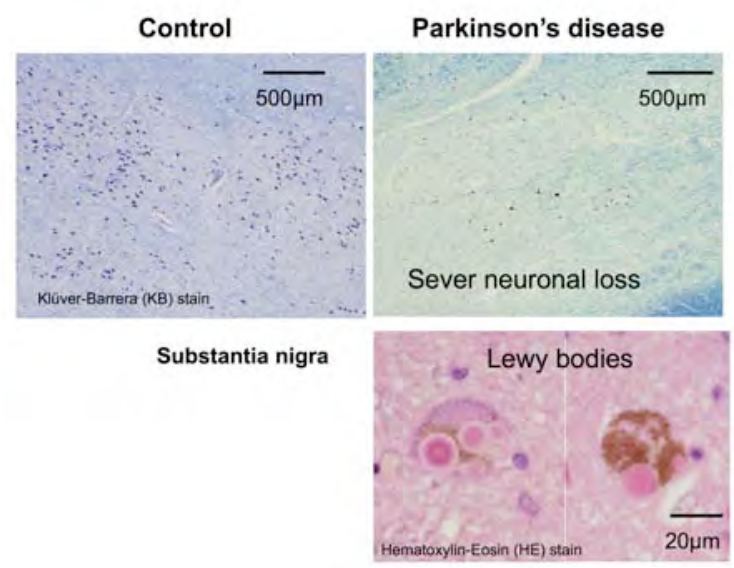

Figure 2. The substantia nigra of a patient with Parkinson's disease shows severe loss of neurons as compared with a control subject. Some remaining neurons represent Lewy bodies.

of alpha-ketoglutarate dehydrogenase complex (KGDHC) in the pigmented neurons of the substantia nigra (Hattori et al., 1991; Mizuno et al., 1994) have been reported in affected patients. In the substantia nigra, decreased activity of catalase and peroxidase (Ambani et al., 1975) and increased amounts of protein carbonyls, 8hydroxy-2'-deoxyguanosine (8-OHdG)/8-hydroxyguanine (8-OHG), 4-hydroxynonenal-lysine and malondialdehyde-lysine (MDAL) (Alam et al., 1997a,b; Zhang et al., 1999; Dalfo et al., 2005) have been reported.

1-Methyl-4-phenyl-1,2,3,6-tetrahydropyridine (MPTP) was the first human parkinsonian agent to be characterized. It is converted to 1-methyl-4phenylpyridinium $\left(\mathrm{MPP}^{+}\right)$by monoamine oxidase $B$ in astrocytes. $\mathrm{MPP}^{+}$damages mitochondrial complex I of dopaminergic neurons after transfer by the dopamine transporter, and increased calcium permeability of the mitochondrial membrane induces free radicals (Smeyne et al., 2005). $\mathrm{MPP}^{+}$has been used to induce selective degeneration of dopaminergic neurons in an experimental model of PD (Nakamura et al., 2000). In addition, rotenone, 6-hydroxydopamine (6-OHDA), paraquat and annonacin have been used as noxious agents to create in vivo models of PD (Fornai et al., 2003; Champy et al., 2004; Bove et al., 2005) (Figure 3). Dopamine and dopamine quinones themselves are considered to be causes of oxidative stress. PINK1 (PTEN-induced putative kinase 1) maintains mitochondrial function 


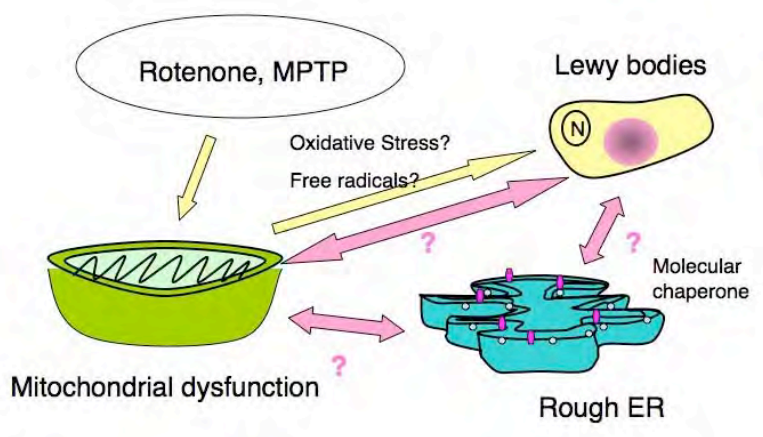

Figure 3. Possible relationship between mitochondria, rough ER and Lewy body formation.

and the gene is causative in some familial PD (Valente et al., 2004).

\section{Unfolded protein retention and endoplasmic reticulum stress}

Alpha-synuclein was found as a main component of the Lewy bodies and the gene was found to be a causative gene of a rare autosomal dominant PD (Polymeropoulos et al., 1997). It has been reported that multiplication of the gene was to be the cause of the disease (Singleton et al., 2003). It has been suggested that aggregates of alpha-synuclein cause potentiation of oxidative stress, possibly with interaction with iron.
Synuclein was considered to be degraded in the proteasome. Knockout of the $26 \mathrm{~S}$ proteasome in the dopaminergic neurons induced "pale bodies", which is reported to be a prodrome of the Lewy bodies (Bedford et al., 2008).

Parkin and UCHL-1 are considered essential for ubiquitination of the unfolded proteins, and the gene mutations were found in some familial PD. It is considered that oxidative stress may lead a combination of Parkin and DJ-1, and the combination suffocates unfolded protein degradation (Kitada et al., 1998; Bonifati et al., 2003). $\mathrm{Mg}$ has also been reported to inhibit spontaneous and iron-induced aggregation of alpha-synuclein (Golts et al., 2002) (Figure 4).

\section{Low Mg and Parkinson's disease model}

In the course of investigations into the pathogenesis of the PDC, the present authors performed an experiment in which rats were exposed to restricted intake of $\mathrm{Ca}$ and/or $\mathrm{Mg}$ over two generations. This resulted in severe loss of dopaminergic neurons exclusively in the substantia nigra in 1-year-old rats that had been subjected to continuously low $\mathrm{Mg}$ intake (onefifth of the normal level) over generations

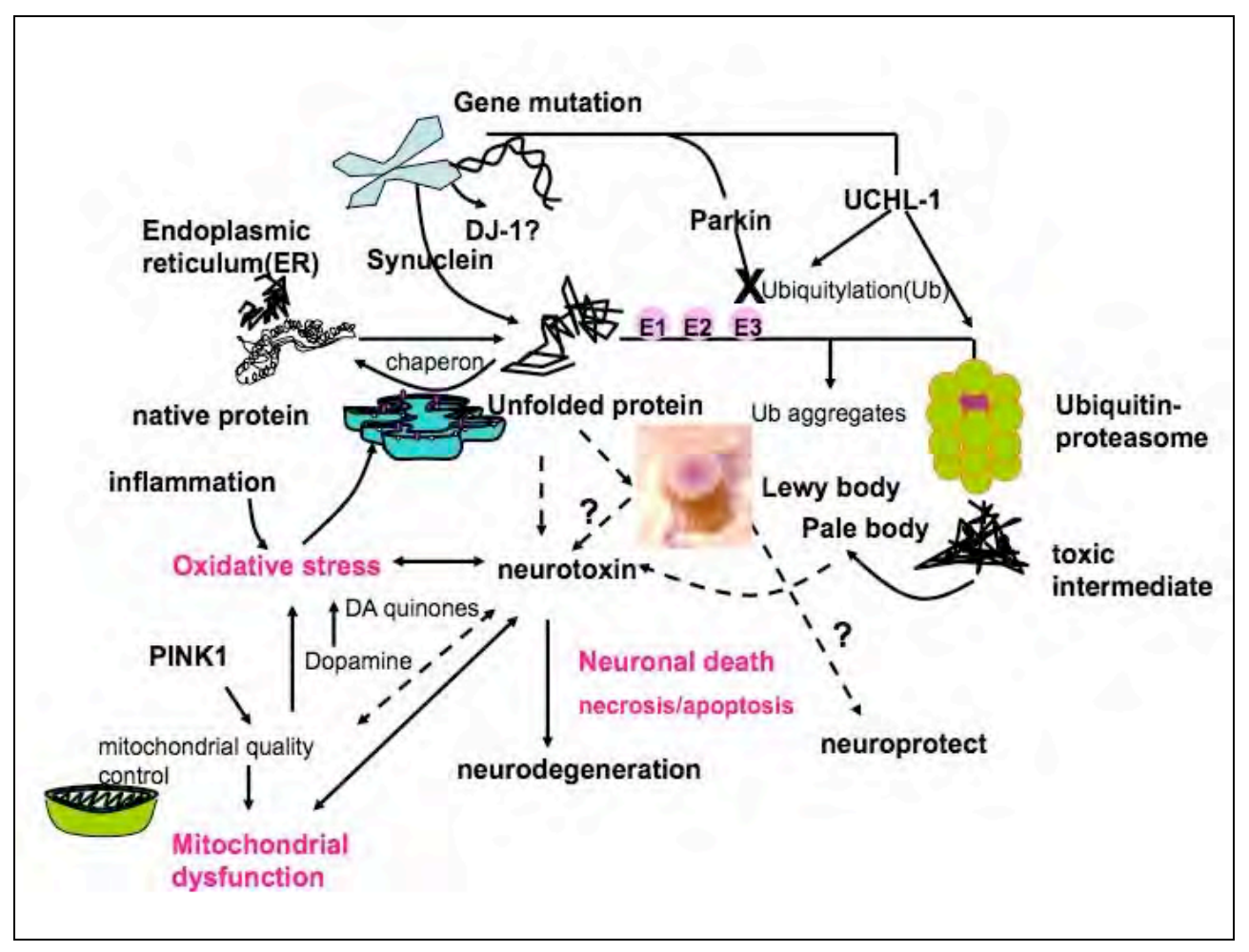

Figure 4. Scheme of possible pathomechanisms of dopaminergic neuron death in Parkinson's disease. 
(Oyanagi et al., 2006). This finding suggested a deep concern of low $\mathrm{Mg}$ intake over several generations to the pathogenesis of degeneration of the substantia nigra in humans.

\section{Therapeutic possibility by Mg for Parkinson's disease}

\section{As a blocker of the glutamatergic NMDA receptor}

$\mathrm{Mg}$ controls cytochrome $\mathrm{c}$ release in mitochondria (Eskes et al., 1998), and decreases the activity of N-methyl-D-aspartate (NMDA) receptors in excitotoxicity (Mayer et al., 1984). Mg treatment has also been shown to decrease cerebral infarct volume in rats in vivo (Lyden et al., 2000). The mechanism responsible for the neuroprotective effect of $\mathrm{Mg}$ has been considered to be reduced presynaptic release of the neurotrans-mitter glutamate (Lin et al., 2002), and blockade of the glutamatergic NMDA receptor (Nowak et al., 1984)(Figure 5). A relationship between decreased $\mathrm{Mg}$ concentration in serum and migraine has been reported in humans, and it has been suggested that migraine might be caused by hypersensitivity of the NMDA receptor to glutamic acid and certain other neuro-excitatory amino acids due to $\mathrm{Mg}$ depletion (Cojocaru et al., 2006). A decrease of cytosolic free $\mathrm{Mg}$ in the occipital lobe of PD patients has also been demonstrated by phosphorus magnetic response spectroscopy (Barbiroli et al., 1999).

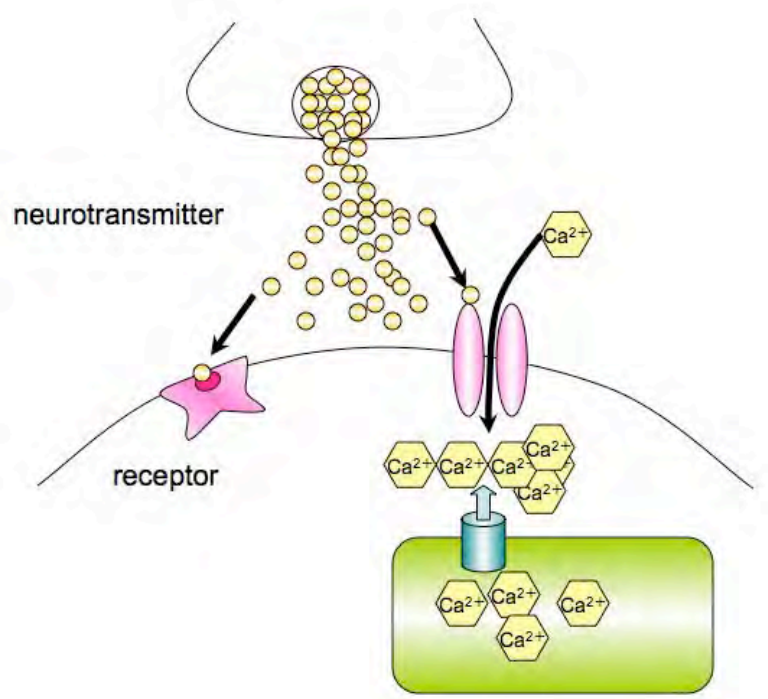

Figure 5. Metallic elements and neuron conduction.
Substantia nigra (rats, 1 year old)
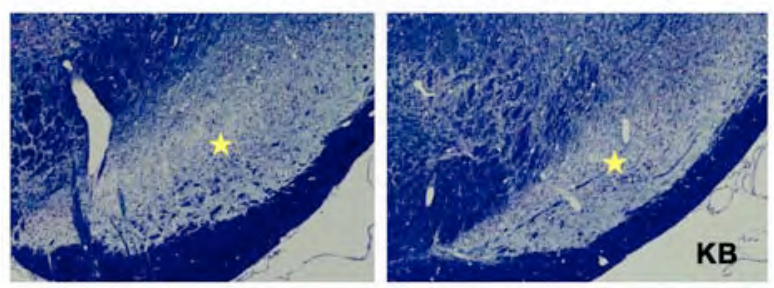

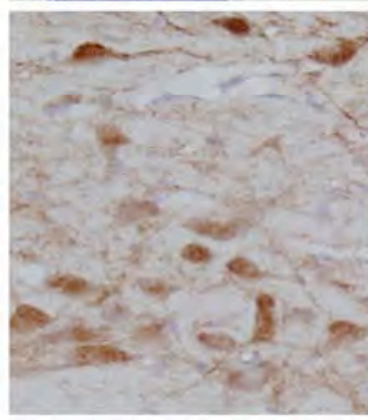

Control

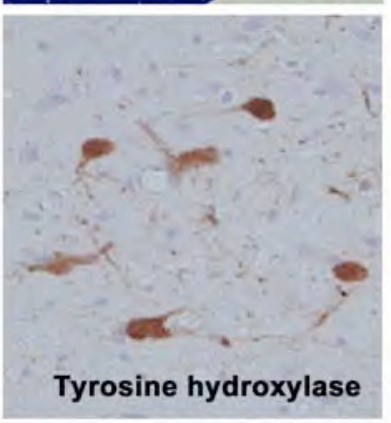

Mg deficiency
Figure 6. Severe atrophy and selective loss of dopaminergic neurons in the substantia nigra in rats with low $\mathrm{Mg}$ over generations (Oyanagi $\mathrm{K}$, et al., 2006).

\section{As an inhibiter of oxidative stress}

The present authors conducted a study to clarify the effects of $\mathrm{Mg}$ administration in a rat PD model involving culture of ventral mesencephalicstriatal cells with 1-methyl-4-phenylpyridinium $\left(\mathrm{MPP}^{+}\right)$, based on recent evidence for significant loss of dopaminergic neurons exclusively in the substantia nigra of 1-year-old rats after exposure to low $\mathrm{Mg}$ intake over generations (Oyanagi et al., 2006) (Figure 6). The results indicated that $\mathrm{Mg}$ might protect dopaminergic neurons in the substantia nigra from degeneration. The concentration of $\mathrm{Mg}$ in the culture medium varied from $0.8 \mathrm{mM}$, corresponding to the control condition, to $4.0 \mathrm{mM}$. Effects were estimated by counting the number of surviving dopaminergic neurons immunopositive for tyrosine hydroxylase and measuring the length of dopaminergic neurites. An increase in the concentration of $\mathrm{Mg}$ to $1.2 \mathrm{mM}$ significantly inhibited the toxicity of $\mathrm{MPP}^{+}$, and a concentration of $4.0 \mathrm{mM}$ completely prevented any decrease in the number of dopaminergic neurons. The length of dopaminergic neurites was significantly preserved in the presence of $\mathrm{Mg}$ at 1.2 and $4.0 \mathrm{mM}$. An increase in the concentration of $\mathrm{Mg}$ to 1.2 and $4.0 \mathrm{mM}$ led to a significant amelioration in the length of dopaminergic neurites after $\mathrm{MPP}^{+}$toxicity (Figure 7). 

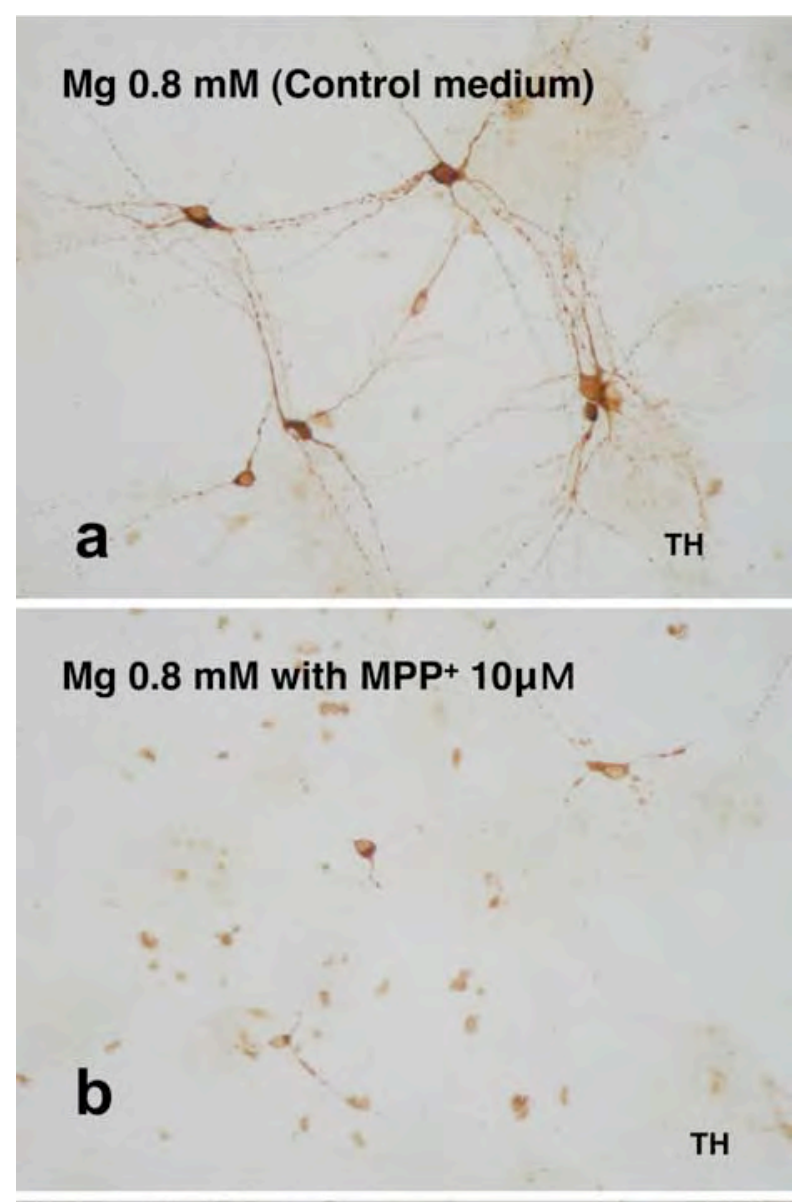

\section{Mg 1.2 mM with MPP+ $10 \mu \mathrm{M}$}

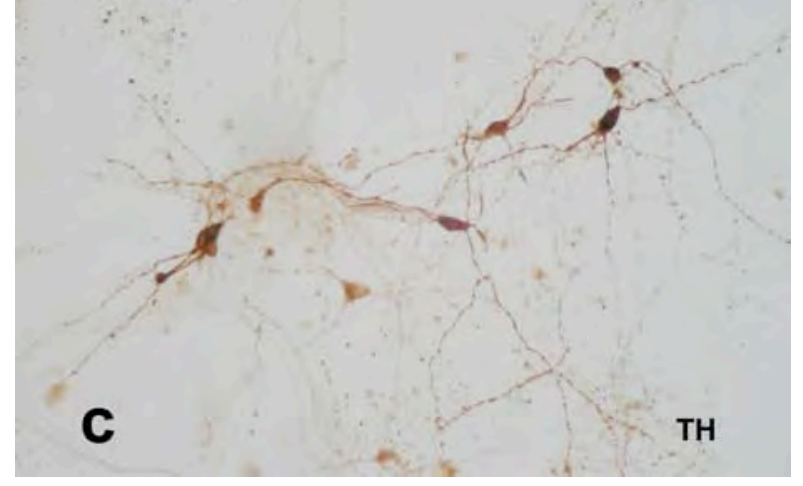

Figure 7. Prevention of $\mathrm{MPP}^{+}$toxicity by $\mathrm{Mg}$ in a Parkinson model (Hashimoto et al., 2008). a: normal condition of cultured nigral dopaminergic neurons. $\mathbf{b}$ : severe loss of dopaminergic neurons and the neuritis after $\mathrm{MPP}^{+}$administration. c: An increase in the concentration of $\mathrm{Mg}$ to $1.2 \mathrm{mM}$ significantly inhibited the toxicity of MPP ${ }^{+}$.
This was the first report to document a significant and striking effect of $\mathrm{Mg}$ for prevention of neurite and neuron pathology, and also amelioration of neurite pathology in a PD model. In addition, an increase in the $\mathrm{Mg}$ concentration to 1.2, 2.0, and $4.0 \mathrm{mM}$ did not induce any degenerative features in the cultured dopaminergic cells, suggesting that a $\mathrm{Mg}$ concentration of up to $4.0 \mathrm{mM}$ in the extracellular space might not induce any neuron degeneration in humans. $\mathrm{Mg}$ oxide per os has often been used as a laxative for patients with $P D$, but is reportedly not absorbed in the bowels, thus not affecting the serum concentration of $\mathrm{Mg}$ (Sakimura et al., 1998). Recent studies by the authors using mice also established that no significant alteration was found in the CSF of B6 mice injected intraperitoneally with $\mathrm{Mg}$, even though the serum $\mathrm{Mg}$ concentration was significantly increased (Sun et al., 2009). Further research is necessary to find $\mathrm{Mg}$ compounds that can easily be absorbed in the bowels and pass through the blood-brain barrier, like Mg-Lthreonate (Slutsky et al., 2010) and besides, via transporters that can carry Mg through the bowel mucosa, blood-brain barrier and plasma membrane of neurons.

\section{Acknowledgements}

The authors are indebted to Dr. M. Yasui, Yasui Clinic, Wakayama, Japan, Dr. K. Nishi, Nishi Clinic, Tokyo, Japan, Dr. J. Nagasao, Ms. E. Kawakami, Dr. L. Sun and Dr. Y. Piao, Department of Neuropathology, Tokyo Metropolitan Institute for Neuroscience, Tokyo, Japan, Dr. S. Takahama, Graduate School of Frontier Biosciences, Osaka University, Osaka, Japan, Ms. Y. Kosugi, Department of Environmental Health and Toxicology, Division of Environmental Health, Tokyo Metropolitan Institute of Public Health, Tokyo, Japan for their valuable advice and technical assistance. This work was supported in part by grants from the Japanese Ministry of Education, Science, Sports and Culture (Basic Research (C) \#20500330 to TH), a Yujin Memorial Grant (to KO) and The Salt Science Research Foundation, No. 1028 (to KO). 


\section{References}

Alam ZI, Daniel SE, Lees AJ, Marsden CD, Jenner P, Halliwell B (1997a) A generalized increase in protein carbonyls in the brain in Parkinson's but incidental Lewy body disease. J Neurochem 69:1326-9.

Alam ZI, Jenner A, Daniel SE, Lees AJ, Cairns N, Marsden CD, Jenner P, Halliwell B (1997b) Oxidative DNA damage in Parkinsonian brain; An apparent selective increase in 8-Hydroxyguanine levels in substantia nigra. J Neurochem 69:1196-203.

Ambani LM, Van Woert MH, Murphy S (1975) Brain peroxidase and catalase in Parkinson disease. Arch Neurol 32:114-8.

Barbiroli B, Martinelli P, Patuelli A, Lodi R, lotti S, Cortelli P, Montagna P (1999) Phosphorus magnetic resonance spectroscopy in multiple system atrophy and Parkinson's disease. Mov Disord 14:430-5.

Bedford L, Hay D, Devoy A, Paine S, Powe DG, Seth R, Gray $T$, Topham I, Fone K, Rezvani N, Mee M, Soane T, Layfield R, Sheppard PW, Ebendal T, Usoskin D, Lowe J, Mayer RJ (2008) Depletion of 265S proteasomes in mouse brain neurons caused neurodegeneration and Lewy-like inclusions resembling human pale bodies. J Neurosci 28:8189-98.

Bocca B, Alimonti A, Senofonte O, Pino A, Violante N, Petrucci F, Sancesario G, Forte G (2006) Metal changes in CSF and peripheral compartments of parkinsonian patients. J Neurol Sci 248:23-30.

Bonifati V, Rizzu P, van Baren MJ, Schaap O, Breedveld GJ, Krieger E, Dekker MC, Squitieri F, Ibanez $P$, Joosse $M$, van Dongen JW, Vanacore $N$, van Swieten JC, Brice A, Meco G, van Duijn CM, Oostra BA, Heutink P (2003) Mutations in the DJ-1 gene associated with autosomal recessive early-onset parkinsonism. Science 299:256-9.

Bove J, Prou D, Perier C, Przedborski S (2005) Toxininduced models of Parkinson's disease.

Neurotherapeutics 2:484-94.

Champy P, Hoglinger GU, Feger J, Gleye C, Hocquemiller R, Laurents A, Guerineau V, Laprevote O, Medija F, Lombes A, Michel P P, Lannuzel A, Hirsch EC, Ruberg M (2004) Annonacin, a lipophilic inhibitior of mitochondrial complex I, induces nigral and striatal neurodegeneration in rats. $J$ Neurochem 88:63-9.

Chen K-M, Chase TN (1985) Parkinsonism-dementia. In: Handbook of Clinical Neurology Vol. 49 (Vinken PJ, Bruyn GW, Klawans HL, eds), Amsterdam, Elsevier, pp. 167-83.
Cojocaru M, Cojocaru IM, Muuroi C, Botezat M, Lazar $L$, Duruta A (2006) Serum magnesium concentrations in migraine with aura. In: Advances in Magnesium Research: New Data (Nechifor M, Porr PJ, eds), Cluj Napoca, Casa Cartii de Stiinta Press, pp 31-5.

Dalfo E, Protero-Otin M, Ayala V, Martinez A, Pamplona R, Ferrer I (2005) Evidence of oxidative stress in the neocortex in incidental Lewy body disease. J Neuropathol Exp Neurol 64:816-30.

Eskes R, Antonsson B, Osen-Sand A, Montessuit S, Richter C, Sadoul R, Mazzei G, Nichols A, Martinou JC (1998) Bax-induced cytochrome $C$ release from mitochondria is independent of the permeability transition pore but highly dependent on $\mathrm{Mg}^{2+}$ ions. $J$ Cell Biol 143:217-24.

Fornai F, Lenzi P, Gesi M, Ferrucci M, Lazzeri G, Busceti $\mathrm{CL}$, Ruffoli R, Soldani $\mathrm{P}$, Ruggieri $\mathrm{S}$, Alessandri MG, Paparelli A (2003) Fine structure and biochemical mechanisms underlying nigrostriatal inclusions and cell death after proteasome inhibition. $J$ Neurosci 23:8955-66.

Garruto RM, Yanagihara R, Gajdusek DC. Arion DM (1984) Concentration on heavy metals and essential minerals in garden soil and drinking water in the Western Pacific. In: Amyotrophic Lateral Sclerosis in Asia and Oceania (Chen KM, Yase Y, eds), Taipei, National Taiwan University, 265-330.

Golts N, Snyder H, Frasier M, theisler C, Choi P, Wolozin B (2002) Magnesium inhibits spontaneous and iron-induced aggregation of alpha-synuclein. J Biol Chem 277:16116-23.

Hasegawa M, Arai T, Akiyama H, Nonaka T, Hiroshi Mori, Hashimoto T, Yamazaki M, Oyanagi K (2007) TDP-43 is deposited in the Guam parkinsonismdementia complex brains. Brain 130:1386-94.

Hattori N, Tanaka M, Ozawa T, Mizuno Y (1991) Immunohistochemical Studies on complexes I, II, III, and VI of mitochondoria in Parkinson's disease. Ann Neurol 30:563-71.

Hirano A, Kurland LT, Krooth RS, Lessell S (1961a) Parkinsonism-dementia complex, an endemic disease on the island of Guam. I. Clinical features. Brain 84:642-61.

Hirano A, Malamud N, Kurland LT (1961b)

Parkinsonism-dementia complex, an endemic disease on the Island of Guam. II. Pathological features. Brain 84:662-79. 
Jellinger KA (1999) The role of iron in neurodegeneration : prospects for pharmacotherapy of Parkinson's disease. Drugs Aging 14:115-40.

Kitada T, Asakawa S, Hattori N, Matsumine $\mathrm{H}$, Yamamura Y, Minoshima S, Yokochi M, Mizuno Y, Shimizu N (1998) Mutations in the parkin gene cause autosomal recessive juvenile parkinsonism. Nature 392:605-8.

Lewy FH (1912) Paralysis agitans. In: Pathologische Anatomi (Lewandowsky M, ed). Handbuch der Neurologie, vol. 3. Berlin, Springer, 920-33.

Lin JY, Chung SY, Lin MC, Cheng FC (2002) Effects of magnesium sulfate on energy metabolites and glutamate in the cortex during focal cerebral ischemia and reperfusion in the gerbil monitored by a duralprobe microdialysis technique. Life Sci 71:803-11.

Lyden P, Wahlgren NG (2000) Mechanisms of action of neuroprotectants in stroke. J Stroke Cerebrovasc Dis 9:9-14.

Mayer ML, Westbrook GL, Guthrie PB (1984) Voltagedependent block by $\mathrm{Mg}^{2+}$ of NMDA responses in spinal cord neurones. Nature 309:261-3.

Mizuno Y, Matuda S, Yoshino $\mathrm{H}$, Mori $\mathrm{H}$, Hattori N, Ikebe S (1994) An immunohistochemical study on alpha-ketoglutarate dehydrogenase complex in Parkinson's disease. Ann Neurol 35:204-10.

Nakamura K, Bindokas VP, Marks JD, Wright DA, Frim DM, Miller RJ, Kang UJ (2000) The selective toxicity of 1-methyl-4-phenylpyridinium to dopaminergic neurons: the role of mitochondrial complex I and reactive oxygen species revisited. Mol Pharmacol 58: 271-8.

Nowak L, Bregestovski P, Ascher P, Herber A, Prochiantz A (1984) Magnesium gates glutamateactivated channels in mouse central neurons. Nature 307:462-5.

Oyanagi K (2005) The nature of the parkinsonismdementia complex and amyotrophic lateral sclerosis of Guam and magnesium deficiency. Parkinson Rel Dis 11:S17-S23.

Oyanagi, K, Kawakami E, Kikuchi-Horie K, Ohara K, Ogata K, Takahama S, Wada M, Kihira T, Yasui M (2006) Magnesium deficiency over generations in rats with special references to the pathogenesis of the parkinsonism-dementia complex and amyotrophic lateral sclerosis of Guam. Neuropathology 26:115-28.
Oyanagi K, Makifuchi T, Ohtoh T, Chen K-M, Gajdusek DC, Chase TN (1997) Distinct pathological features of the Gallyas- and tau-positive glia in the parkinsonismdementia complex and amyotrophic lateral sclerosis of Guam. J Neuropathol Exp Neurol 56:308-16.

Parkinson J (1817) An Essay on the Shaking Palsy. (Neely and Jones, ed) Whittingham and Rowland for Sherwood, London.

Polymeropoulos $\mathrm{MH}$, Lavedan $\mathrm{C}$, Leroy $\mathrm{E}$, Ide $\mathrm{SE}$, Dehejia A, Dutra A, Pike B, Root H, Rubinstein J, Boyer $R$, StenroosES, Chandrasekharappa S, Asanassiadou A, Papapetropoulos T, Johnston WG, Lazzarini AM, Duvoisin RC, Di lorio G, Golbe LI, Nussbaum RL (1997) Mutation in the alpha-synuclein gene identified in families with Parkinson's disease. Science 276:2045-7.

Sakimura K, Machino H, Miyoshi K, Nakata K, Ueda K, Ide M, Minegishi A, Harada Y (1998) Clinical evaluation of YO-106 (magnesium oxide tablets) in the treatment of Habitual constipation-A cross over study with Magnesium oxide powders (JP)- Jpn Pharmacol Ther 26:1027-53.

Saris NE, Mervaala E, Karppanen H, Khawaja JA, Lewenstam A (2000) Magnesium. An update on physiological, clinical and analytical aspects. Clin Chim Acta 294:1-26.

Singleton $A B$, Farrer $M$, Johnson J, Singleton A, Hague $S$, Kachergus J, Hulihan $M$, Peuralinna T, Dutra $A$, Nussbaum R, Lincoln S, Crawley A, Hanson M, Maraganore D, Adler C, Cookson MR, Muenter M, Baptista M, Miller D, Blancato J, Hardy J, Gwinn-Hardy K (2003) Alpha-synclein locus triplication causes Parkinson's disease. Science 302:841

Slutsky I, Abumaria N, Wu LJ, Huang C, Zhang L, Li B, Zhao X, Govindarajan A, Zhao MG, Zhuo M, Tonegawa $S$, Liu G (2010) Enhancement of learning and memory by elevating brain magnesium. Neuron 65:165-77.

Smeyne RJ, Jackson-Lewis V (2005) The MPTP model of Parkinson's disease. Brain Res. Mol Brain Res 134:57-66.

Sun L, Kosugi Y, Kawakami E, Piao Y-S, Hashimoto T, Oyanagi K (2009) Magnesium concentration in the cerebrospinal fluid of mice and its response to changes in serum magnesium concentration. Magnes Res 22:266-72.

Tretiakoff C (1919) Contribution a l'etude del l'Anatomie pathologique du Locus Niger de Soemmering avec quelques deduction relatives a la pathogenie des troubles du tonus musculaire et de la maladie de Parkinson. Theses de Paris. 
Uitti Rj, Rajput AH, Rozdilsky B, Bickis M, Wollin T, Yuen WK (1989) Regional metal concentrations in Parkinson's disease, other chronic neurological diseases, and control brains. Can J Neurol Sci 16:310-4.

Valente EM, Abou-Sleiman PM, Caputo V, Muqit MMK, Harvey K, Gispert S, Ali Z, Del Turco D, Bentivoglio AR, Healy DG, Albanese A, Nussbaum R, GonzálezMaldonado R, Deller T, Salvi S, Cortelli P, Gilks WP, Latchman DS, Harvey RJ, Dallapiccola B, Auburger G, Wood NW (2004) Hereditary Early-Onset Parkinson's Disease Caused by Mutations in PINK1. Science 304:1158-60.

Yamazaki M, Hasegawa M, Mori O, Murayama S, Tsuchiya K, Ikeda K, Chen K-M, Katayama Y, Oyanagi K (2005) Tau-positive fine granules in the cerebral white matter: a novel finding among tauopathies exclusive to parkinsonism-dementia complex of Guam. J Neuropathol Exp Neurol 64:1-8.
Yase Y (1978) ALS in the Kii peninsula: one possible etiological hypothesis. In: Amyotrophic lateral sclerosis (Tsubaki T, Toyokura Y, eds), Tokyo, University of Tokyo Press, pp. 307-18.

Yoritaka A, Hattori N, Uchida K, Tanaka M, Stadtman ER, Mizuno K (1996) Immunohistochemical detection of 4-hydroxynonenal protein adducts in Parkinson's disease. Proc Natl Acad Sci USA 93:2696-701.

Zhang J, Perry G, Smith MA, Robertson D, Olson SJ, Graham DG, Montine TJ (1999) Parkinson's disease is associated with oxidative damage to cytoplasmic DNA and RNA in substantia nigra neurons. Am J Pathol 154:1423-9. 


\section{Section 3}

Involvement of Magnesium in Psychiatric Diseases 



\title{
Magnesium in Alzheimer's disease
}

\author{
Dehua Chui, ${ }^{1, *}$ \' Zheng Chen, ${ }^{2, *}$ Jia Yu, ${ }^{2, *}$ Honglin Zhang, ${ }^{2}$ Weishan Wang, ${ }^{2}$ Yuetao Song, ${ }^{2}$ Huan Yang ${ }^{1}$ and \\ Liang Zhou ${ }^{1}$
}

${ }^{1}$ Neuroscience Research Institute \& Department of Neurobiology, Key Laboratory for Neuroscience, Ministry of Education \& Ministry of Public Health, Health Science Center, Peking University, Beijing 100191, China.

2 Department of Psychiatry \& Institute for Geriatric Clinic and Rehabilitation, Beijing Geriatric Hospital, Beijing 100095, China.

* Dehua Chui, Z. Chen and J. Yu contributed equally to this work.

$\triangle$ dchui@bjmu.edu.cn

\begin{abstract}
Alzheimer's disease (AD) is the most common form of dementia. It is characterized by a progressive cognitive impairment clinically, and excessive deposits of aggregated amyloid- $\beta$ (A $\beta$ ) peptides pathologically. Environmental factors, including nutrition and metal elements, are implicated in the pathophysiology of AD. Magnesium (Mg) affects many biochemical mechanisms vital for neuronal properties and synaptic plasticity, including the response of $\mathrm{N}$-methyl $\mathrm{D}$-aspartate (NMDA) receptors to excitatory amino acids, stability and viscosity of the cell membrane, and antagonism of calcium. $\mathrm{Mg}$ levels were found to be decreased in various tissues of $A D$ patients and negatively correlated with clinical deterioration. Moreover, $\mathrm{Mg}$ was demonstrated to modulate the trafficking and processing of amyloid- $\beta$ precursor protein, which plays a central role in the pathogenesis of AD. Here, we review in vitro and in vivo data that indicated a role for magnesium in many biological and clinical aspects of $A D$.
\end{abstract}

\section{Alzheimer's disease}

Alzheimer's disease (AD) is the most prevalent neurodegenerative disease in elderly people, affecting approximate $6-8 \%$ of all individuals over the age of 65 years. $A D$ is characterized by progressive cognitive impairment and distinct neuropathological lesions in the brain, including intracellular neurofibrillary tangles, and extracellular, parenchymal and cerebrovascular senile plaques (Braak and Braak, 1991). Senile plaques are mainly constituted of a 39-42 amino acid peptide, amyloid- $\beta$ protein $(A \beta)$ (Glenner and Wong, 1984; Masters et al., 1985), which is generally accepted as being neurotoxic and playing a central role in the pathogenesis of neuronal dysfunction and synaptic failure in Alzheimer's disease (Selkoe, 1991; Hardy and Selkoe, 2002). $A \beta$ is derived from full-length amyloid- $\beta$ precursor protein (APP) (Kang et al., 1987; Qi-Takahara et al., 2005), which is a type I trans-membrane protein composed of a large extracellular domain, a short transmembrane domain, and a cytoplasmic tail, by sequential proteolytic cleavages by $\beta$-secretase and $\gamma$ secretase. The $\beta$-cleavage of APP, catalysed by the well characterized transmembrane aspartyl protease $\beta$-site APP-cleaving enzyme (BACE)
(Hussain et al., 1999; Sinha et al., 1999; Yan et al., 1999; Haniu et al., 2000), cleaves APP at the NH2terminus of the $A \beta$ sequence (Seubert et al., 1993) to generates a soluble version of APP (SAPP) and a 99-residue $\mathrm{COOH}$-terminal fragment (CTF $\beta$ or C99) which remains membrane bound.

C99 is further cleaved to release $A \beta$ of varying lengths, predominantly $A \beta 40$ and $A \beta 42$ (Selkoe, 2001; Hussain et al., 1999; Price et al., 1998; Sinha et al., 1999; Christensen et al., 2004), by an atypical aspartyl protease, $\gamma$-secretase complex which contains at least four different proteins, namely Aph-1, nicastrin, presenilin, and Pen-2 (De Strooper, 2003; Edbauer et al., 2003). Proteolysis by $\mathrm{Y}$-secretase is heterogeneous; most of the full-length $A \beta$ species produced is a 40residue peptide $(A \beta 40)$, whereas a small proportion is a 42-residue $\mathrm{COOH}$-terminal variant (Aß42) (Esler and Wolfe, 2001). However, prior processing of APP by $\alpha$-secretase precludes the formation of the neurotoxic $A \beta$. It cuts APP within the $A \beta$ region (between residues Lys 16 and Leu17 of $A \beta$ ), generating a SAPP $\alpha$ and a membraneanchored 83-residue C-terminal fragment (CTF $\alpha$ or (83), which is also a substrate of $\gamma$-secretase (Esch et al., 1990; Sisodia, 1992). $\alpha$-Secretase is thought to be a metalloprotease, such as TNF- $\alpha$ 
converting enzyme (TACE) or a disintegrin and metalloprotease 10 (ADAM10) (Lammich et al., 1999). Secreted APP exerts proliferative actions in a variety of cell types as well as neurotropic and neuroprotective effects (Mucke et al., 1996).

Synaptic failure in $A D$ is caused by accumulation and oligomerization of $A \beta 42$ in limbic and association cortices (Selkoe, 2002). Mutations in the APP gene or presenilin (PS) 1 or 2 genes, which cause an autosomal dominant early onset familial $A D$ ( $<5 \%$ of $A D$ patients), increases the relative production of $A \beta 42$ (Wiltfang et al., 2001). In the majority of patients with so-called sporadic late-onset $A D$, an age-dependent accumulation of $A \beta$, caused by disturbed dynamic balance between anabolic and catabolic activities, has been implicated (Selkoe, 1999; 2001b). Also, environmental factors, such as metallic elements may play a protective or disruptive role in the pathogenesis of $A D$ (for review, see Adlard and Bush, 2006; Shcherbatykh and Carpenter, 2007). Different metals may be involved in multiple aspects of the disease process, such as the regulation of APP gene expression and mRNA translation, the proteolytic processing of APP, the aggregation and degradation of $A \beta$, and the formation of neurofibrillary tangles. Heavy metals (e.g. lead, mercury and cadmium) are neurotoxic and associated with intellectual impairment (Bleecker et al., 2005). Recent studies have implicated lead exposure in the subsequent elevation of APP and $A \beta$ in animals (Basha et al., 2005b) as well as in the aggregation of synthetic $A \beta_{1-40}$ in vitro (Basha et al., 2005a). In the case of aluminium, another "toxic" metal, its relevance to $A D$ is ascribed to the involvement in the formation of paired helical filaments (PHF), the aggregation and toxicity of $A \beta$, and the generation of oxidative species (for review, see Gupta et al., 2005). Transition metals (e.g. copper, zinc, and iron, which are essential in cell biology) can induce $A \beta$ aggregation (Huang et al., 2004; Mantyh et al., 1993) and are found concentrated in and around the amyloid plaques in the AD brain (Lovell et al., 1998). Disturbed homeostasis of these biometals in the AD brain (decreased copper levels, and increased concentrations of iron, zinc, and manganese) has been reported (Cornett et al., 1998; Deibel et al., 1996). An imbalance of zinc and copper has been shown to significantly alter APP processing and $A \beta$ generation in relevant animal models (Bayer et al., 2003; Borchardt et al., 1999; Phinney et al., 2003; Sparks and Schreurs, 2003; Lee et al., 2002; Friedlich et al., 2004).

\section{Neurological function of magnesium}

The magnesium ion, $\mathrm{Mg}^{2+}$, is the second most abundant intracellular cation, serving to stabilize nucleic acid and protein structure (Subirana et al., 2003; Brion and Westhof, 1997), and regulating over 300 enzymes as a cofactor (Romani et al., 1992; 1993; Zhao et al., 2002), including ATPrelated enzymatic reactions (Hirata et al., 2002; Ko et al., 1999). Physiological concentrations of $\mathrm{Mg}$ are essential for synaptic conduction, and required for normal functioning of the nervous system. It has various effects at different concentrations on intellectual and neuronal functions via many bio-chemical mechanisms, including NMDA-receptor responses to excitatory amino acids and calcium influx (Nowak et al., 1984; Mayer et al., 1984; Vandenberg et al., 1987; Matsuda et al., 1987), inhibition of calcium channels (Iseri and French, 1984) and glutamate release (Lin et al., 2002), effects on cell membrane fluidity and stability (Ebel and Gunther, 1980), and toxic effects of calcium (Alvarez-Leefmans et al., 1987). These mechanisms have important roles in chronic neuronal degeneration and subsequent development of dementia.

The role of $\mathrm{Mg}$ in degenerative diseases has been the focus of increased attention in recent years. Continuous low $\mathrm{Mg}$ intake for two generations induces exclusive loss of dopaminergic neurons in rats (Oyanagi, 2005), and may support the $\mathrm{Mg}$ hypothesis in the pathogenesis of parkinsonismdementia complex (PDC) of Guam. Mg supplementation prevents the loss of dopaminergic neurons and ameliorates neurite pathology in a PD model, indicating a role of $\mathrm{Mg}$ in protection of dopaminergic neurons in the substantia nigra from degeneration (Oyanagi et al., 2006; Hashimoto et al., 2008). Also, Mg at concentrations $>0.75 \mathrm{mM}$ inhibits the aggregation of $\alpha$-synuclein, induced either spontaneously or by incubation with iron (Golts et al., 2002). Microinjection of magnesium into cells caused microtubule disassembly (Prescott et al., 1988). $\mathrm{Mg}^{2+}$ and $\mathrm{Ca}^{2+}$ effectively induced formation of approximately $340 \mathrm{kD}$ aggregates of paired helical filament tau (PHF-tau) obtained from 
corticobasal degeneration (CBD) and $A D$ but not normal tau proteins isolated from fetal and adult brains, as determined by sodium dodecyl sulphate (SDS)-polyacrylamide gel electrophoresis and immunoblotting (Yang et al., 1999). This finding suggests regional elevation of these ions may trigger pathological deposition of PHF-tau in certain neurodegenerative disorders.

\section{Magnesium in AD}

Recent evidence suggests that Mg was implicated in the pathogenesis of $A D$. Mg levels were decreased in the serum and brain tissues of $A D$ patients in clinical, experimental and autopsy studies (Durlach, 1990; Glick, 1990a; Lemke, 1995; Andrási et al., 2000; 2005; Vural et al., 2010). Moreover, serum Mg levels in AD patients negatively correlated with the Global Deterioration Scale (GDS) and the Clinical Dementia Rating (CDR) (Cilliler et al., 2007). A causal relationship between low $\mathrm{Mg}$ in hippocampal neurons and impairment of learning was also demonstrated in aged rats (Landfield et al., 1984). Magnesium deficiency can lead to specific impairments in emotional memory (Bardgett et al., 2005; Bardgett et al., 2007), while magnesium therapy facilitates cognitive function recovery following brain injury; however, there are task and dosedependent aspects to this recovery (Enomoto et al., 2005; Hoane, 2005; Hoane, 2007). Increasing brain magnesium leads to the enhancement of both short-term synaptic facilitation and longterm potentiation and improves learning and memory functions in rats (Slutsky et al., 2010). Interestingly, treatment of dementia patients with nutritional $\mathrm{Mg}$ support efficiently improved memory and other symptoms (Glick et al., 1990b). However, therapeutic administration of $\mathrm{Mg}$ is still controversial regarding the treatment of $A D$, and high doses of Mg may have potential detrimental side effects (Clark and Brown, 1992; Fung et al., 1995; Hallak, 1998; Ladner and Lee, 1999).

Neuronal degeneration occurs in PS1 mutant mice without extracellular $A \beta$ deposits, suggesting it is caused by the accumulation of intracellular A 342 (Chui et al., 1999). Deposits of intracellular $A \beta 42$ are correlated with apoptotic cell death in $A D$ brains (Chui et al., 2001). The $A \beta$ is derived from APP through sequential cleavages by $\beta$-and $\gamma$-secretases, whose enzymatic activities are tightly controlled by subcellular localization. Thus, delineation of how the intracellular trafficking of these secretases and APP are regulated is important for understanding $A D$ pathogenesis. Although APP trafficking is regulated by multiple factors including PS1 (Cai et al., 2003), a major component of the $\gamma$-secretase complex, and phospholipase D1, a phospholipidmodifying enzyme, APP can reciprocally regulate PS1 trafficking. APP deficiency results in faster transport of PS1 from the trans-Golgi network to the cell surface and increased steady state levels of PS1 at the cell surface, which can be reversed by restoring APP levels (Liu et al., 2009). However, it is not known whether altered magnesium level may also affect APP trafficking or/and processing. Recently, it has been demonstrated that magnesium modulated APP processing in a time- and dose-dependent manner: extracellular magnesium $\left(\left[\mathrm{Mg}^{2+}\right]_{0}\right)$ at high doses increased CTF $\alpha$ level and SAPP $\alpha$ release. In contrast, $\left[\mathrm{Mg}^{2+}\right]_{0}$ at low doses enhanced CTF $\beta$ accumulation and $A \beta$ secretion (Yu et al., 2010). The mechanism of how varying magnesium concentrations led to shifts between $\alpha$ - and $\beta$-secretase cleavage of APP might be partially explained by the evidence that $\left[\mathrm{Mg}^{2+}\right]_{0}$ at high doses promoted retention of APP on plasma membrane, whereas $\left[\mathrm{Mg}^{2+}\right]_{0}$ at low doses reduced cell surface APP level (Yu et al., 2010). All APP family members are predominantly cleaved in the late secretory pathway, including the plasma membrane and endosomes (Yamazaki et al., 1996). Further, different secretase activities show distinct subcellular localization, namely $\alpha$ secretase at the plasma membrane (Lammich et al., 1999; Skovronsky et al., 2000) and $\beta / \gamma$ secretases within endocytic compartments (Vassar et al., 1999; Huse et al., 2000 ; Cupers et al., 2001 ; Kaether et al., 2002; Ray et al., 1999). Because targeting of APP to distinct subcellular compartments determines processing into amyloidogenic or non-amyloidogenic products, much attention has been focused on factors that regulate APP trafficking. Interestingly, several adaptor proteins are known to influence APP transport and processing. For example, Fspondin, a secreted factor that binds to the extracellular domain of APP (Ho and Sudhof, 2004), has been shown to increase levels of cell surface APP, promote $\alpha$-cleavage of APP, and 
decrease $\beta$-cleavage of APP (Hoe et al., 2005). Similarly, the extracellular matrix protein Reelin caused increased surface APP and a preference for $\alpha$-cleavage over $\beta$-cleavage (Hoe et al., 2006b). These findings suggest that trafficking and proteolysis of APP are regulated together. Thus a function of $\left[\mathrm{Mg}^{2+}\right]_{0}$ in APP transport from/to the cell surface might be a possible explanation for its modulation of APP processing. In the light of $\mathrm{Mg}^{2+}$ as an antagonist of the NMDA receptor, our finding is corroborated by the previous report that chronic NMDA receptor activation decreased $\alpha$-secretase-mediated APP processing and increased $A \beta$ production in cultured cortical neurons (Lesne et al., 2005). Furthermore, several lines of evidence suggest that APP metabolism and $A \beta$ levels are closely correlated with neural activity in animals (Fazeli et al., 1994; Turner et al., 2004; Cirrito et al., 2005; 2008) and humans (Buckner et al., 2005). It has been demonstrated that decreasing neuronal activity by high $\left[\mathrm{Mg}^{2+}\right]_{0}\left(10 \mathrm{mM} \quad \mathrm{MgCl}_{2}\right)$ resulted in significant reduction of $A \beta$ secretion, which may involve a change in APP processing (Kamenetz et al., 2003). However, the precise functional mechanism of how magnesium regulates APP transport and whether magnesium interacts with $\alpha$ - and $\beta$-secretase, or regulates enzyme activity, or their subcellular localization, remains undetermined but will be part of our future analysis.

The dose dependent response of SAPP $\alpha$ to increasing $\left[\mathrm{Mg}^{2+}\right]_{0}$ implies high concentrations of $\mathrm{Mg}$ may exert protective effects against $A D$. Various studies have strongly established that secreted SAPP $\alpha$ possesses potent neurotrophic and neuroprotective activities against excitotoxic and oxidative insults (Mattson et al., 1993; Schubert et al., 1993), p53-mediated apoptosis (Xu et al., 1999), and the proapoptotic action of mutant PS1 by activating the transcription factor NF-KB (Guo et al., 1998). Moreover, SAPP $\alpha$ stimulates neurite outgrowth (Small et al., 1994), regulates synaptogenesis (Morimoto et al., 1998), exerts trophic effects on cerebral neurons in culture (Araki et al., 1991), stabilizes neuronal calcium homeostasis and protects hippocampal and cortical neurons against the toxic effects of glutamate and $A \beta$ peptides (Furukawa et al., 1996). It also has been shown that intracerebroventricular administration of secreted forms of SAPPa to amnestic mice has potent memory-enhancing effects and blocks learning deficits induced by scopolamine (Meziane et al., 1998).

Secreted $A \beta$ increased upon low $\left[\mathrm{Mg}^{2+}\right]_{0}(0.0$ and $0.4 \mathrm{mM}$ ) compared with physiological concentration of $\mathrm{Mg}$ (i.e. $0.8 \mathrm{mM}$ ), whereas high $\left[\mathrm{Mg}^{2+}\right]$ 。 $(1.2,1.6,4.0 \mathrm{mM})$ could not significantly lower total extracellular $A \beta$ level (Yu et al., 2010). The data are consistent with several reports showing a dissociation between SAPP $\alpha$ release and $A B$ generation both in vitro or in vivo (Loefler and Huber, 1993; Querfurth et al., 1994; Dyrks et al., 1994; LeBlanc et al., 1998; Rossner et al., 2000), suggesting that there might be a more complex regulatory mechanism of these two processing events of APP. For instance, constitutive activation of PKC in guinea pig brain increased SAPP $\alpha$ secretion without any effect on secreted $A \beta$ (Rossner et al., 2000), suggesting that the $\alpha$ and $\beta$-secretase pathways may be differentially controlled. Because Yu et al., (2010) examined the effects of $\mathrm{Mg}$ only on the pathologically high production of $A \beta$, the modulation of the physiological $A \beta$ production by $\mathrm{Mg}$ needs to be established in future studies. The steady-state level of $A \beta$ peptide is determined by the rate of production from APP via $\beta$ - and $\gamma$-secretases and degradation by the activity of several degradative enzymes, including neprilysin (Hama et al., 2001; Iwata et al., 2001; Shirotani et al., 2001; Leissring et al., 2003; Marr et al., 2004; review see Wang et al., 2006), insulin degrading enzyme (IDE) (Kurochkin et al., 1994; Farris et al., 2003), endothelin-converting enzyme (Eckman et al., 2003) and MMPs (Roher et al., 1994; Backstrom et al., 1996; Leissring et al., 2003). Yu et al., (unpublished data) also found that $\mathrm{Mg}$ deprivation resulted in a $50 \%$ decrease of neprilysin activity without alteration in the protein level of neprilysin and IDE. Thus, the exacerbated accumulation of $A \beta$ induced by $\left[\mathrm{Mg}^{2+}\right]_{0}$ at $0.0 \mathrm{mM}$ resulted from both the enhanced production and aberrant catabolism.

\section{Conclusion}

Magnesium participates in the biochemical mechanisms of neuronal properties and synaptic functions, which are involved in the pathophysiology of neurodegenerative diseases. 
Magnesium was demonstrated to modulate APP trafficking and processing, and its level was found decreased in AD patients. Both clinical and experimental data implicated a role of $\mathrm{Mg}$ in the pathogenesis of AD. Given the prevalence of magnesium inadequacy in the general population (Ford and Mokdad, 2003), magnesium supplementation could constitute a potential novel pharmacological target for the treatment of $A D$ via its action on APP processing.

\section{References}

Adlard PA, Bush Al (2006) Metals and Alzheimer's disease. J Alzheimers Dis 10:145-63.

Alvarez-Leefmans FJ, Giraldez F, Gamino SM (1987) Intracellular free magnesium in excitable cells: its measurement and its biologic significance. Can J Physiol Pharmacol 65:915-25.

Andrási E, Igaz S, Molnar Z, Mako S (2000) Disturbances of magnesium concentrations in various brain areas in Alzheimer's disease. Magnes Res 13:189-96.

Andrási E, Páli N, Molnár Z, Kösel S (2005) Brain aluminum, magnesium and phosphorus contents of control and Alzheimer-diseased patients. J Alzheimers Dis 7:273-84.

Araki W, Kitaguchi N, Tokushima Y, Ishii K, Aratake H, Shimohama S, Nakamura S, Kimura J (1991) Trophic effect of beta-amyloid precursor protein on cerebral cortical neurons in culture. Biochem Biophys Res Commun 181:265-71.

Backstrom JR, Lim GP, Cullen MJ, Tokes ZA (1996) Matrix metalloproteinase-9 (MMP-9) is synthesized in neurons of the human hippocampus and is capable of degrading the amyloid-beta peptide (1-40). J Neurosci 16:7910-9.

Bardgett ME, Schultheis PJ, McGill DL, Richmond RE, Wagge JR (2005) Magnesium deficiency impairs fear conditioning in mice. Brain Res 1038:100-6.

Bardgett ME, Schultheis PJ, Muzny A, Riddle MD, Wagge JR (2007) Magnesium deficiency reduces fearinduced conditional lick suppression in mice. Magnes Res 20:58-65.

\section{Acknowledgement}

This work was supported by the National Natural Science Foundation of China (NSFC; Grants No. 30570533, No. 30670414 and No. 30973145) and the National High Technology Research and Development Program of China (973 Program No. 2006 CB500705 and 863 Program No. 0060102A4031).

Basha MR, Murali M, Siddiqi HK, Ghosal K, Siddiqi OK, Lashuel HA, Ge YW, Lahiri DK, Zawia NH (2005a) Lead $(\mathrm{Pb})$ exposure and its effect on APP proteolysis and Abeta aggregation. FASEB J 19:2083-4.

Basha MR, Wei W, Bakheet SA, Benitez N, Siddiqi HK, Ge YW, Lahiri DK, Zawia NH (2005b) The fetal basis of amyloidogenesis: exposure to lead and latent overexpression of amyloid precursor protein and betaamyloid in the aging brain. $J$ Neurosci 25:823-9.

Bayer TA, Schafer S, Simons A, Kemmling A, Kamer T, Tepest R, Eckert A, Schussel K, Eikenberg O, SturchlerPierrat C, Abramowski D, Staufenbiel M, Multhaup G (2003) Dietary Cu stabilizes brain superoxidemdismutase 1 activity and reduces amyloid Abeta production in APP23 transgenic mice. Proc Natl Acad Sci USA 100:14187-92.

Bleecker ML, Ford DP, Lindgren KN, Hoese VM, Walsh KS, Vaughan CG (2005) Differential effects of lead exposure on components of verbal memory. Occup Environ Med 62:181-7.

Borchardt T, Camakaris J, Cappai R, Masters CL, Beyreuther K, Multhaup G (1999) Copper inhibits betaamyloid production and stimulates the nonamyloidogenic pathway of amyloid-precursor-protein secretion. Biochem J 344:461-7.

Braak H, Braak E (1991) Neuropathological stageing of Alzheimer-related changes. Acta Neuropathol 82:23959.

Brion P, Westhof E (1997) Hierarchy and dynamics of RNA folding. Annu Rev Biophys Biomol Struct 26:11337. 
Buckner RL, Snyder AZ, Shannon BJ, LaRossa G, Sachs $R$, Fotenos AF, Sheline YI, Klunk WE, Mathis CA, Morris JC, Mintun MA (2005) Molecular, structural, and functional characterization of Alzheimer's disease: evidence for a relationship between default activity, amyloid, and memory. J Neurosci 25:7709-17.

Cai D, Leem JY, Greenfield JP, Wang P, Kim BS, Lopes KO, Kim SH, Zheng H, Greengard IP, Sisodia SS, Thinkaran G, Xu H (2003) Presenilin-1 regulates intracellular trafficking and cell surface delivery of beta-amyloid precursor protein. J Biol Chem 278:344654.

Christensen MA, Zhou W, Qing H, Lehman A, Philipsen $S$, Song W (2004) Transcriptional regulation of BACE1, the beta-amyloid precursor protein beta-secretase, by Sp1. Mol Cell Biol 24:865-74.

Chui DH, Dobo E, Makifuchi T, Akiyama H, Kawakatsu S, Petit A, Checler F, Araki W, Takahashi K, Tabira T (2001) Apoptotic neurons in Alzheimer's disease frequently show intracellular Abeta42 labeling. J Alzheimers Dis 3:231-9.

Chui DH, Tanahashi H, Ozawa K, Ikeda S, Checler F, Ueda O, Suzuki H, Araki W, Inoue H, Shirotani K, Takahashi K, Gallyas F, Tabira T (1999) Transgenic mice with Alzheimer presenilin 1 mutations show accelerated neurodegeneration without amyloid plaque formation. Nat Med 5:560-64.

Cilliler AE, Ozturk S, Ozbakir S (2007) Serum magnesium level and clinical deterioration in Alzheimer's disease. Gerontology 53:419-22.

Cirrito JR, Kang JE, Lee J, Stewart FR, Verges DK, Silverio LM, Bu G, Mennerick S, Holtzman DM (2008) Endocytosis is required for synaptic activity-dependent release of amyloid-beta in vivo. Neuron 58:42-51.

Cirrito JR, Yamada KA, Finn MB, Sloviter RS, Bales KR, May PC, Schoepp DD, Paul SM, Mennerick S, Holtzman DM (2005) Synaptic activity regulates interstitial fluid amyloid-beta levels in vivo. Neuron 48:913-22.

Clark BA, Brown RS (1992) Unsuspected morbid hypermagnesemia in elderly patients. Am J Nephrol 12:336-43.

Cornett CR, Markesbery WR, Ehmann WD (1998) Imbalances of trace elements related to oxidative damage in Alzheimer's disease brain. NeuroToxicology 19:339-45.

Cupers P, Bentahir M, Craessaerts K, Orlans I, Vanderstichele H, Saftig P, De SB, Annaert W (2001)

The discrepancy between presenilin subcellular localization and gamma-secretase processing of amyloid precursor protein. J Cell Biol 154:731-40.

De Strooper B (2003) Aph-1, Pen-2, and Nicastrin with Presenilin generate an active gamma-Secretase complex. Neuron 38:9-12.

Deibel MA, Ehmann WD, Markesbery WR (1996) Copper, iron, and zinc imbalances in severely degenerated brain regions in Alzheimer's disease: possible relation to oxidative stress. J Neurol Sci 143: 137-42.

Durlach J (1990) Magnesium depletion and pathogenesis of Alzheimer's disease. Magnes Res $3: 217-8$.

Dyrks T, Monning U, Beyreuther K, Turner J (1994) Amyloid precursor protein secretion and beta $A 4$ amyloid generation are not mutually exclusive. FEBS Lett 349:210-4.

Ebel H, Gunther T (1980) Magnesium metabolism: a review. J Clin Chem Clin Biochem 18:257-70.

Eckman EA, Watson M, Marlow L, Sambamurti K, Eckman CB (2003) Alzheimer's disease beta-amyloid peptide is increased in mice deficient in endothelinconverting enzyme. J Biol Chem 278:2081-4.

Edbauer D, Winkler E, Regula JT, Pesold B, Steiner H, Haass C (2003) Reconstitution of gamma-secretase activity. Nat Cell Biol 5:486-8.

Enomoto T, Osugi T, Satoh H, Mclntosh TK, Nabeshima $\mathrm{T}$ (2005) Pre-Injury magnesium treatment prevents traumatic brain injury-induced hippocampal ERK activation, neuronal loss, and cognitive dysfunction in the radial-arm maze test. J Neurotrauma 22:783-92.

Esch FS, Keim PS, Beattie EC, Blacher RW, Culwell AR, Oltersdorf T, McClure D, Ward PJ (1990) Cleavage of amyloid beta peptide during constitutive processing of its precursor. Science 248:1122-4.

Esler WP, Wolfe MS (2001) A portrait of Alzheimer secretases--new features and familiar faces. Science 293:1449-54.

Farris W, Mansourian S, Chang Y, Lindsley L, Eckman EA, Frosch MP, Eckman CB, Tanzi RE, Selkoe DJ, Guenette $S$ (2003) Insulin-degrading enzyme regulates the levels of insulin, amyloid beta-protein, and the beta-amyloid precursor protein intracellular domain in vivo. Proc Natl Acad Sci USA 100:4162-7. 
Fazeli MS, Breen K, Errington ML, Bliss TV (1994) Increase in extracellular NCAM and amyloid precursor protein following induction of long-term potentiation in the dentate gyrus of anaesthetized rats. Neurosci Lett 169:77-80.

Ford ES, Mokdad AH (2003) Dietary magnesium intake in a national sample of US adults. J Nutr 133:2879-82.

Friedlich AL, Lee JY, van Groen T, Cherny RA, Volitakis I, Cole TB, Palmiter RD, Koh JY, Bush AI (2004) Neuronal zinc exchange with the blood vessel wall promotes cerebral amyloid angiopathy in an animal model of Alzheimer's disease. J Neurosci 24:3453-9.

Fung MC, Weintraub M, Bowen DL (1995) Hypermagnesemia. Elderly over-the-counter drug users at risk. Arch Fam Med 4:718-23.

Furukawa K, Barger SW, Blalock EM, Mattson MP (1996) Activation of K+ channels and suppression of neuronal activity by secreted beta-amyloid-precursor protein. Nature 379:74-78.

Glenner GG, Wong CW (1984) Alzheimer's disease: initial report of the purification and characterization of a novel cerebrovascular amyloid protein. Biochem Biophys Res Commun 120:885-90.

Glick JL (1990a) Dementias: the role of magnesium deficiency and an hypothesis concerning the pathogenesis of Alzheimer's disease. Med Hypotheses 31:211-25.

Glick JL (1990b) Use of magnesium in the management of dementias. Med Sci Res 18:831-3.

Golts N, Snyder H, Frasier M, Theisler C, Choi P, Wolozin B (2002) Magnesium Inhibits Spontaneous and Iron-induced Aggregation of a-Synuclein. J Biol Chem 277:16116-23.

Guo Q, Robinson N, Mattson MP (1998) Secreted betaamyloid precursor protein counteracts the proapoptotic action of mutant presenilin-1 by activation of NF-kappa $\beta$ and stabilization of calcium homeostasis. J Biol Chem 273:12341-51.

Gupta VB, Anitha S, Hegde ML, Zecca L, Garruto RM, Ravid R, Shankar SK, Stein R, Shanmugavelu P, Jagannatha Rao KS (2005) Aluminium in Alzheimer's disease: are we still at a crossroad? Cell Mol Life Sci 62: 143-58.

Hallak M (1998) Effect of parenteral magnesium sulfate administration on excitatory amino acid receptors in the rat brain. Magnes Res 11:117-31.
Hama E, Shirotani K, Masumoto H, Sekine-Aizawa $Y$, Aizawa H, Saido TC (2001) Clearance of extracellular and cell-associated amyloid beta peptide through viral expression of neprilysin in primary neurons. J Biochem 130:721-6.

Haniu M, Denis P, Young Y, Mendiaz EA, Fuller J, Hui JO, Bennett BD, Kahn S, Ross S, Burgess T, Katta V, Rogers G, Vassar R, Citron M (2000) Characterization of Alzheimer's beta -secretase protein BACE. A pepsin family member with unusual properties. J Biol Chem 275:21099-106.

Hardy J, Selkoe DJ (2002) The amyloid hypothesis of Alzheimer's disease: progress and problems on the road to therapeutics. Science 297:353-6.

Hashimoto T, Nishi K, Nagasao J, Tsuji S, Oyanagi K (2008) Magnesium exerts both preventive and ameliorating effects in an in vitro rat Parkinson disease model involving 1-methyl-4-phenylpyridinium (MPP+) toxicity in dopaminergic neurons. Brain Res 1197:14351.

Hirata A, Hirata F (2002) DNA chain unwinding and annealing reactions of lipocortin (annexin) I heterotetramer: regulation by $\mathrm{Ca}(2+)$ and $\mathrm{Mg}(2+)$. Biochem Biophys Res Commun 291:205-9.

Ho A, Sudhof TC (2004) Binding of F-spondin to amyloid-beta precursor protein: a candidate amyloidbeta precursor protein ligand that modulates amyloidbeta precursor protein cleavage. Proc Natl Acad Sci USA 101:2548-53.

Hoane MR (2005) Treatment with magnesium improves reference memory but not working memory while reducing GFAP expression following traumatic brain injury. Restor Neurol Neurosci 23:67-77.

Hoane MR (2007) Assessment of cognitive function following magnesium therapy in the traumatically injured brain. Magnes Res 20:229-36.

Hoe HS, Tran TS, Matsuoka Y, Howell BW, Rebeck GW (2006) DAB1 and Reelin effects on amyloid precursor protein and ApoE receptor 2 trafficking and processing. J Biol Chem 281:35176-85.

Hoe HS, Wessner D, Beffert U, Becker AG, Matsuoka Y, Rebeck GW (2005) F-spondin interaction with the apolipoprotein E receptor ApoEr2 affects processing of amyloid precursor protein. Mol Cell Biol 25:9259-68.

Huang X, Moir RD, Tanzi RE, Bush Al, Rogers JT (2004) Redox-active metals, oxidative stress, and Alzheimer's disease pathology. Ann NY Acad Sci 1012:153-63. 
Huse JT, Pijak DS, Leslie GJ, Lee VM, Doms RW (2000) Maturation and endosomal targeting of beta-site amyloid precursor protein-cleaving enzyme. The Alzheimer's disease beta-secretase. J Biol Chem 275:33729-37.

Hussain I, Powell D, Howlett DR, Tew DG, Meek TD, Chapman C, Gloger IS, Murphy KE, Southan CD, Ryan DM, Smith TS, Simmons DL, Walsh FS, Dingwall C, Christie $G$ (1999) Identification of a novel aspartic protease (Asp 2) as $\beta$-secretase. Mol Cell Neurosci 14:419-27.

Iseri LT, French JH (1984) Magnesium: nature's physiologic calcium blocker. Am Heart J 108:188-193.

Iwata N, Tsubuki S, Takaki Y, Shirotani K, Lu B, Gerard NP, Gerard C, Hama E, Lee HJ, Saido TC (2001) Metabolic regulation of brain Abeta by neprilysin. Science 292:1550-2.

Kaether C, Lammich S, Edbauer D, Ertl M, Rietdorf J, Capell A, Steiner H, Haass C (2002) Presenilin-1 affects trafficking and processing of betaAPP and is targeted in a complex with nicastrin to the plasma membrane. $J$ Cell Biol 158:551-61.

Kamenetz F, Tomita T, Hsieh H, Seabrook G, Borchelt D, Iwatsubo T, Sisodia S, Malinow R (2003) APP processing and synaptic function. Neuron 37:925-37.

Kang J, Lemaire HG, Unterbeck A, Salbaum JM, Masters CL, Grzeschik KH, Multhaup G, Beyreuther K, Muller-Hill B (1987) The precursor of Alzheimer's disease amyloid A4 protein resembles a cell-surface receptor. Nature 325:733-6.

Ko YH, Hong S, Pedersen PL (1999) Chemical mechanism of ATP synthase. Magnesium plays a pivotal role in formation of the transition state where ATP is synthesized from ADP and inorganic phosphate. J Biol Chem 274:28853-6.

Kurochkin IV, Goto S (1994) Alzheimer's beta-amyloid peptide specifically interacts with and is degraded by insulin degrading enzyme. FEBS Lett 345:33-7.

Ladner CJ, Lee JM (1999) Reduced high-affinity agonist binding at the $\mathrm{M}(1)$ muscarinic receptor in Alzheimer's disease brain: differential sensitivity to agonists and divalent cations. Exp Neurol 158:451-8.

Lammich S, Kojro E, Postina R, Gilbert S, Pfeiffer R, Jasionowski M, Haass C, Fahrenholz F (1999) Constitutive and regulated alpha-secretase cleavage of Alzheimer's amyloid precursor protein by a disintegrin metalloprotease. Proc Natl Acad Sci USA 96:3922-7.
Landfield PW, Morgan GA (1984) Chronically elevating plasma Mg2+ improves hippocampal frequency potentiation and reversal learning in aged and young rats. Brain Res 322:167-71.

LeBlanc AC, Koutroumanis M, Goodyer CG (1998) Protein kinase $C$ activation increases release of secreted amyloid precursor protein without decreasing Abeta production in human primary neuron cultures. J Neurosci 18:2907-13.

Lee JY, Cole TB, Palmiter RD, Suh SW, Koh JY (2002) Contribution by synaptic zinc to the gender-disparate plaque formation in human Swedish mutant APP transgenic mice. Proc Natl Acad Sci USA 99:7705-10.

Leissring MA, Farris W, Chang AY, Walsh DM, Wu X, Sun X, Frosch MP, Selkoe DJ (2003) Enhanced proteolysis of beta-amyloid in APP transgenic mice prevents plaque formation, secondary pathology, and premature death. Neuron 40:1087-93.

Lemke MR (1995) Plasma magnesium decrease and altered calcium/magnesium ratio in severe dementia of the Alzheimer type. Biol Psychiatry 37:341-3.

Lesne S, Ali C, Gabriel C, Croci N, MacKenzie ET, Glabe CG, Plotkine M, Marchand-Verrecchia C, Vivien D, Buisson A (2005) NMDA receptor activation inhibits alpha-secretase and promotes neuronal amyloid-beta production. J Neurosci 25:9367-77.

Lin JY, Chung SY, Lin MC, Cheng FC (2002) Effects of magnesium sulfate on energy metabolites and glutamate in the cortex during focal cerebral ischemia and reperfusion in the gerbil monitored by a dualprobe microdialysis technique. Life Sci 71:803-11.

Liu Y, Zhang YW, Wang X, Zhang $\mathrm{H}$, You X, Liao FF, Xu H (2009) Intracellular trafficking of presenilin 1 is regulated by beta-amyloid precursor protein and phospholipase D1. J Biol Chem 284:12145-52.

Loeffler J, Huber G (1993) Modulation of beta-amyloid precursor protein secretion in differentiated and nondifferentiated cells. Biochem Biophys Res Commun 195:97-103.

Lovell MA, Robertson JD, Teesdale WJ, Campbell JL, Markesbery WR (1998) Copper, iron and zinc in Alzheimer's disease senile plaques. J Neurol Sci 158:47-52.

Mantyh PW, Ghilardi JR, Rogers S, DeMaster E, Allen CJ, Stimson ER, Maggio JE (1993) Aluminum, iron, and zinc ions promote aggregation of physiological concentrations of $\beta$-amyloid peptide. J Neurochem 61:1171-4. 
Marr RA, Guan H, Rockenstein E, Kindy M, Gage FH, Verma I, Masliah E, Hersh LB (2004) Neprilysin regulates amyloid Beta peptide levels. J Mol Neurosci 22:5-11.

Masters CL, Simms G, Weinman NA, Multhaup G, McDonald BL, Beyreuther K (1985) Amyloid plaque core protein in Alzheimer disease and Down syndrome. Proc Natl Acad Sci USA 82:4245-9.

Matsuda H, Saigusa A, Irisawa H (1987) Ohmic conductance through the inwardly rectifying $\mathrm{K}$ channel and blocking by internal Mg2+. Nature 325:156-9.

Mattson MP, Cheng B, Culwell AR, Esch FS, Lieberburg I, Rydel RE (1993) Evidence for excitoprotective and intraneuronal calcium-regulating roles for secreted forms of the beta-amyloid precursor protein. Neuron 10:243-54.

Mayer ML, Westbrook GL, Guthrie PB (1984) Voltagedependent block by $\mathrm{Mg} 2+$ of NMDA responses in spinal cord neurones. Nature 309:261-3.

Meziane H, Dodart JC, Mathis C, Little S, Clemens J, Paul SM, Ungerer A (1998) Memory-enhancing effects of secreted forms of the beta-amyloid precursor protein in normal and amnestic mice. Proc Natl Acad Sci USA 95:12683-8.

Morimoto T, Ohsawa I, Takamura C, Ishiguro M, Kohsaka S (1998) Involvement of amyloid precursor protein in functional synapse formation in cultured hippocampal neurons. J Neurosci Res 51:185-95.

Mucke L, Abraham CR, Masliah E (1996) Neurotrophic and neuroprotective effects of hAPP in transgenic mice. Ann N Y Acad Sci 777:82-8.

Nowak L, Bregestovski P, Ascher P, Herbet A, Prochiantz A (1984) Magnesium gates glutamateactivated channels in mouse central neurones. Nature 307:462-5.

Oyanagi K (2005) The nature of the parkinsonismdementia complex and amyotrophic lateral sclerosis of Guam and magnesium deficiency. Parkinsonism and Related Disorders 11:S17-23.

Oyanagi K, Kawakami E, Kikuchi-Horie K, Ohara K, Ogata K, Takahama S, Wada M, Kihira T, Yasui M (2006) Magnesium deficiency over generations in rats with special references to the pathogenesis of the Parkinsonism-dementia complex and amyotrophic lateral sclerosis of Guam. Neuropathology 26:115-28.

Phinney AL, Drisaldi B, Schmidt SD, Lugowski S, Coronado V, Liang Y, Horne P, Yang J, Sekoulidis J,
Coomaraswamy J, Chishti MA, Cox DW, Mathews PM, Nixon RA, Carlson GA, St George-Hyslop P, Westaway D (2003) In vivo reduction of amyloid-beta by a mutant copper transporter. Proc Natl Acad Sci USA 100:14193-8.

Prescott AR, Comerford JG, Magrath R, Lamb NJC, Warn RM (1988) Effects of elevated intracellular magnesium on cytoskeletal integrity. J Cell Science 89:321-9.

Price DL, Sisodia SS, Borchelt DR (1998) Genetic neurodegenerative diseases: the human illness and transgenic models. Science 282:1079-83.

Qi-Takahara Y, Morishima-Kawashima M, Tanimura Y, Dolios G, Hirotani N, Horikoshi Y, Kametani F, Maeda $M$, Saido TC, Wang R, Ihara Y (2005) Longer forms of amyloid beta protein: implications for the mechanism of intramembrane cleavage by gamma-secretase. J Neurosci 25:436-45.

Querfurth HW, Selkoe DJ (1994) Calcium ionophore increases amyloid beta peptide production by cultured cells. Biochemistry 33:4550-61.

Ray WJ, Yao M, Mumm J, Schroeter EH, Saftig P, Wolfe M, Selkoe DJ, Kopan R, Goate AM (1999) Cell surface presenilin-1 participates in the gamma-secretase-like proteolysis of Notch. J Biol Chem 274:36801-7.

Roher AE, Kasunic TC, Woods AS, Cotter RJ, Ball MJ, Fridman R (1994) Proteolysis of A beta peptide from Alzheimer disease brain by gelatinase A. Biochem Biophys Res Commun 205:1755-61.

Romani A, Marfella C, Scarpa A (1993) Regulation of magnesium uptake and release in the heart and in isolated ventricular myocytes. Circ Res 72:1139-48.

Romani A, Scarpa A (1992) Regulation of cell magnesium. Arch Biochem Biophys 298:1-12. Romani AM, Scarpa A (2000) Regulation of cellular magnesium. Front Biosci 5:D720-34.

Rossner S, Beck M, Stahl T, Mendla K, Schliebs R, Bigl V (2000) Constitutive overactivation of protein kinase $C$ in guinea pig brain increases alpha-secretory APP processing without decreasing beta-amyloid generation. Eur J Neurosci 12:3191-200.

Schubert D, Behl C (1993) The expression of amyloid beta protein precursor protects nerve cells from betaamyloid and glutamate toxicity and alters their interaction with the extracellular matrix. Brain Res 629:275-82. 
Selkoe DJ (1991) The molecular pathology of Alzheimer's disease. Neuron 6:487-98.

Selkoe DJ (1999) Translating cell biology into therapeutic advances in Alzheimer's disease. Nature 399:A23-31.

Selkoe DJ (2001a) Alzheimer's disease: genes, proteins, and therapy. Physiol Rev 81:741-66.

Selkoe DJ (2001b) Clearing the brain's amyloid cobwebs. Neuron 32:177-80.

Selkoe DJ (2002) Alzheimer's disease is a synaptic failure. Science 298:789-91.

Seubert $\mathrm{P}$, Oltersdorf T, Lee MG, Barbour R, Blomquist C, Davis DL, Bryant K, Fritz LC, Galasko D, Thal LJ (1993) Secretion of beta-amyloid precursor protein cleaved at the amino terminus of the beta-amyloid peptide. Nature 361:260-63.

Shcherbatykh I, Carpenter DO (2007) The role of metals in the etiology of Alzheimer's disease. J Alzheimers Dis 11:191-205.

Shirotani K, Tsubuki S, Iwata N, Takaki Y, Harigaya W, Maruyama K, Kiryu-Seo S, Kiyama H, Iwata H, Tomita T, Iwatsubo T, Saido TC (2001) Neprilysin degrades both amyloid beta peptides 1-40 and 1-42 most rapidly and efficiently among thiorphan- and phosphoramidon-sensitive endopeptidases. J Biol Chem 276:21895-901.

Sinha S, Anderson JP, Barbour R, Basi GS, Caccavello R, Davis D, Doan M, Dovey HF, Frigon N, Hong J, Jacobson-Croak K, Jewett $N$, Keim P, Knops J, Lieberburg I, Power M, Tan H, Tatsuno G, Tung J, Schenk D, Seubert $P$, Suomensaari SM, Wang S, Walker D, Zhao J, McConlogue L, John V (1999) Purification and cloning of amyloid precursor protein betasecretase from human brain. Nature 402:537-40.

Sisodia SS (1992) Beta-amyloid precursor protein cleavage by a membrane-bound protease. Proc Natl Acad Sci USA 89:6075-9.

Skovronsky DM, Moore DB, Milla ME, Doms RW, Lee VM (2000) Protein kinase C-dependent alphasecretase competes with beta-secretase for cleavage of amyloid-beta precursor protein in the trans-golgi network. J Biol Chem 275:2568-75.

Slutsky I, Abumaria N, Wu LJ, Huang C, Zhang L, Li B, Zhao X, Govindarajan A, Zhao MG, Zhuo M, Tonegawa S, Liu G (2010) Enhancement of learning and memory by elevating brain magnesium. Neuron 65:165-77.
Small DH, Nurcombe V, Reed G, Clarris H, Moir R, Beyreuther K, Masters CL (1994) A heparin-binding domain in the amyloid protein precursor of Alzheimer's disease is involved in the regulation of neurite outgrowth. J Neurosci 14:2117-27.

Sparks DL, Schreurs BG (2003) Trace amounts of copper in water induce beta-amyloid plaques and learning deficits in a rabbit model of Alzheimer's disease. Proc Natl Acad Sci USA 100:11065-9.

Subirana JA, Soler-Lopez M (2003) Cations as hydrogen bond donors: a view of electrostatic interactions in DNA. Annu Rev Biophys Biomol Struct 32:27-45.

Turner AJ, Fisk L, Nalivaeva NN (2004) Targeting amyloid-degrading enzymes as therapeutic strategies in neurodegeneration. Ann N Y Acad Sci 1035:1-20.

Vandenberg CA (1987) Inward rectification of a potassium channel in cardiac ventricular cells depends on internal magnesium ions. Proc Natl Acad Sci USA $84: 2560-4$.

Vassar R, Bennett BD, Babu-Khan S, Kahn S, Mendiaz $E A$, Denis $P$, Teplow DB, Ross $S$, Amarante $P$, Loeloff $R$, Luo $Y$, Fisher $S$, Fuller J, Edenson S, Lile J, Jarosinski MA, Biere AL, Curran E, Burgess T, Louis JC, Collins F, Treanor J, Rogers G, Citron M (1999) Beta-secretase cleavage of Alzheimer's amyloid precursor protein by the transmembrane aspartic protease BACE. Science 286:735-41.

Vural H, Demirin H, Kara Y, Eren I, Delibas N (2010) Alterations of plasma magnesium, copper, zinc, iron and selenium concentrations and some related erythrocyte antioxidant enzyme activities in patients with Alzheimer's disease. J Trace Elements Med Biol 24:169-73.

Wang DS, Dickson DW, Malter JS (2006) Beta-Amyloid degradation and Alzheimer's disease. J Biomed Biotechnol 2006:58406.

Wiltfang J, Esselmann $\mathrm{H}$, Cupers $\mathrm{P}$, Neumann $\mathrm{M}$, Kretzschmar H, Beyermann M, Schleuder D, Jahn H, Ruther E, Kornhuber J, Annaert W, De SB, Saftig P (2001) Elevation of beta-amyloid peptide 2-42 in sporadic and familial Alzheimer's disease and its generation in PS1 knockout cells. J Biol Chem 276:42645-57.

Wolf FI, Torsello A, Fasanella S, Cittadini A (2003) Cell physiology of magnesium. Mol Aspects Med 24:11-26. 
Xu X, Yang D, Wyss-Coray T, Yan J, Gan L, Sun Y, Mucke $L$ (1999) Wild-type but not Alzheimer-mutant amyloid precursor protein confers resistance against p53mediated apoptosis. Proc Natl Acad Sci USA 96:754752.

Yamazaki T, Koo EH, Selkoe DJ (1996) Trafficking of cell-surface amyloid beta-protein precursor. II. Endocytosis, recycling and lysosomal targeting detected by immunolocalization. J Cell Sci 109:9991008.

Yan R, Bienkowski MJ, Shuck ME, Miao H, Tory MC, Pauley AM, Brashier JR, Stratman NC, Mathews WR, Buhl AE, Carter DB, Tomasselli AG, Parodi LA, Heinrikson RL, Gurney ME (1999) Membraneanchored aspartyl protease with Alzheimer's disease beta-secretase activity. Nature 402:533-7.
Yang LS, Ksiezak-Reding H (1999) Ca2+ and Mg2+ selectively induce aggregates of PHF-tau but not normal human tau. J Neurosci Res 55:36-43.

Yu J, Sun M, Chen Z, Lu J, Liu Y, Zhou L, Xu X, Fan D, Chui D (2010) Magnesium modulates amyloid-beta protein precursor trafficking and processing. J Alzheimers Dis 20:1091-106.

Zhao J, Wang WN, Tan YC, Zheng Y, Wang ZX (2002) Effect of $\mathrm{Mg}(2+)$ on the kinetics of guanine nucleotide binding and hydrolysis by $\mathrm{Cdc} 42$. Biochem Biophys Res Commun 297:653-8. 



\title{
Magnesium and stress
}

\author{
Magdalena D. Cuciureanu ${ }^{1, \otimes}$ and Robert Vink ${ }^{2}$ \\ ${ }^{1}$ Department of Pharmacology, “Gr. T. Popa” University of Medicine and Pharmacy, lasi, 700115, Romania. \\ ${ }^{2}$ Centre for Neuroscience Research, School of Medical Sciences, The University of Adelaide, South Australia 5005, Australia. \\ $\triangle$ magdacuciur@gmail.com
}

\begin{abstract}
Magnesium status is highly associated with stress levels, with both stress and hypomagnesemia potentiating each other's negative effects. Indeed, hypomagnesemia has been associated with stressful conditions such as photosensitive headache, fibromyalgia, chronic fatigue syndrome, audiogenic stress, cold stress, and physical stress, amongst others. The role of magnesium in these conditions is unclear, although a number of potential mechanisms for magnesium's action have been identified including via the glutamatergic, serotonergic, and adrenergic neurotransmitter systems, as well as via several neurohormones. The current review examines the link between magnesium deficiency and stress, focusing on the association between magnesium and various stress pathologies, magnesium's potential interaction with stress pathways, and magnesium's effects on the brain.
\end{abstract}

\section{Introduction}

At the beginning of the twentieth century, Walter Cannon pioneered research concerning the importance of the sympathetic nervous system in adaptation of the body (Quick, 1994). While Claude Bernard was the first who defined the term of "milieu interieur" as being "the constancy of the internal environment" (see Cameron, 2007), it was Walter Cannon who coined the terms "homeostasis" and "fight-orflight response" (Quick, 1994). Subsequently, Hans Selye in 1936 adapted a concept from physics describing the resistance of a body to applied pressure in order to define the concept of stress (Neylan, 1998). General adaptation syndrome, as he called it, is the non-specific response of the body to any demand for change. It consists of three stages of adaptation: an initial brief alarm reaction, a prolonged period of resistance, and finally, the stage of exhaustion and death. Although there is no generally accepted definition, stress can be understood to be a complex adaptive biochemical, physiological, psychological and gene expression change of the organism (stress response) triggered by a stimulus (stressor) that was interpreted by the brain as being dangerous (McEwen, 2008b; Kantorovich et al., 2008).

The neuroendocrine response to stress initiates hyperventilation, elevated blood pressure, increased heart rate, a sudomotor response (sweating), increased blood flow to skeletal muscles, and perturbations of gut function (the 'fight or flight' defence reaction) in order to enable body survival. More, the amygdala activates neurons in the hippocampus and neocortex, where threatening stimuli are associated with fear, in order to adapt future behaviour to avoid danger (Rupniak, 2005).

To further refine the stress concept, Sterling and Eyer (1981) proposed the term allostasis to describe the process of achieving stability through change. As compared to homeostatic values (blood oxygen, blood $\mathrm{pH}$, and body temperature), which have to be kept within a narrow range, the allostatic mediators (hormones of the hypothalamic-pituitary-adrenal axis, catecholamines, and cytokines) may vary during daily and seasonal routines (McEwen, 2008b; Ablin and Buskila, 2010; Billard, 2006).

Nowadays, the profound implications of stress in human pathology are unanimously recognized and represent a dynamic field of research. There is a continued interest in identifying new possibilities to alleviate stress and improve quality of life in a fast-changing, modern world.

The idea that magnesium $(\mathrm{Mg})$ supplementation modifies the effect of chronic stress dates back to 1981 (Classen, 1981). This hypothesis is 
certainly feasible given the cation's ubiquitous distribution and role throughout the body. Indeed, $\mathrm{Mg}$ is the fourth most abundant cation in the body and the second most abundant intracellular cation. It is involved in a wide variety of cellular processes including aerobic and anaerobic metabolism, all bioenergetic reactions, regulation of metabolic pathways, signal transduction, ion channels activity, cell proliferation, differentiation, apoptosis, angiogenesis, and membrane stabilization (Nishizawa et al., 2007; Szewczyk et al., 2008; Turner and Vink 2007; Wolf et al., 2007). At a biochemical level, more than 325 enzymes are $\mathrm{Mg}$ dependent, many of which are nervous system enzymes, thus reflecting the important role potentially played by $\mathrm{Mg}$ in CNS physiological and pathological function (Eby and Eby, 2006).

\section{Magnesium interaction with stress pathways}

Acute stress has been shown to be associated with increased plasma $\mathrm{Mg}$ levels and increased urinary Mg excretion (Murck, 2002; Whyte et al., 1987). The shift of Mg from the intracellular to the extracellular space initially plays a protective role in order to diminish the adverse effects of stress, but extended periods of stress result in progressive $\mathrm{Mg}$ deficit and deleterious consequences for health (Seelig, 1994). Moreover, stress and hypomagnesemia potentiate each other's negative effects in a veritable pathogenic vicious circle. Low $\mathrm{Mg} / \mathrm{Ca}$ ratios augment the release of catecholamines in response to stress (Caddell et al., 1986; Seelig, 1994). Fatty acids resulting from adrenergic-induced lipolysis form undissociated $\mathrm{Mg}$ soaps which further exacerbates $\mathrm{Mg}$ depletion (Seelig, 1994). Mg deficiency also favours the release of vasoconstrictive and platelet aggregating factors (derived from fatty acid metabolism and endothelium), increases the thromboxane $B 2$ to prostaglandin $12\left(\mathrm{TxB}_{2} / \mathrm{PgI}_{2}\right)$ ratio and enhances intravascular blood coagulation (Ceremuzynski et al., 1978; Franz 2004; Dong et al., 2008; Soma et al., 1988; Sontia and Touyz, 2007). Most interestingly, Mg deficiency itself doesn't induce specific pathology but reduces the tolerance to secondary stress (Tejero-Taldo et al., 2006).

The active transport systems between plasma and cerebrospinal fluid enable elevated and relatively constant $\mathrm{Mg}$ concentrations in the CSF (typically $1.1 \mathrm{mmol} / \mathrm{l}$ in CSF compared to 0.8 $\mathrm{mmol} / \mathrm{l}$ in plasma) (Oppelt et al., 1963; Murck 2002). There are reports that parenteral administration of magnesium sulphate augments cerebrospinal fluid $\mathrm{Mg}$ concentration by $20-25 \%$ in approximately 4 hours (Ko et al., 2001; Muir, 2002), although recent reports suggest that $\mathrm{Mg}$ may not readily cross the intact blood brain barrier (McKee et al., 2005; see Chapter 3).

With respect to mechanisms of action, $\mathrm{Mg}$ affects a number of neurotransmitter systems. It inhibits the release of excitatory neurotransmitters and also acts as a voltage-gated antagonist at the glutamate, N-methyl-Daspartate (NMDA) receptor. $\mathrm{Mg}$ also antagonizes calcium entry via voltage-gated channels of all types. In addition to exhibiting agonist properties at $\gamma$-aminobutyric acid A receptors, it is an angiotensin $\|$ receptor antagonist. $\mathrm{Mg}$ inhibits calcium/protein kinase $C$ related neurotransmission, increases reuptake of glutamate via stimulation of the $\mathrm{Na}^{+} / \mathrm{K}^{+}$ATPase, and is involved in mitochondrial ATP-dependent potassium channel activity (Bednarczyk et al., 2005; Eby and Eby, 2006; Muir 2002; Murck, 2002). Furthermore, Mg inhibits the phosphorylation activity of glycogen synthase kinase3 (GSK-3), increases brain-derived neurotrophic factor (BDNF) expression and enhances the CAMP response element-binding (CREB)/BDNF pathway via the serotonergic system, all mechanisms related to the antidepressant-like activity of this ion (Szewczyk et al., 2008).

There is also an increasing body of evidence indicating that psychological stress promotes oxidative stress, mainly by auto-oxidation of catecholamines. Several studies have shown that psychological stress exacerbates lipid peroxidation, increases production of markers of oxidative damage of DNA (8-oxo-7,8dihydroguanine), and decreases plasma antioxidant activity (Seelig, 1994; Sivonova et al., 2004). Many of these processes are antagonized by Mg (Cernak et al., 2000; Muir, 2002; Nishizawa et al., 2007).

A stressor (either physical or psychological) initially activates the hypothalamic-pituitaryadrenal (HPA) axis and the autonomic nervous system. Activating these systems leads to 
release of catecholamines from sympathetic nerves and the adrenal medulla, and of corticotropin-releasing factor (CRF) and vasopressin (AVP) from parvocellular neurons. Seconds later, adrenocorticotropic hormone (ACTH) is secreted from the anterior pituitary gland and stimulates release of glucocorticoids from the adrenal cortex (Rupniak, 2005; Carrasco and Van de Kar, 2003). CRF is a neurotransmitter involved in the coordination of the endocrine, autonomic, behavioural and immune responses to stress, and whose administration elicits stress-like effects (De Souza et al., 1991; Carrasco and Van de Kar, 2003; Cratty and Birkle, 1999). Magnesium interacts either directly or indirectly with the activity of a number of these neurotransmitters and neurohormones. For example, Cratty and Birkle (1999) showed that glutamate-stimulated CRF release is antagonized by the addition of $\mathrm{MgCl}_{2}$ to the incubation medium. $\mathrm{Mg}$ stabilizes CRF receptor binding and is directly correlated to the number of CRF binding sites (Perrin et al., 1986). On the other hand, Mg stimulates the $\mathrm{Na}^{+} / \mathrm{K}^{+}$ATPase, which decreases CRF-receptor sensitivity (De Souza, 1995).

$\mathrm{Mg}$ also decreases the release of ACTH and modulates adrenocortical sensitivity to this hormone. As intracerebroventricular administration of angiotensin II (ATII) increases the secretion of ACTH and AVP via CRF, it is presumed that $\mathrm{Mg}$ induces a suppression of HPA-axis activity, at least partially, through antagonism of ATII effects (Murck, 2002). Intracerebroventricular administration of artificial CSF with low $\mathrm{Mg}$ concentration induces an increase in cortisol secretion in cats (Garcy and Marotta, 1978). In stressful circumstances, $\mathrm{MgSO}_{4}$ administration reduces the release of AVP (Jee et al., 2009), a neuropeptide that plays an important role in the generation of emotions, social behaviour (aggression), and learning and memory (Neumann et al., 2010; Ebner et al., 2000).

Low blood Mg levels have been associated with raised brain noradrenaline (NA) content in mice selectively bred for hypomagnesemia (Amyard et al., 1995). Such low Mg levels in blood have also been associated with increased catecholamine release in response to noise stress in rats, and with significantly increased urinary excretion
(+200\%) of NA (Caddell et al., 1986; Henrotte, 1997). Also, it has been proven that $\mathrm{Mg}$ exerts a direct suppressive effect on locus coeruleus activity and that poor $\mathrm{Mg}$ status increases sensitivity to stress (Henrotte et al., 1997).

Another pathway influenced by $\mathrm{Mg}$ is the serotoninergic system. Mg acts like a cofactor for tryptophan hydroxylase, intervenes in serotonin receptor binding in vitro and exhibits a direct enhancing effect on $5-\mathrm{HT}_{1 \mathrm{~A}}$ serotonin receptor transmission (Szewczyk et al., 2008; Mizoguchi et al., 2008; Abaamrane et al., 2009). Microdialysis studies showed that stressors can change the extracellular levels of serotonin in different brain areas including the hypothalamus, amygdala, frontal cortex and raphe nuclei (Fujino et al., 2002; Funada and Hara, 2001). Serotonin is involved in neuroendocrine regulation of stress through actions on prolactin, oxytocin, AVP, CRF and ACTH activity (Jorgensen, 2007). HPA axis dysregulation and sensitization of $5-\mathrm{HT}_{2}$ receptor-mediated signalling induced by CRF implicates chronic stress in the aetiology of depression and anxiety (Szewczyk et al., 2008; Magalhaes et al., 2010).

It is also tempting to speculate that the protective effect of the oestrogens in stress are partially mediated by $\mathrm{Mg}$ (Carrasco and Van de Kar, 2003). For example, It is known that oestrogens mediate the shift of $\mathrm{Mg}$ from plasma to cells and increases intracellular levels of $\mathrm{Mg}$ (Galland, 1991; Seelig, 1993; Seelig et al., 2004). Likewise, serum levels of $\mathrm{Mg}^{2+}$ and total magnesium were inversely correlated with oestrogen concentration level (MuneyyirciDelale et al., 1999). In postmenopausal women oestrogen replacement therapy suppresses HPA axis responses to an emotional stressor (Dayas et al., 2000).

Besides cortisol, prolactin, is regarded as a measurable marker of a coping strategy to "psychological stress" (Sobrinho, 2003). Confirming that $\mathrm{Mg}$ levels can modulate prolactin secretion during stress, pretreatment with Mg-aspartate significantly decreased, in a dose-dependent manner, the prolactin plasma concentration of turkey pullets exposed to immobilization stress compared to non-treated birds (Ali et al., 1987). In addition, Mg acts like a positive allosteric modulator for oxytocin 
binding to the receptor, and in this way facilitates the neuropeptide's action to alleviate stress (Gimpl et al., 2008; Labuschagne et al., 2010; Uvnas-Moberg and Petersson, 2005).

\section{Magnesium deficiency, stress and miscellaneous pathologies}

Clinical manifestations of hypomagnesemia include neuromuscular irritability and weakness (tremors, fasciculations, tetany and positive Chvostek's and Trousseau's signs, although some of these features may be due to concomitant hypocalcemia), headaches, focal seizures, hyperemotionality, generalized anxiety, panic attack disorders, insomnia, fatigue, and asthenia (Touyz, 2004; Wacker and Parisi, 1968; Eby and Eby, 2006; Rayssiguieret al., 1990; Durlach et al., 1997). For symptomatic patients, plasma magnesium, erythrocyte magnesium, plasma calcium and daily magnesiuria, calciuria and magnesium retention test should be evaluated (Durlach et al., 1997; Cundy, 2008). The alleviation of clinical manifestations by oral supplementation with $5 \mathrm{mg}$ of magnesium $/ \mathrm{kg} /$ day confirms the diagnosis (Durlach et al., 1997).

Experimental data indicate that Mg-deficient rats exhibit slightly increased plasma corticosterone levels, increased irritability and aggressive behaviour, and higher mortality compared to controls (Caddell, 2001; Henrotte et al., 1997). Recent data also indicate that genetic polymorphism of $\mathrm{Na}^{+} / \mathrm{Mg}^{2+}$ exchanger activity influences $\mathrm{Mg}^{2+}$ efflux from erythrocytes (Feillet-Coudray et al., 2004). Extensive work regarding the genetic predisposition for $\mathrm{Mg}$ deficiency has been carried out by Henrotte et al., (1990) who showed that both major histocompatibility complex ( $\mathrm{MHC}$ ) associated genes (HLA and $\mathrm{H}-2$ ) and non-MHC genes are important contributing factors for $\mathrm{Mg}$ status. In humans, Henrotte and Levy-Leboyer (1985) also found an interesting link between HLA-B35, type A personality and low $\mathrm{Mg}^{2+}$ status. HLA-B35 individuals are more frequently found among stress-sensitive type $A$ behaviour subjects and exhibit lower red blood cell Mg levels than noncarriers. Moreover, these individuals show impaired cytotoxicity and higher titres to antibodies after anti-influenza vaccination, data that establish a connection between genetic background, $\mathrm{Mg}$ deficiency, stress and autoimmunity. It is generally accepted that, in response to a psychological stressor, type $A$ persons exhibit increased release of catecholamines and cortisol, which consequently lowers $\mathrm{Mg}$ levels and increases cardiovascular risk as compared to type B behaviour (Henrotte et al., 1985; Matthews, 1982).

Experimental Mg deficiency has also been associated with disrupted sleep patterns. In rats, $\mathrm{Mg}$ deficiency is associated with an increased amplitude in daily variation of sleep and slowwave sleep delta power, and has been noted to shorten life span and lower reproductive ability (Chollet et al., 2001; Motta et al., 1998). In humans, chronic sleep deprivation is associated with progressively decreasing levels of intracellular $\mathrm{Mg}$, reduced duration of cardiopulmonary exercise and increased hypersensitivity of the chronotropic response to sympathetic nervous stimulation (Omiya et al., 2009). Intriguingly, Mg depletion is associated with decreased melatonin and its supplementation alleviates the symptoms (Billyard et al., 2006; Depoortere et al., 1993; Held et al., 2002). In elderly subjects, $\mathrm{Mg}$ administration (10 $\mathrm{mmol}$ and $20 \mathrm{mmol}$ each for 3 days followed by $30 \mathrm{mmol}$ for 14 days) significantly increased slow wave sleep, renin levels during the total night, and aldosterone levels in the second half of the night, but decreased cortisol levels in the first part of the night (Held et al., 2002).

Photosensitive headache is another condition that is exacerbated by stress and Mg deficiency. Even wearing of tinted spectacles indoors is considered to be a valid indicator of psychological distress. Patients most susceptible to develop this condition include photophobic individuals that experience ophthalmological discomfort during visual stress tests. Sarchielli et al., (1992) showed that sufferers of migraine with and without aura, and tension-type headaches, exhibit significantly lower levels of serum and salivary $\mathrm{Mg}$ concentrations. Hypomagnesaemia raises the sensitivity of cerebral arteries to $\mathrm{CO}_{2}$ which in turn favours cerebral vasospasm and headache (Thomas et al., 1994). Despite being controversial, some authors recommend intravenous administration of 1 gram of $\mathrm{MgSO}_{4}$ for the treatment of migraine headache (Durlach et al., 2005). 
Fibromyalgia is considered a stress-related disorder, with both the onset and the exacerbation of the syndrome associated with intensely stressful periods. Patients with fibromyalgia frequently exhibit chronic, widespread pain caused by the increase in the processing and handling of pain by the CNS (Ablin and Buskila, 2010). It has been shown that there is an inverse correlation between $\mathrm{Mg}$ levels and clinical parameters in patients with fibromyalgia (Sendur et al., 2008). The beneficial role of $\mathrm{Mg}$ in this pathology is presumably based on its antagonistic effect upon NMDA receptors, receptors that are significantly involved in the process of central sensitization (Galland, 1991). Various studies have also shown that patients with a chronic fatigue condition closely linked to fibromyalgia exhibit low Mg levels that are correlated with total antioxidant capacity of blood and GSH concentrations, but not with lipid peroxidation in vitro (Galland, 1991; Manuel y Keenoy et al., 2000). In these patients, weekly intramuscular injections of 1 gram of $\mathrm{MgSO}_{4}$ resulted in a significant improvement in energy levels, pain, and emotional reactions as measured by the Nottingham health profile score (Cox et al., 1991; Murck, 2002). Durlach et al., (2002) suggest that fibromyalgia and chronic fatigue syndrome are clinical forms of magnesium depletion associated with dysfunction of the biological clock.

An interesting correlation has been reported between $\mathrm{Mg}$ deficiency and attention-deficit/ hyperactivity disorder (ADHD). Elevated subjective stress levels and stress intolerance is often mentioned as part of the clinical presentation of ADHD, and often these patients present high post-stress cortisol concentrations (Hirvikoski et al., 2009). In a study examining 116 children, $95 \%$ of children with this pathology exhibited Mg deficiency (Kozielec and StarobratHermelin, 1997). Although evidence regarding the improvement of ADHD symptoms by $\mathrm{Mg}$ supplementation is mixed, some studies indicate that a Mg-B6 regimen over at least two months significantly alleviated hyperactivity and hyperemotivity/aggressiveness and improved attention at school (Mousain-Bosc et al., 2006; Rucklidge et al., 2009). Interestingly, ADHD symptoms reappear in a few weeks after the cessation of the treatment, together with a decrease in erythrocyte $\mathrm{Mg}$ values (Mousain-
Bosc et al., 2006). A Mg-B6 regimen has also been shown to be effective in the treatment of Tourette's syndrome. Tourette's syndrome is associated with increased vulnerability to stress, and $\mathrm{Mg}$ deficit is considered to be a central precipitating event (Carrasco and Van de Kar, 2003; Grimaldi, 2002). Hypomagnesemia is linked with exacerbated neural excitability, increased anxiety, orofacial tardive dyskinesia, and migraine, symptoms that are alleviated by the administration of a combination of 0.5 $\mathrm{mEq} / \mathrm{kg} \mathrm{Mg}$ and $2 \mathrm{mg} / \mathrm{kg}$ vitamin B6 (GarciaLopez et al., 2009; Stendig-Lindberg et al., 1998).

An inverse correlation between audiogenic stress and erythrocyte $\mathrm{Mg}$ levels has been observed (Galland, 1991), and low Mg status has been linked to self-reported noise sensitivity, noise-induced emotional lability and noiseinduced feelings of tenseness (Galland, 1991). Notably, exposure to long-term road traffic noise in both children and adults leads to elevated night time secretion of noradrenaline and cortisol when compared to controls (Ising and Braun, 2000). $\mathrm{Mg}$ administration is effective in diminishing ototoxicity (Abaamrane et al., 2009; Ehrenberger and Felix, 1995) as well as in alleviating transportation stress (Tang et al., 2008; Peeters et al., 2005). Cold stress also induces a decrease in Mg levels in CSF and raises the incidence of clinical manifestations (Matsui, 2007). Interestingly, a correlation has been described between $\mathrm{Mg}$ levels (in serum and peritoneal fluid) and stress perception (Jung et al., 2010; Garalejic et al., 2010) in relatively healthy, adult women unable to conceive but without identified organic causes of sterility.

It is now well known that $\mathrm{Mg}$ deficiency facilitates epileptiform discharges in hippocampal slices (Gutierrez et al., 1999) and in audiogenic seizures (Bac et al., 1998). Hippocampal neurons are more sensitive to low $\mathrm{Mg}$ than neurons from other brain regions and their responses to $\mathrm{Mg}$ deficiency differ depending on the developmental period (Furukawa et al., 2009). Human studies show that stress augments seizure frequency and severity in epileptic patients (Sawyer and Escayg, 2010).

Data from a second examination of the Copenhagen City Heart Study showed that selfreported high stress intensity and weekly stress 
were associated with almost a doubled risk of fatal stroke (Truelsen et al., 2003). In ischemic or hypoxic tissue, $\mathrm{Mg}$ antagonizes calcium's effects and decreases reactive oxygen species production through phopholipases, lipoxygenase and cyclooxygenase pathways. Likewise, in conditions associated with cerebral ischemia, $\mathrm{Mg}$ deficiency promotes vasoconstriction of cerebral and coronary arteries, as well as anoxic depolarizations and cortical spreading depression. Accordingly, Mg supplementation potentially plays a neuroprotective role (Turner et al., 2004). Mechanical stress such as membrane stretch or osmotic cell swelling can activate TRPM7-like channels (Numata et al., 2007), which are involved in $\mathrm{Mg}$ transport. These channels are members of the ubiquitously expressed melastatin-related subfamily of transient receptor potential (TRP) channels, and represent essential mediators of anoxic neuronal death (Aarts and Tymianski, 2005; Penner and Fleig, 2007). Low intracellular levels of $\mathrm{Mg}$, a process that exacerbates the effects of stress on the organism, also activate TRPM7 channels.

Conscious, critically ill patients experience unprecedented levels of stress, both physical and psychological. However, even unconscious, comatose or sedated patients can exhibit stress initiated by peripheral stimuli that directly activate limbic structures prior to cortical involvement. These patients exhibit multiple organ dysfunction and veritable endocrine chaos (Papathanassoglou et al., 2010). In these patients, hypomagnesemia is often associated with hypokalemia and is considered to aggravate the prognosis. Under these conditions, prolongation of QT interval constitutes a useful biomarker of electrolyte imbalance and the requirement for replacement therapy (Whitted et al., 2010). It is noteworthy that hypomagnesemia has been linked with a decrease in insulin sensitivity which can also affect the neurological outcome of critically ill patients (Polderman et al., 2003). In critically ill patients, a daily $\mathrm{Mg}$ supplementation index higher than 1 gram/day is associated with a lower mortality rate. $\mathrm{Mg}$ supplementation is also recommended for patients exposed to therapeutic hypothermia who often exhibit hypomagnesemia due to polyuresis (Dabbagh et al., 2006; Polderman et al., 2003).
In a model of intraperitoneal sepsis, $\mathrm{MgSO}_{4}$ administration $(750 \mu \mathrm{mol} / \mathrm{kg})$ attenuated increased blood-brain barrier permeability and caused a reduction in brain edema formation (Esen et al., 2005). Similar results have been observed in experimental traumatic brain injury (Turner and Vink, 2007) where intravenous administration of $\mathrm{MgSO}_{4}(250 \mu \mathrm{mol} / \mathrm{kg})$ also significantly decreased the incidence of posttraumatic depression/anxiety as compared to non-treated animals (Fromm et al., 2004).

Stress also influences the susceptibility to neurodegenerative disorders such as multiple sclerosis and Alzheimer's disease (Brown et al., 2006a; 2006b; Esch et al., 2002a). Proinflammatory effects associated with stress are exacerbated and exert deleterious actions upon the neurons, including increased IL6 levels that initiate microglial activation. Increased iNos levels are also expressed during critical periods and the elevated stress hormones themselves impair hippocampal neurogenesis and memory (Esch et al., 2002a; 2002b). Moreover, Mg deficiency increases neuronal calcium influx and consequently augments NO production that is associated with cytotoxic effects (Eby and Eby, 2006). Recent data also indicate a possible link between acute and sudden psychological stress and the appearance of malignant primitive brain tumours (Cabaniols et al., 2010). Furthermore, long-term survivors of brain cancer continue to experience elevated levels of stress as the fear of recurrence increases with time (Keir et al., 2007). Epidemiological data have described an inverse relationship between $\mathrm{Mg}$ content in drinking water and incidence of some types of cancers (Maier et al., 2007). A protective effect of fresh vegetable and fruit intake has also been noted. Although the role of $\mathrm{Mg}$ in carcinogenesis is yet to be established, it's involvement in DNA stabilization has been described (Anastassopoulou and Theophanides, 2002; Anghileri, 2009; Wolf et al., 2009).

Individuals with cardiovascular pathology are most susceptible to the concerted effects of stress and $\mathrm{Mg}$ deficiency. The inotropic and chronotropic response induced by catecholamine release produces an oxygen debt in the myocardium. In a hypoxic tissue, $\mathrm{Mg}$ shifts from the intracellular to the extracellular space, and 
calcium enters into cells, leading to increased risk of arrhythmias and cardiac deterioration (Seelig, 1989). Interestingly, $\mathrm{MgSO}_{4}$ is successfully used in the treatment of pheochromocytoma, a condition associated with excessive release of catecholamine. In this situation, Mg exhibits simultaneous beta-agonist effects and alpha-adrenergic antagonist actions, improves diastolic dysfunction and reduces episodes of arrhythmia (James, 2009). Encouraging results have also been obtained using $\mathrm{Mg}$ therapy in two newly described cardiomyopathic syndromes: Irukandji syndrome and stress cardiomyopathy. Irukandji syndrome is caused by envenomisation from a jellyfish (Carukia barnesi), which induces excessive release of endogenous catecholamines. Administration of $\mathrm{MgSO}_{4}$ alleviates pain and the cardiovascular response in Irukandji syndrome but there is still a need for evidence-based guidelines in the management of this condition (Tiong, 2009; Nickson et al., 2009; Barnett et al., 2005; Corkeron et al., 2004). Stress cardiomyopathy (also referred as Takotsubo cardiomyopathy or transient left ventricular apical ballooning syndrome) is an acute reversible apical ventricular dysfunction provoked by elevated levels of catecholamines. These stress hormones enable the shift of $\beta 2$-adrenoceptor transducing signal from Gs protein to Gi protein causing negative inotropic effects (Lyon et al., 2008). While there is no specific treatment for this condition, some authors reported beneficial results with $\mathrm{Mg}$ administration (Akashi et al., 2008).

Considerable evidence now supports the fact that $\mathrm{Mg}$ presents beneficial effects in different types of surgical stress, including induction and maintenance of pneumoperitoneum, endotracheal intubation, cardiopulmonary bypass, occlusionreperfusion surgery and middle ear surgery (James 2009; Jee et al., 2009; Puri et al., 1998; Ashton et al., 1991; Delhumeau et al., 1995; Manrique et al., 2010; Dorman et al., 2000; Kurian and Paddikkala 2010; Ryu et al., 2009). Considering the fact that pain induces the release of stress mediators, the blockade of neuronal NMDA receptors by $\mathrm{Mg}$ provides additional positive effects. Even if $\mathrm{Mg}$ is not a potent analgesic per se, several authors have shown that Mg potentates the analgesic effect of traditionally used pain relievers, reduces their requirements and decreases the incidence of postoperative shivering (Dabbagh et al., 2009; Lysakowski et al., 2007). Following carotid endarterectomy or cardiac surgery, Mg therapy improves cognitive outcome and prevents postoperative arrhythmias, especially in patients with contraindications for beta-blockers (Mack et al., 2009; Dabrowski et al., 2008; Davis et al.,).

In athletes who are subjected to severe physical stress, Mg favourably stabilizes the membrane integrity by binding with phosphate groups of phospholipids located in cell and organelle membranes (Golf et al., 1998). In these subjects, $17 \mathrm{mmol}$ magnesium supplementation per day lowered serum cortisol levels and augmented venous $\mathrm{O}_{2}$ partial pressure leading to better performance. Recent studies indicated that magnesium intake should be at least 260 $\mathrm{mg} /$ day for male and $220 \mathrm{mg} /$ day for female athletes (Nielsen and Lukaski, 2006). According to Couzy and colleagues (1990), Mg overdosage in professional sportsmen may lead to hypozincemia. The overtraining syndrome described in athletes is also a stress-related condition that designates the exhaustion stage of Selye's general adaptation syndrome. This syndrome is characterized by impairment of psychological processing, systemic inflammatory response, and HPA dysfunction (Angeli et al., 2004). Magnesium deficiency has been reported in this condition.

\section{Stress and the brain}

The brain is very susceptible to stress, largely because corticosterone binds at this level to high affinity mineralocorticoid receptors (Abaamrane et al., 2009) and to glucocorticoid receptors (GR), albeit with 10-fold lower affinity. The CA1 and dentate gyrus neurons of the hippocampus express high amounts of both receptor types, whereas CA3 pyramidal neurons primarily express mineralocorticoid receptors (Karst and Joels, 2003). A stress-induced rise in corticosteroid levels modulates short-term memory, generally by promoting storage of information that is emotionally related to the stressful event, but by suppressing information that is not associated with it (Lupien and Maheu, 2007). Glucocorticoids contribute to the consolidation of contextual fear conditioning, but can also lead to memory impairments (Kolber et 
al., 2008; Leon-Carrion et al., 2009). The inability to remember important data worsens the individual's stress and makes the subject even more vulnerable. Animal studies also show that stress-induced increases in NA levels modulates declarative memory according to an inverted-Ushaped function by enhancing the memory for information unrelated to the source of stress (Lupien and Maheu, 2007). A strong link between noradrenergic activation, glucocorticoid effects and basolateral amygdala function has been described (McGaugh and Roozendaal, 2002; Baldi and Bucherelli, 2005).

While acute stress affects memory in a reversible way, chronic stress results in deleterious changes in the hippocampus, especially an atrophy of dendrites of pyramidal neurons in the CA3 region of the hippocampus, and in other brain regions (prefrontal cortex, amygdala, brain stem and pons). By antagonising NMDA receptors, Mg prevents acute glucocorticoid effects on neurogenesis (McEwen, 2008a; Slutsky et al., 2010). Mg removal from the extracellular medium produces hyperexcitability and cellular death in cultured hippocampal neurons through a mechanism based on NMDA receptor activation and Raf-MEK-MSK1 signalling pathway (Hughes et al., 2003). Moreover, through the effects upon P-glycoprotein, $\mathrm{Mg}$ modulates the access of corticosteroids to the brain (Murck, 2002). Consistent with these data, $\mathrm{Mg}$ deficiency represents an important factor in the pathogenesis of aging defined by decreased adaptability to stress, alteration of hippocampus functioning, failure to turn off the hypothalamopituitary-adrenal axis and hyperadrenoglucocorticism (Durlach et al., 1993; Steptoe, 2007).

Stress exposure results in a long-lasting sensitization of the HPA axis to subsequent novel stressors. Exposure to a single stressor induces CRF-dependent depression of postsynaptic NMDA receptors that allows glutamate synapses in the paraventricular nucleus of the hypothalamus to undergo a short-term potentiation (STP) (Kuzmiski et al., 2010). There is a growing body of evidence that $\mathrm{Mg}$ plays an important role in synaptic plasticity and is involved in long-term potentiation (LTP) and long-term depression (LTD) of synaptic transmission (Billard, 2006). LTP is induced by cortical stimulation in Mg-free solutions and it is associated with massive calcium influx through NMDA-type receptors (Nakano et al., 2010). Diamond et al., (2007) suggested that complex interactions between LTP and LTD processes in the hippocampus contribute to the storage of emotional memories and stress-induced amnesia. Whether $\mathrm{Mg}$ can modulate effects of stress on LTP in the hippocampus, amygdala and prefrontal cortex remains to be established. Magnesium-Lthreonate administration in rats increases the density of synaptophysin-/synaptobrevin-positive puncta in DG and CA1 subregions of the hippocampus, which contributes to improvement of cognitive functions (learning abilities, working memory, short- and long-term memory in rats) (Slutsky et al., 2010). Mg also acts synergistically with the NMDA antagonist memantine on hippocampal LTP, and there are data that indicate that $\mathrm{Mg}$ leads to an improvement of cognitive function in dementia (Danysz et al., 2000; Frankiewicz et al., 1996).

$\mathrm{Mg}$ is also involved in long-term memory formation through NA activated pathways. Saturation by free $\mathrm{Mg}$ increases the affinity of adenylate cyclase for adrenaline and excessive stress affects the stability of this enzyme and produces loss of activity. Moreover, by activating adenylate cyclase in fat tissues, stress increases the production of chelating metabolites like $\mathrm{ATP}^{4-}$ or citrate ${ }^{-}$, which reduce the level of free $\mathrm{Mg}^{2+}$ (Bennun, 2010).

\section{Magnesium during the developmental period}

A number of studies have now suggested that magnesium and stress may play an important regulatory role in the early developmental period. For example, in rhesus monkeys, stressful pregnancy reduced the hippocampal volume and impaired neurogenesis in the dentate gyrus of the offspring (Bac et al., 1995; Coe et al., 2003). An acoustic startle protocol applied during the 24-weeks gestation time was associated with increased levels of cortisol and reduced exploratory behaviour in the 3-year-old descendants. Another study examining the newborn's response to moderate surgical trauma showed that neonates are more susceptible to stress than infants (Okur et al., 1995). Although there were not significant differences between infants and neonates 
regarding the elevations of cortisol and growth hormones after surgical stress, $\mathrm{Mg}$ concentrations declined more in newborns compared to the other group.

Both experimental and clinical data also suggest that fetal $\mathrm{Mg}$ depletion (as a consequence of maternal deficiency) and stress during critical developmental periods are involved in the pathogeny of sudden infant death syndrome (Caddell, 2001; Durlach et al., 2004). Durlach et al., (2002) also emphasize the importance of chrono-pathological stress due to primary hypofunction of the biological clock and the pineal dysfunction. Among the exogenous stressors that may lead to this syndrome, it is worth mentioning parental smoking, maternal alcoholism, environmental pollutants, bottle feeding, sleeping position, bedding, wrapping and ambient temperature (Durlach et al., 2004).

In fetal guinea pig brain, maternal $\mathrm{MgSO}_{4}$ administration during hypoxic stress exhibits neuroprotective effects by ameliorating nuclear membrane peroxidation and nuclear DNA fragmentation (Maulik et al., 2001). Additionally, maternal $\mathrm{MgSO}_{4}$ treatment before anticipated early preterm birth has proven to reduce the risk of cerebral palsy in surviving infants (Marlow, 2009). Given the "anti-stress" properties of Mg, this discovery once more emphasizes the importance of maternal Mg status on the neural, hormonal and behavioural outcome of the progenitors.

Finally, children hospitalized for respiratory pathology and who received sporadic parental visits exhibited lower serum $\mathrm{Mg}$ level and increased excretion of this cation compared to children who received constant parental support (Bednarek et al., 2004). There are also data that suggest Mg deficiency in children brought up in stressful orphanage conditions may lead to a lower intelligence quotient (Manrique et al., 2010; Papadopol et al., 2001).

\section{Magnesium and self-damaging behaviour}

In order to cope better with stress, individuals exhibit variable behavioural responses ranging from improving lifestyle to self-damage (e.g. smoking, drinking, overeating) or risk-taking. It is well known that susceptibility to stress among the population is diverse and there is a minority who exhibit an extreme response to stress in respect to intensity, features and extension of time. The myriad of individual differences regarding stress responses are mainly a result of genetic background, developmental processes (both prenatal and postnatal), and lifetime experiences (McEwen, 2007).

Individuals exhibiting self-damaging behaviour are more likely to present hypomagnesemia as alcohol itself depletes the organism of $\mathrm{Mg}$ (Romani, 2008). Depletion of $\mathrm{Mg}$ induced in rat astrocytes by alcohol produces disturbances in cytoplasmic and mitochondrial bioenergetic pathways leading to $\mathrm{Ca}^{2+}$ overload, ischemia and stroke (Altura and Altura, 1994). Eating disorders are also commonly linked to hypomagnesemia that may or may not be associated with hypokalemia, hypocalcemia and hypophosphatemia (Laird Birmingham 2010; Touyz, 2004). This is particularly concerning where the normal western diet is already low in magnesium. For example, in most countries, the magnesium RDA for women varies from 240 to $400 \mathrm{mg} /$ day and for adult men varies from 310 to $420 \mathrm{mg} /$ day. However, $\mathrm{Mg}$ intake in $70 \%$ of the western population is less than $400 \mathrm{mg} \mathrm{Mg}^{2+}$ per day, and for approximately $20 \%$ of the population this cation intake is less than one-half of the recommended dose (Eby and Eby, 2006). Persons with inadequate nutrition that often accompanies chronic stressful lifestyle need $\mathrm{Mg}$ supplementation as increased intake of carbohydrates, fats, exaggerated consumption of coffee and sodas reduce the Mg levels in the organism (Seelig, 1994; Nishimuta, 2007).

$\mathrm{Mg}$ administration can provide beneficial effects by decreasing the susceptibility of stressinduced drug relapse. $\mathrm{Mg}$ decreases activity of central glutamatergic synapses, especially those involved in the reward system that is activated by stress (Gilman, 2007; Nechifor, 2008), and displays a modulatory effect for opioid receptor binding (Turner and Vink, 2007). Furthermore, $\mathrm{Mg}$ administration indirectly influences hippocampal neurogenesis through its effect upon corticosteroid hormones, and in this way may decrease the drug-seeking behaviour in drugaddicted persons (Noonan et al., 2010). 


\section{Magnesium and substance $P$}

Several authors have recently suggested that many of the consequences of $\mathrm{Mg}$ deficiency in stress may be explained by the release of substance $P$. Substance $P$ is a neuropeptide that preferably activates tachykinin NK1 receptors. It is considered to be involved both in early (4-7 days) and late ( 3 weeks) phases of the systemic response to $\mathrm{Mg}$ deficiency. In the early days after the onset of a Mg-restricted diet, low concentrations of this cation diminishes the Mggated blockade of NMDA receptor channels, which leads to the release of substance $P$ and calcitonin gene-related peptide from the sensory $C$ fibres. The increased circulating levels of these neuropeptides initiate an important "neurogenic inflammation" characterized by raised levels of inflammatory cells, inflammatory cytokines and augmented production of reactive oxygen and nitrogen species (Kramer et al., 2009; TejeroTaldo et al., 2006). Brain microinjection of substance $P$ activates stress-processing pathways and induces defensive respiratory, cardiovascular, gastrointestinal and psychological changes, as well as stimulating corticosterone secretion by the adrenal glands (Rupniak, 2005). Furthermore, in a model of chronic psychosocial stress in shrews, the NK1

\section{References}

Aarts MM, Tymianski M (2005) TRPM7 and ischemic CNS injury. Neuroscientist 11:116-23.

Abaamrane L, Raffin F, Gal M, Avan P, Sendowski I (2009) Long-term administration of magnesium after acoustic trauma caused by gunshot noise in guinea pigs. Heart Res 247:137-45.

Ablin JN, Buskila D (2010) Emerging therapies for fibromyalgia: an update. Expert Opin Emerging Drugs 15:521-33.

Akashi YJ, Goldstein DS, Barbaro G, Ueyama T (2008) Takotsubo cardiomyopathy: a new form of acute, reversible heart failure. Circulation 118:2754-62.

Ali BH, Silsby JL, el Halawani ME (1987) The effect of magnesium aspartate, xylazine and morphine on the receptor antagonist SLV-323 alleviated dentate cytogenesis and diminished the decrease of hippocampal volume induced by stress (Czeh et al., 2005). Thus, substance $P$ is now widely considered to be involved in the clinical expression of stress.

\section{Conclusion}

$\mathrm{Mg}$ still represents an emerging research field despite being entered into medicine in the $\mathrm{XVII}{ }^{\text {th }}$ century as a constituent of Epsom salts (Durlach 2007). Although much has been discovered, many aspects of magnesium still remain unclear and await discovery. Undoubtedly, the future will uncover new $\mathrm{Mg}$ compounds of medicinal value, will further characterize $\mathrm{Mg}$ as a common factor in more stress-related pathologies, and will result in the discovery of more $\mathrm{Mg}$ transporters, while better methods for the determination of intracellular levels of the cation will pave the way for an improvement in simple diagnosis of true $\mathrm{Mg}^{2+}$ deficiency.

\section{Acknowledgements}

Magdalena Cuciureanu was a recipient of a 2010 Australian Go8 European Fellowship. immobilization-induced increase in the levels of prolactin in turkey plasma. J Vet Pharmacol Ther 10:119-26.

Altura BM, Altura BT (1994) Role of magnesium and calcium in alcohol-induced hypertension and strokes as probed by in vivo television microscopy, digital image microscopy, optical spectroscopy, 31P-NMR, spectroscopy and a unique magnesium ion-selective electrode. Alcohol Clin Exp Res 18:1057-68.

Amyard N, Leyris A, Monier C, Frances H, Boulu RG, Henrotte JG (1995) Brain catecholamines, serotonin and their metabolites in mice selected for low (MGL) and high (MGH) blood magnesium levels. Magnes Res 8:5-9. 
Anastassopoulou J, Theophanides T (2002)

Magnesium-DNA interactions and the possible relation of magnesium to carcinogenesis. Irradiation and free radicals. Crit Rev Oncol Hematol 42:79-91.

Angeli A, Minetto M, Dovio A, Paccotti P (2004) The overtraining syndrome in athletes: a stress-related disorder. J Endocrinol Invest 27:603-12.

Anghileri LJ (2009) Magnesium, calcium and cancer. Magnes Res 22:247-55.

Ashton WB, James MF, Janicki P, Uys PC (1991) Attenuation of the pressor response to tracheal intubation by magnesium sulphate with and without alfentanil in hypertensive proteinuric patients undergoing caesarean section. Brit J Anaes 67:741-7.

Bac P, Pages N, Herrenknecht C, Teste JF (1995) Inhibition of mouse-killing behaviour in magnesiumdeficient rats: effect of pharmacological doses of magnesium pidolate, magnesium aspartate, magnesium lactate, magnesium gluconate and magnesium chloride. Magnes Res 8:37-45.

Bac P, Maurois P, Dupont C, Pages N, Stables JP, Gressens P, Evrard P, Vamecq J (1998) Magnesium deficiency-dependent audiogenic seizures (MDDASs) in adult mice: a nutritional model for discriminatory screening of anticonvulsant drugs and original assessment of neuroprotection properties. J Neurosci 18:4363-73.

Baldi E, Bucherelli C (2005) The inverted "u-shaped" dose-effect relationships in learning and memory: modulation of arousal and consolidation. Nonlinearity Biol Toxicol Med 3:9-21

Barnett FI, Durrheim DN, Speare R, Muller R (2005) Management of Irukandji syndrome in northern Australia. Rural Remote Health 5:369.

Bednarczyk P, Dolowy K, Szewczyk A (2005) Matrix $\mathrm{Mg} 2+$ regulates mitochondrial ATP-dependent potassium channel from heart. FEBS Lett 579:1625-32.

Bednarek A, Pasternak K, Sztanke M, Boguszewska A (2004) Influence of admission procedure and hospitalization form on the value of magnesium concentration in serum, blood cells and urine in children hospitalizated due to pneumonia or obstructive bronchiolitis. Magnes Res 17:94-101.

Bennun A (2010) Characterization of the norepinephrine-activation of adenylate cyclase suggests a role in memory affirmation pathways Overexposure to epinephrine inactivates adenylate cyclase, a causal pathway for stress-pathologies. Bio Systems 100:87-93.

Billard JM (2006) Ageing, hippocampal synaptic activity and magnesium. Magnes Res 19:199-215.

Billyard AJ, Eggett DL, Franz KB (2006) Dietary magnesium deficiency decreases plasma melatonin in rats. Magnes Res 19:157-61.

Brown RF, Tennant CC, Sharrock M, Hodgkinson S, Dunn SM, Pollard JD (2006a) Relationship between stress and relapse in multiple sclerosis: Part I. Important features. Mult Scler 12:453-64.

Brown RF, Tennant CC, Sharrock M, Hodgkinson S, Dunn SM, Pollard JD (2006b) Relationship between stress and relapse in multiple sclerosis: Part II. Direct and indirect relationships. Mult Scler 12:465-75.

Cabaniols C, Giorgi R, Chinot O, Ferahta N, Spinelli V, Alla P, Barrie M, Lehucher-Michel MP (2010) Links between private habits, psychological stress and brain cancer: a case-control pilot study in France. $J$ Neurooncol 103:307-16.

Caddell J, Kupiecki R, Proxmire DL, Satoh P, Hutchinson $B$ (1986) Plasma catecholamines in acute magnesium deficiency in weanling rats. J Nutr 116:1896-901

Caddell JL (2001) A triple-risk model for the sudden infant death syndrome (SIDS) and the apparent lifethreatening episode (ALTE): the stressed magnesium deficient weanling rat. Magnes Res 14:227-38.

Cameron OG (2007) Pharmacological Treatments of Stress, In: Encyclopedia of Stress (Fink G, ed.) 2nd Edn, Oxford, Academic Press, p113-8.

Carrasco GA, Van de Kar LD (2003) Neuroendocrine pharmacology of stress. Eur J Pharmacol 463:235-72.

Ceremuzynski L, Herbaczynska-Cedro K, BroniszewskaArdelt B, Nauman J, Nauman A, Wozniewicz B, Lawecki $J$ (1978) Evidence for the detrimental effect of adrenaline infused to healthy dogs in doses imitating spontaneous secretion after coronary occlusion. Cardiovasc Res 12:179-89.

Cernak I, Savic V, Kotur J, Prokic V, Kuljic B, Grbovic D, Veljovic M (2000) Alterations in magnesium and oxidative status during chronic emotional stress. Magnes Res 13:29-36.

Chollet D, Franken P, Raffin Y, Henrotte JG, Widmer J, Malafosse A, Tafti M (2001) Magnesium involvement in sleep: genetic and nutritional models. Behav Genet 31:413-25. 
Classen HG (1981) [Effect of the current magnesium status on the development of stress ulcers and myocardial necroses]. Fortschr Med 99:1303-6.

Coe CL, Kramer M, Czeh B, Gould E, Reeves AJ, Kirschbaum C, Fuchs E (2003) Prenatal stress diminishes neurogenesis in the dentate gyrus of juvenile rhesus monkeys. Biol Psychiatry 54:1025-34.

Corkeron M, Pereira P, Makrocanis C (2004) Early experience with magnesium administration in Irukandji syndrome. Anaesth Intensive Care 32:666-9.

Couzy F, Lafargue P, Guezennec CY (1990) Zinc metabolism in the athlete: influence of training, nutrition and other factors. Int J Sports Med 11:263-6

Cox IM, Campbell MJ, Dowson D (1991) Red blood cell magnesium and chronic fatigue syndrome. Lancet 337:757-60.

Cratty MS, Birkle DL (1999) N-methyl-D-aspartate (NMDA)-mediated corticotropin-releasing factor (CRF) release in cultured rat amygdala neurons. Peptides 20:93-100.

Cundy T GA, Reid I.R., (2008) Calcium, phosphate and magnesium. In: Clinical biochemistry: Metabolic and Clinical Aspects (De William J and Marshall SKB, eds.) 2nd edn, Edinburgh, Churchill Livingstone Elsevier.

Czeh B, Pudovkina O, van der Hart MG, Simon M, Heilbronner U, Michaelis T, Watanabe T, Frahm J, Fuchs E (2005) Examining SLV-323, a novel NK1 receptor antagonist, in a chronic psychosocial stress model for depression. Psychopharmacol (Berl) 180:548-57.

Dabbagh A, Elyasi H, Razavi SS, Fathi M, Rajaei S (2009) Intravenous magnesium sulfate for post-operative pain in patients undergoing lower limb orthopedic surgery. Acta Anaesthesiol Scand 53:1088-91.

Dabbagh OC, Aldawood AS, Arabi YM, Lone NA, Brits R, Pillay M (2006) Magnesium supplementation and the potential association with mortality rates among critically ill non-cardiac patients. Saudi Med J 27:821-5.

Dabrowski W, Rzecki Z, Sztanke M, Visconti J, Wacinski P, Pasternak K (2008) The efficiency of magnesium supplementation in patients undergoing cardiopulmonary bypass: changes in serum magnesium concentrations and atrial fibrillation episodes. Magnes Res 21:205-17.

Danysz W, Parsons CG, Mobius HJ, Stoffler A, Quack G (2000) Neuroprotective and symptomatological action of memantine relevant for Alzheimer's disease-a unified glutamatergic hypothesis on the mechanism of action. Neurotox Res 2:85-97.

Davis EM, Packard KA, Hilleman DE (2010)

Pharmacologic prophylaxis of postoperative atrial fibrillation in patients undergoing cardiac surgery: beyond beta-blockers. Pharmacotherapy 30:749, $274 \mathrm{e}-318 \mathrm{e}$.

Dayas CV, Xu Y, Buller KM, Day TA (2000) Effects of chronic oestrogen replacement on stress-induced activation of hypothalamic-pituitary-adrenal axis control pathways. J Neuroendocrinol 12:784-94.

De Souza EB, Grigoriadis DE, Webster EL (1991) Role of brain, pituitary and spleen corticotropin-releasing factor receptors in the stress response. Methods Achiev Exp Pathol 14:23-44.

De Souza EB (1995) Corticotropin-releasing factor receptors: physiology, pharmacology, biochemistry and role in central nervous system and immune disorders. Psychoneuroendocrinol 20:789-819.

Delhumeau A, Granry JC, Cottineau C, Bukowski JG, Corbeau JJ, Moreau X (1995) [Comparison of vascular effects of magnesium sulfate and nicardipine during extracorporeal circulation]. Annales Francaises d'anesthesie et de Reanimation 14:149-53.

Depoortere H, Francon D, Llopis J (1993) Effects of a magnesium-deficient diet on sleep organization in rats. Neuropsychobiol 27:237-45.

Diamond DM, Campbell AM, Park CR, Halonen J, Zoladz PR (2007) The temporal dynamics model of emotional memory processing: a synthesis on the neurobiological basis of stress-induced amnesia, flashbulb and traumatic memories, and the YerkesDodson law. Neural Plast 2007:60803.

Dong JF, Cruz MA, Aboulfatova K, Martin C, Choi H, Bergeron AL, Martini SR, Kroll MH, Kent TA (2008) Magnesium maintains endothelial integrity, upregulates proteolysis of ultra-large von Willebrand factor, and reduces platelet aggregation under flow conditions. Thromb Haemost 99:586-93.

Dorman BH, Sade RM, Burnette JS, Wiles HB, Pinosky ML, Reeves ST, Bond BR, Spinale FG (2000) Magnesium supplementation in the prevention of arrhythmias in pediatric patients undergoing surgery for congenital heart defects. Amer Heart J 139:522-8. 
Durlach J (2007) Overview of magnesium research: history and current trends. In: New Perspectives in Magnesium Research: Nutrition and Health (Nishizawa Y, Mori H, Durlach J, eds.) London, Springer-Verlag, p310.

Durlach J, Bac P, Durlach V, Bara M, Guiet-Bara A (1997) Neurotic, neuromuscular and autonomic nervous form of magnesium imbalance. Magnes Res 10:169-95.

Durlach J, Durlach V, Bac P, Rayssiguier Y, Bara M, Guiet-Bara A (1993) Magnesium and ageing. II. Clinical data: aetiological mechanisms and pathophysiological consequences of magnesium deficit in the elderly. Magnes Res 6:379-94.

Durlach J, Pages N, Bac P, Bara M, Guiet-Bara A, Agrapart C (2002) Chronopathological forms of magnesium depletion with hypofunction or with hyperfunction of the biological clock. Magnes Res 15:263-8.

Durlach J, Pages N, Bac P, Bara M, Guiet-Bara A (2004) New data on the importance of gestational $\mathrm{Mg}$ deficiency. Magnes Res 17:116-25.

Durlach J, Pages N, Bac P, Bara M, Guiet-Bara A (2005) Headache due to photosensitive magnesium depletion. Magnes Res 18:109-22

Ebner K, Wotjak CT, Landgraf R, Engelmann M (2000) A single social defeat experience selectively stimulates the release of oxytocin, but not vasopresssin, within the septal area of male rats. Brain Res 872:87-92.

Eby GA, Eby KL (2006) Rapid recovery from major depression using magnesium treatment. Med Hypotheses 67:362-70.

Ehrenberger K, Felix D (1995) Receptor pharmacological models for inner ear therapies with emphasis on glutamate receptors: a survey. Acta Otolaryngol 115:236-40.

Esch T, Stefano GB, Fricchione GL, Benson H (2002a) Stress-related diseases - a potential role for nitric oxide. Med Sci Monit 8:103-18.

Esch T, Stefano GB, Fricchione GL, Benson H (2002b) The role of stress in neurodegenerative diseases and mental disorders. Neuro Endocrinol Lett 23:199-208.

Esen F, Erdem T, Aktan D, Orhan M, Kaya M, Eraksoy $\mathrm{H}$, Cakar N, Telci L (2005) Effect of magnesium sulfate administration on blood-brain barrier in a rat model of intraperitoneal sepsis: a randomized controlled experimental study. Crit Care 9:R18-23.
Feillet-Coudray C, Coudray C, Wolf FI, Henrotte JG, Rayssiguier Y, Mazur A (2004) Magnesium metabolism in mice selected for high and low erythrocyte magnesium levels. Metabolism 53:660-5.

Frankiewicz T, Potier B, Bashir Zl, Collingridge GL, Parsons CG (1996) Effects of memantine and MK-801 on NMDA-induced currents in cultured neurones and on synaptic transmission and LTP in area CA1 of rat hippocampal slices. Br J Pharmacol 117:689-97.

Franz KB (2004) A functional biological marker is needed for diagnosing magnesium deficiency. J Amer Coll Nutr 23:738S-41S.

Fromm L, Heath DL, Vink R, Nimmo AJ (2004)

Magnesium attenuates post-traumatic depression/anxiety following diffuse traumatic brain injury in rats. J Amer Coll Nutr 23:529S-33S.

Fujino K, Yoshitake T, Inoue O, Ibii N, Kehr J, Ishida J, Nohta H, Yamaguchi M (2002) Increased serotonin release in mice frontal cortex and hippocampus induced by acute physiological stressors. Neurosci Lett 320:91-5.

Funada M, Hara C (2001) Differential effects of psychological stress on activation of the 5hydroxytryptamine- and dopamine-containing neurons in the brain of freely moving rats. Brain Res 901:247-51.

Furukawa Y, Kasai N, Torimitsu K (2009) Effect of Mg2+ on neural activity of rat cortical and hippocampal neurons in vitro. Magnes Res 22:174S-81S

Galland L (1991) Magnesium, stress and neuropsychiatric disorders. Magnesium Trace Elements 10:287-301.

Garalejic E, Bojovic-Jovic D, Damjanovic A, Arsic B, Pantic I, Turjacanin-Pantelic D, Perovic M (2010) Hamilton anxiety scale (HAMA) in infertile women with endometriosis and its correlation with magnesium levels in peritoneal fluid. Psychiatria Danubina 22:64-7.

Garcia-Lopez R, Perea-Milla E, Garcia CR, Rivas-Ruiz F, Romero-Gonzalez J, Moreno JL, Faus V, Aguas Gdel C, Diaz JC (2009) New therapeutic approach to Tourette Syndrome in children based on a randomized placebocontrolled double-blind phase IV study of the effectiveness and safety of magnesium and vitamin B6. Trials 10:16. 
Garcy AM, Marotta SF (1978) Effects of cerebroventricular perfusion with monovalent and divalent cations on plasma cortisol of conscious cats. Neuroendocrinol 26:32-40.

Gilman S (2007) Neurobiology of Disease, 1st edn, Elsevier Academic Press, New York.

Gimpl G, Reitz J, Brauer S, Trossen C (2008) Oxytocin receptors: ligand binding, signalling and cholesterol dependence. Prog Brain Res 170:193-204.

Golf SW, Bender S, Gruttner J (1998) On the significance of magnesium in extreme physical stress. Cardiovas Drugs Therapy 12 Suppl 2:197-202.

Grimaldi BL (2002) The central role of magnesium deficiency in Tourette's syndrome: causal relationships between magnesium deficiency, altered biochemical pathways and symptoms relating to Tourette's syndrome and several reported comorbid conditions. Med Hypotheses 58:47-60.

Gutierrez R, Armand V, Schuchmann S, Heinemann U (1999) Epileptiform activity induced by low $\mathrm{Mg} 2+$ in cultured rat hippocampal slices. Brain Research 815:294-303.

Held K, Antonijevic IA, Kunzel H, Uhr M, Wetter TC, Golly IC, Steiger A, Murck H (2002) Oral Mg(2+) supplementation reverses age-related neuroendocrine and sleep EEG changes in humans.

Pharmacopsychiatry 35:135-43.

Henrotte JG, Franck G, Santarromana M, Frances H, Mouton D, Motta R (1997) Mice selected for low and high blood magnesium levels: a new model for stress studies. Physiol Behav 61:653-8.

Henrotte JG, Levy-Leboyer C (1985) [Is type A behavior modulated by genetic factors regulating magnesium and zinc metabolism? Working hypotheses and preliminary results]. Magnesium 4:295-302.

Henrotte JG, Pla M, Dausset J (1990) HLA- and H-2associated variations of intra- and extracellular magnesium content. Proc Natl Acad Sci USA:1894-8.

Henrotte JG, Plouin PF, Levy-Leboyer C, Moser G, Sidoroff-Girault N, Franck G, Santarromana M, Pineau $M$ (1985) Blood and urinary magnesium, zinc, calcium, free fatty acids, and catecholamines in type $A$ and type B subjects. J Amer Coll Nutr 4:165-72.

Hirvikoski T, Lindholm T, Nordenstrom A, Nordstrom AL, Lajic S (2009) High self-perceived stress and many stressors, but normal diurnal cortisol rhythm, in adults with ADHD (attention-deficit/hyperactivity disorder). Horm Behav 55:418-24.

Hughes JP, Staton PC, Wilkinson MG, Strijbos PJ, Skaper SD, Arthur JS, Reith AD (2003) Mitogen and stress response kinase-1 (MSK1) mediates excitotoxic induced death of hippocampal neurones. J Neurochem 86:25-32.

Ising H, Braun C (2000) Acute and chronic endocrine effects of noise: Review of the research conducted at the Institute for Water, Soil and Air Hygiene. Noise Health 2:7-24.

James MF (2009) Magnesium: an emerging drug in anaesthesia. Brit J Anaesth 103:465-7.

Jee D, Lee D, Yun S, Lee C (2009) Magnesium sulphate attenuates arterial pressure increase during laparoscopic cholecystectomy. Brit J Anaesth 103:4849.

Jorgensen HS (2007) Studies on the neuroendocrine role of serotonin. Dan Med Bull 54:266-88.

Jung KI, Ock SM, Chung JH, Song CH (2010) Associations of serum $\mathrm{Ca}$ and $\mathrm{Mg}$ levels with mental health in adult women without psychiatric disorders. Biol Trace Element Research 133:153-61.

Kantorovich V, Eisenhofer G, Pacak K (2008) Pheochromocytoma: an endocrine stress mimicking disorder. Annals NY Acad Sci 1148:462-8.

Karst H, Joels M (2003) Effect of chronic stress on synaptic currents in rat hippocampal dentate gyrus neurons. J Neurophysiol 89:625-33.

Keir ST, Swartz JJ, Friedman HS (2007) Stress and longterm survivors of brain cancer. Support Care Cancer $15: 1423-8$

Ko SH, Lim HR, Kim DC, Han YJ, Choe H, Song HS (2001) Magnesium sulfate does not reduce postoperative analgesic requirements. Anesthesiol 95:640-6.

Kolber BJ, Roberts MS, Howell MP, Wozniak DF, Sands MS, Muglia LJ (2008) Central amygdala glucocorticoid receptor action promotes fear-associated $\mathrm{CRH}$ activation and conditioning. Proc Natl Acad Sci USA 105:12004-9.

Kozielec T, Starobrat-Hermelin B (1997) Assessment of magnesium levels in children with attention deficit hyperactivity disorder (ADHD). Magnes Res 10:143-8. 
Kramer JH, Spurney C, lantorno M, Tziros C, Mak IT, Tejero-Taldo MI, Chmielinska JJ, Komarov AM, Weglicki WB (2009) Neurogenic inflammation and cardiac dysfunction due to hypomagnesemia. Am J Med Sci 338:22-7.

Kurian GA, Paddikkala J (2010) N-acetylcysteine and magnesium improve biochemical abnormalities associated with myocardial ischaemic reperfusion in South Indian patients undergoing coronary artery bypass grafting: a comparative analysis. Singapore Med J 51:381-8.

Kuzmiski JB, Marty V, Baimoukhametova DV, Bains JS (2010) Stress-induced priming of glutamate synapses unmasks associative short-term plasticity. Nat Neurosci 13:1257-64.

Labuschagne I, Phan KL, Wood A, Angstadt M, Chua P, Heinrichs M, Stout JC, Nathan PJ (2010) Oxytocin attenuates amygdala reactivity to fear in generalized social anxiety disorder. Neuropsychopharmacol 35:2403-13.

Laird Birmingham CJ (2010) Complications of nutritional therapy. In: Medical Management of Eating Disorders): Cambridge: University Press, 59-72.

Leon-Carrion J, Atutxa AM, Mangas MA, Soto-Moreno A, Pumar A, Leon-Justel A, Martin-Rodriguez JF, Venegas $\mathrm{E}$, Dominguez-Morales MR, Leal-Cerro A (2009) A clinical profile of memory impairment in humans due to endogenous glucocorticoid excess. Clin Endocrinol (Oxf) 70:192-200.

Lupien SJ, Maheu FS (eds) (2007) Memory and Stress. Academic Press: Oxford.

Lyon AR, Rees PS, Prasad S, Poole-Wilson PA, Harding SE (2008) Stress (Takotsubo) cardiomyopathy-a novel pathophysiological hypothesis to explain catecholamine-induced acute myocardial stunning. Nat Clin Pract Cardiovasc Med 5:22-9.

Lysakowski C, Dumont L, Czarnetzki C, Tramer MR (2007) Magnesium as an adjuvant to postoperative analgesia: a systematic review of randomized trials. Anesthes Analges 104:1532-9.

Mack WJ, Kellner CP, Sahlein DH, Ducruet AF, Kim GH, Mocco J, Zurica J, Komotar RJ, Haque R, Sciacca R, Quest DO, Solomon RA, Connolly ES, Heyer EJ (2009) Intraoperative magnesium infusion during carotid endarterectomy: a double-blind placebo-controlled trial. J Neurosurg 110:961-7

Magalhaes AC, Holmes KD, Dale LB, Comps-Agrar L, Lee D, Yadav PN, Drysdale L, Poulter MO, Roth BL, Pin
JP, Anisman H, Ferguson SS (2010) CRF receptor 1 regulates anxiety behavior via sensitization of 5-HT2 receptor signaling. Nat Neurosci 13:622-9.

Maier JA, Nasulewicz-Goldeman A, Simonacci M, Boninsegna A, Mazur A, Wolf FI (2007) Insights into the mechanisms involved in magnesium-dependent inhibition of primary tumor growth. Nutr Cancer 59:192-8.

Manrique AM, Arroyo M, Lin Y, El Khoudary SR, Colvin E, Lichtenstein S, Chrysostomou C, Orr R, Jooste E, Davis P, Wearden P, Morell V, Munoz R (2010) Magnesium supplementation during cardiopulmonary bypass to prevent junctional ectopic tachycardia after pediatric cardiac surgery: a randomized controlled study. J Thorac Cardiovasc Surg 139:162-9.

Manuel y Keenoy B, Moorkens G, Vertommen J, Noe M, Neve J, De Leeuw I (2000) Magnesium status and parameters of the oxidant-antioxidant balance in patients with chronic fatigue: effects of supplementation with magnesium. J Amer Coll Nutr 19:374-82.

Marlow N (2009) Review: antenatal magnesium sulphate prevents cerebral palsy in preterm infants. Evid Based Med 14:141.

Matsui T (2007) Significance of Magnesium in Animals (Yoshiki Nishizawa, Mori H, Durlach J, eds.) London: Springer-Verlag.

Matthews KA (1982) Psychological perspectives on the type A behavior pattern. Psychol Bull 91:293-323.

Maulik D, Qayyum I, Powell SR, Karantza M, Mishra OP, Delivoria-Papadopoulos M (2001) Post-hypoxic magnesium decreases nuclear oxidative damage in the fetal guinea pig brain. Brain research 890:130-6.

McEwen BS (2007) Stress, Definitions and Concepts of. In: Encyclopedia of Stress (Fink, ed.) 2nd ed., vol. 3: Oxford, Academic Press, 653.

McEwen BS (2008a) Central effects of stress hormones in health and disease: Understanding the protective and damaging effects of stress and stress mediators. Eur J Pharmacol 583:174-85.

McEwen W (2008b) Allostasis and Allostatic Load. In: Encyclopedia of Stress (Fink G, ed.) 2nd ed., vol. I: Oxford: Academic Press, 135-41.

McGaugh JL, Roozendaal B (2002) Role of adrenal stress hormones in forming lasting memories in the brain. Curr Opin Neurobiol 12:205-10. 
Mckee JA, Brewer RP, Macy GE, Borel CO, Reynolds JD, Warner DS (2005) Magnesium neuroprotection is limited in humans with acute brain injury. Neurocrit Care 2:342-51.

Mizoguchi K, Shoji H, Ikeda R, Tanaka Y, Tabira T (2008) Persistent depressive state after chronic stress in rats is accompanied by HPA axis dysregulation and reduced prefrontal dopaminergic neurotransmission. Pharmacol Biochem Behav 91:170-5.

Motta R, Louis JP, Frank G, Henrotte JG (1998) Unexpected association between reproductive longevity and blood magnesium levels in a new model of selected mouse strains. Growth Dev Aging 62:37-45.

Mousain-Bosc M, Roche M, Polge A, Pradal-Prat D, Rapin J, Bali JP (2006) Improvement of neurobehavioral disorders in children supplemented with magnesium-vitamin B6. I. Attention deficit hyperactivity disorders. Magnes Res 19:46-52.

Muir KW (2002) Magnesium in stroke treatment. Postgrad Med J 78:641-5.

Muneyyirci-Delale O, Nacharaju VL, Dalloul M, Altura BM, Altura BT (1999) Serum ionized magnesium and calcium in women after menopause: inverse relation of estrogen with ionized magnesium. Fertil Steril 71:869-72.

Murck H (2002) Magnesium and affective disorders. Nutr Neurosci 5:375-89.

Nakano T, Doi T, Yoshimoto J, Doya K (2010) A kinetic model of dopamine- and calcium-dependent striatal synaptic plasticity. PLoS Comput Biol 6:e1000670.

Nechifor M (2008) Magnesium in drug dependences. Magnes Res 21:5-15.

Neumann ID, Veenema AH, Beiderbeck DI (2010)

Aggression and anxiety: social context and neurobiological links. Front Behav Neurosci 4:12.

Neylan (1998) Hans Selye and the Field of Stress Research. J Neuropsychiatry Clin Neurosci 10:230-1.

Nickson CP, Waugh EB, Jacups SP, Currie BJ (2009) Irukandji syndrome case series from Australia's Tropical Northern Territory. Ann Emerg Med 54:395403.

Nielsen FH, Lukaski HC (2006) Update on the relationship between magnesium and exercise. Magnes Res 19:180-9.
Nishimuta M KN, Morikuni E, Matsuzaki N, Yoshioka YH, Yamada H, Kitajima H, Takeyama H (2007) Magnesium Requirement and Affecting Factors (Nishizawa Y, Mori H, Durlach J, eds.) London, Springer-Verlag, p94-103.

Nishizawa Y, Morii H, Durlach J (2007) New Perspectives in Magnesium Research, Nutrition and Health (Nishizawa Y, Mori H, Durlach J, eds.) London, Springer-Verlag.

Noonan MA, Bulin SE, Fuller DC, Eisch AJ (2010) Reduction of adult hippocampal neurogenesis confers vulnerability in an animal model of cocaine addiction. $J$ Neurosci 30:304-15.

Numata T, Shimizu T, Okada Y (2007) Direct mechanostress sensitivity of TRPM7 channel. Cell Physiol Biochem 19:1-8.

Okur H, Kucukaydin M, Ustdal KM (1995) The endocrine and metabolic response to surgical stress in the neonate. J Pediatr Surg 30:626-5.

Omiya K, Akashi YJ, Yoneyama K, Osada N, Tanabe K, Miyake F (2009) Heart-rate response to sympathetic nervous stimulation, exercise, and magnesium concentration in various sleep conditions. Int J Sport Nutr Exercise Metabol 19:127-35.

Oppelt WW, Maclntyre I, Rall DP (1963) Magnesium exchange between blood and cerebrospinal fluid. Am J Physiol 205:959-62.

Papadopol V, Tuchendria E, Palamaru I (2001) Magnesium and some psychological features in two groups of pupils (magnesium and psychic features). Magnes Res 14:27-32

Papathanassoglou ED, Giannakopoulou M, Mpouzika M, Bozas E, Karabinis A (2010) Potential effects of stress in critical illness through the role of stress neuropeptides. Nurs Crit Care 15:204-16.

Peeters E, Neyt A, Beckers F, De Smet S, Aubert AE, Geers R (2005) Influence of supplemental magnesium, tryptophan, vitamin $C$, and vitamin E on stress responses of pigs to vibration. J Animal Sci 83:1568-80.

Penner R, Fleig A (2007) The Mg2+ and Mg(2+)nucleotide-regulated channel-kinase TRPM7. Handb Exp Pharmacol:313-28.

Perrin MH, Haas Y, Rivier JE, Vale WW (1986) Corticotropin-releasing factor binding to the anterior pituitary receptor is modulated by divalent cations and guanyl nucleotides. Endocrinol 118:1171-9. 
Polderman KH, van Zanten AR, Girbes AR (2003) The importance of magnesium in critically ill patients: a role in mitigating neurological injury and in the prevention of vasospasms. Intensive Care Med 29:1202-3.

Puri GD, Marudhachalam KS, Chari P, Suri RK (1998) The effect of magnesium sulphate on hemodynamics and its efficacy in attenuating the response to endotracheal intubation in patients with coronary artery disease. Anesthes Analges 87:808-11.

Quick S (1994) Walter Bradford Cannon: Pioneer of stress research. Int J Stress Management I:141-3

Rayssiguier Y, Guezennec CY, Durlach J (1990) New experimental and clinical data on the relationship between magnesium and sport. Magnes Res 3:93-102.

Romani AM (2008) Magnesium homeostasis and alcohol consumption. Magnes Res 21:197-204.

Rucklidge JJ, Johnstone J, Kaplan BJ (2009) Nutrient supplementation approaches in the treatment of ADHD. Expert Rev Neurother 9:461-76.

Rupniak NMJ (2005) Substance P (NK1 receptor) antagonists. In: Handbook of Stress and the Brain Part 2: Stress: Integrative and Clinical Aspects (Steckler T, Kalin NH, Reul JMHM, eds) Elsevier, New York, 42335.

Ryu JH, Sohn IS, Do SH (2009) Controlled hypotension for middle ear surgery: a comparison between remifentanil and magnesium sulphate. Brit J Anaesthes 103:490-5.

Sarchielli P, Coata G, Firenze C, Morucci P, Abbritti G, Gallai V (1992) Serum and salivary magnesium levels in migraine and tension-type headache. Results in a group of adult patients. Cephalalgia 12:21-7.

Sawyer NT, Escayg A (2010) Stress and epilepsy: multiple models, multiple outcomes. J Clin Neurophysiol 27:445-52.

Seelig M (1989) Cardiovascular consequences of magnesium deficiency and loss: pathogenesis, prevalence and manifestations--magnesium and chloride loss in refractory potassium repletion. Amer J Cardiol 63:4G-21G.

Seelig MS (1993) Interrelationship of magnesium and estrogen in cardiovascular and bone disorders, eclampsia, migraine and premenstrual syndrome. J Amer Coll Nutr 12:442-58.
Seelig MS (1994) Consequences of magnesium deficiency on the enhancement of stress reactions; preventive and therapeutic implications (a review). J Amer Coll Nutr 13:429-46.

Seelig MS, Altura BM, Altura BT (2004) Benefits and risks of sex hormone replacement in postmenopausal women. J Amer Coll Nutr 23:482S-96S.

Sendur OF, Tastaban E, Turan Y, Ulman C (2008) The relationship between serum trace element levels and clinical parameters in patients with fibromyalgia. Rheumatol Int 28:1117-21.

Sivonova M, Zitnanova I, Hlincikova L, Skodacek I, Trebaticka J, Durackova Z (2004) Oxidative stress in university students during examinations. Stress (Amsterdam, Netherlands) 7:183-8

Slutsky I, Abumaria N, Wu LJ, Huang C, Zhang L, Li B, Zhao X, Govindarajan A, Zhao MG, Zhuo M, Tonegawa $S$, Liu G (2010) Enhancement of learning and memory by elevating brain magnesium. Neuron 65:165-77.

Sobrinho LG (2003) Prolactin, psychological stress and environment in humans: adaptation and maladaptation. Pituitary 6:35-9.

Soma M, Cunnane SC, Horrobin DF, Manku MS, Honda M, Hatano M (1988) Effects of low magnesium diet on the vascular prostaglandin and fatty acid metabolism in rats. Prostaglandins 36:431-41.

Sontia B, Touyz RM (2007) Role of magnesium in hypertension. Arch Biochem Biophys 458:33-9.

Stendig-Lindberg G, Moran D, Shapiro Y (1998) How significant is magnesium in thermoregulation? J Basic Clinical Physiol Pharmacol 9:73-85.

Sterling P, Eyer J (1981) Biological basis of stress related mortality. Soc Sci Med E 15, 3-42.

Steptoe A (2007) Stress Effects, Overview. In: Encyclopedia of Stress (Fink G, ed.) 2nd edn, vol. 3: Oxford, Academic Press, 599-600.

Szewczyk B, Poleszak E, Sowa-Kucma M, Siwek M, Dudek D, Ryszewska-Pokrasniewicz B, RadziwonZaleska M, Opoka W, Czekaj J, Pilc A, Nowak G (2008) Antidepressant activity of zinc and magnesium in view of the current hypotheses of antidepressant action. Pharmacol Rep 60:588-9. 
Tang R, Yu B, Zhang K, Chen D (2008) Effects of supplementing two levels of magnesium aspartate and transportation stress on pork quality and gene expression of micro-calpain and calpastatin of finishing pigs. Arch Animal Nutr 62:415-25.

Tejero-Taldo MI, Kramer JH, Mak lu T, Komarov AM, Weglicki WB (2006) The nerve-heart connection in the pro-oxidant response to Mg-deficiency. Heart Failure Rev 11:35-44.

Thomas J, Tomb E, Thomas E, Faure G (1994) Migraine treatment by oral magnesium intake and correction of the irritation of buccofacial and cervical muscles as a side effect of mandibular imbalance. Magnes Res 7:123-7.

Tiong K (2009) Irukandji syndrome, catecholamines, and mid-ventricular stress cardiomyopathy. Eur J Echocardiogr 10:334-6.

Touyz RM (2004) Magnesium in clinical medicine. Front Biosci 9:1278-93.

Truelsen T, Nielsen N, Boysen G, Gronbaek M (2003) Self-reported stress and risk of stroke: the Copenhagen City Heart Study. Stroke 34:856-62.

Turner R, Vink R (2007) Magnesium in the Central Nervous System. In: New Perspectives in Magnesium Research: Nutrition and Research (Yoshiki Nishizawa HM, Mori, H, Durlach J, eds.) London, Springer-Verlag, p338-58.
Turner RJ, Dasilva KW, O'Connor C, van den Heuvel C, Vink R (2004) Magnesium gluconate offers no more protection than magnesium sulphate following diffuse traumatic brain injury in rats. J Am Coll Nutr 23: 541S$4 \mathrm{~S}$.

Uvnas-Moberg K, Petersson M (2005) [Oxytocin, a mediator of anti-stress, well-being, social interaction, growth and healing]. Z Psychosom Med Psychother 51:57-80.

Wacker WE, Parisi AF (1968) Magnesium metabolism. N Engl J Med 278:712-7.

Whitted AD, Stanifer JW, Dube P, Borkowski BJ, Yusuf J, Komolafe BO, Davis RC, Jr., Soberman JE, Weber KT (2010) A dyshomeostasis of electrolytes and trace elements in acute stressor states: impact on the heart. Am J Med Sci 340:48-53.

Whyte KF, Addis GJ, Whitesmith R, Reid JL (1987) Adrenergic control of plasma magnesium in man. Clin Sci (Lond) 72:135-8.

Wolf FI, Maier JA, Nasulewicz A, Feillet-Coudray C, Simonacci M, Mazur A, Cittadini A (2007) Magnesium and neoplasia: from carcinogenesis to tumor growth and progression or treatment. Arch Biochem Biophys 458:24-32.

Wolf FI, Trapani V, Cittadini A, Maier JA (2009) Hypomagnesaemia in oncologic patients: to treat or not to treat? Magnes Res 22:5-9. 


\title{
Magnesium in neuroses and neuroticism
}

\author{
Victoria Papadopol ${ }^{1, \otimes}$ and Mihai Nechifor ${ }^{2}$ \\ ${ }^{1}$ Regional Centre of Public Health, lasi, Romania. \\ ${ }^{2}$ Department of Pharmacology, "Gr. T. Popa” University of Medicine and Pharmacy, lasi, Romania. \\ $\triangle$ vicpapadopol@yahoo.com
}

\begin{abstract}
Neuroses are a frequently encountered group of psychiatric diseases with a symptomatology including neuronal hyperexcitability, anxiety, panic, phobic reactions, quite frequent tiredness, attention deficit, anorexia or bulimia, and sleep disorders, amongst others. The main pathogenic mechanisms involved in neurosis are increased activity of the glutamatergic cerebral systems (the increased synthesis, presynaptic release and action upon NMDA receptors), an intense catecholamine release, and the decrease of GABAergic cerebral systems activity. In most neurotic patients there is a reduction of plasma and intracellular magnesium concentration. Magnesium administration decreases anxiety, panic and phobia and ameliorates the attention deficit and sleep disorders. We consider that magnesium acts mainly by: a) the reduction of presynaptic glutamate release; b) the reduction of NMDA receptor activity by competing with calcium at NMDA receptor coupled calcium channels; $c$ ) the positive allosteric modulator effect at the level of some metabotropic presynaptic glutamate receptors, thereby decreasing presynaptic glutamate release and stimulating GABA release; and d) the decrease of catecholamine release by a direct presynaptic effect under the action of some factors including calcium. The stimulation of catecholamine release under stressful conditions leads to increased magnesium waste that is an essential event in the appearance of the functional cerebral changes characteristic of neurosis symptomatology. Unlike neuroses, neuroticism is not pathologic, but rather a normal state characterized by the dominance of some neuropsychological symptoms. There are data that show that the level of magnesium concentration is lower in subjects with high scores of neuroticism compared with other subjects.
\end{abstract}

\section{Introduction}

Magnesium is a nutrient essential for the health of the whole organism, including the brain. It is the fourth most abundant mineral in the body. Due to its relationships with more than 300 biochemical reactions, it is possible that magnesium is involved in more aspects of human body make-up and metabolism than any other mineral. Because the usual diet has drifted away from green leafy vegetables and unprocessed grains in favour of more refined and often nutrient-poor food options, magnesium inadequacy has become quite common in many areas of the world. One study shows that $68 \%$ of Americans are magnesium deficient ( $w w w . u s d a . g o v)$.

The role of magnesium is complex and its deficiency is implicated in a number of nonspecific neuropsychological changes such as agitation, fear, anxiety, depression, dizziness, poor attention, insomnia, and restlessness. Some of these symptoms characterize the mental illness known as neurosis.

\section{Neuroses}

Neuroses - characteristics and classification

Neurosis is a functional disorder of the central nervous system (CNS) generally characterized by excessive anxiety without evidence of neurologic disease, sometimes accompanied by defensive or immature behaviours. It is a general term describing a group of widespread mental illnesses also known as neurotic disorders, and thus, those suffering from them are said to be neurotics. It is also an emotional disorder that affects a part of the personality. Neuroses include a large variety of psychiatric disorders exhibiting a range of symptoms such as phobias, anxiety, tics and others. These disorders are quite a heterogeneous entity that is difficult to delimit. The myriad of factors that have been implicated makes the outline of a single psychopathological theory of 
neurosis difficult, and this has convinced some modern authors to remove the term. Although the American Diagnostic and Statistic Manual of Mental Disorders (DSM) has eliminated the category of neurosis, many authors still refer to them. Sometimes it is named anxiety neurosis or anxiety and phobic disorders. Anxiety, one of the most common symptoms of neurosis, is a physical symptom that frequently includes palpitations, nausea, chest pain, sweating, and trembling, amongst others.

Some authors consider that the main characteristics of neuroses are the existence of psychic, somatic and behavioural troubles without anatomical and psychological changes, with the patient being aware of the pathology of their symptoms but unable to control them (although social adaptation is satisfactory except in serious forms of the disorder). There is also an obvious but variable response to therapy (medicines, psychotherapy, relaxing techniques, hypnosis). Historically, the definition and classification of neuroses were frequently restructured according to different explicative theories. Therefore, the neuroses-related terminology is very variable. Essentially, neurotic symptoms are considered as a symbolic expression of an internal drama that the subject is unable to control because its essential elements drop out of their consciousness. Some authors include the social phobias, agoraphobias, hypochondria, panic disorder and even hysteria in neuroses. Generally, neurosis means poor ability to adapt to ones environment, an inability to change one's life pattern, and the inability to develop a richer, more complex, more satisfying personality (Boeree, 2002). Some professionals use the term to describe anxious symptoms and associated behaviour, or to describe the range of mental illnesses outside of the psychiatric disorders. Others use the term neurosis to describe the internal process itself that triggers the anxiety characteristics.

The International Classification of Diseases (ICD) 10 (World Health Organisation, 1992) grouped the neurotic disorders together with stress and somatophorm disturbances because of their historical association with the term neurosis, and because of their association with psychological causes. The concept of neurosis was not the major organizing principle. The combination of symptoms is common (most frequent being the associated depression-anxiety), especially in the less serious variants of these disturbances that are met in the first instance. ICD-10 introduces this type of disorder in the chapter "Neurotic, stress-related and somatoform disorders":

a) phobic anxiety disorders: agoraphobia with panic attacks, agoraphobia without panic attacks, social phobia, specific phobia, other phobic anxiety disorders (specified and unspecified)

b) other anxiety disorders: panic disorder, generalized anxiety disorder, mixed anxiety and depressive disorder, other anxiety disorders (specified and unspecified)

c) obsessive-compulsive disorders

d) reactions to severe stress, and adjustment disorders

e) dissociative disorders

f) somatoform disorders

g) other neurotic disorders.

Excessive anxiety and fear are common symptoms of neurotic disorders. According to Boeree (2002), the signs and symptoms of neurosis refer to a variety of psychological problems involving persistent experience of negative affects including anxiety, sadness and depression, anger, irritability, mental confusion, low sense of self-worth, behavioural symptoms such as phobic avoidance, vigilance, impulsive and compulsive acts, lethargy, cognitive problems such as unpleasant and disturbing thoughts, repetition of thoughts and obsessions, habitual fantasizing, negativity and cynicism. Neurosis or neurotic disorder can vegetatively affect the person's ability to function, thus affecting the activities of daily living. Some factors (education, culture) may serve to override any predisposing physiological conditions, or to exacerbate them. An essential point concerns the triggering stressors in a person's life, which lead to the various emotional, behavioural, and cognitive symptoms of neurosis.

\section{Relationship between magnesium and neuroses}

There are studies that show the involvement of magnesium in the pathogeny of most symptoms that can appear in neuroses. The magnesium level in the brain is more stable and decreases more slowly than in other tissues, but small decreases have important influences on neuronal 
functioning. Taking into account the existence of some symptoms, both in magnesium deficiency and in neurosis (latent tetany, hyperventilation syndrome, anxiety, hyper emotionality, fatigue and sometimes migraine, dizziness, nervous fits such as panic attack, particularly, the sensation of a "lump in the throat"), one can speculate that a relationship between magnesium deficiency and neurosis exists.

The most frequent and characteristic form of magnesium deficit disorders in the central nervous system is nervous hyperexcitability (Durlach, 1998; Durlach et al., 1997), and hence nervous forms of magnesium deficiency represent the most commonly seen form in clinical practice whatever the age. Nervous hyperexcitability is, at the same time, one of the most frequent features of neuroses. Oral administration of magnesium leads to the correction of the symptomatology and the normalization of the electropolygraphic modifications, ECG and other static and dynamic parameters.

Panic is an essential symptom of neuroses. A few studies have determined the plasma concentration of magnesium and other bivalent cations of panic disorder patients. Nahar et al.,
(2010) did not find significant differences in the magnesium plasma concentration in panic disorder patients versus the control group, but the intracellular concentrations were not determined. ATPase activity was studied in the erythrocytes of the patients with phobic neurosis. Sechenov (1975) found an increased activity of the $\mathrm{Mg}^{2+}$ ATPase in these patients but a decreased activity of the $\mathrm{Na}^{+} / \mathrm{K}^{+}$ATPase as compared with the control group. The significance of these changes is not clear.

In anxiety neurosis, there was not only a decrease in magnesium concentration, but also modified quantitative proportions between magnesium and other blood plasma or cytoplasmic components. It was shown that the blood fatty acids/magnesium ratio was increased and the magnesium level was decreased in anxiety neurotic patients (lakushev et al., 1989).

Regarding the mechanism by which magnesium reduces panic and anxiety, we consider that the main elements are decreased glutamate action and increased action of the GABAergic systems. Magnesium reduces anxiety by a number of mechanisms (Figure 1). These include reducing actions at the level of the calcium channel

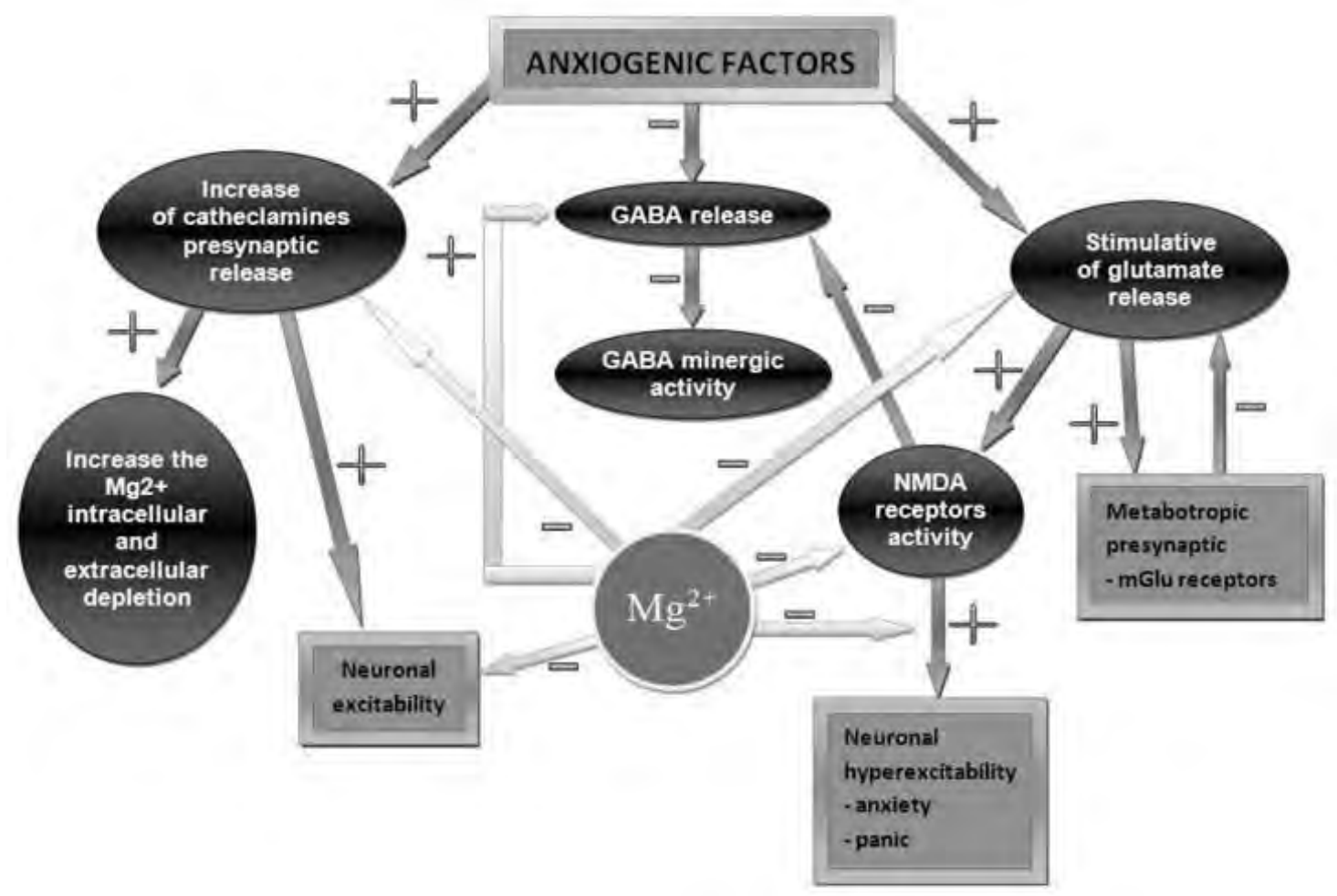

Figure 1. Magnesium mechanisms of action in anxiety and panic. -, inhibition; +, stimulation (increase). 
coupled with NMDA receptors, reducing the presynaptic cerebral release of epinephrine and nor-epinephrine, increasing GABA concentration in some brain areas, and acting as a modulator of glutamate release at the presynaptic level.

Glutamate is the most important excitatory neurotransmitter in mammalian brain. It acts at three major receptor types including the NMDA ionotropic receptor coupled to a $\mathrm{Ca}^{2+}$ channel, the AMPA (alpha-amino-3 hydroxy-5 methyl-4isoxazole propionic acid) receptor, and the metabotropic glutamate receptor (mGluR) group (at least 8 subtypes). Glutamate metabotropic receptors are divided into three groups, group I including mGluR1 and mGluR5 subtypes, group II including mGluR2 and mGluR3 subtypes, and group III including mGluR4, mGluR6, mGluR7 and mGluR8 subtypes.

The most important postsynaptic receptor population for glutamate is the NMDA receptors. Dorsomedial hippocampal neurons express both NMDA and AMPA receptors for glutamate (Bailey et al., 2003; Goren et al., 2003). Magnesium doesn't have a noticeable influence upon AMPA receptors and the existing data implicate the NMDA hypothalamic receptors in producing anxiety and panic disorders. Magnesium does reduce the effect of NMDA receptor stimulation and this is the main mechanism of its anxiolytic effect (Coan and Collingridge, 1985). Chronic stress involved in the pathogeny of some neuroses increases the hypothalamic NMDA receptor expression (Lee et al., 2003).

The mGluRs are a family of G-protein coupled receptors that have a widespread expression in the CNS (Niswender and Conn, 2010). They have a modulator role in synaptic transmission and in normal excitability in the brain, playing a key role in the modulation of glutamatergic activity, glutamate secretion and presynaptic release, GABAergic system activity and in regulation of neuroendocrine activity. The normal and pathological implications of the activation of these receptors are very different. Glutamate action on these brain receptors is important in normal and in pathologic situations such as fear, anxiety, addiction, panic, withdrawal syndrome, and posttraumatic stress disorders. Notably, divalent cations modulate the activity of mGluRs (Francesconi and Duvoisin, 2004). A mounting and important role in not only in anxiety, but also in other CNS disorders, is attributed to malfunction of the mGluRs. Regarding the symptoms in neurosis (anxiety, fear, cognitive impairment, working memory), the mGluR2 and the mGluR7 are the most important types of mGluRs and they are highly expressed in hippocampus (Desai, 1992). The mGluR7, in particular, has a wide distribution in the brain, and is preferentially localized to presynaptic axon terminals in the amygdala and hippocampus (Masugi et al., 1999). This receptor has a low affinity for glutamate, and Niswender and Conn (2010) consider that it only activates in the case of overstimulation by glutamate, and reduces glutamatergic activity. Selective stimulation of mGluR7 induced anxiolytic-like effects in mice by enhancement of GABAergic neurotransmission, and by reduction of glutamate synthesis and action (Stachowicz et al., 2008). The lack of mGluR7 caused a deficit in fear response and conditioned taste aversion (Masugi et al., 1999). Magnesium is important for mGluR7 receptor activity and may explain the enhancement of anxiety in hypomagnesemia (Niswender and Conn, 2010).

The agonists of some mGluRs are promising drugs in the future treatment of some psychiatric disorders. The agonists and positive allosteric modulators of group I mGluRs might treat anxiety disorders (Krystal et al., 2010). The mGluR group II agonists block fear learning when they are administrated into the amygdala prior to training (Walker and Davis, 2002). Mares et al., (2010) showed in immature rats that some mGluRs are involved in production of anxiolysis. Magnesium is also a positive allosteric modulator for some mGluRs.

The ratio between glutamate-induced excitatory activity and GABAergic inhibition in some brain areas (such as the amygdala, dorsomedial hypothalamus and cortex) plays an essential role in behaviour and imbalances are involved in the pathogenic mechanism of anxiety, panic disorder and phobia. Experimental chronic inhibition of GABA synthesis and enhancement of glutamatergic activity in the dorsomedial hypothalamus induced panic-like responses in rats (Johnson and Shekhar, 2006). Glutamate NMDA receptors are the most important receptor group involved in experimental lactate-induced 
panic-like responses in rats, but other receptors for glutamate are also involved.

Carbamazepine, an antiepileptic drug, is also used for the treatment of many nonepileptic disorders, including anxiety. The anxiolytic effects of carbamazepine seem to be mediated, at least in part, by interactions with the GABAergic system because muscimol (a $G_{A B A_{A}}$ receptor agonist) enhanced the carbamazepine anxiolytic effect. The anxiogenic effect of NMDA administration was also reversed by carbamazepine (Rezvanfard et al., 2009). In our studies (Nechifor et al., 2005; 2007), carbamazepine administration in therapeutic doses in human subjects increased the intracellular magnesium level. We believe that the anxiolytic effect of carbamazepine is due, at least in part, to the increased intracellular magnesium concentration.

GABA antagonizes glutamate hyperexcitation in some brain regions such as the dorsomedial hypothalamus and protects against anxiety and panic disorders (Millan, 2003). Favouring the idea that the GABAergic system plays an important role in preventing and reducing panic and anxiety, a low GABA concentration in brain has been associated with anxiety behaviours in rats and mice. In rats, chronic administration of 1allylglicine (a GABA synthesis inhibitor) in the dorsomedial hypothalamus induced panic disorder and enhanced panic-like behaviour produced by sodium lactate infusion (Schekhar et al., 1996; 2003). A reduction of GABAergic system activity has also been shown in non-medicated panic disorder patients. The subjects without major depression had a $22 \%$ reduction of the total occipital cortex GABA concentration compared with the normal control group (Goddard et al., 2001). Crestani et al., (1999) and Goddard et al., (2001) also showed that impaired brain GABA and benzodiazepine receptor activity is involved in phobic behaviours in mice. Glutamatergic system hyperactivity is also involved in other symptoms of neurosis, for example the dysregulation of the fear memory, the extinction of the fear memory and post-stress neuroses. An NMDA receptor agonist, Dcycloserine $(5 \mathrm{mg} / \mathrm{kg})$, blocked fear extinction in rats (Yang et al., 2007).

The catecholamines are also involved in fear and panic disorders. The injection of an $\alpha 1$-blocker in the dorsomedial hypothalamus blocks panic-like responses of animals (Johnson and Shekhar, 2006). The systemic administration of yohimbine induced panic-like responses, by blocking presynaptic $\alpha 2$-receptors and increasing epinephrine and norepinephrine release (Lowry et al., 2003). Yohimbine administration in healthy subjects increases the plasma level of norepinephrine, increases adrenergic activity, and results in increased anxiety and nervousness in the patients. Charney et al., (1984) found a significant positive correlation between the plasma level of the norepinephrine metabolite, 3methoxy-4-hydroxyphenylglicol (MHPG), and patient anxiety and panic attacks. Notably, magnesium reduces epinephrine and norepinephrine synthesis and release, decreases anxiety and could prevent the panic attacks.

Magnesium deficiency, even when mild, increases susceptibility to various types of neurologic and psychological stressors in healthy human subjects and diverse groups of patients. Repletion of deficiency reverses this increased stress sensitivity, and pharmacologic loading of magnesium salts induces resistance to neuropsychological stressors. Mild magnesium deficiency appears to be common among patients with disorders considered functional or neurotic and appears to contribute to a symptom complex that includes asthenia, sleep disorders, irritability, hyperarousal, spasm of striated and smooth muscle, and hyperventilation (Galland, 1991-1992). Stress, being a frequent cause of neurosis, influences magnesium concentration in the organism. Adrenergic stress induces a shift of magnesium from the intracellular to the extracellular space and increases urinary magnesium loss. In contrast, magnesium deficiency increases the vulnerability of human body to stress and the damage induced by stress (Galland, 1991). Magnesium deficiency also increases neuronal excitability, not only by increasing the excitatory effect of glutamate mediated by NMDA receptors, but possibly by increasing the calcium current in pre- and postsynaptic membranes and by alteration of the $\mathrm{Na}^{+} / \mathrm{K}^{+}$ATPases (Morris, 1992).

Glutamate induces a powerful stimulation of norepinephrine release in the amygdala and in hippocampal slices (Fink et al., 1992). $\mathrm{Mg}^{2+}$ ions and MK-801 (dizocilpine, an NMDA receptor 
antagonist) reduced the NMDA-evoked overflow of norepinephrine. Norepinephrine release related to the stimulation of AMPA receptors by glutamate does not seem to be influenced by $\mathrm{Mg}^{2+}$. Since the activation of mGluR4 and mGluR7 induces a decrease in glutamate release from presynaptic areas (Schrader and Tasker, 1997), it is possible that presynaptic mGluRs can thereby influence norepinephrine release.

Another important problem is the frequency of sleep disturbances in patients with neurosis. There are data that support the possible involvement of magnesium in sleep regulation. In rats, magnesium deficiency is associated with a decrease in sleep time. Magnesium content in four brain areas was also highly and positively correlated with the length of sleep periods (Chollet et al., 2000). Erythrocyte magnesium concentrations are low in adult human subjects with chronic sleep deprivation $(1.1 \pm 0.4 \mathrm{mg} / \mathrm{dl})$ versus a control group $(1.8 \pm 0.4 \mathrm{mg} / \mathrm{dl}$ ) (Tanabe et al., 1997). The reduction of sleep time is frequently associated with chronic fatigue. Chronic sleep deprivation is associated with an increase of plasma catecholamine release and with a decrease of intracellular magnesium level. These changes occur relatively quickly. In healthy male students (20-24 years aged), 4 weeks of partial sleep deprivation was sufficient for the appearance of these changes in the intracellular concentration of magnesium, and in the plasma epinephrine and norepinephrine concentrations. The students with chronic sleep deprivation over the 4 -week period (sleep was $<80 \%$ compared to ordinary days) had significantly decreased intracellular magnesium levels (Takase et al., 2004a; 2004b).

Experimental magnesium restricted diets in rats also increases the cerebral dopamine and norepinephrine concentration. This rise is associated with a decrease of sleep time and an increase in electroencephalographic wakefulness (Poenaru et al., 1984). Magnesium deficiency is accompanied by a decrease of sleep duration and by an increase of brain dopamine level (Chollet et al., 2000). These data strongly support the reduction of cerebral catecholamine release by magnesium. The effect of $\mathrm{MgSO}_{4}(0.5 \mathrm{mg}$ $\mathrm{MgSO}_{4} / \mathrm{h}$ between 20.30 hours till 7.00 hours) on sleep electroencephalogram (EEG, recorded between 23.00 and 7.00 hours) in healthy adult men showed an increase of the third sleep cycle with unchanged delta power throughout the night (Murch and Steiger, 1998). The activation of GABA receptors is very important for the initiation and maintenance of nor-rapid-eye movement (NREM) sleep. Magnesium stimulates the activity of cerebral GABAergic systems by behaving as a modulator of GABA receptors, increasing their activity.

Magnesium has a favourable effect with respect to sleep onset and maintenance and also tiredness, which appears as a consequence of sleep deprivation. In their studies of chronic sleep deprivation, Tanabe et al., (1998) reported a reduction of intracellular magnesium level. This reduction was associated with a decreased exercise tolerance. Specifically, when subjects were submitted to a bicycle ergometer cardiopulmonary exercise test, the sleepdeprived subjects had a decreased exercise tolerance. The administration of $100 \mathrm{mg}$ magnesium orally per day for 1 month improved the exercise tolerance. There was no difference between the sleep-deprived patients and the normal sleep patients regarding the peak oxygen uptake and anaerobic threshold. The mechanism through which chronic sleep deprivation can reduce intracellular magnesium concentration is unknown, but we consider that the excess catecholamines that appear in sleep deprivation could accelerate magnesium disposal. Hornyak et al., (1998) showed that magnesium therapy can be useful in periodic leg movements related to insomnia. Magnesium was administered orally, $12.4 \mathrm{mmol}$ in the evening during 4-6 weeks. After magnesium administration, periodic limb movements during sleep decreased significantly and the total sleep duration increased.

Attention deficit and hyperactivity disorders (ADHD) are only found in school children and in many patients with neurosis. In these patients, the plasma and erythrocyte magnesium level is low. In an animal experimental model of attention deficit, the hippocampal glutamatestimulated release of norepinephrine was significantly higher. The stimulatory effect of glutamate on norepinephrine release was reduced by $1 \mu \mathrm{M}$ CNQX (an AMPA receptor antagonist) suggesting that in this case the AMPA receptor stimulation by glutamate plays an important role. The NMDA receptor antagonist 
MK-801 $(10 \mu \mathrm{M})$ did not reduce the glutamatestimulated release of norepinephrine (Howells and Russell, 2008). An involvement of dopamine in attention deficit disorder from the neurotic patient is also possible, because in patients with ADHD there is dysfunction of the dopaminergic system. In an experimental rat model for ADHD, glutamate-stimulated dopamine release in the substantia nigra is higher. Warton et al., (2009) consider that abnormal dopaminergic system function in ADHD could be the result of a change in dopamine central neurons (dopamine synthesis, dopamine transporters or dopamine receptors), or an indirect effect of imbalanced glutamate regulation of dopaminergic neurons. We think that a similar process also occurs in the case of attention deficit in the neurotic patients. Magnesium can directly reduce dopamine release at the presynaptic level and can also reduce the stimulatory effect of glutamate on dopamine release. Treatment with Mag-B6 was useful in attention deficit syndrome and in hyperactivity treatment (Nogovitsina and Levitina, 2006; 2007). Mag-B6 improved behaviour, decreased the level of anxiety and aggression, increased attention and corrected the magnesium homeostasis in children with ADHD.

Both bulimia and anorexia are also symptoms found in patients with neurosis. In patients with anorexia nervosa (DSM-IV criteria), a decreased magnesium level was found compared to the control group. About $60 \%$ of the patients had low serum magnesium (Birmingham et al., 2004).

In neurotic and stressed patients, sexual dysfunction (reduced libido and sexual potency) is frequently present. This dysfunction is largely caused by increased prolactin (PRL) synthesis. It was experimentally shown that immobilization and other forms of stress (also implicated in the pathogeny of some cases of neuroses) increased PRL secretion. Pretreatment of the animals with magnesium aspartate in doses of $100 \mathrm{mg}, 200 \mathrm{mg}$ and $400 \mathrm{mg}$ significantly decreased the stressinduced PRL secretion in a dose-dependent manner (Ali et al., 1987). D-aspartate increased PRL secretion by stimulation of NMDA receptors. In the fish S. Mossambicus, magnesium also lowered PRL secretion (Bonga et al., 1983).

There are a number of mechanisms by which magnesium may reduce PRL levels in neurosis, thereby lowering or preventing the effects of this anterior pituitary hormone. It can directly reduce secretion of PRL at the level of secretory cells, it can inhibit stimulation by prolactin releasing hormone at the pituitary level to decrease PRL secretion (Kasahara et al., 1993), it can reduce the stimulatory effect of glutamate on pituitary PRL release, and finally, magnesium can reduce the stimulatory effect of calcium ions on PRL secretion. In cultures of adult female rats, pituitary cell glutamate significantly increased the rate of PRL release. This release was augmented 4-fold after elimination of magnesium from the perfusate (Login, 1990). Both NMDA (100 $\mu \mathrm{M})$ and kainate $(100 \mu \mathrm{M})$ increased PRL secretion by NMDA receptor stimulation, whereas AMPA receptor agonists did not modify PRL secretion and release. The antagonism between magnesium and the calcium channels coupled to the NMDA receptors is the essential factor in reduction of $\mathrm{PRL}$ secretion by this bivalent cation.

\section{Magnesium treatment of neuroses}

There are a limited number of studies that show a beneficial effect of magnesium in treatment of the different symptoms of the diseases known as neurotic disorders. Using mice, Poleszak et al., (2004) demonstrated that doses of 20 and $30 \mathrm{mg}$ magnesium/kg (doses which did not affect locomotor activity) produced anxiolytic-like effects measured using the plus-maze test. These doses were acutely or chronically administered and resulted in at least a $58 \%$ increase in serum magnesium concentration. Magnesium induced the anxiolytic-like effects without tolerance to these activities, suggesting a potential anxiolytic activity in these disorders in human.

Singewald et al., (2004) showed in an animal experiment (mice) that magnesium depletion leads to enhanced depression- and anxietyrelated behaviour, which was further validated by the reversibility of the behavioural changes by known antidepressant and anxiolytic substances. Using a rat model, Fromm et al., (2004) demonstrated a reduced incidence of anxiety after traumatic brain injury using magnesium sulphate as an interventional treatment. The study of Bockova et al., (1992) demonstrated that the combination of an anxiolytic with a magnesium salt (lactate) resulted in a more rapid reduction of the symptoms of anxious depressive neurosis. 
Taborska (1995) showed the curative effect of magnesium salts in patients suffering panic disorders and latent tetany. The symptoms of panic disorder are, with few exceptions, virtually identical with those of latent tetany. The author examined 20 patients treated in a psychiatric outpatient unit for panic disorder, examining for latent tetany plus serum and red blood cell magnesium. He found a concomitant incidence of latent tetany with a decreased level of intracellular magnesium. Remission of problems common to both nosological entities occurred in $90 \%$ of cases, in response to therapy with magnesium salts.

A double-blind, randomized, placebo-controlled study evaluated the efficacy and safety of a fixed combination containing two plant extracts and magnesium in the treatment of 264 patients presenting generalized anxiety of mild-tomoderate intensity. It was demonstrated that the preparation was safe and more effective than placebo (Hanus et al., 2004).

The studies of Durlach et al., (1997) concluded that the primary or secondary, acute or chronic nervous forms of magnesium deficiency induced by insufficient magnesium intake remain reversible over a long period by simply normalizing the magnesium intake. Untreated chronic forms may however bring irreversible organic disorders.

Physiological oral magnesium supplementation $(5 \mathrm{mg} / \mathrm{kg} /$ day) is simple and can be carried out via the diet or with magnesium salts. This treatment is able to cure all the functional symptoms of magnesium deficiency, including signs of neuromuscular hyperexcitability and psychiatric symptoms, which frequently mimic a neurotic pattern. It is necessary to highlight the curative and preventive importance of oral physiological maternal magnesium supplementation, not only during pregnancy, but also in the child throughout life from infancy to older age, to possibly prevent the so-called constitutional factor of neurolability.

We should emphasize that the nervous consequences of magnesium deficiency remain functional with an atomical integrity for a long time. They are, however, completely reversible since they can be restored to normal with simple oral physiological magnesium supplementation (Durlach et al., 1987; 1997).

\section{Neuroticism}

Neuroticism is a fundamental personality trait in the study of psychology. Unlike neurosis, it is not pathological in itself but is a tendency that can become pathological in certain conditions. It is also a risk factor for "internalizing" mental disorders such as phobia, depression, panic disorders and other anxiety disorders, traditionally called neuroses (Hettema et al., 2006). Neuroticism is an enduring tendency to experience negative emotional states like hyperemotivity, hyperexcitability, anxiety, anger, impulsivity and other psychosomatic manifestations. It is sometimes called emotional instability. Individuals who score high on neuroticism are more likely than the average to experience such feelings as anxiety, anger, guilt and depressed mood (Matthews and Deary, 1998). They are emotionally reactive and respond to events that would not affect most people. Moreover, their reactions tend to be more intense than normal. They are more likely to interpret ordinary situations as threatening, and minor frustrations as hopelessly difficult. Their negative emotional reactions tend to persist for unusually long periods of time, which means they are often in a bad mood. These problems in emotional regulation can diminish a neurotic's ability to think clearly, make decisions, and cope effectively with stress.

Neuroticism appears to be related to physiological differences in the brain. Hans Eysenck theorized that neuroticism is a function of activity in the limbic system, and his research suggests that people who score highly on measures of neuroticism have a more reactive sympathetic nervous system, and are more sensitive to environmental stimulation (Eysenck and Eysenck, 1985). Behavioural genetics researchers have found that a significant portion of the variability on measures of neuroticism can be attributed to genetic factors (Viken et al., 1994). As Boeree (2002) shows, neuroticism may be a predisposing physiological condition for neurosis. 
Table 1. Mean magnesium values $(\mathrm{mmol} / \mathrm{L}$ ) in children from an orphanage (Group I) and a state school (Group II). Adapted from Papadopol et al., 2001.

\begin{tabular}{|l|c|c|c|c|}
\hline & Group I & Group II & t-test & $\mathrm{p}$ \\
\hline Serum magnesium & $0.83+/-0.09 *$ & $0.78+/-0.06$ & 3.76 & $<0.001$ \\
Erythrocyte magnesium & $1.82+/-0.18$ & $1.91+/-0.18$ & 3.75 & $<0.001$ \\
\hline
\end{tabular}

$*$ : mean \pm standard deviation; $p$ : significance level

Table 2. Comparison of outcomes of personality traits. Adapted from Papadopol et al., 2001.

\begin{tabular}{|l|l|c|c|c|}
\hline & Steps of personality traits & $\begin{array}{c}\text { Group I } \\
\text { (number of } \\
\text { cases) }\end{array}$ & $\begin{array}{c}\text { Group II } \\
\text { (number of } \\
\text { cases) }\end{array}$ & \\
\hline Psychoticism & Pathological & 12 & 11 & $\chi^{2}=7.87$ \\
& Pathological tendencies & 9 & 19 & $\mathrm{p}=0.019$ \\
& Normal & 63 & 39 & \\
\hline Neuroticism & Pathological & 18 & 6 & $\chi^{2}=8.75$ \\
& Pathological tendencies & 23 & 12 & $\mathrm{p}=0.012$ \\
& Normal & 43 & 51 & \\
\hline Extraversion & Pathological & 25 & 14 & $\chi^{2}=0.53$ \\
& Pathological tendencies & 44 & 17 & $\mathrm{p}=0.766$ \\
& Normal & 38 & \\
\hline
\end{tabular}

p: significance level.

\section{Relationship between magnesium and neuroticism}

As has already been shown, magnesium deficiency manifests at the central nervous system level as hyperemotivity, hyperexcitability, anxiety, and impulsivity, amongst others, and these are also observed in neuroticism. A relationship between magnesium deficiency and neuroticism is therefore suggested. Some studies (Tuchendria et al., 1998) suggest that magnesium deficiency can be a causal factor of neurotic tendencies in elderly persons. In a group of 80 aged persons, all cases with erythrocyte magnesium deficit presented two or more neurotic tendencies, most frequent being the depressive ones.

There are also a few studies on magnesium deficiency in children and its relationship with personality traits. Specifically, this relationship in children and teenagers was examined in two groups of pupils from different socioeconomic backgrounds, namely an orphanage (group I) and a regular state school (group II) (Papadopol at al, 2001). Magnesium level and personality features were compared, including psychoticism, neuroticism and extraversion. With regard to the magnesium level (Table 1), one can see a discord between serum magnesium levels and erythrocyte levels. It is known that serum values of magnesium are less stable than erythrocyte values, being quite easily influenced by factors such as stress or physical activity that increases magnesium requirements. Serum magnesium may be normal in spite of a significant magnesium deficiency. The children from the orphanage had a more uniform lifestyle, with a more regular program of eating, sleeping and physical activities. Nutrition is also very important for the tissue magnesium levels. The nutrition of the children from the orphanage is characterized by a satisfactory, uniform level for all the children, while the nutrition of the children who 
Table 3. Relationship between magnesium deficit and neuroticism in children from an orphanage (Group I) and a state school (Group II). Adapted from Papadopol et al., 2001.

\begin{tabular}{|l|c|c|c|c|}
\hline & Group & $\mathrm{r}$ & $\mathrm{n}$ & $\mathrm{p}$ \\
\hline Serum magnesium & $\mathrm{I}$ & +0.25 & 84 & $\mathbf{0 . 0 2 3}$ \\
Erythrocyte magnesium & $\mathrm{II}$ & +0.26 & 67 & $\mathbf{0 . 0 3 5}$ \\
\hline
\end{tabular}

$r$ : Pearson correlation index; $n$ : number of subjects; $p$ : significance level.

live with their families is very variable but generally better because of socioeconomic differences.

The distribution of the subjects with respect to personality features in the two groups differed significantly in terms of psychoticism and neuroticism (Table 2). The more numerous pathological tendencies of group I were probably a consequence of the unfavourable emotional climate from the institutionalized milieu. An investigation of the relationship between magnesium levels and personality features pointed out only one significant difference between the two groups, namely neuroticism

\section{References}

Ali BH, Silsby JL, el Halawani ME (1987) The effect of magnesium aspartate, xylazine and morphine on the immobilization-induced increase in the levels of prolactin in turkey plasma. J Vet Pharmacol Ther 10:119-26.

Bailey TW, Nicol GD, Schild JH, DiMicco JA (2003) Synaptic and membrane properties of neurons in the dorsomedial hypothalamus. Brain Res 985:150-62.

Birmingham CL, Puddicombe D, Hlynsky J (2004) Hypomagnesemia during refeeding in anorexia nervosa. Eat Weight Disord 9:236-7.

Bockova E, Hronek J, Kolomaznik M, Polackova J, Curdova NO (1992) Potentiation of the effects of anxiolytics with magnesium salts. Cesk Psychiatr 88:141-4.

Boeree G (2002) A bio-social theory of neurosis. http: //webspace.ship.edu/cgboer/genpsyneurosis.html.
(Table 3). The positive correlation in magnesium deficit-neuroticism suggests that magnesium deficit may be a causal factor in this negative aspect of personality.

In a study by Nizankovska-Blaz et al., (1993) on 249 girls and boys aged 5-15 years, serum magnesium level was decreased in 24 children and neurotic reactions or concentration disturbances were observed in 21 of them.

In conclusion, all the available clinical and experimental data prove the involvement and importance of magnesium in neuroses and neuroticism.

Bonga W, Löwik CJ, van der Meij JC (1983) Effects of external Mg2+ and Ca2+ on branchial osmotic water permeability and prolactin secretion in the teleost fish Sarotherodon mossambicus. Gen Comp Endocrinol 52:222-31.

Charney DS, Heninger GR, Breier A (1984) Noradrenergic function in panic anxiety. Effects of yohimbine in healthy subjects and patients with agoraphobia and panic disorder. Arch Gen Psychiatry 41:751-63.

Chollet D, Franken P, Raffin Y, Malafosse A, Widmer J, Tafti M (2000) Blood and brain magnesium in inbred mice and their correlation with sleep quality. Am J Physiol Regul Integr Comp Physiol 279:R2173-8.

Coan EJ, Collingridge GL (1985) Magnesium ions block an $\mathrm{N}$-methyl-D-aspartate receptor-mediated component of synaptic transmission in rat hippocampus. Neurosci Lett 53:21-6. 
Crestani F, Lorez M, Baer K, Essrich C, Benke D, Laurent JP, Belzung C, Fritschy KM, Lucher IH (1999) Decreased GABA receptor clustering results in enhanced anxiety and a bias for treatment. $J$ Neurosci 2:833-9.

Desai MA, Smith TS, Conn PJ (1992) Multiple metabotropic glutamate receptors regulate hippocampal function. Synapse 12:206-13.

Durlach J, Bac P, Durlach V, Bara M, Guiet-Bara A (1997) Neurotic, neuromuscular and autonomic nervous form of magnesium imbalance. Magnes Res 10:169-95

Durlach, J., Poenaru, S., Rouhani, S., Bara, M. \& GuietBara, A (1987) The control of central neural hyperOexcitability in magnesium deficiency. In: Nutrients and Brain Function. (Essman WB, ed), Basel, Karger, 48-71.

Durlach J (1998) Aetiology and pathophysiology of central nervous hyperexcitability due to various type of magnesium deficit: magnesium deficiency and magnesium depletion. Abstr $6^{\text {th }}$ Europ Magnes Congress, Budapest (Abstract).

Duvoisin RM, Pfankuch T, Wilson JM, Grabell J, Chhajlani V, Brown DG, Johnson E, Raber J (2010) Acute pharmacological modulation of mGluR8 reduces measures of anxiety. Behav Brain Res 212:168-73.

Eysenck HJ, Eysenck MW (1985) Personality and individual differences: A natural science approach. Perspectives on individual differences. New York, Plenum Press (Springer).

Fink K, Schultheiss R, Göthert M (1992) Stimulation of noradrenaline release in human cerebral cortex mediated by N-methyl-D-aspartate (NMDA) and nonNMDA receptors. Br J Pharmacol 106:67-72.

Francesconi A, Duvoisin RM (2004) Divalent cations modulate the activity of metabotropic glutamate receptors. J Neurosci Res 75:472-9.

Fromm L, Heath DL, Vink R, Nimmo AJ (2004) Magnesium attenuates post-traumatic depression/anxiety following diffuse traumatic brain injury in rats. J Am Coll Nutr 23:529S-533S.

Galland L (1991) Magnesium, stress and neuropsychiatric disorders. Magnes Trace Elem 10: 287-301.

Goddard AW, Mason GF, Almai A, Rothman DL, Behar KL, Petroff OA, Charney DS, Krystal JH (2001) Reductions in occipital cortex GABA levels in panic disorder detected with 1h-magnetic resonance spectroscopy. Arch Gen Psychiatry 58:556-61.

Gören MZ, Yananli HR, Berkman K, Onat F, Aker R (2003) The influence of dorsomedial hypothalamic nucleus on contralateral paraventricular nucleus in NMDA-mediated cardiovascular responses. Brain Res 968:219-26.

Hanus M, Lafon J, Mathieu M (2004) Double-blind, randomised, placebo-controlled study to evaluate the efficacy and safety of a fixed combination containing two plant extracts (Crataegus oxyacantha and Eschscholtzia californica) and magnesium in mild-tomoderate anxiety disorders. Curr Med Res Opin 20:6371.

Hettema JM, Neale MC, Myers JM, Prescott CA, Kendler KS (2006) A population-based twin study of the relationship between neuroticism and internalizing disorders. Am J Psychiatry 163:857-64.

Hornyak M, Voderholzer U, Hohagen F, Berger M, Riemann D (1998) Magnesium therapy for periodic leg movements-related insomnia and restless legs syndrome: an open pilot study. Sleep 21:501-5.

Howells FM, Russell VA (2008) Glutamate-stimulated release of norepinephrine in hippocampal slices of animal models of attention-deficit/hyperactivity disorder (spontaneously hypertensive rat) and depression/anxiety-like behaviours (Wistar-Kioto rat) Brain Res 1200:107-15.

lakushev VS, Ruzhov AA, Mironova EV (1989) Changes in the concentration of nonesterified fatty acids and magnesium in emotional stress. Fiziol Zh SSSR Im I M Sechenova 75:1146-50.

International Classification of Diseases-10 (1992) World Health Organization, Geneva.

Johnson PL, Shekhar A (2006) Panic-prone state induced in rats with GABA dysfunction in the dorsomedial hypothalamus is mediated by NMDA receptors. J Neurosci 26:7093-104.

Kasahara K, Tasaka K, Masumoto N, Nishizaki T, Mizuki J, Tahara M, Miyake A, Tanizawa O (1993) High magnesium concentration inhibits ligand-stimulated calcium influx and hormone secretion in rat pituitary lactotropes with involvement of intracellular free magnesium. Biochem Biophys Res Commun 197:92-9. 
Krystal JM, Mathew SJ, D'Souza DC, Garakani A, Gunduz-Bruce H, Charney DS (2010) Potential psychiatric applications of metabotropic glutamate receptor agonists and antagonists. CNS Drugs 24: 66993.

Lee PR, Brady D, Koenig Jl (2003) Corticosterone alters $\mathrm{N}$-methyl-D-aspartate receptor subunit mRNA expression before puberty. Brain Res Mol Brain Res 115:55-62.

Login IS (1990) Direct stimulation of pituitary prolactin release by glutamate. Life Sci 47:2269-75.

Lowry CA, Plant A, Shanks N, Ingram CD, Lightman SL (2003) Anatomical and functional evidence for a stress-responsive, monoamine-accumulating area in the dorsomedial hypothalamus of the adult rat brain Horm Behav 43:254-62.

Mares $\mathrm{P}$, Mikulecká A, Tichá K, Lojková-Janecková D, Kubová H (2010) Metabotropic glutamate receptors as a target for anticonvulsant and anxiolytic action in immature rats. Epilepsia 51, Suppl 3:24-6.

Masugi M, Yokoi M, Shigemoto R, Muguruma K, Watanabe Y, Sansig G, van der Putten H, Nakanishi S (1999) Metabotropic glutamate receptor subtype 7 ablation causes deficit in fear response and conditioned taste aversion. J Neurosci 19:955-63.

Matthews G, Deary IJ (1998) Personality Traits, Cambridge, Cambridge University Press.

Millan MJ (2003) The neurobiology and control of anxious states. Prog Neurobiol 71:83-244.

Morris ME (1992) Brain and CSF magnesium concentrations during magnesium deficit in animals and humans: neurological symptoms. Magnes Res 5:303-13.

Murck H, Steiger A (1998) Mg2+ reduces ACTH secretion and enhances spindle power without changing delta power during sleep in men - possible therapeutic implications. Psychopharmacology (Berl) 137:247-52.

Nahar Z, Azad MA, Rahman MA, Rahman MA, Bari W, Islam SN, Islam MS, Hasnat A (2010) Comparative analysis of serum manganese, zinc, calcium, copper and magnesium level in panic disorder patients. Biol Trace Elem Res 133:284-90.

Nechifor M (2007) Magnesium in Psychoses. In: New perspectives in Magnesium Research - Nutrition and Health (Yoshiki Nishizuwa, Hirotoshi Mori, Jean Durlach, eds) London, SpringenVerlag, pp 369-77.
Nechifor M, Vaideanu C, Mindreci I, Palamaru I, Boistenu P (2005) Research on plasmatic and tissular concentration of some bivalent cations in patients with bipolar disorders. In: Abstract book of $5^{\text {th }}$ International Symposium on Trace Elements in HumanNew Perspectives (Ermidon Pollet S, Pollet S, eds) Athens Publishing House, p18.

Niswender CM, Conn PJ ( 2010) Metabotropic glutamate receptors: physiology, pharmacology, and disease. Annu Rev Pharmacol Toxicol 50:295-322.

Nizankowska-Blaz T, Korczowski R, Zys K, Rybak A (1993) Level of magnesium in blood serum in children from the province of Rzeszow. Wiad Lek 46:120-2.

Nogovitsina OR, Levitina EV (2006) Effect of MAGNE$B 6$ on the clinical and biochemical manifestations of the syndrome of attention deficit and hyperactivity in children. Eksp Klin Farmakol 69:74-7.

Nogovitsina OR, Levitina EV (2007) Neurological aspects of the clinical features, pathophysiology, and corrections of impairments in attention deficit hyperactivity disorder. Neurosci Behav Physiol 37:199202.

Papadopol V, Tuchendria E, Palamaru I ( 2001) Magnesium and some psychological features in two groups of pupils (magnesium and psychic features). Magnes Res 14:27-32.

Poenaru S, Rouhani S, Durlach J, Aymard N, Belkahla F, Rayssiguier Y, lovino M (1984) Vigilance states and cerebral monoamine metabolism in experimental magnesium deficiency. Magnesium 3:145-51.

Poleszak E, Szewczyk B, Kedzierska E, Wlaź P, Pilc A, Nowak G (2004) Antidepressant- and anxiolytic-like activity of magnesium in mice. Pharmacol Biochem Behav 78:7-12.

Rezvanfard M, Zarrindast MR, Bina P (2009) Role of ventral hippocampal GABA $(A)$ and NMDA receptors in the anxiolytic effect of carbamazepine in rats using the elevated plus maze test. Pharmacology 84:356-66.

Schrader LA, Tasker JG (1997) Presynaptic modulation by metabotropic glutamate receptors of excitatory and inhibitory synaptic inputs to hypothalamic magnocellular neurons. J Neurophysiol 77:527-36.

Sechenov SI (1975) Activity of Mg2+, N+ and K+ stimulated ATPase in erythrocytes of patients with phobic neurosis and the effect of lithium carbonate in enzymatic activity. Vopr Med Khim 21:283-5. 
Shekhar A, Keim SR, Simon JR, McBride WJ (1996) Dorsomedial hypothalamic GABA dysfunction produces physiological arousal following sodium lactate infusions. Pharmacol Biochem Behav 55:24956.

Shekhar A, Sajdyk TJ, Gehlert DR, Rainnie DG (2003) The amygdala, panic disorder, and cardiovascular responses. Ann N Y Acad Sci 985:308-25.

Singewald N, Sinner C, Hetzenauer A, Sartori SB, Murck H (2004) Magnesium-deficient diet alters depression- and anxiety-related behavior in mice-influence of desipramine and Hypericum perforatum extract. Neuropharmacology 47:1189-97.

Stachowicz K, Brañski P, Kłak K, van der Putten $\mathrm{H}$, Cryan JF, Flor PJ, Andrzej P (2008) Selective activation of metabotropic G-protein-coupled glutamate 7 receptor elicits anxiolytic-like effects in mice by modulating GABAergic neurotransmission. Behav Pharmacol 19:597-603.

Takase B, Akima T, Satomura K, Ohsuzu F, Mastui T, Ishihara M, Kurita A (2004a) Effects of chronic sleep deprivation on autonomic activity by examining heart rate variability, plasma catecholamine, and intracellular magnesium levels. Biomed Pharmacother 58 Suppl 1:S35-9.

Takase B, Akima T, Uehata A, Ohsuzu F, Kurita A (2004b) Effect of chronic stress and sleep deprivation on both flow-mediated dilation in the brachial artery and the intracellular magnesium level in humans. Clin Cardiol 27:223-7.

Tanabe K, Osada N, Suzuki N, Nakayama M, Yokoyama Y, Yamamoto A, Oya M, Murabayashi T, Yamamoto M, Omiya K, Itoh H, Murayama M (1997) Erythrocyte magnesium and prostaglandin dynamics in chronic sleep deprivation. Clin Cardiol 20:265-8.
Tanabe K, Yamamoto A, Suzuki N, Osada N, Yokoyama Y, Samejima H, Seki A, Oya M, Murabayashi T, Nakayama M, Yamamoto M, Omiya K, Itoh H, Murayama M (1998) Efficacy of oral magnesium administration on decreased exercise tolerance in a state of chronic sleep deprivation. Jpn Circ J 62:341-6.

Taborska V (1995) Incidence of latent tetany in patients with panic disorder. Cesk Psychiatr 91:183-90.

Tuchendria E, Palamaru I, Papadopol V, Duda R, Laszlo S, Rusu L (1996) Magnesium deficit: a possible causal factor of csome neurotic tendencies in elderly persons. (Abstract) Magnes Res 9:232-3.

Viken RJ, Rose RJ, Kaprio J, Koskenvuo M (1994) A developmental genetic analysis of adult personality: extraversion and neuroticism from 18 to 59 years of age. J Pers Soc Psychol 66:722-30.

Walker DL, Davis M (2002) The role of amygdala glutamate receptors in fear learning, fear-potentiated startle, and extinction. Pharmacol Biochem Behav 71:379-92.

World Health Organization (1992) International Classification of Diseases-10, World Health Organization, Geneva.

Warton FL, Howells FM, Russell VA (2009) Increased glutamate-stimulated release of dopamine in substantia nigra of a rat model for attentiondeficit/hyperactivity disorder--lack of effect of methylphenidate. Metab Brain Dis 24:599-613.

Yang YL, Chao PK, Ro LS, Wo YY, Lu KT (2007) Glutamate NMDA receptors within the amygdala participate in the modulatory effect of glucocorticoids on extinction of conditioned fear in rats.

Neuropsychopharmacol 32:1042-51. 



\title{
Magnesium, hyperactivity and autism in children
}

\author{
Marianne Mousain-Bosc, ${ }^{1, \bigotimes}$ Christian Siatka $^{1}$ and Jean-Pierre Bali ${ }^{2}$ \\ ${ }^{1}$ Ecole de l'ADN, Museum d'Histoire Naturelle de Nîmes, 19, Grand'rue, 30000 Nimes, France. \\ ${ }^{2}$ Université Montpellier I, 34000 Montpellier, France. \\ $\triangle$ mariannemousain@yahoo.fr
}

\begin{abstract}
For many years, magnesium has been described as a crucial factor for cellular activity. In this chapter, a brief overview of pharmacology and genetics of magnesium transport will be followed by a review of clinical and biological studies of $\mathrm{Mg}$-vitamin B6 supplementation in attention deficit/hyperactivity disorder (ADHD) and autism (autistic spectrum disorders family, ASD) in children. Although no study carried out on a rational basis has been published to date, some experimental and/or clinical works support a positive effect of such therapy in these pathologies. All the individual observations report a decrease in hyperactivity and a stabilization of scholarly behaviour with treatment. These data strongly support the need for a controlled study to confirm or invalidate these assumptions.
\end{abstract}

\section{Introduction}

Magnesium is the second most abundant intracellular cation in the body. Its main action is to regulate enzyme activity, to control the activity of various calcium and potassium channels, and to promote membrane stabilization. It is also responsible for the maintenance of the transmembrane gradients of sodium and potassium. Magnesium depletion is known to be associated with many clinical diseases including hypocalcemia, hypokalemia, cardiac arrhythmias, neuromuscular excitability, hypertension, atherosclerosis, and osteoporosis. Some evidence indicates that magnesium could also be involved in neurological diseases such as attention deficit/hyperactivity disorder and autism. However, no direct study has been published to confirm this assumption.

Attention deficit/hyperactivity disorder (ADHD) and autism (autistic spectrum disorders, ASD; pervasive developmental disorders, PDD) are different neurological disorders which have been described for many years, and in which the involvement of a deficient magnesium pathway could be suspected given the presence of active transport for such cations through transient receptor potential melastatin (TRPM) channels in the brain.

ADHD is the most common neurobehavioral disorder presenting for treatment in youth.
Children with ADHD are "a group at risk" as far as their further emotional and social development and educational possibilities are concerned (Spencer et al., 2002). An effective intervention for many hyperactive children, besides methylphenidate and other psycho-stimulant drugs, is the use of vitamin B6 (pyridoxine) and magnesium $(\mathrm{Mg})$. For over 30 years, parents have given high doses of pyridoxine and $\mathrm{Mg}$ to their children and have observed decreased physical aggression and improved social responsiveness. However, up until today, very few studies have reported a possible association between magnesium supplementation, ADHD symptoms, and the $\mathrm{Mg}$ status of the children. The first such study (Liebscher and Liebscher, 2003) suggested that patients with ADHD should be considered as potentially Mg-deficient as opposed to an incorrect interpretation of the serum $\mathrm{Mg}$ test (tetanic patients have lower $\mathrm{Mg}$ values than normals). Other studies from Kozielec et al., for the first time reported an intra-erythrocyte magnesium deficiency in ADHD children (Kozielec and Starobrat-Hermelin, 1997). We also published similar data (Mousain-Bosc et al., 2004, 2006). However, it is not a true "Mg deficiency" with clinically associated respiratory repletion, as is observed in familial hypomagnesaemia with secondary hypocalcemia (Shalev et al., 1998; Mousain-Bosc et al., 2004). More precisely, it may be called "intracellular Mg deficiency" affecting mainly neural transmission, which is very sensitive to such ionic variations. 
Another group of neuronal diseases in which magnesium has been implicated is ASD/PDD/ autism. Studies from 18 different research groups have shown that vitamin B6 and $\mathrm{Mg}$ are beneficial to about half of autistic individuals, with no significant adverse effects. Eleven of these studies involved a double-blind, placebo controlled design and have documented decreases in behavioural problems, improvements in appropriate behaviour, and normalisation of brain wave activity and urine biochemistry. There is also evidence that $\mathrm{B} 6$ and $\mathrm{Mg}$ may reduce seizure activity. Parent reports confirm improvement in attention, learning, speech/language, and visual contact. More recently, in a pilot study of a moderate dose, multivitamin/mineral supplement for children with autistic spectrum disorder, Adams and Holloway (2004) found significant improvements in sleep and gastrointestinal problems compared to the placebo group. Despite all these data, the intervention using $\mathrm{Mg}-\mathrm{B6}$ remains controversial and contradictory studies have been published (Vink, 2001; Helpern, 1993; Mousain-Bosc et al., 2006; Shalev et al., 1998).

New data reporting a possible association between Mg-B6 supplementation, neurobehavioural symptoms, and $\mathrm{Mg}$ status of children have subsequently been published, opening a new way for research in this domain. This chapter will present a review of essential works published in this domain.

\section{Magnesium transport and pharmacology of magnesium}

\section{Magnesium transport}

The protein involved in $\mathrm{Mg}$ permeable channels belongs to the family of TRPM proteins (Schmidt and Taylor 1988; Chubanov et al., 2004). The transient receptor potential (TRP) superfamily consists of a large number of cation channels that are mostly permeable to both monovalent and divalent cations. The 28 mammalian TRP channels can be subdivided into six main subfamilies: the TRPC (canonical), TRPV (vanilloid), TRPM (melastatin), TRPP (polycystin), TRPML (mucolipin) and the TRPA (ankyrin) groups. TRP channels are expressed in almost every tissue and cell type and play an important role in the regulation of various cell functions. Currently, significant scientific effort is being devoted to understanding the physiology of TRP channels and their relationship to human diseases. At this point, only a few channelopathies in which defects in TRP genes are the direct cause of cellular dysfunction have been identified. In addition, mapping of TRP genes to susceptible chromosome regions (e.g. translocations, breakpoint intervals, increased frequency of polymorphisms) has been considered as being suggestive of the involvement of these channels in hereditary diseases. Moreover, a strong indication of involvement of TRP channels in several diseases comes from the correlations between the levels of channel expression and the disease symptoms. Finally, TRP channels are involved in some systemic diseases due to their role as targets for irritants, inflammation products, and xenobiotic toxins. The analysis of transgenic models allows further extrapolations of TRP channel deficiency to human physiology and disease.

Two receptors, TRPM6 and TRPM7, have been identified as $\mathrm{Mg}$ permeable ion channels and their specificity is due to their link with protein kinase. This is the reason why they are defined as chanzymes. Theses chanzymes are involved in $\mathrm{Mg}$ reabsorption in kidney and intestine. The intake of $\mathrm{Mg}$ is driven by a transmembrane potential that facilitates the entry of the cation through the TRPM6 channel at the apical part of epithelial cells. The hypothesis is in favor of a $\mathrm{Na}$-dependent flux through ATP-dependent mechanisms (De Franceschi et al., 2000; Nilius et al., 2007).

In the kidney, different transport pathways for $\mathrm{Mg}$ exist along the nephron. The majority of filtered $\mathrm{Mg}$ is reabsorbed in the thick ascending limb of the loop of Henle via the paracellular route. Observations using genetic studies in affected individuals disclosed the first molecular components of epithelial Mg transport: the tight junction protein paracellin-1 (claudin-16) which was discovered as a key player in paracellular $\mathrm{Mg}$ and calcium reabsorption in the thick ascending limb of the loop of Henle and the distal convoluted tubule (De Franceschi et al., 2000; Nilius et al., 2007). Mutations of this protein lead to familial hypomagnesemia with hypercalciuria and nephrocalcinosis, a combined urinary $\mathrm{Mg}$ and calcium wasting which almost invariably leads to progression to end stage renal disease.

The discovery of TRPM receptors involved in $\mathrm{Mg}$ homeostasis (Borella et al., 1993; Montell, 2003) serves to identify the genes involved in primary 
inherited hypomagnesaemia by genetic engineering and molecular cloning. Several genes encoding proteins are directly involved in renal $\mathrm{Mg}$ handling. The family of paracellin-1 (PCLN-1) proteins is directly involved in $\mathrm{Mg}$ and calcium reabsorption in the distal convoluted tubule (DCT). Diseases such as hypomagnesaemia with secondary hypocalcaemia (HSH) are observed. Reabsorption of $\mathrm{Mg}$ in the DCT is active and transcellular. The renal $\mathrm{Mg}$ leak in HSH patients is due to the role of TRPM 6 in active transcellular $\mathrm{Mg}$ reabsorption in the DCT. The strong interaction between TRPM6 and TRPM7 involves the apical $\mathrm{Mg}$ channel responsible for its uptake from urine into DCT cells. In HSH patients, renal $\mathrm{Mg}$ wasting not only contributes to the development of hypomagnesemia in the postnatal period, but also prevents an adequate conservation of absorbed $\mathrm{Mg}$ under supplementation. The secondary hypocalcemia is due to an inhibition of parathyroid hormone caused by profound hypomagnesemia. Affected children typically manifest during the first months of life with generalised convulsions or signs of increased neuromuscular excitability like muscle spasms or tetany. Reduced clinical symptoms and the normalization of calcium homeostasis are guaranteed by immediate intravenous magnesium administration of followed by a long-term substitution with high oral doses of magnesium.

$\mathrm{HSH}$ can also be characterized as an autosomal recessive disease due to the gene mapped on chromosome 9q22. Mutational studies on the TRPM 6 receptor have shown the same type of $\mathrm{HSH}$. So, TRPM 6 is identified as a Mg permeable ion channel that causes a combined defect of intestinal and renal magnesium transport.

The TRPM6 protein is $52 \%$ homologous to TRPM7 and was studied together with TRPM7 in order to study their regulation. They were functionally characterized as a constitutively active ion channel permeable to a variety of cations like calcium and Mg. So, TRPM6 and TRPM7 are closely related members of the TRPM ion channel family named after its founding member, melastatin. The pathophysiologic consequences of a TRPM6 defect for $\mathrm{Mg}$ transport in intestine and distal convoluted tubule are important. In the intestine, an active transcellular transport and a passive paracellular pathway were identified. Both TRPM6 and TRPM7 proteins share the unique feature of an atypical kinase domain at their C-terminus, for which they have been termed chanzymes (Basso et al., 2000; Schlingmann and Gudermann, 2005). The apical entry into the epithelial cell is a basolateral extrusion mechanism that links $\mathrm{Mg}$ export to sodium influx. These transport processes yield curvilinear kinetics for intestinal Mg absorption. In addition, it has been described that an increased intake of oral magnesium enhances passive paracellular absorption. It can therefore be supposed that HSH patients with defective transcellular Mg transport are able to achieve at least subnormal serum magnesium levels and relief of symptoms.

Electrophysiological and biochemical analyses identified TRPM7 as an important player in cellular $\mathrm{Mg}$ homeostasis. The critical role of TRPM6 in epithelial Mg transport emerged from the discovery of loss-of-function mutations in patients with a severe form of hereditary $\mathrm{HSH}$. TRPM6 plays an important role as an influx pathway for Mg (Schmidt and Taylor, 1988; Chubanov et al., 2004).

In summary, TRP channels have a multifunctional role and are involved in many fundamental cell functions in physiopathology and diseases. The TRP superfamily is implicated in channelopathies involving an activation of a membrane cation channel. The TRPM subfamilies are distinct families, whereas both TRPM6 and TRPM7 (serine /threonine kinase) exhibit high/variable permeability to calcium and $\mathrm{Mg}$ and are regulated through intracellular levels of Mg and Mg-ATP. The defects in these ion channels supposedly cause various diseases described as channel-opathies. The genetic defect in TRP channels has been identified as the direct cause of hereditary disease. Mutations on the TRPM6 gene are likened to human proteinuric kidney disease. TRPM7, the closest relative of TRPM6, is also implicated in some neurodegenerative diseases such as amyotrophic lateral sclerosis and Parkinson's disease.

\section{Involvement of magnesium in membrane stability and gene expression}

Membrane stability is influenced by many parameters including ionic conductance, ionic fluxes and Mg (Ebel and Gunther, 2005; Wolf et al., 2009). Because of the activity of the superfamily of TRP channels, the effect of $\mathrm{Mg}$ is 
observed on many membranes. It has been reported that the concentration of extracellular Mg can affect blood flow, blood pressure and vascular reactivity in intact mammals (Schmidt and Taylor, 1988, Van der Wiijst et al., 2009). The red cell membrane properties have also been explored; $\mathrm{Mg}$ regulates stability and extends the gross elasticity of the red cell membrane. Magnesium-depleted cells also undergo structural changes on heating below the temperature at which vesiculation sets in.

There is a drastic change in ionic flux through the outer and inner cell membranes both in the impaired membranes of cancer, and in $\mathrm{Mg}$ deficiency. Researchers from the School of Public Health at the University of Minnesota have just concluded that diets rich in $\mathrm{Mg}$ reduced the occurrence of colon cancer (Chakraborti et al., 2002; Aaron, 2006). In another study (Macdonald et al., 2004; Susanna et al., 2005) women with the highest magnesium intake demonstrated a $40 \%$ lower risk of developing cancer than those with the lowest intake of the mineral.

Magnesium linked to the phospholipids, and more particularly to phosphatidylserine, reduces membrane fluidity and increases the stability of pre-synaptic vesicle membranes. Lecithins (phosphatidylcholines) and phosphatidylserines are brain nutrients that modulate acetylcholine metabolism, and act to stimulate memory. A 2003 study (Demougeot et al., 2004) describes the lecithins as promoting the synthesis and the working of neurotransmitters involved in memory processes. The mechanism of beneficial action was linked to activation of phospholipase systems in the hippocampus by choline.

In obstetric and embryonic research, different $\mathrm{Mg}$ salts have been studied, such as $\mathrm{MgCl}_{2}, \mathrm{Mg}$ acetate and $\mathrm{Mg}$-citrate. They are known to increase and decrease the membrane stability on the two faces of the amnion. $\mathrm{MgSO}_{4}, \mathrm{Mg}$-lactate and Mg-nitrate increased the stability on the maternal side, but decreased it on the fetal side. Thus, addition of $\mathrm{Mg}$ salts modifies human amniotic membrane stability.

In response to a $\mathrm{Mg}$ deficiency, five gene alterations could be identified using DNA arrays: osteopontin, the cholecystokinin A receptor, connexin 45, a growth hormone receptor and
BAG1. Other fundamental studies in bacteria characterized an 'M-box', a genetic switch that is sensitive to cellular $\mathrm{Mg}$ levels through the conformation of its newly synthesized RNA. This $\mathrm{Mg}$ sensing ribo-switch controls transcription termination in front of a $\mathrm{Mg}$ transporter gene. Magnesium therefore acts directly as a genetic regulator in genetic expression of RNA in order to control metal ion homeostasis (De Rose, 2007). As $\mathrm{Mg}$ has been shown to activate gene expression, the mitochondrial genome must be affected. Treatment of mild mitochondrial dysfunction may include administration of mitochondrial cofactors like magnesium (Martin et al., 2007).

\section{Action of magnesium on synaptic facilitation}

In 2006, a review article (Billard, 2006) indicated that $\mathrm{Mg}$ is involved in age-related deficits in transmitter release, neuronal excitability, and some forms of synaptic plasticity such as longterm depression of synaptic transmission. Further studies presented by Slutsky et al., (2010) show that $\mathrm{Mg}$ is essential for maintaining normal body and brain functions.

Learning and memory are fundamental brain functions affected by dietary and environmental factors. Increasing brain $\mathrm{Mg}$ using a newly developed $\mathrm{Mg}$ compound (Mg-L-threonate) leads to the enhancement of learning ability, working memory, and short and long-term memory in rats. Functionally, $\mathrm{Mg}$ increased the number of functional pre-synaptic release sites, while it reduced their release probability. These findings suggest that an increase in brain $\mathrm{Mg}$ enhances both short-term synaptic facilitation and longterm potentiation and improves learning and memory functions. Magnesium impacts upon the release of neurotransmitters, and other mediators or modulators (Slutsky et al., 2010).

\section{Magnesium in blood}

The study of the regulation of $\mathrm{Mg}$ has gained particular interest in the last decades thanks to the molecular characterization of specific magnesium transporters and the exploitation of molecular biology techniques to clarify their cellular and physiological function(s). Magnesium can be detected both in the plasma in the cationic form $\left(\mathrm{Mg}^{2+}\right)$ and in blood cells, namely in erythrocytes. All these assays are today well described and can be performed in many laboratories. In contrast, experimental tools to 
trace cellular $\mathrm{Mg}^{2+}$ and to define its homeostasis in living cells have not witnessed corresponding progress. It was not until recently that efforts were made to design more appropriate fluorescent indicators that could translate the advances of live imaging techniques into the field of $\mathrm{Mg}^{2+}$ research (Trapani et al., 2010).

Phosphorus magnetic resonance spectroscopy also offers an opportunity to measure in vivo the free cytosolic $\mathrm{Mg}^{2+}$ of different tissues. In particular, this technique has been employed in human brain and in skeletal muscle providing new hints on $\mathrm{Mg}^{2+}$ homeostasis and on its involvement in cellular bioenergetics. In skeletal muscle it has been shown that the changes in free $\mathrm{Mg}^{2+}$ concentration occurring during contraction and in post-exercise recovery are mainly due to the influence of cytosolic $\mathrm{pH}$. The possibility of assessing the free cytosolic $\mathrm{Mg}^{2+}$ concentration in the human brain offered the chance of studying the involvement of $\mathrm{Mg}^{2+}$ in different neurological pathologies, and particularly in those where defective mitochondrial energy production represents the primary causative factor in the pathogenesis. Moreover, it has also been shown that the measurement of brain $\mathrm{Mg}^{2+}$ can help in the differential diagnosis of neurodegenerative diseases sharing common clinical features, such as Multiple System Atrophy and Parkinson's disease (Ebel et al., 2004; lotti and Malucelli, 2008).

Magnesium concentrations in plasma and cells could be affected by diet, disease and genetic factors. It is known that carcinogenesis induces disturbances in $\mathrm{Mg}$ distribution, which cause $\mathrm{Mg}$ mobilization through blood cells and $\mathrm{Mg}$ depletion in non-neoplastic tissues. Magnesium deficiency seems to be carcinogenic, and in the case of solid tumours, a high level of supplemental Mg inhibits carcinogenesis. Both carcinogenesis and $\mathrm{Mg}$ deficiency increase the plasma membrane permeability and fluidity. Scientists have in fact found out that there is much less Mg binding to membrane phospholipids of cancer cells, than to normal cell membranes (Wolf et al., 2008).

A progressive elevation in external $\mathrm{Mg}$ levels in blood will produce a progressive inhibition of most contractile elements. The contractile response observed upon withdrawal of external $\mathrm{Mg}$ are dependent upon calcium concentrations and the polarity of the membrane. This response is not related to an inhibition of the $\mathrm{Na}^{+} / \mathrm{K}^{+}$ ATPase. It is known that $\mathrm{Mg}$, in combination with $\mathrm{Na}^{+}$and $\mathrm{K}^{+}$, play a major role in regulating blood pressure and arteriolar tone (Altura et al., 1978).

Magnesium concentration in plasma and cells could also be affected by genetic disease. The genetic factors involved in the regulation of $\mathrm{Mg}$ homeostasis were studied in low (MgL) and high $(\mathrm{MgH})$ magnesium status strains of mice. In this model, magnesium-deficiency affects plasma, erythrocyte and urine $\mathrm{Mg}$ concentrations in similar proportions in the two strains (Schlingmann et al., 2002; Montell, 2005; Günter, 2007; Ozgo et al., 2007)

\section{Clinical symptoms of ADHD syndrome}

\section{Clinical cases}

ADHD has been described since the beginning of the twentieth century and was called hyperkinesia or "psycho-motor instability". It affects 3 to $9 \%$ of the population of children and adults (FeilletCoudray, 2005; Biederman, 2006). The clinical picture is that of a hyperirritable, impulsive, aggressive child with an attention deficit. The diagnosis is basically done at six years of age when the child presents at primary school. These symptoms impair emotional development and can lead to serious social and family disorders. In adults, statistics show a powerful link between ADHD and serious problems in life. These findings support the idea that when diagnosed in the community, ADHD is a clinically significant and highly disabling disorder in adults and must be treated very early in children. However, parents' and children's histories should be studied. In parents, behavioural disorders such as hyperactivity, aggressiveness, emotional lability, stress, or maternal spasmophilia may be present. During pregnancy, several symptoms can be observed including weariness, stress, anxiety and breakdown, arterial hypertension, muscle cramps, contractions, sleeping disorders, diet deficiency, and twin pregnancy.

The first signs of ADHD generally appear during the first year of life and include sleeping disorders (short sleeping and waking to a small intensity noise), spasm of the sob, tremor of the hands and the arms, intense emotionality, frequent tears, hyper-excitability, frequent fidgeting or squirming, inability to keep one's seat, inclination to put their 
life in danger, climbing, and an inability to see potential danger. The adaptation to nursery school at three or four years of age becomes rapidly difficult, with hyper-excitability, impulsivity and difficulty in being a good listener.

At six years of age, all the clinical symptoms of ADHD as described in the DMS-IV manual (Findling et al., 1997) are present, including:

- hyperactivity: fidgets with hands or feet, or squirms in seat; gets up from seat when expected to remain seated; runs about or climbs when it is not appropriate; is "on the go" or often acts as if "driven by a motor"; talks excessively; blurts out answers before questions have been finished; has difficulty in awaiting their turn; interrupts or intrudes on others; has an inclination to put their life in danger.

- impulsivity: becomes aggressive, doesn't control their movements; gets angry and has difficulty stopping this aggression.

- attention deficit: symptoms of inattention should be present for at least six months and when children are attending primary school; does not give close attention to detail or makes careless mistakes in schoolwork or other activities; has trouble maintaining attention on tasks or play activities; does not follow instructions and fails to finish schoolwork, chores or duty in the work place (not due to opposite behaviour or lack of understanding instructions but due to slowness to execute the tasks); has trouble organizing activities; avoids dislikes or does not want to do things that take a lot of mental effort for a long period of time; loses things needed for tasks and activities (toys, pencils, books or tools); is often easily inattentive; is often forgetful in daily activities and has memory difficulties.

In addition, other clinical symptoms frequently occur and must been considered as main symptoms. These include:

- sleeping disorders: mild sleeping problems; cannot put to sleep; has nightmares, broken sleep; is frightened by the night and awakes weary; very frequent abdominal pains occurring with stress.

- unexplained weariness: weariness begins in the morning with slowness to stand up, to get dressed, and abnormal weariness in sports activities.

- feeling of faintness with or without losing consc- iousness; twitches in the face or in the breathing reflect hyper-excitability; gnaws one's nails; anxiety, stress, mood disorders, easily upset; loss of trust and loss of respect of oneself; often breaks down; is afraid of people and frightened to die. The medical examination reveals a Chvostek's signs in $66 \%$ sign of hyper-excitable children.

In the clinical description of ADHD, biological data are usually not evoked. However, for instance, hyper-excitability can be revealed by an intracellular $\mathrm{Mg}$ deficiency since $\mathrm{Mg}$ has been shown to be involved in the control of some nervous system processes.

\section{Clinical Case Study 1}

In the first months of her life, patient OCE presented signs of hyper-excitability. She twiddled her fingers, jumped to a lower level noise and woke often. A prescribed calcium therapy was not effective and the tremors persisted. Alternate calcium and Mg therapy improved all the signs.

At five years, before beginning primary school, patient OCE again presented signs of hyperexcitability. She was fidgety and didn't stay in her seat. Sleeping disorders reappeared where she awoke very often and slept very little. She was impulsive and slapped children, although it was not intentional. She didn't control herself and was subject to terrible fits of anger. The possibility of $\mathrm{Mg}$ deficiency was raised since her mother has suffered from "spasmophilia" for some years. Both OCE and her mother showed a decrease in erythrocyte Mg levels.

After two months of Mg-B6 treatment, OCE felt better. She became cool, good, less capricious, didn't play the fool, and her sleeping was calm. Erythrocyte $\mathrm{Mg}$ increased and the therapy was accordingly stopped. Some months later, all the ADHD signs rapidly reappeared including attention deficit, hyperactivity, sleeping disorders and impulsiveness. Biological control confirms the diagnosis of $\mathrm{Mg}$ metabolism disorder (Mg imbalance), a "genetic disease". The Mg-B6 treatment was recommenced with symptoms resolving. However, each time OCE stopped taking the treatment, ADHD symptoms returned! OCE received $\mathrm{Mg}$ therapy over the eight-year period made up of $6 \mathrm{mg} / \mathrm{kg} / \mathrm{day} \mathrm{Mg}$ and $0.6 \mathrm{mg} / \mathrm{kg} /$ day vitamin B6 (Figure 1). 


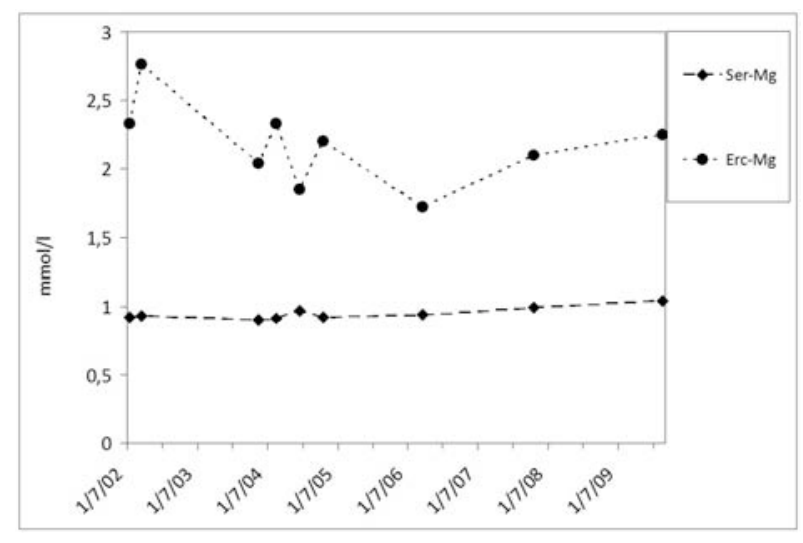

Figure 1. Evolution of biological parameters for magnesium in the case of patient OCE (born February, 1997) during the follow-up (ADHD syndrome). Ser-Mg: total serum magnesium concentrations in $\mathrm{mmol} / \mathrm{l}$; Erc-Mg: magnesium concentrations in erythrocytes (obtained after red cell lysis) in $\mathrm{mmol} / \mathrm{l}$.

\section{Clinical Case Study 2}

Patient NIC was seventeen years old when he first came to the consulting office. In the first years of his life, he demonstrated sleeping disorders, restless sleep, frequent tears, had a delay with communication, was an emotional child, uneasy, over-excitable, and a little aggressive. Upon admission to secondary school, behavioural disorders persisted. He was impulsive, didn't control himself, was aggressive and was dyslexic.

A diagnosis of ADHD was made and psychostimulant treatment was prescribed over three years. He never took the prescribed dosage because his physician (his grandfather) and his pharmacist had warned the parents of the risks of this medication (methylphenidate). Rapidly, he presented side effects to this treatment, including sleeping disorders, muscular pains, weariness, "feeling lethargic", behavioural disorders, "became as a zombie", headaches, palpitations, anxiety attacks, breakdowns, and "was frightened to die". His parents were worried by these side effects, and read our publications at the beginning of 2007. The levels of erythrocyte $\mathrm{Mg}$, calcium, and ionized calcium had dramatically decreased. The parents decided to stop the psychostimulant treatment. A Mg supplementation (300 mg) spectacularly improved patient NIC's behaviour. In some weeks, NIC became cool and more relaxed. An excellent participation in scholarly activities and a better concentration in tasks resulted in excellent scholastic results in four months. NIC received the "congratulations of the schoolboard" at the end of 2007! NIC could control himself, was not aggressive, had no behavioural disorders, and had self-confidence. In six months, all the biological disturbances were normalized. At the school of "masonry for historic monuments", NIC has excellent assessments - "a good student and hardworker"! NIC received a Mg supplementation for more than three years and each time when he tried to decrease the dose, clinical symptoms reappeared.

The disease, called hypomagnesemia with secondary hypocalcemic, is heritable. NIC's sister was also emotional and hypersensitive while his brother was hyper-excitable, always "on the go " and had both sleeping and behavioural disorders. She had Mg deficiency and now feels better with $\mathrm{Mg}$ therapy. His mother presented with cramps, muscular pains, pins and needles in the hands, stress and migraines. A biological profile showed an erythrocyte Mg deficiency. After six months of $\mathrm{Mg}$ therapy, the signs disappeared. The maternal grandfather suffers from Alzheimer's disease. NIC's father was emotionally stressed, uneasy and for several years experienced sleeping disorders, heart disease, attention deficit, and above all memory disorders. He is only forty years of age! $\mathrm{He}$ had an erythrocyte Mg deficiency and after six months of the same treatment, their memory improved (Figure 2).

In March 2007, the methylphenidate treatment was stopped and the magnesium therapy was set up. A clear behavioral improvement was observed. However, after the three years of magnesium therapy, all biological data remain stable. This result could suggest an alteration of the $\mathrm{Mg}$ transport. It encouraged us to perform additional experiments on $\mathrm{Mg}$ channels in order to check their expression in various tissues.

\section{Experimental Study Number 1}

In order to study relationships between hyperactivity symptoms and Erc-Mg levels, we designed an open study on 40 children with ADHD syndrome. For ethical reasons, we chose not to perform a double-blind study against either psychostimulants (methylphenidate) or placebo. We felt that parents would not support such a design. 


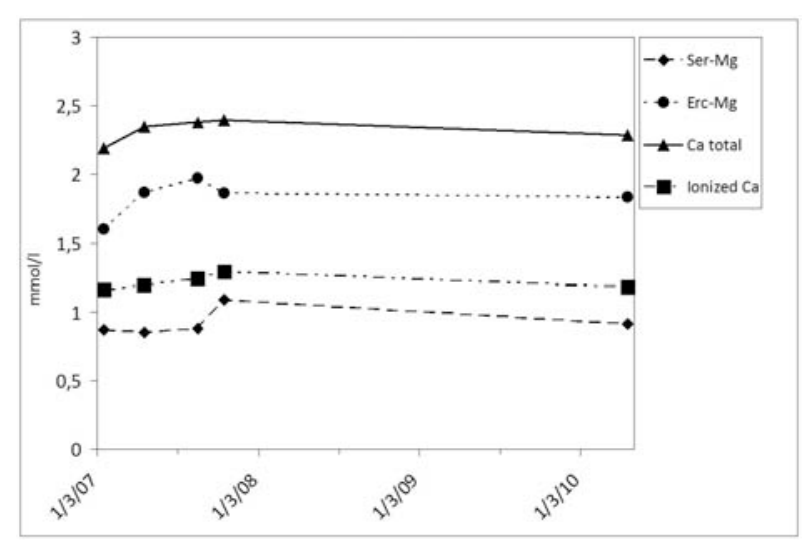

Figure 2. Evolution of biological parameters for magnesium and calcium in the case of patient NIC during the follow-up (ADHD syndrome). Ser-Mg: total serum magnesium concentrations in $\mathrm{mmol} / \mathrm{l}$; Erc-Mg: magnesium concentrations in erythrocytes (obtained after red cell lysis) in $\mathrm{mmol} / \mathrm{l}$; Total Ca: total serum calcium concentrations in $\mathrm{mmol} / \mathrm{l}$; lonized calcium concentrations in $\mathrm{mmol} / \mathrm{l}$ in serum.

In addition, psychostimulants were found to alter Mg homeostasis (Tolbert et al., 1993). Our results (Mousain-Bosc et al., 2004) showed a statistically significant improvement of the symptoms after Mg-B6 supplementation, together with a rise in Erc-Mg values.

In this study, a slight but significant intraerythrocyte $\mathrm{Mg}$ depletion was evident in ADHD patients together with a concomitant decrease in intracellular calcium concentrations. As we know, $\mathrm{Mg}$ is essential for normal central activity and Erc$\mathrm{Mg}$ could be representative of intracellular $\mathrm{Mg}^{2+}$ concentrations. A decrease in Erc-Mg without changes in serum $\mathrm{Mg}$ concentrations could be interpreted as an alteration in a $\mathrm{Mg}$ transporter $\left(\mathrm{Na}^{+} / \mathrm{Mg}^{2+}\right.$ exchanger) in erythrocytes with concomitant effects on neuronal $\mathrm{Mg}$ concentrations. The difficulty in achieving normal Erc-Mg values under Mg-B6 treatment supports this hypothesis. In addition, supplementation with $\mathrm{Mg}$ pidolate was found to decrease $\mathrm{Na}^{+} / \mathrm{Mg}^{2+}$ exchanger activity with a concomitant rise in $\mathrm{Mg}$ and $\mathrm{K}^{+}$contents of erythrocytes in sickle cell disease (DMS-IV). Erc-Mg was described as a controversial biological parameter for the monitoring of $\mathrm{Mg}$ deficiency, in contrast to others (Borella et al., 1993; Vink et al., 2009) who consider Erc-Mg as a suitable index. Moreover, Basso et al., (2000) and Helpern et al., (1998) present Erc-Mg as not useful for the monitoring of individual changes. We think that in this last study, a 3-week treatment of $\mathrm{Mg}$ without B6 was too short to induce a durable increase in Erc-Mg (vitamin B6 was described as enhancing Mg entry into the cell). In any case, in our hands, Erc-Mg measurements were standardized and it appears as a potent indicator of cellular Mg deficiency.

Calcium and $\mathrm{Mg}$ cellular contents classically follow the same pathway - when Mg increased, calcium also increased. This may explain the significant correlation between Erc-Mg and intracellular calcium values as well as the fact that in children who have low intracellular calcium values, $\mathrm{Mg}$ therapy increased intracellular calcium levels. It can be hypothesized that a genetic factor, which modulates $\mathrm{Na}^{+} / \mathrm{Mg}^{2+}$ exchanger activity, may be important in the regulation of $\mathrm{Mg}$ metabolism (Heath and Vink, 1998).

We also found that increased hyperactivity and decreased scholastic attention were associated with decreased Erc-Mg values. This observation was supported by the fact that Mg-B6 supplementation induced a rise in Erc-Mg values and a concomitant improvement of the clinical symptoms. What are the respective roles of pyridoxine and $\mathrm{Mg}$ in these observations? It was classically accepted that $\mathrm{Mg}$ is administered with pyridoxine to decrease irritable side-effects of the B6 therapy. We show here evidence of the role of $\mathrm{Mg}$ itself in this therapy. Previous data support this observation. In ADHD disorders, in which disruptive behaviour with hyperactivity was found, psychostimulants are used to improve mental health, probably by increasing synaptic noradrenaline activity. In children who received methylphenidate, a significant increase (6\%) in plasma $\mathrm{Mg}$ concentrations was found depending on the dosage of the drug, showing a relationship between improvement of hyperactivity and $\mathrm{Mg}$ metabolism (Schmidt et al., 1994 (Tolbert et al., 1993). More recently, in autistic children with behavioural disorders and hyperactivity (Zilbovicius et al., 2000; Schmidt and Taylor, 1988; Chakraborti et al., 2002), positive emission tomography (PET) has shown a significant decrease in cerebral blood flow localized at the temporal lobe level in $76 \%$ of the children examined. Taken together with the fact that intraerythrocyte free $\mathrm{Mg}^{2+}$ is associated with increased blood pressure (Zilbovicius et al., 2000) and that 
brain from rats fed with low $\mathrm{Mg}$ diets are more susceptible to permanent brain focal ischemia (Gervais et al., 2004), we can hypothesize that intracellular $\mathrm{Mg}^{2+}$ deficiency could be responsible, at least in part, of some central activity disorders observed in these children.

The duration of the treatment necessary to get significant improvement seems to be about 8 weeks. Since the cause of this deficiency is yet unknown, and since the symptoms reappeared when the Mg-B6 diet was stopped, the treatment must be maintained for a long time. In addition, while it was difficult to find an evident biological link between central disorders and Erc-Mg values, this biological parameter could be used to select, among the large population of children with hyperactive symptoms, a small population with behavioural abnormalities that is relevant for a Mg-B6 diet. It is evident that another accessible $\mathrm{Mg}$ store, more significant for central disorders, has to be found.

In conclusion, this study provides additional information about the therapeutic role of a Mg-B6 regimen in children with ADHD. This effect seems to be associated, at least in part, to a cellular $\mathrm{Mg}^{2+}$ deficiency, as evidenced by intra-erythrocyte $\mathrm{Mg}$ measurements. Installing a Mg-B6 supplementation for some weeks restored higher intraerythrocyte $\mathrm{Mg}^{2+}$ values and significantly reduced the clinical symptoms of these diseases. As chronic Mg deficiency was shown to be associated to hyperactivity, irritability, sleep disturbances, and low scholar attention, besides other traditional therapeutic treatment, a $\mathrm{Mg}$ supplementation could be required in children with ADHD.

\section{Etiology and risk factors for ADHD}

The etiology of ADHD is not yet known, although the causes are considered multifactorial:

- genetics: twin studies indicate that heritability ranges from $60 \%$ to $90 \%$. Various genes are currently being studied ( $\mathrm{Mg}$ is involved in gene expression and essential for membrane stability) (Ozgo et al., 2007).

- brain abnormalities: it is believed that the dopaminergic system is involved in ADHD. Magnesium has an impact by reducing the release of neurotransmitters and other mediators.
Magnesium also stabilizes the membranes (Nemoto et al., 2006).

- environmental and perinatal factors: studies in rats suggest that a magnesium-deficient diet influences not only mineral metabolism but also protein metabolism. Growth retardation results from the low food intake that is induced by magnesium deficiency, which provokes an alteration in energy metabolism. But the protein nutritional status in Mg-deficient rats is restored by dietary $\mathrm{Mg}$ supplementation in seven days. In women, maternal $\mathrm{Mg}$ intake has an immediate effect on placental vascular flow. Magnesium sulphate reduces the vasoconstriction effect of angiotensin II in human placenta. Reduced placental vascular flow is at least, in part, responsible for placental insufficiency and intrauterine growth retardation. Magnesium deficiency increases the risks of miscarriage, premature delivery, fetal growth retardation, twin pregnancy, thyroid dysfunction and stress (due to illness/depression leading to a loss of $\mathrm{Mg}$ by the kidneys, and increased $\mathrm{Mg}$ requirements)(Takaya et al., 2006).

- essential fatty acid imbalance: nutritional factors such as essential fatty-acid (EFA) deficiencies have been associated with ADHD. The principal omega3 fatty acid in the brain, DHA, is highly accumulated in nervous tissue membranes and is important for neural function. Studies of diet showed that children with ADHD consumed equal amounts of omega- 3 and omega- 6 fatty acid, compared to control children. However, ADHD children had significantly lower levels of DHA and total omega- 3 fatty acids, higher omega- 6 fatty acids, and lower ratios of omega-3/omega- 6 fatty acids, compared to control children. These results suggest that adolescents with ADHD have abnormal EFA profiles, which are not explained by differences in intake. The role of $\mathrm{Mg}$ will be evoked in the hypothesis of imbalance of essential fatty acids. Vitamin B6 is also an important cofactor for numerous metabolic reactions including metabolism of serotonin, GABA (gammaamino-butyric acid) and dopamine (Colter et al., 2008, Gonon, 2009).

\section{Treatment of ADHD}

Conventional therapy is often multimodal including behavioural therapies and medications. Approved drugs for ADHD are psychostimulants 


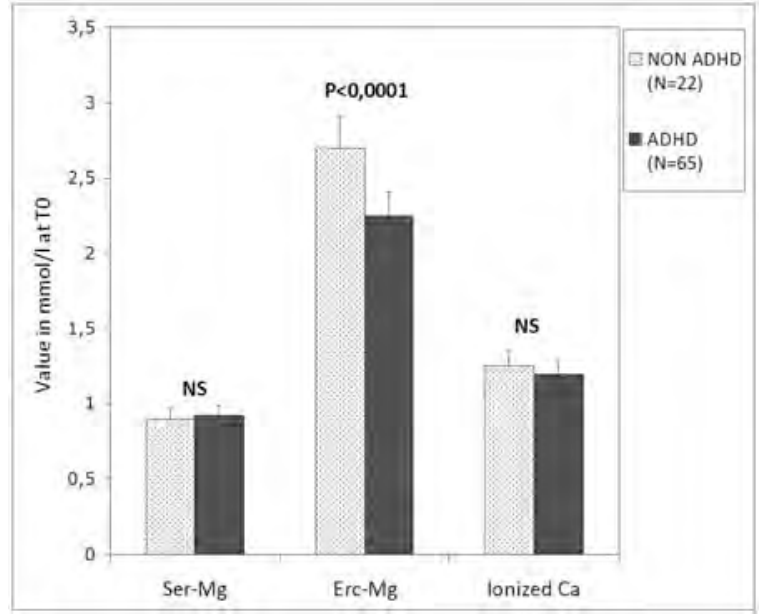

Figure 3. Biological data obtained at the first visit for 65 ADHD and 22 non-ADHD children. Ser-Mg: total serum magnesium concentrations in $\mathrm{mmol} / \mathrm{l}$; Erc-Mg: magnesium concentrations in erythrocyte (obtained after red cell lysis) in $\mathrm{mmol} / \mathrm{I}$. Only Erc$\mathrm{Mg}$ appeared to be significantly lower than before treatment (statistically significant at $p<0.05$ ).

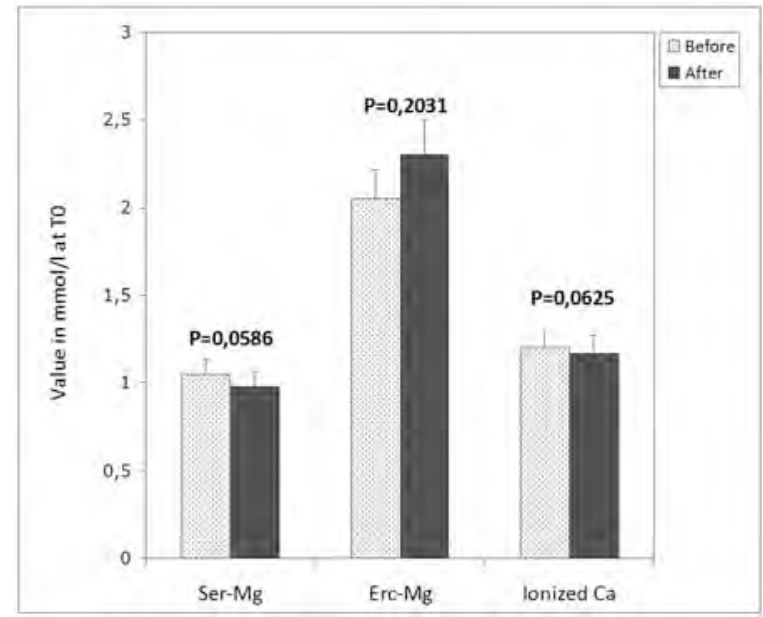

Figure 4. Biological data obtained 3 months after the first visit for the same children. Ser-Mg: total serum magnesium concentrations in $\mathrm{mmol} / \mathrm{l}$; ErcMg: magnesium concentrations in erythrocyte (obtained after red cell lysis) (in mmol/l). Erc-Mg recovered normal values (statistically significant at $\mathrm{p}<0.05)$.

(amphetamine derivatives), including methylphenidate. Psychostimulant medications approved by the U.S. Food and Drug Administration (FDA) include methylphenidate (Ritalin ${ }^{\circledR}$, Concerta ${ }^{\circledR}$ ), and, more recently, atomoxetine (Strattera ${ }^{\circledR}$ ).

In 2008, a study by the National Centre of Scientific Research (CNRS) in France (Gonon, 2009) concluded that "although psychostimulants alleviate the core symptoms of attention deficit hyperactivity disorder $(A D H D)$, recent studies confirm that their impact on the long-term outcomes of ADHD children is null. Psychostimulants enhance extracellular dopamine". Numerous review articles assert that they correct an underlying dopaminergic deficit of genetic origin. This dopamine-deficit theory of ADHD is often based upon an overly simplistic dopaminergic theory of reward. Psychostimulant medication does not improve long-term academic outcomes of the ADHD children. Therefore, this hypothesis should not be put forward to bias ADHD management towards psychostimulants. The American Journal of Psychiatry in June 2009 published a study entitled "Sudden death and use of stimulant medications in youths" funded, in part, by the FDA and the National Institute for Mental Health (Gould et al., 2009). Side effects are described for psychostimulant medications over some years.

As we have seen previously, dietary factors and $\mathrm{Mg}$ deficiency can play a significant role in the etiology of ADHD syndrome. In our study, $\mathrm{Mg}$ deficiency was found in $89 \%$ of children with ADHD, whereas only $23 \%$ of non-ADHD children present with low erythrocyte $\mathrm{Mg}$ deficiency (Table1).

\section{Clinical symptoms of autistic spectrum disorders (ASD)}

The prevalence of autism increases continuously and remains an extreme challenge to clinical researchers. It is a neurobiological condition having its origins in a disturbance of the cellular structure of the brain during pregnancy. Generally speaking, there is no "cure" for such children and the holy grail of finding a neuropharmacological reversal of symptoms is currently being researched worldwide.

For some years, one must insist on the screening of the early signs of autism during the two first years of the life as described in the DMS-IV manual:

- Relationship disorders; child is "too good" with very few smiles.

- Visual contact disorders; missing or poor visual attention; doesn't look at the parents; look is shifty. 
Table 1.

\begin{tabular}{|l|l|l|l|}
\hline \multicolumn{2}{|c|}{ Value in mmol/l } & \multicolumn{1}{c|}{ Non ADHD (N=22) } & \multicolumn{1}{c|}{ ADHD (N=85) } \\
\hline \multicolumn{2}{|c|}{ Threshold value } & \multicolumn{2}{c|}{$\%$ children < threshold } \\
\hline Ser-Mg & 0.85 & 0.00 & 27.69 \\
\hline Erc-Mg & 2.46 & 22.73 & 81.54 \\
\hline lonized Ca & 1.18 & 0.00 & 19.05 \\
\hline \multicolumn{2}{|c|}{ At least one criterion under threshold } & $23.81(5 / 21)$ & $88.89(56 / 63)$ \\
\hline
\end{tabular}

- Difficulty in listening; seems not to hear; delayed communication; doesn't repeat words.

- Motor affectation; doesn't take the toys; stereotyped movements of the hands; sits and walks belatedly; hypo-tonicity or hyper-tonicity.

- $\quad$ Sleeping disorders; broken sleep; crying.

- $\quad$ Feeding difficulties; slobbers; cannot swallow; refuses food.

The major feature of autism spectrum disorder is that the symptoms only manifest themselves after the age of eighteen months and before the age of three. It is considered like a regression of development (Burn, 2009). We can explain that ASD are disorders of brain function and not of brain structure.

After three years, the clinical picture of ASD becomes typical:

- Impairment of social interactions.

- visual contact: doesn't look at the parents and the siblings; gives a blank look; look is shifty.

- connection with equals: has no interest with the parents, with the friends at the primary school; seems to be "in their bubble".

- delight partitions: has no emotional relationship with the family, cannot express their pleasure for the event; does not show social reciprocity in the form of an answer to a human presence.

- Loss of communication.

- delayed communication: speaks belatedly; some sounds or some syllables are emitted late; few spontaneous verbal exchanges; lack of creativity in thought.

- no communication: doesn't repeat words.
- stereotypical language: repeats the same word sometimes without significance.

- social mimicking: cannot reproduce something after a long time earnestly trying.

- $\quad$ Stereotyped restricted behavior.

- stereotyped interest: has very poor interest or repeats the same answer for something.

- customs.

- handling things: difficulty to take the things, to take toys, and can have abnormal movements.

- motor affectation: to walk, moves with difficulty; to bring something to somebody, cannot take initiative.

- Abnormal or delayed functioning.

- language.

- social interactions.

- symbolic games: doesn't understand the rules of the game; cannot dream up the game.

- behavioural disorders like sleeping disorders, aggressiveness, impulsivity; attention deficit; cannot listen.

Magnesium metabolism could be involved in autism, autism spectrum disorder or pervasive developmental disorder (Rimland et al., 1978). Magnesium prevents against encephalopathy and developmental delay (Doyle et al., 2008).

\section{Clinical Case Study 3}

Patient ARN, three years old, presents all the clinical signs of ASD, an autistic disorder that began after two years of age. The adaptation at the nursery school became difficult. His mother describes behavioural disorders, with incessant restlessness, doesn't listen, puts own life in danger, let's go the mother's hand in the street, crosses the street without looking around, has important sleeping and communication disorders, 
doesn't speak, is always squealing, and his glance is shifty. At school, the headmaster's evidence was spectacular - "ARN become isolated, doesn't draw, has no interest with the activities, doesn't listen about the forbidden subjects. He doesn't speak, expresses himself by shouting, and his glance is shifty". During the examination, patient ARN was nervous, restless, tetanized, he clenched his fists and shook his hands. In the face of all these clinical symptoms, the possibility of a $\mathrm{Mg}$ deficiency is raised. His mother has presented a $\mathrm{Mg}$ deficiency for several years.

With Mg-B6 therapy, $(6 \mathrm{mg} / \mathrm{kg} /$ day of $\mathrm{Mg}$ and 0.6 $\mathrm{mg} / \mathrm{kg} /$ day of vitamin B6), ARN improved quickly month after month, probably because $\mathrm{Mg}$ supplementation was set up very early in life before the age of four years. ARN becomes cool, obedient, wants to get dressed alone, put his shoes on, takes interest in the family activities, is looking to manipulate the computer, begins drawing, doesn't cry, says some words and some sentences. At school, he begins to participate in the games, takes a book, shows the pictures and repeats the words. The improvements are spectacular. After six months of treatment, he can pronounce complete sentences and draws. After eighteen months of therapy, the parents stopped therapy, however the behavioural disorders reappeared with incessant restlessness at home and school in two weeks. Another attempt to stop the treatment was also unsuccessful where he became tired and depressed. The parents now do not want to stop the treatment anymore.

Today, patient ARN is twelve, doesn't present a behavioural disorder and communication and language is almost normal. He begins high school and has taken Mg-B6 supplementation for eight years (additional biological data: karyotype is $\mathbf{4 6}$ $X Y$; fragile $X$ negative).

\section{Clinical Case Study 4}

Patient FLO was four years old when the diagnosis of typical autism was made. When he was seven years old, the parents were informed about our work on $\mathrm{Mg}$ and autism. He presented communication disorders, could not emit sounds, didn't talk, had abnormal visual reactions, didn't look someone in the eyes, had a blank look and his look was shifty. The behavioural disorders were important with many anxiety attacks, a difficulty to calm, impulsiveness and constant movement with fits of anger. He had no creativity for games and didn't know how to play. Erythrocyte $\mathrm{Mg}$ was low. The same observation was made in his parents (Figures 5-7), despite them having a balanced diet including fishes, meats, fruits and vegetables.

After two months of treatment with $6 \mathrm{mg} / \mathrm{kg} / \mathrm{day}$ magnesium and $0.6 \mathrm{mg} / \mathrm{kg} /$ day vitamin $\mathrm{B} 6$, patient FLO was more cool, stayed at school with pleasure, began to talk with little sentences, was less in "his bubble", and was looking for his father when he came back from work. He was beginning to play with friends. After three years of treatment, FLO talks appropriately, understands the language, goes to a normal school where his reading skills have developed. During the first discussion, the parents advise that they want a third child. They both have evidence of important $\mathrm{Mg}$ depletion. A preventive Mg supplementation both before the desired pregnancy and during the pregnancy seemed to be essential, and indeed allowed good development of the baby without baby blues in the mother. The little sister is now two years old, bright, very much smiling and is always in a good mood! (Additional biological data: search for mutation in the gene neuroligine NLGN3 was negative; search for mutation in the gene neuroligine NLGN $4 \mathrm{X}$ was also negative).

\section{Experimental Study Number 2}

In order to study the effect of Mg-B6 for treating social, communication and behavioural responses of children with pervasive developmental disorders (PDD) or autism in connection with the $\mathrm{Mg} / \mathrm{calcium}$ status of the child, we designed an open study on 33 children with PDD syndrome. Our results showed a statistically significant improvement of the symptoms after Mg-B6 supplementation together with a rise in Erc-Mg values (Mousain-Bosc et al., 2006).

Intraerythrocyte $\mathrm{Mg}$ (Erc-Mg), serum $\mathrm{Mg}$ (s-Mg) and blood ionized calcium (i-Ca) were measured at different times. Clinical symptoms of PDD were scored (0 to 4). In contrast to s-Mg or i-Ca, PDD children exhibited significantly lower Erc-Mg values than controls (1.26 times; 16/33). The MgB6 regimen led to an increase in Erc-Mg values (1.18 times, 11/17) and this supplementation improved PDD symptoms in the large majority of 


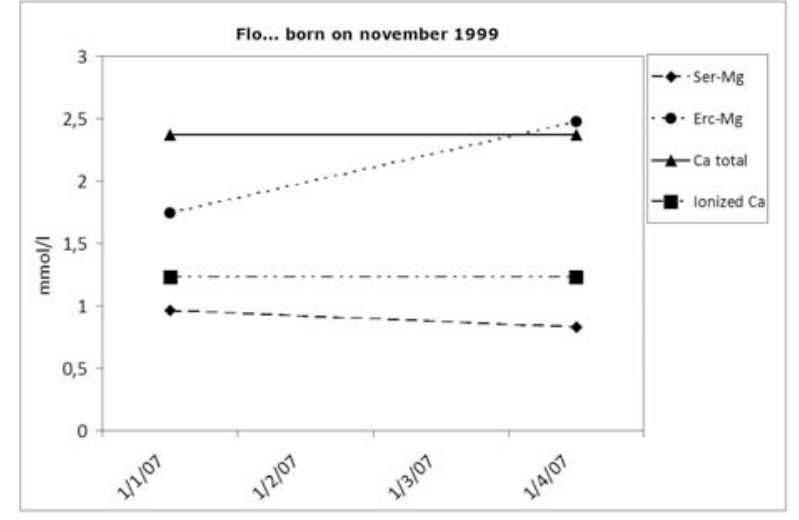

Figures 5. Evolution of biological parameters for magnesium and calcium in the case of patient FLO (born November 1999) during the follow-up (ASD/PED syndrome). Ser-Mg: total serum magnesium concentrations in $\mathrm{mmol} / \mathrm{l}$; Erc-Mg: magnesium concentrations in erythrocytes (obtained after red cell lysis) in $\mathrm{mmol} / \mathrm{l}$; Total Ca: total serum calcium concentrations in $\mathrm{mmol} / \mathrm{l}$; lonized calcium concentrations in $\mathrm{mmol} / \mathrm{l}$ in serum.

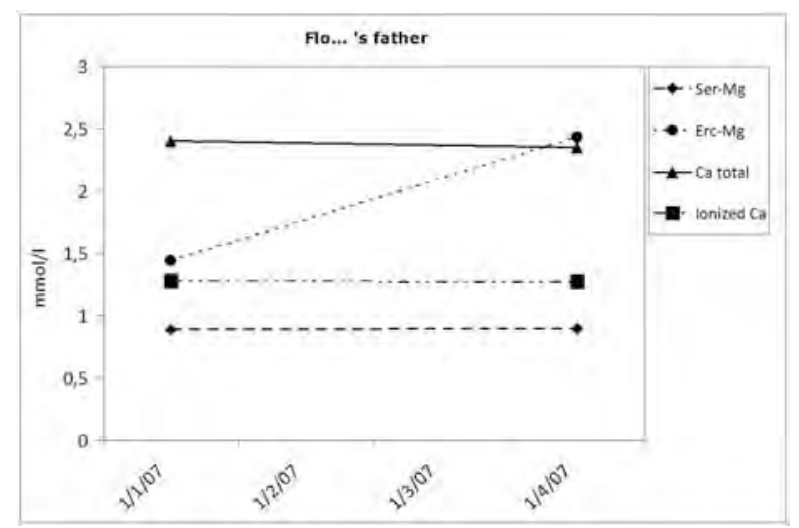

Figure 6. Evolution of biological parameters for magnesium and calcium in the case of patient FLO's father during the follow-up. Ser-Mg: total serum magnesium concentrations in $\mathrm{mmol} / \mathrm{l}$; Erc$\mathrm{Mg}$ : magnesium concentrations in erythrocytes (obtained after red cell lysis) in $\mathrm{mmol} / \mathrm{l}$; Total $\mathrm{Ca}$ : total serum calcium concentrations in $\mathrm{mmol} / \mathrm{l}$; lonized calcium concentrations in $\mathrm{mmol} / \mathrm{l}$ in serum.

children with no adverse effects: social interactions $(23 / 33, p<0.0001)$, communication (24/33, $p<0.0001)$, stereotypical restricted behaviour (18/33, $p<0.0001)$, and abnormal/ delayed functioning $(17 / 33, p<0.0001) ; 15 / 33$ children improved in the first three groups of

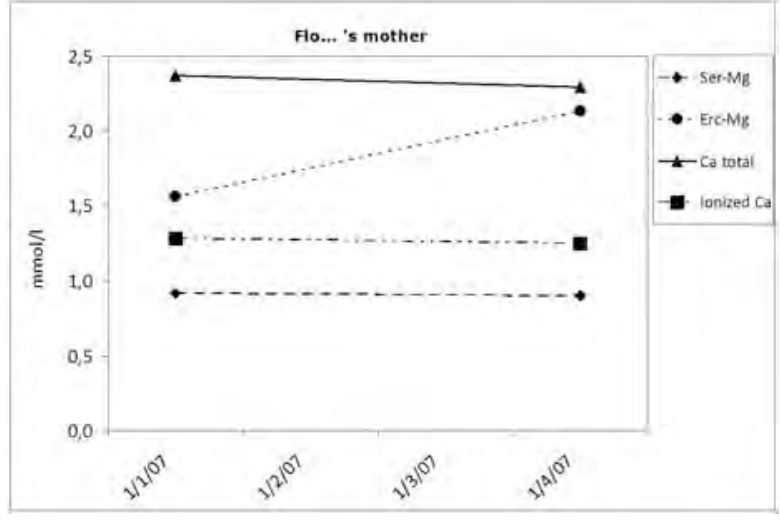

Figure 7. Evolution of biological parameters for magnesium and calcium in the case of patient FLO's mother during the follow-up. Ser-Mg: total serum magnesium concentrations in mmol/l; ErcMg: magnesium concentrations in erythrocytes (obtained after red cell lysis) in $\mathrm{mmol} / \mathrm{l}$; Total $\mathrm{Ca}$ : total serum calcium concentrations in $\mathrm{mmol} / \mathrm{l}$; lonized calcium concentrations in $\mathrm{mmol} / \mathrm{l}$ in serum.

symptoms. When the Mg-B6 treatment was stopped, PDD symptoms reappeared in a few weeks. A statistically significant relationship was found in Erc-Mg values from children before treatment and their mothers.

The neurobiological basis of a $\mathrm{Mg} / \mathrm{vit} \mathrm{B} 6$ supplementation supposes the existence of an impaired neuronal Mg pathway which could be reversed with Mg-B6 therapy. As we have previously discussed, $\mathrm{Mg}$ acts as an ionic membrane regulator and modulator of ion transfer through membrane channels. In brain, it has been shown that traumatic injury causes a decline in $\mathrm{Mg}^{2+}$ concentrations, focally as well as in the blood circulation, and contributes to the development of neurologic deficit (Vink et al., 2009). Similarly, brain ischaemia caused a decline in intracellular free $\mathrm{Mg}^{2+}$ concentrations (Basso et al., 2000) and $\mathrm{Mg}$ salt administration improves motor outcome in this situation (Ebel and Gunther, 2005). One of its most important modes of action is to inhibit the glutamate N-methyl-Daspartate (NMDA) channel (Zilbovicius et al., 2000). The activity of this channel generates an influx of calcium and, in turn, leads to excitotoxic cell death and apoptosis (Borella et al., 1993). In the same way, abnormal dietary deficiency of $\mathrm{Mg}$ as well as abnormalities in Mg metabolism play important roles in different types of heart 
diseases, and $\mathrm{Mg}$ influences catecholamine signalling in such diseases (Gervais et al., 2004).

Recently, in primary autistic children, positive emission tomography (PET) has been used to demonstrate a significant decrease in cerebral blood flow localized to the temporal lobes in 16/21 of children (Zilbovicius et al., 2000; Macdonald et al., 2004; Demougeot et al., 2004). Taken together with the fact that $\mathrm{Mg}$ has been shown to increase blood pressure (Macdonald et al., 2004) and that brain from rats fed with low $\mathrm{Mg}$ diets are more susceptible to permanent brain focal ischaemia (Demougeot et al., 2004), we can hypothesize that intracellular $\mathrm{Mg}^{2+}$ depletion could be responsible, at least in part, of some central activity disorders observed in PDD/autistic children.

In our study, an intra-erythrocyte $\mathrm{Mg}$ depletion was evidenced in almost half of the PDD children. To explain such a phenomenon, two hypotheses can be proposed: (i) a metabolic inhibition of membrane $\mathrm{Na}^{+} / \mathrm{K}^{+}$ATPase (observed in autism (Kurup and Kurup, 2003) with concomitant rise in intracellular calcium and decrease in intracellular $\mathrm{Mg}^{2+}$; (ii) a genetic defect in magnesium transport through plasma membrane $\left(\mathrm{Na}^{+}-\mathrm{Mg}^{2+}\right.$ exchanger (Ebel et al., 2004; Ebel and Gunther, 2005) or TRPM chanzymes (Montell, 2005). As Erc-Mg can be considered as representative of some intracellular $\mathrm{Mg}^{2+}$ concentrations, a decrease in Erc-Mg without changes in serum $\mathrm{Mg}$ concentration could be interpreted as an alteration of $\mathrm{Mg}^{2+}$ transport through the plasma membrane. The demonstration that TRPM7 is critical for $\mathrm{Mg}^{2}$ homeostasis evoked the possibility that mutation of TRPM channels may cause disease in humans as a result of reduced intracellular $\mathrm{Mg}^{2+}$ levels. Indeed, mutations were found in the case of hypomagnesemia with secondary hypocalcemia (Schlingmann et al., 2008) and, in this case, symptoms associated with TRPM6 mutations were improved by supplementation with high $\mathrm{Mg}$ doses, in agreement with increased $\mathrm{Mg}$ entry through a passive mode of $\mathrm{Mg}$ influx. This genetic hypothesis was also supported by our own data showing a positive correlation between low Erc$\mathrm{Mg}$ values in PDD children and their mothers. Similarly, Feillet-Coudray et al., (2006) have found that, in mice genetically selected for low magnesium levels, $\mathrm{Mg}$ efflux from erythrocytes was significantly increased. The genetic regulation of erythrocyte $\mathrm{Mg}$ content depends on the modification of $\mathrm{Mg}$ influx (Schlingmann et al., 2002). To confirm such a hypothesis, a genetic study of a PDD child's family has clearly to be developed.

When PDD children were supplemented with MgB6 treatment, Erc-Mg values more or less increased in only $65 \%$ of children. The impairment to get normal Erc-Mg values under MgvitB6 treatment supports the hypothesis of a defect in $\mathrm{Mg}$ transport in erythrocytes. In sickle cell disease, $\mathrm{Mg}$ pidolate supplementation was found to decrease $\mathrm{Na}^{+} / \mathrm{Mg}^{2+}$ exchanger activity with a partial rise in $\mathrm{Mg}$ and $\mathrm{K}^{+}$content of erythrocytes (De Franceschi et al., 2000). Doses of MgB6 and the duration of treatment, which have not been taken into account in our study, could also explain such an observation. Concerning the respective roles of pyridoxine and $\mathrm{Mg}$ in these observations, it was classically accepted that $\mathrm{Mg}$ is associated with pyridoxine to decrease irritable side-effects of the B6 therapy and that B6 is the main factor involved in the improvement of clinical symptoms in autistic patients. Following Erc-Mg values during $\mathrm{Mg}-\mathrm{B} 6$ treatment, we bring here evidence of the role of $\mathrm{Mg}$ itself in this therapy.

Mg-B6 treatment of PDD children was shown to improve symptoms of the disease. Three of the four main groups of clinical signs described in DSM-IV were significantly reduced and, for the first time, we found that $8 / 12$ of children who improved under treatment showed higher Erc-Mg values. Persons only slightly deficient in $\mathrm{Mg}$ become irritable, highly-strung, sensitive to noise, hyperexcitable, apprehensive and beligerent. If the deficiency was more severe or prolonged, they may develop twitching, tremors, irregular pulse, insomnia, muscle weakness, jerkiness, and leg and foot cramps. These symptoms can also be found in some cases of PDD/autism. Although this study was an open, non-controlled study, we found a relationship between clinical signs of PDD/autism and a biological parameter, namely Erc-Mg. However, we were unable to establish any correlation between improvement of symptoms and increase in Erc-Mg. Various possibilities might explain this lack of correlation. Firstly, Erc-Mg is probably not the best biological parameter to follow the relationship between $\mathrm{Mg}$ 
homeostasis and the neurological dysfunction of PDD/autism. Contradictory reports have been published on the use of Erc-Mg as index of $\mathrm{Mg}^{2+}$ status (Borella et al., 1993, Basso et al., 2000) and new biological tests that could help to study genetic alterations of $\mathrm{Mg}$ transport (lymphocytes, etc.) have to be tested. Secondly, other neurofunctional disorders may be involved in autism, such as a decrease in temporal blood flow. Even if low Erc-Mg levels have been correlated with a decrease in blood pressure, there is no evidence to associate blood pressure and cerebral blood flow in all cases.

In conclusion, this study presents new information about the therapeutic role of a MgB6 regimen in children with PDD syndrome. This effect seems to be associated, at least in part, to a cellular $\mathrm{Mg}$ depletion as evidenced by intraeythrocyte Mg measurements. Children with pervasive developmental disorders (including autism) exhibit low Erc-Mg levels. Parents frequently showed similar low Erc-Mg values suggesting a genetic defect in $\mathrm{Mg}$ transport. Installing a Mg-B6 supplementation for some weeks restored intraerythrocyte $\mathrm{Mg}$ values and significantly reduced the clinical symptoms of these diseases.

\section{Etiology: role of magnesium during pregnancy}

Since developmental disorders appear early during fetal development, $\mathrm{Mg}$ therapy could be justified even during pregnancy. The efficacy of $\mathrm{Mg}$ supplementation is more important if treatment is in the early years of life. Magnesium activates protein and amino acid synthesis and Mg deficiency leads to fetal growth retardation by reducing the nutritive utilization of protein as a result of decreased protein absorption and synthesis. Another study found a low cerebral blood flow in the temporal lobe probably due to low Mg levels in the cells (Zilbovicius et al., 2000).

\section{Effect of magnesium in fragile- $X$ syndrome}

Fragile- $X$ syndrome is an $X$-linked disorder characterized primarily by speech delay and moderate mental retardation and neurobehavioural disorders. The incidence of fragile $X$ syndrome is estimated at $1 / 4000-1 / 6000$ in males and half that for females (Wiesner et al., 2004). This disease is linked to the mutation of the FMR1 gene located on the $X$ chromosome, characterized by expansion (CGG amplification) at the FRAXA site (Xq27.3) in the non-coding region of the first exon (Weisman-Shomer et al., 2002). These authors also showed that two factors were indispensable for the stabilization of the CGG tetraplex: magnesium and ATP.

We report a single case of a child referred to our pediatrics unit for a behavioural disorder and for whom Fragile $X$ syndrome had previously been genetically confirmed. Considering the genetic origin of this disease, performing the clinical and biological evaluation of the patient's family completed our work. Both the patient and his affected family members were given a $\mathrm{Mg}$ and vitamin B6 supplementation and evaluated over three years. The family were followed clinically and biologically for the three years, including the children with the mental retardation, pervasive development disorder or attention deficit hyperactivity disorder. All children carried a mutation produced by expansion at the FRAXA site at $\mathrm{Xq} 27.3$ and showed $\mathrm{Mg}$ and calcium disorders (normal serum $\mathrm{Mg}$ concentration, but decreased erythrocyte $\mathrm{Mg}$ and plasma ionized $\left.\mathrm{Ca}^{2+}\right)$. The mother presented a premutation by expansion at the FRAXA site at Xq 27.3 with symptoms of $\mathrm{Mg}$ depletion (emotivity, asthenia, stress). $\mathrm{Mg}-\mathrm{B} 6$ supplementation $(6 \mathrm{mg} / \mathrm{kg} /$ day $\mathrm{Mg}$ and $0.6 \mathrm{mg} / \mathrm{kg} /$ day vitamin B6, orally) for three years reduced clinical symptoms in the mother and improved the behaviour of the children (aggressiveness, lack of attention at school) concomitant with an increase of intraerythrocyte $\mathrm{Mg}$. When the Mg-B6 treatment was stopped for two months in one of the children, clinical symptoms reappeared.

In 2007, the hypothesis was put forward that Fragile $X$ syndrome (Fra- $X$ ) is caused by the transcriptional silencing of the FMR1 gene that encodes the Fra-X mental retardation protein (FMRP) (Dolen et al., 2007; Hayashi et al., 2007). However, the pathogenesis of this disease is unknown. According to one proposal, many psychiatric and neurological symptoms of Fra- $X$ result from the unchecked activation of mGluR5, a metabotropic glutamate receptor. To test this hypothesis, FMR1 mutant mice presenting a 50\% reduction in mGluR5 expression were generated and studied in terms of the range of phenotypes with relevance to the human disorder. Results 
demonstrated that mGluR5 contributes significantly to the pathogenesis of the disease, a finding that has significant therapeutic implications for Fragile $X$ and related developmental disorders. In line with this observation, Hou et al., (2006) demonstrated that mGluR-LTD induces a transient, translationdependent increase in FMRP that is rapidly degraded by the ubiquitin-proteasome pathway.

The successful management of this disease by Mg- B6 supplementation observed in the present study, with an early and late evaluation at 3 years, supports the argument that a deficit in $\mathrm{Mg}$ influences the appearance of Fra- $X$ and that by replacing this $\mathrm{Mg}$, the disease symptoms may be managed. Berthelot's group (Martin et al., 2007) has previously demonstrated in rats that a deficit in $\mathrm{Mg}$ acts by reducing the level of proteasomes, protein complexes that act at the level of the mGluR5 receptor already implicated in this disease. Conversely, Picado et al., (1994) noted an increase in the levels of $\mathrm{Mg} / \mathrm{NA}$ in patients with behavioural problems and for whom hypertension was diagnosed. It has been demonstrated that for behavioural disorders in which hyperactivity and autism in particular are involved, for which current treatment regimens are difficult to standardize, management of disease symptoms with Mg-B6 supplementation is possible (Mousain-Bosc et al., 2006). Magnesium depletion represents one possible explanation for the appearance of Fra-X syndrome. Studies show that Mg-B6 supplementation given to subjects improved their behavioural disorders. The presence of a genetic mutation together with $\mathrm{Mg}$ deficiency in mothers may constitute an indication for regular evaluation of $\mathrm{Mg}$ status, and supplementation with Mg-B6 during pregnancy. We present herein some unpublished data obtained with 3 cases of Fra-X abnormality (poster presentation in Gordon Research Conference, Ventura, CA, March 2008).

\section{Conclusions}

This review brings additional information about the therapeutic role of a $\mathrm{Mg}-\mathrm{B} 6$ regimen in children with ADHD or ASD/autism syndrome. This effect seems to be associated, at least in part, to a cellular Mg depletion as evidenced by intraeythrocyte $\mathrm{Mg}$ measurements. Children with ADHD or PDD/ASD (pervasive developmental disorders/autistic spectrum disorders), including autism, exhibit low Erc-Mg levels. Parents frequently showed similar low Erc-Mg values suggesting a genetic defect in $\mathrm{Mg}$ transport. Installing a Mg-B6 supplement-ation for some weeks restored higher intraerythrocyte $\mathrm{Mg}$ values and significantly reduced the clinical symptoms of these diseases.

However, in a recent meta-analysis of all studies, Nye et al., (2010) arrived at the same conclusion as what they published in 2005: "Due to the small number of studies, and the methodological quality of studies, no recommendation can be advanced regarding the use of $B 6-\mathrm{Mg}$ as a treatment for autism. There is simply not sufficient evidence to demonstrate treatment efficacy". Together with the fact that both the American Psychiatric Association and the American Academy of Pediatrics have stated that megavitamin treatment for learning disabilities and autism is not justified, it helps explain the dearth of clinical studies investigating the use of magnesium and vitamin B6 (Mg-B6) in the treatment of autism and autistic spectrum disorders.

Magnesium is known to be crucial for brain activity and its involvement in the prevention of neurobehavioural diseases seems to be established. As a clinical double-blind study with Mg-B6 treatment over placebo cannot be accepted for regulatory and ethical reasons, it was suggested to put children under an "alternative treatment" before the conventional drug therapy. We hope that the combined use of new tools to measure intracerebral $\mathrm{Mg}^{2+}$ levels as proposed by the group of lotti et al., (2008), and a more specific clinical evaluation, will help to improve the outcome of children with these pathologies.

\section{Acknowledgements}

The authors would like to express their thanks to Professor J.P. Rapin (Dijon) who help them in the conception of the work. 


\section{References}

Adams JB, Holloway C (2004) Pilot study of a moderate dose multivitamin/mineral supplement for children with autistic spectrum disorder. J Altern Complement Med 10:1033-9.

Alexander RT, Hoenderop JG, Bindels RJ (2008) Molecular determinants of magnesium homeostasis: insights from human disease. J Am Soc Nephrol 19:14518.

Altura BM, Altura BT (1978) Magnesium and vascular tone and reactivity. Blood Vessels 15:5-16.

Association Psychiatric Association (1994) Diagnostic and Statistical Manual of Mental Disorders DSM-IV-TR Fourth Edition: American Psychiatric Association, Arlington, USA.

Basso LE, Ubbink JB, Delport R (2000) Erythrocyte magnesium concentration as an index of magnesium status: a perspective from a magnesium supplementation study. Clin Chim Acta 291:1-8.

Biederman J, Faraone SV, Spencer TJ, Mick E, Monuteaux MC, Aleardi M (2006) Functional impairments in adults with self-reports of diagnosed ADHD: A controlled study of 1001 adults in the community. J Clin Psychiatry 67:524-40.

Billard JM (2006) Ageing, hippocampal synaptic activity and magnesium. Magnes Res 19:199-215.

Borella P, Ambrosini G, Concari M, Bargellini A (1993) Is magnesium content in erythrocytes suitable for evaluating cation retention after oral physiological supplementation in marginally magnesium-deficient subjects? Magnes Res 6:149-53.

Burn R (2009) The Effect of Magnesium Deficiency on Brain Function: Autism Spectrum Disorder, Neurology, Genetics and Remedial Solutions. The Autism Centre, Carmarthenshire, UK.

Chakraborti S, Chakraborti T, Mandal M, Mandal A, Das $S$, Ghosh S (2002) Protective role of magnesium in cardiovascular diseases: a review. Mol Cell Biochem 238:163-79.

Chubanov V, Waldegger S, Mederos y Schnitzler M, Vitzthum H, Sassen MC, Seyberth HW, Konrad M, Gudermann T (2004) Disruption of TRPM6/TRPM7 complex formation by a mutation in the TRPM6 gene causes hypomagnesemia with secondary hypocalcemia. Proc Natl Acad Sci USA 101:2894-9.
Colter AL, Cutler C, Meckling KA (2008) Fatty acid status and behavioural symptoms of attention deficit hyperactivity disorder in adolescents: a case-control study. Nutr J 7:8.

De Franceschi L, Bachir D, Galacteros F, Tchernia G, Cynober T, Neuberg D, Beuzard Y, Brugnara C (2000) Oral magnesium pidolate: effects of long-term administration in patients with sickle cell disease. $\mathrm{Br} \mathrm{J}$ Haematol 108:284-9.

De Jesus Moreno Moreno M (2003) Cognitive improvement in mild to moderate Alzheimer's dementia after treatment with the acetylcholine precursor choline alfoscerate: a multicenter, double-blind, randomized, placebo-controlled trial. Clin Ther 25:178-93.

Demougeot C, Bobillier-Chaumont S, Mossiat C, Marie C, Berthelot A (2004) Effect of diets with different magnesium content in ischemic stroke rats. Neurosci Lett 362:17-20.

DeRose VJ (2007) Sensing cellular magnesium with RNA. Nat Chem Biol 3:693-4.

Dolen G, Osterweil E, Rao BS, Smith GB, Auerbach BD, Chattarji S, Bear MF (2007) Correction of fragile X syndrome in mice. Neuron 56:955-62.

Doyle LW, Crowther CA, Middleton P, Marret S (2007) Magnesium sulphate for women at risk of preterm birth for neuroprotection of the fetus. Cochrane Database Syst Rev:CD004661.

Ebel H, Gunther $\mathrm{T}$ (2005) $\mathrm{Na}^{+} / \mathrm{Mg}^{2+}$ antiport in erythrocytes of spontaneously hypertensive rats: role of $\mathrm{Mg}^{2+}$ in the pathogenesis of hypertension. Magnes Res 18:175-85.

Ebel H, Kreis R, Gunther T (2004) Regulation of $\mathrm{Na}^{+} / \mathrm{Mg}^{2+}$ antiport in rat erythrocytes. Biochim Biophys Acta 1664:150-60.

Feillet-Coudray C, Coudray C, Wolf Fl, Henrotte JG, Rayssiguier Y, Mazur A (2004) Magnesium metabolism in mice selected for high and low erythrocyte magnesium levels. Metabolism 53:660-5.

Feillet-Coudray C, Trzeciakiewicz A, Coudray C, Rambeau M, Chanson A, Rayssiguier Y, Opolski A, Wolf FI, Mazur A (2006) Erythrocyte magnesium fluxes in mice with nutritionally and genetically low magnesium status. Eur J Nutr 45:171-7.

Fergusson JW RJ, O'Laughlin JW, Banks CV (1964) Simultaneous spectrophotometric determination of calcium and magnesium with chlorophosphonazo III. Anal Chem 36:796-99. 
Findling RL, Maxwell K, Scotese-Wojtila L, Huang J, Yamashita T, Wiznitzer M (1997) High-dose pyridoxine and magnesium administration in children with autistic disorder: an absence of salutary effects in a doubleblind, placebo-controlled study. J Autism Dev Disord 27:467-78.

Folsom AR, Hong CP (2006) Magnesium intake and reduced risk of colon cancer in a prospective study of women. Am J Epidemiol 163:232-5.

Gervais H, Belin P, Boddaert N, Leboyer M, Coez A, Sfaello I, Barthelemy C, Brunelle F, Samson Y, Zilbovicius $M$ (2004) Abnormal cortical voice processing in autism. Nat Neurosci 7:801-2.

Gonon F (2009) The dopaminergic hypothesis of attention-deficit/hyperactivity disorder needs reexamining. Trends Neurosci 32:2-8.

Gould MS, Walsh BT, Munfakh JL, Kleinman M, Duan N, Olfson M, Greenhill L, Cooper T (2009) Sudden death and use of stimulant medications in youths. $\mathrm{Am} \mathrm{J}$ Psychiatry 166:992-1001.

Gunther T (2007) $\mathrm{Na}^{+} / \mathrm{Mg}^{2+}$ antiport in non-erythrocyte vertebrate cells. Magnes Res 20:89-99.

Hayashi ML, Rao BS, Seo JS, Choi HS, Dolan BM, Choi SY, Chattarji S, Tonegawa S (2007) Inhibition of p21activated kinase rescues symptoms of fragile $X$ syndrome in mice. Proc Natl Acad Sci USA 104:1148994.

Heath DL, Vink R (1998) Neuroprotective effects of $\mathrm{MgSO} 4$ and $\mathrm{MgCl} 2$ in closed head injury: a comparative phosphorus NMR study. J Neurotrauma 15:183-9.

Helpern JA, Vande Linde AM, Welch KM, Levine SR, Schultz LR, Ordidge RJ, Halvorson HR, Hugg JW (1993) Acute elevation and recovery of intracellular $\left[\mathrm{Mg}^{2+}\right]$ following human focal cerebral ischemia. Neurology 43:1577-81.

Hou L, Antion MD, Hu D, Spencer CM, Paylor R, Klann E (2006) Dynamic translational and proteasomal regulation of fragile $X$ mental retardation protein controls mGluR-dependent long-term depression. Neuron 51:441-54.

lotti S, Malucelli E (2008) In vivo assessment of $\mathrm{Mg}^{2+}$ in human brain and skeletal muscle by 31P-MRS. Magnes Res 21:157-62.

Jordt SE, Ehrlich BE (2007) TRP channels in disease. Subcell Biochem 45:253-71.
Kidd PM (2002) Autism, an extreme challenge to integrative medicine. Part 2: medical management. Altern Med Rev 7:472-99.

Kozielec T, Starobrat-Hermelin B (1997) Assessment of magnesium levels in children with attention deficit hyperactivity disorder (ADHD). Magnes Res 10:143-8.

Kuriyama S, Kamiyama M, Watanabe M, Tamahashi S, Muraguchi I, Watanabe T, Hozawa A, Ohkubo T, Nishino Y, Tsubono Y, Tsuji I, Hisamichi S (2002) Pyridoxine treatment in a subgroup of children with pervasive developmental disorders. Dev Med Child Neurol 44:2846.

Lelord G, Callaway E, Muh JP (1982) Clinical and biological effects of high doses of vitamin B6 and magnesium on autistic children. Acta Vitaminol Enzymol 4:27-44.

Liebscher DH, Liebscher DE (2004) About the misdiagnosis of magnesium deficiency. J Am Coll Nutr 23:730S-1S.

Macdonald RL, Curry DJ, Aihara Y, Zhang ZD, Jahromi BS, Yassari R (2004) Magnesium and experimental vasospasm. J Neurosurg 100:106-10.

Martin H, Staedtler F, Lamboley C, Adrian M, Schumacher MM, Chibout SD, Laurant P, Richert L, Berthelot A (2007) Effects of long-term dietary intake of magnesium on rat liver transcriptome. Magnes Res 20:259-65.

Martineau J, Barthelemy C, Cheliakine C, Lelord G (1988) Brief report: an open middle-term study of combined vitamin B6-magnesium in a subgroup of autistic children selected on their sensitivity to this treatment. J Autism Dev Disord 18:435-47.

Montell C (2003) $\mathrm{Mg}^{2+}$ homeostasis: the $\mathrm{Mg}^{2+}$ nificent TRPM chanzymes. Curr Biol 13:R799-801.

Montell C (2005) The TRP superfamily of cation channels. Sci STKE 2005:re3.

Mousain-Bosc M, Roche M, Polge A, Pradal-Prat D, Rapin J, Bali JP (2006) Improvement of neurobehavioral disorders in children supplemented with magnesiumvitamin B6. II. Pervasive developmental disorder-autism. Magnes Res 19:53-62.

Mousain-Bosc M, Roche M, Rapin J, Bali JP (2004) Magnesium VitB6 intake reduces central nervous system hyperexcitability in children. J Am Coll Nutr 23:545S-8S. 
Murza KA, Pavelko SL, Malani MD, Nye C (2010) Vitamin B 6-magnesium treatment for autism: the current status of the research. Magnes Res 23:115-7.

Nemoto T, Matsuzaki H, Uehara M, Suzuki K (2006) Magnesium-deficient diet-induced reduction in protein utilization in rats is reversed by dietary magnesium supplementation. Magnes Res 19:19-27.

Nilius B (2007) TRP channels in disease. Biochim Biophys Acta 1772:805-12.

Nilius B, Owsianik G, Voets T (2008) Transient receptor potential channels meet phosphoinositides. EMBO J 27:2809-16.

Nye C, Brice A (2005) Combined vitamin B6-magnesium treatment in autism spectrum disorder. Cochrane Database Syst Rev:CD003497.

Ozgo M, Bayle D, Zimowska W, Mazur A (2007) Effect of a low magnesium diet on magnesium status and gene expression in the kidneys of mice selected for high and low magnesium erythrocyte levels. Magnes Res 20:14853.

Picado MJ, de la Sierra A, Aguilera MT, Coca A, UrbanoMarquez A (1994) Increased activity of the $\mathrm{Mg}^{2+} / \mathrm{Na}^{+}$ exchanger in red blood cells from essential hypertensive patients. Hypertension 23:987-91.

Rimland B, Callaway E, Dreyfus P (1978) The effect of high doses of vitamin $B 6$ on autistic children: a doubleblind crossover study. Am J Psychiatry 135:472-5.

Sanjad SA, Hariri A, Habbal ZM, Lifton RP (2007) A novel PCLN-1 gene mutation in familial hypomagnesemia with hypercalciuria and atypical phenotype. Pediatr Nephrol 22:503-8.

Schlingmann KP, Weber S, Peters M, Niemann Nejsum L, Vitzthum $\mathrm{H}$, Klingel $\mathrm{K}$, Kratz $\mathrm{M}$, Haddad E, Ristoff $\mathrm{E}$, Dinour D, Syrrou M, Nielsen S, Sassen M, Waldegger S, Seyberth HW, Konrad M (2002) Hypomagnesemia with secondary hypocalcemia is caused by mutations in TRPM6, a new member of the TRPM gene family. Nat Genet 31:166-70.

Schlingmann KP, Gudermann T (2005) A critical role of TRPM channel-kinase for human magnesium transport. J Physiol 566:301-8.

Schmidt ME, Kruesi MJ, Elia J, Borcherding BG, Elin RJ, Hosseini JM, McFarlin KE, Hamburger S (1994) Effect of dextroamphetamine and methylphenidate on calcium and magnesium concentration in hyperactive boys. Psychiatry Res 54:199-210.
Schmidt CJ, Taylor VL (1988) Release of

$[3 \mathrm{H}]$ norepinephrine from rat hippocampal slices by $\mathrm{N}$ methyl-D-aspartate: comparison of the inhibitory effects of $\mathrm{Mg}^{2+}$ and MK-801. Eur J Pharmacol 156:11120.

Schmitz C, Perraud AL, Johnson CO, Inabe K, Smith MK, Penner R, Kurosaki T, Fleig A, Scharenberg AM (2003) Regulation of vertebrate cellular $\mathrm{Mg}^{2+}$ homeostasis by TRPM7. Cell 114:191-200.

Shalev H, Phillip M, Galil A, Carmi R, Landau D (1998) Clinical presentation and outcome in primary familial hypomagnesaemia. Arch Dis Child 78:127-30.

Slutsky I, Abumaria N, Wu LJ, Huang C, Zhang L, Li B, Zhao X, Govindarajan A, Zhao MG, Zhuo M, Tonegawa S, Liu G (2010) Enhancement of learning and memory by elevating brain magnesium. Neuron 65:165-77.

Spencer TJ, Biederman, J., Wilens, T.E. \& Faraone, S.V (2002) Overview and neurobiology of attentiondeficit/hyperactivity disorder. J Clin Psychiatry 63:3-9.

Starobrat-Hermelin B, Kozielec T (1997) The effects of magnesium physiological supplementation on hyperactivity in children with attention deficit hyperactivity disorder (ADHD). Positive response to magnesium oral loading test. Magnes Res 10:149-56.

Takaya J, Yamato F, Kaneko K (2006) Possible relationship between low birth weight and magnesium status: from the standpoint of "fetal origin" hypothesis. Magnes Res 19:63-9.

Tolbert L, Haigler T, Waits MM, Dennis T (1993) Brief report: lack of response in an autistic population to a low dose clinical trial of pyridoxine plus magnesium. $J$ Autism Dev Disord 23:193-9.

Trapani V, Farruggia G, Marraccini C, lotti S, Cittadini A, Wolf FI (2010) Intracellular magnesium detection: imaging a brighter future. Analyst 135:1855-66.

Van der Wiijst J, Hodenrop, JGJ, Bindels, RJM (2009) Epithelial $\mathrm{Mg}^{2+}$ channel TRPM6: insight into the molecular regulation. Magnes Res 22:127-32.

Vink R (2001) Magnesium in traumatic brain injury: past findings and future directions, In: Advances in Magnesium Research: Nutrition and Health / Y. Rayssiguier, A. Mazur, J. Durlach (eds.) John Libbey and Company, London, pp. 405-12.

Vink R, Cook NL, van den Heuvel C (2009) Magnesium in acute and chronic brain injury: an update. Magnes Res 22:158S-62S. 
Voets T, Nilius B, Hoefs S, van der Kemp AW, Droogmans G, Bindels RJ, Hoenderop JG (2004) TRPM6 forms the $\mathrm{Mg}^{2+}$ influx channel involved in intestinal and renal $\mathrm{Mg}^{2+}$ absorption. J Biol Chem 279:19-25.

Walder RY, Landau D, Meyer P, Shalev H, Tsolia M, Borochowitz Z, Boettger MB, Beck GE, Englehardt RK, Carmi R, Sheffield VC (2002) Mutation of TRPM6 causes familial hypomagnesemia with secondary hypocalcemia. Nat Genet 31:171-4.

Weisman-Shomer P, Cohen E, Fry M (2002) Distinct domains in the CArG-box binding factor $A$ destabilize tetraplex forms of the fragile $X$ expanded sequence d(CGG)n. Nucleic Acids Res 30:3672-81.

Wiesner GL, Cassidy SB, Grimes SJ, Matthews AL, Acheson LS (2004) Clinical consult: developmental delay/fragile X syndrome. Prim Care 31:621-5.
Wolf Fl, Trapani V, Cittadini A (2008) Magnesium and the control of cell proliferation: looking for a needle in a haystack. Magnes Res 21:83-91.

Wolf FI, Trapani V, Cittadini A, Maier JA (2009)

Hypomagnesaemia in oncologic patients: to treat or not to treat? Magnes Res 22:5-9.

Zilbovicius $\mathrm{M}$, Boddaert $\mathrm{N}$, Belin $\mathrm{P}$, Poline JB, Remy $\mathrm{P}$, Mangin JF, Thivard L, Barthelemy C, Samson Y (2000) Temporal lobe dysfunction in childhood autism: a PET study. Positron emission tomography. Am J Psychiatry 157:1988-93. 


\title{
Magnesium in psychoses (schizophrenia and bipolar disorders)
}

\author{
Mihai Nechifor
}

Department of Pharmacology, "Gr.T.Popa” University of Medicine and Pharmacy, lasi, Romania. \nechif@yahoo.com

\begin{abstract}
Schizophrenia and bipolar disorders are two of the most severe CNS conditions. Changes in plasma and intracellular magnesium concentration, as well as in other bivalent cations, have been found in both psychoses. Our data, as well as that of other authors, has shown that schizophrenic, paranoid patients admitted in the acute state and without previous treatment, have significantly decreased intracellular magnesium levels compared to healthy subjects. Therapy with haloperidol (a typical antipsychotic) or with risperidone (an atypical antipsychotic) both significantly raised the intracellular magnesium concentration without causing significant changes in plasma magnesium concentration. The increase in intracellular magnesium concentration was positively correlated with the improvement in clinical symptomatology. We consider that magnesium acts foremost by reducing glutamate release and by its action upon NMDA receptors, and results in an augmentation in the activity of the GABAergic systems. Unlike the hypothesis that only implicates zinc deficits in the pathogeny of schizophrenia, we consider that both intracellular magnesium and extracellular zinc deficits are equally involved in schizophrenia pathogeny. In patients with untreated bipolar disorder, our data showed a significant decrease in intracellular magnesium concentration and plasma zinc concentration during the manic episode. Therapy with mood modulators (carbamazepine and valproic acid) increased total intracellular magnesium and plasma zinc concentrations without having a significant effect on total plasma magnesium concentration. Other data showed that lithium also increases intracellular magnesium concentration. The fact that mood modulators with different mechanisms of action have in common the increase of intracellular magnesium concentration is an argument to consider this augmentation as an important element of their mechanism of action.
\end{abstract}

\section{Introduction}

Psychoses are amongst the most severe diseases of the CNS. They are diseases in thought processes and behaviour leading to a loss of contact with reality. Various neurological and psychiatric pathologies are associated with changes of plasma and intracellular magnesium and other bivalent cations. Such changes in magnesium concentrations have been observed in brain injury, stroke, headaches, epilepsy, major depression and others (Altura and Altura, 1999; Durlach and Bac, 1997). Schizophrenia and bipolar psychosis present an increasing incidence.

\section{Schizophrenia}

Schizophrenia is a chronic and severe brain disorder whose incidence is quite difficult to evaluate. Nonetheless, there are data that show that the prevalence of this disease could arrive at $0.5 \%$ of population (Kirkbride et al., 2008;
Takei et al., 1992). It is a psychiatric illness that results in difficulty in telling the difference between real things and ideas and non-real ideas, which causes disturbed thinking and strong and inappropriate emotions. Four basic types of schizophrenia have been described:

a) catatonic schizophrenia;

b) disorganized schizophrenia (hebephrenic schizophrenia);

c) paranoid schizophrenia;

d) undifferentiated schizophrenia.

Identifying the changes in magnesium concentration in schizophrenic patients can be difficult, and sometimes contradictory, as a result of several factors including diversity of types of disease, unknown time of onset of the disease, and particularities of the disease evolution with periods of reduced intensity in occurring symptoms. In all circumstances, the association of other diseases can also modify intra and extracellular concentrations of magnesium and other bivalent cations. 
Some authors have shown increased plasma $\mathrm{Mg}$ concentrations in schizophrenic patients. Specifically, Jabotinsky-Rubin et al., (1993) consider that schizophrenic patients present increased $\mathrm{Mg}$ plasma concentrations, but that haloperidol administration reduces $\mathrm{Mg}$ levels. These authors also show that decreased $\mathrm{Mg}$ concentration is involved in the generation of extrapyramidal side effects of haloperidol, and consider that in schizophrenia, hypermagnesemia might be correlated with some failure of the catecholamine systems in the CNS. Gattaz et al., (1983) found a higher $\mathrm{Mg}$ level in the CSF of the schizophrenic patients. It was stated that schizophrenia appears to increase magnesium concentration in CSF, which was associated with decreased neuronal cGMP concentration.

In contrast, other authors didn't find any differences in magnesium concentration between healthy subjects and schizophrenic patients. In the studies of Kornhuber et al., (1994), post-mortem determination of $\mathrm{Mg}$ concentrations in brain didn't show any differences between the schizophrenic patient group and a control group. Athanassesnas (1983) indicated that there was no difference in plasma $\mathrm{Mg}^{2+}$ and $\mathrm{Ca}^{2+}$ concentrations in drug free schizophrenic patients.

Finally, other research has shown low $\mathrm{Mg}^{2+}$ concentration in the CSF of acute schizophrenic patients, but that the levels increase in remitted schizophrenics (Levine et al., 1996). Other authors indicated that patients with chronic schizophrenia present magnesium deficiency (Kirov et al., 1990; Kanofsky and Sandyk, 1991). Magnesium deficiency was also noted in psychosis induced by cancer chemotherapy (Matzen and Martin, 1985).

Our studies (Nechifor et al., 2004) showed that intraerythrocyte total magnesium concentration is significantly lower in patients with paranoid schizophrenia before treatment, as compared to normal subjects. The level of intraerythrocyte magnesium was $4.82 \pm 0.32 \mathrm{mg} / \mathrm{dl}$ in acute paranoid schizophrenia patients as compared to $5.82 \pm 0.11 \mathrm{mg} / \mathrm{dl}$ in the normal control group $(p<0.05)$. In this study, there were no differences between plasma magnesium concentrations of schizophrenic patients and the healthy subjects
(18.2 $\pm 11 \mathrm{mg} / \mathrm{L}$ in schizophrenic patients; $18.7 \pm$ $2.17 \mathrm{mg} / \mathrm{L}$ in the control group).

Kirov and Tsachev (1990) observed a rise in plasma magnesium level in schizophrenic patients in clinic remission, but other studies haven't found any correlation between the decrease in plasma magnesium concentration and the symptoms of schizophrenic patients (Kirov et al., 1994).

The analysis of extracellular magnesium and other bivalent cation concentrations in postmortem schizophrenic patients in a variety of cerebral areas (hippocampus, amygdala, caudate nucleus, cortex) showed significant differences between the drug-free schizophrenic group and treated schizophrenic patients. There weren't significant differences regarding magnesium concentration, but drug-free schizophrenic patients presented an increased copper level and a reduced zinc level.

We tested the effects of two different antipsychotic drugs (haloperidol and risperidone) in therapeutic doses on plasma and erythrocyte total magnesium concentrations in acute, paranoid, adult schizophrenic patients. Our data (Nechifor et al., 2004) showed that both typical antipsychotic drugs (haloperidol and risperidone) induced a rise in the intraerythrocyte magnesium concentration and also improved the clinical symptoms in paranoid schizophrenia patients. The data indicated a rise in erythrocyte magnesium concentration after haloperidol treatment from $4.82 \pm 0.32 \mathrm{mg} / \mathrm{dl}$ to $5.46 \pm$ $0.19 \mathrm{mg} / \mathrm{dl} \quad(p<0.05)$ and after risperidone treatment to $5.28 \pm 0.21 \mathrm{mg} / \mathrm{dl}(p<0.05)$. The antipsychotic drugs tested have very different mechanisms of action. Haloperidol is a strong blocker of D1, D2 and $\alpha 1$ receptors, and a weak blocker of $5-\mathrm{HT}_{2 \mathrm{~A}}$ receptors. Risperidone is a strong blocker of D2 receptors and $5-\mathrm{HT}_{2 \mathrm{~A}}$ receptors, but a very weak blocker of $D 1$ receptors.

Renn et al., (2010) also found an increased magnesium level in schizophrenic patients treated with antipsychotics, which is in agreement with our data. These data favour the idea that an increase in magnesium concentration is not a peripheral phenomenon, but an essential element for the mechanism of action of the antipsychotic drugs utilized in 
schizophrenia treatment. However, there is also contrary information whereby treatment with classic neuroleptics decrease plasma concentrations of magnesium, and chronic treatment with neuroleptics may even produce hypomagnesemia in some schizophrenics (Alexander et al., 1979).

Regarding the involvement of variations in magnesium concentration in the etiopathogeny of psychosis, we should take into account that intracellular magnesium plays more roles than extracellular magnesium, and intracellular total magnesium and free $\mathrm{Mg}^{2+}$ are compartmentalized between cell organelles and the cytosol (Gunther et al., 2006). In terms of the glutamatergic hypothesis of schizophrenia that involves the imbalance between glutamate and dopamine with an excess of glutamate (Krebs, 1992), an increase in magnesium concentration induced by typical and atypical neuroleptic administration is mainly involved in reduction of NMDA receptor activation by glutamate. In this circumstance, $\mathrm{Mg}^{2+}$ reduces the neuronal $\mathrm{Ca}^{2+}$ entrance through $\mathrm{Ca}^{2+}$ channels coupled with NMDA receptors. We suppose that $\mathrm{Mg}^{2+}$ acts in the same way in the experimental phencyclidine model of psychosis. We believe that the increase in cell $\mathrm{Mg}$ level is important for the antipsychotic action of haloperidol and risperidone. The effect of magnesium could be produced by decreasing the neuronal response to glutamate stimulation of NMDA receptors, decreasing the presynaptic release of glutamate, decreasing formation of peroxide radicals, and increasing GABAergic activity and glycine synthesis; glycine inhibits catecholamine release (Johnson et al., 1994).

The increase in magnesium concentration can reduce anxiety, hallucinations and agitation whereas hypomagnesemia could exacerbate anxiety and hallucinations. Ang et al., (1993) considered that hypomagnesemia is one of the causes of antipsychotic treatment resistance. The improvement of the clinical state and the increase in intracellular magnesium concentration leads to the idea that magnesium might be involved in the development of the antipsychotic drugs' therapeutic effect. The positive effect of increased magnesium concentration could be explained by the decreased activity of NMDA receptors, using the same reasoning that the excessive activity of some glutamatergic brain systems are involved in some psychotic symptoms.

Some post-mortem studies in human subjects have also showed abnormalities of GABAergic systems in schizophrenia patients. There is evidence for a deficit in GABA-containing interneurons in the prefrontal cortex (Beasley et al., 2002; Reynolds et al., 2001). There are biochemical and morphological arguments for a deficit in GABAergic systems in schizophrenia and this deficit is limited to the parvum-albumin class of GABAergic interneurons (Blum and Mann, 2002). It is possible that by increasing neuronal magnesium concentration with antipsychotic drugs, GABAergic systems are activated and the partial deficit of the GABAergic cortical systems are corrected. Regarding the mechanisms by which magnesium can increase the activity of the GABAergic systems, there are at least two possibilities: by enhancing vesicular GABA-transporter activity (Gerstein et al., 2005) or by modulation of $\mathrm{GABA}_{\mathrm{A}}$ receptor activity (Stanley et al., 1995). There is evidence that magnesium potentiates the function of native and recombinant $\mathrm{GABA}_{A}$ receptors and Moykkyven et al., (2001) suggested a putative $\mathrm{Mg}$ binding site on the $\mathrm{GABA}_{\mathrm{A}}$ receptor complex.

One of the most promising therapeutic prospects is the use of drugs acting at metabotropic glutamate receptors. Krystal et al., (2010) described the potential of mGluR2 and mGluR5 agonists and allosteric positive modulators in the treatment of schizophrenia. Magnesium has a positive modulator effect at the level of some mGlu receptors. We believe that the increase in intracellular magnesium concentration after antipsychotic drug treatment allows magnesium to exert this modulator role, especially at mGluR5 and mGluR2, and possibly at mGluR7 level. By these means, magnesium not only reduces the glutamate action at the level of NMDA receptors, but also can increase the GABAergic activity and reduce presynaptic glutamate release.

Autism is one of the main symptoms of schizophrenia but there are also patients with autism (especially children) who are not considered to be schizophrenic. In autistic children that are not schizophrenic, Strambi et al., (2006) showed that there are no differences 
in intracellular $\mathrm{Mg}$ in autistic children versus normal children. Unlike autistic children, patients with schizophrenia have significantly lower plasma and intracellular $\mathrm{Mg}$ concentrations compared to normal subjects. We consider this fact as an argument for a different pathogeny of autism compared with schizophrenia. Regarding the effect of treatment with vitamin-B6-magnesium in children with autism syndrome (non-schizophrenic), there are contradictory data. Nyl and Brice (2002) maintain that in these children (a small number of cases studied), a favourable effect of this treatment might exist. However, Findling (1997) showed no significant differences between the treated and placebo group.

Eating disorders and cognitive dysfunction has also been identified in schizophrenia (Wobrock et al., 2009). The serum levels of glutamate and glutamine in schizophrenic people with anorexia and cognitive impairment were significantly higher than those in the control group. Based on these findings, Nakazato et al., (2010) hypothesized that changes in the glutamate concentration are involved in the mechanism of cognitive impairment and anorexia of schizophrenic patients. We are in agreement with this point of view and we consider that magnesium, by decreasing the action of glutamate upon NMDA receptors, could contribute to the improvement of these symptoms in schizophrenic patients.

Although the etiopathogeny of schizophrenia is not sufficiently known, we consider that an increase in magnesium concentration by antipsychotic therapy is not just a secondary phenomenon, but is part of the mechanism of action of antipsychotics. We consider that the data above indicates that the changes of intracellular magnesium concentration are important for neuroleptic drug action in schizophrenic patients, and the fact that antipsychotic drugs with different chemical structures and mechanisms of action induce the increase in intracellular magnesium levels, further supports this idea. Contrary to Andrews (1992), who supported only the zinc deficiency theory of schizophrenia, we consider that intracellular magnesium and plasma zinc deficiency play an important role in the pathogenic mechanism of schizophrenia. The increase in intracellular magnesium concentration is important, and is not just a side effect of the therapeutic action of typical and atypical antipsychotics.

\section{Bipolar disorders}

Bipolar disorder (BD), also called manicdepressive psychosis, is an affective psychosis. It is a recurrent and severe psychiatric disease characterized by episodes of mania followed by depression and by mood instability. Without adequate treatment, $\mathrm{BD}$ is associated with high rates of mortality. BD is considered a relatively frequent psychiatric disease. Vacheron-Trystan et al., (2004) consider that the prevalence of BD over a lifetime is around $1 \%$ in the general population. There are several forms of BD. According to DSM IV, the first type of BD (the most frequent one) has a dominance of manic symptoms, associated with periods of depression. According to DSM IV, there are two main subtypes of BD: type I BD with at least one manic episode or several manic and depressive episodes, and type II BD which is related to patients enduring recurrent depressive episodes.

There is some data, some contradictory, regarding magnesium and other bivalent cation concentrations in patients with bipolar disorder, although they haven't all been made in the same type of BD or at the same stage of this disease. Decreasing $\mathrm{Mg}$ level could exacerbate anxiety, weakness and other symptoms in BD patients (Kirov and Tsachev, 1990). Frazer et al., (1983) found an increased level of erythrocyte magnesium concentration in patients with bipolar disorder before hospitalization. Contrary to this, George et al., (1994) highlight significant changes of magnesium concentration in CSF of BD patients and cannot establish a correlation between the disease outcome and this cation concentration. Some authors have reported a decreased plasma magnesium concentration in $B D$ patients compared to healthy control (Herzberg and Herzberg, 1977) while others found a disturbed ratio between plasma calcium and magnesium concentrations. An increased calcium/magnesium ratio was correlated with manic agitation (Carman et al., 1979). The current medication is mood modulators, the 
most commonly used drug from this category being lithium, and some anticonvulsive substances that have proven to be effective in $\mathrm{BD}$, such as carbamazepine, sodium valproate, and lamotrigine (Arban et al., 2005). More rarely used in the treatment of $B D$ are gabapentine and topiramate. Also, some atypical antipsychotics such as olanzapine, clozapine and risperidone have been administered in BD.

Lithium was and remains one of the most utilized mood modulators in BD therapy, especially in patients with the dominance of manic symptoms, although its therapeutic utility has a tendency to diminish (Gay and Olie, 2005). The data describing the influence of lithium administration upon serum magnesium concentrations are variable. Most studies show that repeated lithium salt administration increases magnesium concentration. Lithium increases the intracellular magnesium concentration by competing with magnesium for some intracellular binding sites (Leyden et al., 2000). Some data demonstrate $\mathrm{Li}^{+} / \mathrm{Mg}^{2+}$ competition at therapeutic intracellular $\mathrm{Li}^{+}$concentration (after treatment) in human neuroblastoma SH-SY5Y cells (Abukhdeir et al., 2003). After experimental loading of neuroblastoma cells with 1-2 mM of extracellular $\mathrm{Li}^{+}$, the intracellular free magnesium concentration was significantly higher. With respect to $\mathrm{Li}^{+}$and $\mathrm{Mg}^{+}$competition at some intracellular sites, the existing data have identified the following molecules as sites of competition: G-protein transducin in the guanosine 5'-diphosphate bound conformation (Srinivasan et al., 2004); inositol monophosphatase; glycogen synthase kinase 3 (GSK3); fructose 1,6 biphosphatase; biphosphate nucleotidase (Gould et al., 2004); and finally, ATP and ADP phosphate binding sites. It is also possible that other intracellular binding sites for $\mathrm{Li}^{+} / \mathrm{Mg}^{2+}$ competition exist. Rybakowski and Szaynerman (1976), hypothesized that magnesium could increase the intracellular influx of lithium. This could be another means of magnesium involvement in the mechanism by which lithium reduces the clinical symptoms from bipolar disorders.

Our data (Nechifor et al., 2006; 2007) show that adult patients with bipolar disorder type I presenting acute manic attacks and with no previous treatment exhibit lower intracellular magnesium levels than the control group. Plasma zinc concentration was also significantly lower $(0.72 \pm 0.02 \mathrm{mg} / \mathrm{l}$ versus $89 \pm 0.12 \mathrm{mg} / \mathrm{l}$ $(p<0.05)$. There were no significant differences between patients with bipolar disorder type I and the control group with respect to plasma total magnesium concentration.

Carbamazepine treatment $(600 \mathrm{mg} /$ day per os for 4 weeks) and sodium valproate $(900 \mathrm{mg} /$ day for 4 weeks) significantly increased intracellular magnesium levels from $45.0 \pm 1.87 \mathrm{mg} / \mathrm{l}$ before treatment to $52.02 \pm 0.9 \mathrm{mg} / \mathrm{l}$ after sodium valproate, and $53.72 \pm 2.18 \mathrm{mg} / \mathrm{l}$ after carbamazepine $(\mathrm{p}<0.05)$. Plasma magnesium concentration wasn't significantly modified but there was a significant rise in plasma zinc concentration (Nechifor et al., 2005; 2006). Walden et al., (1993) showed that magnesium deficiency decreased the beneficial effect of carbamazepine in the treatment of epilepsies but we think that hypomagnesemia also decreases the effect carbamazepine in the treatment of affective disorders. Magnesium oxide increases the verapamil maintenance therapy in mania (Giannini et al., 2000). This fact favours the idea that an increase in magnesium concentration is important, maybe essential for the therapeutic effect of some drugs used in BD treatment. Magnesium valproate reduces the hyperactivity in an animal model of mania. This effect of magnesium valproate could be abolished by bicuculline, suggesting that actions on postsynaptic GABA may also be involved in the antimanic action of magnesium valproate (Cao and Peng, 1993).

We consider that the increase in intracellular magnesium concentration is an important feature of carbamazepine, valproic acid and other mood modulators used in BD treatment. We also consider that transmembrane calcium influx plays an important role in the development of some psychiatric disorders. The calcium channel antagonists (verapamil and others) increase the carbamazepine effect. Magnesium, acting like a natural calcium antagonist on some ionic channels, is a factor that contributes at the pharmacodynamic effect of some mood modulators. In contrast, calcium ions have an antagonistic effect on carbamazepine action. Carbamazepine reduces the neuronal excitability and glutamate release 


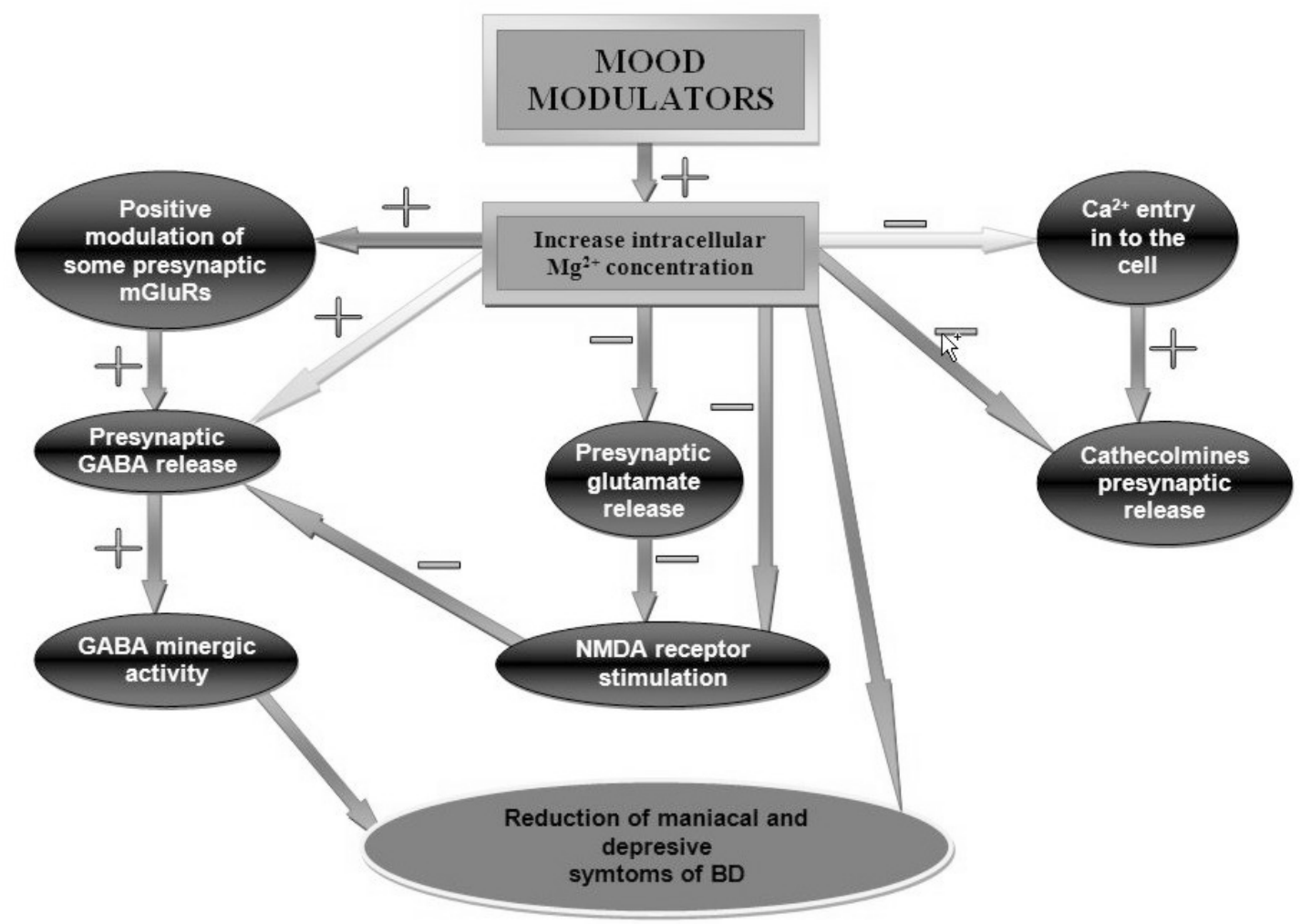

Figure 1. The mechanism of magnesium's involvement in mood modulator action. $+=$ stimulation; - = inhibition

and we consider that this effect is due, at least in part, by increasing magnesium concentration. The increase in intracellular magnesium concentration at the neuronal level is important for all the mood modulator drugs utilized in BD therapy, and not only for lithium (Nechifor et al., 2008). Some of the possible mechanisms describing magnesium's involvement in mood modulator action are presented in Figure 1. Gobbi and Janiri (2006) showed that magnesium valproate significantly modulated the response induced by NMDA-receptor stimulation. Chuinard et al., (1990) showed that magnesium aspartate administration was effective in stabilizing the mood of rapid-cycling $B D$, which favours the idea that the increase in magnesium concentration is an important factor of lithium and other mood modulators mechanism of action. Heiden et al., (1999) administered magnesium sulphate iv as supplementary treatment in severe acute mania.

In conclusion, we suggest that the increase in plasma zinc level and the erythrocyte magnesium level is not a peripheral phenomenon but is involved in the mechanism by which some mood modulators can reduce clinical symptoms of bipolar disorders, not only the manic episodes of BD but also the depressive periods. The changes in intra and extracellular plasma magnesium concentrations and other bivalent cations are an important part of the mechanism of action of some mood modulators and other psychotropic drugs. 


\section{References}

Abukhdeir AM, Layden BT, Minadeo N, Bryant FB, Stubbs EB Jr, Mota De Freitas D (2003) Effect of chronic $\mathrm{Li}^{+}$treatment on free intracellular $\mathrm{Mg}^{2+}$ on human neuroblastoma SH-SY5Y cells. Bipolar Disord 5:6-13.

Alexander DR, Deeb M, Bitar F, Antun F (1986) Sodium-potassium, magnesium and calcium ATPase activities in erythrocyte membranes from maniacdepressive patients responding to lithium. Biol Psychiatry 21:997-1007.

Altura BM, Altura BT (1999) Association of alcohol in brain injury, headaches and stroke with brain-tissue and serum levels of ionized magnesium. Alcohol 19:119-30.

Andrews RC (1992) An update of the zinc deficiency theory of schizophrenia. Identification of the sex determininig system as the site of action of reproductive zinc deficiency. Med Hypotheses 38:28491.

Ang AN, Ko SM, Tan CH (1995) Calcium, magnesium and psychotic symptoms in a girl with idiopathic hypoparathyroidism. Psychoso. Med 57:299-302.

Athanassenas G, Papadopoulos E, Kourkoubas A, Tsitourides S, Gabriel Hoidas E, Frangos E (1983) Serum calcium and magnesium levels in chronic schizophrenics. J Clin Psychopharmacol 3:212-216.

Arban R, Marian G, Brackenborough K, Winyard L, Wilson A, Gerard P, Large C (2005) Evaluation of the effects of lamotrigine, valproate and carbamazepine in a rodent model of mania. Behav Brain Res 158:123-32.

Beasley CL, Zhang ZJ, Patten I, Reynolds GP (2002) Selective deficits in prefrontal cortical GABAergic neurons in schizophrenia defined by the presence of calcium-binding proteins. Biol. Psychiatry 52:708-15.

Blum BP, Mann JJ (2002) The GABAergic system in schizophrenia. Int J Neuropsychopharmacol 5:159-79.

Cao BJ, Peng NA.(1993) Magnesium valproate attenuates hyperactivity induced by dexamphetaminechlordiazepoxide mixture in rodents. Eur J Pharmacol 237:177-81.

Carman JS, Post R, Runkle DC, Bunney Jr. WE, Wyatt RJ (1979) Increased serum calcium and phosphorus with the switch into maniac or excited psychotic state. $\mathrm{Br} J$ Psychiatry 135:55-61.
Chouinard G, Beauclai I, Geiser R, Etienne P (1990) A pilot study of magnesium aspartate hydrochloride (Magnesiocard) as a mood stabilized for a rapid cycling bipolar affective disorder patients. Prog Neuropsychopharmacol Biol Psychiatry 14:171-80.

Durlach J, Bac P (1997) Mechanisms of action in the nervous system in Magnesium deficiency and dementia, In Mineral and Neurotoxicity (Yasui M, Strong MJ, Ota K, Verit MA, eds) CRR Press, Boca Raton, 27-38.

Frazer A, Ramsey TA, Swann A (1983) Plasma and erythrocyte in affective disorders. J Affect Disorder 5:103-13.

Findling RL, Maxwell K, Scotese-Wojtila L, Huang J, Yamashita T, Wiznitzer M (1997) High-dose pyridoxine and magnesium administration in children with autistic disorder: an absence of salutary effect sin a double-blind placebo-controlled study. J Autism Dev Disord 27:467-78.

Gattaz WF, Kattermann R, Gattaz D, Beckmann H (1983) Magnesium and calcium in the CSF of schizophrenic patients and healthy controls: correlations with cyclic GMP. Biol Psychiatry 18:935-9.

Gay C, Olie JP (2005) Management of bipolar disorders. Rev Prat 55:513-22.

George MS, Rosenstein D, Rubinow DR, Kling MA, Post RM (1994) CSF magnesium in affective disorders lack correlation with clinical course of treatment. Psychiatry Res 5:139-46.

Giannini AJ, MacKenzie AM, Melemis SM, Ventresco J, Condon M (2000) Magnesium oxide augmentation of verapamil maintenance therapy in mania. Psychiatry Res 93:83-7.

Gerstein M, Huleihel M, Mane R, Stilman M, Kashtuzki I. Hallak M, Golan H (2005) Remodelling of hippocampal GABAergic system in adult offspring after maternal hypoxia and magnesium sulphate load: immunohistochemical study. Exp Neurol 196:18-29.

Gobbi G. Janiri L (2006) Sodium and magnesium valproate in vivo modulate glutamatergic and GABAergic synapses in the medial prefrontal cortex. Psychopharmacol 185:255-262. 
Gould TD, Quiroz JA, Singh J, Zarate CA, Manji HK (2004) Emerging experimental therapeutics for bipolar disorder: insights from the molecular and cellular actions of current mood stabilizers. Mol Psychiatry 9:734-55.

Gunther T (2006) Concentration, compartmentation and metabolic function of intracellular free $\mathrm{Mg}^{2+}$. Magnes Res 19:225-36.

Heiden A, Frey R, Presslich O, Blasbichle T, Smetana R, Kasper $S$ (1999) Treatment of severe mania with intravenous magnesium sulphate as a supplementary therapy. Psychiatry Res 3:236-46.

Herzberg L, Herzberg B (1977) Mood change and magnesium. A possible interaction between magnesium and lithium. J New Ment Dis 163:423-26.

Jabotisky-Rubin K, Durst R, Levitin LA (1993) Effects of haloperidol on human plasma magnesium. $J$ Psychiatry Res 27:155-9.

Johnson D, Blandina P, Goldfarb J (1994) Glycine inhibition of glutamate-evoked release of norepinephrine in hypothalamus is strycnineinsensitive. Brain Res 650:70-4.

Kanofsky JD, Sandyk R (1991) Magnesium deficiency in chronic schizophrenia. Int J Neurosci 61:87-90.

Kirkbride JB, Boydell J, Ploubidis GB, Morgan C, Dazzan P, McKenzie K, Murray RM, Jones PB (2008) Testing the association between the incidence of schizophrenia and social capital in an urban area. Psychol Med 38:1083-94.

Kirov GK, Birch NJ, Steadman P, Ramsey RG (1994) Plasma magnesium levels in a population of psychiatric patients: correlation with symptoms. Neuropsychobiology 30:73-8.

Kirov GK, Tsachev KN (1990) Magnesium, schizophrenia and maniac-depressive disease, Neuropsychobiology 23:79-81.

Kornhuber J, Lange KW, Kruzik P, Rausch WD, Gabriel E, Jellinger K, Riederer P (1994) Iron, copper, zinc, magnesium and calcium in post-mortem brain tissue from schizophrenic patients. Biol Psychiatry 36:31-4.

Krebs MO (1995)Glutamatergic hypothesis of schizophrenia: psychoses induced by phencyclidine and cortical-subcortical imbalance. Encephale 21:5818.
Krystal JH, Mathew SJ, D'souza DC. Garakani A, Gunduz-Bruce H, Charney DS (2010) Potential psychiatric applications of metabotropic glutamate receptor agonists and antagonists. CNS Drugs 24:6693.

Layden B, Diven C, Minadeo N, Bryant FB, Mota de Freitas $D(2000) \mathrm{Li}^{+} / \mathrm{Mg}^{2+}$ competition at therapeutic intracellular $\mathrm{Li}^{+}$levels in human neuroblastoma $\mathrm{SH}-$ SY5Y cells. Bipolar Dis 2:2000-4.

Levine J, Rapoport A, Mashiah M, Dolev E (1996) Serum and cerebrospinal levels of calcium and magnesium in acute versus remitted schizophrenic patients. Neuropsychobiology 33:169-172.

Matzen TA, Martin RL (1985) Magnesium deficiency in psychosis induced by cancer chemotherapy. Biol Psychiatry 20:788-94.

McLean MJ, McDonald RL (1986) Sodium valproate but not ethosuximide produces use and voltage dependent limitation of high-frequency repetitive firing of action-potentials of mouse central neurons in cell culture. J Pharmacol Exp Ther 238:727-38.

Moykkyen T, Uusi-Oukari M, Heikkla J, Lovinger DM, Luddens $\mathrm{H}$, Korpi ER (2001) Magnesium potentiation of the function of native and recombinant GABA-A receptors. Neuroreport 12:2175-79.

Murck H (2002) Magnesium and affective disorders, Nutr Neurosci 5:375-89.

Nakazato M, Hashimoto K, Schmidt U, Tchanturia K, Campbell IC, Collier DA, Iyo M, Treasure J (2010) Serum glutamine, set-shifting ability and anorexia nervosa. Ann Gen Psychiatry 9:29-43.

Nechifor M (2007) Magnesium in Psychoses, in New Perspectives in Magnesium Research - Nutrition and Health, (Nishizawa Y, Mori H, Durlach J, eds) SpringerVerlag, London, pp 369-77.

Nechifor M (2008) Interaction between magnesium and psychotropic drugs. Magnes Res 21:97-100.

Nechifor M, Vaideanu C, Mindreci I, Borza C (2006) Variation of magnesium concentrations in psychoses, In: Advances in Magnesium Research-New Data (Porr P J, Nechifor M, Durlach J, eds) John Libbey Eurotext, Paris, pp 25-30.

Nechifor M, Vaideanu C, Palamaru I, Borza C, Mindreci I (2004) The influence of some antipsychotics on erythrocyte magnesium and plasma magnesium, calcium, copper and zinc in patients with paranoid schizophrenia. J Am Coll Nutr 23:5493-519. 
Nechifor M, Vaideanu C, Mandreci I, Palamaru I, Boisteanu P (2005) Research on plasma and erythrocyte concentration of some bivalent cations in patients with bipolar disorders, In (Ermidu-Pollet S., Pollet S. eds.) Proceedings Book of 3rd International Symposium on Trace Elements in Humans - New Perspectives, Athens, pp 150-159.

Nye C, Brice A (2005) Combined vitamin B6magnesium treatment in autism spectrum disorder. Cochrane Database Syst Rev 19:CD003497.

Renn JH, Yang NP, Choup P (2010) Effects of plasma magnesium and prolactin in quantitative ultrasound measurements of heel bone among schizophrenic patients. Muscoloskeletal Disord 1:35-52.

Reynolds GP, Zhang ZJ, Beasley CL (2001) Neurochemical correlates of cortical GABAergic deficits in schizophrenia: selective losses of calcium binding protein immunoreactivity. Brain Res Bull 55:579-84.

Rybakowski J, Szaynerman Z (1974) Lithiummagnesium relationship in red blood cells during lithium prophylaxis. Pharmakopsychiatr Neuropsychopharmacol 9:242-6.

Srinivasan C, Toon J, Amari L, Abukhdeir AM, Hamm H, Geraldes CF, Ho YK, Mota De Freitas D (2004) Competition between lithium and magnesium ions for the G-protein transducin in the guanosine $5^{\prime}$ - diphosphate bound conformation. J Inorg Biochem 98:691-701.

Staley KJ, Soldo BL, Proctor WR (1995) Ionic mechanisms of neuronal excitation by inhibitory $\mathrm{GABA}_{A}$ receptors. Science 269:977-81.

Stambi M, Longini M, Hayek J, Berni S, Macucci F, Scalacci E, Vezzosi P (2006) Magnesium profile in autism. Biol Trace Elem Res 109:97-104.

Takei N, Lewis G, Sham PC, Murray RM (1992) Ageperiod-cohort analysis of the incidence of schizophrenia. Br J Psychiatry 26:963-73.

Vacheron-Trystram MN, Braitman A, Cheref S, Affray L (2004) Antipsychotics in Bipolar disorders. Encephale 30:417-24.

Warden J, Grunze H, Mayer A, Dusing ., Schirrmacher K, Liu Z, Bingmann D. (1993)Calcium antagonistic effects of carbamazepine in epileptics and affective psychoses. Neuropsychobiology 27:171-5.

Wobrock T, Ecker UK, Scheerk H, Schneider-Axmann T, Falkdi P, Gruber O (2009) Cognitive impairment of executive function as a core symptom of schizophrenia. World J Biol Psychiatry 10:442-51. 



\title{
Magnesium and major depression
}

\author{
George A. Eby, ${ }^{1, \bigotimes}$ Karen L. Eby ${ }^{1}$ and Harald Murck $^{2}$ \\ ${ }^{1}$ George Eby Research Institute, 14909-C 2109 Paramount Avenue, Austin, Texas 78704, USA. \\ ${ }^{2}$ Clinic of Psychiatry and Psychotherapy, University of Marburg, 35043 Marburg, Germany. \\ $\square$ george.eby "at" george-eby-research.com
}

\begin{abstract}
The treatment of major depression (MD) is still a major unmet medical need in the majority of patients. Sixty percent of cases of MD are treatment-resistant depression (TRD), showing that classical treatments for MD are poorly effective to non-effective. Magnesium has been largely removed from processed foods, especially refined grains, in the Western world, harming the brain and causing mood disorders. Magnesium deficiency causes N-methyl-D-aspartate (NMDA) coupled calcium channels to be biased towards opening which causes neuronal injury and neurological dysfunction, which we believe results in MD. Oral administration of $\mathrm{Mg}$ to animals produced antidepressant-like effects that were comparable to those of antidepressant drugs. Cerebral spinal fluid (CSF) Mg has been found to be low in suicidal TRD. The first report of $\mathrm{Mg}$ treatment for agitated depression was published in 1921 showing success in 220 out of $\mathbf{2 5 0}$ cases. One $\mathbf{2 0 0 8}$ randomized clinical trial showed that $\mathbf{M g}$ was as effective as the tricyclic antidepressant imipramine in treating MD. Intravenous and oral Mg protocols have been reported to rapidly terminate MD safely and without side effects. Brain $\mathrm{Mg}$ deficiency reduces serotonin levels, and antidepressant drugs have been shown to have the action of raising brain Mg. Excessive calcium, glutamate and aspartate intake can greatly worsen MD. We believe that, when taken together, there is more than sufficient evidence to implicate inadequate dietary $\mathrm{Mg}$ as contributing to the cause of $\mathrm{MD}$, and we suggest that physicians prescribe $\mathrm{Mg}$ for its prevention and treatment.
\end{abstract}

\section{Incidence of major depression}

Neuropsychiatric disorders account for $36 \%$ of all non-communicable illnesses. They are the leading cause of all disability (more than twice that of cardiovascular diseases and malignant neoplasms) in the United States and Canada. Depressive disorders cause $40 \%$ of all neuropsychiatric disorders (World Health Report, 2004). Without magnesium (Mg) treatment, major depression (MD) is expected to affect up to 25 percent of the American population at some point in their lives. Besides core symptoms of depression, i.e. depressed mood and lack of energy, other symptoms may vary, but nevertheless have strong effects on patient quality of life. These include increased or decreased appetite and weight and sleep disturbances, in particular difficulties falling asleep, maintaining sleep and, in melancholic depression, early morning awakening. Emotional reactivity can be very different with increases in sensitivity to social challenges, easy upset, sadness on the one hand and an unresponsive mood, which leaves patients with "feelings of feelinglessness" on the other hand. Feelings of guilt and sadness can be very pronounced, leading to suicidal ideation and finally suicide itself.

\section{Increasing incidence of depression}

Americans are developing MD at higher rates and younger ages than ever before. People born around 1900 rarely had childhood or early adult depression, and only about one percent ever developed depression. People born between 1935 and 1944 had a one percent incidence of depression by age 15 , a 2 percent rate of depression by age 25 , and 9 percent incidence by age 45 . People born in 1955, had a one percent incidence of depression by age 15, a 6 percent incidence by age 25 , and a lifetime incidence of 25 percent (Meyer and Quenzar, 2005).

The onset of depression has greatly increased in incidence, and it is affecting people much earlier in their lives during the late 20th century and early 21st century than before the 20th century (Meyer and Quenzar, 2005). The reasons for 
these findings are not quite clear, but inadequate dietary $\mathrm{Mg}$ is prevalent in America and is the most likely cause. There is probably a real increase in depression, coupled with a change in diagnostic habits, which has to do with the element of stigma. Lower stigma leads to a higher rate of diagnosis. Modern antidepressant drugs are better tolerated than first generation antidepressive treatments that, in terms of a riskbenefit analysis, makes less severely affected subjects candidates for psychopharmacological intervention. Unfortunately, antidepressant and anti-anxiety medications commonly in use have a tendency to promote suicide, especially in young people (Stone, 2009).

\section{Classical depression treatments}

Among those who seek professional help for clinical depression, some patients find relief for their condition using therapies based on modification of monoaminergic systems. Newer generation antidepressants are now used and they include selective serotonin reuptake inhibitors (SSRIs), combined serotonin/noradrenaline reuptake inhibitors, and drugs that interact with monoaminergic receptors such as mirtazapine. First generation antidepressants like monoamine oxidase inhibitors (MAOIs) and tricyclic antidepressants are now rarely used because they are not well tolerated.

\section{Treatment-resistant depression}

A large proportion of the burden caused by depression is attributable to treatment-resistant depression (TRD). Only half of patients who are treated with a first line pharmacological therapy of an SSRI respond and only 30\% experience remission (Trivedi et al., 2006). If a second drug also fails, a commonly used criterion for TRD is fulfilled. Treatment-resistant depression is highly recurrent with as many as $80 \%$ of those requiring multiple treatments relapsing within a year of achieving remission. For those with a more protracted illness, the probability of recovery within 10 years is about $40 \%$. Patients with TRD are more likely to suffer from comorbid physical and mental disorders, to experience marked and protracted functional impairment, and to incur higher medical and mental healthcare costs. Thus, in order to reduce the substantial burden caused by depression, TRD is a central focus of medical research (Fekadu et al., 2009). Apart from the complete non-responders there are many patients who fulfil criteria of "remission" but still have symptoms severe enough to interfere with their quality of life, i.e. residual symptoms (Nierenberg et al., 2010). Besides quality of life implications of residual symptoms, their presence predicts relapse of depressive disorders. This shows that even in the group of remitted subjects an unmet medical need remains.

\section{Markers and risk factors of major depression}

Major depression is a disorder that has both psychological and physiological causes and risk factors. Because psychological causes may trigger pathophysiologic mechanisms, there is overlap. In this article we are focusing primarily on the physiology of MD with some reference of MD as a stress-related disorder. We also focus on systems that may be related to $\mathrm{Mg}$-dependent regulation.

\section{Biological markers of depression or depression- vulnerability}

There are certain biomarkers which have been described in patients with depression and in "high risk" populations; meaning those who never have had MD, but who have a close relative that does (Lauer et al., 1995; Modell et al., 1998). These biomarkers include an increased stress hormone axis (HPA-axis), a higher density of rapid eye movements in sleep EEGs and less slow-wave sleep (SWS), particularly in the first sleep cycle (Krieg et al., 2001). Lower SWS and higher HPA axis activity were also identified as risk factors for a relapse in patients, who were in remission after a depressive episode (Hatzinger et al., 2004). Not all studies confirm these points, in particular with regard to HPA axis findings (Ising et al., 2005). This suggests biological variability in subjects with a risk for depression.

A more recently identified potential marker for depression is aldosterone, which is increased in patients with depression (Emanuele et al., 2005; Murck et al., 2003). This may be of importance, as aldosterone is a primary regulator of $\mathrm{Mg}$. As both aldosterone and cortisol are regulated by ACTH there could be a correlation between the two phenomena (Murck, 2002). 
Typical sleep disturbances occur in MD including falling asleep, staying asleep and early morning awakening, or hypersomnia and excessive napping. Sleep EEG patterns have been identified for MD. Rapid eye movement (REM) sleep changes occur with a shorter latency to the first occurrence of REM, a higher number of eye movements during REM sleep, i.e. a higher REM density, and in some subjects reduced duration of slow wave sleep (Steiger and Kimura, 2010; Thase et al., 1997). Subjective reports of sleep disturbances may also be predictive of a depressive episode. A review of eight epidemiological studies concluded that insomnia, lasting for a period of 2 weeks, was predictive for development of depression in the subsequent 1 3 years (Riemann and Voderholzer, 2003). Insomnia at baseline and 1 year post-baseline compared with those with no sleep complaints was strongly predictive of a future MD (adjusted odds ratio, $\mathrm{OR}=39.8 ; \mathrm{P}<0.01$ ) (Ford and Kamerow, 1989). Another study (van den Berg et al., 2007) found a similar association for hypersomnia. Sleep disturbances and neuroendocrine data are correlated, in particular hypercortisolism is correlated with the number of awakenings and reduced SWS (Hubain et al., 1998).

Several cardiovascular factors, especially low systolic blood pressure, are risk factors for MD (Gilmore et al., 1995; Paterniti et al., 2000). Subjects with MD show lower heart rate variability (HRV) (Udupa et al., 2007). Insomnia is related to a lower HRV (Spiegelhalder et al., 2010). Heart rate variability appears to increase with successful treatment. Reduced HRV is a risk factor for depression in patients with coronary heart disease (Pizzi et al., 2008).

Inflammatory markers are an additional area of potentially useful predictive markers for depression. Plasma interleukin 6 (IL-6), TNF $\alpha$ and prostaglandin E2 (PDE2) are of relevance (Murck et al., 2004; Zorrilla et al., 2001). Elevated inflammatory markers are related to nonresponse to the most frequently used antidepressants today, i.e. SSRIs (Eller et al., 2008; O'Brien et al., 2007). Anti-inflammatory agents appear to have anti-depressant effects, as in the case of TNFa-antagonists (Uguz et al., 2009), cyclooxygenase inhibition (Muller et al., 2006) and omega-3 fatty acids (Murck et al., 2004).

\section{Changes in dietary magnesium}

Against the widespread belief that Western countries have the best-fed people on planet Earth, there is evidence that serious deficits in dietary Mg in the Western world are related to an increased risk for MD and biological markers of depression. For a long time it was not accepted that food could have any influence on brain structure or its function including cognitive, mood and intellectual development. It is now absolutely certain that $\mathrm{Mg}$ plays vital roles in all major metabolisms in oxidation-reduction and in ionic regulation, among its other roles in the brain (Bourre, 2006) and in mood disorders Dietary magnesium intake has steadily declined over the preceding century, due to: a) the practice of refining grain to make processed foods; b) making dietary choices low in magnesium-rich foods such as whole grains, rice and wheat bran, nuts, seeds, chocolate, peanuts, peanut butter and green leafy vegetables; c) strong chemical sequestration of metals during food processing; and d) complete removal of minerals from drinking water processed by distillation, de-salination and reverse osmosis so prevalent in the Middle East, Australia and elsewhere. Only $16 \%$ of the original $\mathrm{Mg}$ and $24 \%$ of the original zinc found in whole wheat remain in refined wheat (Anonymous, 2002). These circumstances reduced average bioavailable $\mathrm{Mg}$ consumption from $450 \mathrm{mg}$ in the 19th century and before, to $250 \mathrm{mg}$ per day or less in the 20th and early 21st centuries, resulting in significant and unhealthy $\mathrm{Mg}$ deficiency in the majority of the population (Seelig and Rosanoff, 2003). Approximately $68 \%$ of U.S. adults consume less than the U.S. recommended daily allowance (RDA) of Mg (420 mg / day for men, $320 \mathrm{mg} /$ day for women), with $19 \%$ consuming less than half of the RDA (Jacka et al., 2009). Deficiency of dietary $\mathrm{Mg}$ has been related to depressive disorders (Rasmussen et al., 1989; Hashizume and Mori, 1990). The pathological signs of Mgdeficiency and possible reasons for its development have been reviewed earlier (Morris, 1992; Durlach, 1984; Durlach et al., 1995; Durlach et al., 1997; Durlach, 2002). A recent study confirmed the epidemiological relationship between low dietary $\mathrm{Mg}$ intake and the risk of developing depression (Jacka et al., 2009). Conversely, extensive marketing and resultant consumption of excitotoxic glutamates and 
aspartates have very greatly increased over the same period, even though there is a strong connection between dietary and endogenous excitotoxin excess and neurological dysfunction and mental illness (Blaylock, 1999; Walton et al., 1993).

\section{Regulation of brain magnesium}

The concentration of $\mathrm{Mg}$ within the brain results from a highly regulated process. Its concentration in cerebrospinal fluid (CSF) is higher than that in plasma, pointing to the existence of active transport systems between these compartments (Morris, 1992). Magnesium moves out of CSF into blood by passive diffusion and bulk filtration (Oppelt et al., 1963). These dynamics result in relative stability of intracerebral $\mathrm{Mg}$ concentration even in case of Mg depletion. Up to 40 days of $\mathrm{Mg}$ depletion led only to a small global intracerebral decrease in $\mathrm{Mg}$ concentration in rats, however important regional differences existed (Poenaru et al., 1997). The brainstem seemed particularly vulnerable to $\mathrm{Mg}$ depletion. Increasing $\mathrm{Mg}$ administration had no strong influence on CSF Mg concentrations. Only small changes in CSF $\mathrm{Mg}$ after prolonged supplementation were reported in dogs (Kemeny et al., 1961; Schain, 1964). However, chronic dietary Mg deficits led to proportional changes in CSF and brain cellular Mg (Chutkow, 1974).

It is of importance to note that metabolism of $\mathrm{Mg}$ depends on endocrine parameters. Administration of aldosterone led to an enhancement of urinary excretion of $\mathrm{Mg}$ in humans (Horton and Biglieri, 1962). Acute $\beta$-adrenergic stimulation with adrenaline or salbutamol reduced plasma $\mathrm{Mg}$ in humans (Whyte et al., 1987), pointing to an adrenergic mechanism of $\mathrm{Mg}$ regulation. The parathyroid is influenced by $\mathrm{Mg}$ in its function, and it regulates $\mathrm{Mg}$ levels and distribution (Zofkova and Kancheva, 1995). Inadequate central nervous system (CNS) concentration of $\mathrm{Mg}^{2+}$ has a critical level below which neurological dysfunction occurs (Yasui et al., 1997; Langley, 1991).

Acute emotional stress, which involves an activation of the SNS and the HPA axis, led to an increase in $\mathrm{Mg}$ excretion in humans (Grases et al., 2006) and experimental animals (Altura et al., 1992), the latter resulting from reduced tissue and serum Mg. Swim stress in animals led to an activation of several stress hormone systems and an increase in $\mathrm{Mg}$ in plasma, but a decrease in brain Mg (Poleszak et al., 2005). This shows that tissue $\mathrm{Mg}$ is more relevant than plasma $\mathrm{Mg}$. Stress induced decrease in brain $\mathrm{Mg}$ could be counteracted with additional administration of the tricyclic antidepressant imipramine. This finding is complicated by observation that coadministration of both imipramine and $\mathrm{Mg}$ has a beneficial behavioural effect, but does not counteract the reduction of brain $\mathrm{Mg}$ concentration resulting from swim stress. Therefore, increased sympathetic nervous system (SNS) activity could lead to increased $\mathrm{Mg}$ excretion. This suggests that stressful activities result in lowered Mg content of the CNS and potentially and increased risk of depression.

Similarly, thyroid function is related to $\mathrm{Mg}$ regulation. In 135 non-medicated patients with mild to moderate major depressive disorder, a significant correlation between mean serum $\mathrm{Mg}^{2+}$ levels and mean circulating T4 thyroid level was observed. There was no significant correlation between any other thyroid indices and either serum $\mathrm{Mg}^{2+}$ or $\mathrm{Ca}^{2+}$. The thyroid axis is known to regulate $\mathrm{Mg}$ metabolism possibly by regulation of transport of $\mathrm{Mg}^{2+}$ from extracellular to intracellular fluid compartments. For T4, and to a lesser extent serum $\mathrm{Mg}^{2+}$, the most consistent changes were observed with response to antidepressant treatment (Joffe et al., 1996).

\section{Changes of serum magnesium in major depression}

Our following review of serum $\mathrm{Mg}$ and depression shows greatly conflicting results, perhaps because $99 \%$ of $\mathrm{Mg}$ is intracellular with only $1 \%$ being found in serum (Mann and Truswell, 2002), and perhaps because some of the work used magnesium oxide, which is not bioavailable (Firoz and Graber, 2001; Lindberg et al., 1990; Walker et al., 2003; Enya et al., 2004).

In patients with low serum $\mathrm{Mg}$, depressive symptoms have been observed (Hashizume and Mori, 1990). Deficits of $\mathrm{Mg}$ may result from inadequate intake, malabsorption, or renal loss of $\mathrm{Mg}$ that occurs in certain disease states such as alcoholism and diabetes, and with certain drug therapies (antidiuretics, aminoglycosides, fluoro- 
quinolones, cisplatin, digoxin, cyclosporin, amphotericin B) (Morris, 1992). Resulting Mgdeficiencies may lead to depressive symptoms which are secondary to an underlying somatic disease, like diabetes mellitus (BarraganRodriguez et al., 2007). In depressed patients, an early study reported a decrease in total plasma $\mathrm{Mg}$, but not in ionized $\mathrm{Mg}$, with an increase in total $\mathrm{Mg}$ occurring after treatment with electroconvulsive therapy or tryptophan (Frizel et al., 1969). Higher serum (Bjorum, 1972) and plasma $\mathrm{Mg}$ levels have been reported in patients with recurrent depression independent from their actual state, i.e. as a trait marker (Cade, 1964; Widmer et al., 1992). Widmer stressed the importance of gender and clinical subtypes of depressive disorder. They also reported an association between increases in plasma $\mathrm{Mg}$ and severity of depression and of plasma cortisol (Widmer et al., 1995). Another group reported that depressive symptoms were positively correlated with serum $\mathrm{Mg}$ in longstanding depression and remission, but not in acute depression (Linder et al., 1989). A further study shows the same relation in patients with unipolar depression (Hasey et al., 1993). In other studies, this finding could not be confirmed (Naylor et al., 1972; George et al., 1994). A further study compared serum $\mathrm{Mg}$ and serum $\mathrm{Ca} / \mathrm{Mg}$ ratio of 145 drug free patients with MD. They compared MD patients to a group of non-mood disordered patients without finding any difference between groups (Young et al., 1996). In another study no changes in absolute values of plasma $\mathrm{Mg}$ concentration could be observed in patients with a variety of psychiatric disorders. An association between the amount of $\mathrm{Mg}$ plasma disturbance (either higher or lower than normal) and severity of clinical disturbance was shown (Kirov et al., 1994). Research subjects, who did not meet criteria of any specific psychiatric disorder, were studied to explore their experiences with depression, anxiety and stress in relation to $\mathrm{Mg}$ levels. Subjects were not differentiated on the basis of high vs low $\mathrm{Ca} / \mathrm{Mg}$ ratios, but on the basis of $\mathrm{Mg}$ tertiles. The group in the middle tertile had the lowest levels of depression and stress in comparison to upper and lower tertiles (Jung et al., 2010). A more recent study found a decreased serum $\mathrm{Mg}$ concentration in patients with more severe depression (Hamilton depression score $>23$ ), whereas no change was observed for less severely affected subjects
(Nechifor, 2009). The studies of Kirov et al., (1994) and Jung et al., (2010) could point to the fact that closer specification of depressive disorders may be required. Gender differences (with lower concentrations in women) exist in plasma Mg concentrations in patients with depression (Herzberg and Herzeberg, 1977; Herzberg and Bold, 1972; George et al., 1994), possibly contributing to the variability seen in the mentioned studies. Furthermore, it appears that medication should be controlled, as antidepressant compounds have been reported to influence $\mathrm{Mg}$ metabolism, as measured as changes in erythrocyte $\mathrm{Mg}$ concentration in the course of antidepressant treatment (Nechifor, 2009). It is unclear if this is a direct effect of the compounds (sertraline and amitriptyline) or an indirect effect of clinical improvement of subjects.

In patients with depression, an increase in aldosterone concentration has been observed (Murck et al., 2002a). Under physiologic conditions an increase in aldosterone concentration should lead to an increased excretion of $\mathrm{Mg}$. Reports of an increase in serum $\mathrm{Mg}$ points to a disturbance in $\mathrm{Mg}$ regulation in these patients, which may also involve a higher grade of $\mathrm{Mg}$ mobilization from intracellular pools. On the other hand a decreased sensitivity of mineralocorticoid receptors (MR), which are the primary responsible receptor for aldosterone regulation in the kidney, has been reported. This points to a potentially complex $\mathrm{Mg}$ dysregulation. It would be of importance to determine the relationship between $\mathrm{Mg}$ and aldosterone concentration in order to clarify this issue.

In contrast to peripheral changes in plasma or serum, changes within the CNS point to a brain $\mathrm{Mg}$ deficiency. In subjects with MD, a decrease in CSF Mg concentration has been confirmed (Banki et al., 1986; Banki et al., 1985). A more recent study found an increase in the $\mathrm{Ca} / \mathrm{Mg}$ ratio of CSF in patients with depression (Levine et al., 1999). Most recently, post-mortem studies in patients that had depression showed a reduced $\mathrm{Mg}$ concentration in brain tissue (Nowak et al., 2010). Human brain Mg measurements by phosphorus nuclear magnetic resonance spectroscopy (NMR) have demonstrated a reduced $\mathrm{Mg}$ concentration in depressed patients who were refractory to treatment with an SSRI (losifescu et al., 2005). 
This method provides the best means to determine $\mathrm{Mg}$ concentrations in specific anatomical areas in living brains.

\section{Functional impact of magnesium}

An examination of the role of $\mathrm{Mg}$ in neurons provides some insight into the cause and possible treatment of MD. Weston in 1921 reported that Meltzer and Auer first showed in 1905 that the primary effect of Mg treatment upon nerve cells was that of paralysis without any preceding excitation, and the effect seemed to be exclusively of an inhibitory character (Weston, 1921-1922).

\section{Monoaminergic systems and magnesium}

The locus coeruleus (LC) is the principal anatomical structure of the noradrenergic system. Its activity is related to that of the SNS, and it shows overactivity in melancholic depression. Magnesium infusion led to an increase in circulating noradrenaline (NA) in humans in one study (Leppert et al., 1994), whereas no changes in catecholamines were reported in another (Zofkova et al., 1993). Magnesium deficiency on the other hand increased plasma catecholamines in response to noise stress in rats (Caddell et al., 1986). Mice selectively bred for low blood $\mathrm{Mg}$ levels showed a significantly higher brain NA content without a change in dopamine, HVA, and serotonin compared to those with high Mg levels (Amyard et al., 1995). This was interpreted as a higher sensitivity to stress in mice with low blood $\mathrm{Mg}$ content. A suppressive effect of $\mathrm{Mg}$ on LC activity has been demonstrated (Shiekhattar and AstonJones, 1992) and interpreted as an NMDA antagonistic mechanism. Magnesium deficiency reduced behavioural activity from dopaminergic activation by amphetamine and apomorphine (Kantak, 1988). The antidepressant-like effects of $\mathrm{Mg}$ are dependent on its interaction with serotonergic, noradrenergic and dopaminergic receptors (Cardoso et al., 2009).

\section{Glutamatergic system and magnesium}

Magnesium depletion is specifically deleterious to neurons by causing NMDA-coupled calcium channels to be biased towards opening (Sapolsky, 1992). At normal neuronal resting membrane potentials, pores of the NMDA glutamate-gated ion channels are blocked by $\mathrm{Mg}^{2+}$ ions (Bear et al., 2001; Mark et al., 2001; Kandel et al., 1995; McMenimen, 2006). The ion channel of the NMDA receptor complex is subject to voltagedependent regulation by $\mathrm{Mg}^{2+}$ ions (Mark et al., 2001; Alberts et al., 2002; Decollogne et al., 1997). Importantly, different types of NMDA receptors exist, on the basis of their constitution subunits. Besides NR1 subunits, which are constitutional for all subtypes, NR2 elements define their diversity. NR2 exists in flavours of NR2A, NR2B, NR2C and NR2D. Of particular interest, NMDA receptors which contain NR2A and NR2B receptors, are more sensitive to voltage sensitive Mg block (Kuner and Schoepfer, 1996). This direct interaction of $\mathrm{Mg}$ with the NMDA receptor intracellular mechanism influences its activity. In hippocampal synaptosomes, the Mg block of the NMDA dependent ion channel is removed by activation of protein kinase $C$ (PKC) without changing membrane potential (Pittaluga et al., 2000). Intracellular administration of a PKC agonist accordingly potentiated NMDA receptor function in cultured hippocampal neurons (Xiong et al., 1998). This could lead to a feed-forward cycle; an NMDA dependent $\mathrm{Ca}^{2+}$ current may increase PKC activity leading to further release of the $\mathrm{Mg}$-block of the NMDA dependent ion-current. Mg also has a direct influence on PKC function. The catalytic subunit of PKC requires $\mathrm{Mg}$ as a cofactor (Hannun and Bell, 1990), and deactivation of PKC by adenosine triphosphate (ATP) depends on the presence of $\mathrm{Mg}$ (Wolf et al., 1985). Besides influencing NMDAergic neurotransmission, $\mathrm{Mg}$ depletion affects hippocampal excitability via non-NMDAergic Ca currents, which can also be suppressed by verapamil, a Ca-channel blocker (Pohl et al., 1992; Walden et al., 1992). These findings, when put together, illustrate several possible mechanisms for $\mathrm{Mg}$ to limit the kindling state via modulation of different elements of the Ca-PKC-second messenger system.

Normally operating NMDA receptors admit into neurons only the amount of $\mathrm{Ca}^{2+}$ that is vital to their function, but abnormally functioning NMDA receptors (due to inadequate $\mathrm{Mg}$ ) increase influx of cellular $\mathrm{Ca}^{2+}$ beyond manageable levels leading to the generation of toxic reactive oxygen species and of toxic amounts of nitric oxide (NO) radicals (Blaylock, 1999; Mark, 2001; Carafoli, 2005). 


\section{Stress hormone systems (HPA and RAAS) and magnesium}

Acute intravenous $\mathrm{Mg}$ administration decreased adrenocorticotrophic hormone (ACTH) secretion in healthy subjects (Murck and Steiger, 1998), but did not lead to a change in cortisol secretion (Zofkova et al., 1993; Murck and Steiger, 1998). Subchronic oral administration of $\mathrm{Mg}$ in healthy elderly subjects led to a decrease in cortisol concentration without a change in ACTH (Held et al., 2002). Chronic oral administration of highdose $\mathrm{Mg}$ leads to considerable reductions in cortisol and lowered stress responsiveness. Both effects are in line with a decrease in central corticotropin-releasing hormone release, but differential effects occur at the level of the adrenal cortex. The possibility of an NMDA antagonistic effect of $\mathrm{Mg}$ has been discussed in the context of hippocampal function. Both systems also influence endocrine regulation and hypo-thalamic activity.

\section{Inflammatory system and magnesium}

Magnesium depletion has been studied for its effects on inflammatory markers. Feeding rats Mg-depleted food led to a nearly 3-fold increase in plasma IL-6, a significant increase of fibrinogen and other acute phase proteins and a significant reduction in $\mathrm{Zn}$ (Malpuech-Brugere et al., 2000). $\mathrm{Mg}$ deficiency also amplifies endotoxin-induced lethality in rats, which was correlated with increased TNF $\alpha$ production (Malpuech-Brugere et al., 1999). In rat models, $\mathrm{Mg}$ deficiency led to an increase in thromboxane (TBX2) and prostaglandin E2 (PGE2) (Nigam et al., 1986; Soma et al., 1988). For a review of the correlation between $\mathrm{Mg}$ deficiency and inflammatory changes, see Nielsen et al., (2010). Of considerable importance is that NMDA-ergic mechanisms may activate inflammatory mechanisms (Muller et al., 2009).

\section{Magnesium in animal and human research}

In the following sections we summarize data concerning effects of $\mathrm{Mg}$ in animals and preliminary findings in humans. One important issue to keep in mind is the unresolved question: Does $\mathrm{Mg}$ treat a $\mathrm{Mg}$ deficit or is $\mathrm{Mg}$ to be regarded as a pharmacological agent, i.e. independent of a pre-existing deficit? We will not be able to answer this question on the basis of current data. Nevertheless, in the long run it is important to identify specific characteristics of subjects who may benefit from $\mathrm{Mg}$ treatment. Most subjects having MD treatable with magnesium also have, or have had, other disorders that are also treatable with magnesium (Eby, 1999-2010).

The functional systems described above have been recognized as potential pharmacological targets for treatment of depression. All current commercial antidepressants are based on the hypothesis of a monoamine dysfunction. On the other hand, NMDA receptor antagonists, and in particular those for the NR2B subtype, have demonstrated clinical efficacy (Berman et al., 2000; Preskorn et al., 2008). The HPA axis has long been recognized as a potential target for antidepressants (Flores et al., 2006; Holsboer, 2000; Jahn et al., 2004). More recently, antiinflammatory mechanisms like COX-2 inhibitors (Muller et al., 2006) and TNFa-antagonists (Uguz et al., 2009) have been demonstrated. In the following text we report behavioural effects by $\mathrm{Mg}$ in animal models as well as human clinical data. The specific mechanism of these effects by $\mathrm{Mg}$ has yet to be elucidated.

\section{Animal Models and magnesium}

Animal models have provided insight into roles of stress on Mg status, effects of Mg deficiency, and effects of $\mathrm{Mg}$ treatment in depression.

$\mathrm{Mg}$ is involved in behavioural control in rats. $\mathrm{Mg}$ deficiency led to a reduction in offensive behaviour and an increase in defensive behaviour (Kantak, 1988). Apomorphine- and Lamphetamine mediated behaviour was suppressed by $\mathrm{Mg}$ deficiency, suggesting that $\mathrm{Mg}$ is an important factor for the stimulatory action of catecholamines, possibly at the postsynaptic site. In the forced swim test, a screening test for possible antidepressive potency of substances, $\mathrm{Mg}$ reduced immobility similar to the noradrenaline reuptake inhibitor imipramine (Decollogne et al., 1997). Compared to control mice fed a normal diet, mice receiving a low $\mathrm{Mg}$ diet ( $10 \%$ of daily requirement) for several weeks displayed increased immobility time in the forced swim test, indicating enhanced depression-like behaviour. In addition, partial magnesium depletion feeds increased anxiety-related 
behaviour in the light/dark and open field test, while locomotor activity and motor coordination was not influenced. Magnesium depletion led to depression and anxiety-related behaviour in mice. These changes due to $\mathrm{Mg}$ deficiency were prevented by coadministration of desipramine or hypericum extract (Singewald et al., 2004). A dependency of intracellular $\mathrm{Mg}$ and behavioural regulation appears to exist. Mice selected for low vs high erythrocyte Mg levels showed pronounced neurobiological differences (Henrotte et al., 1997). Low $\mathrm{Mg}$ mice showed a more restless behaviour, a more aggressive behaviour under stressful conditions and higher brain and urine NA levels compared to mice with high erythrocyte $\mathrm{Mg}$. It is not clear if a correlation between intraerythrocyte and intraneuronal $\mathrm{Mg}$ concentration exists.

Of importance is that $\mathrm{Mg}$ deficiency leads to depression-like changes and that $\mathrm{Mg}$ administration has "therapeutic" effects on depression-like behaviour. Magnesium administration led to anxiolytic effects in the elevated plus maze, and antidepressant like effects in the forced swim test (Poleszak et al., 2004). In stressed rats, $\mathrm{Mg}$ and imipramine acted synergistically on immobility time as measured in the forced swim test, i.e. non-efficacious doses of both compounds combined led to a positive behavioural response (Poleszak et al., 2006). The mechanism is complex and appears to involve NMDAergic (Poleszak et al., 2008), GABAergic (Poleszak, 2008) and serotoninergic (Poleszak, 2007) modulation.

\section{Human studies and magnesium}

There are a number of open label studies on therapeutic effects of $\mathrm{Mg}$ administration in treating depression, depression-like and anxiety illnesses. As early as 1921 a report on the therapeutic effect of $\mathrm{Mg}$ was published in the first issue of the American Journal of Psychiatry (and immediately forgotten). In 220 out of 250 doses of magnesium sulphate (one or two cc of a 25 or 50 percent solution) given hypodermically to 50 patients having agitated depression, with several having various other agitated states, treatment caused patients to relax and sleep from four to six hours resulting in a $90 \%$ success rate for Mg (Weston, 1921-22). The sedative side effects from giving too much $\mathrm{Mg}$ were quickly and easily reversed by giving similar amounts of calcium chloride given hypodermically.

Later studies showed a beneficial effect of $\mathrm{Mg}$ in treatment of rapid cycling bipolar disorder in ten patients (Chouinard et al., 1990) in an open label study for periods up to 32 weeks. Magnesium was found to produce clinical results at least equivalent to those of lithium in $50 \%$ of patients. The possibility that $\mathrm{Mg}$ could replace or improve efficacy of lithium without side effects as a preventive treatment of manic-depressive illness was suggested.

In tests of magnesium lactate and vitamin B6 (pyridoxine) in treatment of 25 patients with anxiety-depression and epilepsy, the combination exerted positive non-specific influences on the patients' mental state, especially with regard to affective disorders. Effects emerged on day 14 of treatment and achieved a statistically significant level by day 28. Treatment was well tolerated and did not cause any side effects (Kalim et al., 2004).

Further support comes from improvement of a subject with the rare hereditary disorder of Gitelman's syndrome, which leads to a lack of reabsorption of electrolytes including $\mathrm{Mg}$. Intravenous supplement of magnesium sulphate (20 mEq/day dissolved in $100 \mathrm{ml}$ normal saline given over 2 hours each day for 3 days) immediately terminated both depression and paraesthesia, suggesting that hypomagnesemia played a role in clinical manifestations of depression (Enya et al., 2004). Oral magnesium oxide treatment was ineffective and promoted diarrhoea. Magnesium oxide should not be given in treatment of mental illnesses since it is not bioavailable generally and it does not pass the blood-brain barrier.

Mood stabilizing properties of $\mathrm{Mg}$ have been demonstrated in case reports in patients with mania (Pavlinac et al., 1979). In an open study with intravenous magnesium sulphate used as a supplementary therapy to lithium, benzodiazepines, and neuroleptics in mania, marked clinical improvement was observed in the 10 patients included. This was accompanied with a significant reduction in requirement for neuroleptic and benzodiazepine (Heiden et al., 1999). In a further open trial in mania, the effect of a $\mathrm{Mg}$-verapamil combination was compared 
with that of verapamil alone, and the combination was superior for manic symptoms (Giannini et al., 2000).

Four case histories were presented showing rapid recovery (less than 7 days) from MD using 125$300 \mathrm{mg}$ of magnesium (as glycinate and taurinate) with each meal and at bedtime (Eby and Eby, 2006). Related and accompanying mental illnesses in these case histories, which were also benefited, included traumatic brain injury, headache, suicidal ideation, anxiety, irritability, insomnia, cocaine, alcohol and tobacco abuse, hypersensitivity to calcium, shortterm memory loss and IQ loss. Post partum depression (PPD) appeared prevented in several case histories with $\mathrm{Mg}$, wherein the patients had previously had severe PPD without $\mathrm{Mg}$. The possibility that magnesium deficiency is the main cause of most MD and related mental health problems, including IQ loss and addiction, was described as enormously important to public health and was recommended for immediate further study (Eby and Eby, 2006). Magnesium was usually claimed as effective for treatment of depression in general use, by depressives who had read "Depression Treatment: A Cure for Depression using Magnesium?" on the Internet (Eby, 1999-2010).

To date only one randomized, double blind, controlled trial exists concerning effects of $\mathrm{Mg}$ administration on MD. Magnesium chloride $\left(\mathrm{MgCl}_{2}\right)$ was evaluated in the treatment of newly diagnosed depression. Twenty-three elderly patients with type 2 diabetes and hypomagnesemia were enrolled and randomly allocated to receive orally either $50 \mathrm{~mL}$ of a $\mathrm{MgCl}_{2}$ $5 \%$ solution (equivalent to $450 \mathrm{mg}$ of elemental $\mathrm{Mg}$ ) or $50 \mathrm{mg}$ imipramine daily during 12 weeks. Widowhood or divorce in the previous six months, alcoholism, degenerative illnesses of the nervous central system, previous or current treatment with antidepressants, chronic diarrhoea, use of diuretics, and reduced renal function were exclusion criteria. At the end of the treatment period, depression scores were identical between groups. Serum Mg levels were significantly higher in subjects receiving $\mathrm{MgCl}_{2}$ than in subjects receiving imipramine $(p=0.0005)$ (Barragán-Rodríguez et al., 2008). Several studies in indications that involve depressed mood exist. In a double-blind, placebo-controlled trial in chronic fatigue syndrome, a disorder related to atypical depression, weekly intramuscular injections of $2 \mathrm{ml}$ magnesium sulphate (50\%) or placebo for 6 weeks showed significant superiority of $\mathrm{Mg}$ over placebo on energy levels, pain, and emotional reactions as measured by the Nottingham health profile score (Cox et al., 1991).

In another placebo controlled study of premenstrual syndrome, which has depressive elements, effects of oral $\mathrm{Mg}$ for the duration of two menstrual cycles was observed (Facchinetti et al., 1991). The medication consisted of $3 \times$ $360 \mathrm{mg} \mathrm{Mg}$ in the form of Mg-pyrrolidone carboxylic acid. It was administered from day 15 of the menstrual cycle to onset of menstrual flow. Mg showed a significant superiority to placebo in the total score of the Menstrual Distress Questionnaire score and especially the cluster "negative affect". In the same indication, combination of $200 \mathrm{mg} \mathrm{Mg}$ and $50 \mathrm{mg}$ vitamin B6, but not individual treatments alone, administered daily for the duration of one menstrual cycle in a Latin square design was superior compared to placebo on premenstrual symptoms such as nervous tension, mood swings, irritability and anxiety (De Souza et al., 2000). Another study using a crossover design found only an improvement in symptoms related to fluid retention in women taking $200 \mathrm{mg} \mathrm{Mg}$ or placebo in the second menstrual cycle (Walker et al., 1998).

\section{Adverse effect of calcium in depression}

Decreases in CSF calcium accompanied mood elevation and motor activation in depressed patients. Similarly, decreases in CSF calcium occurred during acute psychotic agitation or mania. Periodic recurrences of such agitated states were accompanied at their onset by transient increases in serum calcium and phosphorus. Several observations suggested that such serum ion shifts triggered the more enduring and opposite shifts in CSF calcium and, in turn, manic behaviour. Progressive restriction of dietary calcium was earlier reported to mitigate and finally abolish both rhythmic rises in serum calcium and periodic agitated episodes. A modest oral calcium lactate supplement (approximately one additional U.S. Recommended Daily Allowance) intensified 
agitation and worsened depression. In manic patients, symptomatology grew significantly and substantially worse during 2 to 6 weeks of oral vitamin D administration.

On the other hand, in 12 patients, subcutaneous injections of synthetic salmon calcitonin decreased serum calcium and phosphorus, increased cerebrospinal fluid calcium, and augmented depressive symptomatology. Salmon calcitonin, which lowers blood calcium levels, also decreased quantified motor activity, frequency and severity of periodic agitated episodes, serum creatine phosphokinase and prolactin, and nocturnal sleep, while vitamin $D$ or calcium lactate raised them (Carman and Wyatt, 1979). A dietary supplement of $500 \mathrm{mg}$ calcium immediately and severely worsened MD, which was extinguishable within one hour by orally treating with $400 \mathrm{mg}$ gel capsules of $\mathrm{Mg}$ as magnesium glycinate (Eby and Eby, 2006).

In a study of electrolytes in CSF from depressives, a positive correlation was found between calcium concentration and symptom severity in hospitalized depressed patients. CSF calcium levels tended to decrease as patients improved. In rapidly cycling patients, CSF calcium was higher during depression than during mania (Jimersom et al., 1979).

Major depression was found to be accompanied by hyperactivity of subcellular calcium signalling, and any means of reducing pathological neuronal calcium ion flow to reduce pathological nitric oxide/NMDA neuronal output would have antidepressant effects (Paul, 2001). Hypercalcemia from excessive vitamin D intake, hyperparathyroidism or other causes has been suggested to lead to clinical depression by lowering brain Mg (Keddie, 1987).

\section{Biomarkers of magnesium and magnesium- related functions}

Although there is considerable interest in understanding roles of $\mathrm{Mg}$ in brain chemistry and explanation of its role there, we found little consensus as to the value of $\mathrm{Mg}$ measurements in blood (serum, plasma or cellular) as a diagnostic tool useful in treating depression. CSF concentrations of $\mathrm{Mg}$ in depression were also conflicted, although there appeared agreement that CSF $\mathrm{Mg}^{2+}$ values were low only in severe depression associated with suicide attempts, supporting the notion that $\mathrm{Mg}$ would be useful specifically for TRD, which is often much more severe than treatable depression.

Since $99 \%$ of $\mathrm{Mg}$ is intracellular, serum $\mathrm{Mg}$ testing of the remaining $1 \%$ in serum produces misleading results (Mann and Truswell, 2002). Although serum values less than $0.9 \mathrm{mmol} / \mathrm{Mg}$ demonstrate $\mathrm{Mg}$ deficiency in non-depressives (Liebscher and Liebscher, 2004), it is often normal or elevated in depression. Consequently, serum tests are of negative utility due to their misleading nature, and red blood cell tests are also of questionable, perhaps negative, value. Although various tests for $\mathrm{Mg}$ deficiency and calcium excess appear desirable, the response to $\mathrm{Mg}$ treatment with calcium reduction is always the deciding criteria.

Brain compartments of $\mathrm{Mg}$ are isolated from blood and bone compartments requiring different analytical means. Measuring brain $\mathrm{Mg}$ can be safely accomplished in humans using phosphorus NMR spectroscopy (losifescu et al., 2008; lotti and Maulcelli, 2008). This test needs to be validated by repeated trials as a reliable means to determine low brain $\mathrm{Mg}$ in diagnosis of low brain $\mathrm{Mg}$ as cause of MD.

A health warning is therefore warranted regarding potential misinterpretation of "normal" serum $\mathrm{Mg}$. In case of doubt, restoration of $\mathrm{Mg}$ stores in deficient patients is simple, tolerable, and inexpensive and can be clinically beneficial (Ismail et al., 2010).

\section{Recommendations concerning magnesium- supplementation}

Dietary fibres affect intestinal absorption of $\mathrm{Mg}$. Inulin, a prebiotic that supports bifidobacteria and lactobacilli, has been confirmed to be important in absorption of $\mathrm{Mg}$ in the large intestines (Scholz-Ahrens and Schrezenmeir, 2007; Rondón et al., 2008) nearly doubling $\mathrm{Mg}$ absorption and reducing tendency to diarrhoea, but increasing gas, while taking $\mathrm{Mg}$. Large doses of psyllium seed husks greatly reduced absorption of Mg (Asvarujanon et al., 2004) and gum arabic significantly increased both intestinal and renal excretion of Mg (Nasir et al., 2008), and 
both should be avoided. Indole-3-carbinol (I3C) (200 mg/meal) was found in a case study to greatly reduce the necessity for frequent $\mathrm{Mg}$ treatment to prevent relapse (Eby and Eby, 2006).

Useful Mg compounds are those that have a sufficiently low first stability constant to be bioavailable and include magnesium acetate, chloride, citrate, gluconate, glycinate, lactate, malate, oxalate, succinate, sulphate and tartrate (Furia, 1968). The bioavailability of magnesium oxide is extremely limited, ranging from $0-4 \%$ (Firoz and Graber, 2001; Lindberg et al., 1990; Walker et al., 2003; Enya et al., 2004). Studies in which magnesium oxide is used produce misleading results when compared with results obtained using other compounds of $\mathrm{Mg}$ for the same indication.

\section{Future clinical research}

We are concerned that there were only a few clinical trials of $\mathrm{Mg}$ for human depression found. We suggest that future trials be of larger populations in formal, double blind, placebocontrolled clinical trials, or comparative trials against a proven antidepressant such as imipramine $50 \mathrm{mg}$ daily. Only when evidence of efficacy has become clearer are physicians likely to adopt Mg for MD.

From reports reviewed herein, we suggest that in future clinical research in depression that the initial treatment utilize iv drip magnesium sulphate in the manner of Enya et al., (2004), (20 $\mathrm{mEq} /$ day dissolved in $100 \mathrm{ml}$ normal saline given over 2 hours each day for 3 days), to provide an expected rapid ( $<3$ days) initial induction of remission. Treatment is to be followed by an oral bioavailable $\mathrm{Mg}(125-300 \mathrm{mg} \quad 4 \mathrm{t} / \mathrm{d})$ maintenance treatment in the manner of Eby and Eby (2006) or Barragán-Rodríguez (2008) using bioavailable $\mathrm{Mg}$ compounds with emphasis on magnesium glycinate and taurinate. Magnesium oxide must not be used since it is nonbioavailable (Firoz and Graber, 2001; Lindberg et al., 1990; Walker et al., 2003; Enya et al., 2004). During iv administration, cardiac monitoring should be performed. Protocols for iv administration of magnesium sulphate have been widely used for treatment of eclampsia. The antidote to magnesium overdose is calcium gluconate or calcium chloride. Since $99 \%$ of $\mathrm{Mg}$ is intracellular, serum $\mathrm{Mg}$ testing of the remaining $1 \%$ found in serum will produce misleading results (Mann and Truswell, 2002) and it should not be used in future research.

\section{Impediments to success and precautions}

We are concerned that there are some impediments to proper absorption of $\mathrm{Mg}$ in large therapeutic doses by the oral route with intestinal issues, especially inflammatory bowel disease (Galland, 1988) and diarrhoea being primary concerns. Large doses of oral $\mathrm{Mg}$ without calcium may exponentially increase Candida albicans intestinal growth (Holmes et al., 1991), causing or worsening diarrhoea and impairing absorption of $\mathrm{Mg}$, while adding calcium may greatly and immediately worsen depression. Candida albicans overgrowth may be prevented or treated naturally by greatly reducing intake of sugars and treating with large amounts of Bacillus Coagulans probiotic and biotin. We are very concerned that magnesium oxide will be given with failure to respond becoming highly evident, thus damaging the reputation of $\mathrm{Mg}$ as an effective treatment for depression. Do not give magnesium oxide because it has been shown to be not bioavailable. Additionally, magnesium aspartate and magnesium glutamate have neurotoxic ligands and they must be avoided to prevent worsening depression.

\section{Conclusions}

Lack of definitive, large-scale, double-blind, placebo-controlled clinical trials is the limiting factor for making strong treatment recommendations using $\mathrm{Mg}$. Countering that point of view is the good safety of $\mathrm{Mg}$ compared to side-effect prone antidepressant and anti-anxiety medications.

From evidence reviewed here and from the 2010 review by Eby and Eby, the relationship between low $\mathrm{Mg}$ intake as a risk factor for MD appears to have extremely important preventative and treatment implications. A dietary supplement of 600 to $800 \mathrm{mg} /$ day $\mathrm{Mg}$ (other than magnesium oxide) should be a universal prevention strategy.

Mechanisms, which lead to CNS Mg depletion, should be further studied in an effort to discover 
new targets and medication for MD. Evidence for a $\mathrm{Mg}$ regulation effect of psychoptropic drugs already exists, however the exact mechanism is unclear. Reasonable candidates go beyond monoaminergic mechanisms and may include manipulation of the renin-angiotensinaldosterone system and, less studied, specific $\mathrm{Mg}$ transport mechanisms. The best direct measurement of brain $\mathrm{Mg}$ is by phosphorus NMR spectroscopy, but this will probably be reserved for research purposes.

Although more research is clearly needed, we suggest that it is past time to give $\mathrm{Mg}$ in appropriate doses and to reduce intake of calcium, glutamate and aspartate for the

\section{References}

ABC Homeopathy (2010) Magnesia Muriatica. http://www.abchomeopathy.com/r.php/Mag-m/mind (accessed August 15, 2010).

Alberts B, Johnson A, Lewis J, Raff M, Roberts K, Walter P (2002) Molecular Biology of the Cell 4th ed. New York, NY, Garland Science, 615-658.

Altura BM, Altura BT, Gebrewold A, Ising H, Gunther T (1992) Noise-induced hypertension and magnesium in rats: relationship to microcirculation and calcium. $J$ Appl Physiol 72:194-202.

Amyard N, Leyris A, Monier C, Francès H, Boulu RG, Henrotte JG (1995) Brain catecholamines, serotonin and their metabolites in mice selected for low (MGL) and high (MGH) blood magnesium levels. Magnes Res 8:5-9.

Anonymous (2002) Heart gains from whole grains. Harv Heart Lett 13:2-4.

Asvarujanon P, Ishizuka S, Hara H (2004) Inhibitory effects of psyllium on rat mineral absorption were abolished by reduction of viscosity with partial hydrolysis. Biosci Biotechnol Biochem 68:1737-42.

Banki CM, Arato M, Kilts CD (1986) Aminergic studies and cerebrospinal fluid cations in suicide. Annals of the New York Academy of Sciences 487:221-30.

Banki CM, Vojnik M, Papp Z, Balla KZ, Arato M (1985) Cerebrospinal fluid magnesium and calcium related to prevention and treatment of MD, especially TRD, and anxiety, since we can expect rapid improvements in patient health and major reductions in patient expenses without side effects. We did not pay attention to those early $\mathrm{Mg}$ and brain pioneers Meltzer and Auer in 1905 and other magnesium researchers over the last 100 years and we may pay the price today.

\section{Acknowledgements}

We thank the George and Patsy Eby Foundation for financial support. We thank Mikaila Bell for assistance. We have no financial interests or conflicts of interest in any aspect of this research. amine metabolites, diagnosis, and suicide attempts. Biol Psychiatry 20:163-71.

Barragán-Rodríguez L, Rodríguez-Morán M, GuerreroRomero F (2008) Efficacy and safety of oral magnesium supplementation in the treatment of depression in the elderly with type 2 diabetes: a randomized, equivalent trial. Magnes Res 21:218-23.

Bear MF, Connors BW, Paradiso MA (2001) Neurotransmitter Systems In: Neuroscience Exploring the brain, 2nd edn, Baltimore MD, Lippincott Williams \& Wilkins, pp 131-62.

Berman RM, Cappiello A, Anand A, Oren DA, Heninger GR, Charney DS, Krystal JH (2000) Antidepressant effects of ketamine in depressed patients. Biol Psychiatry 47:351-4.

Bjorum N (1972) Electrolytes in blood in endogenous depression. Acta Psychiatr Scand 48:59-68.

Blaylock RL (1999) Food additive excitotoxins and degenerative brain disorders. Medical Sentinel 4:2125.

Bourre JM (2006) Effects of nutrients (in food) on the structure and function of the nervous system: update on dietary requirements for brain Part 1: micronutrients. J Nutr Health Aging 10:377-85.

Caddell J, Kupiecki R, Proxmire DL, Satoh P, Hutchinson $B$ (1986) Plasma catecholamines in acute magnesium deficiency in weanling rats. J Nutr 116:1896-901. 
Cade JF (1964) A significant elevation of plasma magnesium levels in schizophrenia and depressive states. Med J Aust 1:195-6.

Carafoli E (2005) Calcium - a universal carrier of biological signals. FEBS J 272:1073-89.

Cardoso CC, Lobato KR, Binfaré RW, Ferreira PK, Rosa $A O$, Santos AR, Rodrigues AL (2009) Evidence for the involvement of the monoaminergic system in the antidepressant-like effect of magnesium. Prog Neuropsychopharmacol Biol Psychiatry 33:235-42.

Carman JS, Wyatt RJ (1979) Calcium: bivalent cations in the bivalent psychoses. Biol Psychiatry 14:295-336.

Chouinard G, Beauclair L, Geiser R, Etienne P (1990) A pilot study of magnesium aspartate hydrochloride (Magnesiocard) as a mood stabilizer for rapid cycling bipolar affective disorder patients. Prog Neuropsychopharmacol Biol Psychiatry 14:171-80.

Chutkow JG (1974) Metabolism of magnesium in central nervous system. Relationship between concentrations of magnesium in cerebrospinal fluid and brain in magnesium deficiency. Neurology 24:7807.

Cox IM, Campbell MJ, Dowson D (1991) Red blood cell magnesium and chronic fatigue syndrome. Lancet 337(8744):757-60.

Decollogne S, Tomas A, Lecerf C, Adamowicz E, Seman M (1997) NMDA receptor complex blockade by oral administration of magnesium: comparison with MK801. Pharmacol Biochem Behav 58:261-8.

De Souza MC, Walker AF, Robinson PA, Bolland K (2000) A synergistic effect of a daily supplement for 1 month of $200 \mathrm{mg}$ magnesium plus $50 \mathrm{mg}$ vitamin B6 for the relief of anxiety-related premenstrual symptoms: a randomized, double-blind, crossover study. J Womens Health Gend Based Med 9:131-9.

Durlach J and Bac P (1997) Mechanisms of action on the nervous system in magnesium deficiency and dementia. Yasui, M Strong, MJ, Ota, K and Verity MA (eds) In: Mineral and Metal Neurotoxicology, Boca Raton, New York, CRC Press, pp 201-9.

Durlach J, Durlach V (1984) Speculations on hormonal controls of magnesium homeostasis: a hypothesis. Magnesium 3:109-31.

Durlach J, Durlach V, Bara M, Guiet-Bara A (1995) Diverse applications of magnesium therapy In: Berthon G (ed) Handbook of Metal-Ligand Interactions in Biologic Fluids, Bioinorganic Medicine New York, NY, Marcel Dekker, pp 1116-24.

Durlach J, Pagès N, Bac P, Bara M, Guiet-Bara A, Agrapart C (2002) Chronopathological forms of magnesium depletion with hypofunction or with hyperfunction of the biological clock. Magnes Res 15:263-8.

Eby GA (1999-2010) Depression Treatment: A Cure for Depression using Magnesium? http://george-ebyresearch.com/html/depression-anxiety.html (accessed August 15, 2010).

Eby GA, Eby KL (2006) Rapid recovery from major depression using magnesium treatment. Med Hypotheses 67:362-70.

Eby GA, Eby KL (2010) Magnesium for treatmentresistant depression: a review and hypothesis. Med Hypotheses 74:649-60.

Eller T, Vasar V, Shlik J, Maron E (2008) Proinflammatory cytokines and treatment response to escitalopram in major depressive disorder. Prog Neuropsychopharmacol Biol Psychiatry 32:445-50.

Emanuele E, Geroldi D, Minoretti P, Coen E, Politi P (2005) Increased plasma aldosterone in patients with clinical depression. Arch Med Res 36:544-8.

Enya $M$, Kanoh $Y$, Mune $T$, Ishizawa $M$, Sarui $H$, Yamamoto M, Takeda N, Yasuda K, Yasujima M, Tsutaya S, Takeda J (2004) Depressive state and paresthesia dramatically improved by intravenous MgSO4 in Gitelman's syndrome. Intern Med 43:410-4.

Facchinetti F, Borella P, Sances G, Fioroni L, Nappi RE, Genazzani AR (1991) Oral magnesium successfully relieves premenstrual mood changes. Obstet Gynecol 78:177-81.

Fekadu A, Wooderson SC, Markopoulo K, Donaldson C, Papadopoulos A, Cleare AJ (2009) What happens to patients with treatment-resistant depression? A systematic review of medium to long-term outcome studies. J Affect Disord 116(1-2):4-11.

Firoz M, Graber M (2001) Bioavailability of US commercial magnesium preparations. Magnes Res 14:257-62.

Flores BH, Kenna H, Keller J, Solvason HB, Schatzberg AF (2006) Clinical and biological effects of mifepristone treatment for psychotic depression. Neuropsychopharmacology 31:628-36. 
Ford DE, Kamerow DB (1989) Epidemiologic study of sleep disturbances and psychiatric disorders. An opportunity for prevention? JAMA 262:1479-84.

Frizel D, Coppen A, Marks V (1969) Plasma magnesium and calcium in depression. Br J Psychiatry 115:1375-7.

Furia TE. 1968 Sequestrants in Foods - Chapter 7 In: Furia, TA (ed), Handbook of Food Additives Cleveland, $\mathrm{OH}$, The Chemical Rubber Co, pp 289-312.

Galland L (1988) Magnesium and inflammatory bowel disease. Magnesium 7:78-83.

George MS, Rosenstein D, Rubinow DR, Kling MA, Post RM (1994) CSF magnesium in affective disorder: lack of correlation with clinical course of treatment. Psychiatry Res 51:139-46.

Giannini AJ, Nakoneczie AM, Melemis SM, Ventresco J, Condon M (2000) Magnesium augmentation of verapamil maintenance therapy in mania. Psychiatry Res 93:83-7.

Gilmore C, Green BH, Copeland JR, Dewey ME, Davidson IA, Saunders PA, Sharma V (1995) Blood pressure as a risk factor for depression in elderly people: a prospective study. Acta psychiatrica Scandinavica 91:126-9.

Grases G, Pérez-Castelló JA, Sanchis $P$, Casero A, Perelló J, Isern B, Rigo E, Grases F (2006): Anxiety and stress among science students. Study of calcium and magnesium alterations. Magnes Res 19:102-6.

Hannun YA, Bell RM (1990) Rat brain protein kinase C. Kinetic analysis of substrate dependence, allosteric regulation, and autophosphorylation. J Biol Chem 265:2962-72.

Hasey GM, D'Alessandro E, Cooke RG, Warsh JJ (1993) The interface between thyroid activity, magnesium, and depression: a pilot study. Biol Psychiatry 33:133-5.

Hashizume N, Mori M (1990) An analysis of hypermagnesemia and hypomagnesemia. Jpn J Med 29:368-72.

Hatzinger M, Hemmeter UM, Brand S, Ising M, Holsboer-Trachsler E (2004) Electroencephalographic sleep profiles in treatment course and long-term outcome of major depression: association with DEX/CRH-test response. J Psychiatr Res 38:453-65.

Heiden A, Frey R, Presslich O, Blasbichler T, Smetana R, Kasper S (1999) Treatment of severe mania with intravenous magnesium sulphate as a supplementary therapy. J Psychiatry Res 89:239-46.
Held K, Antonijevic IA, Künzel H, Uhr M, Wetter TC, Golly IC, Steiger A, Murck H (2002) Oral Mg( ${ }^{2+}$ ) supplementation reverses age-related neuroendocrine and sleep EEG changes in humans.

Pharmacopsychiatry 35:135-43.

Henrotte JG, Franck G, Santarromana M, Francès H, Mouton D, Motta R (1997) Mice selected for low and high blood magnesium levels: a new model for stress studies. Physiol Behav 61:653-8.

Herzberg L, Bold AM (1972) Sex difference in mean serum-magnesium levels in depression. Lancet 1(7760):1128-9.

Herzberg L, Herzeberg B (1977) Mood change and magnesium. A possible interaction between magnesium and lithium? J Nerv Ment Dis 165:423-6.

Holmes AR, Cannon RD, Shepherd MG (1991) Effect of calcium ion uptake on Candida albicans morphology. FEMS Microbiol Lett 61:187-93.

Holsboer F (2000) The corticosteroid receptor hypothesis of depression. Neuropsychopharmacology 23:477-501.

Hubain PP, Staner L, Dramaix M, Kerkhofs M, Papadimitriou G, Mendlewicz J, Linkowski P (1998) The dexamethasone suppression test and sleep electroencephalogram in nonbipolar major depressed inpatients: a multivariate analysis. Biol Psychiatry 43:220-9.

losifescu DV, Bolo NR, Nierenberg AA, Jensen JE, Fava $M$, Renshaw PF (2008) Brain bioenergetics and response to triiodothyronine augmentation in major depressive disorder. Biol Psychiatry 63:1127-34.

losifescu DV, Nierenberg AA, Mischoulon D, Perlis RH, Papakostas GI, Ryan JL, Alpert JE, Fava M (2005) An open study of triiodothyronine augmentation of selective serotonin reuptake inhibitors in treatmentresistant major depressive disorder. J Clin Psychiatry 66:1038-42.

lotti S, Malucelli E (2008) In vivo assessment of $\mathrm{Mg}^{2+}$ in human brain and skeletal muscle by 31P-MRS. Magnes Res 21:157-62.

Ising M, Lauer CJ, Holsboer F, Modell S (2005) The Munich vulnerability study on affective disorders: premorbid neuroendocrine profile of affected highrisk probands. J Psychiatr Res 39:21-8. 
Ismail Y, Ismail AA, Ismail AA (2010) The underestimated problem of using serum magnesium measurements to exclude magnesium deficiency in adults; a health warning is needed for "normal" results. Clin Chem Lab Med 48:323-7.

Jacka FN, Overland S, Stewart R, Tell GS, Bjelland I, Mykletun A (2009) Association between magnesium intake and depression and anxiety in communitydwelling adults: the Hordaland Health Study. Aust NZ J Psychiatry 43:45-52.

Jahn H, Schick M, Kiefer F, Kellner M, Yassouridis A, Wiedemann K (2004) Metyrapone as additive treatment in major depression: a double-blind and placebo-controlled trial. Arch Gen Psychiatry 61:123544.

Jimerson DC, Post RM, Carman JS, van Kammen DP, Wood JH, Goodwin FK, Bunney WE Jr. (1979) CSF calcium: clinical correlates in affective illness and schizophrenia. Biol Psychiatry 14:37-51.

Joffe RT, Levitt AJ, Young LT (1996) The thyroid, magnesium and calcium in major depression. Biol Psychiatry 40:428-9.

Jung KI, Ock SM, Chung JH, Song CH (2010) Associations of serum $\mathrm{Ca}$ and $\mathrm{Mg}$ levels with mental health in adult women without psychiatric disorders. Biol Trace Elem Res 133:153-61.

Kalinin VV, Zheleznova EV, Rogacheva TA, Sokolova LV, Polianskiĭ DA, Zemlianaia AA, Nazmetdinova DM (2004) [A use of Magne-B6 in the treatment of anxiety-depressive states in patients with epilepsy]. $Z h$ Nevrol Psikhiatr Im S S Korsakova 104:51-5.

Kandel ER, Schwartz JH, Jessell TM (1995) Synaptic integration In: Essentials of neural science and behaviour. Stamford, CT, Appleton \& Lange, pp 21941.

Kantak KM (1988) Magnesium deficiency alters aggressive behaviour and catecholamine function. Behav Neurosci 102:304-11.

Keddie KM (1987) Severe depressive illness in the context of hypervitaminosis D. Br J Psychiatry 150:3946.

Kemeny A, Boldizsar H, Pethes G (1961) The distribution of cations in plasma and cerebrospinal fluid following infusion of solution of salts of sodium, potassium magnesium and calcium. J New Drugs 7:218-27.
Kirov GK, Birch NJ, Steadman P, Ramsey RG (1994)

Plasma magnesium levels in a population of psychiatric patients: correlations with symptoms. Neuropsychobiology 30:73-8.

Krieg JC, Lauer CJ, Schreiber W, Modell S, Holsboer F (2001) Neuroendocrine, polysomnographic and psychometric observations in healthy subjects at high familial risk for affective disorders: the current state of the 'Munich vulnerability study'. J Affect Disord 62:337.

Kuner T, Schoepfer R (1996) Multiple structural elements determine subunit specificity of $\mathrm{Mg}^{2+}$ block in NMDA receptor channels. J Neurosci 16:3549-3558. Langley WF, Mann D (1991) Skeletal buffer function and symptomatic magnesium deficiency. Med Hypotheses 34:62-5.

Lauer CJ, Schreiber W, Holsboer F, Krieg JC (1995) In quest of identifying vulnerability markers for psychiatric disorders by all-night polysomnography. Arch Gen Psychiatry 52:145-53.

Leppert J, Myrdal U, Hedner T, Edvinsson L, Tracz Z, Ringqvist I (1994) Effect of magnesium sulfate infusion on circulating levels of noradrenaline and neuropeptide-Y-like immunoreactivity in patients with primary Raynaud's phenomenon. Angiology 45:63745.

Levine J, Stein D, Rapoport A, Kurtzman L (1999) High serum and cerebrospinal fluid $\mathrm{Ca} / \mathrm{Mg}$ ratio in recently hospitalized acutely depressed patients.

Neuropsychobiology 39:63-70.

Liebscher DH, Liebscher DE (2004) About the misdiagnosis of magnesium deficiency. J Am Coll Nutr 23:730S-1S.

Lindberg JS, Zobitz MM, Poindexter JR, Pak CY (1990) Magnesium bioavailability from magnesium citrate and magnesium oxide. J Am Coll Nutr 9:48-55.

Linder J, Brismar K, Beck-Friis J, Saaf J, Wetterberg L (1989) Calcium and magnesium concentrations in affective disorder: difference between plasma and serum in relation to symptoms. Acta Psychiatr Scand 80:527-37.

Malpuech-Brugère $C$, Nowacki $W$, Daveau $M$, Gueux $E$, Linard C, Rock E, Lebreton J, Mazur A, Rayssiguier Y (2000) Inflammatory response following acute magnesium deficiency in the rat. Biochim Biophys Acta 1501:91-8. 
Malpuech-Brugere C, Nowacki W, Rock E, Gueux E, Mazur A, Rayssiguier Y (1999) Enhanced tumor necrosis factor-alpha production following endotoxin challenge in rats is an early event during magnesium deficiency. Biochim Biophys Acta 1453:35-40.

Mann J, Truswell AS (2002) Essentials of Human Nutrition. 2nd ed. New York, NY, Oxford University Press, p 141.

Mark LP, Prost RW, Ulmer JL, Smith MM, Daniels DL, Strottmann JM, Brown WD Hacein-Bey L (2001) Pictorial review of glutamate excitotoxicity: Fundamental concepts for neuroimaging. Am J Neuroradiol 22:1813-24.

McMenimen KA, Dougherty DA, Lester HA, Petersson EJ (2006) Probing the $\mathrm{Mg}^{2+}$ blockade site of an $\mathrm{N}$ methyl-D-aspartate (NMDA) receptor with unnatural amino acid mutagenesis. ACS Chem Biol 1:227-34.

Meyer JS, Quenzer LF (2005) Psychopharmacology: Drugs the Brain and Behavior. Sunderland MA, Sinauer Associates Inc, pp 163-82.

Morris ME (1992) Brain and CSF magnesium concentrations during magnesium deficit in animals and humans: neurological symptoms. Magnes Res 5:303-13.

Muller N, Myint AM, Schwarz MJ (2009) The impact of neuroimmune dysregulation on neuroprotection and neurotoxicity in psychiatric disorders-relation to drug treatment. Dialogues Clin Neurosci 11:319-32.

Müller N, Schwarz MJ, Dehning S, Douhe A, Cerovecki A, Goldstein-Müller B, Spellmann I, Hetzel G, Maino K, Kleindienst N, Möller HJ, Arolt V, Riedel M (2006) The cyclooxygenase- 2 inhibitor celecoxib has therapeutic effects in major depression: results of a double-blind, randomized, placebo controlled, add-on pilot study to reboxetine. Mol Psychiatry 11:680-4.

Murck H (2002) Magnesium and affective disorders. Nutr Neurosci 5:375-89.

Murck H, Held K, Ziegenbein M, Kunzel H, Koch K, Steiger A (2003) The renin-angiotensin-aldosterone system in patients with depression compared to controls-a sleep endocrine study. BMC Psychiatry 3:15.

Murck H, Song C, Horrobin DF, Uhr M (2004) Ethyleicosapentaenoate and dexamethasone resistance in therapy-refractory depression. Int J Neuropsychopharmacol 7:341-9.

Murck H, Steiger A (1998) $\mathrm{Mg}^{2+}$ reduces ACTH secretion and enhances spindle power without changing delta power during sleep in men -- possible therapeutic implications. Psychopharmacology (Berl) 137:247-52.

Nasir O, Artunc F, Saeed A, Kambal MA, Kalbacher H, Sandulache D, Boini KM, Jahovic N, Lang F (2008) Effects of gum arabic (Acacia senegal) on water and electrolyte balance in healthy mice. J Ren Nutr 18:2308.

Naylor GJ, Fleming LW, Stewart WK, McNamee HB, Le Poidevin D (1972) Plasma magnesium and calcium levels in depressive psychosis. Br J Psychiatry 120:6834.

Nechifor M (2009) Magnesium in major depression. Magnes Res 22:163S-6S.

Nielsen FH (2010) Magnesium, inflammation, and obesity in chronic disease. Nutr Rev 68:333-40.

Nierenberg AA, Husain MM, Trivedi MH, Fava M, Warden D, Wisniewski SR, Miyahara S, Rush AJ (2010) Residual symptoms after remission of major depressive disorder with citalopram and risk of relapse: a STAR*D report. Psychological Medicine 40:41-50.

Nigam S, Averdunk R, Gunther T (1986) Alteration of prostanoid metabolism in rats with magnesium deficiency. Prostaglandins Leukot Med 23:1-10.

Nowak G, Poleszak E, Sowa-Kucma M, Pilc A (2010) Magnesium and Glutamate interaction in depression and antidepressant therapy. Biological Psychiatry 67:195S.

O'Brien SM, Scully P, Fitzgerald P, Scott LV, Dinan TG (2007) Plasma cytokine profiles in depressed patients who fail to respond to selective serotonin reuptake inhibitor therapy. J Psychiatr Res 41:326-31.

Oppelt WW, Maclntyre I, Rall DP (1963) Magnesium exchange between blood and cerebrospinal fluid. Am J Physiol. 205:959-62.

Paterniti S, Verdier-Taillefer $\mathrm{MH}$, Geneste C, Bisserbe JC, Alperovitch A (2000) Low blood pressure and risk of depression in the elderly. A prospective communitybased study. Br J Psychiatry 176:464-467.

Paul IA (2001) Antidepressant activity and calcium signaling cascades. Hum Psychopharmacol 16:71-80. Pavlinac D, Langer R, Lenhard L, Deftos L (1979) Magnesium in affective disorders. Biol Psychiatry 14:657-61. 
Pittaluga A, Bonfanti A, Raiteri M (2000) Somatostatin potentiates NMDA receptor function via activation of InsP(3) receptors and $\mathrm{PKC}$ leading to removal of the $\mathrm{Mg}\left({ }^{2+}\right)$ block without depolarization. Br J Pharmacol 130:557-66.

Pizzi C, Manzoli L, Mancini S, Costa GM (2008) Analysis of potential predictors of depression among coronary heart disease risk factors including heart rate variability, markers of inflammation, and endothelial function. Eur Heart J 29:1110-7.

Poenaru S, Manicom R, Rouhani S, Aymard P, Bajenaru $\mathrm{O}$, Rayssiguier $\mathrm{Y}$, Emmanouillidis E, Gueux E, Nkanga $\mathrm{N}$, Durlach J, Dall'ava J (1997) Stability of brain content of magnesium in experimental hypomagnesemia. Brain Res 769:329-32.

Pohl M, Straub H, Speckmann EJ (1992) Low magnesium-induced epileptiform discharges in guinea pig hippocampal slices: depression by the organic calcium antagonist verapamil. Brain Res 577:29-35.

Poleszak E (2007) Modulation of antidepressant-like activity of magnesium by serotonergic system. $J$ Neural Transm 114:1129-34.

Poleszak E, Szewczyk B, Kedzierska E, Wlaz P, Pil A, Nowak G (2004) Antidepressant- and anxiolytic-like activity of magnesium in mice. Pharmacol Biochem Behav 78:7-12.

Poleszak E, Szewczyk B, Wlaź A, Fidecka S, Wlaź P, Pilc A, Nowak G (2008) D-serine, a selective glycine/Nmethyl-D-aspartate receptor agonist, antagonizes the antidepressant-like effects of magnesium and zinc in mice. Pharmacol Rep 60:996-1000.

Poleszak E, Wlaź P, Kedzierska E, Nieoczym D, Wyska E, Szymura-Oleksiak J, Fidecka S, Radziwoń-Zaleska M, Nowak G (2006) Immobility stress induces depressionlike behaviour in the forced swim test in mice: effect of magnesium and imipramine. Pharmacol Rep 58:746-52.

Poleszak E, Wlaź P, Kedzierska E, Nieoczym D, Wróbel A, Fidecka S, Pilc A, Nowak G (2007) NMDA/glutamate mechanism of antidepressant-like action of magnesium in forced swim test in mice. Pharmacol Biochem Behav 88:158-64.

Poleszak E, Wlaź P, Szewczyk B, Kedzierska E, Wyska E, Librowski T, Szymura-Oleksiak J, Fidecka S, Pilc A, Nowak G (2005) Enhancement of antidepressant-like activity by joint administration of imipramine and magnesium in the forced swim test: Behavioral and pharmacokinetic studies in mice. Pharmacol Biochem Behav 81:524-9.
Preskorn SH, Baker B, Kolluri S, Menniti FS, Krams M, Landen JW (2008) An innovative design to establish proof of concept of the antidepressant effects of the NR2B subunit selective N-methyl-D-aspartate antagonist, $\mathrm{CP}-101,606$, in patients with treatmentrefractory major depressive disorder. J Clin Psychopharmacol 28:631-7.

Rasmussen HH, Mortensen PB, Jensen IW (1989) Depression and magnesium deficiency. Int J Psychiatry Med 19:57-63.

Riemann D, Voderholzer U (2003) Primary insomnia: a risk factor to develop depression? J Affect Disord 76:255-9.

Rondón LJ, Rayssiguier Y, Mazur A (2008) Dietary inulin in mice stimulates $\mathrm{Mg}^{2+}$ absorption and modulates TRPM6 and TRPM7 expression in large intestine and kidney. Magnes Res 21:224-31.

Sapolsky RM (1992) Stress the aging brain and the mechanisms of neuron death. Cambridge, MA, A Bradford Book, The MIT Press, pp 192.

Schain RJ (1964) Cerebrospinal Fluid and Serum Cation Levels. Arch Neurol 11:330-3.

Scholz-Ahrens KE, Schrezenmeir J (2007) Inulin and oligofructose and mineral metabolism: the evidence from animal trials. J Nutr 137(11 Suppl):2513S-23S.

Seelig MS, Rosanoff A (2003) The Magnesium Factor. New York, NY Avery Publishing Group, pp 5.

Shealy CN (1992) The neurochemistry of depression. Amer J Pain Management 2:13-6.

Shiekhattar R, Aston-Jones G (1992) NMDA-receptormediated sensory responses of brain noradrenergic neurons are suppressed by in vivo concentrations of extracellular magnesium. Synapse 10:103-9.

Singewald N, Sinner C, Hetzenauer A, Sartori SB, Murck H (2004) Magnesium-deficient diet alters depression- and anxiety-related behaviour in mice-influence of desipramine and Hypericum perforatum extract. Neuropharmacology 47:1189-97.

Soma M, Cunnane SC, Horrobin DF, Manku MS, Honda M, Hatano M (1988) Effects of low magnesium diet on the vascular prostaglandin and fatty acid metabolism in rats. Prostaglandins 36:431-41.

Spiegelhalder K, Fuchs L, Ladwig J, Kyle SD, Nissen C, Voderholzer U, Feige B, Riemann D (2010) Heart rate and heart rate variability in subjectively reported insomnia. J Sleep Res 20:137-45. 
Steiger A, Kimura M (2010) Wake and sleep EEG provide biomarkers in depression. J Psychiatr Res 44:242-52.

Stone $\mathrm{M}$, Laughren $\mathrm{T}$, Jones $\mathrm{ML}$, Levenson $\mathrm{M}$, Holland PC, Hughes A, Hammad TA, Temple R, Rochester G (2009) Risk of suicidality in clinical trials of antidepressants in adults: analysis of proprietary data submitted to US Food and Drug Administration. BMJ 339:b2880.

Thase ME, Kupfer DJ, Fasiczka AJ, Buysse DJ, Simons $A D$, Frank E (1997) Identifying an abnormal electroencephalographic sleep profile to characterize major depressive disorder. Biol Psychiatry 41:964-73.

Trivedi MH, Rush AJ, Wisniewski SR, Nierenberg AA, Warden D, Ritz L, Norquist G, Howland RH, Lebowitz B, McGrath PJ, Shores-Wilson K, Biggs MM, Balasubramani GK, Fava M; STAR*D Study Team (2006) Evaluation of outcomes with citalopram for depression using measurement-based care in STAR*D: implications for clinical practice. Am J Psychiatry 163:28-40

Udupa K, Sathyaprabha TN, Thirthalli J, Kishore KR, Lavekar GS, Raju TR, Gangadhar BN (2007) Alteration of cardiac autonomic functions in patients with major depression: a study using heart rate variability measures. J Affect Disord 100:137-41.

Uguz F, Akman C, Kucuksarac S, Tufekci O (2009) Antitumor necrosis factor-alpha therapy is associated with less frequent mood and anxiety disorders in patients with rheumatoid arthritis. Psychiatry Clin Neurosci 63:50-5.

van den Berg JF, Tulen JH, Neven AK, Hofman A, Miedema HM, Witteman JC, Tiemeier H (2007) Sleep duration and hypertension are not associated in the elderly. Hypertension 50:585-89.

Walden J, Grunze H, Bingmann D, Liu Z, Düsing R (1992) Calcium antagonistic effects of carbamazepine as a mechanism of action in neuropsychiatric disorders: studies in calcium dependent model epilepsies. Eur Neuropsychopharmacol 2:455-62.

Walker AF, De Souza MC, Vickers MF, Abeyasekera S, Collins ML, Trinca LA (1998) Magnesium supplementation alleviates premenstrual symptoms of fluid retention. J Womens Health 7:1157-65.

Walker AF, Marakis G, Christie S, Byng M (2003) Mg citrate found more bioavailable than other $\mathrm{Mg}$ preparations in a randomised double-blind study. Magnes Res 16:183-91.
Walton RG, Hudak R, Green-Waite RJ (1993) Adverse reactions to aspartame: double-blind challenge in patients from a vulnerable population. Biol Psychiatry 34:13-7.

Weston PG (1921-22) Magnesium as a sedative. Am J Psychiatry 1:637-8.

Whyte KF, Addis GJ, Whitesmith R, Reid JL (1987) Adrenergic control of plasma magnesium in man. Clin Sci (Lond) 72:135-8.

Widmer J, Bovier P, Karege F, Raffin $\mathrm{Y}$, Hilleret $\mathrm{H}$, Gaillard JM, Tissot R (1992) Evolution of blood magnesium, sodium and potassium in depressed patients followed for three months. Neuropsychobiology 26:173-9.

Widmer J, Henrotte JG, Raffin $\mathrm{Y}$, Bovier P, Hilleret $\mathrm{H}$, Gaillard JM (1995) Relationship between erythrocyte magnesium plasma electrolytes and cortisol and intensity of symptoms in major depressed patients. $J$ Affect Disord 34:201-9.

World Health Report 2004: Changing History Annex Table 3: Burden of disease in DALYs by cause, sex and mortality stratum in WHO regions estimates for 2002. Geneva, The World Health Organization.

Xiong ZG, Raouf R, Lu WY, Wang LY, Orser BA, Dudek EM, Browning MD, MacDonald JF (1998) Regulation of $\mathrm{N}$-methyl-D-aspartate receptor function by constitutively active protein kinase C. Mol Pharmacol 54:1055-63.

Young LT, Robb JC, Levitt AJ, Cooke RG, Joffe RT (1996) Serum $\mathrm{Mg}^{2+}$ and $\mathrm{Ca}^{2+} / \mathrm{Mg}^{2+}$ ratio in major depressive disorder. Neuropsychobiology 34:26-8.

Zofkova I, Kancheva RL (1995) The relationship between magnesium and calciotropic hormones. Magnes Res 8:77-84.

Zofková I, Lamberg-Allardt C, Kancheva RL, Stárka L (1993) Effect of hypermagnesemia on the adenohypophyseal-gonadal function, parathyroid hormone secretion and some other hormonal indicators. Horm Metab Res. 25:29-33.

Zorrilla EP, Luborsky L, McKay JR, Rosenthal R, Houldin A, Tax A, McCorkle R, Seligman DA, Schmidt K (2001) The relationship of depression and stressors to immunological assays: a meta-analytic review. Brain Behavior immunity 15:199-226. 


\title{
Magnesium in drug abuse and addiction
}

\author{
Mihai Nechifor $\bowtie$ \\ Department of Pharmacology, "Gr. T. Popa” University of Medicine and Pharmacy, lasi, Romania. \\ \mechif@yahoo.com
}

\begin{abstract}
Addiction to different substances is considered to be a psychiatric disorder. Magnesium reduces the intensity of addiction to opiates and psychostimulants (cocaine, amphetamine, nicotine, and others). It also decreases the auto-administration of cocaine and the relapse into cocaine and amphetamine intake, as well as reducing the experimental addiction to morphine, cocaine and other substances in animals. In heroin addicts, alcohol consumers and other drug abusers, the plasma and intracellular magnesium concentration is lower compared to healthy subjects. We consider that one of the mechanisms by which magnesium reduces the consumption of some highly addictive substances is its moderate effect of stimulating the reward system. However, other main mechanisms involved in magnesium's action are the reduction of dopamine and glutamate release at presynaptic terminals in the brain, the decrease of NO synthase activity, the stimulation of GABAergic system activity, the reduction of postsynaptic NMDA receptor activity, and the reduction of some neuromediators released by $\mathrm{Ca}^{2+}$ and acting at calcium channels. Apart from the action of magnesium ions during emerging addiction, administration of this cation after the appearance of withdrawal syndrome reduces the intensity of the clinical symptoms. There are data that show that stress increases the vulnerability of people to develop addiction to different substances, and also reduces drug-free time and increases the incidence of relapse in heroin addicts. Stress increases catecholamine release and stimulates magnesium release from the body. This decrease in magnesium concentration is one of the important factors that hastens relapse.
\end{abstract}

\section{Introduction}

Drug dependence is today considered a chronic medical illness (Kosten, 1998) producing significant changes in the biochemistry and function of the brain (McLellan et al., 2000). Koob and Le Moal (2001) presented the neurobiology of addiction from the perspective of allostasis, whereby addiction is considered a cycle of progressively increasing dysregulation of reward systems, producing compulsive use of drugs with the loss of control over drug-taking.

There is a large group of substances that result in more or less intense addiction, which is characterized by three major features: compulsive use (intake), craving, and withdrawal syndrome (when administration is stopped). The number of substances for which a more or less intense dependence was signalled is relatively high and is growing continuously. These include the opiates (morphine, heroin, etc.), the psychodysleptics (LSD), alcohol, cannabinoids and psychostimulants.
The main brain structures involved in development of drug dependence are the nucleus accumbens, ventral tegmentum, the periductal grey substance, the mesolimbic system, and the nucleus coeruleus. Dysfunction in the mesolimbic system, nucleus accumbens, prefrontal cortex, and ventral tegmental area are considered involved in the mechanism of drug abuse disorders (Miguel- Hidalgo, 2009).

The molecular mechanisms involved in addiction are complicated but the main chemical neuromediators involved are dopamine, glutamate, serotonin, endogenous opioid peptides, nitric oxide (NO) and others. The glutamatergic, dopaminergic and opioid mechanisms are considered the most involved (Grass and Olive, 2007). These mechanisms not only involve the neurons but also the neuroglia. There are many factors that can influence the intensity of addiction or withdrawal syndrome symptoms. Amongst them are magnesium and other bivalent cations (Ruiz Martinez et al., 1990). 


\section{Opiates}

Opiates are the cause of one of the most powerful and frequent addictions, with heroin intake, especially, resulting in unique medical and social problems. There are data that show that magnesium decreases the intensity of opioid addiction, with administration of $\mathrm{Mg}$ acetate (0.5 $\mathrm{mEq} / \mathrm{kg} /$ day) reducing the experimental physical dependence (Nechifor et al., 2004b). The intensity of symptoms in naloxone-induced withdrawal syndrome reduced, even when magnesium administration was stopped during the period of withdrawal syndrome. Administration of $\mathrm{Mg}$ aspartate $(732 \mathrm{mg} /$ day) for 12 weeks in heroin-addictive patients was also beneficial (Daini et al., 2006; Karakiewicz et al., 2007). Magnesium can potentially reduce the intensity of addiction through a number of mechanisms (Figure 1), including:
- decreasing dopamine synthesis and presynaptic release in brain;

- decreasing the activity of glutamate NMDA receptors;

- decreasing the activity of brain NOS and NO synthesis;

- modulating the opioid coupling at brain $\mu$ receptors;

- increasing glutamate metabolism (as the main excitatory amino acid involved in addiction) by enhancing glutamate decarboxylase activity;

- increasing GABAergic activity in some brain areas by increasing the vesicular GABA transporter synthesis (Gerstein et al., 2005).

Magnesium also potentiates the function of $\mathrm{GABA}_{\mathrm{A}}$ receptors suggesting a putative $\mathrm{Mg}^{2+}$ binding site on the $G A B A_{A}$ receptor protein (Möykkynen et al., 2001).

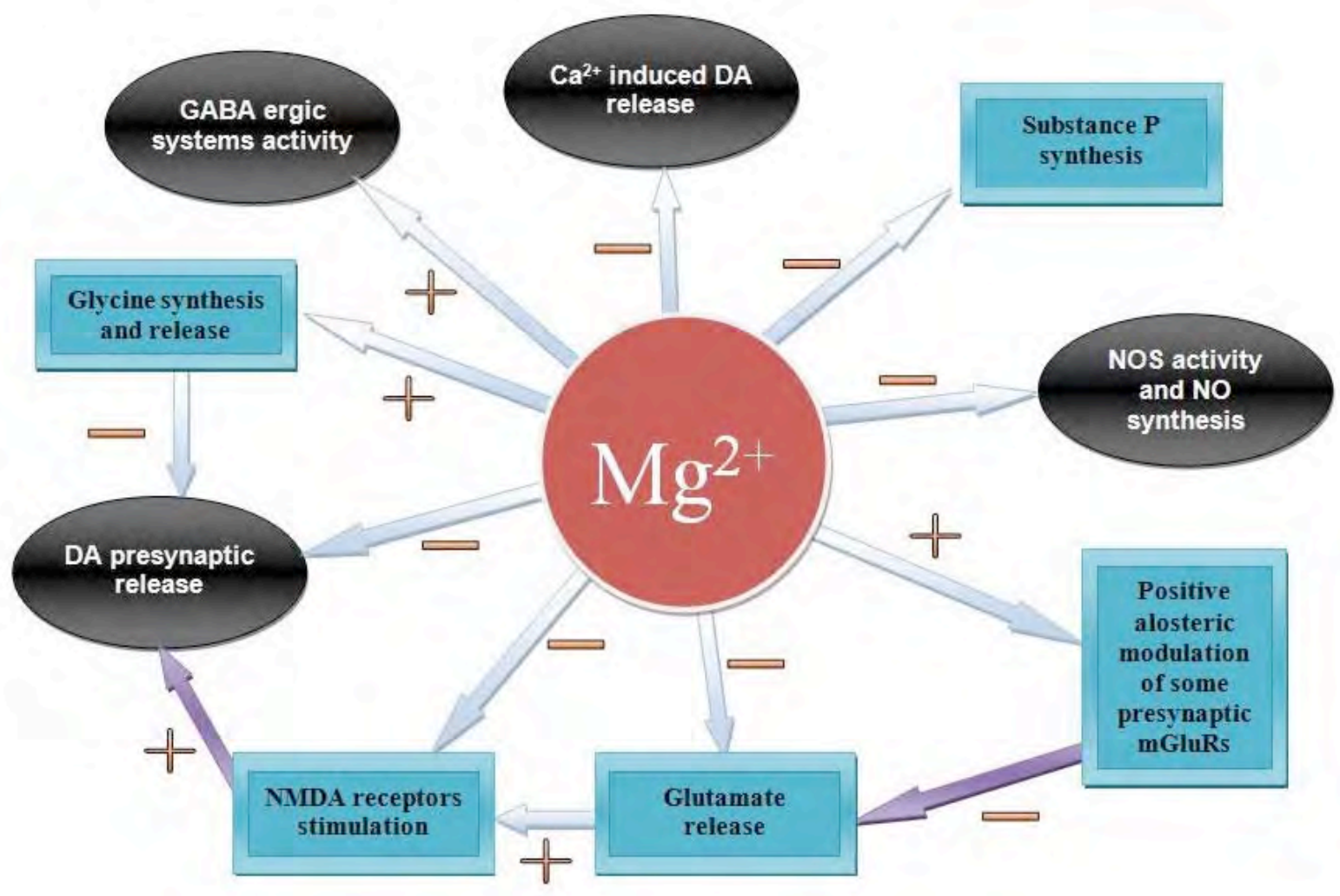

Figure 1. Mechanisms by which magnesium reduces the intensity of opiate addiction. $-=$ inhibition; $+=$ stimulation; DA = dopamine; NOS = nitric oxide synthase. 
Dopamine is considered the most important molecule in development of pharmacodependence. Indeed, substances that result in pharmacodependence strongly increase the level of dopamine in the midbrain. As an example, morphine produces a dose-dependent increase in dopamine-containing neurons in the substantia nigra and ventral tegmentum in rat brain (Trulson and Arasteh, 1985). Magnesium can reduce dopamine release in some brain structures through direct presynaptic action at the level of some dopaminergic synapses, by inhibiting calcium induced brain dopamine release, and by decreasing the stimulatory action of glutamate upon dopamine release. Brain dopamine level in mice is significantly increased following icv administration of $\mathrm{CaCl}_{2}$. Magnesium, an antagonist to calcium, inhibits the dopamine release (Sutoo and Akiyama, 2000).

There are NMDA receptors at the level of some dopaminergic nerve endings whose stimulation also increases dopamine release. The reduction in NMDA receptor stimulation by magnesium can reduce dopamine release induced by the addictive substance (Chéramy et al., 1994). Extracellular $\mathrm{Mg}^{2+}$ blocks NMDA ion channels in a voltage dependent manner and increases the receptor affinity for glycine (Paoletti et al., 1995). Glycine inhibits the glutamate-evoked release of noradrenaline, and possibly other catecholamines (Johnson et al., 1994). In this way, the facilitating effect of magnesium on glycine linkage at its binding sites could reduce the glutamate stimulating effect of presynaptic catecholamine release (dopamine release being an essential step of addiction development). A selective inhibitor of the glycine transporter (Gly T1) significantly increased dopamine release (Bennett and Gronier, 2005). This fact indicates that glycine reduces dopamine release, in this way decreasing the intensity of addictive processes. Notably, magnesium increases both glycine synthesis and release.

Vaupel et al., (1995) showed that nitric oxide synthase (NOS) inhibitors (L-nitroarginine, Lnitroarginine methyl ester) reduce several signs of opiate withdrawal (Kimes et al., 1993). This fact supports the involvement of nitric oxide in the pathogenic signs of withdrawal syndrome.
$\mathrm{Mg}^{2+}$ also inhibits NOS and we consider that in this way, it also reduces the intensity of symptoms from withdrawal syndrome.

Chronic morphine administration decreases the NMDA receptor sensibility to $\mathrm{Mg}$ binding. The glutamatergic systems provide important regulation of dopamine function, while the GABA system can modulate basal levels and stimulate dopamine and glutamate release. The idea of a major role of the glutamatergic system in morphine addiction has also been proposed (Sekiya et al., 2004) on the basis that icv administration of DLBOA (a potent glutamate transporter inhibitor) to morphine-dependent rats significantly facilitates the expression of naloxone-precipitated withdrawal. $\mathrm{Mg}$ action is mainly due to its effects on neuronal activity, but also impacts upon neuroglial activity. Glial cells from the CNS play an important role in regulation of glutamatergic transmission. Some substances that give rise to addiction alter the functions of astrocytes while others affect glial cell functions (Miguel-Hidalgo, 2009). Astrocyte involvement in the control of glutamate uptake and release is important for the development of addiction.

Substance $\mathrm{P}$ and its NK1 receptors also play a role in opiate addiction and in stress (Commons et al., 2010). This neuropeptide modulates NMDA receptor responses to glutamate stimulation (Parker et al., 1998). Magnesium reduces the synthesis and action of substance $P$ and we believe that in this way, it can decrease the intensity of addiction. Stress induces magnesium depletion and increases the vulnerability to development of addiction or of relapse. We consider that low magnesium levels play an important role in this vulnerability.

\section{Psychostimulants}

Psychostimulants are a group of substances with various chemical structures and different mechanisms of actions, partly common, partly different, which results in a strong activation of some CNS processes. All psychostimulants induce a certain degree of addiction, but its intensity is very different. The most utilized psychostimulants are cocaine and its derivatives, amphetamines, caffeine and nicotine. 


\section{Cocaine}

In cocaine abusers, magnesium reduced the craving for this substance (Margolin et al., 1992). Cocaine craving scores were $78 \%$ lower in those taking magnesium than in patients taking placebo. $\mathrm{Mg}$ also reduced cocaine selfadministration in patients and cocaine consumption in rats (Kantak et al., 1998). In cocaine addicts, the plasma level of $\mathrm{Mg}^{2+}$ is higher than in heroin addicts (Tonioni et al., 2009). NMDA receptors are essential for cocaine action in brain. NMDA antagonists and some NMDAcoupled ion channel blockers (like magnesium) may modify the cocaine effects (Kantak et al., 1998).

Our data shows that magnesium never determines dependence. $\mathrm{Mg}$ has a moderate effect of stimulating the brain reward system. $\mathrm{MgC1}_{2}$ results in an increase in time spent in the conditioning compartment in the case of conditioned place preference (CPP)(Nechifor et al., 2010). We consider that, apart from other mechanisms, the small stimulation on the brain reward system is important in realizing magnesium's effect of reducing cocaine consumption.

There are also data showing that genes are important for the behaviour of drug abusers who have a strong genetic determination. The allelic variations associated with the dopamine transporter showed a relationship with the paranoia of cocaine-dependent people (Gellertnet et al., 1999). We consider that genetic deficiencies in magnesium transport at the level of the neuronal membrane may be involved in the speed of addiction development and in the severity of withdrawal syndrome.

\section{Caffeine}

Caffeine is one of the most consumed psychoactive substances, although the existence of caffeine dependence is controversial (Huges et al., 1992; Daly and Fredholm, 1998). There are data suggesting that caffeine results in a clinical dependence syndrome very similar to that produced by other psychoactive substances, as well as from the point of view of the caffeineinduced craving (Ogawa and Ueki, 2007). Caffeine has only weak reinforcing proprieties, but withdrawal syndrome is a reality and is similar to that produced by other drugs of abuse. Rats chronically exposed to $1 \mathrm{~g} / \mathrm{L}$ caffeine a day for 20 days developed caffeine withdrawal syndrome (Dingle et al., 2008).

Caffeine blocks both $A_{1}$ and $A_{2}$ adenosine receptors. Chronic administration of xanthines (caffeine, theophylline) causes a significant increase in the number of adenosine, nicotine and serotoninergic receptors in the brain. It also increases the number of L-type calcium channels in the neuronal membrane. The blockade of both $A_{1}$ and $A_{2}$ receptors is necessary for the full spectrum of caffeine's pharmacologic effects.

The $\mathrm{Ca}^{2+}$ ions from the endoplasmic reticulum are very important for normal cell function. Any change in intracellular calcium concentration can have an impact on neuronal activity. Caffeine enhances the intracellular $\mathrm{Ca}^{2+}$ peak. This effect is higher in young animals and is altered with aging (Alshuaib et al., 2006). Caffeine-releasable calcium ions may stimulate glutamate synthesis and release, which increases NMDA receptor activity. Wang (2007) showed that caffeinemediated glutamate release is produced by activation of protein kinase $C$ pathways and involves an interaction between caffeine and presynaptic adenosine $A_{1}$ receptors. The importance of caffeine action on adenosine receptors also comes out of the fact that these receptors act as regulators of neurotransmitter release in the brain (Sebastiao and Ribeiro, 2009). Adenosine stimulates all types of adenosine receptors. The omnipresence of $A_{1}, A_{2}$ and $A_{3}$ receptors in CNS neurons and neuroglia reflect the major role of adenosine in modulating synaptic activity in many brain regions, including those involved in dependence.

Blockade of adenosine $A_{1 A}$ and $A_{2 A}$ receptors is involved in the development of caffeine addiction. Burgalassi et al., (2009) showed that a substantial number of heavy caffeine drinkers satisfy the research criteria for dependence. Adenosine $A_{1}$ and $A_{2}$ receptors have a greater involvement in addiction processes, as well as development of withdrawal syndrome, rather than in development of caffeine dependence.

Adenosine and adenosine receptors are also involved in morphine dependence and opioid withdrawal. Adenosine is able to reduce the dosedependent, naloxone-precipitated withdrawal in 
guinea pigs exposed to morphine. Caffeine significantly increases naloxone-precipitated withdrawal (Capasso, 2000). Research on guinea pig isolated ileum exposed to morphine showed that the contraction induced by naloxone was increased by caffeine ( $P_{1}$ antagonist) in a concentration dependent manner (Capasso and Loizzo, 2001). The caffeine effect is amplified by low $\mathrm{Mg}^{2+}$ concentration.

$A_{1}$ receptor antagonists such as caffeine increase the presynaptic release of dopamine and glutamate. This is thought to be the main mechanism for caffeine dependence. $A_{1}$ receptors are located in presynaptic areas of glutamatergic neurons and $\mathrm{Mg}$ can reduce caffeine dependence by blocking NMDA coupled $\mathrm{Ca}^{2+}$ channel activity, reducing the capacity of $\mathrm{A}_{1}$ receptor antagonists (such as caffeine) to stimulate glutamate and dopamine release, or by reducing the glutamate stimulation of dopamine presynaptic release. It is not very clear if caffeine enhances magnesium elimination from the human body.

\section{Amphetamine}

Amphetamine is a very potent sympathomimetic agent in stimulating the CNS. The main mechanism of action is by release of biogenic amines from their storage sites in presynaptic parts of central synapses. The dependence and stereotypical behaviour associated with amphetamine is induced by stimulation of dopamine release. Disturbance of perception and psychotic behaviour may be due to release of serotonin and dopamine in the mesolimbic system (Hoffman, 2001). There is also a small stimulating effect of amphetamine on some serotonin receptors. Increased intraneuronal $\mathrm{Mg}^{2+}$ concentration is thought to reduce the intensity of psychostimulant addiction. Evidence in favour is the fact that $\mathrm{Li}^{+}$(which increases intracellular magnesium concentration) has an antagonistic effect with amphetamine at the level of the nucleus accumbens. $\mathrm{Li}^{+}$also decreased dopaminergic transmission stimulated by amphetamine (Gray et al., 1997). After administration of $1 \mathrm{mg} / \mathrm{kg}$ amphetamine, dopamine levels increase by $427 \%$ versus the basal level. This dopamine release is calcium potentiated. The replacement of calcium by magnesium reduces the response to amphetamine administration (Warburton et al.,
1996). It was also remarked that structural brain abnormalities are associated with amphetamine abuse. These differences included lower cortical gray matter quantity and higher striatal volume than in normal subjects (Berman et al., 2008). We do not know how magnesium influences these modifications.

\section{Nicotine}

Existing data shows that nicotine addiction develops in chronic smokers with over 10-20 cigarettes/day. Chronic smoking decreases the level of serum magnesium (Niemela et al., 1997; Nechifor et al., 2004a), while magnesium administration decreases the number of smoked cigarettes as well as nicotine addiction. Specifically, 2 ampoules/day Magne-B6 administration for four weeks significantly decreased the number of cigarettes smoked by heavy smokers (smokers with over 20 cigarettes/day) (Nechifor et al., 2004a). The Fagerstrom score was significantly reduced in the smoker group that received magnesium, from $7.93 \pm 0.17$ before magnesium to $6.78 \pm 0.18$ after magnesium $(p<0.05) . \mathrm{Mg}^{2+}$ can potentially reduce nicotine addiction by:

- acting as a partial antagonist of calcium entry into neurons, thereby decreasing glutamate release and glutamatergic transmission, which is stimulated by nicotine;

- decreasing nicotine-induced pre-synaptic release of dopamine and other catecholamines;

- increasing magnesium concentration in the neuron producing a decrease in sodium concentration. This decreases the stimulant effect of nicotine on nicotine receptors.

- decreasing the nicotine addictive effect by diminishing the nicotine effect on GABA synthesis. Nicotine diminishes GABA synthesis and release in some brain areas by stimulation of nicotine presynaptic receptors;

- enhancing some of the GABA effects and diminishing some effects of the excitatory amino acids in drug dependence. GABA antagonizes some of the glutamate-induced stimulatory effects of NMDA receptors.

Like nicotine, synaptically released acetylcholine stimulates nicotinic receptors and in this way 
enhances glutamatergic activity (Guo et al., 2005). The release of DA from the nucleus accumbens and substantia nigra was stimulated by glutamate, and nicotine enhanced this release (Marien et al., 1983). Such DA secretion induced by glutamate and nicotine is $\mathrm{Ca}^{2+}$ dependent and was inhibited by $\mathrm{Mg}^{2+}$. We think that this is an essential mechanism for magnesium action to reduce the nicotine dependence.

\section{Ethanol dependence}

Alcohol induced dependence is one of the most widespread dependencies. Abuse and addiction depend, at least partially, on the activation of mesolimbic dopaminergic systems. The activation of these systems is achieved directly by ethanol, but also by acetaldehyde, which results from ethanol metabolism. Acetaldehyde increases dopaminergic neuronal activity in the nucleus accumbens, ventral tegmental area and in other parts of the CNS (Foddai et al., 2004; Diana et al., 2008). Alcohol-dehydrogenase drastically inhibits the effects of ethanol on dopaminergic neurons of the ventral tegmental area (VTA). We consider that magnesium could reduce the stimulating effect of ethanol on dopaminergic systems by directly reducing the presynaptic release of dopamine, but also by decreasing acetaldehyde production.

There is important evidence implicating the endogenous opioids in the processes of reward and reinforcement (Gianoulakis, 2009). Endogenous opioids, like morphine, induce an increase of dopamine concentration in the nucleus accumbens, which is considered the most important structure for drug addiction. This is considered a common effect for many drugs involved in abuse. Ethanol increases betaendorphin release. The stimulation of opioid receptors (especially of $\mu$ receptors) seems to be important for ethanol addiction, with low morphine doses increasing ethanol consumption (Hertz, 1997). Magnesium reduces receptor binding of morphine and other $\mu$ receptor agonists (Mendez et al., 2001; Rodriguez et al., 1992). This way, magnesium could reduce the stimulation of dopamine synthesis produced by large quantities of opioid peptides, which themselves are induced by ethanol in the nucleus accumbens and VTA. We therefore consider that Mg could decrease ethanol addiction and hypomagnesemia could increase alcohol consumption. Mendez et al., (2001) showed that the reinforcing properties of ethanol may be partially mediated by ethanol regulation of $\mu$ receptors in dopaminergic neurons from mesolimbic systems. A modulation of $\mu$ receptors from the frontal and prefrontal cortex is also possible.

There are also findings that suggest that mGluR5 receptors modulate ethanol self-administration in rats (Schroeder et al., 2005). Dopamine is the most important neurotransmitter involved in the reward mechanism and it influences the development of alcohol dependence and relapse. Two polymorphisms of the D2 dopamine receptors seem to suffer 2.5 times more risk to develop ethanol dependence (Prasad et al., 2010). Embry and Lippman (1987) considered that magnesium deficiency plays an important role in the alcohol-withdrawal system. There are data that show that magnesium administration decreases the intensity of symptoms from the withdrawal syndrome. The alcohol induces hypomagnesemia and increases urinary magnesium loss. Low concentrations of ethanol deplete free intracellular magnesium (Babu et al., 1999). Consistent with Shane and Flink (1992), we believe that magnesium deficiency is not only involved in the intensity of alcohol withdrawal, but also in the development of alcohol dependence.

The NMDA receptors from the nucleus accumbens and the mesolimbic structures are involved in drug reward and reinforcement. Ethanol sensitivity of NMDA receptors from the nucleus accumbens is important for ethanolinduced neuroadaptation of the reward system. Chronic alcohol administration results in an increased glutamate binding to the NMDA receptors (Hu and Ticku, 1995). This highlights the importance of glutamate action in development of alcohol addiction. The activation of NMDA receptors in rats increases during the alcohol withdrawal syndrome (Sanna et al., 1993). Electrolyte abnormalities are common in chronic alcoholics and during alcohol withdrawal syndrome (Stasinkyniene, 2002). Alcohol consumption is one of the major causes for hypomagnesemia, which is one of the most important cation imbalances in alcoholic patients and during withdrawal (Carl and Halzbach, 1994). 
In alcoholic patients, urinary magnesium loss increases 2-3 fold (Romain, 2008) and brain intracellular $\mathrm{Mg}^{2+}$ level are reduced (Li et al., 2001; Pasternak, 1999). Low ethanol concentrations deplete type 2 astrocytes of intracellular free magnesium (Babu et al., 1999). The $\mathrm{Mg}^{2+}$ dependent decay off-rate of NMDA miniature synaptic currents (mEPSes) was also significantly reduced by ethanol (Zhang et al., 2005), suggesting that ethanol not only contributes to increase the release of magnesium from the body, but also to increase magnesium's effect at the NMDA receptor level.

$\mathrm{Mg}^{2+}$ ions modulate neuronal excitability and are involved in alcohol-related behaviours. UusiOukari et al., (2001) found that the putative $\mathrm{Mg}^{2+}$ binding sites differ between alcohol insensitive (AT) and alcohol sensitive (ANT) rats lines. In the presence of GABA, the effect of low $\mathrm{Mg}$ concentration was higher in cerebral cortex and in the caudate-putamen of AT rats than in the ANT animals. Alcohol sensitive rats have alterations of the alpha 6 subunit - containing $G_{A B A_{A}}$ receptors. It is possible that these receptors might be involved in the sensitivity of different lines of rats to alcohol and also in the different sensibility of animals to sedative drugs (Uusi-Oukari M et al., 2001). Magnesium administration only during the withdrawal syndrome of ethanol addicts reduced the clinical symptoms.

\section{Hallucinogens (psychedelic substances)}

Hallucinogens are substances that induce hallucinations, disorders of thinking and delusions. The most potent hallucinogen is LSD (lysergic acid diethylamide) that produces a significant hallucinogen effect at a dose of as little as 25-50 ug. Other psychedelic drugs are derivatives with indolaminic structure (psylocibine, DMT and others) and substances with phenethylaminic structure (mescaline, MDMA, and DOM). The precise mechanism of action of these substances is not yet known, but a positive correlation between the relative affinity of hallucinogens for serotonin $5-\mathrm{HT}_{2}$ receptors and their potency to induce hallucination has been observed (Titeler et al., 1988).

The hallucinogens (LSD, phencyclidine, psilocybin, mescaline, DMT and others) give a strong psychic dependence, but the concept of addiction is controversial. The presence of withdrawal from hallucinogens has not been clearly established because these drugs don't seem to induce physical dependence. The most utilized hallucinogen is LSD.

There are data that favour the idea that all phencyclidine receptors in the brain are associated with NMDA receptors. Notably, there is an interaction of L-glutamate and magnesium with the phencyclidine recognition site in the brain (Loo et al., 1987). The agonists of NMDA receptors induce a high affinity state in the phencyclidine receptors. This is another possibility by which $\mathrm{Mg}^{2+}$ can influence phencyclidine action. $\mathrm{Mg}^{2+}$ inhibits MK-801 (a ligand for the NMDA ion channel phencyclidine site) binding in the cortex (Chahal et al., 1998). It is possible for magnesium to also decrease phencyclidine action this way. However, Rothman et al., (1989) demonstrated the existence of a high-affinity, phencyclidine binding site associated with the dopamine reuptake carrier. The hallucinogen could therefore influence synaptic dopamine concentration. It is still unknown how $\mathrm{Mg}^{2+}$ influences dopamine reuptake.

Regarding other hallucinogens such as psilocin (from Psilocybe mushrooms) and phenylethylamine, subchronic experimental intoxication in rats disturbs magnesium concentrations (Majdanik et al., 2007). Low magnesium concentration also resulted from chronic mescaline and LSD administration. Hypomagnesemia is associated with an increased intracellular entrance of $\mathrm{Ca}^{2+}$ in these conditions. In cerebral vasospasm, which appears in chronic hallucinogen intoxication, both magnesium and the calcium antagonist verapamil block this effect (Altura and Altura, 1983). The NMDA receptor is coupled with an ion channel and has regulatory sites for phencyclidine, glycine, and also for magnesium and zinc. In this way, it might be possible for an interaction between magnesium and phencyclidine to exist with respect to the activity of NMDA receptors.

Lerma et al., (1991) suggest that interactions between $\mathrm{Mg}^{2+}$ and phencyclidine at the level of the NMDA channels are competitive. Indeed, 0.5 $\mathrm{mM} \mathrm{Mg}$ caused a four-fold decrease in phencyclidine potency. In vitro incubation of 
phencyclidine stimulated brain slides with 1.2 $\mathrm{mM} \mathrm{MgC1} 1_{2}$ shows that $\mathrm{Na}$ efflux produced by the NMDA receptor stimulation is decreased.

\section{Benzodiazepines}

Magnesium aspartate decreases benzodiazepine addiction (lorazepam, alprazolam, or bromazepam) (Hantouche et al., 1998). The decrease in addiction intensity was manifested as prolonged delay in benzodiazepine reintake, reduction of withdrawal intensity, and reduction of anxiety during benzodiazepine discontinuation. In benzodiazepine withdrawal syndrome, strong anxiety is present. This phenomenon is associated with a potentiation of AMPA receptor activity and AMPA receptor currents in hippocampal pyramidal neurons. Calcium/ calmodulin-dependent protein kinase (PK) II has a contribution by enhancing the glutamatergic synaptic activity during benzodiazepine withdrawal (Shen et al., 2010). In some patients that received $\mathrm{Mg} \mathrm{L}$-aspartate, cessation of benzodiazepines was obtained without onset of withdrawal syndrome. It is possible that magnesium ions acting at the level of AMPA receptors influence the calcium/calmodulin dependent PK II to reduce the anxiety and other clinical symptoms present in benzodiazepine withdrawal syndrome.

\section{Cannabinoids}

Delta 9-tetrahydrocannabinol is the principal psychoactive compound in marijuana, although it is widely used in a number of forms. It induces an important psychic dependence, but only a modest physical dependence. Bac and Germain Fattal (2006) showed that magnesium deficiency increased the neurotoxicity of THC at low doses in rats. Hyperagressiveness and THC induced muricidal behaviour in rats was also increased by magnesium deficiency. Otherwise, there is very little data regarding the influence of magnesium and other bivalent cations in cannabinoid addiction.

\section{Conclusion}

Regarding the involvement of magnesium in addiction, we consider that is important not only at the neuronal level, but also at neuroglial level. According to data that shows that the glial cells are involved in addictive behaviour, mainly through the regulation of glutamate transport and activity, we believe that magnesium acts at the neuroglial level by reducing the action of glutamate. There are NMDA receptors at the glial level (in oligodendrocytes, microglia and astrocytes) (Verkhratsky and Kirchhoff, 2007), and at both these receptors and the neuronal NMDA receptor level, there is a calcium channel that can be influenced by magnesium.

We consider that the ability of magnesium to reduce addiction to different substances and reduce the intensity of addiction to different substances is essentially related to the association of two different factors - its ability to produce a moderate stimulation of the brain reward system (Nechifor et al., 2010), and its capacity to reduce the activity of glutamatergic substances, importantly involved in compulsive use disorders.

Compulsive drug-taking behaviour is an important characteristic element in addiction. The existence of a strong reinforcement activity is a determining factor for drug addiction (Koob and Bloom, 1988). Reducing the intensity of dependence on different compounds involves reducing their reinforcing proprieties. Responsereinforcement learning is dependent on NMDA receptor activation (Kelley et al., 1997). We consider that magnesium reduces reinforcing proprieties of different compounds by action on these NMDA receptors. 


\section{References}

Alshuaib WB, Cherian SP, Hasan MY, Fahim MA (2006) Modulation of neuronal [Ca2+]i by caffeine is altered with aging. Int J Dev Neurosci 24:389-94.

Altura BM, Altura BT (1983) Pharmacologic inhibition of cerebral vasospasm in ischemia, hallucinogen ingestion, and hypomagnesemia: barbiturates, calcium antagonists, and magnesium. Am J Emerg Med 1:18090.

Babu AN, Cheng TP, Zhang A, Altura BT, Altura BM (1999) Low concentrations of ethanol deplete type-2 astrocyte of intracellular free magnesium. Brain Res Bull 50:59-62.

Bac P, German-Fattal M (2006), Potentiation of Delta9tetrahydrocannabinol (THC) effects by magnesium deficiency in the rat. Ann Pharm Fr 64:207-13.

Bennet S., Gronier B (2005), Modulation of striatal dopamine release in vitro by agonists of the glycine $B$ site of NMDA receptors. Interactions with antypsychotics. Eur J Pharmacol 527:52-9.

Berman S, O'Neill J, Fears S, Bartzokis G, London ED (2008) Abuse of amphetamines and structural abnormalities in the brain. Ann NY Acad Sci 1141:195220.

Burgalassi A, Ramacciotti CE, Bianchi M, Coli E, Polese L, Bondi E, Massimetti G, Dell'osso L (2009) Caffeine consumption among eating disorder patients: epidemiology, motivations, and potential of abuse. Eat Weight Disord 14:212-18.

Carl G, Holzbach E (1994) Reversible hypokalemia and hypomagnesemia during alcohol withdrawal syndrome. Nervenarzt 6:206-11.

Capasso A (2000) Adenosine receptors are involved in the control of acute naloxone-precipitated withdrawal: in vitro evidence. Life Sci 66:873-83.

Capasso A, Loizzo A (2001) Purinoreceptors are involved in the control of acute morphine withdrawal. Life Sci 69:2179-88.

Chahal H, D'Souza SW, Barson AJ, Slater P (1998) Modulation by magnesium of $\mathrm{N}$-methyl-D-aspartate receptors in developing human brain. Arch Dis Child Fetal Neonatal Ed 78:F116-20.

Cheramy A, L'Hirondel M, Godehen G, Artaud F, Glowinsky J (1998) Direct and indirect presynaptic control of dopamine release by excitatory amino acids. Amino Acids 14:63-9.
Commons KG (2010) Neuronal pathways linking substance $P$ to drug addiction and stress. Brain Res 1314:175-82.

Daini S, Tonioni F, Barra A, Lai C, Lacerenza R, Sgambato A, Bria P, Cittadini A (2006) Serum magnesium profile in heroin addicts -according to psychiatric comorbidity. Magnes Res 19:162-6.

Daly JW, Fredholm BB (1998) Caffeine- an atypical drug of dependence. Drug Alcohol Depend 51:199206.

Diana M, Peana AT, Sirca D, Lintas A, Melis M, Enrico P (2008) Crucial role of acetaldehyde in alcohol activation of the mesolimbic dopamine system. Ann N Y Acad Sci 1139:307-17.

Dingle RN, Dreumont-Boudreau SE, Lolordo VM (2008) Caffeine dependence in rats: effects of exposure duration and concentration. Physiol Behav 95:252-57.

Embry CK, Lippmann S (1987) Use of magnesium sulfate in alcohol withdrawal. Am Fam Physician 35:167-70.

Foddai M, Dosia G, Spiga S, Diana M (2004) Acetaldehyde increases dopaminergic neuronal activity in the VTA. Neuropsychopharmacology 29:5306.

Gass JT, Olive MF (2008) Glutamatergic substrates of drug addiction and alcoholism. Biochem Pharmacol 75:218-65.

Gerstein M, Huleihel M, Mane R, Stilman M, Kashtuzki I, Hallak M, Golan H (2005) Remodeling of hippocampal GABAergic system in adult offspring after maternal hypoxia and magnesium sulphate load: immunohistochemical study. Exp Neurol 196:18-29.

Gelernter J, Cubells JF, Kidd JR, Pakstis AJ, Kidd KK (1999) Population studies of polymorphisms of the serotonin transporter protein gene. Am J Med Genet 88:61-6.

Gianoulakis C (2009) Endogenous opioids and addiction to alcohol and other drugs of abuse. Curr Top Med Chem 9:999-1001.

Gray JA, Moran PM, Grigoryan G, Peters SL, Young AM, Joseph MH (1997) Latent inhibition: the nucleus accumbens connection revisited. Behav Brain Res 88:27-34. 
Guo JZ, Liu Y, Sorenson EM, Chiappinelli VA (2005) Synaptically released and exogenous ACh activates different nicotinic receptors to enhance evoked glutamatergic transmission in the lateral geniculate nucleus. J Neurophysiol 94:2549-60.

Hantouche EG, Guelfi JD, Comet D (1998) Alpha-betha L-aspartate magnesium in treatment of chronic benzodiazepine abuse: controlled and double blind study versus placebo. Encephale 24:469-79.

Hernandes MS, De Magaihaes L, Troncone LRP (2005) Glycine stimulates the release of labelled acethylcholine but not dopamine nor glutamate from superfused rat striatal tissue. Brain Res Bull 26:418-30.

Herz A (1997) Endogenous opioid systems and alcohol addiction. Psychopharmacology (Berl) 129:99-111.

Hoffman BB (2001) Cathecolamines, sympathomimetic drugs, and adrenergic receptor antagonists, In: Goodman \& Gilman's The Pharmacological Basis of Therapeutics (Hardman JG, Limbird LE, eds.) Tenth International Edition, New York, Mc Graw Hill, 215-68.

Hu X, Ticku MK (1995) Chronic ethanol treatment upregulates the NMDA receptor function and binding in mammalian cortical neurons. Mol Brain Research 30:347-56

Hughes JR, Oliveto AH, Helzer JE, Higgins ST, Bickel WK (1992) Should caffeine abuse, dependence, or withdrawal be added to DSM-IV and ICD-10? Am J Psychiatry 149:33-40.

Kantak KM, Edwards MA, O'Connor TP (1998) Modulation of the discriminative stimulus and ratealtering effects of cocaine by competitive and noncompetitive N-methyl-D-aspartate antagonists. Pharmacol Biochem Behav 59:159-69.

Karakiewicz B, Kozielec T, Brodowski ., Chlubek D, Noceri I, Starczewski A, Brodowska A, Laszczylnska M (2007) Serum magnesium concentration in drug addicted patients. Magnes Res 20:55-7.

Kelley AE, Smith-Roe SL, Holahan MR (1997) Responsereinforcement learning is dependent on $\mathrm{N}$-methyl-Daspartate receptor activation in the nucleus accumbens core. Proc Natl Acad Sci USA 94:12174-9.

Kimes AS, Vaupel DB, London ED (1993) Atenuation of some signs of opioid withdrawal by inhibitors of nitric oxide synthase. Psychopharmacology 112:521-24.

Koob GF, Bloom FE (1988) Cellular and molecular mechanisms of drug dependence. Science 242:715-23.
Koob GF, Le Moal M (2001) Drug addiction, dysregulation of reward, and allostasis. Neuropsychopharmacology 24:97-129.

Kosten TR (1998) Addiction as a brain disease. Am J Psychiatry 155:711-13.

Lerman J, Zukin RS, Bennett MV (1991) Interaction of $\mathrm{Mg} 2+$ and phencyclidine in use-dependent block of NMDA channels. Neurosci Lett 123:187-91.

Li W, Zheng T, Babu A., Altura BT, Gupta RK, Altura BM (2001) Importance of magnesium ions in development of tolerance to ethanol: studies on cultured cerebral vascular smooth muscle cells, type 2 astrocytes and intact rat brain. Brain Res Bull 56:153-8.

Li-Smerin Y, Johnson JW (1996) Effects of intracellular $\mathrm{Mg} 2+$ on channel gating and steady-state responses of the NMDA receptor in cultured rat neurons. $J$ Physiol 491:137-50.

Loo PS, Braunwalder AF, Lehmann J, Williams M, Sills MA (1987) Interaction of L-glutamate and magnesium with phencyclidine recognition sites in rat brain: evidence for multiple affinity states of the phencyclidine/N-methyl-D-aspartate receptor complex. Mol Pharmacol 32:820-30.

Majdanik S, Borowiak K, Brzezńska M, MachoyMokrzyńska A (2007) Concentration of selected microelements in blood serum of rats exposed to the action of psilocin and phenylethylamine. Ann Acad Med Stetin 53:153-8.

Marien M, Brien J, Jhamandas K (1983) Regional release of $3 \mathrm{H}$ dopamine from rat brain in vitro effects of opioids on release induced by potassium, nicotine and I-glutamic acid. Can J Physiol Pharmacol 61:43-60.

Margolin A, Kantak K, Copenhaver M, Avant SSK (2003) A preliminary controlled investigation of magnesium Laspartate hydrochloride for illicit cocaine and opiate use in methadone-maintained patients. J Addict Dis 22:49-61.

McLellan AT, Lewis DC, O'Brien CP, Kleber HD (2000) Drug dependence, a chronic medical illness. JAMA 284:1689-95.

Méndez M, Leriche M, Calva JC (2001) Acute ethanol administration differentially modulates mu opioid receptors in the rat meso-accumbens and mesocortical pathways. Brain Res Mol Brain Res 94:148-56. 
Miguel-Hidalgo JJ (2009) The role of glial cells in drug abuse. Curr Drug Abuse Rev 2:72-82.

Moykkyen T, Uusi-Oukari M, Heikkila J, Lovinger DM, Luddens H, Korpi ER (2001) Magnesium potentiation of the function of native and recombinant GABA A receptors. Neuroreport 12:2175-9.

Nechifor M, Chelarescu D, Mândreci I, Cartas N (2004) Magnesium influence on nicotine pharmacodependence and smoking. Magnes Res 17:176-81.

Nechifor M, Chelarescu D, Miftode M (2004) Magnesium influence on morphine-induced pharmacodependence in rats. Magnes Res 17:7-13.

Nechifor M, Chelarescu D, Ciubotariu D (2010) The influence of magnesium-induced stimulation of the reward system. Magnesium Res 23:41-7.

Niemela JE, Cecco SA, Rehak NN, Elin RJ (1997) The effect of smoking on the serum ionized magnesium concentration is method-dependent. Arch Pathol Lab Med 121:1087-92.

Ogawa N, Ueki H (2007) Clinical importance of caffeine dependence and abuse. Psychiatry Clin Neurosci 61:263-8.

Paoletti P, Neyton J, Ascher P (1995) Glycineindependent and subunit-specific potentiation of NMDA response by extra cellular $\mathrm{Mg}^{2+}$. Neuron 15:1109-20.

Parker D, Zhang W, Grillner S (1998) Substance P modulates NMDA responses and causes long-term protein synthesis -dependent modulation of the lamprey locomotor network. J Neurosci 18:4800-13.

Pasternak K (1999) Tissue concentrations of magnesium in rats receiving various dosages of ethano. Magnes Res 12:167-70.

Prasad P, Ambekar A, Vaswani M (2010) Dopamine D2 receptor polymorphisms and susceptibility to alcohol dependence in Indian males: a preliminary study. BMC Med Genet 11:24.

Rodriguez FD, Bardaji E, Trayno Jr R (1992) Differential effects of $\mathrm{Mg}^{2+}$ and other divalent cation on the binding of tritiated opioid ligands. J Neurochem 591:467-72.

Romani AM (2008) Magnesium homeostasis and alcohol consumption. Magnes Res 21:197-204.

Rothman RB, Reid AA, Monn JA, Jacobson AE, Rice KC (1989) The psychotomimetic drug phencyclidine labels two high affinity binding sites in guinea pig brain: evidence for $\mathrm{N}$-methyl-D-aspartate-coupled and dopamine reuptake carrier-associated phencyclidine binding sites. Mol Pharmacol 36:887-96.

Ruiz Martinez M, Gil Extremera B, Maldonaldo MD, Cantero-Hinojosa J, Moreno-Abadia V (1990) Klin Wochenschr 68:507-11.

Sanna E, Serra M, Cossu A, Colombo G, Follesa P, Cuccheddu T, Concas A, Biegio G (1993) Chronic ethanol intoxication induces differential effects in GABA A and NMDA receptor function in the rat brain. Alcoholism: Clin Exp Research 17:115-23.

Schroeder JP, Overstreet DH, Hodge CW (2005) The mGluR5 antagonist MPEP decreases operant ethanol self-administration during maintenance and after repeated alcohol-deprivations in alcohol-preferring $(P)$ rats. Psychopharmacology 179:262-7.

Sebastião AM, Ribeiro JA (2009) Tuning and finetuning of synapses with adenosine. Curr Neuropharmacol 7:180-94.

Sekiya Y, Nakagawa T, Ozawa T, Minami M, Satoh M (2004) Facilitation of morphine withdrawal symptoms and morphine-induced conditioned place preference by a glutamate transporter inhibitor DL-threo-betabenzyloxyaspartate in rats. Eur J Pharmacol 485:20110.

Shane SR, Flink EB (1991-1992) Magnesium deficiency in alcohol addiction and withdrawal. Magnes Trace Elem 10:263-8.

Shen G, Van Sickle BJ, Tietz El (2010) Calcium /calmodulin-dependent protein kinase II mediates hippocampal glutamatergic plasticity during benzodiazepine withdrawal. Neuropsychopharmacol 35:1890-9.

Stasiukyniene V (2002) Blood plasma potassium, sodium and magnesium level in chronic alcoholism during alcohol withdrawal. Medicina (Kannas) 38:8925.

Rosenzweig-Haugbol S, Ebert B, Ulrichsen J (2005) Upregulation of glutamate receptor subtypes during alcohol withdrawal in rats. Alcohol Alcoholism 40:8995.

Sutoo D, Akiyama K (2000) Effect of magnesium on calcium-dependent brain function that prolongs ethanol-induced sleeping time in mice. Neurosci Lett 204:5-8. 
Titeler M, Lyon RA, Glennon RA (1988) Radioligand binding evidence implicates the brain $5-\mathrm{HT} 2$ receptors as a site of action for LSD and phenylisopropyl-amine hallucinogens. Psychopharmacology 94:213-6.

Tonioni F, Martinotti G, Barra A, Martinelli D, Autullo G, Rinaldi C, Tedeschi C, Janiri L, Bria P (2009) Cocaine use disorders and serum magnesium profile, Neuropsychobiology 53:159-64.

Trulson ME, Arasteh K (1985) Morphine increases the activity of midbrain dopamine neurons in vitro. Eur J Pharmacol 114:105-9.

Uusi-Oukari M, Makela R, Soini S, Korpi ER (2001) Cation modulation of GABA A receptors in brain section of AT and ANT rats. Alcohol 25:69-75.

Vaupel DB, Kimes AS, London ED (1995) Nitric oxide synthase inhibitors. Preclinical studies of potential use for the treatment of opioid withdrawal.

Neuropsychopharmacology 13:315-322

Verkhratsky A, Kirchhoff F (2007) NMDA Receptors in glia. Neuroscientist 13:28-37.
Wang SJ (2007) Caffeine facilitation of glutamate release from rat cerebral cortex nerve terminals (synaptosomes) through activation protein kinase $\mathrm{C}$ pathway: an interaction with presynaptic adenosine A1 receptors. Synapse 61:401-11.

Warburton EC, Mitchell SN, Joseph MH (1996) Calcium dependence of sensitised dopamine release in rat nucleus accumbens following amphetamine challenge: implications for the disruption of latent inhibition. Behav Pharmacol 7:119-29.

West O, Roderique-Davies G (2008) Development and initial validation of a caffeine craving questionnaire. $J$ Psychopharmacol 22:80-91.

Xing H, Azimi-Zonooz A, Shuttleworth CW, Connor JA (2004) Caffeine releasable stores of $\mathrm{Ca}^{2+}$ show depletion prior to the final steps in delayed CA1 neuronal death. J Neurophysiol 92:2960-7.

Zhang TA, Hendricson AW, Morrisett RA (2005) Dual synaptic sites of D1-dopaminergic regulation of ethanol sensitivity of NMDA receptors in nucleus accumbens. Synapse 58:30-44. 